\title{
Photoinduced Fragmentation Borylation of Cyclic Alcohols and Hemiacetals
}

Chao Shu, Rudrakshula Madhavachary, Adam Noble,* and Varinder K. Aggarwal*

School of Chemistry, University of Bristol, Cantock’s Close, Bristol BS8 1TS, U.K.

a.noble@bristol.ac.uk, v.aggarwal@bristol.ac.uk

\section{Table of Contents}

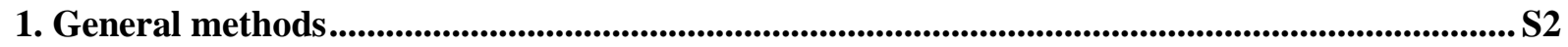

1.1. Solvents, Reagents, Glassware and Reaction Setup ............................................................... 2

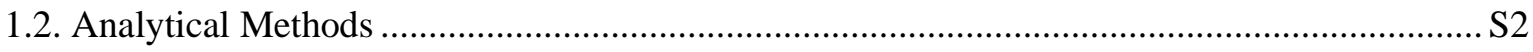

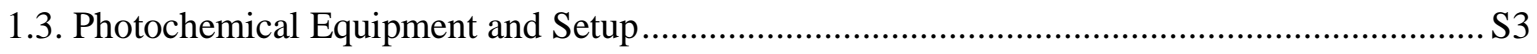

2. Additional Optimization Studies...................................................................................................................... S4

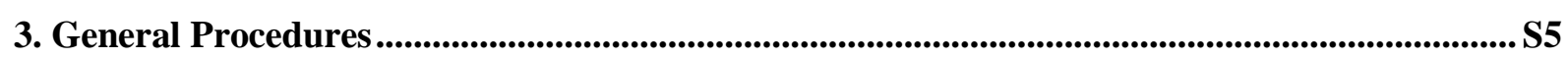

3.1. General Procedure 1 (GP1): Synthesis of $N$-Alkoxyphthalimides from Ketones ..................... S5

3.2. General Procedure 2 (GP2): Synthesis of $N$-Alkoxyphthalimides from Cyclic Enol Ethers .... S6

3.3. General Procedure 3 (GP3): Fragmentation Borylation Reaction.......................................... S6

4. Substrate Synthesis and Characterization ................................................................................................... S7

5. Unsuccessful Substrates ............................................................................................................................... S45

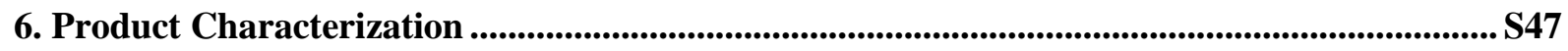

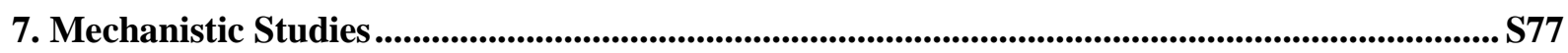

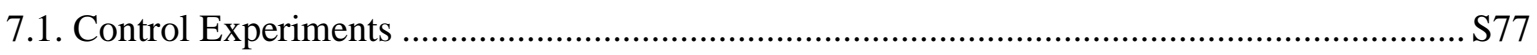

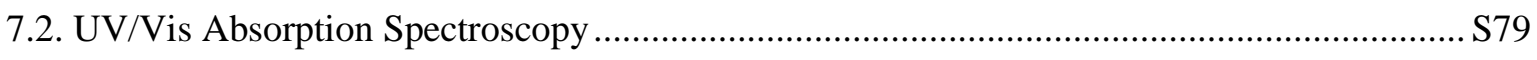

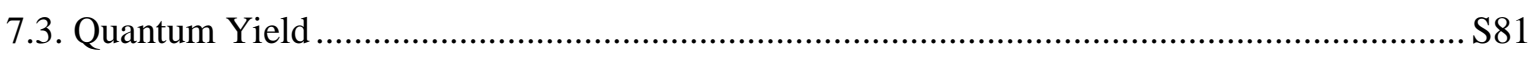

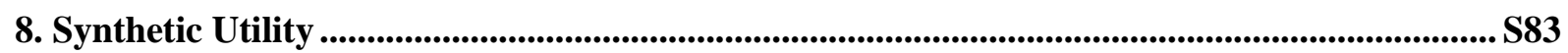

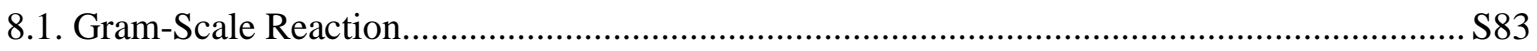

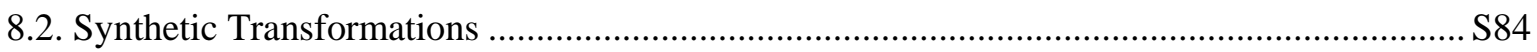

8.3. One-Pot Activation/Fragmentation Borylation of Cyclic Enol Ethers ...................................S87

9. References ....................................................................................................................................................................... S88

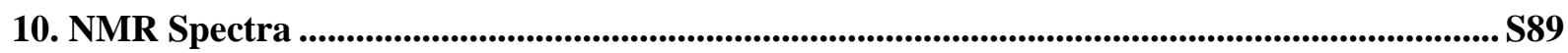




\section{General methods}

\subsection{Solvents, Reagents, Glassware and Reaction Setup}

Unless otherwise stated, all reactions were conducted under an inert atmosphere of $\mathrm{N}_{2}$ in flame dried glassware using standard Schlenk techniques. Air- and moisture-sensitive liquids and solutions were transferred via syringe into the reaction vessels through rubber septa. Unless otherwise specified, all reagents were purchased at highest commercial quality and used as received. Non-anhydrous solvents were purchased (unless specified) at the highest commercial quality and used as received. Anhydrous $N, N$-dimethylformamide (DMF), $N, N$-dimethylacetamide (DMAc), and methanol were purchased from Acros and used as received. Dichloromethane (DCM) and tetrahydrofuran (THF) were dried on an Anhydrous Engineering alumina column drying system.

\subsection{Analytical Methods}

Chromatography: Flash column chromatography was carried out using Sigma-Aldrich silica gel (60 A, 230-400 mesh, 40-63 $\mu \mathrm{m}$ ) or a Biotage IsoleraTM flash purification system. Reactions were followed by thin-layer chromatography (TLC) where practical, using aluminium-backed Merck Kieselgel 60 F254 fluorescent treated silica gel plates, which were visualised under UV light or by staining with aqueous basic potassium permanganate, acidic $p$-anisaldehyde solution in ethanol, or phosphomolybdic acid solution in ethanol.

IR: IR spectra were recorded on neat compounds using a Perkin Elmer (Spectrum One) FT-IR spectrometer (ATR sampling accessory). Selected absorbances $\left(v_{\max }\right.$, expressed in $\mathrm{cm}^{-1}$ ) are reported and denoted w. (weak), m. (medium) or s. (strong).

${ }^{1}$ H NMR: Spectra were recorded on Jeol ECS (400 MHz), Jeol ECZ (400 MHz or Bruker Avance (400 $\mathrm{MHz}$ or $500 \mathrm{MHz}$ ) instruments. Chemical shifts $(\delta)$ are quoted in parts per million (ppm) and referenced to the appropriate NMR solvent peak(s). Coupling constants $(J)$ are given in Hertz $(\mathrm{Hz})$ and refer to apparent multiplicities $(\mathrm{s}=$ singlet, $\mathrm{br} . \mathrm{s}=$ broad singlet, $\mathrm{d}=$ doublet, $\mathrm{t}=$ triplet, $\mathrm{q}=$ quartet, $\mathrm{p}=$ pentet, $\mathrm{m}=$ multiplet, $\mathrm{dd}=$ doublet of doublets, etc.). The ${ }^{1} \mathrm{H}$ NMR spectra are reported as follows: chemical shift (multiplicity, coupling constants, number of protons).

${ }^{13}$ C NMR: Spectra were recorded on a Jeol ECS (101 MHz), Jeol ECZ (101 MHz or Bruker Avance $(101 \mathrm{MHz}$ or $126 \mathrm{MHz}$ ) instruments. Chemical shifts $(\delta)$ are quoted in parts per million (ppm) and referenced to the appropriate NMR solvent peak(s). 
HRMS: High resolution mass spectra were recorded on a Bruker Daltronics MicroTOF II (ESI), Thermo Scientific Orbitrap (ESI, APCI) or Thermo Scientific QExactive (EI). Only molecular ion peaks $\left([\mathrm{M}+\mathrm{H}]^{+}\right.$or $[\mathrm{M}+\mathrm{Na}]^{+}$for ESI and $\mathrm{APCI} ; \mathrm{M}^{+}$for $\left.\mathrm{EI}\right)$ are reported.

M.p.: Melting points were recorded in degrees Celsius $\left({ }^{\circ} \mathrm{C}\right)$, using a Stuart SMP30 melting point apparatus and are reported uncorrected.

GC-MS: Gas chromatography-mass spectrometry was recorded on an Agilent 6890 Series GC and 5973 detector using a HP-5MS UI column (15 m x $0.25 \mathrm{~mm}$ x $0.25 \mu \mathrm{m})$.

\subsection{Photochemical Equipment and Setup}

The blue LED lamps were $40 \mathrm{~W}$ Kessil A160WE Tuna Blue LED Aquarium Lights (used with the colour dial turned fully anticlockwise and the intensity dial turned fully clockwise).

All photochemical reactions were carried out at room temperature (r.t.) with fan assisted cooling to maintain a temperature of $25-30{ }^{\circ} \mathrm{C}$.

Reaction set-up: The reaction vials were positioned $5 \mathrm{~cm}$ from a single $40 \mathrm{~W}$ Kessil LED lamp with a fan positioned above the reaction (Figure S1).

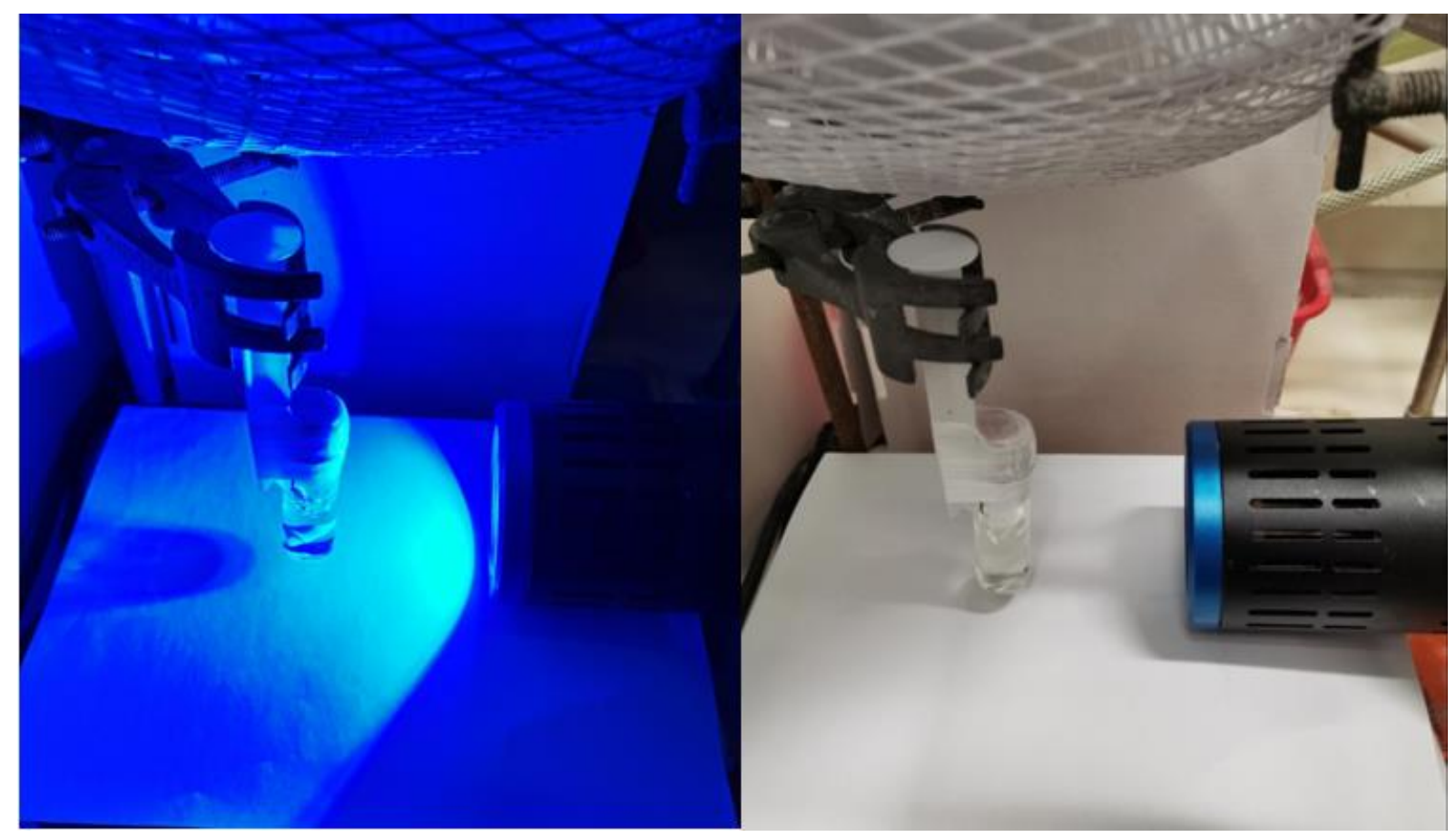

Figure S1. Photochemical reaction setup. 


\section{Additional Optimization Studies}

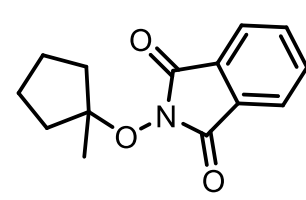

1a

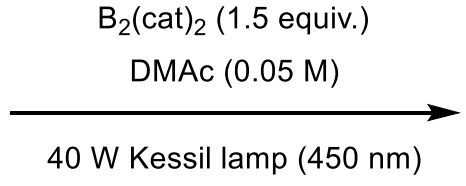

r.t., $20 \mathrm{~h}$

then pinacol, $\mathrm{Et}_{3} \mathrm{~N}$

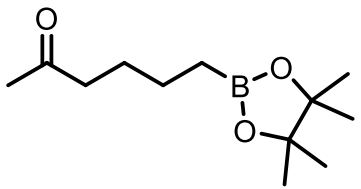

2

\begin{tabular}{|c|c|c|}
\hline Entry & Deviation from standard conditions & $\%$ yield $2^{\text {[a] }}$ \\
\hline 1 & none & 90 \\
\hline 2 & 2.0 equiv. of $\mathrm{B}_{2}(\mathrm{cat})_{2}$ & 88 \\
\hline 3 & $\mathbf{1 b}$ instead of $\mathbf{1 a}$ & 0 \\
\hline 4 & $\mathbf{1 c}$ instead of $\mathbf{1 a}$ & 33 \\
\hline 5 & DMAc (0.1 M) & 75 \\
\hline 6 & DMAc (0.2 M) & 61 \\
\hline 7 & blue LED strips (450 nm) & 90 \\
\hline 8 & 390 nm Kessil lamp (PR160L) & 75 \\
\hline 9 & 1.2 equiv. of $\mathrm{B}_{2}(\mathrm{cat})_{2}$ at $60^{\circ} \mathrm{C}$ (no light) & 40 \\
\hline 10 & 2.0 equiv. of $\mathrm{B}_{2}(\mathrm{cat})_{2}$ at $60^{\circ} \mathrm{C}$ (no light) & 50 \\
\hline 11 & 1.2 equiv. of $\mathrm{B}_{2}(\mathrm{OH})_{4}$ at $60^{\circ} \mathrm{C}$ (no light) & 7 \\
\hline
\end{tabular}

Reaction conditions: 1a $(0.10 \mathrm{mmol}), \mathrm{B}_{2}(\mathrm{cat})_{2}(0.12 \mathrm{mmol}), \mathrm{DMAc}(0.05 \mathrm{M}, 2.0 \mathrm{~mL}), 40 \mathrm{~W}$ Kessil Lamp (Maximum wavelength $=450 \mathrm{~nm})$, $\mathrm{N}_{2}$, r.t., $20 \mathrm{~h}$; then pinacol $(0.3 \mathrm{mmol}), \mathrm{Et}_{3} \mathrm{~N}(0.25 \mathrm{~mL}), 1 \mathrm{~h}$. [a] Measured by ${ }^{1} \mathrm{H}$ NMR analysis of the crude reaction mixture using diethyl phthalate as an internal standard.<smiles>CC1(ON2C(=O)CCC2=O)CCCC1</smiles>

1b<smiles>CC1(ON2C(=O)c3c(Cl)c(Cl)c(Cl)c(Cl)c3C2=O)CCCC1</smiles>

$1 c$<smiles>c1ccc2c(c1)OB(B1Oc3ccccc3O1)O2</smiles>

$\mathrm{B}_{2}(\mathrm{cat})_{2}$ 


\section{General Procedures}

\subsection{General Procedure 1 (GP1): Synthesis of $N$-Alkoxyphthalimides from Ketones}

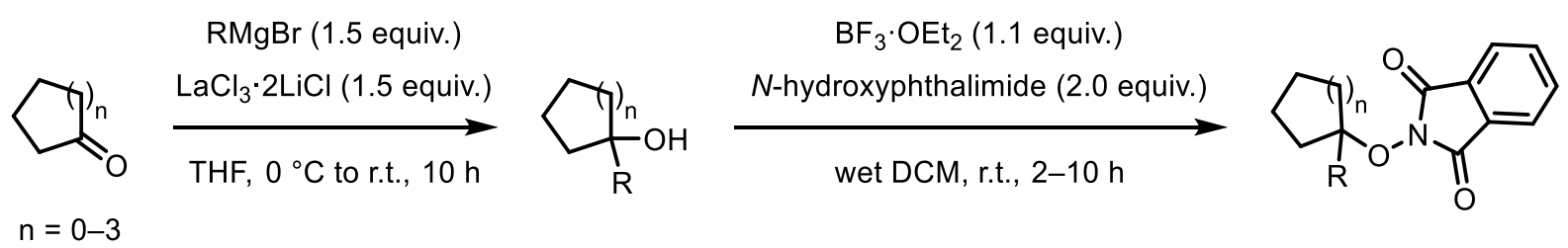

GP1-A) Following a modified literature procedure: ${ }^{1}$ To a flame-dried, $\mathrm{N}_{2}$-flushed $100 \mathrm{~mL}$ Schlenk flask equipped with a septum and a magnetic stirrer bar was placed $\mathrm{LaCl}_{3} \cdot 2 \mathrm{LiCl}(0.6 \mathrm{M}$ in THF, 1.5 equiv.) at $0{ }^{\circ} \mathrm{C} . \mathrm{RMgBr}$ (1.5 equiv.) was added dropwise, and the resulting mixture was stirred for $30 \mathrm{~min}$ at room temperature. The reaction mixture was cooled to $0{ }^{\circ} \mathrm{C}$, the ketone $(6.0 \mathrm{mmol}, 1.0$ equiv. $)$ was added and the reaction was allowed to warm to room temperature and stirred for $2-10 \mathrm{~h}$. When complete conversion was observed by TLC analysis, saturated aqueous $\mathrm{NH}_{4} \mathrm{Cl}(20 \mathrm{~mL})$ and water $(20 \mathrm{~mL})$ were added. The aqueous layer was extracted with DCM $(3 \times 40 \mathrm{~mL})$, and the combined extracts were dried over $\mathrm{MgSO}_{4}$, filtered, and concentrated under reduced pressure. The crude residue was purified by flash column chromatography to give the pure tertiary alcohol for use in the next step.

GP1-B) Following a modified literature procedure: ${ }^{2}$ A mixture of tertiary alcohol (5.0 mmol, 1.0 equiv.) and $N$-hydroxyphthalimide (2.0 equiv.) in wet DCM $(0.5 \mathrm{M})$ was cooled to $0{ }^{\circ} \mathrm{C}$ before $\mathrm{BF}_{3} \cdot \mathrm{OEt}_{2}(1.1-$ 2.0 equiv.) was added dropwise. The reaction mixture was allowed to warm to room temperature and stirred for $2-10 \mathrm{~h}$. When complete conversion was observed by TLC analysis, saturated aqueous $\mathrm{Na}_{2} \mathrm{CO}_{3}$ $(30 \mathrm{~mL})$ was added. The mixture was extracted with DCM $(3 \times 40 \mathrm{~mL})$ and the combined extracts were dried over $\mathrm{MgSO}_{4}$, filtered, and concentrated under reduced pressure. The residue was then purified with flash column chromatography (EtOAc/pentane or EtOAc/hexane) to afford the desired $\mathrm{N}$ alkoxyphthalimides. 


\subsection{General Procedure 2 (GP2): Synthesis of $N$-Alkoxyphthalimides from Cyclic Enol}

Ethers

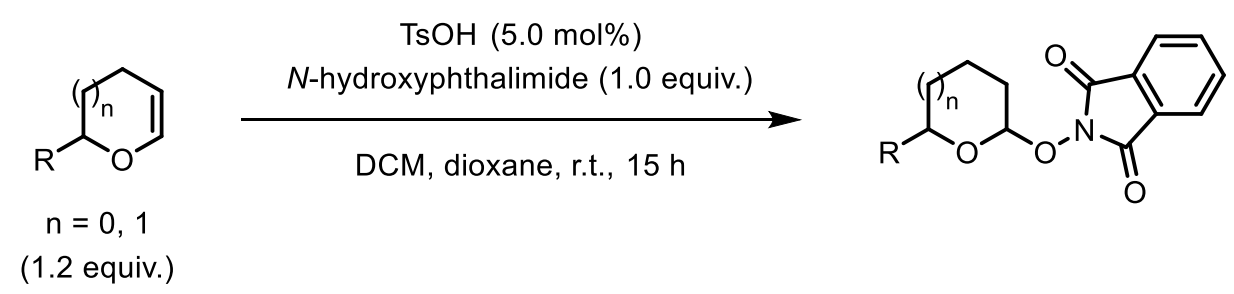

Following a modified literature procedure. ${ }^{3} \mathrm{~A}$ mixture of cyclic enol ether (1.2 equiv.) and $\mathrm{N}$ hydroxyphthalimide (1.0 equiv.) in DCM/dioxane (1:1) was cooled to $0{ }^{\circ} \mathrm{C}$, then para-toluenesulfonic acid ( $\mathrm{TsOH}, 5.0 \mathrm{~mol} \%$ ) was added to the solution. The reaction mixture was stirred at room temperature for $15 \mathrm{~h}$, then solvent was concentrated under reduced pressure. The residue was then purified with flash column chromatography chromatography (EtOAc/pentane or EtOAc/hexane) to afford the desired $\mathrm{N}$-alkoxyphthalimides.

\subsection{General Procedure 3 (GP3): Fragmentation Borylation Reaction}

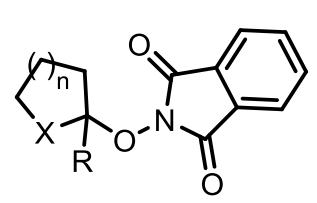

$\mathrm{n}=0,1,2,3$

$\mathrm{X}=\mathrm{CH}_{2}, \mathrm{O}$

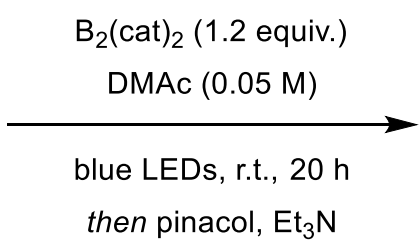

then pinacol, $\mathrm{Et}_{3} \mathrm{~N}$<smiles>[R]C(=O)CCCCB1OC(C)(C)C(C)(C)O1</smiles>

$N$-Alkoxyphthalimide ( $0.50 \mathrm{mmol}, 1.0$ equiv.) and $\mathrm{B}_{2}$ (cat) $)_{2}$ (1.2 equiv.) were added to an $\mathrm{N}_{2}$-flushed 7 $\mathrm{mL}$ vial containing a magnetic stirrer bar. Dry DMAc $(0.05 \mathrm{M})$ was added and the headspace of the vial was purged with a gentle stream of $\mathrm{N}_{2}$ for approximately $10 \mathrm{~min}$. The vial was tightly sealed and stirred under blue LED irradiation for $20 \mathrm{~h}$ (see Figure $\mathbf{S 1}$ for experimental setup). Irradiation was stopped and a solution of pinacol (3.0 equiv.) in $\mathrm{Et}_{3} \mathrm{~N}$ (20.0 equiv.) was added to the reaction mixture, which was stirred at room temperature for a further $1 \mathrm{~h}$. The reaction mixture was diluted with DCM (40 mL) and washed with water $(3 \times 30 \mathrm{~mL})$ and brine $(20 \mathrm{~mL})$, dried over $\mathrm{MgSO}_{4}$, filtered, and concentrated under reduced pressure. The crude material was purified by flash column chromatography (EtOAc/pentane or EtOAc/hexane) to afford the boronic ester product. 


\section{Substrate Synthesis and Characterization}

\section{2-((1-Methylcyclopentyl)oxy)isoindoline-1,3-dione (1a)}

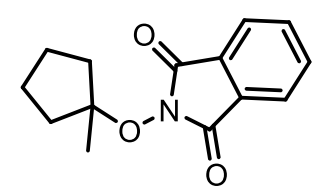

Prepared following GP1-B: 1-Methylcyclopentan-1-ol (2.00 g, 20.0 mmol, 1.0 equiv.) was treated with $N$-hydroxyphthalimide $(6.52 \mathrm{~g}, 40.0 \mathrm{mmol}, 2.0$ equiv. $)$ and $\mathrm{BF}_{3} \cdot \mathrm{OEt}_{2}(3.12 \mathrm{~g}, 22.0 \mathrm{mmol}, 1.1$ equiv. $)$ in wet DCM $(0.3 \mathrm{M})$ at room temperature for $2 \mathrm{~h}$. Purification by column chromatography (20\% EtOAc/hexane) gave 1a $(1.47 \mathrm{~g}, 6.00 \mathrm{mmol}, 30 \%)$ as pale-yellow oil.

TLC: $\mathrm{R}_{\mathrm{f}}=0.40\left(20 \%\right.$ EtOAc/hexane, $\mathrm{KMnO}_{4}$ stain $)$.

${ }^{1} \mathbf{H}$ NMR $\left(400 \mathrm{MHz}, \mathrm{CDCl}_{3}\right): \delta_{\mathrm{H}} 7.86-7.80(\mathrm{~m}, 2 \mathrm{H}), 7.78-7.72(\mathrm{~m}, 2 \mathrm{H}), 2.18-2.08(\mathrm{~m}, 2 \mathrm{H}), 2.08-$ $1.97(\mathrm{~m}, 2 \mathrm{H}), 1.74-1.64(\mathrm{~m}, 2 \mathrm{H}), 1.64-1.55(\mathrm{~m}, 2 \mathrm{H}), 1.47$ (s, 3H) ppm. See spectrum.

${ }^{13} \mathrm{C}$ NMR $\left(101 \mathrm{MHz}, \mathrm{CDCl}_{3}\right): \delta_{\mathrm{C}} 165.9,134.4,129.4,123.4,97.9,38.0,24.5,24.3 \mathrm{ppm}$. See spectrum. IR (film) $v_{\max }$ : 2967, 2875, 1792, 1733(s), 1467, 1447, 1350, 1318, 1106, 967, 877, $703 \mathrm{~cm}^{-1}$. HRMS (ESI) m/z: [M + Na $]^{+}$Calcd for $\mathrm{C}_{14} \mathrm{H}_{15} \mathrm{NNaO}_{3}$ 268.0944; Found 268.0950.

\section{1-((1-Methylcyclopentyl)oxy)pyrrolidine-2,5-dione (1b)}<smiles>CC1(ON2C(=O)CCC2=O)CCCC1</smiles>

Prepared following a modified GP1-B: 1-Methylcyclopentan-1-ol (600 mg, $6.00 \mathrm{mmol}, 1.0$ equiv.) was treated with $N$-hydroxysuccinimide (1.38 g, $12.0 \mathrm{mmol}, 2.0$ equiv.) and $\mathrm{BF}_{3} \cdot \mathrm{OEt}_{2}(937 \mathrm{mg}, 6.60 \mathrm{mmol}$, 1.1 equiv.) in wet DCM $(0.5 \mathrm{M})$ at room temperature for $2 \mathrm{~h}$. Purification by column chromatography (20\% EtOAc/hexane) gave $\mathbf{1 b}(197 \mathrm{mg}, 0.60 \mathrm{mmol}, 10 \%)$ as colourless oil.

TLC: $\mathrm{R}_{\mathrm{f}}=0.40\left(20 \% \mathrm{EtOAc} / \mathrm{hexane}, \mathrm{KMnO}_{4}\right.$ stain $)$.

${ }^{1} \mathbf{H}$ NMR (400 MHz, $\left.\mathrm{CDCl}_{3}\right): \delta_{\mathrm{H}} 2.72(\mathrm{~s}, 4 \mathrm{H}), 2.09-2.01(\mathrm{~m}, 2 \mathrm{H}), 1.99-1.91(\mathrm{~m}, 2 \mathrm{H}), 1.69-1.60(\mathrm{~m}$, $2 \mathrm{H}), 1.58-1.50(\mathrm{~m}, 2 \mathrm{H}), 1.38(\mathrm{~s}, 3 \mathrm{H}) \mathrm{ppm}$. See spectrum.

${ }^{13}$ C NMR (101 MHz, $\left.\mathrm{CDCl}_{3}\right): \delta_{\mathrm{C}} 173.2,98.2,38.1,25.6,24.3,24.2$ ppm. See spectrum. IR (film) $v_{\max }: 2968,2876,1786,1721(\mathrm{~s}), 1443,1432,1354,1181,1066,815 \mathrm{~cm}^{-1}$. 
HRMS (ESI) m/z: [M + Na $]^{+}$Calcd for $\mathrm{C}_{10} \mathrm{H}_{15} \mathrm{NNaO}_{3} 220.0944$; Found 220.0948.

\section{4,5,6,7-Tetrachloro-2-((1-methylcyclopentyl)oxy)isoindoline-1,3-dione (1c)}<smiles>CC1(ON2C(=O)c3c(Cl)c(Cl)c(Cl)c(Cl)c3C2=O)CCCC1</smiles>

Prepared following a modified GP1-B: 1-Methylcyclopentan-1-ol (600 mg, $6.00 \mathrm{mmol}, 1.0$ equiv.) was treated with 4,5,6,7-tetrachloro-2-hydroxy-isoindole-1,3-dione (3.61 g, $12.0 \mathrm{mmol}, 2.0$ equiv.) and $\mathrm{BF}_{3} \cdot \mathrm{OEt}_{2}(937 \mathrm{mg}, 6.60 \mathrm{mmol}, 1.1$ equiv. $)$ in wet DCM $(0.5 \mathrm{M})$ at room temperature for $2 \mathrm{~h}$. Purification by column chromatography (10\% EtOAc/hexane) gave 1c (483 mg, $1.26 \mathrm{mmol}, 21 \%)$ as white solid.

TLC: $\mathrm{R}_{\mathrm{f}}=0.40\left(20 \% \mathrm{EtOAc} / \mathrm{hexane}, \mathrm{KMnO}_{4}\right.$ stain $)$.

${ }^{1} \mathbf{H}$ NMR $\left(400 \mathrm{MHz}, \mathrm{CDCl}_{3}\right): \delta_{\mathrm{H}} 2.13-1.97(\mathrm{~m}, 4 \mathrm{H}), 1.73-1.64(\mathrm{~m}, 2 \mathrm{H}), 1.64-1.55(\mathrm{~m}, 2 \mathrm{H}), 1.46(\mathrm{~s}$, 3H) ppm. See spectrum.

${ }^{13} \mathrm{C}$ NMR (101 MHz, $\left.\mathrm{CDCl}_{3}\right): \delta_{\mathrm{C}} 161.4,140.6,129.8,125.0,98.9,38.0,24.4,24.3 \mathrm{ppm}$. See spectrum. IR (film) $v_{\max }: 2969,2875,1794,1735(\mathrm{~s}), 1572,1447,1365,1320,1197,1122,1028,889,730 \mathrm{~cm}^{-1}$.

HRMS (ESI) m/z: [M + Na] ${ }^{+}$Calcd for $\mathrm{C}_{14} \mathrm{H}_{11} \mathrm{Cl}_{4} \mathrm{NNaO}_{3}$ 403.9385; Found 403.9380 .

M.p.: $115-117^{\circ} \mathrm{C}$.

\section{2-(1-Methylcyclobutoxy)isoindoline-1,3-dione (3a)}

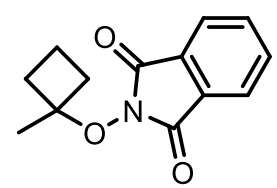

Prepared according to GP1: $\mathrm{LaCl}_{3} \cdot 2 \mathrm{LiCl}(9.0 \mathrm{mmol}, 1.5$ equiv.), methylmagnesium bromide $(9.0 \mathrm{mmol}$, 1.5 equiv.), and cyclobutanone ( $420 \mathrm{mg}, 6.00 \mathrm{mmol}, 1.0$ equiv.) were stirred at room temperature for 2 h. Purification by column chromatography (20\% EtOAc/hexane) gave 1-methylcyclobutan-1-ol (300 $\mathrm{mg}, 3.48 \mathrm{mmol}, 58 \%$ ) as a bright yellow oil. 1-Methylcyclobutan-1-ol (300 mg, $3.48 \mathrm{mmol}, 1.0$ equiv.) was then treated with $N$-hydroxyphthalimide (1.14 g, $6.96 \mathrm{mmol}, 2.0$ equiv.) and $\mathrm{BF}_{3} \cdot \mathrm{OEt}_{2}(544 \mathrm{mg}$, $3.83 \mathrm{mmol}, 1.1$ equiv.) in wet DCM $(0.5 \mathrm{M})$ at room temperature for $3 \mathrm{~h}$. Purification by column chromatography (20\% EtOAc/hexane) gave 3a (472.0 mg, $2.04 \mathrm{mmol}, 58 \%)$ as a white solid. 
TLC: $\mathrm{R}_{\mathrm{f}}=0.51\left(20 \%\right.$ EtOAc/hexane, $\mathrm{KMnO}_{4}$ stain $)$.

${ }^{1} \mathbf{H}$ NMR $\left(400 \mathrm{MHz}, \mathrm{CDCl}_{3}\right): \delta_{\mathrm{H}} 7.87-7.82(\mathrm{~m}, 2 \mathrm{H}), 7.78-7.73(\mathrm{~m}, 2 \mathrm{H}), 2.56-2.45(\mathrm{~m}, 2 \mathrm{H}), 2.02-$ $1.93(\mathrm{~m}, 2 \mathrm{H}), 1.76(\mathrm{dtt}, J=11.1,10.2,3.0 \mathrm{~Hz}, 1 \mathrm{H}), 1.59-1.48(\mathrm{~m}, 1 \mathrm{H}), 1.53(\mathrm{~s}, 3 \mathrm{H})$ ppm. See spectrum. ${ }^{13}$ C NMR (101 MHz, $\left.\mathrm{CDCl}_{3}\right): \delta_{\mathrm{C}} 165.3,134.4,129.2,123.5,87.7,33.6,23.0,11.8 \mathrm{ppm}$. See spectrum. IR (film) $v_{\max }$ : 2972, 2940, 1786, 1732(s), 1466, 1355, 1248, 1185, 1152, 964, 877, 704cm.

HRMS (ESI) m/z: [M + H] ${ }^{+}$Calcd for $\mathrm{C}_{13} \mathrm{H}_{14} \mathrm{NO}_{3}$ 232.0968; Found 232.0976.

M.p.: $110-112^{\circ} \mathrm{C}$.

\section{2-(1-Ethylcyclobutoxy)isoindoline-1,3-dione (4a)}

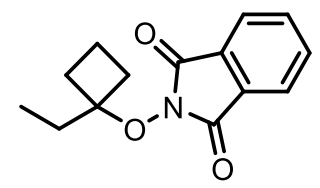

Prepared according to GP1: $\mathrm{LaCl}_{3} \cdot 2 \mathrm{LiCl}(9.0 \mathrm{mmol}, 1.5$ equiv.), methylmagnesium bromide $(9.0 \mathrm{mmol}$, 1.5 equiv.), and cyclobutanone ( $420 \mathrm{mg}, 6.00 \mathrm{mmol}, 1.0$ equiv.) were stirred at room temperature for 10 h. Purification by column chromatography (20\% EtOAc/hexane) gave 1-ethylcyclobutan-1-ol (300 mg, $3.00 \mathrm{mmol}, 50 \%)$ as a bright yellow oil. 1-Ethylcyclobutan-1-ol (300 mg, $3.00 \mathrm{mmol}, 1.0$ equiv.) was then treated with $N$-hydroxyphthalimide ( $979 \mathrm{mg}, 6.00 \mathrm{mmol}, 2.0$ equiv.) and $\mathrm{BF}_{3} \cdot \mathrm{OEt}_{2}(468.6 \mathrm{mg}, 3.30$ mmol, 1.1 equiv.) in wet DCM $(0.5 \mathrm{M})$ at room temperature for $3 \mathrm{~h}$. Purification by column chromatography (20\% EtOAc/hexane) gave $4 \mathbf{a}(427.0 \mathrm{mg}, 1.74 \mathrm{mmol}, 58 \%)$ as a yellow oil.

TLC: $\mathrm{R}_{\mathrm{f}}=0.51\left(20 \% \mathrm{EtOAc} /\right.$ hexane, $\mathrm{KMnO}_{4}$ stain $)$.

${ }^{1} \mathbf{H}$ NMR $\left(400 \mathrm{MHz}, \mathrm{CDCl}_{3}\right): \delta_{\mathrm{H}} 7.88-7.82(\mathrm{~m}, 2 \mathrm{H}), 7.79-7.73(\mathrm{~m}, 2 \mathrm{H}), 2.43-2.30(\mathrm{~m}, 2 \mathrm{H}), 2.08-$ $1.97(\mathrm{~m}, 2 \mathrm{H}), 1.87(\mathrm{q}, J=7.3 \mathrm{~Hz}, 2 \mathrm{H}), 1.73(\mathrm{dtt}, J=11.1,10.3,3.3 \mathrm{~Hz}, 1 \mathrm{H}), 1.55-1.42(\mathrm{~m}, 1 \mathrm{H}), 1.14$ (t, $J=7.3 \mathrm{~Hz}, 3 \mathrm{H})$ ppm. See spectrum.

${ }^{13} \mathrm{C}$ NMR (101 MHz, $\left.\mathrm{CDCl}_{3}\right): \delta_{\mathrm{C}} 165.6,134.4,129.3,123.5,90.7,31.0,29.1,12.3,7.7 \mathrm{ppm} . \underline{\text { See }}$ spectrum.

IR (film) $v_{\max }: 2970,2942,1791,1733(\mathrm{~s}), 1467,1369,1187,973,905,878,729 \mathrm{~cm}^{-1}$.

HRMS (ESI) m/z: [M + Na $]^{+}$Calcd for $\mathrm{C}_{14} \mathrm{H}_{15} \mathrm{NNaO}_{3}$ 268.0944; Found 268.0935.

M.p.: $112-113^{\circ} \mathrm{C}$. 


\section{2-(1-Butylcyclobutoxy)isoindoline-1,3-dione (5a)}

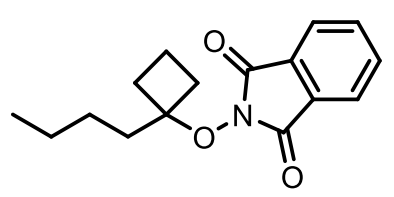

To a flame-dried, $\mathrm{N}_{2}$-flushed $100 \mathrm{~mL}$ Schlenk flask equipped with a septum and a magnetic stirrer bar was added cyclobutanone (700 mg, $10.0 \mathrm{mmol}, 1.0$ equiv.) and THF (20 mL). The solution was cooled to $-78{ }^{\circ} \mathrm{C}$ and $n$-butyllithium (1.6 M in hexanes, $25 \mathrm{mmol}, 2.5$ equiv.) was added dropwise. The reaction mixture was allowed to warm to room temperature and stirred for $15 \mathrm{~h}$. Saturated aqueous $\mathrm{NH}_{4} \mathrm{Cl}(20$ $\mathrm{mL})$ and water $(20 \mathrm{~mL})$ were added. The aqueous layer was extracted with DCM $(3 \times 40 \mathrm{~mL})$, and the combined extracts were dried over $\mathrm{MgSO}_{4}$, filtered, and concentrated under reduced pressure. The crude product was purified by column chromatography (20\% EtOAc/hexane) to give 1-butylcyclobutan-1-ol (765 $\mathrm{mg}, 5.96 \mathrm{mmol}, 66 \%)$ as a bright yellow oil.

According to GP1-B: 1-Butylcyclobutan-1-ol (641.0 mg, $5.00 \mathrm{mmol}, 1.0$ equiv.) was then treated with $N$-hydroxyphthalimide (1.63 g, $10.0 \mathrm{mmol}, 2.0$ equiv.) and $\mathrm{BF}_{3} \cdot \mathrm{OEt}_{2}(781 \mathrm{mg}, 5.50 \mathrm{mmol}, 1.1$ equiv.) in wet DCM $(0.5 \mathrm{M})$ at room temperature for $4 \mathrm{~h}$. Purification by column chromatography $(20 \%$ EtOAc/hexane) gave $\mathbf{5 a}(720.0 \mathrm{mg}, 2.63 \mathrm{mmol}, 53 \%)$ as a yellow oil.

TLC: $\mathrm{R}_{\mathrm{f}}=0.51\left(20 \% \mathrm{EtOAc} / \mathrm{hexane}, \mathrm{KMnO}_{4}\right.$ stain $)$.

${ }^{1} \mathbf{H}$ NMR $\left(400 \mathrm{MHz}, \mathrm{CDCl}_{3}\right): \delta_{\mathrm{H}} 7.88-7.82(\mathrm{~m}, 2 \mathrm{H}), 7.78-7.73(\mathrm{~m}, 2 \mathrm{H}), 2.41-2.28(\mathrm{~m}, 2 \mathrm{H}), 2.08-$ $1.97(\mathrm{~m}, 2 \mathrm{H}), 1.87-1.79(\mathrm{~m}, 2 \mathrm{H}), 1.71(\mathrm{dtt}, J=11.0,10.3,3.3 \mathrm{~Hz}, 1 \mathrm{H}), 1.65-1.55(\mathrm{~m}, 2 \mathrm{H}), 1.54-$ $1.43(\mathrm{~m}, 1 \mathrm{H}), 1.44-1.34(\mathrm{~m}, 2 \mathrm{H}), 0.96(\mathrm{t}, J=7.3 \mathrm{~Hz}, 3 \mathrm{H}) \mathrm{ppm} . \underline{\text { See spectrum. }}$

${ }^{13}$ C NMR (101 MHz, $\left.\mathrm{CDCl}_{3}\right): \delta_{\mathrm{C}} 165.6,134.4,129.3,123.5,90.4,36.2,31.4,25.5,23.1,14.1,12.3$ ppm. See spectrum.

IR (film) $v_{\max }: 2954,2871,1791,1731(\mathrm{~s}), 1466,1370,1351,1187,1079,975,878,701 \mathrm{~cm}^{-1}$.

HRMS (ESI) m/z: [M + Na $]^{+}$Calcd for $\mathrm{C}_{16} \mathrm{H}_{19} \mathrm{NNaO}_{3}[\mathrm{M}+\mathrm{Na}]^{+}$296.1257; Found 296.1263.

M.p.: $74-75^{\circ} \mathrm{C}$.

\section{2-(1-Nonylcyclobutoxy)isoindoline-1,3-dione (6a)}

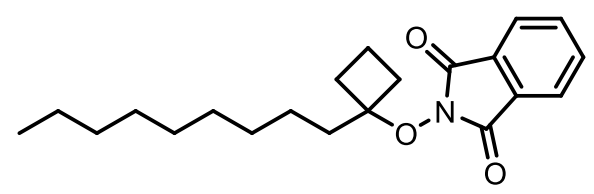

Prepared according to GP1: $\mathrm{LaCl}_{3} \cdot 2 \mathrm{LiCl}$ ( $9.0 \mathrm{mmol}$, 1.5 equiv.), nonylmagnesium bromide (9.0 mmol, 1.5 equiv.), and cyclobutanone ( $420 \mathrm{mg}, 6.00 \mathrm{mmol}, 1.0$ equiv.) were stirred at room temperature for 10 
h. Purification by column chromatography (20\% EtOAc/hexane) gave 1-nonylcyclobutan-1-ol (1.15 g, $5.79 \mathrm{mmol}, 96 \%$ ) as a bright yellow oil. 1-Nonylcyclobutan-1-ol (992 mg, $5.00 \mathrm{mmol}, 1.0$ equiv.) was then treated with $N$-hydroxyphthalimide $\left(1.63 \mathrm{~g}, 10.0 \mathrm{mmol}, 2.0\right.$ equiv.) and $\mathrm{BF}_{3} \cdot \mathrm{OEt}_{2}(781 \mathrm{mg}, 5.50$ mmol, 1.1 equiv.) in wet DCM $(0.5 \mathrm{M})$ at room temperature for $4 \mathrm{~h}$. Purification by column chromatography (20\% EtOAc/hexane) gave 6a (699.0 mg, $2.03 \mathrm{mmol}, 41 \%)$ as a yellow oil.

TLC: $\mathrm{R}_{\mathrm{f}}=0.51\left(20 \%\right.$ EtOAc/hexane, $\mathrm{KMnO}_{4}$ stain $)$.

${ }^{1} \mathbf{H}$ NMR $\left(400 \mathrm{MHz}, \mathrm{CDCl}_{3}\right): \delta_{\mathrm{H}} 7.88-7.82(\mathrm{~m}, 2 \mathrm{H}), 7.78-7.73(\mathrm{~m}, 2 \mathrm{H}), 2.41-2.29(\mathrm{~m}, 2 \mathrm{H}), 2.08-$ $1.97(\mathrm{~m}, 2 \mathrm{H}), 1.86-1.78(\mathrm{~m}, 2 \mathrm{H}), 1.78-1.64(\mathrm{~m}, 1 \mathrm{H}), 1.67-1.55(\mathrm{~m}, 2 \mathrm{H}), 1.57-1.41(\mathrm{~m}, 1 \mathrm{H}), 1.41$ $-1.21(\mathrm{~m}, 12 \mathrm{H}), 0.88(\mathrm{~d}, J=7.0 \mathrm{~Hz}, 3 \mathrm{H}) \mathrm{ppm}$. See spectrum.

${ }^{13} \mathrm{C}$ NMR $\left(101 \mathrm{MHz}, \mathrm{CDCl}_{3}\right): \delta_{\mathrm{C}} 165.6,134.4,129.3,123.5,90.5,36.5,31.9,31.5,30.1,29.6,29.3$, 23.3, 22.7, 14.1, 12.3 ppm. See spectrum.

IR (film) $v_{\max }: 2923,2871,1792,1732(\mathrm{~s}), 1467,1370,1250,1187,1113,1057,1033,904,704 \mathrm{~cm}^{-1}$.

HRMS (ESI) m/z: [M + Na $]^{+}$Calcd for $\mathrm{C}_{21} \mathrm{H}_{29} \mathrm{NNaO}_{3}$ 366.2040; Found 366.2034.

\section{2-(1-Octadecylcyclobutoxy)isoindoline-1,3-dione (7a)}

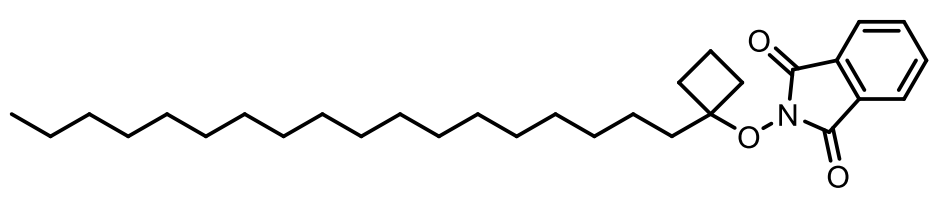

Prepared according to GP1: $\mathrm{LaCl}_{3} \cdot 2 \mathrm{LiCl}$ (9.0 mmol, 1.5 equiv.), octadecylmagnesium chloride (9.0 mmol, 1.5 equiv.), and cyclobutanone ( $420 \mathrm{mg}, 6.00 \mathrm{mmol}, 1.0$ equiv.) were stirred at room temperature for $10 \mathrm{~h}$. Purification by column chromatography (20\% EtOAc/hexane) gave 1-octedecylcyclobutan-1ol (1.62 g, $5.01 \mathrm{mmol}, 84 \%)$ as a bright yellow oil. 1-Octadecylcyclobutan-1-ol (1.62 mg, $5.00 \mathrm{mmol}$, 1.0 equiv.) was then treated with $N$-hydroxyphthalimide (1.63 g, $10.0 \mathrm{mmol}, 2.0$ equiv.) and $\mathrm{BF}_{3} \cdot \mathrm{OEt}_{2}$ (781 mg, $5.50 \mathrm{mmol}, 1.1$ equiv.) in wet DCM $(0.5 \mathrm{M})$ at room temperature for $4 \mathrm{~h}$. Purification by column chromatography (20\% EtOAc/hexane) gave 7a (1.50 g, $3.19 \mathrm{mmol}, 64 \%)$ as a pale yellow solid.

TLC: $\mathrm{R}_{\mathrm{f}}=0.51\left(20 \%\right.$ EtOAc/hexane, $\mathrm{KMnO}_{4}$ stain $)$.

${ }^{1} \mathbf{H}$ NMR $\left(400 \mathrm{MHz}, \mathrm{CDCl}_{3}\right): \delta_{\mathrm{H}} 7.88-7.82(\mathrm{~m}, 2 \mathrm{H}), 7.78-7.73(\mathrm{~m}, 2 \mathrm{H}), 2.41-2.30(\mathrm{~m}, 2 \mathrm{H}), 2.08-$ $1.98(\mathrm{~m}, 2 \mathrm{H}), 1.85-1.79(\mathrm{~m}, 2 \mathrm{H}), 1.77-1.66(\mathrm{~m}, 1 \mathrm{H}), 1.66-1.57(\mathrm{~m}, 2 \mathrm{H}), 1.55-1.43(\mathrm{~m}, 1 \mathrm{H}), 1.40$ $-1.20(\mathrm{~m}, 30 \mathrm{H}), 0.88(\mathrm{~d}, J=7.0 \mathrm{~Hz}, 3 \mathrm{H}) \mathrm{ppm} . \underline{\text { See spectrum. }}$.

${ }^{13} \mathrm{C}$ NMR $\left(101 \mathrm{MHz}, \mathrm{CDCl}_{3}\right): \delta_{\mathrm{C}} 165.6,134.4,129.3,123.5,90.5,36.5,31.9,31.5,30.1,29.7,29.7$, 29.6, 29.6, 29.4, 23.3, 22.7, 14.1, 12.4 ppm. See spectrum. 
IR (film) $v_{\max }: 2974,2922,1792,1734(\mathrm{~s}), 1467,1406,1372,1250,1056,904,878,726 \mathrm{~cm}^{-1}$.

HRMS (ESI) m/z: [M + Na $]^{+}$Calcd for $\mathrm{C}_{30} \mathrm{H}_{47} \mathrm{NNaO}_{3}$ 492.3448; Found 492.3445 .

M.p.: $64-66^{\circ} \mathrm{C}$.

\section{2-(1-Isobutylcyclobutoxy)isoindoline-1,3-dione (8a)}

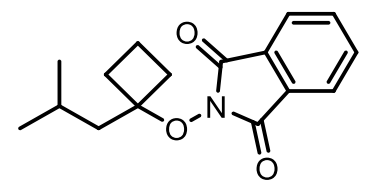

Prepared according to GP1: $\mathrm{LaCl}_{3} \cdot 2 \mathrm{LiCl}$ (9.0 mmol, 1.5 equiv.), isobutylmagnesium bromide (9.0 mmol, 1.5 equiv.), and cyclobutanone ( $420 \mathrm{mg}, 6.00 \mathrm{mmol}, 1.0$ equiv.) were stirred at room temperature for $4 \mathrm{~h}$. Purification by column chromatography (20\% EtOAc/hexane) gave 1-isobutylcyclobutan-1-ol (650 mg, $5.07 \mathrm{mmol}, 85 \%)$ as a bright yellow oil. 1-Isobutylcyclobutan-1-ol (641 mg, $5.00 \mathrm{mmol}, 1.0$ equiv.) was then treated with $N$-hydroxyphthalimide (1.63 g, $10.0 \mathrm{mmol}, 2.0$ equiv.) and $\mathrm{BF}_{3} \cdot \mathrm{OEt}_{2}$ (781 $\mathrm{mg}, 5.50 \mathrm{mmol}, 1.1$ equiv. $)$ in wet DCM $(0.5 \mathrm{M})$ at room temperature for $4 \mathrm{~h}$. Purification by column chromatography (20\% EtOAc/hexane) gave $\mathbf{8 a}(612 \mathrm{mg}, 2.24 \mathrm{mmol}, 45 \%)$ as a pale yellow solid.

TLC: $\mathrm{R}_{\mathrm{f}}=0.51\left(20 \%\right.$ EtOAc/hexane, $\mathrm{KMnO}_{4}$ stain $)$.

${ }^{1} \mathbf{H}$ NMR $\left(400 \mathrm{MHz}, \mathrm{CDCl}_{3}\right): \delta_{\mathrm{H}} 7.88-7.83(\mathrm{~m}, 2 \mathrm{H}), 7.79-7.73(\mathrm{~m}, 2 \mathrm{H}), 2.37-2.26(\mathrm{~m}, 2 \mathrm{H}), 2.19-$ $2.01(\mathrm{~m}, 3 \mathrm{H}), 1.83(\mathrm{~d}, J=6.5 \mathrm{~Hz}, 2 \mathrm{H}), 1.69-1.59(\mathrm{~m}, 1 \mathrm{H}), 1.55-1.42(\mathrm{~m}, 1 \mathrm{H}), 1.05(\mathrm{~d}, J=6.7 \mathrm{~Hz}$, 6H) ppm. See spectrum.

${ }^{13}$ C NMR (101 MHz, $\left.\mathrm{CDCl}_{3}\right): \delta_{\mathrm{C}} 165.7,134.4,129.4,123.5,90.7,45.0,32.3,24.7,24.1,12.5$ ppm. $\underline{\text { See }}$ spectrum.

IR (film) $v_{\max }: 2973,2923,1792,1733(\mathrm{~s}), 1468,1406,1371,1264,1187,1056,904,725,704 \mathrm{~cm}^{-1}$.

HRMS (ESI) m/z: [M + Na $]^{+}$Calcd for $\mathrm{C}_{16} \mathrm{H}_{19} \mathrm{NNaO}_{3}$ 296.1257; Found 296.1260.

M.p.: $75-77^{\circ} \mathrm{C}$. 


\section{2-(1-Isopentylcyclobutoxy)isoindoline-1,3-dione (9a)}

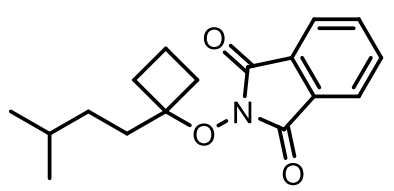

Prepared according to GP1: $\mathrm{LaCl}_{3} \cdot 2 \mathrm{LiCl}(9.0 \mathrm{mmol}, 1.5$ equiv.), isopentylmagnesium bromide (9.0 mmol, 1.5 equiv.), and cyclobutanone ( $420 \mathrm{mg}, 6.00 \mathrm{mmol}, 1.0$ equiv.) were stirred at room temperature for $10 \mathrm{~h}$. Purification by column chromatography (20\% EtOAc/hexane) gave 1-isopentylcyclobutan-1ol (800 mg, $5.63 \mathrm{mmol}, 94 \%)$ as a bright yellow oil. 1-Isopentylcyclobutan-1-ol (711 mg, $5.00 \mathrm{mmol}$, 1.0 equiv.) was then treated with $N$-hydroxyphthalimide ( $1.63 \mathrm{~g}, 10.0 \mathrm{mmol}, 2.0$ equiv.) and $\mathrm{BF}_{3} \cdot \mathrm{OEt}_{2}$ (781 mg, $5.50 \mathrm{mmol}, 1.1$ equiv.) in wet DCM $(0.5 \mathrm{M})$ at room temperature for $4 \mathrm{~h}$. Purification by column chromatography (20\% EtOAc/hexane) gave 9a (850 mg, $2.96 \mathrm{mmol}, 59 \%$ ) as a white solid.

TLC: $\mathrm{R}_{\mathrm{f}}=0.51\left(20 \%\right.$ EtOAc/hexane, $\mathrm{KMnO}_{4}$ stain $)$.

${ }^{1} \mathbf{H}$ NMR $\left(400 \mathrm{MHz}, \mathrm{CDCl}_{3}\right): \delta_{\mathrm{H}} 7.88-7.83(\mathrm{~m}, 2 \mathrm{H}), 7.78-7.73(\mathrm{~m}, 2 \mathrm{H}), 2.41-2.29(\mathrm{~m}, 2 \mathrm{H}), 2.08-$ $1.97(\mathrm{~m}, 2 \mathrm{H}), 1.88-1.79(\mathrm{~m}, 2 \mathrm{H}), 1.78-1.65(\mathrm{~m}, 1 \mathrm{H}), 1.64-1.56(\mathrm{~m}, 1 \mathrm{H}), 1.55-1.44(\mathrm{~m}, 3 \mathrm{H}), 0.95$ $(\mathrm{d}, J=6.4 \mathrm{~Hz}, 6 \mathrm{H}) \mathrm{ppm}$. See spectrum.

${ }^{13} \mathrm{C}$ NMR $\left(101 \mathrm{MHz}, \mathrm{CDCl}_{3}\right): \delta_{\mathrm{C}} 165.6,134.4,129.3,123.5,90.5,34.3,32.1,31.5,28.5,22.6,12.4$ ppm. $\underline{\text { See spectrum. }}$.

IR (film) $v_{\max }: 2950,2856,1790,1730(\mathrm{~s}), 1466,1365,1188,1115,1079,978,878,701 \mathrm{~cm}^{-1}$.

HRMS (ESI) m/z: [M + Na $]^{+}$Calcd for $\mathrm{C}_{17} \mathrm{H}_{21} \mathrm{NNaO}_{3}$ 310.1414; Found 310.1421.

M.p.: $84-86^{\circ} \mathrm{C}$.

\section{2-(1-Phenethylcyclobutoxy)isoindoline-1,3-dione (10a)}

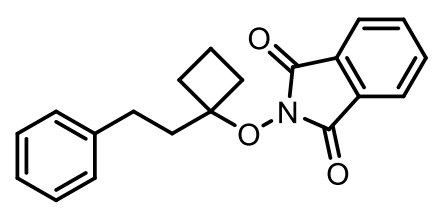

Prepared according to GP1: $\mathrm{LaCl}_{3} \cdot 2 \mathrm{LiCl}(9.0 \mathrm{mmol}$, 1.5 equiv.), phenethylmagnesium bromide (9.0 mmol, 1.5 equiv.), and cyclobutanone ( $420 \mathrm{mg}, 6.00 \mathrm{mmol}, 1.0$ equiv.) were stirred at room temperature for $10 \mathrm{~h}$. Purification by column chromatography (20\% EtOAc/hexane) gave 1-phenethylcyclobutan-1ol (1.04 g, $5.92 \mathrm{mmol}, 98 \%)$ as a bright yellow oil. 1-Phenethylcyclobutan-1-ol (881.5 mg, $5.00 \mathrm{mmol}$, 1.0 equiv.) was then treated with $N$-hydroxyphthalimide $\left(1.63 \mathrm{~g}, 10.0 \mathrm{mmol}, 2.0\right.$ equiv.) and $\mathrm{BF}_{3} \cdot \mathrm{OEt}_{2}$ 
(781 mg, $5.50 \mathrm{mmol}, 1.1$ equiv.) in wet DCM $(20 \mathrm{~mL})$ at room temperature for $4 \mathrm{~h}$. Purification by column chromatography (20\% EtOAc/hexane) gave 10a (340 $\mathrm{mg}, 1.05 \mathrm{mmol}, 21 \%)$ as a yellow oil.

TLC: $\mathrm{R}_{\mathrm{f}}=0.50$ (20\% EtOAc/hexane, $\mathrm{KMnO}_{4}$ stain) .

${ }^{1} \mathbf{H}$ NMR $\left(400 \mathrm{MHz}, \mathrm{CDCl}_{3}\right): \delta_{\mathrm{H}} 7.91-7.83(\mathrm{~m}, 2 \mathrm{H}), 7.80-7.73(\mathrm{~m}, 2 \mathrm{H}), 7.33-7.27(\mathrm{~m}, 4 \mathrm{H}), 7.23-$ $7.17(\mathrm{~m}, 1 \mathrm{H}), 3.02-2.96(\mathrm{~m}, 2 \mathrm{H}), 2.47-2.38(\mathrm{~m}, 2 \mathrm{H}), 2.18-2.12(\mathrm{~m}, 2 \mathrm{H}), 2.12-2.04(\mathrm{~m}, 2 \mathrm{H}), 1.80$ (qt, $J=10.4,3.5 \mathrm{~Hz}, 1 \mathrm{H}), 1.64-1.52(\mathrm{~m}, 1 \mathrm{H}) \mathrm{ppm} . \underline{\text { See spectrum }}$.

${ }^{13} \mathrm{C}$ NMR (101 MHz, $\left.\mathrm{CDCl}_{3}\right): \delta_{\mathrm{C}} 165.6,142.5,134.5,129.3,128.5,128.4,125.7,123.5,90.1,38.6$, $31.4,29.7,12.5 \mathrm{ppm}$. See spectrum.

IR (film) $v_{\max }$ : 2981, 2958, 1791, 1733(s), 1610, 1497, 1467, 1454, 1371, 1250, 1187, 1057, 1033, 877, $701 \mathrm{~cm}^{-1}$.

HRMS (ESI) m/z: [M + Na $]^{+}$Calcd for $\mathrm{C}_{20} \mathrm{H}_{19} \mathrm{NNaO}_{3}$ 344.1257; Found 344.1252.

\section{5-(1-((1,3-Dioxoisoindolin-2-yl)oxy)cyclobutyl)pentyl 4-methylbenzenesulfonate (11a)}

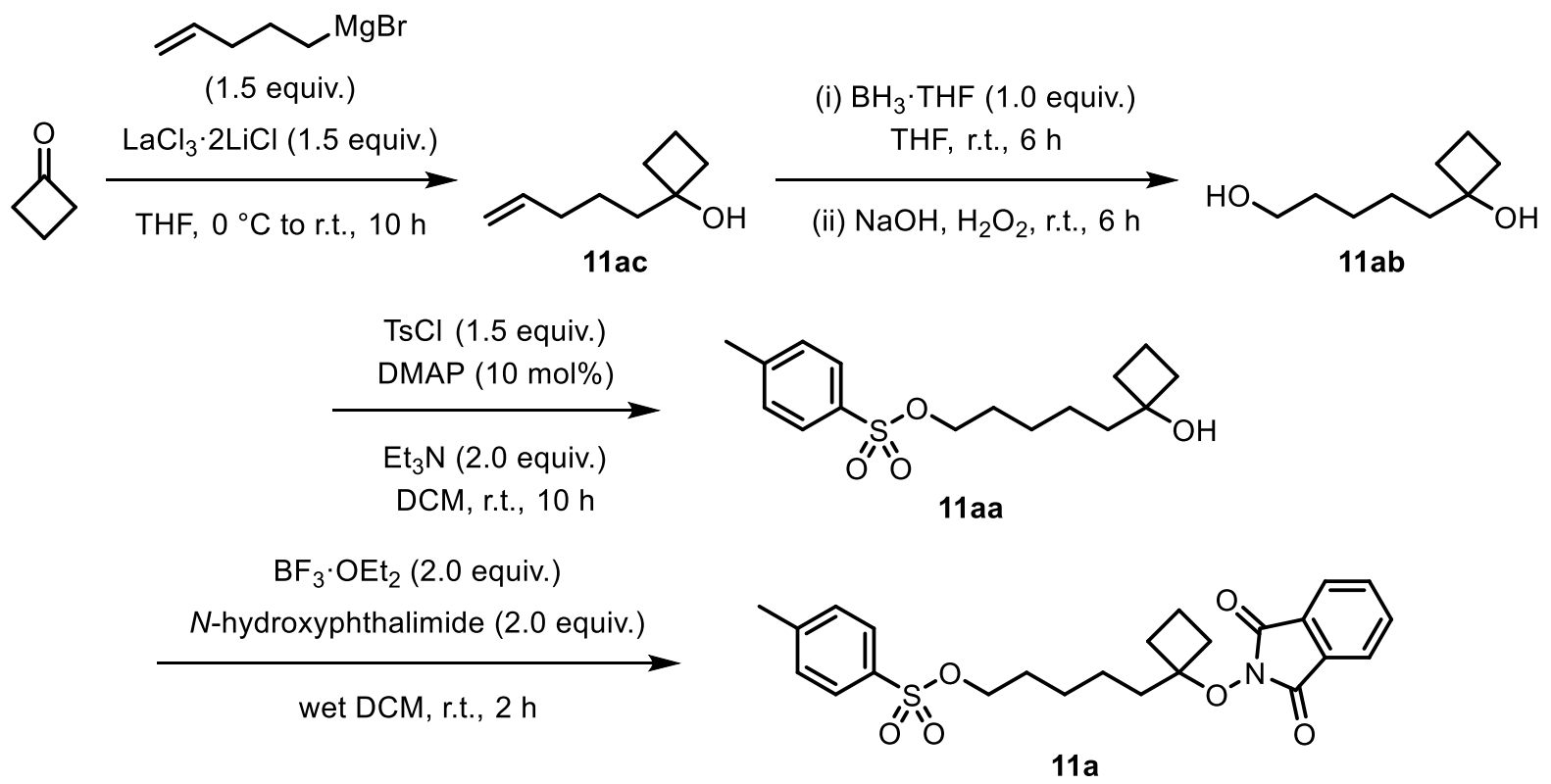

Tertiary alcohol 11ac was prepared according to GP1-A: $\mathrm{LaCl}_{3} \cdot 2 \mathrm{LiCl}$ (18 mmol, 1.5 equiv.), pent-4en-1-ylmagnesium bromide (18 mmol, 1.5 equiv.), and cyclobutanone ( $841 \mathrm{mg}, 12.0 \mathrm{mmol}, 1.0$ equiv.) were stirred at room temperature for $10 \mathrm{~h}$. Purification by column chromatography (20\% EtOAc/hexane) gave 11ac (1.68 g, $12.0 \mathrm{mmol}, 99 \%)$ as a bright yellow oil.

Diol 11ab was prepared following a modified literature procedure: ${ }^{4}$ To a solution of 11ac $(2.10 \mathrm{~g}, 15.0$ mmol, 1.0 equiv.) in dry THF (20 mL) was added borane (1.0 M in THF, $15 \mathrm{~mL}, 1.0$ equiv.) dropwise at $0{ }^{\circ} \mathrm{C}$ under a $\mathrm{N}_{2}$ atmosphere. The reaction was stirred for $1 \mathrm{~h}$ at $0{ }^{\circ} \mathrm{C}$ and $6 \mathrm{~h}$ at room temperature. The reaction was cooled to $0{ }^{\circ} \mathrm{C}$ and water $(10 \mathrm{~mL})$ was added dropwise followed by aqueous $\mathrm{NaOH}(3 \mathrm{~N}$, 
$12 \mathrm{~mL})$ and $\mathrm{H}_{2} \mathrm{O}_{2}(30 \%$ aqueous, $10 \mathrm{~mL})$. The resulting mixture was stirred for $0.5 \mathrm{~h}$ at $0{ }^{\circ} \mathrm{C}$ and $6 \mathrm{~h}$ at room temperature. The solution was saturated with solid $\mathrm{NaCl}$ and extracted with $\mathrm{Et}_{2} \mathrm{O}(3 \times 50 \mathrm{~mL})$. The combined organic extracts were washed with brine, dried over $\mathrm{MgSO}_{4}$, filtered, and concentrated under reduced pressure. Purification by column chromatography (40\% EtOAc/hexane) afforded 11ab as a $(1.66 \mathrm{~g}, 10.5 \mathrm{mmol}, 70 \%)$ colorless oil.

To a solution of 11ab ( $316 \mathrm{mg}, 2.0 \mathrm{mmol}, 1.0$ equiv.) and triethylamine ( $0.40 \mathrm{~g}, 4.0 \mathrm{mmol}, 2.0$ equiv.) in dry DCM $(30 \mathrm{~mL})$ was slowly added para-toluenesulfonyl chloride $(\mathrm{TsCl}, 572 \mathrm{mg}, 3.0 \mathrm{mmol}, 1.5$ equiv.) and 4-(dimethylamino)pyridine (DMAP, $24 \mathrm{mg}, 0.20 \mathrm{mmol}, 10 \mathrm{~mol} \%$ ) at $0{ }^{\circ} \mathrm{C}$. The solution was stirred at room temperature for $10 \mathrm{~h}$. Then, the reaction mixture was diluted with water $(50 \mathrm{~mL})$ and extracted with DCM $(3 \times 50 \mathrm{~mL})$. The combined organic extracts were washed with brine, dried over $\mathrm{MgSO}_{4}$, filtered, and concentrated under reduced pressure. Purification by column chromatography (20\% EtOAc/hexane) afforded 11 aa (540 $\mathrm{mg}, 1.73 \mathrm{mmol}, 86 \%)$ as a yellow oil.

$N$-Alkoxyphthalimide 11a was prepared from 11aa according to GP1-B: Cyclobutanol 11aa (469 mg, $1.50 \mathrm{mmol}, 1.0$ equiv.) was treated with $N$-hydroxyphthalimide (489 mg, $3.00 \mathrm{mmol}, 2.0$ equiv.) and $\mathrm{BF}_{3}$. $\mathrm{OEt}_{2}(426 \mathrm{mg}, 3.00 \mathrm{mmol}, 2.0$ equiv. $)$ in wet DCM $(20 \mathrm{~mL})$ at room temperature for $2 \mathrm{~h}$. Purification by column chromatography (20\% EtOAc/hexane) gave 11a $(500 \mathrm{mg}, 1.09 \mathrm{mmol}, 72 \%)$ as a pale-yellow oil.

TLC: $\mathrm{R}_{\mathrm{f}}=0.40\left(20 \% \mathrm{EtOAc} / \mathrm{hexane}, \mathrm{KMnO}_{4}\right.$ stain $)$.

${ }^{1} \mathbf{H}$ NMR $\left(400 \mathrm{MHz}, \mathrm{CDCl}_{3}\right): \delta_{\mathrm{H}} 7.88-7.80(\mathrm{~m}, 2 \mathrm{H}), 7.83-7.76(\mathrm{~m}, 2 \mathrm{H}), 7.79-7.72(\mathrm{~m}, 2 \mathrm{H}), 7.38-$ $7.30(\mathrm{~m}, 2 \mathrm{H}), 4.06(\mathrm{t}, J=6.5 \mathrm{~Hz}, 2 \mathrm{H}), 2.44(\mathrm{~s}, 3 \mathrm{H}), 2.39-2.27(\mathrm{~m}, 2 \mathrm{H}), 2.02-1.91(\mathrm{~m}, 2 \mathrm{H}), 1.81-$ $1.65(\mathrm{~m}, 5 \mathrm{H}), 1.64-1.51(\mathrm{~m}, 2 \mathrm{H}), 1.53-1.42(\mathrm{~m}, 1 \mathrm{H}), 1.44-1.34(\mathrm{~m}, 2 \mathrm{H}) \mathrm{ppm}$. See spectrum.

${ }^{13} \mathrm{C}$ NMR $\left(101 \mathrm{MHz}, \mathrm{CDCl}_{3}\right): \delta_{\mathrm{C}} 165.5,144.6,134.5,133.3,129.8,129.2,127.9,123.5,90.1,70.7$, 36.1, 31.3, 28.8, 25.7, 22.6, 21.6, 12.3 ppm. See spectrum.

IR (film) $v_{\max }: 2973,2923,1791,1732(\mathrm{~s}), 1598,1467,1356,1187,1176,1056,1033,877,705 \mathrm{~cm}^{-1}$.

HRMS (ESI) m/z: [M + Na] ${ }^{+}$Calcd for $\mathrm{C}_{24} \mathrm{H}_{27} \mathrm{NNaO}_{6} \mathrm{~S} 480.1451$; Found 480.1458 . 


\section{5-(1-((1,3-Dioxoisoindolin-2-yl)oxy)cyclobutyl)pentyl 4-methylbenzenesulfonate (12a)}
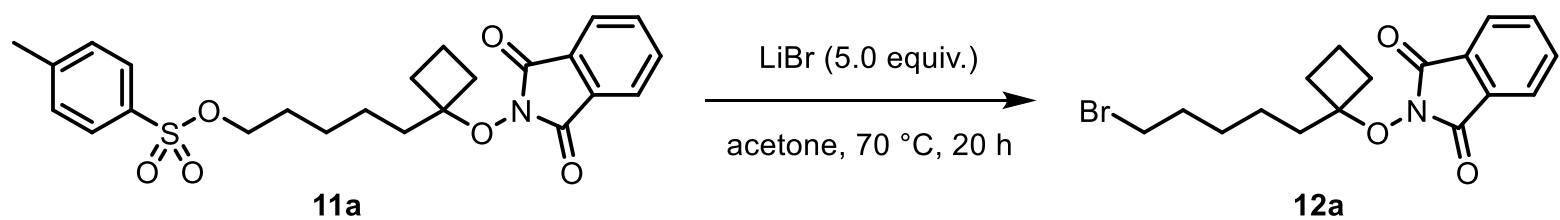

To a solution of $N$-alkoxyphthalimide 11a (137 mg, $0.299 \mathrm{mmol}, 1.0$ equiv.) in acetone ( $5 \mathrm{~mL}$ ) was added $\mathrm{LiBr}\left(130 \mathrm{mg}, 1.50 \mathrm{mmol}, 5.0\right.$ equiv.) at room temperature. The reaction was stirred at $70{ }^{\circ} \mathrm{C}$ for $20 \mathrm{~h}$. After allowing to cool to room temperature, DCM $(20 \mathrm{~mL})$ and water $(20 \mathrm{~mL})$ was added. The aqueous layer was extracted with DCM $(3 \times 30 \mathrm{~mL})$, the combined organic layers were dried over $\mathrm{MgSO}_{4}$, filtered, and concentrated under reduced pressure. The residue was purified by column chromatography (20\% EtOAc/hexane) to give product 12a $(88 \mathrm{mg}, 0.24 \mathrm{mmol}, 80 \%)$ as a pale-yellow oil.

TLC: $\mathrm{R}_{\mathrm{f}}=0.50\left(20 \%\right.$ EtOAc/hexane, $\mathrm{KMnO}_{4}$ stain $)$.

${ }^{1} \mathbf{H}$ NMR $\left(400 \mathrm{MHz}, \mathrm{CDCl}_{3}\right): \delta_{\mathrm{H}} 7.90-7.81(\mathrm{~m}, 2 \mathrm{H}), 7.81-7.72(\mathrm{~m}, 2 \mathrm{H}), 3.45(\mathrm{t}, J=6.8 \mathrm{~Hz}, 2 \mathrm{H}), 2.41$ $-2.31(\mathrm{~m}, 2 \mathrm{H}), 2.05-1.97(\mathrm{~m}, 2 \mathrm{H}), 1.96-1.90(\mathrm{~m}, 2 \mathrm{H}), 1.87-1.81(\mathrm{~m}, 2 \mathrm{H}), 1.79-1.62(\mathrm{~m}, 3 \mathrm{H}), 1.56$ $-1.46(\mathrm{~m}, 3 \mathrm{H}) \mathrm{ppm}$. See spectrum.

${ }^{13} \mathrm{C}$ NMR $\left(101 \mathrm{MHz}, \mathrm{CDCl}_{3}\right): \delta_{\mathrm{C}} 165.6,134.5,129.3,123.5,90.2,36.2,34.0,32.8,31.4,28.6,22.5$, $12.4 \mathrm{ppm} . \underline{\text { See spectrum. }}$.

IR (film) $v_{\max }: 2978,2923,1791,1732(\mathrm{~s}), 1467,1372,1251,1057,903,725 \mathrm{~cm}^{-1}$.

HRMS (ESI) m/z: [M + Na $]^{+}$Calcd for $\mathrm{C}_{17} \mathrm{H}_{20} \mathrm{BrNNaO}_{3}$ 388.0519; Found 388.0533. 


\section{3-(1-((1,3-Dioxoisoindolin-2-yl)oxy)cyclobutyl)propyl benzoate (13a)}
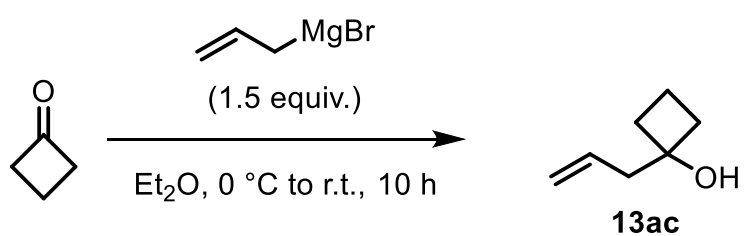

(i) $\mathrm{BH}_{3} \cdot \mathrm{THF}(1.0$ equiv.)

THF, r.t., $6 \mathrm{~h}$

(ii) $\mathrm{NaOH}, \mathrm{H}_{2} \mathrm{O}_{2}$, r.t., $6 \mathrm{~h}$
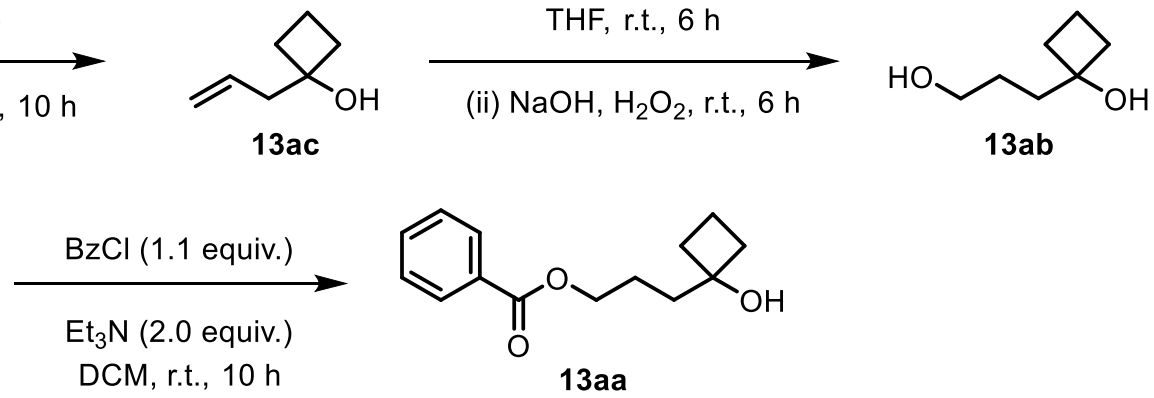

$\mathrm{BF}_{3} \cdot \mathrm{OEt}_{2}$ (2.0 equiv.)

$N$-hydroxyphthalimide (2.0 equiv.)

wet DCM, r.t., $10 \mathrm{~h}$

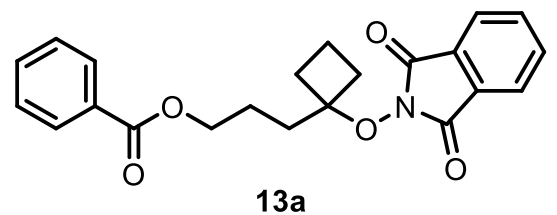

To a flame-dried, $\mathrm{N}_{2}$-flushed $50 \mathrm{~mL}$ Schlenk flask equipped with a septum and a magnetic stirrer bar was placed cyclobutanone (701 mg, $10.0 \mathrm{mmol}, 1.0$ equiv.) in dry $\mathrm{Et}_{2} \mathrm{O}(20 \mathrm{~mL})$ at $0{ }^{\circ} \mathrm{C}$. Allylmagnesium bromide (15.0 mmol, 1.50 equiv.) was added dropwise and the resulting mixture was stirred for $10 \mathrm{~h}$ at room temperature. Saturated aqueous $\mathrm{NH}_{4} \mathrm{Cl}(15 \mathrm{~mL})$ was added. The layers were separated, the aqueous layer was extracted with DCM $(3 \times 50 \mathrm{~mL})$, the combined organic layers were dried over $\mathrm{MgSO}_{4}$, filtered, and concentrated under reduced pressure. The crude product was purified by column chromatography (20\% EtOAc/hexane) to give 13ac (800 mg, $7.13 \mathrm{mmol}, 71 \%$ ) as a bright yellow oil.

To a solution of $13 \mathbf{a c}(1.68 \mathrm{~g}, 15.0 \mathrm{mmol}, 1.0$ equiv.) in dry THF $(20 \mathrm{~mL})$ was added borane $(1.0 \mathrm{M}$ in THF, $15 \mathrm{~mL}, 1.0$ equiv.) dropwise at $0{ }^{\circ} \mathrm{C}$ under a $\mathrm{N}_{2}$ atmosphere. The reaction was stirred for $1 \mathrm{~h}$ at 0 ${ }^{\circ} \mathrm{C}$ and $6 \mathrm{~h}$ at room temperature. The reaction was cooled to $0{ }^{\circ} \mathrm{C}$ and water $(10 \mathrm{~mL})$ was added dropwise followed by aqueous $\mathrm{NaOH}(3 \mathrm{~N}, 12 \mathrm{~mL})$ and $\mathrm{H}_{2} \mathrm{O}_{2}(30 \%$ aqueous, $10 \mathrm{~mL})$. The resulting mixture was stirred for $0.5 \mathrm{~h}$ at $0{ }^{\circ} \mathrm{C}$ and $6 \mathrm{~h}$ at room temperature. The solution was saturated with solid $\mathrm{NaCl}$ and extracted with $\mathrm{Et}_{2} \mathrm{O}(3 \times 50 \mathrm{~mL})$. The combined organic extracts were washed with brine, dried over $\mathrm{MgSO}_{4}$, filtered, and concentrated under reduced pressure. Purification by column chromatography (40\% EtOAc/hexane) afforded 13ab as a colorless oil (1.24 g, $9.52 \mathrm{mmol}, 63 \%)$.

To a solution of 13ab $(1.82 \mathrm{~g}, 14.0 \mathrm{mmol}, 1.0$ equiv. $)$ and triethylamine ( $2.83 \mathrm{~g}, 28.0 \mathrm{mmol}, 2.0$ equiv.) in dry DCM (40 mL) was slowly added benzoyl chloride $(2.17 \mathrm{~g}, 15.4 \mathrm{mmol}, 1.1$ equiv. $)$ at $0{ }^{\circ} \mathrm{C}$. The solution was stirred at room temperature for $10 \mathrm{~h}$. Then, the reaction mixture was diluted with water (50 $\mathrm{mL})$ and extracted with DCM $(3 \times 30 \mathrm{~mL})$. The combined organic extracts were washed with brine, dried over $\mathrm{MgSO}_{4}$, filtered, and concentrated under reduced pressure. Purification by column chromatography (20\% EtOAc/hexane) afforded 13aa (2.45 g, $10.5 \mathrm{mmol}, 75 \%)$ as a yellow oil. 
$N$-Alkoxyphthalimide 13a was prepared from 13aa according to GP1-B: Cyclobutanol 13aa (2.34 g, $10.0 \mathrm{mmol}, 1.0$ equiv.) was treated with $N$-hydroxyphthalimide ( $3.26 \mathrm{~g}, 20.0 \mathrm{mmol}, 2.0$ equiv.) and $\mathrm{BF}_{3} \cdot \mathrm{OEt}_{2}(2.84 \mathrm{~g}, 20.0 \mathrm{mmol}, 2.0$ equiv. $)$ in wet $\mathrm{DCM}(50 \mathrm{~mL})$ at room temperature for $10 \mathrm{~h}$. Purification by column chromatography (20\% EtOAc/hexane) gave 13a $(2.47 \mathrm{~g}, 6.51 \mathrm{mmol}, 65 \%)$ as a yellow oil.

TLC: $\mathrm{R}_{\mathrm{f}}=0.51\left(20 \%\right.$ EtOAc/hexane, $\mathrm{KMnO}_{4}$ stain $)$.

${ }^{1} \mathbf{H}$ NMR $\left(400 \mathrm{MHz}, \mathrm{CDCl}_{3}\right): \delta_{\mathrm{H}} 8.09-8.05(\mathrm{~m}, 2 \mathrm{H}), 7.88-7.82(\mathrm{~m}, 2 \mathrm{H}), 7.79-7.73(\mathrm{~m}, 2 \mathrm{H}), 7.58-$ $7.53(\mathrm{~m}, 1 \mathrm{H}), 7.47-7.41(\mathrm{~m}, 2 \mathrm{H}), 4.44(\mathrm{t}, J=6.5 \mathrm{~Hz}, 2 \mathrm{H}), 2.46-2.35(\mathrm{~m}, 2 \mathrm{H}), 2.20-2.11(\mathrm{~m}, 2 \mathrm{H})$, $2.09-1.97(\mathrm{~m}, 4 \mathrm{H}), 1.78(\mathrm{dtt}, J=11.1,10.2,3.4 \mathrm{~Hz}, 1 \mathrm{H}), 1.61-1.48(\mathrm{~m}, 1 \mathrm{H}) \mathrm{ppm}$. See spectrum.

${ }^{13} \mathrm{C}$ NMR $\left(101 \mathrm{MHz}, \mathrm{CDCl}_{3}\right): \delta_{\mathrm{C}} 166.7,165.5,134.5,132.8,130.5,129.6,129.3,128.3,123.5,89.9$, $65.3,32.9,31.4,23.0,12.4$ ppm. See spectrum.

IR (film) $v_{\max }$ : 2953, 1792, 1733(s), 1714, 1602, 1468, 1452, 1372, 1276, 1187, 1113, 978, $904 \mathrm{~cm}^{-1}$.

HRMS (ESI) m/z: [M + Na $]^{+}$Calcd for $\mathrm{C}_{22} \mathrm{H}_{21} \mathrm{NNaO}_{5}$ 402.1312; Found 402.1306.

\section{3-(1-((1,3-Dioxoisoindolin-2-yl)oxy)cyclobutyl)propyl 1-naphthoate (14a)}
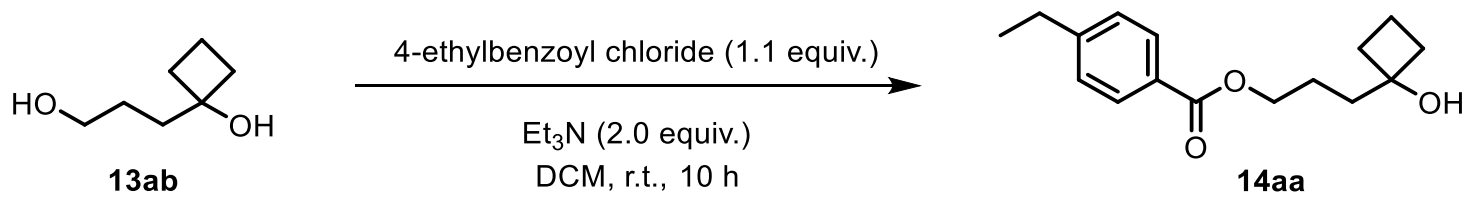

$\mathrm{BF}_{3} \cdot \mathrm{OEt}_{2}$ (2.0 equiv.)

$N$-hydroxyphthalimide (2.0 equiv.)

wet DCM, r.t., $2 \mathrm{~h}$

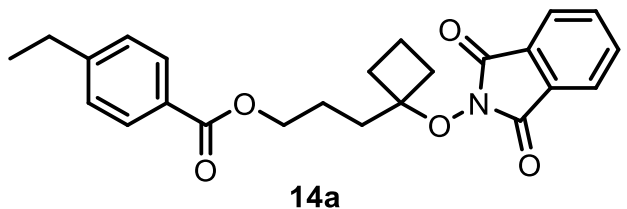

To a solution of 13ab (326 mg, $2.5 \mathrm{mmol}, 1.0$ equiv.) and triethylamine ( $0.50 \mathrm{~g}, 5.0 \mathrm{mmol}, 2.0$ equiv.) in dry DCM $(20 \mathrm{~mL})$ was slowly added 4-ethylbenzoyl chloride $\left(0.46 \mathrm{~g}, 2.8 \mathrm{mmol}, 1.1\right.$ equiv.) at $0{ }^{\circ} \mathrm{C}$. The solution was stirred at room temperature for $10 \mathrm{~h}$. Then, the reaction mixture was diluted with water $(50 \mathrm{~mL})$ and extracted with DCM $(3 \times 50 \mathrm{~mL})$. The combined organic extracts were washed with brine, dried over $\mathrm{MgSO}_{4}$, filtered, and concentrated under reduced pressure. Purification by column chromatography (20\% EtOAc/hexane) gave 14aa (483 mg, $1.84 \mathrm{mmol}, 74 \%)$ as a yellow oil.

$N$-Alkoxyphthalimide 14a was prepared from 14aa according to GP1-B: Cyclobutanol 14aa (472 $\mathrm{mg}$, $1.80 \mathrm{mmol}, 1.0$ equiv.) was treated with $N$-hydroxyphthalimide (587 mg, $3.60 \mathrm{mmol}, 2.0$ equiv.) and $\mathrm{BF}_{3} \cdot \mathrm{OEt}_{2}(511 \mathrm{mg}, 3.60 \mathrm{mmol}, 2.0$ equiv. $)$ in wet $\mathrm{DCM}(20 \mathrm{~mL})$ at room temperature for $2 \mathrm{~h}$. Purification by column chromatography (20\% EtOAc/hexane) gave 14a (445 mg, $1.10 \mathrm{mmol}, 61 \%$ ) as a yellow oil. 
TLC: $\mathrm{R}_{\mathrm{f}}=0.30\left(20 \% \mathrm{EtOAc} / \mathrm{hexane}, \mathrm{KMnO}_{4}\right.$ stain $)$.

${ }^{1} \mathbf{H}$ NMR $\left(400 \mathrm{MHz}, \mathrm{CDCl}_{3}\right): \delta_{\mathrm{H}} 8.01-7.94(\mathrm{~m}, 2 \mathrm{H}), 7.88-7.80(\mathrm{~m}, 2 \mathrm{H}), 7.81-7.71(\mathrm{~m}, 2 \mathrm{H}), 7.29-$ $7.22(\mathrm{~m}, 2 \mathrm{H}), 4.41(\mathrm{t}, J=6.5 \mathrm{~Hz}, 2 \mathrm{H}), 2.70(\mathrm{q}, J=7.6 \mathrm{~Hz}, 2 \mathrm{H}), 2.45-2.35(\mathrm{~m}, 2 \mathrm{H}), 2.19-2.10(\mathrm{~m}$, 2H), $2.09-1.96(\mathrm{~m}, 4 \mathrm{H}), 1.77(\mathrm{dtt}, J=11.2,10.2,3.3 \mathrm{~Hz}, 1 \mathrm{H}), 1.54(\mathrm{dp}, J=11.1,8.8 \mathrm{~Hz}, 1 \mathrm{H}), 1.25(\mathrm{t}$, $J=7.6 \mathrm{~Hz}, 3 \mathrm{H}) \mathrm{ppm} . \underline{\text { See spectrum. }}$.

${ }^{13} \mathrm{C}$ NMR $\left(101 \mathrm{MHz}, \mathrm{CDCl}_{3}\right): \delta_{\mathrm{C}} 166.7,165.5,149.6,134.5,129.7,129.3,128.0,127.8,123.5,89.9$, 65.0, 32.9, 31.4, 28.9, 23.0, 15.2, 12.4 ppm. See spectrum.

IR (film) $v_{\max }$ 2972, 2901, 1791, 1733(s), 1611, 1468, 1453, 1374, 1276, 1056, 904, $725 \mathrm{~cm}^{-1}$.

HRMS (ESI) m/z: [M + Na $]^{+}$Calcd for $\mathrm{C}_{24} \mathrm{H}_{25} \mathrm{NNaO}_{5} 430.1625$; Found 430.1615.

\section{3-(1-((1,3-Dioxoisoindolin-2-yl)oxy)cyclobutyl)propyl 4-chlorobenzoate (15a)}

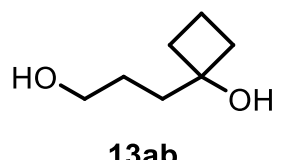

$13 \mathrm{ab}$

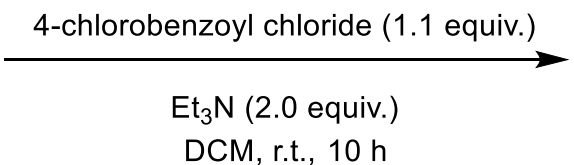

DCM, r.t., $10 \mathrm{~h}$

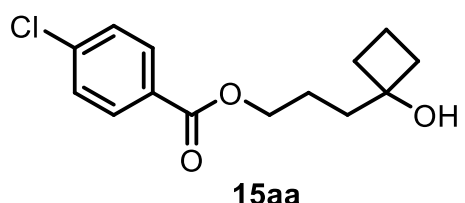

15 aa

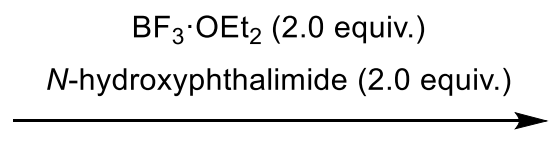

wet DCM, r.t., $8 \mathrm{~h}$

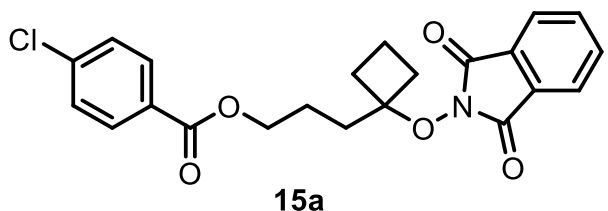

To a solution of 13ab (260 mg, $2.0 \mathrm{mmol}, 1.0$ equiv.) and triethylamine ( $0.40 \mathrm{~g}, 4.0 \mathrm{mmol}, 2.0$ equiv.) in dry DCM (20 mL) was slowly added 4-chlorobenzoyl chloride $\left(0.38 \mathrm{~g}, 2.2 \mathrm{mmol}, 1.1\right.$ equiv.) at $0{ }^{\circ} \mathrm{C}$. The solution was stirred at room temperature for $10 \mathrm{~h}$. Then, the reaction mixture was diluted with water $(50 \mathrm{~mL})$ and extracted with DCM $(3 \times 50 \mathrm{~mL})$. The combined organic extracts were washed with brine, dried over $\mathrm{MgSO}_{4}$, filtered, and concentrated under reduced pressure. Purification by column chromatography (20\% EtOAc/hexane) gave 15aa (526 mg, $1.96 \mathrm{mmol}, 98 \%$ ) as a yellow oil.

$N$-Alkoxyphthalimide 15a was prepared from 15aa according to GP1-B: Cyclobutanol 15aa (483.7 mg, $1.80 \mathrm{mmol}, 1.0$ equiv.) was treated with $N$-hydroxyphthalimide (587 mg, $3.6 \mathrm{mmol}, 2.0$ equiv.) and $\mathrm{BF}_{3} \cdot \mathrm{OEt}_{2}(511 \mathrm{mg}, 3.60 \mathrm{mmol}, 2.0$ equiv. $)$ in wet $\mathrm{DCM}(20 \mathrm{~mL})$ at room temperature for $8 \mathrm{~h}$. Purification by column chromatography (40\% EtOAc/hexane) gave 15a (410 mg, $0.99 \mathrm{mmol}, 55 \%$ ) as a white solid.

TLC: $\mathrm{R}_{\mathrm{f}}=0.40$ (30\% EtOAc/hexane, $\mathrm{KMnO}_{4}$ stain) . 
${ }^{1} \mathbf{H}$ NMR $\left(400 \mathrm{MHz}, \mathrm{CDCl}_{3}\right): \delta_{\mathrm{H}} 8.04-7.96(\mathrm{~m}, 2 \mathrm{H}), 7.89-7.80(\mathrm{~m}, 2 \mathrm{H}), 7.82-7.72(\mathrm{~m}, 2 \mathrm{H}), 7.45-$ $7.37(\mathrm{~m}, 2 \mathrm{H}), 4.43(\mathrm{t}, J=6.5 \mathrm{~Hz}, 2 \mathrm{H}), 2.47-2.35(\mathrm{~m}, 2 \mathrm{H}), 2.21-2.10(\mathrm{~m}, 2 \mathrm{H}), 2.10-1.94(\mathrm{~m}, 4 \mathrm{H})$, $1.79(\mathrm{dtt}, J=11.0,10.2,3.4 \mathrm{~Hz}, 1 \mathrm{H}), 1.55(\mathrm{dp}, J=11.2,8.8 \mathrm{~Hz}, 1 \mathrm{H}) \mathrm{ppm}$. See spectrum.

${ }^{13} \mathrm{C}$ NMR $\left(101 \mathrm{MHz}, \mathrm{CDCl}_{3}\right): \delta_{\mathrm{C}} 165.7,165.5,139.2,134.5,130.9,129.2,128.9,128.6,123.5,89.8$, $65.5,32.8,31.4,22.9,12.4$ ppm. See spectrum.

IR (film) $v_{\max }:$ 2972, 2923, 1790, 1733(s), 1591, 1468, 1372, 1273, 1057, 1033, 906, 878, $726 \mathrm{~cm}^{-1}$.

HRMS (ESI) m/z: [M + Na $]^{+}$Calcd for $\mathrm{C}_{22} \mathrm{H}_{20} \mathrm{NClNaO}_{5}$ 436.0922; Found 436.0902.

M.p.: $86-88^{\circ} \mathrm{C}$.

\section{3-(1-((1,3-Dioxoisoindolin-2-yl)oxy)cyclobutyl)propyl 3-bromobenzoate (16a)}
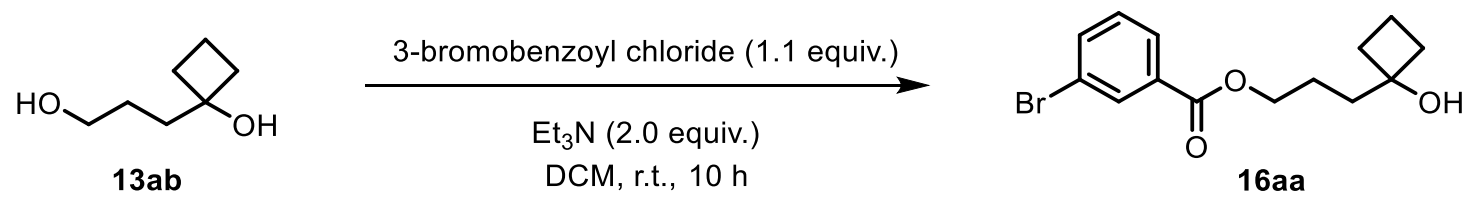

$\mathrm{BF}_{3} \cdot \mathrm{OEt}_{2}$ (2.0 equiv.)

$N$-hydroxyphthalimide (2.0 equiv.)

wet DCM, r.t., $10 \mathrm{~h}$

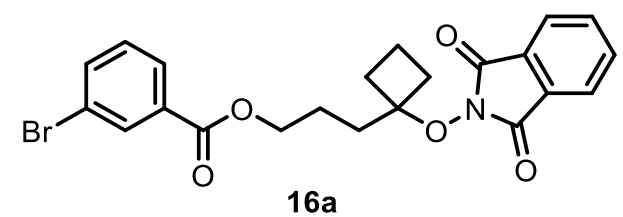

To a solution of 13ab (391 mg, $3.0 \mathrm{mmol}, 1.0$ equiv.) and triethylamine ( $0.60 \mathrm{~g}, 6.0 \mathrm{mmol}, 2.0$ equiv.) in dry DCM $(20 \mathrm{~mL})$ was slowly added 3-bromobenzoyl chloride $(0.72 \mathrm{~g}, 3.3 \mathrm{mmol}, 1.1$ equiv. $)$ at $0{ }^{\circ} \mathrm{C}$. The solution was stirred at room temperature for $10 \mathrm{~h}$. Then, the reaction mixture was diluted with water $(50 \mathrm{~mL})$ and extracted with DCM $(3 \times 30 \mathrm{~mL})$. The combined organic extracts were washed with brine, dried over $\mathrm{MgSO}_{4}$, filtered, and concentrated under reduced pressure. Purification by column chromatography (20\% EtOAc/hexane) gave 16aa (917.5 mg, $2.93 \mathrm{mmol}, 97 \%$ ) as a yellow oil.

$N$-Alkoxyphthalimide 16a was prepared from 16aa according to GP1-B: Cyclobutanol 16aa (783 mg, $2.50 \mathrm{mmol}, 1.0$ equiv.) was treated with $N$-hydroxyphthalimide $(816 \mathrm{mg}, 5.00 \mathrm{mmol}, 2.0$ equiv.) and $\mathrm{BF}_{3} \cdot \mathrm{OEt}_{2}(710 \mathrm{mg}, 5.00 \mathrm{mmol}, 2.0$ equiv.) in wet $\mathrm{DCM}(20 \mathrm{~mL})$ at room temperature for $10 \mathrm{~h}$. Purification by column chromatography (40\% EtOAc/hexane) gave 16a (846 mg, $1.84 \mathrm{mmol}, 74 \%$ ) as a pale-yellow oil.

TLC: $\mathrm{R}_{\mathrm{f}}=0.40\left(30 \%\right.$ EtOAc/hexane, $\mathrm{KMnO}_{4}$ stain) .

${ }^{1} \mathbf{H}$ NMR $\left(400 \mathrm{MHz}, \mathrm{CDCl}_{3}\right): \delta_{\mathrm{H}} 8.20(\mathrm{t}, J=1.7 \mathrm{~Hz}, 1 \mathrm{H}), 8.03-7.96(\mathrm{~m}, 1 \mathrm{H}), 7.89-7.81(\mathrm{~m}, 2 \mathrm{H}), 7.81$ $-7.72(\mathrm{~m}, 2 \mathrm{H}), 7.71-7.64(\mathrm{~m}, 1 \mathrm{H}), 7.32(\mathrm{t}, J=7.8 \mathrm{~Hz}, 1 \mathrm{H}), 4.44(\mathrm{t}, J=6.6 \mathrm{~Hz}, 2 \mathrm{H}), 2.47-2.35(\mathrm{~m}$, 
2H), $2.21-2.11(\mathrm{~m}, 2 \mathrm{H}), 2.09-1.95(\mathrm{~m}, 4 \mathrm{H}), 1.80(\mathrm{dtt}, J=11.1,10.2,3.4 \mathrm{~Hz}, 1 \mathrm{H}), 1.62-1.48(\mathrm{~m}$, 1H) ppm. See spectrum.

${ }^{13} \mathrm{C}$ NMR $\left(101 \mathrm{MHz}, \mathrm{CDCl}_{3}\right): \delta_{\mathrm{C}} 165.5,165.3,135.8,134.5,132.6,132.4,129.9,129.3,128.2,123.6$, $122.4,89.9,65.7,32.8,31.4,23.0,12.4$ ppm. See spectrum.

IR (film) $v_{\max }: 2973,2922,1790,1733(\mathrm{~s}), 1570,1468,1372,1281,1254,1057,1032,904,726 \mathrm{~cm}^{-1}$.

HRMS (ESI) m/z: [M + Na] ${ }^{+}$Calcd for $\mathrm{C}_{22} \mathrm{H}_{20} \mathrm{BrNNaO}_{5}$ 480.0417; Found 480.0404.

\section{3-(1-((1,3-Dioxoisoindolin-2-yl)oxy)cyclobutyl)propyl 1-naphthoate (17a)}
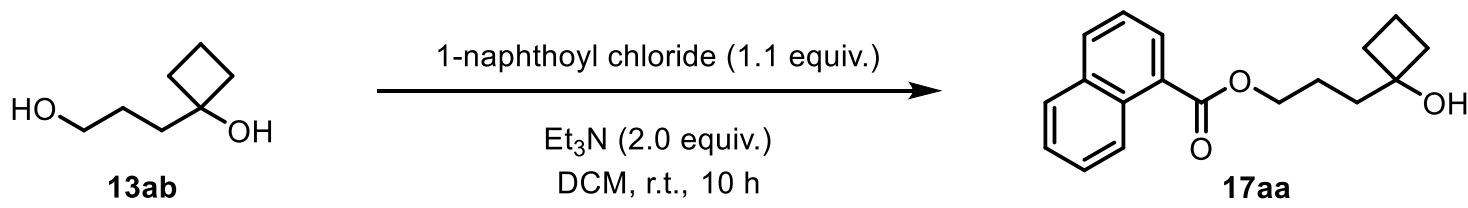

$\mathrm{BF}_{3} \cdot \mathrm{OEt}_{2}$ (2.0 equiv.)

$N$-hydroxyphthalimide ( 2.0 equiv.)

wet DCM, r.t., $6 \mathrm{~h}$

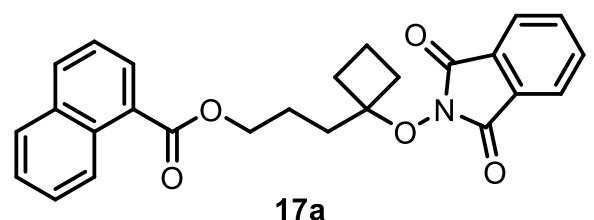

To a solution of 13ab $(260 \mathrm{mg}, 2.00 \mathrm{mmol}, 1.0$ equiv. $)$ and triethylamine ( $0.40 \mathrm{~g}, 4.0 \mathrm{mmol}, 2.0$ equiv.) in dry DCM $(20 \mathrm{~mL})$ was slowly added 1-naphthoyl chloride $(0.42 \mathrm{~g}, 2.2 \mathrm{mmol}, 1.1$ equiv. $)$ at $0{ }^{\circ} \mathrm{C}$. The solution was stirred at room temperature for $10 \mathrm{~h}$. Then, the reaction mixture was diluted with water (50 $\mathrm{mL})$ and extracted with DCM $(3 \times 30 \mathrm{~mL})$. The combined organic extracts were washed with brine, dried over $\mathrm{MgSO}_{4}$, filtered, and concentrated under reduced pressure. Purification by column chromatography (20\% EtOAc/hexane) gave 17aa (443 mg, $1.56 \mathrm{mmol}, 78 \%$ ) as a yellow oil.

$N$-Alkoxyphthalimide 17a was prepared from 17aa according to GP1-B: Cyclobutanol 17aa (427 mg, $1.50 \mathrm{mmol}, 1.0$ equiv.) was treated with $N$-hydroxyphthalimide ( $489 \mathrm{mg}, 3.00 \mathrm{mmol}, 2.0$ equiv.) and $\mathrm{BF}_{3} \cdot \mathrm{OEt}_{2}(426 \mathrm{mg}, 3.00 \mathrm{mmol}, 2.0$ equiv. $)$ in wet $\mathrm{DCM}(20 \mathrm{~mL})$ at room temperature for $6 \mathrm{~h}$. Purification by column chromatography (40\% EtOAc/hexane) gave 17a (335 mg, $0.78 \mathrm{mmol}, 52 \%$ ) as a yellow oil.

TLC: $\mathrm{R}_{\mathrm{f}}=0.35$ (20\% EtOAc/hexane, $\mathrm{KMnO}_{4}$ stain) .

${ }^{1} \mathbf{H}$ NMR (400 MHz, $\left.\mathrm{CDCl}_{3}\right): \delta_{\mathrm{H}} 8.94(\mathrm{dd}, J=8.7,1.1 \mathrm{~Hz}, 1 \mathrm{H}), 8.22(\mathrm{dd}, J=7.3,1.3 \mathrm{~Hz}, 1 \mathrm{H}), 8.02(\mathrm{~d}$, $J=8.3 \mathrm{~Hz}, 1 \mathrm{H}), 7.90-7.87(\mathrm{~m}, 1 \mathrm{H}), 7.86-7.81(\mathrm{~m}, 2 \mathrm{H}), 7.78-7.72(\mathrm{~m}, 2 \mathrm{H}), 7.64-7.59(\mathrm{~m}, 1 \mathrm{H})$, $7.56-7.48(\mathrm{~m}, 2 \mathrm{H}), 4.54(\mathrm{t}, J=6.5 \mathrm{~Hz}, 2 \mathrm{H}), 2.48-2.36(\mathrm{~m}, 2 \mathrm{H}), 2.27-2.18(\mathrm{~m}, 2 \mathrm{H}), 2.11-2.01(\mathrm{~m}$, 4H), 1.78 (dtt, $J=11.1,10.3,3.4 \mathrm{~Hz}, 1 \mathrm{H}), 1.55(\mathrm{dp}, J=11.1,8.6 \mathrm{~Hz}, 1 \mathrm{H}) \mathrm{ppm}$. See spectrum. 
${ }^{13} \mathrm{C}$ NMR (101 MHz, $\left.\mathrm{CDCl}_{3}\right): \delta_{\mathrm{C}} 167.6,165.5,134.5,133.8,133.2,131.4,130.1,129.3,128.5,127.7$, $127.4,126.1,125.9,124.5,123.5,89.9,65.4,33.0,31.4,23.1,12.4$ ppm. See spectrum.

IR (film) $v_{\max }$ : 2973, 2922, 2901, 1791, 1732(s), 1709(s), 1509, 1467, 1405, 1393, 1372, 1242, 1196, $1133,1051,906,783,727 \mathrm{~cm}^{-1}$.

HRMS (ESI) m/z: [M + Na $]^{+}$Calcd for $\mathrm{C}_{26} \mathrm{H}_{23} \mathrm{NNaO}_{5}$ 452.1468; Found 452.1448.

\section{3-(1-((1,3-Dioxoisoindolin-2-yl)oxy)cyclobutyl)propyl furan-2-carboxylate (18a)}
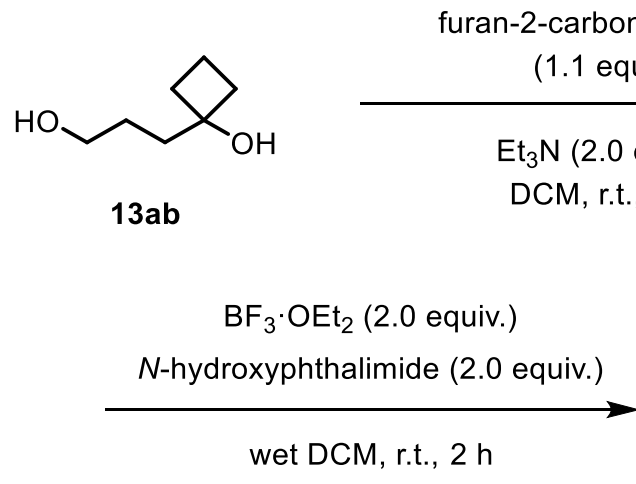

furan-2-carbonyl chloride

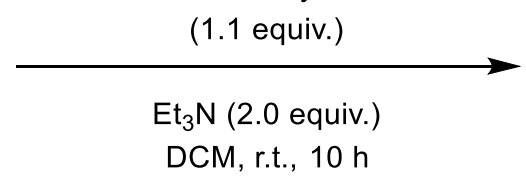

DCM, r.t., $10 \mathrm{~h}$

18 aa

To a solution of 13ab (260 mg, $2.00 \mathrm{mmol}, 1.0$ equiv.) and triethylamine ( $0.40 \mathrm{~g}, 4.0 \mathrm{mmol}, 2.0$ equiv.) in dry DCM $(20 \mathrm{~mL})$ was slowly added furan-2-carbonyl chloride $(0.28 \mathrm{~g}, 2.2 \mathrm{mmol}, 1.1$ equiv. $)$ at $0{ }^{\circ} \mathrm{C}$. The solution was stirred at room temperature for $10 \mathrm{~h}$. Then, the reaction mixture was diluted with water $(50 \mathrm{~mL})$ and extracted with DCM $(3 \times 30 \mathrm{~mL})$. The combined organic extracts were washed with brine, dried over $\mathrm{MgSO}_{4}$, filtered, and concentrated under reduced pressure. Purification by column chromatography (20\% EtOAc/hexane) gave 18aa (381 mg, $1.69 \mathrm{mmol}, 85 \%)$ as a yellow oil.

$N$-Alkoxyphthalimide 18a was prepared from 18aa according to GP1-B: Cyclobutanol 18aa (337 mg, $1.50 \mathrm{mmol}, 1.0$ equiv.) was treated with $N$-hydroxyphthalimide (489 mg, $3.00 \mathrm{mmol}, 2.0$ equiv.) and $\mathrm{BF}_{3} \cdot \mathrm{OEt}_{2}(426 \mathrm{mg}, 3.00 \mathrm{mmol}, 2.0$ equiv. $)$ in wet $\mathrm{DCM}(20 \mathrm{~mL})$ at room temperature for $2 \mathrm{~h}$. Purification by column chromatography (40\% EtOAc/hexane) gave $\mathbf{1 8 a}(249 \mathrm{mg}, 0.67 \mathrm{mmol}, 45 \%)$ as a colourless oil.

TLC: $\mathrm{R}_{\mathrm{f}}=0.40$ (30\% EtOAc/hexane, $\mathrm{KMnO}_{4}$ stain) .

${ }^{1} \mathbf{H}$ NMR $\left(400 \mathrm{MHz}, \mathrm{CDCl}_{3}\right): \delta_{\mathrm{H}} 7.88-7.79(\mathrm{~m}, 2 \mathrm{H}), 7.80-7.71(\mathrm{~m}, 2 \mathrm{H}), 7.56(\mathrm{dd}, J=1.8,0.9 \mathrm{~Hz}$, $1 \mathrm{H}), 7.18(\mathrm{dd}, J=3.5,0.8 \mathrm{~Hz}, 1 \mathrm{H}), 6.49(\mathrm{dd}, J=3.5,1.7 \mathrm{~Hz}, 1 \mathrm{H}), 4.40(\mathrm{t}, J=6.6 \mathrm{~Hz}, 2 \mathrm{H}), 2.43-2.33$ (m, 2H), $2.16-2.07(\mathrm{~m}, 2 \mathrm{H}), 2.07-1.99(\mathrm{~m}, 2 \mathrm{H}), 1.98-1.92(\mathrm{~m}, 2 \mathrm{H}), 1.76(\mathrm{dtt}, J=11.1,10.2,3.4 \mathrm{~Hz}$, $1 \mathrm{H}), 1.52(\mathrm{dp}, J=11.1,8.8 \mathrm{~Hz}, 1 \mathrm{H}) \mathrm{ppm}$. See spectrum. 
${ }^{13} \mathrm{C}$ NMR (101 MHz, $\left.\mathrm{CDCl}_{3}\right): \delta_{\mathrm{C}} 165.5,158.8,146.2,144.8,134.5,129.2,123.5,117.7,111.7,89.8$, 65.2, 32.7, 31.3, 23.0, 12.3 ppm. See spectrum.

IR (film) $v_{\max }: 2973,2923,1791,1732(\mathrm{~s}), 1581,1469,1372,1297,1183,1117,1056,906,726 \mathrm{~cm}^{-1}$.

HRMS (ESI) m/z: [M + Na $]^{+}$Calcd for $\mathrm{C}_{20} \mathrm{H}_{19} \mathrm{NNaO}_{6}$ 392.1105; Found 392.1104.

\section{3-(1-((1,3-Dioxoisoindolin-2-yl)oxy)cyclobutyl)propyl thiophene-3-carboxylate (19a)}
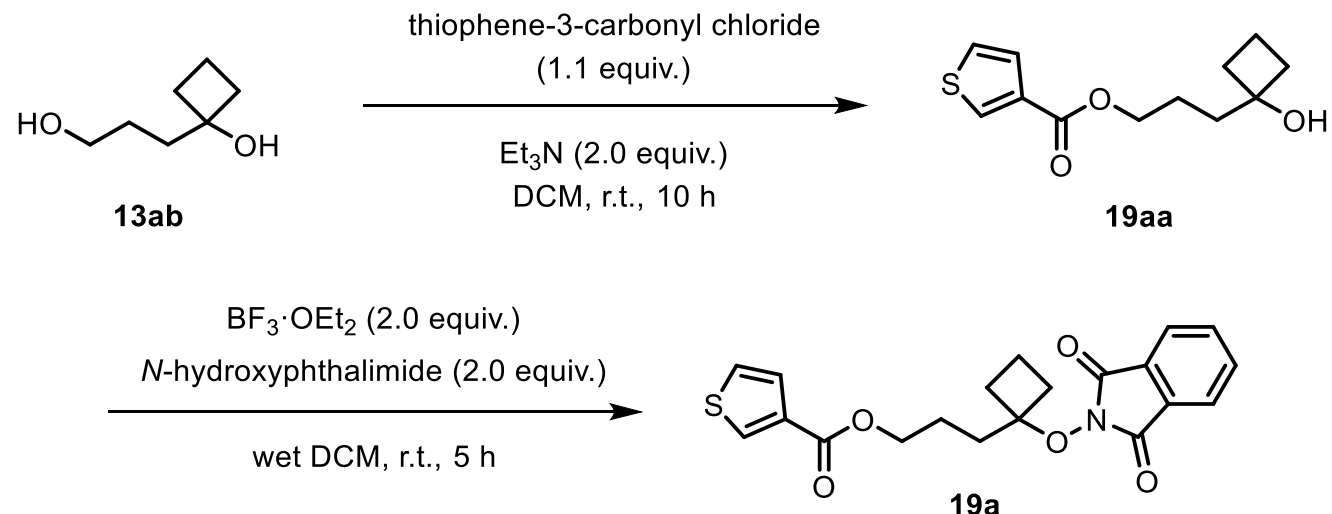

To a solution of 13ab (176 mg, $1.50 \mathrm{mmol}, 1.0$ equiv.) and triethylamine ( $0.30 \mathrm{~g}, 3.0 \mathrm{mmol}, 2.0$ equiv. $)$ in dry DCM (20 mL) was slowly added thiophene-3-carbonyl chloride (242 mg, $1.65 \mathrm{mmol}, 1.1$ equiv.) at $0{ }^{\circ} \mathrm{C}$. The solution was stirred at room temperature for $10 \mathrm{~h}$. Then, the reaction mixture was diluted with water $(50 \mathrm{~mL})$ and extracted with DCM $(3 \times 30 \mathrm{~mL})$. The combined organic extracts were washed with brine, dried over $\mathrm{MgSO}_{4}$, filtered, and concentrated under reduced pressure. Purification by column chromatography (20\% EtOAc/hexane) gave 19aa (290 mg, $1.21 \mathrm{mmol}, 80 \%)$ as a yellow oil.

$N$-Alkoxyphthalimide 19a was prepared from 19aa according to GP1-B: Cyclobutanol 19aa (288 mg, $1.20 \mathrm{mmol}, 1.0$ equiv.) was treated with $N$-hydroxyphthalimide (391 mg, $2.40 \mathrm{mmol}, 2.0$ equiv.) and $\mathrm{BF}_{3} \cdot \mathrm{OEt}_{2}(341 \mathrm{mg}, 2.40 \mathrm{mmol}, 2.0$ equiv. $)$ in wet $\mathrm{DCM}(20 \mathrm{~mL})$ at room temperature for $5 \mathrm{~h}$. Purification by column chromatography (20\% EtOAc/hexane) gave 19a (240 mg, $0.62 \mathrm{mmol}, 52 \%$ ) as a yellow oil.

TLC: $\mathrm{R}_{\mathrm{f}}=0.45$ (40\% EtOAc/hexane, $\mathrm{KMnO}_{4}$ stain).

${ }^{1} \mathbf{H}$ NMR $\left(400 \mathrm{MHz}, \mathrm{CDCl}_{3}\right): \delta_{\mathrm{H}} 8.12(\mathrm{dd}, J=3.1,1.2 \mathrm{~Hz}, 1 \mathrm{H}), 7.89-7.80(\mathrm{~m}, 2 \mathrm{H}), 7.81-7.72(\mathrm{~m}$, 2H), $7.54(\mathrm{dd}, J=5.1,1.2 \mathrm{~Hz}, 1 \mathrm{H}), 7.30(\mathrm{dd}, J=5.1,3.1 \mathrm{~Hz}, 1 \mathrm{H}), 4.38(\mathrm{t}, J=6.5 \mathrm{~Hz}, 2 \mathrm{H}), 2.45-2.35$ (m, 2H), $2.17-2.09(\mathrm{~m}, 2 \mathrm{H}), 2.08-2.00(\mathrm{~m}, 2 \mathrm{H}), 2.00-1.93(\mathrm{~m}, 2 \mathrm{H}), 1.77(\mathrm{dtt}, J=11.2,10.3,3.4 \mathrm{~Hz}$, 1H), 1.54 (dp, $J=11.1,8.8 \mathrm{~Hz}, 1 \mathrm{H}) \mathrm{ppm}$. See spectrum.

${ }^{13} \mathrm{C}$ NMR $\left(101 \mathrm{MHz}, \mathrm{CDCl}_{3}\right): \delta_{\mathrm{C}} 165.5,162.9,134.5,133.9,132.5,129.3,127.9,125.9,123.5,89.9$, 64.9, 32.8, 31.4, 23.0, $12.4 \mathrm{ppm}$. See spectrum.

IR (film) $v_{\max }: 2949,1791,1734(\mathrm{~s}), 1711(\mathrm{~s}), 1610,1523,1467,1370,1264,1187,1103,877 \mathrm{~cm}^{-1}$. 
HRMS (ESI) m/z: [M + Na] ${ }^{+}$Calcd for $\mathrm{C}_{20} \mathrm{H}_{19} \mathrm{NNaO}_{5} \mathrm{~S}$ 408.0876; Found 408.0869.

\section{3-(1-((1,3-Dioxoisoindolin-2-yl)oxy)cyclobutyl)propyl 2-(thiophen-2-yl)acetate (20a)}
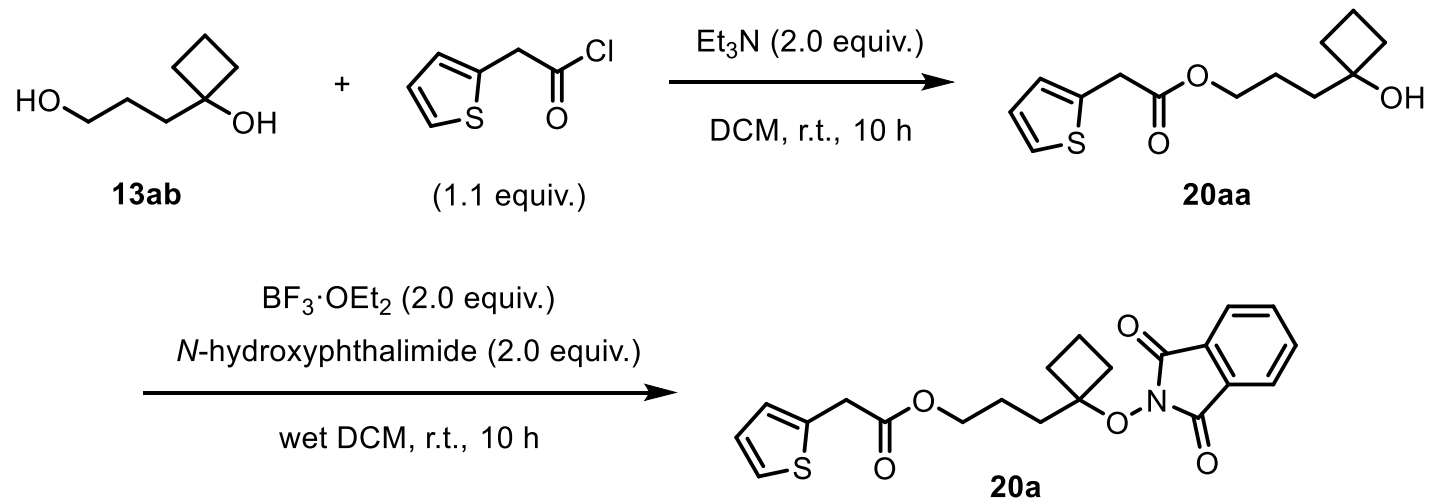

To a solution of 13ab (391 mg, $3.00 \mathrm{mmol}, 1.0$ equiv.) and triethylamine ( $0.60 \mathrm{~g}, 6.0 \mathrm{mmol}, 2.0$ equiv.) in dry DCM (20 mL) was slowly added 2-thiopheneacetyl chloride (0.53 g, $3.3 \mathrm{mmol}, 1.1$ equiv.) at $0{ }^{\circ} \mathrm{C}$. The solution was stirred at room temperature for $10 \mathrm{~h}$. Then, the reaction mixture was diluted with water $(50 \mathrm{~mL})$ and extracted with DCM $(3 \times 30 \mathrm{~mL})$. The combined organic extracts were washed with brine, dried over $\mathrm{MgSO}_{4}$, filtered, and concentrated under reduced pressure. Purification by column chromatography (40\% EtOAc/hexane) gave 20aa (462 mg, $1.81 \mathrm{mmol}, 60 \%$ ) as a yellow oil.

$N$-Alkoxyphthalimide 20a was prepared from 20aa according to GP1-B: Cyclobutanol 20aa (458 mg, $1.80 \mathrm{mmol}, 1.0$ equiv.) was treated with $N$-hydroxyphthalimide (587 mg, $3.60 \mathrm{mmol}, 2.0$ equiv.) and $\mathrm{BF}_{3} \cdot \mathrm{OEt}_{2}(511 \mathrm{mg}, 3.60 \mathrm{mmol}, 2.0$ equiv.) in wet DCM $(20 \mathrm{~mL})$ at room temperature for $10 \mathrm{~h}$. Purification by column chromatography (40\% EtOAc/hexane) gave 20a (216 mg, $0.54 \mathrm{mmol}, 30 \%$ ) as a yellow oil.

TLC: $\mathrm{R}_{\mathrm{f}}=0.26\left(40 \%\right.$ EtOAc/hexane, $\mathrm{KMnO}_{4}$ stain $)$.

${ }^{1} \mathbf{H}$ NMR $\left(400 \mathrm{MHz}, \mathrm{CDCl}_{3}\right): \delta_{\mathrm{H}} 7.88-7.82(\mathrm{~m}, 2 \mathrm{H}), 7.79-7.74(\mathrm{~m}, 2 \mathrm{H}), 7.22-7.17(\mathrm{~m}, 1 \mathrm{H}), 6.97-$ $6.92(\mathrm{~m}, 2 \mathrm{H}), 4.24(\mathrm{t}, J=6.4 \mathrm{~Hz}, 2 \mathrm{H}), 3.86(\mathrm{~s}, 2 \mathrm{H}), 2.42-2.29(\mathrm{~m}, 2 \mathrm{H}), 2.05-1.95(\mathrm{~m}, 4 \mathrm{H}), 1.91-$ $1.83(\mathrm{~m}, 2 \mathrm{H}), 1.75(\mathrm{dtt}, J=11.2,10.3,3.4 \mathrm{~Hz}, 1 \mathrm{H}), 1.50(\mathrm{dp}, J=11.3,8.8 \mathrm{~Hz}, 1 \mathrm{H}) \mathrm{ppm}$. See spectrum.

${ }^{13} \mathrm{C}$ NMR $\left(101 \mathrm{MHz}, \mathrm{CDCl}_{3}\right): \delta_{\mathrm{C}} 170.5,165.5,135.2,134.5,129.2,126.8,126.7,124.9,123.5,89.8$, $65.4,35.5,32.7,31.3,22.8,12.3$ ppm. See spectrum.

IR (film) $v_{\max }: 2953,1790,1731(\mathrm{~s}), 1610,1467,1370,1325,1256,1185,1113,974,906,877 \mathrm{~cm}^{-1}$.

HRMS (ESI) m/z: [M + Na $]^{+}$Calcd for $\mathrm{C}_{21} \mathrm{H}_{21} \mathrm{NNaO}_{5} \mathrm{~S}$ 422.1033; Found 422.1029. 


\section{3-(1-((1,3-Dioxoisoindolin-2-yl)oxy)cyclobutyl)propyl cyclopropanecarboxylate (21a)}
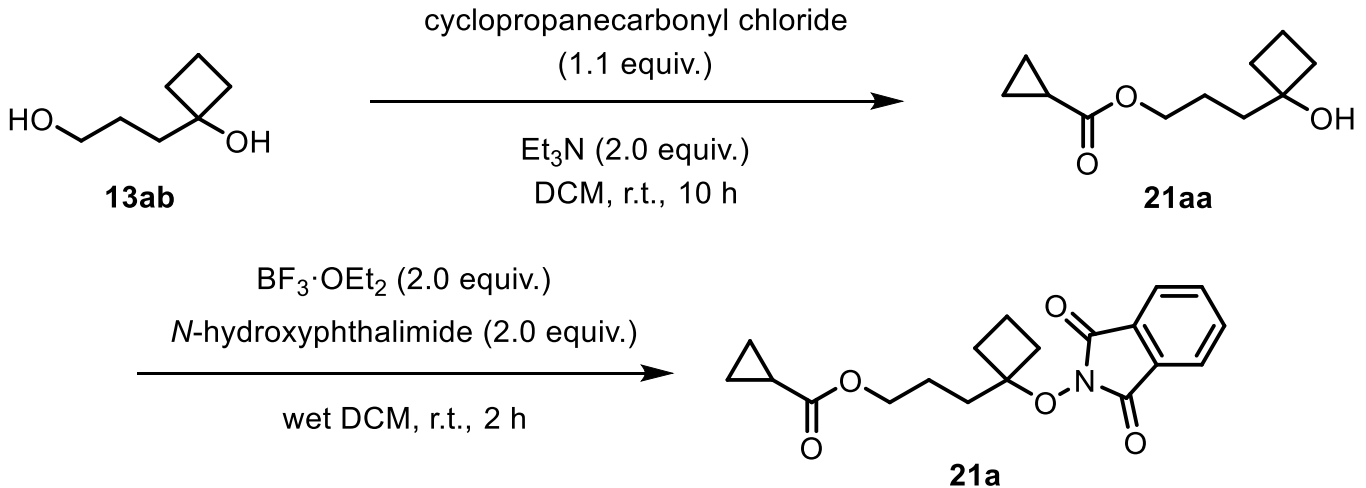

To a solution of 13ab $(260 \mathrm{mg}, 2.00 \mathrm{mmol}, 1.0$ equiv.) and triethylamine ( $0.40 \mathrm{~g}, 4.0 \mathrm{mmol}, 2.0$ equiv.) in dry DCM (20 mL) was slowly added cyclopropanecarbonyl chloride (0.23 g, $2.2 \mathrm{mmol}, 1.1$ equiv.) at $0{ }^{\circ} \mathrm{C}$. The solution was stirred at room temperature for $10 \mathrm{~h}$. Then, the reaction mixture was diluted with water $(50 \mathrm{~mL})$ and extracted with DCM $(3 \times 30 \mathrm{~mL})$. The combined organic extracts were washed with brine, dried over $\mathrm{MgSO}_{4}$, filtered, and concentrated under reduced pressure. Purification by column chromatography (20\% EtOAc/hexane) gave 21aa (376 mg, $1.89 \mathrm{mmol}, 95 \%$ ) as a yellow oil.

$N$-Alkoxyphthalimide 21a was prepared from 21aa according to GP1-B: Cyclobutanol 21aa (357 mg, $1.80 \mathrm{mmol}, 1.0$ equiv.) was treated with $N$-hydroxyphthalimide (587 mg, $3.60 \mathrm{mmol}, 2.0$ equiv.) and $\mathrm{BF}_{3} \cdot \mathrm{OEt}_{2}(511 \mathrm{mg}, 3.60 \mathrm{mmol}, 2.0$ equiv. $)$ in wet $\mathrm{DCM}(20 \mathrm{~mL})$ at room temperature for $2 \mathrm{~h}$. Purification by column chromatography (20\% EtOAc/hexane) gave $\mathbf{2 1 a}(308 \mathrm{mg}, 0.90 \mathrm{mmol}, 50 \%$ ) as a white solid.

TLC: $\mathrm{R}_{\mathrm{f}}=0.45$ (20\% EtOAc/hexane, $\mathrm{KMnO}_{4}$ stain).

${ }^{1} \mathrm{H}$ NMR $\left(400 \mathrm{MHz}, \mathrm{CDCl}_{3}\right): \delta_{\mathrm{H}} 7.90-7.80(\mathrm{~m}, 2 \mathrm{H}), 7.81-7.72(\mathrm{~m}, 2 \mathrm{H}), 4.17(\mathrm{t}, J=6.4 \mathrm{~Hz}, 2 \mathrm{H}), 2.43$ $-2.32(\mathrm{~m}, 2 \mathrm{H}), 2.07-1.96(\mathrm{~m}, 4 \mathrm{H}), 1.93-1.87(\mathrm{~m}, 2 \mathrm{H}), 1.75(\mathrm{dtt}, J=11.1,10.2,3.3 \mathrm{~Hz}, 1 \mathrm{H}), 1.65-$ $1.57(\mathrm{~m}, 1 \mathrm{H}), 1.52(\mathrm{dp}, J=11.1,8.7 \mathrm{~Hz}, 1 \mathrm{H}), 1.02-0.97(\mathrm{~m}, 2 \mathrm{H}), 0.87-0.81(\mathrm{~m}, 2 \mathrm{H}) \mathrm{ppm} . \underline{\text { See }}$ spectrum.

${ }^{13} \mathrm{C}$ NMR $\left(101 \mathrm{MHz}, \mathrm{CDCl}_{3}\right): \delta_{\mathrm{C}} 175.0,165.5,134.5,129.3,123.5,89.9,64.7,32.7,31.4,22.9,12.9$, 12.3, 8.3 ppm. See spectrum.

IR (film) $v_{\max }: 2973,2923,1792,1734(\mathrm{~s}), 1468,1406,1372,1254,1056,1033,903,724 \mathrm{~cm}^{-1}$.

HRMS (ESI) m/z: [M + Na $]^{+}$Calcd for $\mathrm{C}_{19} \mathrm{H}_{21} \mathrm{NNaO}_{5}$ 366.1312; Found 366.1313.

M.p.: $95-97^{\circ} \mathrm{C}$. 


\section{5-(1-((1,3-Dioxoisoindolin-2-yl)oxy)cyclobutyl)pentyl $\left(1 R^{*}, 2 R^{*}\right)$-2-phenylcyclopropane-1- carboxylate (22a)}<smiles>CCN(CC)CC(=O)C1C[C@H]1c1ccccc1</smiles>
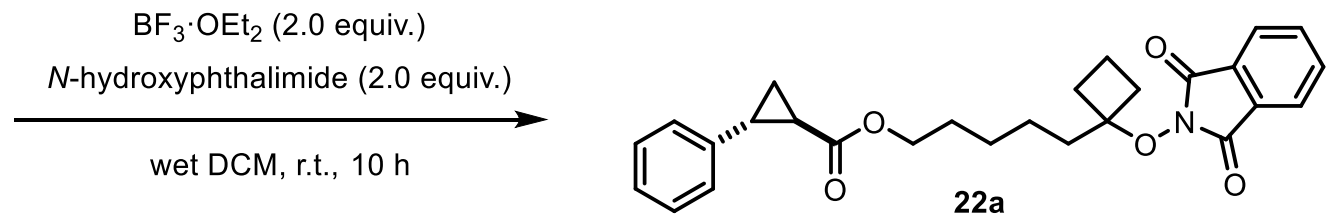

To a solution of 11ab (316 mg, $2.00 \mathrm{mmol}, 1.0$ equiv.) and triethylamine ( $0.40 \mathrm{~g}, 4.0 \mathrm{mmol}, 2.0$ equiv.) in dry DCM (30 mL) was slowly added trans-2-phenyl-1-cyclopropanecarbonyl chloride (397 mg, 2.20 mmol, 1.1 equiv.) at $0{ }^{\circ} \mathrm{C}$. The solution was stirred at room temperature for $10 \mathrm{~h}$. Then, the reaction mixture was diluted with water $(20 \mathrm{~mL})$ and extracted with DCM $(3 \times 30 \mathrm{~mL})$. The combined organic extracts were washed with brine, dried over $\mathrm{MgSO}_{4}$, filtered, and concentrated under reduced pressure. Purification by column chromatography (20\% EtOAc/hexane) gave 22aa (480 mg, $1.59 \mathrm{mmol}, 79 \%$ ) as a yellow oil.

$N$-Alkoxyphthalimide 22a was prepared from 22aa according to GP1-B: Cyclobutanol 22aa (453 mg, $1.50 \mathrm{mmol}, 1.0$ equiv.) was treated with $N$-hydroxyphthalimide (489 mg, $3.00 \mathrm{mmol}, 2.0$ equiv.) and $\mathrm{BF}_{3} \cdot \mathrm{OEt}_{2}$ (426 mg, $3.00 \mathrm{mmol}, 2.0$ equiv.) in wet DCM $(20 \mathrm{~mL}$ ) at room temperature for $10 \mathrm{~h}$. Purification by column chromatography (20\% EtOAc/hexane) gave 22a (441.2 mg, $0.98 \mathrm{mmol}, 65 \%$ ) as a pale-yellow oil.

TLC: $\mathrm{R}_{\mathrm{f}}=0.42\left(40 \%\right.$ EtOAc/hexane, $\mathrm{KMnO}_{4}$ stain $)$.

${ }^{1} \mathbf{H}$ NMR $\left(400 \mathrm{MHz}, \mathrm{CDCl}_{3}\right): \delta_{\mathrm{H}} 7.89-7.79(\mathrm{~m}, 2 \mathrm{H}), 7.80-7.71(\mathrm{~m}, 2 \mathrm{H}), 7.32-7.22(\mathrm{~m}, 2 \mathrm{H}), 7.23-$ $7.14(\mathrm{~m}, 1 \mathrm{H}), 7.13-7.07(\mathrm{~m}, 2 \mathrm{H}), 4.14(\mathrm{t}, J=6.7 \mathrm{~Hz}, 2 \mathrm{H}), 2.52(\mathrm{ddd}, J=9.2,6.5,4.2 \mathrm{~Hz}, 1 \mathrm{H}), 2.42-$ $2.30(\mathrm{~m}, 2 \mathrm{H}), 2.06-1.98(\mathrm{~m}, 2 \mathrm{H}), 1.92(\mathrm{ddd}, J=8.4,5.3,4.2 \mathrm{~Hz}, 1 \mathrm{H}), 1.88-1.80(\mathrm{~m}, 2 \mathrm{H}), 1.78-1.64$ (m, 5H), $1.62-1.57(\mathrm{~m}, 1 \mathrm{H}), 1.56-1.42(\mathrm{~m}, 3 \mathrm{H}), 1.36-1.24(\mathrm{~m}, 1 \mathrm{H}) \mathrm{ppm}$. See spectrum.

${ }^{13}$ C NMR $\left(101 \mathrm{MHz}, \mathrm{CDCl}_{3}\right): \delta_{\mathrm{C}} 173.5,165.5,140.1,134.4,129.3,128.4,126.4,126.2,123.5,90.3$, $64.9,36.3,31.4,28.6,26.4,26.1,24.2,23.0,17.0,12.3$ ppm. See spectrum.

IR (film) $v_{\max }: 2946,2901,1792,1733(\mathrm{~s}), 1606,1498,1467,1409,1265,1185,1079,974,904,726$, $704 \mathrm{~cm}^{-1}$.

HRMS (ESI) m/z: [M + Na $]^{+}$Calcd for $\mathrm{C}_{27} \mathrm{H}_{29} \mathrm{NNaO}_{5}$ 470.1938; Found 470.1931. 


\section{3-(1-((1,3-Dioxoisoindolin-2-yl)oxy)cyclobutyl)propyl 5-bromopentanoate (23a)}
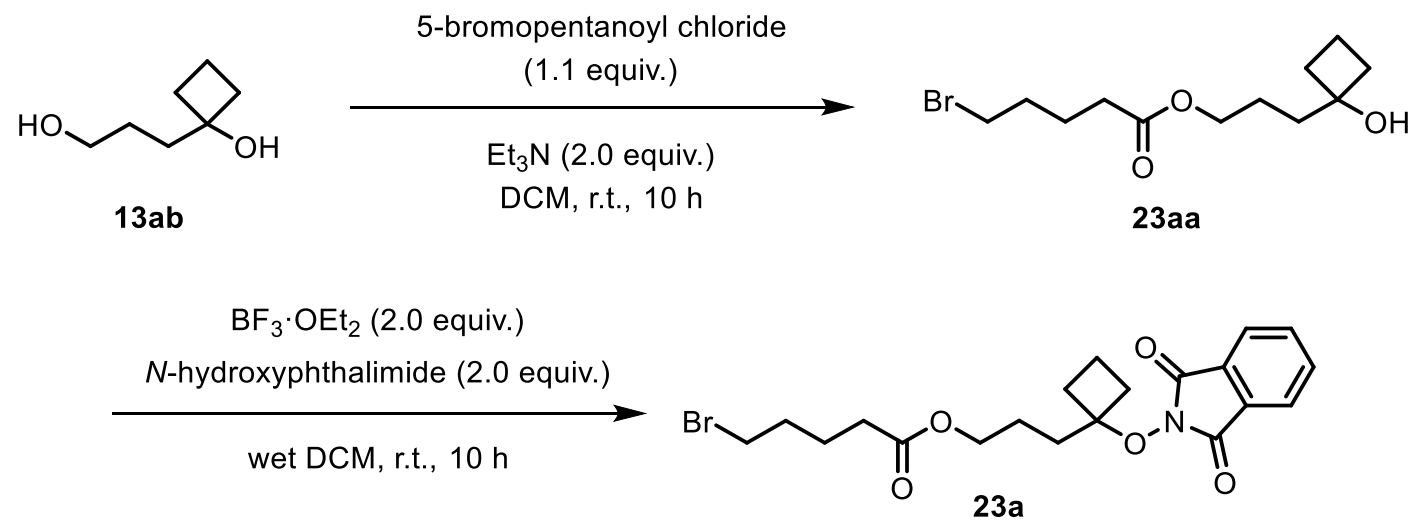

To a solution of 13ab $(260 \mathrm{mg}, 2.00 \mathrm{mmol}, 1.0$ equiv. $)$ and triethylamine ( $0.40 \mathrm{~g}, 4.0 \mathrm{mmol}, 2.0$ equiv.) in dry DCM $(20 \mathrm{~mL})$ was slowly added 5-bromopentanoyl chloride $(0.44 \mathrm{~g}, 2.2 \mathrm{mmol}, 1.1$ equiv. $)$ at $0{ }^{\circ} \mathrm{C}$. The solution was stirred at room temperature for $10 \mathrm{~h}$. Then, the reaction mixture was diluted with water $(50 \mathrm{~mL})$ and extracted with DCM $(3 \times 30 \mathrm{~mL})$. The combined organic extracts were washed with brine, dried over $\mathrm{MgSO}_{4}$, filtered, and concentrated under reduced pressure. Purification by column chromatography (20\% EtOAc/hexane) gave 23aa (502 mg, $1.71 \mathrm{mmol}, 86 \%$ ) as a yellow oil.

$N$-Alkoxyphthalimide 23a was prepared from 23aa according to GP1-B: Cyclobutanol 23aa (440 mg, $1.50 \mathrm{mmol}, 1.0$ equiv.) was treated with $N$-hydroxyphthalimide (489 mg, $3.00 \mathrm{mmol}, 2.0$ equiv.) and $\mathrm{BF}_{3} \cdot \mathrm{OEt}_{2}$ (426 mg, $3.00 \mathrm{mmol}, 2.0$ equiv.) in wet DCM $(20 \mathrm{~mL}$ ) at room temperature for $10 \mathrm{~h}$. Purification by column chromatography (20\% EtOAc/hexane) gave 23a (351 mg, $0.73 \mathrm{mmol}, 49 \%$ ) as a yellow oil.

TLC: $\mathrm{R}_{\mathrm{f}}=0.45\left(20 \%\right.$ EtOAc/hexane, $\mathrm{KMnO}_{4}$ stain $)$.

${ }^{1} \mathbf{H}$ NMR $\left(400 \mathrm{MHz}, \mathrm{CDCl}_{3}\right): \delta_{\mathrm{H}} 7.89-7.80(\mathrm{~m}, 2 \mathrm{H}), 7.81-7.72(\mathrm{~m}, 2 \mathrm{H}), 4.18(\mathrm{t}, J=6.4 \mathrm{~Hz}, 2 \mathrm{H}), 3.41$ $(\mathrm{t}, J=6.6 \mathrm{~Hz}, 2 \mathrm{H}), 2.43-2.32(\mathrm{~m}, 2 \mathrm{H}), 2.36(\mathrm{t}, J=7.2 \mathrm{~Hz}, 2 \mathrm{H}), 2.05-1.95(\mathrm{~m}, 4 \mathrm{H}), 1.95-1.85(\mathrm{~m}$, $4 \mathrm{H}), 1.84-1.70(\mathrm{~m}, 3 \mathrm{H}), 1.52(\mathrm{dp}, J=11.1,8.8 \mathrm{~Hz}, 1 \mathrm{H}) \mathrm{ppm}$. See spectrum.

${ }^{13} \mathrm{C}$ NMR $\left(101 \mathrm{MHz}, \mathrm{CDCl}_{3}\right): \delta_{\mathrm{C}} 173.2,165.5,134.5,129.2,123.5,89.8,64.7,33.3,33.0,32.7,32.0$, 31.3, 23.5, 22.9, 12.3 ppm. See spectrum.

IR (film) $v_{\max }: 2972,2901,1791,1732,1467,1372,1252,1187,1056,904,726 \mathrm{~cm}^{-1}$.

HRMS (ESI) m/z: [M + Na ${ }^{+}$Calcd for $\mathrm{C}_{20} \mathrm{H}_{24} \mathrm{NBrNaO}_{5}$ 460.0730; Found 460.0740. 


\section{3-(1-((1,3-Dioxoisoindolin-2-yl)oxy)cyclobutyl)propyl $(3 r, 5 r, 7 r)$-adamantane-1-carboxylate}

(24a)
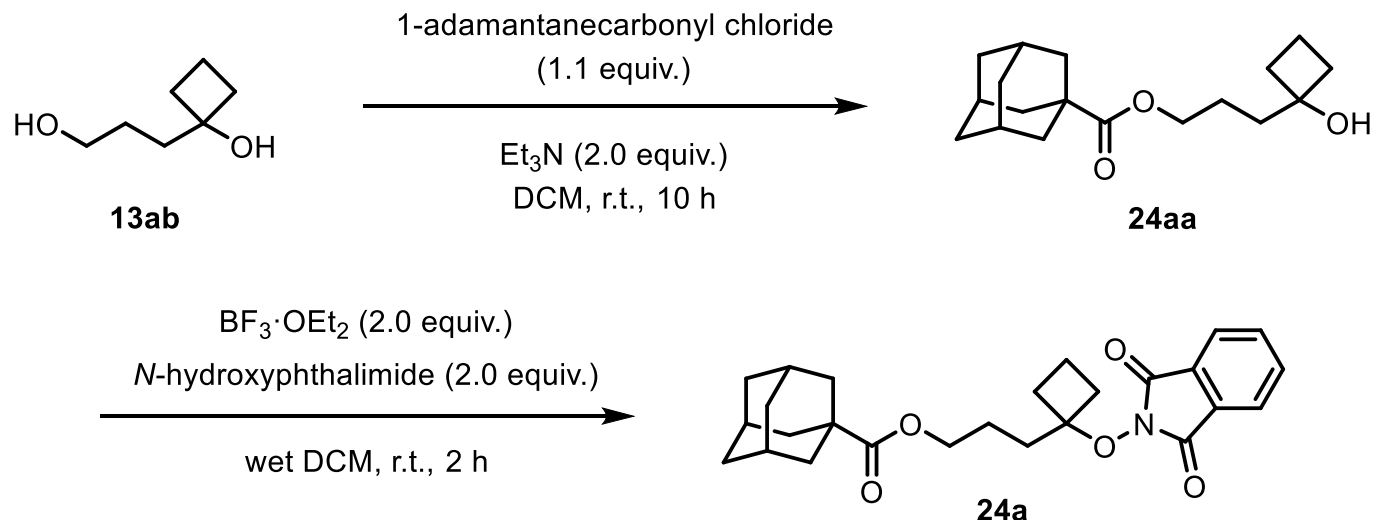

To a solution of 13ab (260 mg, $2.00 \mathrm{mmol}, 1.0$ equiv.) and triethylamine ( $0.40 \mathrm{~g}, 4.0 \mathrm{mmol}, 2.0$ equiv.) in dry DCM (20 mL) was slowly added 1-adamantanecarbonyl chloride (0.43 g, $2.2 \mathrm{mmol}, 1.1$ equiv.) at $0{ }^{\circ} \mathrm{C}$. The solution was stirred at room temperature for $10 \mathrm{~h}$. Then, the reaction mixture was diluted with water $(50 \mathrm{~mL})$ and extracted with DCM $(3 \times 30 \mathrm{~mL})$. The combined organic extracts were washed with brine, dried over $\mathrm{MgSO}_{4}$, filtered, and concentrated under reduced pressure. Purification by column chromatography (20\% EtOAc/hexane) gave 24aa (395 mg, $1.35 \mathrm{mmol}, 68 \%$ ) as a yellow oil.

$\mathrm{N}$-Alkoxyphthalimide 24a was prepared from 24aa according to GP1-B: Cyclobutanol 24aa (380 mg, $1.30 \mathrm{mmol}, 1.0$ equiv.) was treated with $N$-hydroxyphthalimide (424 mg, $2.60 \mathrm{mmol}, 2.0$ equiv.) and $\mathrm{BF}_{3} \cdot \mathrm{OEt}_{2}(0.37 \mathrm{~g}, 2.6 \mathrm{mmol}, 2.0$ equiv. $)$ in wet DCM $(20 \mathrm{~mL})$ at room temperature for $2 \mathrm{~h}$. Purification by column chromatography (40\% EtOAc/hexane) gave $\mathbf{2 4 a}(220 \mathrm{mg}, 0.503 \mathrm{mmol}, 39 \%)$ as a paleyellow oil.

TLC: $\mathrm{R}_{\mathrm{f}}=0.50\left(20 \%\right.$ EtOAc/hexane, $\mathrm{KMnO}_{4}$ stain $)$.

${ }^{1} \mathbf{H}$ NMR $\left(400 \mathrm{MHz}, \mathrm{CDCl}_{3}\right): \delta_{\mathrm{H}} 7.90-7.81(\mathrm{~m}, 2 \mathrm{H}), 7.81-7.72(\mathrm{~m}, 2 \mathrm{H}), 4.15(\mathrm{t}, J=6.3 \mathrm{~Hz}, 2 \mathrm{H}), 2.44$ $-2.33(\mathrm{~m}, 2 \mathrm{H}), 2.06-1.95(\mathrm{~m}, 6 \mathrm{H}), 1.94-1.86(\mathrm{~m}, 8 \mathrm{H}), 1.80-1.66(\mathrm{~m}, 8 \mathrm{H}), 1.51(\mathrm{dp}, J=11.2,8.8$ $\mathrm{Hz}, 1 \mathrm{H}) \mathrm{ppm}$. See spectrum.

${ }^{13} \mathrm{C}$ NMR $\left(101 \mathrm{MHz}, \mathrm{CDCl}_{3}\right): \delta_{\mathrm{C}} 177.8,165.5,134.5,129.3,123.5,89.9,64.3,40.8,38.9,36.5,32.7$, 31.4, 28.0, 22.9, 12.4 ppm. See spectrum.

IR (film) $v_{\max }: 2972,2904,1792,1733(\mathrm{~s}), 1467,1453,1371,1234,1186,1078,906,877,729 \mathrm{~cm}^{-1}$.

HRMS (ESI) m/z: [M + Na $]^{+}$Calcd for $\mathrm{C}_{26} \mathrm{H}_{31} \mathrm{NNaO}_{5}$ 460.2094; Found 460.2085. 


\section{3-(1-((1,3-Dioxoisoindolin-2-yl)oxy)cyclobutyl)propyl (1R,4S)-1,7,7-trimethyl-3-oxo-2- oxabicyclo[2.2.1]heptane-4-carboxylate (25a)}
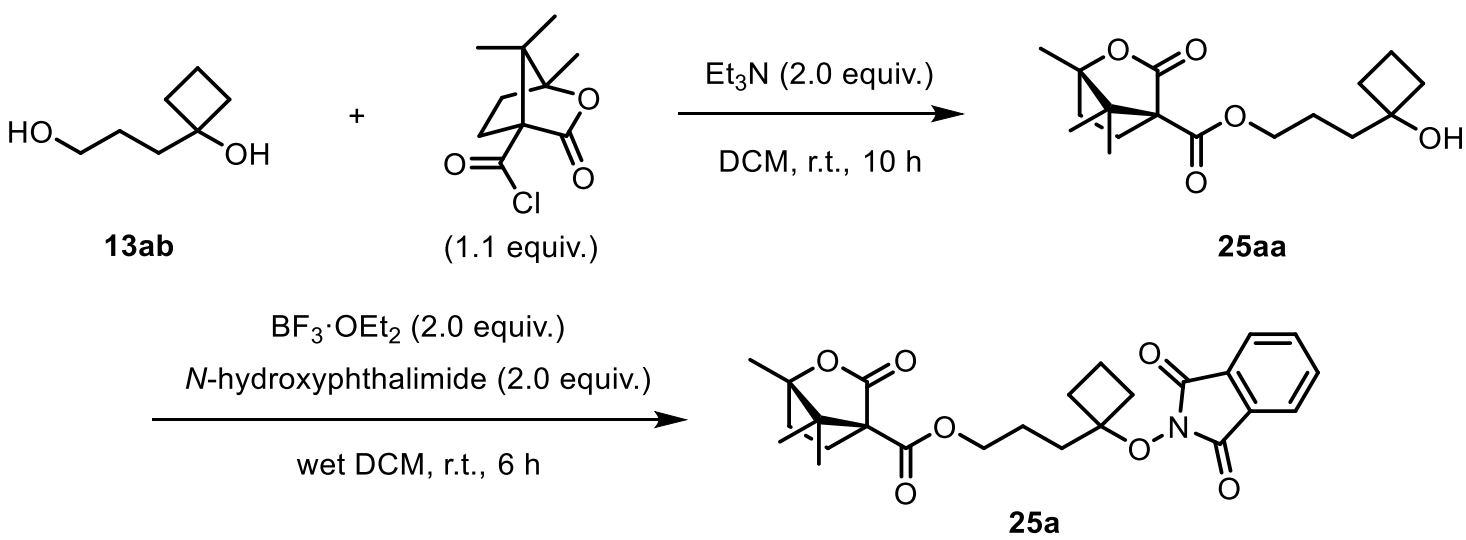

To a solution of 13ab (260 mg, $2.00 \mathrm{mmol}, 1.0$ equiv.) and triethylamine ( $0.40 \mathrm{~g}, 4.0 \mathrm{mmol}, 2.0$ equiv.) in dry DCM (20 mL) was slowly added (1S)-(-)-camphanic chloride $(0.48 \mathrm{~g}, 2.2 \mathrm{mmol}, 1.1$ equiv.) at $0{ }^{\circ} \mathrm{C}$. The solution was stirred at room temperature for $10 \mathrm{~h}$. Then, the reaction mixture was diluted with water $(50 \mathrm{~mL})$ and extracted with DCM $(3 \times 30 \mathrm{~mL})$. The combined organic extracts were washed with brine, dried over $\mathrm{MgSO}_{4}$, filtered, and concentrated under reduced pressure. Purification by column chromatography (40\% EtOAc/hexane) gave 25aa (528 mg, $1.70 \mathrm{mmol}, 85 \%$ ) as a yellow oil.

$\mathrm{N}$-Alkoxyphthalimide 25a was prepared from 25aa according to GP1-B: Cyclobutanol 25aa (466 mg, $1.50 \mathrm{mmol}, 1.0$ equiv.) was treated with $N$-hydroxyphthalimide (489 mg, $3.00 \mathrm{mmol}, 2.0$ equiv.) and $\mathrm{BF}_{3} \cdot \mathrm{OEt}_{2}(426 \mathrm{mg}, 3.00 \mathrm{mmol}, 2.0$ equiv. $)$ in wet $\mathrm{DCM}(20 \mathrm{~mL})$ at room temperature for $6 \mathrm{~h}$. Purification by column chromatography (40\% EtOAc/hexane) gave $\mathbf{2 5 a}$ (342 mg, $0.75 \mathrm{mmol}, 50 \%$ ) as a yellow oil.

TLC: $\mathrm{R}_{\mathrm{f}}=0.25\left(40 \%\right.$ EtOAc/hexane, $\mathrm{KMnO}_{4}$ stain $)$.

${ }^{1} \mathbf{H}$ NMR $\left(400 \mathrm{MHz}, \mathrm{CDCl}_{3}\right): \delta_{\mathrm{H}} 7.87-7.82(\mathrm{~m}, 2 \mathrm{H}), 7.79-7.74(\mathrm{~m}, 2 \mathrm{H}), 4.40-4.30(\mathrm{~m}, 2 \mathrm{H}), 2.45$ (ddd, $J=13.5,10.8,4.3 \mathrm{~Hz}, 1 \mathrm{H}), 2.43-2.32$ (m, 2H), $2.12-1.96(\mathrm{~m}, 5 \mathrm{H}), 1.97-1.88(\mathrm{~m}, 3 \mathrm{H}), 1.77$ $(\mathrm{dtt}, J=11.8,10.9,3.7 \mathrm{~Hz}, 1 \mathrm{H}), 1.69(\mathrm{ddd}, J=13.4,9.4,4.3 \mathrm{~Hz}, 1 \mathrm{H}), 1.52(\mathrm{dp}, J=11.2,8.8 \mathrm{~Hz}, 1 \mathrm{H})$, $1.11(\mathrm{~s}, 3 \mathrm{H}), 1.07$ (s, 3H), 0.98 (s, 3H) ppm. See spectrum.

${ }^{13} \mathrm{C}$ NMR $\left(101 \mathrm{MHz}, \mathrm{CDCl}_{3}\right): \delta_{\mathrm{C}} 178.2,167.5,165.5,134.6,129.2,123.6,91.2,89.7,65.9,54.8,54.1$, 32.7, 31.3, 30.6, 29.0, 22.9, 16.8, 16.8, 12.4, 9.7 ppm. See spectrum.

IR (film) $v_{\max }: 2969,2933,1783(\mathrm{~s}), 1733(\mathrm{~s}), 1469,1448,1397,1314,1273,1169,1063,904 \mathrm{~cm}^{-1}$.

HRMS (ESI) m/z: [M + Na $]^{+}$Calcd for $\mathrm{C}_{25} \mathrm{H}_{29} \mathrm{NNaO}_{7}$ 478.1836; Found 478.1849. 


\section{2-((1-Ethylcyclopentyl)oxy)isoindoline-1,3-dione (26a)}

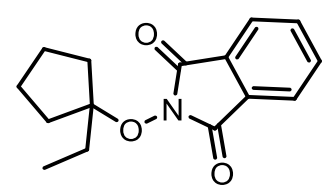

Prepared following GP1-B: 1-Ethylcyclopentan-1-ol (685 mg, $6.00 \mathrm{mmol}, 1.0$ equiv.) was treated with $N$-hydroxyphthalimide (1.63 g, $10.0 \mathrm{mmol}, 1.7$ equiv.) and $\mathrm{BF}_{3} \cdot \mathrm{OEt}_{2}(937 \mathrm{mg}, 6.60 \mathrm{mmol}, 1.1$ equiv.) in wet DCM $(0.5 \mathrm{M})$ at room temperature for $2 \mathrm{~h}$. Purification by column chromatography (20\% EtOAc/hexane) gave $\mathbf{2 6 a}$ (467 mg, $1.80 \mathrm{mmol}, 30 \%$ ) as colourless oil.

TLC: $\mathrm{R}_{\mathrm{f}}=0.40\left(20 \%\right.$ EtOAc/hexane, $\mathrm{KMnO}_{4}$ stain $)$.

${ }^{1} \mathbf{H}$ NMR $\left(400 \mathrm{MHz}, \mathrm{CDCl}_{3}\right): \delta_{\mathrm{H}} 7.85-7.79(\mathrm{~m}, 2 \mathrm{H}), 7.77-7.71(\mathrm{~m}, 2 \mathrm{H}), 2.06-1.93(\mathrm{~m}, 4 \mathrm{H}), 1.80(\mathrm{q}$, $J=7.4 \mathrm{~Hz}, 2 \mathrm{H}), 1.72-1.58(\mathrm{~m}, 4 \mathrm{H}), 1.08(\mathrm{t}, J=7.4 \mathrm{~Hz}, 3 \mathrm{H}) \mathrm{ppm} . \underline{\text { See spectrum. }}$.

${ }^{13} \mathrm{C}$ NMR $\left(101 \mathrm{MHz}, \mathrm{CDCl}_{3}\right): \delta_{\mathrm{C}} 166.1,134.4,129.4,123.3,101.3,34.9,30.2,24.5,9.1 \mathrm{ppm} . \underline{\text { See }}$ spectrum.

IR (film) $v_{\max }$ : 2966, 2943, 1792, 1732(s), 1468, 1369, 1346, 1188, 1079, 965, 904, 726, $704 \mathrm{~cm}^{-1}$.

HRMS (ESI) m/z: [M + Na $]^{+}$Calcd for $\mathrm{C}_{15} \mathrm{H}_{17} \mathrm{NNaO}_{3}$ 282.1101; Found 282.1103.

\section{2-((1-Pentylcyclopentyl)oxy)isoindoline-1,3-dione (27a)}

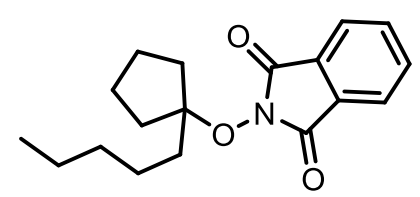

Prepared following GP1-B: 1-Pentylcyclopentan-1-ol (1.79 g, 11.5 mmol, 1.0 equiv.) was treated with $N$-hydroxyphthalimide (3.75 g, $23.0 \mathrm{mmol}, 2.0$ equiv.) and $\mathrm{BF}_{3} \cdot \mathrm{OEt}_{2}(1.80 \mathrm{~g}, 12.7 \mathrm{mmol}, 1.1$ equiv.) in wet DCM $(40 \mathrm{~mL})$ at room temperature for $3 \mathrm{~h}$. Purification by column chromatography (20\% EtOAc/hexane) gave 27a (415.9 mg, $1.38 \mathrm{mmol}, 12 \%)$ as colourless oil.

TLC: $\mathrm{R}_{\mathrm{f}}=0.50\left(20 \% \mathrm{EtOAc} /\right.$ hexane, $\mathrm{KMnO}_{4}$ stain $)$.

${ }^{1} \mathbf{H}$ NMR $\left(400 \mathrm{MHz}, \mathrm{CDCl}_{3}\right): \delta_{\mathrm{H}} 7.86-7.80(\mathrm{~m}, 2 \mathrm{H}), 7.77-7.71(\mathrm{~m}, 2 \mathrm{H}), 2.06-1.92(\mathrm{~m}, 4 \mathrm{H}), 1.78-$ $1.71(\mathrm{~m}, 2 \mathrm{H}), 1.71-1.59(\mathrm{~m}, 4 \mathrm{H}), 1.58-1.49(\mathrm{~m}, 2 \mathrm{H}), 1.40-1.26(\mathrm{~m}, 4 \mathrm{H}), 0.89(\mathrm{~d}, J=7.0 \mathrm{~Hz}, 3 \mathrm{H})$ ppm. See spectrum.

${ }^{13} \mathrm{C}$ NMR $\left(101 \mathrm{MHz}, \mathrm{CDCl}_{3}\right): \delta_{\mathrm{C}} 166.1,134.4,129.4,123.4,100.9,37.7,35.4,32.5,24.5,24.4,22.7$, 14.1 ppm. See spectrum. 
IR (film) $v_{\max }: 2987,2901,1793,1733(\mathrm{~s}), 1468,1372,1187,1078,804,877,724,705 \mathrm{~cm}^{-1}$.

HRMS (ESI) m/z: [M + Na $]^{+}$Calcd for $\mathrm{C}_{18} \mathrm{H}_{23} \mathrm{NNaO}_{3}$ 324.1570; Found 324.1575.

\section{2-(1-Isobutylcyclobutoxy)isoindoline-1,3-dione (28a)}

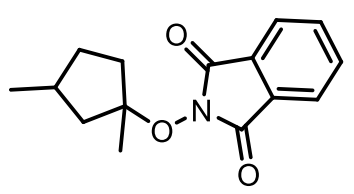

Prepared according to a modified GP1 (the Grignard addition was performed in $\mathrm{Et}_{2} \mathrm{O}$ in the absence of $\mathrm{LaCl}_{3} \cdot 2 \mathrm{LiCl}$ ): 3-Methylcyclopentanone (981 mg, $10.0 \mathrm{mmol}, 1.0$ equiv.) and methylmagnesium bromide (30 mmol, 3.0 equiv.) were stirred in $\mathrm{Et}_{2} \mathrm{O}(30 \mathrm{~mL})$ at room temperature for $10 \mathrm{~h}$. Purification by column chromatography (20\% EtOAc/hexane) gave 1,3-dimethylcyclopentan-1-ol (902 mg, 7.90 mmol, 79\%). 1,3-Dimethylcyclopentan-1-ol (902 mg, $7.90 \mathrm{mmol}, 1.0$ equiv.) was then treated with $N$ hydroxyphthalimide (2.57 g, $15.8 \mathrm{mmol}, 2.0$ equiv.) and $\mathrm{BF}_{3} \cdot \mathrm{OEt}_{2}(1.12 \mathrm{~g}, 7.90 \mathrm{mmol}, 1.0$ equiv.) in wet DCM $(50 \mathrm{~mL})$ at room temperature for $2 \mathrm{~h}$. Purification by column chromatography (20\% EtOAc/hexane) gave $\mathbf{2 8 a}$ (512 mg, $1.98 \mathrm{mmol}, 25 \%, 57: 43$ d.r.) as a yellow oil. The d.r. was determined by ${ }^{1} \mathrm{H}$ NMR analysis of the purified product.

TLC: $\mathrm{R}_{\mathrm{f}}=0.51\left(20 \%\right.$ EtOAc/hexane, $\mathrm{KMnO}_{4}$ stain $)$.

${ }^{1} \mathbf{H}$ NMR $\left(400 \mathrm{MHz}, \mathrm{CDCl}_{3}\right):\left(57: 43\right.$ mixture of two diastereomers) $\delta_{\mathrm{H}} 7.86-7.80(\mathrm{~m}, 2 \mathrm{H}), 7.77-7.72$ (m, 2H), $2.61-2.49(\mathrm{~m}, 0.57 \mathrm{H}), 2.33-1.60(\mathrm{~m}, 5 \mathrm{H}), 1.56-1.49(\mathrm{~m}, 0.43 \mathrm{H}), 1.46(\mathrm{~s}, 1.71 \mathrm{H}), 1.45$ (s, $1.29 \mathrm{H}), 1.29-1.11(\mathrm{~m}, 1 \mathrm{H}), 1.10(\mathrm{~d}, J=6.6 \mathrm{~Hz}, 1.29 \mathrm{H}), 1.03(\mathrm{~d}, J=6.7 \mathrm{~Hz}, 1.71 \mathrm{H}) \mathrm{ppm} . \underline{\text { See spectrum. }}$

${ }^{13}$ C NMR (101 MHz, $\left.\mathrm{CDCl}_{3}\right)$ : (57:43 mixture of two diastereomers): Major diastereomer: $\delta_{\mathrm{C}} 165.9$, 134.4, 129.4, 123.4, 98.2, 47.0, 37.7, 33.4, 33.3, 25.2, 20.6 ppm; Minor diastereomer: $\delta_{\mathrm{C}}$. 165.8, 134.4, 129.4, 123.4, 97.6, 46.4, 38.4, 33.8, 33.1, 25.6, 20.5 ppm. See spectrum.

IR (film) $v_{\max }$ : 2972, 2901, 1792, 1734(s), 1467, 1372, 1265, 1185, 1078, 968, 877, 732, $703 \mathrm{~cm}^{-1}$.

HRMS (ESI) m/z: [M + Na $]^{+}$Calcd for $\mathrm{C}_{15} \mathrm{H}_{17} \mathrm{NNaO}_{3}$ 282.1101; Found 282.1098. 


\section{2-((1-Methyl-3-phenylcyclopentyl)oxy)isoindoline-1,3-dione (29a)}

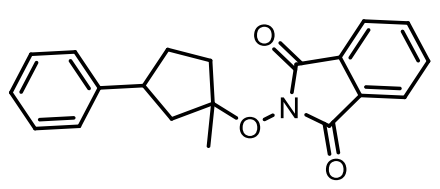

Prepared according to GP1: $\mathrm{LaCl}_{3} \cdot 2 \mathrm{LiCl}(7.5 \mathrm{mmol}, 1.5$ equiv.), methylmagnesium bromide ( $7.5 \mathrm{mmol}$, 1.5 equiv.), and 3-phenylcyclopentan-1-one ( $0.80 \mathrm{~g}, 5.0 \mathrm{mmol}, 1.0$ equiv.) were stirred at room temperature for $10 \mathrm{~h}$. Purification by column chromatography (20\% EtOAc/hexane) gave 1-methyl-3phenylcyclopentan-1-ol $(0.71 \mathrm{mg}, 4.0 \mathrm{mmol}, 80 \%)$ as a bright yellow oil. 1-Methyl-3phenylcyclopentan-1-ol (705 mg, $4.00 \mathrm{mmol}, 1.0$ equiv.) was then treated with $N$-hydroxyphthalimide (1.30 g, $8.00 \mathrm{mmol}, 2.0$ equiv.) and $\mathrm{BF}_{3} \cdot \mathrm{OEt}_{2}(625 \mathrm{mg}, 4.40 \mathrm{mmol}, 1.1$ equiv.) in wet $\mathrm{DCM}(30 \mathrm{~mL})$ at room temperature for $4 \mathrm{~h}$. Purification by column chromatography (20\% EtOAc/hexane) gave 29a (300 $\mathrm{mg}, 0.93 \mathrm{mmol}, 23 \%, 65: 35$ d.r.) as a yellow oil. The d.r. was determined by ${ }^{1} \mathrm{H}$ NMR analysis of the purified product.

TLC: $\mathrm{R}_{\mathrm{f}}=0.51\left(20 \%\right.$ EtOAc/hexane, $\mathrm{KMnO}_{4}$ stain $)$.

${ }^{1} \mathbf{H}$ NMR $\left(400 \mathrm{MHz}, \mathrm{CDCl}_{3}\right)$ : (65:35 mixture of two diastereomers) $\delta_{\mathrm{H}} 7.89-7.82(\mathrm{~m}, 2 \mathrm{H}), 7.79-7.72$ $(\mathrm{m}, 2 \mathrm{H}), 7.37-7.27(\mathrm{~m}, 4 \mathrm{H}), 7.22-7.16(\mathrm{~m}, 1 \mathrm{H}), 3.82(\mathrm{dddd}, J=11.7,9.9,7.7,7.0 \mathrm{~Hz}, 0.65 \mathrm{H}), 3.21$ $-3.11(\mathrm{~m}, 0.35 \mathrm{H}), 2.57-2.21(\mathrm{~m}, 3.35 \mathrm{H}), 2.14-2.06(\mathrm{~m}, 0.35 \mathrm{H}), 1.97$ (ddd, $J=14.3,9.9,4.4 \mathrm{~Hz}$, $0.65 \mathrm{H}), 1.81(\mathrm{dtd}, J=12.5,9.7,7.1 \mathrm{~Hz}, 0.65 \mathrm{H}), 1.75-1.61(\mathrm{~m}, 1 \mathrm{H}), 1.55(\mathrm{~s}, 1.95 \mathrm{H}), 1.55(\mathrm{~s}, 1.05 \mathrm{H})$ ppm. See spectrum.

${ }^{13}$ C NMR (101 MHz, $\left.\mathrm{CDCl}_{3}\right)$ : (65:35 mixture of two diastereomers): Major diastereomer: $\delta_{\mathrm{C}} 165.9$, 145.1, 134.5, 129.4, 128.4, 127.1, 126.0, 123.5, 97.5, 46.7, 43.8, 37.7, 32.7, 24.8 ppm; Minor diastereomer: $\delta_{\mathrm{C}} .165 .9,144.8,134.5,129.4,128.4,127.3,126.1,123.5,97.0,46.3,45.0,38.7,33.8$, 25.6 ppm. See spectrum.

IR (film) $v_{\max }: 2978,1792,1734(\mathrm{~s}), 1493,1467,1264,1186,1107,967,906,728,705 \mathrm{~cm}^{-1}$.

HRMS (ESI) m/z: [M + Na] $]^{+}$Calcd for $\mathrm{C}_{20} \mathrm{H}_{19} \mathrm{NNaO}_{3}$ 344.1257; Found 344.1266.

\section{2-(((1S*,2R*,4R*)-2-Methylbicyclo[2.2.1]heptan-2-yl)oxy)isoindoline-1,3-dione (30a)}

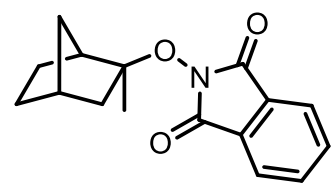

Prepared according to a modified GP1 (the Grignard addition was performed in $\mathrm{Et}_{2} \mathrm{O}$ in the absence of $\mathrm{LaCl}_{3} \cdot 2 \mathrm{LiCl}$ ): 2-Norbornanone (1.10 g, $10.0 \mathrm{mmol}, 1.0$ equiv.) and methylmagnesium bromide (30 
mmol, 3.0 equiv.) were stirred in $\mathrm{Et}_{2} \mathrm{O}(30 \mathrm{~mL})$ at room temperature for $10 \mathrm{~h}$. Purification by column chromatography (20\% EtOAc/hexane) gave $(1 S, 2 R, 4 R)$-2-methylbicyclo[2.2.1]heptan-2-ol (1.01 g, $8.00 \mathrm{mmol}, 80 \%)$ as a bright yellow oil. $(1 S, 2 R, 4 R)$-2-Methylbicyclo[2.2.1]heptan-2-ol (1.00 g, 8.00 mmol, 1.0 equiv.) was then treated with $N$-hydroxyphthalimide $(2.61 \mathrm{~g}, 16.0 \mathrm{mmol}, 2.0$ equiv.) and $\mathrm{BF}_{3} \cdot \mathrm{OEt}_{2}(1.14 \mathrm{~g}, 8.00 \mathrm{mmol}, 1.0$ equiv. $)$ in wet $\mathrm{DCM}(40 \mathrm{~mL})$ at room temperature for $2 \mathrm{~h}$. Purification by column chromatography (20\% EtOAc/hexane) gave 30a (1.52 g, $5.60 \mathrm{mmol}, 70 \%,>97: 3$ d.r.) as a yellow oil. The d.r. was determined by ${ }^{1} \mathrm{H}$ NMR analysis of the purified product.

TLC: $\mathrm{R}_{\mathrm{f}}=0.40\left(20 \% \mathrm{EtOAc} / \mathrm{hexane}, \mathrm{KMnO}_{4}\right.$ stain $)$.

${ }^{1} \mathbf{H}$ NMR $\left(400 \mathrm{MHz}, \mathrm{CDCl}_{3}\right): \delta_{\mathrm{H}} 7.82-7.76(\mathrm{~m}, 2 \mathrm{H}), 7.75-7.69(\mathrm{~m}, 2 \mathrm{H}), 2.39-2.26(\mathrm{~m}, 3 \mathrm{H}), 2.07-$ $2.00(\mathrm{~m}, 1 \mathrm{H}), 1.59-1.43(\mathrm{~m}, 2 \mathrm{H}), 1.41(\mathrm{~s}, 3 \mathrm{H}), 1.34-1.20(\mathrm{~m}, 3 \mathrm{H}), 1.11-1.03(\mathrm{~m}, 1 \mathrm{H}) \mathrm{ppm} . \underline{\text { See }}$ spectrum.

${ }^{13} \mathrm{C}$ NMR $\left(101 \mathrm{MHz}, \mathrm{CDCl}_{3}\right): \delta_{\mathrm{C}} 165.8,134.3,129.3,123.3,96.1,46.3,44.9,37.6,36.7,27.9,23.9$, 21.5 ppm. See spectrum.

IR (film) $v_{\max }$ : 2967, 2883, 1792 1733(s), 1467, 1441, 1378, 1349, 1264, 1188, 1107, 965, $877 \mathrm{~cm}^{-1}$.

HRMS (ESI) m/z: [M + Na $]^{+}$Calcd for $\mathrm{C}_{16} \mathrm{H}_{17} \mathrm{NNaO}_{3}$ 294.1101; Found 294.1097.

\section{2-(((3a $\left.R^{*}, 4 R^{*}, 5 R^{*}, 7 R^{*}, 7 \mathrm{a} R^{*}\right)-5$-Methyloctahydro- $1 H-4,7$-methanoinden-5-} yl)oxy)isoindoline-1,3-dione (31a)

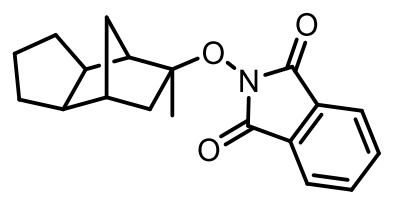

Prepared according to a modified GP1 (the Grignard addition was performed in $\mathrm{Et}_{2} \mathrm{O}$ in the absence of $\left.\mathrm{LaCl}_{3} \cdot 2 \mathrm{LiCl}\right)$ : Tricyclo[5.2.1.0 $\left.0^{2,6}\right]$ decan-8-one $(0.90 \mathrm{~g}, 6.0 \mathrm{mmol}, 1.0$ equiv.) and methylmagnesium bromide (15 mmol, 2.5 equiv.) were stirred in $\mathrm{Et}_{2} \mathrm{O}(30 \mathrm{~mL})$ at room temperature for $15 \mathrm{~h}$. Purification by column chromatography ( $20 \% \mathrm{EtOAc} /$ hexane) gave $(3 \mathrm{a} R, 4 R, 5 \mathrm{~S}, 7 R, 7 \mathrm{a} R)-5$-methyloctahydro- $1 \mathrm{H}-$ 4,7-methanoinden-5-ol $(0.83 \mathrm{~g}, 5.0 \mathrm{mmol}, 83 \%)$ as a bright yellow oil. (3aR,4R,5S,7R,7aR)-5Methyloctahydro-1H-4,7-methanoinden-5-ol (0.83 g, 5.0 mmol, 1.0 equiv.) was then treated with $N$ hydroxyphthalimide (1.63 g, $10.0 \mathrm{mmol}, 2.0$ equiv.) and $\mathrm{BF}_{3} \cdot \mathrm{OEt}_{2}(0.94 \mathrm{~g}, 6.60 \mathrm{mmol}, 1.0$ equiv.) in wet DCM $(30 \mathrm{~mL})$ at room temperature for $3 \mathrm{~h}$. Purification by column chromatography (20\% EtOAc/hexane) gave 31a (0.92 g, $2.95 \mathrm{mmol}, 59 \%$, >97:3 d.r.) as a yellow oil. The d.r. was determined by ${ }^{1} \mathrm{H}$ NMR analysis of the purified product.

TLC: $\mathrm{R}_{\mathrm{f}}=0.40$ (20\% EtOAc/hexane, $\mathrm{KMnO}_{4}$ stain) 
${ }^{1} \mathbf{H}$ NMR $\left(400 \mathrm{MHz}, \mathrm{CDCl}_{3}\right): \delta_{\mathrm{H}} 7.87-7.78(\mathrm{~m}, 2 \mathrm{H}), 7.79-7.69(\mathrm{~m}, 2 \mathrm{H}), 2.18-2.10(\mathrm{~m}, 2 \mathrm{H}), 2.09-$ $2.02(\mathrm{~m}, 2 \mathrm{H}), 2.01-1.91(\mathrm{~m}, 1 \mathrm{H}), 1.90-1.75(\mathrm{~m}, 3 \mathrm{H}), 1.75-1.65(\mathrm{~m}, 1 \mathrm{H}), 1.52-1.46(\mathrm{~m}, 1 \mathrm{H}), 1.45$ $(\mathrm{s}, 3 \mathrm{H}), 1.28-1.14(\mathrm{~m}, 2 \mathrm{H}), 1.11-0.94(\mathrm{~m}, 2 \mathrm{H}) \mathrm{ppm} . \underline{\text { See spectrum. }}$

${ }^{13}$ C NMR (101 MHz, $\left.\mathrm{CDCl}_{3}\right): \delta_{\mathrm{C}} 166.0,134.4,129.4,123.4,96.3,50.7,47.1,44.2,41.8,41.3,32.3$, $31.9,31.3,27.7,21.2 \mathrm{ppm}$. See spectrum.

IR (film) $v_{\max }$ : 2945, 2862, 1792, 1731(s), 1467, 1448, 1347, 1313, 1265, 1186, 1078, 964, $876 \mathrm{~cm}^{-1}$.

HRMS (ESI) m/z: [M + Na $]^{+}$Calcd for $\mathrm{C}_{19} \mathrm{H}_{21} \mathrm{NNaO}_{3}$ 334.1414; Found 334.1407.

\section{2-(Cyclopentyloxy)isoindoline-1,3-dione (32a)}

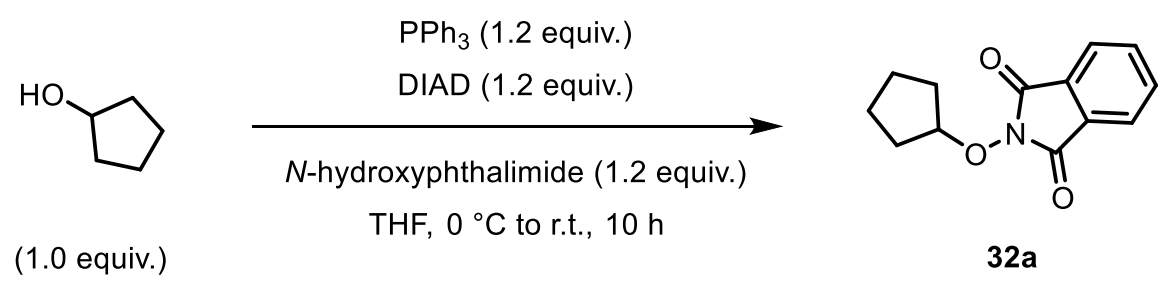

To a solution of cyclopentanol (516 mg, $6.00 \mathrm{mmol}, 1.0$ equiv.), $N$-hydroxyphthalimide $(1.17 \mathrm{~g}, 7.20$ mmol, 1.2 equiv.) and triphenylphosphine ( $1.89 \mathrm{~g}, 7.20 \mathrm{mmol}, 1.2$ equiv. $)$ in $\mathrm{THF}(30 \mathrm{~mL})$ at $0{ }^{\circ} \mathrm{C}$ was added diisopropyl azodicarboxylate $(1.42 \mathrm{~mL}, 7.20 \mathrm{mmol}, 1.0$ equiv.) dropwise. The reaction was allowed to warm to room temperature and stirred for $10 \mathrm{~h}$ before removal of the solvent under reduced pressure. The residue was purified by column chromatography (20\% EtOAc/hexane) to give 32a (902 $\mathrm{mg}, 3.90 \mathrm{mmol}, 65 \%$ ) as a white solid. All recorded spectroscopic data matched those previously reported in the literature. ${ }^{5}$

${ }^{1} \mathbf{H}$ NMR $\left(400 \mathrm{MHz}, \mathrm{CDCl}_{3}\right): \delta_{\mathrm{H}} 7.85-7.79(\mathrm{~m}, 2 \mathrm{H}), 7.76-7.70(\mathrm{~m}, 2 \mathrm{H}), 4.94-4.89(\mathrm{~m}, 1 \mathrm{H}), 2.04-$ $1.86(\mathrm{~m}, 4 \mathrm{H}), 1.82-1.70(\mathrm{~m}, 2 \mathrm{H}), 1.66-1.54(\mathrm{~m}, 2 \mathrm{H}) \mathrm{ppm} . \underline{\text { See spectrum. }}$

${ }^{13}$ C NMR (101 MHz, $\left.\mathrm{CDCl}_{3}\right): \delta_{\mathrm{C}} 164.3,134.3,129.0,123.4,90.3,31.5,23.5$ ppm. See spectrum.

\section{2-((1-Methylcyclohexyl)oxy)isoindoline-1,3-dione (33a)}<smiles>CC1(ON2C(=O)c3ccccc3C2=O)CCCCC1</smiles>

Prepared following GP1-B: 1-Methylcyclohexan-1-ol (1.14 g, 10.0 mmol, 1.0 equiv.) was treated with $N$-hydroxyphthalimide (1.96 g, $12.0 \mathrm{mmol}, 1.2$ equiv. $)$ and $\mathrm{BF}_{3} \cdot \mathrm{OEt}_{2}(1.56 \mathrm{~g}, 11.0 \mathrm{mmol}, 1.1$ equiv. $)$ in 
wet DCM $(0.5 \mathrm{M})$ at room temperature for $3 \mathrm{~h}$. Purification by column chromatography (20\% EtOAc/hexane) gave 33a (1.19 g, $4.60 \mathrm{mmol}, 46 \%)$ as a white solid.

TLC: $\mathrm{R}_{\mathrm{f}}=0.40$ (20\% EtOAc/hexane, $\mathrm{KMnO}_{4}$ stain) .

${ }^{1} \mathbf{H}$ NMR $\left(400 \mathrm{MHz}, \mathrm{CDCl}_{3}\right): \delta_{\mathrm{H}} 7.83-7.77(\mathrm{~m}, 2 \mathrm{H}), 7.75-7.69(\mathrm{~m}, 2 \mathrm{H}), 1.90-1.77(\mathrm{~m}, 4 \mathrm{H}), 1.68-$ $1.59(\mathrm{~m}, 2 \mathrm{H}), 1.47-1.35(\mathrm{~m}, 4 \mathrm{H}), 1.33(\mathrm{~s}, 3 \mathrm{H}) \mathrm{ppm}$. See spectrum.

${ }^{13} \mathrm{C}$ NMR $\left(101 \mathrm{MHz}, \mathrm{CDCl}_{3}\right): \delta_{\mathrm{C}} 165.7,134.3,129.3,123.3,88.3,36.2,25.3,23.5,23.0 \mathrm{ppm} . \underline{\text { See }}$ spectrum.

IR (film) $v_{\max }: 2933,2853,1791,1730(\mathrm{~s}), 1467,1447,1369,1349,1187,1107,968,876,712 \mathrm{~cm}^{-1}$.

HRMS (ESI) m/z: [M + Na $]^{+}$Calcd for $\mathrm{C}_{15} \mathrm{H}_{17} \mathrm{NNaO}_{3}$ 282.1101; Found 282.1090.

M.p.: $96-98^{\circ} \mathrm{C}$.

\section{2-((1,4-Dimethylcyclohexyl)oxy)isoindoline-1,3-dione (34a)}

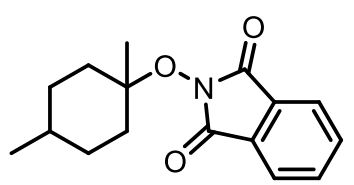

Prepared according to a modified GP1 (the Grignard addition was performed in $\mathrm{Et}_{2} \mathrm{O}$ in the absence of $\mathrm{LaCl}_{3} \cdot 2 \mathrm{LiCl}$ ): 4-Methylcyclohexan-1-one $(673 \mathrm{mg}, 6.00 \mathrm{mmol}, 1.0$ equiv.) and methylmagnesium bromide (15 mmol, 2.5 equiv.) were stirred in $\mathrm{Et}_{2} \mathrm{O}(20 \mathrm{~mL})$ at room temperature for $10 \mathrm{~h}$. Purification by column chromatography (20\% EtOAc/hexane) gave 1,4-dimethylcyclohexan-1-ol (391 mg, 3.42 mmol, 57\%) as a bright yellow oil. 1,4-Dimethylcyclohexan-1-ol (192 mg, $1.50 \mathrm{mmol}, 1.0$ equiv.) was then treated with $N$-hydroxyphthalimide ( $489 \mathrm{mg}, 3.00 \mathrm{mmol}, 2.0$ equiv.) and $\mathrm{BF}_{3} \cdot \mathrm{OEt}_{2}$ (234 mg, 1.65 mmol, 1.1 equiv.) in wet DCM $(20 \mathrm{~mL})$ at room temperature for $2 \mathrm{~h}$. Purification by column chromatography (20\% EtOAc/hexane) gave 34a (163 mg, $0.60 \mathrm{mmol}, 40 \%,>97: 3$ d.r.) as a yellow oil. The d.r. was determined by ${ }^{1} \mathrm{H}$ NMR analysis of the purified product.

TLC: $\mathrm{R}_{\mathrm{f}}=0.51\left(20 \%\right.$ EtOAc/hexane, $\mathrm{KMnO}_{4}$ stain $)$.

${ }^{1} \mathbf{H}$ NMR $\left(400 \mathrm{MHz}, \mathrm{CDCl}_{3}\right): \delta_{\mathrm{H}} 7.86-7.81(\mathrm{~m}, 2 \mathrm{H}), 7.77-7.71(\mathrm{~m}, 2 \mathrm{H}), 1.85-1.79(\mathrm{~m}, 4 \mathrm{H}), 1.78-$ $1.71(\mathrm{~m}, 2 \mathrm{H}), 1.49-1.38(\mathrm{~m}, 1 \mathrm{H}), 1.41(\mathrm{~s}, 3 \mathrm{H}), 1.13-0.97(\mathrm{~m}, 2 \mathrm{H}), 0.90(\mathrm{~d}, J=6.5 \mathrm{~Hz}, 3 \mathrm{H}) \mathrm{ppm}$. See spectrum.

${ }^{13}$ C NMR (101 MHz, $\left.\mathrm{CDCl}_{3}\right): \delta_{\mathrm{C}} 165.8,134.4,129.4,123.4,89.4,35.8,32.4,31.9,21.8,21.6$ ppm. See spectrum. 
IR (film) $v_{\max }: 2949,2869,1790,1733(\mathrm{~s}), 1467,1371,1349,1188,1109,967,904,705 \mathrm{~cm}^{-1}$.

HRMS (ESI) m/z: [M + Na $]^{+}$Calcd for $\mathrm{C}_{16} \mathrm{H}_{19} \mathrm{NNaO}_{3}$ 296.1257; Found 296.1268.

\section{2-((1,4,4-Trimethylcyclohexyl)oxy)isoindoline-1,3-dione (35a)}

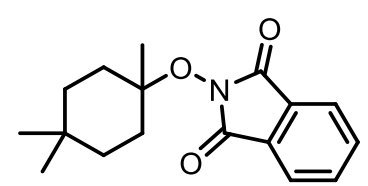

Prepared according to GP1: $\mathrm{LaCl}_{3} \cdot 2 \mathrm{LiCl}(9.0 \mathrm{mmol}, 1.5$ equiv. $)$, methylmagnesium bromide $(9.0 \mathrm{mmol}$, 1.5 equiv.), and 4,4-dimethylcyclohexan-1-one (757 mg, $6.00 \mathrm{mmol}, 1.0$ equiv.) were stirred at room temperature for $10 \mathrm{~h}$. Purification by column chromatography (20\% EtOAc/hexane) gave 1,4,4trimethylcyclohexan-1-ol (797 mg, $5.60 \mathrm{mmol}, 93 \%)$ as a bright yellow oil. 1,4,4-Trimethylcyclohexan1-ol (711 mg, $5.00 \mathrm{mmol}, 1.0$ equiv.) was then treated with $N$-hydroxyphthalimide (1.63 g, $10.0 \mathrm{mmol}$, 2.0 equiv.) and $\mathrm{BF}_{3} \cdot \mathrm{OEt}_{2}(781 \mathrm{mg}, 5.50 \mathrm{mmol}, 1.1$ equiv. $)$ in wet $\mathrm{DCM}(30 \mathrm{~mL})$ at room temperature for $2 \mathrm{~h}$. Purification by column chromatography (20\% EtOAc/hexane) gave 35a (450 mg, $1.57 \mathrm{mmol}$, $31 \%)$ as a yellow solid.

TLC: $\mathrm{R}_{\mathrm{f}}=0.51\left(20 \%\right.$ EtOAc/hexane, $\mathrm{KMnO}_{4}$ stain $)$.

${ }^{1} \mathbf{H}$ NMR $\left(400 \mathrm{MHz}, \mathrm{CDCl}_{3}\right): \delta_{\mathrm{H}} 7.86-7.80(\mathrm{~m}, 2 \mathrm{H}), 7.77-7.71(\mathrm{~m}, 2 \mathrm{H}), 1.95(\mathrm{ddd}, J=13.3,9.0,3.5$ $\mathrm{Hz}, 2 \mathrm{H}), 1.72-1.57$ (m, 4H), 1.34 (s, 3H), 1.27 (ddd, $J=12.3,9.0,3.5 \mathrm{~Hz}, 2 \mathrm{H}), 0.98$ (s, 3H), 0.92 (s, 3H) ppm. See spectrum.

${ }^{13} \mathrm{C}$ NMR $\left(101 \mathrm{MHz}, \mathrm{CDCl}_{3}\right): \delta_{\mathrm{C}} 165.8,134.4,129.3,123.4,88.2,35.7,32.4,29.6,28.3,28.0,23.7$ ppm. See spectrum.

IR (film) $v_{\max }: 2947,2861,1792,1733(\mathrm{~s}), 1467,1366,1350,1265,1189,967,905,727,702 \mathrm{~cm}^{-1}$.

HRMS (ESI) m/z: [M + Na $]^{+}$Calcd for $\mathrm{C}_{17} \mathrm{H}_{21} \mathrm{NNaO}_{3} 310.1414$; Found 310.1412.

M.p.: $76-78^{\circ} \mathrm{C}$. 


\section{2-(((1r,4r)-1-Methyl-4-phenylcyclohexyl)oxy)isoindoline-1,3-dione (36a)}

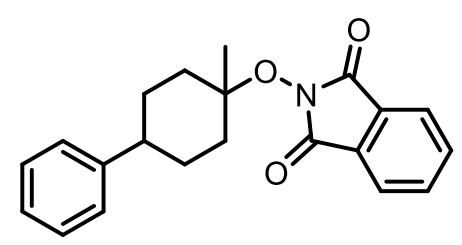

Prepared according to GP1: $\mathrm{LaCl}_{3} \cdot 2 \mathrm{LiCl}$ ( $7.2 \mathrm{mmol}, 1.2$ equiv.), methylmagnesium bromide ( $7.2 \mathrm{mmol}$, 1.2 equiv.), and 4-phenylcyclohexan-1-one (1.05 g, $6.00 \mathrm{mmol}, 1.0$ equiv.) were stirred at room temperature for $10 \mathrm{~h}$. Purification by column chromatography (20\% EtOAc/hexane) gave 1-methyl-4phenylcyclohexan-1-ol (602 mg, $3.16 \mathrm{mmol}, 53 \%)$ as a bright yellow oil. 1-Methyl-4phenylcyclohexan-1-ol (571 mg, $3.00 \mathrm{mmol}, 1.0$ equiv.) was then treated with $N$-hydroxyphthalimide (978 mg, $6.00 \mathrm{mmol}, 2.0$ equiv.) and $\mathrm{BF}_{3} \cdot \mathrm{OEt}_{2}(469 \mathrm{mg}, 3.30 \mathrm{mmol}, 1.1$ equiv.) in wet $\mathrm{DCM}(20 \mathrm{~mL})$ at room temperature for $4 \mathrm{~h}$. Purification by column chromatography (20\% EtOAc/hexane) gave 36a (500 mg, $1.49 \mathrm{mmol}, 50 \%, 85: 15$ d.r.) as a yellow oil. The d.r. was determined by ${ }^{1} \mathrm{H}$ NMR analysis of the purified product.

TLC: $\mathrm{R}_{\mathrm{f}}=0.38\left(20 \%\right.$ EtOAc/hexane, $\mathrm{KMnO}_{4}$ stain $)$.

${ }^{1} \mathbf{H} \mathbf{N M R}\left(400 \mathrm{MHz}, \mathrm{CDCl}_{3}\right)$ : (only the major diastereomer peaks are reported) $\delta_{\mathrm{H}} 7.87-7.81(\mathrm{~m}, 2 \mathrm{H})$, $7.78-7.74(\mathrm{~m}, 2 \mathrm{H}), 7.34-7.27(\mathrm{~m}, 4 \mathrm{H}), 7.23-7.16(\mathrm{~m}, 1 \mathrm{H}), 2.53(\mathrm{tt}, J=12.2,3.3 \mathrm{~Hz}, 1 \mathrm{H}), 2.41-$ $2.26(\mathrm{~m}, 2 \mathrm{H}), 2.24-2.16(\mathrm{~m}, 2 \mathrm{H}), 1.82-1.73(\mathrm{~m}, 2 \mathrm{H}), 1.64-1.52(\mathrm{~m}, 2 \mathrm{H}), 1.34(\mathrm{~s}, 3 \mathrm{H}) \mathrm{ppm} . \underline{\text { See }}$ spectrum.

${ }^{13} \mathbf{C}$ NMR (101 MHz, $\mathrm{CDCl}_{3}$ ): (only the major diastereomer peaks are reported) $\delta_{\mathrm{C}} 165.8,147.2,134.4$, 129.3, 128.3, 127.0, 125.9, 123.4, 85.8, 43.5, 36.6, 29.2, 26.4 ppm. See spectrum.

IR (film) $v_{\max }: 2973,2924,1791,1731(\mathrm{~s}), 1606,1493,1449,1372,1188,1106,1065,967,878 \mathrm{~cm}^{-1}$.

HRMS (ESI) m/z: [M + Na $]^{+}$Calcd for $\mathrm{C}_{21} \mathrm{H}_{21} \mathrm{NNaO}_{3}$ 358.1414; Found 358.1411.

\section{2-((1-Methylcycloheptyl)oxy)isoindoline-1,3-dione (37a)}<smiles>CC1(ON2C(=O)c3ccccc3C2=O)CCCCC1</smiles>

Prepared according to a modified GP1 (the Grignard addition was performed in $\mathrm{Et}_{2} \mathrm{O}$ in the absence of $\mathrm{LaCl}_{3} \cdot 2 \mathrm{LiCl}$ ): Cycloheptanone (1.10 g, $10.0 \mathrm{mmol}, 1.0$ equiv.) and methylmagnesium bromide (15 mmol, 1.5 equiv.) were stirred in $\mathrm{Et}_{2} \mathrm{O}(20 \mathrm{~mL})$ at room temperature for $15 \mathrm{~h}$. Purification by column chromatography (20\% EtOAc/hexane) gave 1-methylcycloheptan-1-ol (850 mg, $6.63 \mathrm{mmol}, 66 \%$ ) as a 
bright yellow oil. 1-Methylcycloheptan-1-ol (650 mg, $5.00 \mathrm{mmol}, 1.0$ equiv.) was then treated with $\mathrm{N}$ hydroxyphthalimide (1.63 g, $10.0 \mathrm{mmol}, 2.0$ equiv.) and $\mathrm{BF}_{3} \cdot \mathrm{OEt}_{2}(781 \mathrm{mg}, 5.50 \mathrm{mmol}, 1.1$ equiv.) in wet DCM $(30 \mathrm{~mL})$ at room temperature for $3 \mathrm{~h}$. Purification by column chromatography (20\% EtOAc/hexane) gave $\mathbf{3 7 a}(273 \mathrm{mg}, 0.99 \mathrm{mmol}, 20 \%)$ as a white solid.

TLC: $\mathrm{R}_{\mathrm{f}}=0.51\left(20 \% \mathrm{EtOAc} / \mathrm{hexane}, \mathrm{KMnO}_{4}\right.$ stain $)$.

${ }^{1} \mathbf{H}$ NMR $\left(400 \mathrm{MHz}, \mathrm{CDCl}_{3}\right): \delta_{\mathrm{H}} 7.85-7.80(\mathrm{~m}, 2 \mathrm{H}), 7.77-7.71(\mathrm{~m}, 2 \mathrm{H}), 2.13-2.05(\mathrm{~m}, 2 \mathrm{H}), 1.87$ $1.77(\mathrm{~m}, 2 \mathrm{H}), 1.76-1.68(\mathrm{~m}, 2 \mathrm{H}), 1.68-1.59(\mathrm{~m}, 2 \mathrm{H}), 1.58-1.50(\mathrm{~m}, 2 \mathrm{H}), 1.45-1.38(\mathrm{~m}, 2 \mathrm{H}), 1.35$ (s, 3H) ppm. See spectrum.

${ }^{13} \mathrm{C}$ NMR (101 MHz, $\left.\mathrm{CDCl}_{3}\right): \delta_{\mathrm{C}} 166.0,134.4,129.4,123.4,92.7,38.9,30.6,26.5,23.0$ ppm. $\underline{\text { See }}$ spectrum.

IR (film) $v_{\max }$ : 2971, 2924, 1791, 1730(s), 1466, 1371, 1348, 1187, 1078, 966, 876, $705 \mathrm{~cm}^{-1}$.

HRMS (ESI) m/z: [M + Na $]^{+}$Calcd for $\mathrm{C}_{16} \mathrm{H}_{19} \mathrm{NNaO}_{3}$ 296.1257; Found 296.1252.

M.p.: $65-67^{\circ} \mathrm{C}$.

2-((Tetrahydrofuran-2-yl)oxy)isoindoline-1,3-dione (38a)

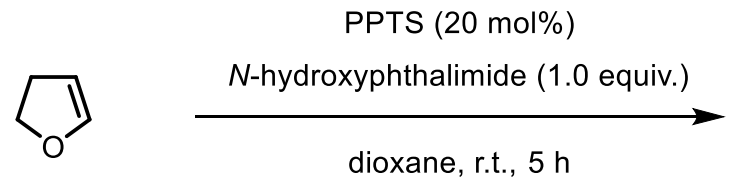

(2.0 equiv.)

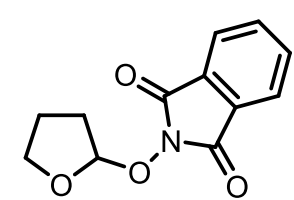

$38 a$

Prepared following a modified literature procedure: ${ }^{6}$ To a solution of 2,3-dihydrofuran $(3.50 \mathrm{~g}, 50.0$ mmol, 2.0 equiv.) in 1,4-dioxane $(20 \mathrm{~mL})$ at room temperature was added $N$-hydroxyphthalimide $(4.10$ g, 25.0 mmol, 1.0 equiv.) and pyridinium para-toluenesulfonate (PPTS, $1.25 \mathrm{~g}, 5.0 \mathrm{mmol}, 0.2$ equiv.). The reaction was stirred at room temperature for $5 \mathrm{~h}$ before being poured onto water $(100 \mathrm{~mL})$. The resulting precipitate was collected by filtration to give product $\mathbf{3 8 a}(4.78 \mathrm{~g}, 20.5 \mathrm{mmol}, 82 \%)$ as a white solid. All recorded spectroscopic data matched those previously reported in the literature. ${ }^{6}$

${ }^{1} \mathbf{H}$ NMR $\left(400 \mathrm{MHz}, \mathrm{CDCl}_{3}\right): \delta_{\mathrm{H}} 7.86-7.77(\mathrm{~m}, 2 \mathrm{H}), 7.77-7.68(\mathrm{~m}, 2 \mathrm{H}), 5.78(\mathrm{~d}, J=4.7 \mathrm{~Hz}, 1 \mathrm{H})$, $4.34(\mathrm{td}, J=8.2,6.3 \mathrm{~Hz}, 1 \mathrm{H}), 4.00(\mathrm{td}, J=8.3,5.7 \mathrm{~Hz}, 1 \mathrm{H}), 2.34-2.18(\mathrm{~m}, 2 \mathrm{H}), 2.16-2.04(\mathrm{~m}, 1 \mathrm{H})$, $2.00-1.90(\mathrm{~m}, 1 \mathrm{H})$ ppm. See spectrum.

${ }^{13}$ C NMR (101 MHz, $\left.\mathrm{CDCl}_{3}\right): \delta_{\mathrm{C}} 163.9,134.3,129.1,123.4,108.8,69.1,30.8,22.5 \mathrm{ppm} . \underline{\text { See spectrum. }}$. 


\section{2-((5-Methyltetrahydrofuran-2-yl)oxy)isoindoline-1,3-dione (39a)}
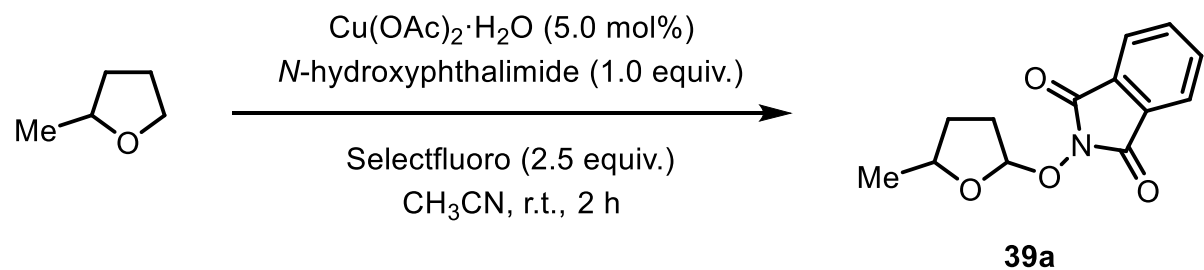

Prepared following a modified literature procedure: ${ }^{7}$ A flame-dried flask was charged with NHPI (326.2 mg, 2.0 mmol, 1.0 equiv.), 2-methyltetrahydrofuran (4 mL), Selectfluor (1.77 g, 5.0 mmol, 2.5 equiv.), $\mathrm{Cu}(\mathrm{OAc})_{2} \cdot \mathrm{H}_{2} \mathrm{O}(20.0 \mathrm{mg}, 5 \mathrm{~mol} \%)$, dry acetonitrile $(20 \mathrm{~mL})$ were added and the resulting mixture being stirred at room temperature for $2 \mathrm{~h}$. Then aqueous $\mathrm{NaHCO}_{3}$ solution $(20 \mathrm{~mL})$ was added to the reaction mixture, the mixture was extracted with DCM $(3 \times 30 \mathrm{~mL})$, and the combined organic layers were dried over $\mathrm{MgSO}_{4}$, filtered, and concentrated under reduced pressure. The residue was purified by column chromatography (20\% EtOAc/hexane) to give product 39a $(242.4 \mathrm{mg}, 0.98 \mathrm{mmol}, 49 \%,>97: 3$ d.r.) as a white solid. The d.r. was determined by ${ }^{1} \mathrm{H}$ NMR analysis of the purified product. All recorded spectroscopic data matched those previously reported in the literature. ${ }^{7}$

${ }^{1} \mathbf{H}$ NMR $\left(400 \mathrm{MHz}, \mathrm{CDCl}_{3}\right): \delta_{\mathrm{H}} 7.84-7.80(\mathrm{~m}, 2 \mathrm{H}), 7.75-7.70(\mathrm{~m}, 2 \mathrm{H}), 5.78(\mathrm{t}, J=3.2 \mathrm{~Hz}, 1 \mathrm{H}), 4.76$ $-4.65(\mathrm{~m}, 1 \mathrm{H}), 2.36-2.20(\mathrm{~m}, 3 \mathrm{H}), 1.56-1.45(\mathrm{~m}, 1 \mathrm{H}), 1.25(\mathrm{~d}, J=6.2 \mathrm{~Hz}, 3 \mathrm{H}) \mathrm{ppm}$. See spectrum. ${ }^{13} \mathrm{C}$ NMR $\left(101 \mathrm{MHz}, \mathrm{CDCl}_{3}\right): \delta_{\mathrm{C}} 163.9,134.2,129.2,123.4,108.9,76.6,30.6,30.2,20.5$ ppm. See spectrum.

\section{2-((5-Phenyltetrahydrofuran-2-yl)oxy)isoindoline-1,3-dione (40a)}<smiles>O=C1c2ccccc2C(=O)N1OC1CCC(c2ccccc2)O1</smiles>

Prepared according to GP2, with 2-phenyl-2,3-dihydrofuran (526 mg, $3.60 \mathrm{mmol}$, 1.2 equiv.). Purification by column chromatography (20\% EtOAc/hexane) gave 40a (566 mg, $1.83 \mathrm{mmol}, 61 \%$, $>97: 3$ d.r.) as a white solid. The d.r. was determined by ${ }^{1} \mathrm{H}$ NMR analysis of the purified product. All recorded spectroscopic data matched those previously reported in the literature. ${ }^{8}$

${ }^{1} \mathbf{H}$ NMR $\left(400 \mathrm{MHz}, \mathrm{CDCl}_{3}\right): \delta_{\mathrm{H}} 7.86-7.77(\mathrm{~m}, 2 \mathrm{H}), 7.77-7.67(\mathrm{~m}, 2 \mathrm{H}), 7.38-7.28(\mathrm{~m}, 4 \mathrm{H}), 7.32-$ $7.22(\mathrm{~m}, 1 \mathrm{H}), 6.01(\mathrm{t}, J=3.2 \mathrm{~Hz}, 1 \mathrm{H}), 5.61(\mathrm{t}, J=7.4 \mathrm{~Hz}, 1 \mathrm{H}), 2.70-2.59(\mathrm{~m}, 1 \mathrm{H}), 2.41-2.35(\mathrm{~m}, 2 \mathrm{H})$, $1.97-1.86(\mathrm{~m}, 1 \mathrm{H}) \mathrm{ppm} . \underline{\text { See spectrum. }}$. 
${ }^{13} \mathrm{C}$ NMR (101 MHz, $\left.\mathrm{CDCl}_{3}\right): \delta_{\mathrm{C}} 163.8,141.0,134.3,129.1,128.5,127.7,126.1,123.4,108.9,81.5$, $31.4,30.6$ ppm. See spectrum.

\section{2-((Tetrahydro-2H-pyran-2-yl)oxy)isoindoline-1,3-dione (41a)}<smiles>O=C1c2ccccc2C(=O)N1OC1CCCCO1</smiles>

Prepared according to GP2, with 3,4-dihydro- $2 H$-pyran (1.61 g, $19.0 \mathrm{mmol}, 1.2$ equiv.). Purification by column chromatography (20\% EtOAc/hexane) gave $41 \mathrm{a}$ (3.36 g, $3.60 \mathrm{mmol}, 85 \%)$ as a white solid. All recorded spectroscopic data matched those previously reported in the literature. ${ }^{9}$

${ }^{1} \mathbf{H}$ NMR $\left(400 \mathrm{MHz}, \mathrm{CDCl}_{3}\right): \delta_{\mathrm{H}} 7.83-7.77(\mathrm{~m}, 2 \mathrm{H}), 7.74-7.68(\mathrm{~m}, 2 \mathrm{H}), 5.40(\mathrm{~s}, 1 \mathrm{H}), 4.54-4.45(\mathrm{~m}$, $1 \mathrm{H}), 3.68-3.60(\mathrm{~m}, 1 \mathrm{H}), 2.13-2.05(\mathrm{~m}, 1 \mathrm{H}), 2.00-1.87(\mathrm{~m}, 1 \mathrm{H}), 1.87-1.77(\mathrm{~m}, 1 \mathrm{H}), 1.75-1.61$ $(\mathrm{m}, 3 \mathrm{H}) \mathrm{ppm}$. See spectrum.

${ }^{13} \mathrm{C}$ NMR $\left(101 \mathrm{MHz}, \mathrm{CDCl}_{3}\right): \delta_{\mathrm{C}} 163.7,134.2,129.1,123.3,103.0,62.2,27.6,24.8,17.6 \mathrm{ppm} . \underline{\text { See }}$ spectrum.

2-((6-Phenyltetrahydro-2H-pyran-2-yl)oxy)isoindoline-1,3-dione (42a)<smiles>O=C1c2ccccc2C(=O)N1OC1CCCC(c2ccccc2)O1</smiles>

Prepared according to GP2, with 2-phenyl-3,4-dihydro-2H-pyran (481 mg, $3.0 \mathrm{mmol}, 1.2$ equiv.). Purification by column chromatography (20\% EtOAc/hexane) gave 42a (509 mg, $1.58 \mathrm{mmol}, 63 \%$, $>97: 3$ d.r.) as a white solid. The d.r. was determined by ${ }^{1} \mathrm{H}$ NMR analysis of the purified product. All recorded spectroscopic data matched those previously reported in the literature. ${ }^{8}$

${ }^{1} \mathbf{H}$ NMR $\left(400 \mathrm{MHz}, \mathrm{CDCl}_{3}\right): \delta_{\mathrm{H}} 7.81-7.76(\mathrm{~m}, 2 \mathrm{H}), 7.71-7.66(\mathrm{~m}, 2 \mathrm{H}), 7.45-7.41(\mathrm{~m}, 2 \mathrm{H}), 7.36-$ $7.31(\mathrm{~m}, 2 \mathrm{H}), 7.28-7.23(\mathrm{~m}, 1 \mathrm{H}), 5.66-5.58(\mathrm{~m}, 2 \mathrm{H}), 2.30-2.10(\mathrm{~m}, 2 \mathrm{H}), 1.99-1.90(\mathrm{~m}, 2 \mathrm{H}), 1.89$ $-1.74(\mathrm{~m}, 2 \mathrm{H}) \mathrm{ppm}$. See spectrum.

${ }^{13}$ C NMR (101 MHz, $\left.\mathrm{CDCl}_{3}\right): \delta_{\mathrm{C}} 163.8,142.0,134.2,129.2,128.4,127.7,126.7,123.4,103.6,73.3$, 32.3, 27.2, $18.1 \mathrm{ppm}$. See spectrum. 


\section{2-((6-((Benzyloxy)methyl)tetrahydro-2H-pyran-2-yl)oxy)isoindoline-1,3-dione (43a)}

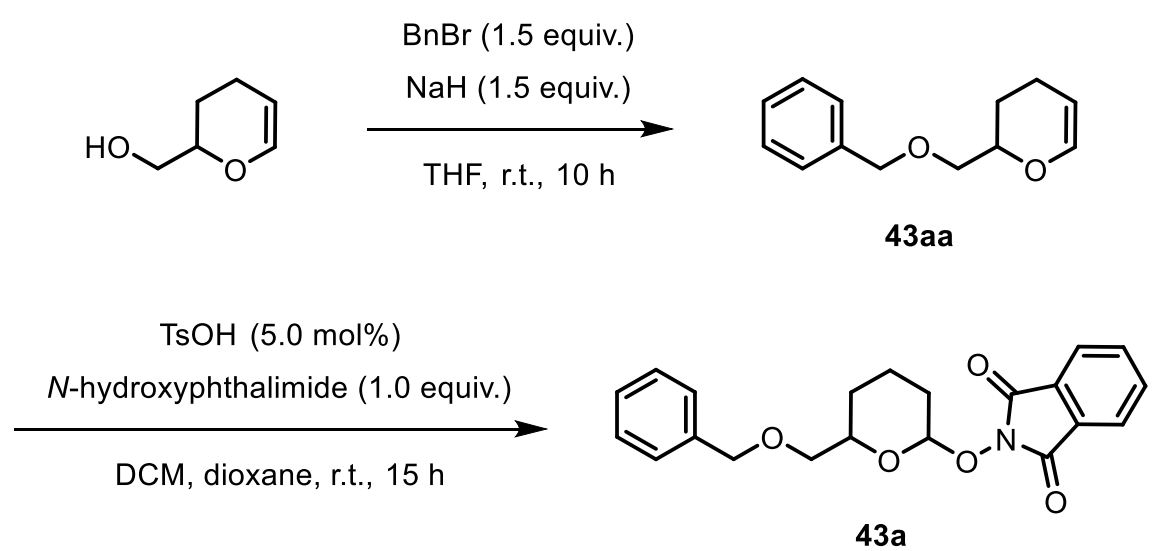

To a solution of (3,4-dihydro-2H-pyran-2-yl)methanol (685 mg, $6.00 \mathrm{mmol}, 1.0$ equiv.) in dry THF (20 $\mathrm{mL})$ at $0{ }^{\circ} \mathrm{C}$ was slowly added $\mathrm{NaH}(0.36 \mathrm{~g}, 9.0 \mathrm{mmol}, 1.5$ equiv.). The reaction was stirred at room temperature for $0.5 \mathrm{~h}$ before adding benzyl bromide ( $1.54 \mathrm{~g}, 9.00 \mathrm{mmol}, 1.5$ equiv.) and the resulting mixture was stirred at room temperature for $10 \mathrm{~h}$. The reaction mixture was diluted with water $(20 \mathrm{~mL})$ and extracted with DCM $(3 \times 30 \mathrm{~mL})$. The combined organic extracts were washed with brine, dried over $\mathrm{MgSO}_{4}$, filtered, and concentrated under reduced pressure. Purification by column chromatography (20\% EtOAc/hexane) afforded 43aa (1.02 g, $5.00 \mathrm{mmol}, 80 \%)$ as a yellow oil.

$N$-alkoxyphthalimide 43a was prepared from 43aa (1.02 g, $4.00 \mathrm{mmol}, 1.2$ equiv.) according to GP2. Purification by column chromatography (20\% EtOAc/hexane) gave 43a ( $0.88 \mathrm{~g}, 2.40 \mathrm{mmol}, 60 \%,>97: 3$ d.r.) as a white solid. The d.r. was determined by ${ }^{1} \mathrm{H}$ NMR analysis of the purified product.

TLC: $\mathrm{R}_{\mathrm{f}}=0.24\left(20 \%\right.$ EtOAc/hexane, $\mathrm{KMnO}_{4}$ stain $)$.

${ }^{1} \mathbf{H}$ NMR $\left(400 \mathrm{MHz}, \mathrm{CDCl}_{3}\right): \delta_{\mathrm{H}} 7.83-7.77(\mathrm{~m}, 2 \mathrm{H}), 7.75-7.69(\mathrm{~m}, 2 \mathrm{H}), 7.34-7.21(\mathrm{~m}, 5 \mathrm{H}), 5.54(\mathrm{~d}$, $J=3.6 \mathrm{~Hz}, 1 \mathrm{H}), 4.80-4.73(\mathrm{~m}, 1 \mathrm{H}), 4.60(\mathrm{~d}, J=12.2 \mathrm{~Hz}, 1 \mathrm{H}), 4.49(\mathrm{~d}, J=12.2 \mathrm{~Hz}, 1 \mathrm{H}), 3.49(\mathrm{~d}, J=$ $4.3 \mathrm{~Hz}, 2 \mathrm{H}), 2.19-2.10(\mathrm{~m}, 1 \mathrm{H}), 2.07-1.94(\mathrm{~m}, 1 \mathrm{H}), 1.86-1.69(\mathrm{~m}, 3 \mathrm{H}), 1.69-1.58(\mathrm{~m}, 1 \mathrm{H}) \mathrm{ppm}$. See spectrum.

${ }^{13}$ C NMR (101 MHz, $\left.\mathrm{CDCl}_{3}\right): \delta_{\mathrm{C}} 163.7,138.5,134.2,129.2,128.2,127.5,127.3,123.4,103.4,73.1$, 72.8, 70.0, 27.2, 26.7, 17.4 ppm. See spectrum.

IR (film) $v_{\max }: 2987,2901,1791,1733(\mathrm{~s}), 1610,1467,1453,1379,1264,1135,1066,878,731 \mathrm{~cm}^{-1}$.

HRMS (ESI) m/z: [M + Na $]^{+}$Calcd for $\mathrm{C}_{21} \mathrm{H}_{21} \mathrm{NNaO}_{5}$ 390.1312; Found 390.1328.

M.p.: $101-103{ }^{\circ} \mathrm{C}$. 


\section{(6-((1,3-Dioxoisoindolin-2-yl)oxy)tetrahydro-2H-pyran-2-yl)methyl acetate (44a)}
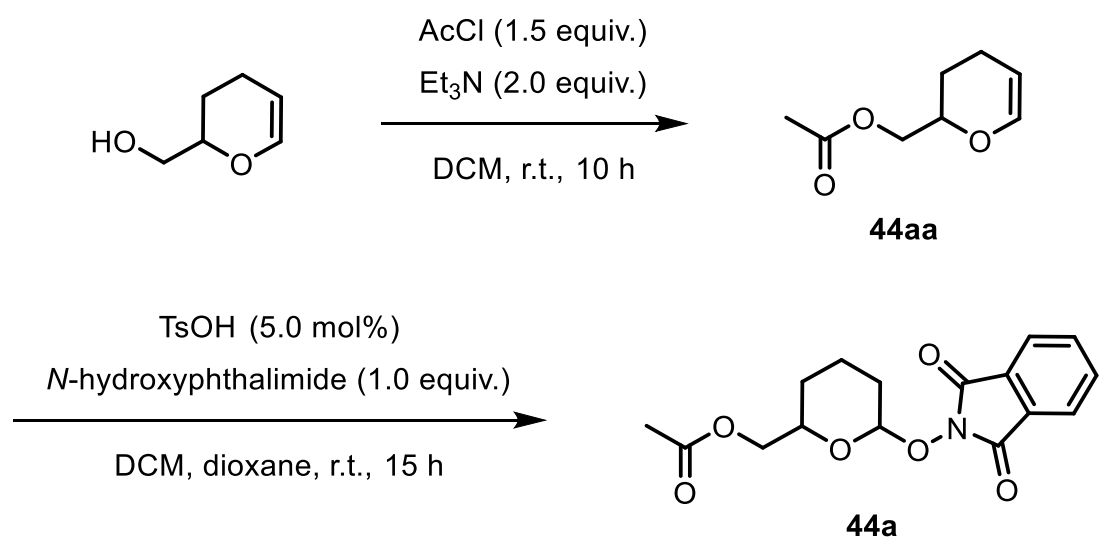

To a solution of (3,4-dihydro-2H-pyran-2-yl)methanol (685 mg, $6.00 \mathrm{mmol}, 1.0$ equiv.) and triethylamine (1.21 g, $12.0 \mathrm{mmol}, 2.0$ equiv.) in dry DCM (20 mL) was slowly added acetyl chloride $(0.71 \mathrm{~g}, 9.0 \mathrm{mmol}, 1.5$ equiv. $)$ at $0{ }^{\circ} \mathrm{C}$. The solution was stirred at room temperature for $10 \mathrm{~h}$ before adding water $(50 \mathrm{~mL})$ and extracting with DCM $(3 \times 30 \mathrm{~mL})$. The combined organic extracts were washed with brine, dried over $\mathrm{MgSO}_{4}$, filtered, and concentrated under reduced pressure. Purification by column chromatography ( $20 \%$ EtOAc/hexane) gave $44 \mathbf{a a}(840 \mathrm{mg}, 5.38 \mathrm{mmol}, 90 \%)$ as a yellow oil. $N$-alkoxyphthalimide 44a was prepared from $44 \mathbf{a a}(780 \mathrm{mg}, 5.00 \mathrm{mmol}, 1.2$ equiv.) according to GP2. Purification by column chromatography (20\% EtOAc/hexane) gave 44a (930 mg, $2.91 \mathrm{mmol}, 70 \%$, $>97: 3$ d.r.) as a white solid. The d.r. was determined by ${ }^{1} \mathrm{H}$ NMR analysis of the purified product.

TLC: $\mathrm{R}_{\mathrm{f}}=0.24\left(20 \%\right.$ EtOAc/hexane, $\mathrm{KMnO}_{4}$ stain $)$.

${ }^{1} \mathbf{H}$ NMR $\left(400 \mathrm{MHz}, \mathrm{CDCl}_{3}\right): \delta_{\mathrm{H}} 7.83-7.73(\mathrm{~m}, 2 \mathrm{H}), 7.75-7.66(\mathrm{~m}, 2 \mathrm{H}), 5.47(\mathrm{~d}, J=3.6 \mathrm{~Hz}, 1 \mathrm{H})$, $4.75(\mathrm{ddt}, J=11.6,5.5,2.7 \mathrm{~Hz}, 1 \mathrm{H}), 4.14(\mathrm{dd}, J=11.8,3.2 \mathrm{~Hz}, 1 \mathrm{H}), 3.96(\mathrm{dd}, J=11.8,6.0 \mathrm{~Hz}, 1 \mathrm{H})$, $2.16-2.08(\mathrm{~m}, 1 \mathrm{H}), 2.06-1.90(\mathrm{~m}, 1 \mathrm{H}), 2.00(\mathrm{~s}, 3 \mathrm{H}), 1.84-1.65(\mathrm{~m}, 3 \mathrm{H}), 1.51-1.39(\mathrm{~m}, 1 \mathrm{H}) \mathrm{ppm}$. See spectrum.

${ }^{13}$ C NMR (101 MHz, $\left.\mathrm{CDCl}_{3}\right): \delta_{\mathrm{C}} 170.8,163.4,134.3,129.0,123.2,103.1,69.1,66.5,26.9,26.2,20.7$, $17.2 \mathrm{ppm} . \underline{\text { See spectrum. }}$

IR (film) $v_{\max }$ : 2956, 2901, 1791, 1733(s), 1468, 1377, 1187, 1083, 968, 704, 725, $703 \mathrm{~cm}^{-1}$.

HRMS (ESI) m/z: [M + Na] $]^{+}$Calcd for $\mathrm{C}_{16} \mathrm{H}_{17} \mathrm{NNaO}_{6}$ 342.0948; Found 342.0944.

M.p.: $112-114{ }^{\circ} \mathrm{C}$. 


\section{(6-((1,3-Dioxoisoindolin-2-yl)oxy)tetrahydro-2H-pyran-2-yl)methyl benzoate (45a)}
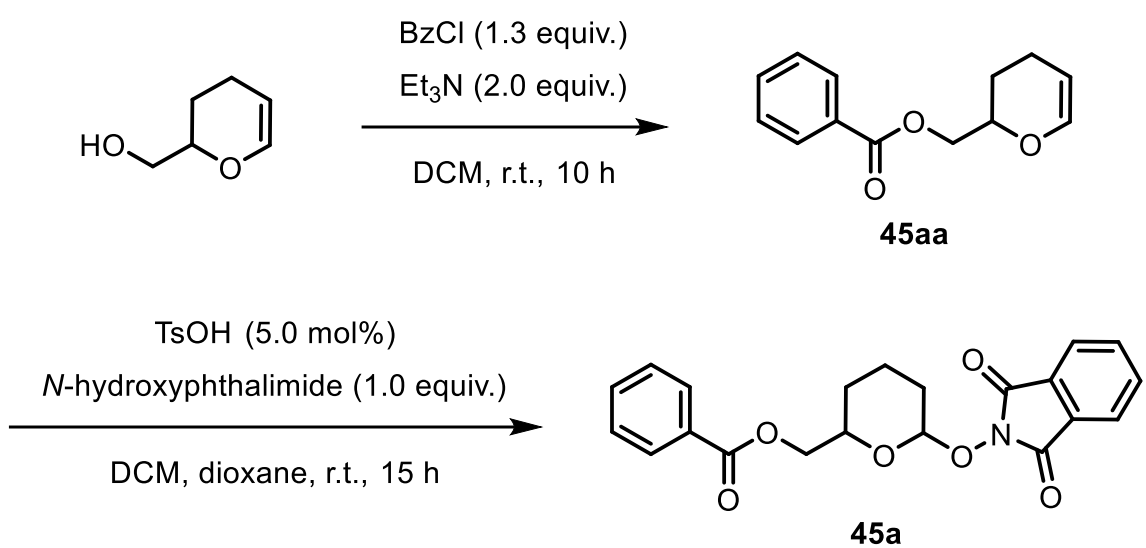

To a solution of (3,4-dihydro-2H-pyran-2-yl)methanol $(685 \mathrm{mg}, 6.00 \mathrm{mmol}, 1.0$ equiv.) and triethylamine (1.21 g, $12.0 \mathrm{mmol}, 2.0$ equiv.) in dry DCM (20 mL) was slowly added benzoyl chloride $\left(1.10 \mathrm{~g}, 7.8 \mathrm{mmol}, 1.3\right.$ equiv.) at $0{ }^{\circ} \mathrm{C}$. The solution was stirred at room temperature for $10 \mathrm{~h}$ before adding water $(50 \mathrm{~mL})$ and extracting with DCM $(3 \times 30 \mathrm{~mL})$. The combined organic extracts were washed with brine, dried over $\mathrm{MgSO}_{4}$, filtered, and concentrated under reduced pressure. Purification by column chromatography (20\% EtOAc/hexane) gave 45aa (1.31 g, $5.99 \mathrm{mmol}, 99 \%$ ) as a yellow oil.

$N$-alkoxyphthalimide 45a was prepared from $45 \mathrm{aa}$ ( $1.31 \mathrm{~g}, 6.00 \mathrm{mmol}, 1.2$ equiv.) according to GP2. Purification by column chromatography (20\% EtOAc/hexane) gave 45a (1.06 g, $2.78 \mathrm{mmol}, 56 \%,>97: 3$ d.r.) as a white solid. The d.r. was determined by ${ }^{1} \mathrm{H}$ NMR analysis of the purified product.

TLC: $\mathrm{R}_{\mathrm{f}}=0.24$ (20\% EtOAc/hexane, $\mathrm{KMnO}_{4}$ stain).

${ }^{1} \mathbf{H}$ NMR $\left(400 \mathrm{MHz}, \mathrm{CDCl}_{3}\right): \delta_{\mathrm{H}} 8.05-7.97(\mathrm{~m}, 2 \mathrm{H}), 7.71-7.65(\mathrm{~m}, 4 \mathrm{H}), 7.56-7.47(\mathrm{~m}, 1 \mathrm{H}), 7.39-$ $7.30(\mathrm{~m}, 2 \mathrm{H}), 5.53(\mathrm{~d}, J=3.5 \mathrm{~Hz}, 1 \mathrm{H}), 5.02-4.91(\mathrm{~m}, 1 \mathrm{H}), 4.38(\mathrm{dd}, J=11.8,3.1 \mathrm{~Hz}, 1 \mathrm{H}), 4.25(\mathrm{dd}, J$ $=11.8,6.6 \mathrm{~Hz}, 1 \mathrm{H}), 2.21-2.14(\mathrm{~m}, 1 \mathrm{H}), 2.11-1.99(\mathrm{~m}, 1 \mathrm{H}), 1.86-1.74(\mathrm{~m}, 3 \mathrm{H}), 1.61-1.49(\mathrm{~m}, 1 \mathrm{H})$ ppm. $\underline{\text { See spectrum. }}$.

${ }^{13} \mathrm{C}$ NMR (101 MHz, $\left.\mathrm{CDCl}_{3}\right): \delta_{\mathrm{C}} 166.4,163.5,134.2,132.7,130.3,129.7,129.0,128.2,123.3,102.9$, 69.2, 67.3, 27.0, 26.5, 17.2 ppm. See spectrum.

IR (film) $v_{\max }$ : 2987, 2901, 1791, 1734(s), 1720, 1468, 1443, 1407, 1379, 1264, 1187, 1066, 1051, 731, $702 \mathrm{~cm}^{-1}$.

HRMS (ESI) m/z: [M + Na $]^{+}$Calcd for $\mathrm{C}_{21} \mathrm{H}_{19} \mathrm{NNaO}_{6}$ 404.1105; Found 404.1104.

M.p.: $151-153{ }^{\circ} \mathrm{C}$. 


\section{2-(((4R,5S,6R)-4,5-Dimethoxy-6-(methoxymethyl)tetrahydro-2H-pyran-2-yl)oxy)isoindoline-}

\section{1,3-dione (46a)}

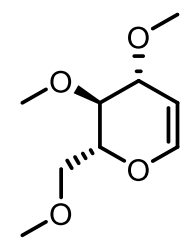

(1.0 equiv.)

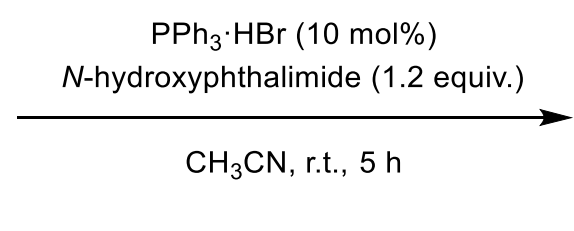

$46 a$

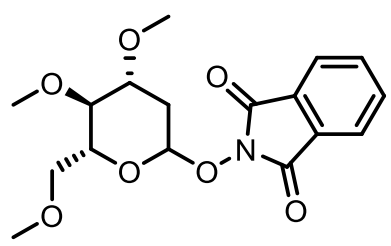

Prepared following a modified literature procedure: ${ }^{10}$ To a stirred solution of tri- $O$-methyl-D-glucal (0.94 g, $5.0 \mathrm{mmol}, 1.0$ equiv.) in acetonitrile $(9 \mathrm{~mL})$ at room temperature were added $\mathrm{N}$ hydroxyphthalimide (0.97 g, $6.0 \mathrm{mmol}, 1.2$ equiv.) and $\mathrm{Ph}_{3} \mathrm{P} \cdot \mathrm{HBr}(171.0 \mathrm{mg}, 0.5 \mathrm{mmol}, 10 \mathrm{~mol} \%)$. After stirring at room temperature for $5 \mathrm{~h}$, the reaction mixture was concentrated under reduced pressure. The residue was purified by column chromatography (20\% EtOAc/hexane) to give 46a (1.4 g, $4.0 \mathrm{mmol}$, $80 \%,>97: 3$ d.r.) as a yellow oil. The d.r. was determined by ${ }^{1} \mathrm{H}$ NMR analysis of the purified product.

TLC: $\mathrm{R}_{\mathrm{f}}=0.26\left(40 \%\right.$ EtOAc/hexane, $\mathrm{KMnO}_{4}$ stain) .

$[\alpha]^{20}{ }^{20}:+80.0\left(c 1.0, \mathrm{CHCl}_{3}\right)$.

${ }^{1} \mathbf{H}$ NMR (400 MHz, $\left.\mathrm{CDCl}_{3}\right): \delta_{\mathrm{H}} 7.83-7.78(\mathrm{~m}, 2 \mathrm{H}), 7.75-7.69(\mathrm{~m}, 2 \mathrm{H}), 5.49(\mathrm{~d}, J=3.8 \mathrm{~Hz}, 1 \mathrm{H})$, $4.44(\mathrm{dt}, J=10.1,2.1 \mathrm{~Hz}, 1 \mathrm{H}), 3.74-3.64(\mathrm{~m}, 2 \mathrm{H}), 3.56(\mathrm{~s}, 3 \mathrm{H}), 3.51(\mathrm{dd}, J=10.8,2.1 \mathrm{~Hz}, 1 \mathrm{H}), 3.48$ (s, 3H), 3.36 (s, 3H), 3.31 (dd, $J=10.2,8.9 \mathrm{~Hz}, 1 \mathrm{H}), 2.62$ (ddd, $J=13.9,5.2,1.3 \mathrm{~Hz}, 1 \mathrm{H}), 1.74$ (ddd, $J$ $=13.9,11.5,4.2 \mathrm{~Hz}, 1 \mathrm{H})$. See spectrum.

${ }^{13} \mathrm{C}$ NMR (101 MHz, $\left.\mathrm{CDCl}_{3}\right): \delta_{\mathrm{C}} 163.4,134.4,129.0,123.5,103.7,79.1,77.8,72.7,70.6,60.4,59.1$, 57.6, $32.8 \mathrm{ppm}$. See spectrum.

IR (film) $v_{\max }: 2977,2860,1733(\mathrm{~s}), 1445,1382,1351,1119,1077,844,749 \mathrm{~cm}^{-1}$.

HRMS (ESI) m/z: [M + Na $]^{+}$Calcd for $\mathrm{C}_{17} \mathrm{H}_{21} \mathrm{NNaO}_{7} 374.1216$; Found 374.1213. 


\section{Unsuccessful Substrates}

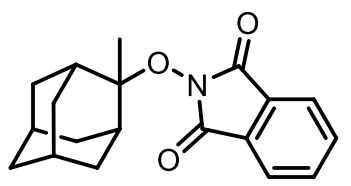

S1

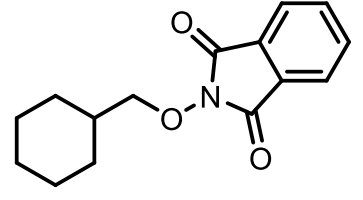

S2

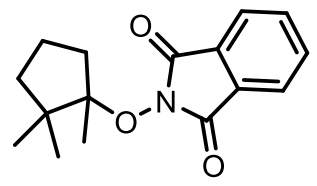

S3

Substrates $\mathbf{S 1}$ and $\mathbf{S 2}$ were prepared and investigated in the fragmentation borylation reaction under our optimized conditions.

Reaction of $\mathbf{S 1}$ resulted in complete conversion but no desired product. The major product was 2-methyl2 -adamantanol, which is likely formed because of slow $\beta$-scission of the oxygen-centered radical intermediate, resulting in competitive borate ester formation via O-borylation. Hydrolysis of the borate ester during work-up provides the alcohol.

Reaction of S2 resulted in almost complete recovered of unreacted S2, with only trace cyclohexyl boronic acid pinacol ester observed by GC-MS.

We attempted to synthesize a tertiary boronic ester by fragmentation borylation of substrate S3. However, all our attempts to prepare $\mathbf{S 3}$ were unsuccessful.

\section{2-(((1r,3r,5r,7r)-2-Methyladamantan-2-yl)oxy)isoindoline-1,3-dione (S1)}

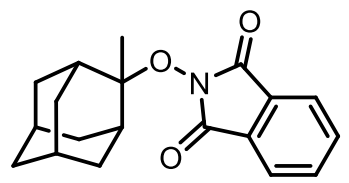

Prepared following GP1-B: 2-Methyl-2-adamantanol (1.07 g, 6.44 mmol, 1.0 equiv.) was treated with $N$-hydroxyphthalimide $(2.10 \mathrm{~g}, 12.9 \mathrm{mmol}, 2.0$ equiv. $)$ and $\mathrm{BF}_{3} \cdot \mathrm{OEt}_{2}(1.01 \mathrm{~g}, 7.08 \mathrm{mmol}, 1.1$ equiv. $)$ in wet DCM $(0.30 \mathrm{M})$ at room temperature for $4 \mathrm{~h}$. Purification by column chromatography (20\% EtOAc/hexane) gave S1 (1.43 g, $4.59 \mathrm{mmol}, 71 \%)$ as a pale-yellow oil.

${ }^{1} \mathbf{H}$ NMR $\left(400 \mathrm{MHz}, \mathrm{CDCl}_{3}\right): \delta_{\mathrm{H}} 7.85-7.76(\mathrm{~m}, 2 \mathrm{H}), 7.77-7.68(\mathrm{~m}, 2 \mathrm{H}), 2.72-2.64(\mathrm{~m}, 2 \mathrm{H}), 2.10-$ $2.06(\mathrm{~m}, 2 \mathrm{H}), 1.93-1.76(\mathrm{~m}, 6 \mathrm{H}), 1.75-1.71(\mathrm{~m}, 2 \mathrm{H}), 1.67-1.58(\mathrm{~m}, 2 \mathrm{H}), 1.39(\mathrm{~s}, 3 \mathrm{H})$. See spectrum.

${ }^{13} \mathrm{C}$ NMR $\left(101 \mathrm{MHz}, \mathrm{CDCl}_{3}\right): \delta_{\mathrm{C}} 165.7,134.3,129.2,123.2,92.3,38.3,37.5,35.9,32.8,27.4,26.9$, 20.8 ppm. See spectrum.

IR (film) $v_{\max }: 2906,1856,1787,1731(\mathrm{~s}), 1608,1443,1351,1185,1102,963,874 \mathrm{~cm}^{-1}$.

HRMS $\left(\mathrm{ESI}^{+}\right) \mathrm{m} / \mathrm{z}:[\mathrm{M}+\mathrm{Na}]^{+}$Calcd. for $\mathrm{C}_{19} \mathrm{H}_{21} \mathrm{NNaO}_{3}$ 334.1414; Found 334.1412. 


\section{2-(Cyclohexylmethoxy)isoindoline-1,3-dione (S2)}

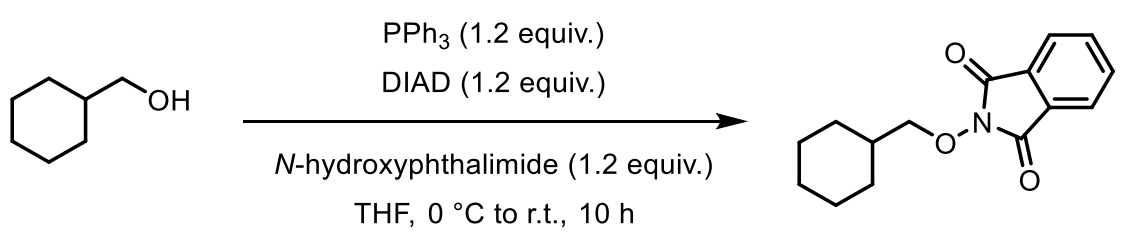

To a solution of cyclohexylmethanol $(685 \mathrm{mg}, 6.00 \mathrm{mmol}, 1.0$ equiv.), $N$-hydroxyphthalimide $(1.17 \mathrm{~g}$, $7.20 \mathrm{mmol}, 1.2$ equiv.) and triphenylphosphine ( $1.89 \mathrm{~g}, 7.20 \mathrm{mmol}, 1.2$ equiv. $)$ in $\mathrm{THF}(30 \mathrm{~mL})$ at $0{ }^{\circ} \mathrm{C}$ was added diisopropyl azodicarboxylate $(1.42 \mathrm{~mL}, 7.20 \mathrm{mmol}, 1.0$ equiv.) dropwise. The reaction was allowed to warm to room temperature and stirred for $10 \mathrm{~h}$ before removal of the solvent under reduced pressure. The residue was purified by column chromatography (20\% EtOAc/hexane) to give product $(1.21 \mathrm{~g}, 4.67 \mathrm{mmol}, 78 \%)$ as a colorless oil.

${ }^{1} \mathbf{H}$ NMR $\left(400 \mathrm{MHz}, \mathrm{CDCl}_{3}\right): \delta_{\mathrm{H}} 7.87-7.78(\mathrm{~m}, 2 \mathrm{H}), 7.78-7.69(\mathrm{~m}, 2 \mathrm{H}), 4.00(\mathrm{~d}, J=6.6 \mathrm{~Hz}, 2 \mathrm{H})$, $1.98-1.90(\mathrm{~m}, 2 \mathrm{H}), 1.90-1.80(\mathrm{~m}, 1 \mathrm{H}), 1.80-1.72(\mathrm{~m}, 2 \mathrm{H}), 1.73-1.65(\mathrm{~m}, 1 \mathrm{H}), 1.35-1.16(\mathrm{~m}, 3 \mathrm{H})$, $1.14-1.01(\mathrm{~m}, 2 \mathrm{H}) \mathrm{ppm} . \underline{\text { See spectrum. }}$.

${ }^{13}$ C NMR $\left(101 \mathrm{MHz}, \mathrm{CDCl}_{3}\right): \delta_{\mathrm{C}} 163.60134 .4,129.0,123.4,83.8,36.8,29.5,26.4,25.6$ ppm. See spectrum.

IR (film) $v_{\max }$ : 2924, 2853, 1780, 1726(s), 1466, 1372, 1186, 1128, 993, 877, $698 \mathrm{~cm}^{-1}$.

HRMS (ESI) m/z: [M + Na $]^{+}$Calcd for $\mathrm{C}_{15} \mathrm{H}_{17} \mathrm{NNaO}_{3}$ 282.1101; Found 282.1099. 


\section{Product Characterization}

6-(4,4,5,5-Tetramethyl-1,3,2-dioxaborolan-2-yl)hexan-2-one (2)<smiles>CC(=O)CCCCB1OC(C)(C)C(C)(C)O1</smiles>

Pinacol boronic ester 2 was prepared following GP3, using $N$-alkoxyphthalimide $1 \mathbf{a}(74 \mathrm{mg}, 0.30 \mathrm{mmol}$, 1.0 equiv.). Flash chromatography (10\% EtOAc/hexane) gave 2 (61 mg, $0.27 \mathrm{mmol}, 90 \%)$ as colourless liquid. All recorded spectroscopic data matched those previously reported in the literature. ${ }^{11}$

${ }^{1} \mathbf{H}$ NMR $\left(400 \mathrm{MHz}, \mathrm{CDCl}_{3}\right): \delta_{\mathrm{H}} 2.40(\mathrm{t}, J=7.4 \mathrm{~Hz}, 2 \mathrm{H}), 2.11(\mathrm{~s}, 3 \mathrm{H}), 1.61-1.52(\mathrm{~m}, 2 \mathrm{H}), 1.45-1.35$ $(\mathrm{m}, 2 \mathrm{H}), 1.23(\mathrm{~s}, 12 \mathrm{H}), 0.77(\mathrm{t}, J=7.8 \mathrm{~Hz}, 2 \mathrm{H}) \mathrm{ppm}$. See spectrum.

${ }^{13} \mathrm{C}$ NMR (101 MHz, $\left.\mathrm{CDCl}_{3}\right): \delta_{\mathrm{C}} 209.3,82.9,43.7,29.7,26.5,24.8,23.6 \mathrm{ppm}$. The carbon attached to boron could not be observed due to quadrupolar relaxation. See spectrum.

\section{5-(4,4,5,5-Tetramethyl-1,3,2-dioxaborolan-2-yl)pentan-2-one (3)}

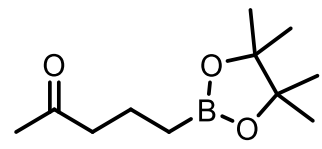

Pinacol boronic ester 3 was prepared following GP3, using $N$-alkoxyphthalimide 3a ( $69 \mathrm{mg}, 0.30 \mathrm{mmol}$, 1.0 equiv.). Flash chromatography (10\% EtOAc/hexane) gave $3(53 \mathrm{mg}, 0.25 \mathrm{mmol}, 84 \%)$ as a colourless liquid. All recorded spectroscopic data matched those previously reported in the literature. ${ }^{12}$

${ }^{1} \mathrm{H}$ NMR (400 MHz, CDCl 3 ): $\delta_{\mathrm{H}} 2.42(\mathrm{t}, J=7.5 \mathrm{~Hz}, 2 \mathrm{H}), 2.11(\mathrm{~s}, 3 \mathrm{H}), 1.69(\mathrm{p}, J=7.7 \mathrm{~Hz}, 2 \mathrm{H}), 1.23$ (s, $12 \mathrm{H}), 0.77$ (t, $J=7.8 \mathrm{~Hz}, 2 \mathrm{H}) \mathrm{ppm}$. See spectrum.

${ }^{13} \mathrm{C} \mathrm{NMR}\left(101 \mathrm{MHz}, \mathrm{CDCl}_{3}\right): \delta_{\mathrm{C}} 209.2,83.0,46.1,29.8,24.8,18.5 \mathrm{ppm}$. The carbon attached to boron could not be observed due to quadrupolar relaxation. See spectrum. 


\section{6-(4,4,5,5-Tetramethyl-1,3,2-dioxaborolan-2-yl)hexan-3-one (4)}

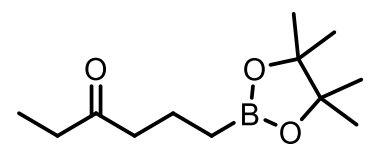

Pinacol boronic ester 4 was prepared following GP3, using $N$-alkoxyphthalimide $\mathbf{4 a}$ (74 mg, $0.30 \mathrm{mmol}$, 1.0 equiv.). Flash chromatography (10\% EtOAc/hexane) gave 4 (53 mg, $0.23 \mathrm{mmol}, 78 \%$ ) as colourless liquid.

TLC: $\mathrm{R}_{\mathrm{f}}=0.45\left(10 \%\right.$ EtOAc/hexane, $\mathrm{KMnO}_{4}$ stain $)$.

${ }^{1} \mathbf{H}$ NMR $\left(400 \mathrm{MHz}, \mathrm{CDCl}_{3}\right): \delta_{\mathrm{H}} 2.39(\mathrm{q}, J=7.3 \mathrm{~Hz}, 2 \mathrm{H}), .39(\mathrm{t}, J=7.5 \mathrm{~Hz}, 2 \mathrm{H}), 1.68(\mathrm{p}, J=7.7 \mathrm{~Hz}$, $1 \mathrm{H}), 1.22(\mathrm{~s}, 12 \mathrm{H}), 1.02(\mathrm{t}, J=7.4 \mathrm{~Hz}, 3 \mathrm{H}), 0.76(\mathrm{t}, J=7.8 \mathrm{~Hz}, 2 \mathrm{H}) \mathrm{ppm}$. See spectrum.

${ }^{13} \mathbf{C}$ NMR $\left(101 \mathrm{MHz}, \mathrm{CDCl}_{3}\right): \delta_{\mathrm{C}} 211.8,83.0,44.6,35.8,24.8,18.6,7.8 \mathrm{ppm}$. The carbon attached to boron could not be observed due to quadrupolar relaxation. See spectrum.

${ }^{11}$ B NMR $\left(128 \mathrm{MHz}, \mathrm{CDCl}_{3}\right): \delta_{\mathrm{B}} 33.8$ (br. s, 1B) ppm. See spectrum.

IR (film) $v_{\max }: 2978,2939,1713(\mathrm{~s}), 1372,1319,1267,1144,968,847,732 \mathrm{~cm}^{-1}$.

HRMS (ESI) m/z: $[\mathrm{M}+\mathrm{H}]^{+}$Calcd for $\mathrm{C}_{12} \mathrm{H}_{24} \mathrm{BO}_{3} 227.1815$; Found 227.1813.

\section{1-(4,4,5,5-Tetramethyl-1,3,2-dioxaborolan-2-yl)octan-4-one (5)}

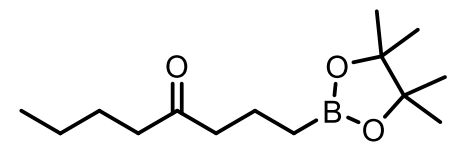

Pinacol boronic ester 5 was prepared following GP3, using $N$-alkoxyphthalimide $\mathbf{5 a}(82 \mathrm{mg}, 0.30 \mathrm{mmol}$, 1.0 equiv.). Flash chromatography (10\% EtOAc/hexane) gave 5 (53 mg, $0.21 \mathrm{mmol}, 70 \%)$ as colourless liquid.

TLC: $\mathrm{R}_{\mathrm{f}}=0.45\left(10 \%\right.$ EtOAc/hexane, $\mathrm{KMnO}_{4}$ stain $)$.

${ }^{1} \mathrm{H} \mathrm{NMR}\left(400 \mathrm{MHz}, \mathrm{CDCl}_{3}\right): \delta_{\mathrm{H}} 2.38(\mathrm{t}, J=7.5 \mathrm{~Hz}, 2 \mathrm{H}), 2.36(\mathrm{t}, J=7.5 \mathrm{~Hz}, 2 \mathrm{H}), 1.71-1.62(\mathrm{~m}, 2 \mathrm{H})$, $1.56-1.47(\mathrm{~m}, 2 \mathrm{H}), 1.33-1.23(\mathrm{~m}, 2 \mathrm{H}), 1.22(\mathrm{~s}, 12 \mathrm{H}), 0.87(\mathrm{t}, J=7.3 \mathrm{~Hz}, 3 \mathrm{H}), 0.75(\mathrm{t}, J=7.8 \mathrm{~Hz}, 2 \mathrm{H})$ ppm. See spectrum.

${ }^{13} \mathrm{C}$ NMR $\left(101 \mathrm{MHz}, \mathrm{CDCl}_{3}\right): \delta_{\mathrm{C}} 211.5,83.0,45.0,42.5,25.9,24.8,22.3,18.5,13.8 \mathrm{ppm}$. The carbon attached to boron could not be observed due to quadrupolar relaxation. See spectrum.

${ }^{11} \mathrm{~B}$ NMR $\left(128 \mathrm{MHz}, \mathrm{CDCl}_{3}\right): \delta_{\mathrm{B}} 33.8$ (br. s, 1B) ppm. See spectrum. 
IR (film) $v_{\max }$ : 2980, 2936, 1709(s), 1407, 1372, 1320, 1143, 1057, 907, $728 \mathrm{~cm}^{-1}$.

HRMS (ESI) m/z: [M + Na $]^{+}$Calcd for $\mathrm{C}_{14} \mathrm{H}_{27} \mathrm{BNaO}_{3}$ 277.1948; Found 277.1945.

\section{1-(4,4,5,5-Tetramethyl-1,3,2-dioxaborolan-2-yl)tridecan-4-one (6)}

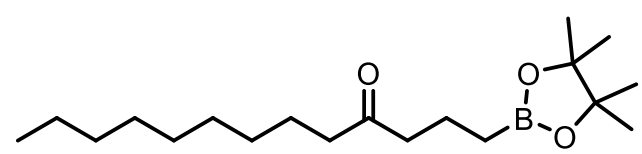

Pinacol boronic ester 6 was prepared following GP3, using $N$-alkoxyphthalimide 6a (103 mg, 0.30 mmol, 1.0 equiv.). Flash chromatography (10\% EtOAc/hexane) gave 6 (60 mg, $0.17 \mathrm{mmol}, 62 \%)$ as colourless liquid.

TLC: $\mathrm{R}_{\mathrm{f}}=0.45$ (10\% EtOAc/hexane, $\mathrm{KMnO}_{4}$ stain).

${ }^{1} \mathbf{H}$ NMR (400 MHz, $\left.\mathrm{CDCl}_{3}\right): \delta_{\mathrm{H}} 2.40(\mathrm{t}, J=7.5 \mathrm{~Hz}, 2 \mathrm{H}), 2.37(\mathrm{t}, J=7.5 \mathrm{~Hz}, 2 \mathrm{H}), 1.74-1.64(\mathrm{~m}, 2 \mathrm{H})$, $1.57-1.52(\mathrm{~m}, 2 \mathrm{H}), 1.31-1.24(\mathrm{~m}, 12 \mathrm{H}), 1.24(\mathrm{~s}, 12 \mathrm{H}), 0.87(\mathrm{~d}, J=7.1 \mathrm{~Hz}, 3 \mathrm{H}), 0.77(\mathrm{t}, J=7.8 \mathrm{~Hz}$, 2H) ppm. See spectrum.

${ }^{13} \mathrm{C}$ NMR $\left(101 \mathrm{MHz}, \mathrm{CDCl}_{3}\right): \delta_{\mathrm{C}} 211.6,83.0,45.0,42.8,31.9,29.4,29.3,29.3,24.8,23.9,22.7,18.6$, $14.1 \mathrm{ppm}$. The carbon attached to boron could not be observed due to quadrupolar relaxation. See spectrum.

${ }^{11} \mathbf{B}$ NMR (128 MHz, $\left.\mathrm{CDCl}_{3}\right): \delta_{\mathrm{B}} 33.8$ (br. s, 1B) ppm. $\underline{\text { See spectrum. }}$

IR (film) $v_{\max }: 2974,2924,1712(\mathrm{~s}), 1455,1407,1371,1319,1144,1056,1033,846,731 \mathrm{~cm}^{-1}$.

HRMS (ESI) m/z: [M + Na $]^{+}$Calcd for $\mathrm{C}_{19} \mathrm{H}_{37} \mathrm{BNaO}_{3}$ 347.2731; Found 347.2736.

\section{1-(4,4,5,5-Tetramethyl-1,3,2-dioxaborolan-2-yl)docosan-4-one (7)}

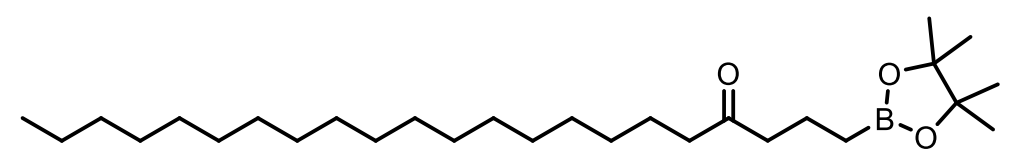

Pinacol boronic ester 7 was prepared following GP3, using $N$-alkoxyphthalimide 7a ( $94 \mathrm{mg}, 0.20 \mathrm{mmol}$, 1.0 equiv.). Flash chromatography (10\% EtOAc/hexane) gave 7 (64 mg, $0.14 \mathrm{mmol}, 71 \%$ ) as colourless liquid.

TLC: $\mathrm{R}_{\mathrm{f}}=0.45$ (10\% EtOAc/hexane, $\mathrm{KMnO}_{4}$ stain) . 
${ }^{1} \mathbf{H}$ NMR (400 MHz, $\left.\mathrm{CDCl}_{3}\right): \delta_{\mathrm{H}} 2.40(\mathrm{t}, J=7.5 \mathrm{~Hz}, 2 \mathrm{H}), 2.37(\mathrm{t}, J=7.5 \mathrm{~Hz}, 2 \mathrm{H}), 1.73-1.63(\mathrm{~m}, 2 \mathrm{H})$, $1.58-1.50(\mathrm{~m}, 2 \mathrm{H}), 1.32-1.24(\mathrm{~m}, 30 \mathrm{H}), 1.24(\mathrm{~s}, 12 \mathrm{H}), 0.88(\mathrm{t}, J=7.0 \mathrm{~Hz}, 3 \mathrm{H}), 0.77(\mathrm{t}, J=7.8 \mathrm{~Hz}$, 2H) ppm. See spectrum.

${ }^{13} \mathrm{C}$ NMR $\left(101 \mathrm{MHz}, \mathrm{CDCl}_{3}\right): \delta_{\mathrm{C}} 211.6,83.0,45.0,42.8,31.9,29.7,29.7,29.6,29.5,29.4,29.4,29.3$, 24.8, 23.9, 22.7, 18.6, $14.1 \mathrm{ppm}$. The carbon attached to boron could not be observed due to quadrupolar relaxation. See spectrum.

${ }^{11} \mathbf{B}$ NMR (128 MHz, $\mathrm{CDCl}_{3}$ ): $\delta_{\mathrm{B}} 33.7$ (br. s, 1B) ppm. See spectrum.

IR (film) $v_{\max }: 2973,2921,2853,1713(\mathrm{~s}), 1466,1407,1372,1319,1144,1056,1033,892,734 \mathrm{~cm}^{-1}$.

HRMS (ESI) m/z: [M + Na $]^{+}$Calcd for $\mathrm{C}_{28} \mathrm{H}_{55} \mathrm{BNaO}_{3}$ 473.4142; Found 473.4121.

\section{6-Methyl-1-(4,4,5,5-tetramethyl-1,3,2-dioxaborolan-2-yl)heptan-4-one (8)}

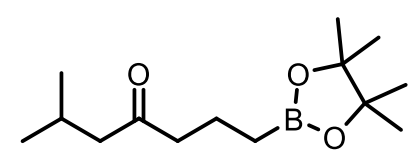

Pinacol boronic ester 8 was prepared following GP3, using $N$-alkoxyphthalimide $\mathbf{8 a}(82 \mathrm{mg}, 0.30 \mathrm{mmol}$, 1.0 equiv.). Flash chromatography (10\% EtOAc/hexane) gave 8 (58 mg, $0.23 \mathrm{mmol}, 76 \%)$ as colourless liquid.

TLC: $\mathrm{R}_{\mathrm{f}}=0.45\left(10 \%\right.$ EtOAc/hexane, $\mathrm{KMnO}_{4}$ stain $)$.

${ }^{1} \mathbf{H}$ NMR $\left(400 \mathrm{MHz}, \mathrm{CDCl}_{3}\right): \delta_{\mathrm{H}} 2.37(\mathrm{t}, J=7.4 \mathrm{~Hz}, 2 \mathrm{H}), 2.25(\mathrm{~d}, J=7.0 \mathrm{~Hz}, 2 \mathrm{H}), 2.17-2.06(\mathrm{~m}, 1 \mathrm{H})$, $1.71-1.64(\mathrm{~m}, 2 \mathrm{H}), 1.23(\mathrm{~s}, 12 \mathrm{H}), 0.89(\mathrm{~d}, J=6.6 \mathrm{~Hz}, 6 \mathrm{H}), 0.76(\mathrm{t}, J=7.8 \mathrm{~Hz}, 2 \mathrm{H}) \mathrm{ppm}$. See spectrum.

${ }^{13} \mathrm{C}$ NMR $\left(101 \mathrm{MHz}, \mathrm{CDCl}_{3}\right): \delta_{\mathrm{C}} 211.1,83.0,51.8,45.6,24.8,24.6,22.6,18.4 \mathrm{ppm}$. The carbon attached to boron could not be observed due to quadrupolar relaxation. See spectrum.

${ }^{11} \mathbf{B}$ NMR (128 MHz, $\mathrm{CDCl}_{3}$ ): $\delta_{\mathrm{B}} 33.8$ (br. s, 1B) ppm. $\underline{\text { See spectrum. }}$

IR (film) $v_{\max }: 2974,2923,1708(\mathrm{~s}), 1468,1372,1320,1144,1057,1033,906,729 \mathrm{~cm}^{-1}$.

HRMS (ESI) m/z: [M + Na $]^{+}$Calcd for $\mathrm{C}_{14} \mathrm{H}_{27} \mathrm{BNaO}_{3}$ 277.1948; Found 277.1945. 


\section{7-Methyl-1-(4,4,5,5-tetramethyl-1,3,2-dioxaborolan-2-yl)octan-4-one (9)}

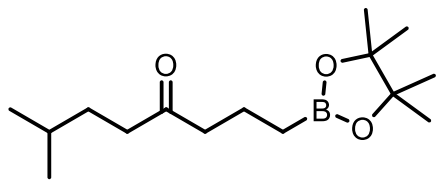

Pinacol boronic ester 9 was prepared following GP3, using $N$-alkoxyphthalimide $9 \mathbf{a}(86 \mathrm{mg}, 0.30 \mathrm{mmol}$, 1.0 equiv.). Flash chromatography (10\% EtOAc/hexane) gave $\mathbf{9}(57 \mathrm{mg}, 0.21 \mathrm{mmol}, 70 \%)$ as colourless liquid.

TLC: $\mathrm{R}_{\mathrm{f}}=0.45\left(10 \%\right.$ EtOAc/hexane, $\mathrm{KMnO}_{4}$ stain $)$.

${ }^{1} \mathrm{H}$ NMR $\left(400 \mathrm{MHz}, \mathrm{CDCl}_{3}\right): \delta_{\mathrm{H}} 2.40(\mathrm{t}, J=7.4 \mathrm{~Hz}, 2 \mathrm{H}), 2.37(\mathrm{t}, J=7.6 \mathrm{~Hz}, 2 \mathrm{H}), 1.73-1.63(\mathrm{~m}, 2 \mathrm{H})$, $1.55-1.40(\mathrm{~m}, 3 \mathrm{H}), 1.23(\mathrm{~s}, 12 \mathrm{H}), 0.87(\mathrm{~d}, J=6.4 \mathrm{~Hz}, 6 \mathrm{H}), 0.76(\mathrm{t}, J=7.8 \mathrm{~Hz}, 2 \mathrm{H}) \mathrm{ppm}$. See spectrum.

${ }^{13}$ C NMR (101 MHz, $\left.\mathrm{CDCl}_{3}\right): \delta_{\mathrm{C}} 211.7,83.0,45.0,40.8,32.7,27.7,24.8,22.3,18.6 \mathrm{ppm}$. The carbon attached to boron could not be observed due to quadrupolar relaxation. See spectrum.

${ }^{11}$ B NMR (128 MHz, $\left.\mathrm{CDCl}_{3}\right)$ : $\delta_{\mathrm{B}} 33.8$ (br. s, 1B) ppm. See spectrum.

IR (film) $v_{\max }: 2979,2958,1709(\mathrm{~s}), 1468,1372,1319,1143,906,728 \mathrm{~cm}^{-1}$.

HRMS (ESI) m/z: [M + Na $]^{+}$Calcd for $\mathrm{C}_{15} \mathrm{H}_{29} \mathrm{BNaO}_{3} 291.2105$; Found 291.2111.

\section{1-Phenyl-6-(4,4,5,5-tetramethyl-1,3,2-dioxaborolan-2-yl)hexan-3-one (10)}

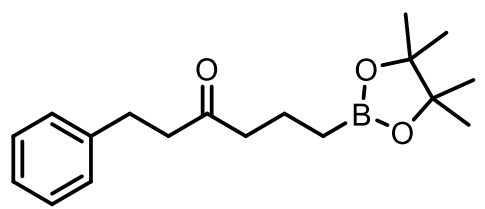

Pinacol boronic ester 10 was prepared following GP3, using $N$-alkoxyphthalimide 10a (32 mg, 0.10 mmol, 1.0 equiv.). Flash chromatography (10\% EtOAc/hexane) gave 10 (20 mg, 0.07 mmol, 67\%) as colourless liquid.

TLC: $\mathrm{R}_{\mathrm{f}}=0.40$ (10\% EtOAc/hexane, $\mathrm{KMnO}_{4}$ stain $)$.

${ }^{1} \mathbf{H}$ NMR $\left(400 \mathrm{MHz}, \mathrm{CDCl}_{3}\right): \delta_{\mathrm{H}} 7.30-7.25(\mathrm{~m}, 2 \mathrm{H}), 7.20-7.16(\mathrm{~m}, 3 \mathrm{H}), 2.89(\mathrm{t}, J=7.7 \mathrm{~Hz}, 2 \mathrm{H}), 2.72$ $(\mathrm{t}, J=7.7 \mathrm{~Hz}, 2 \mathrm{H}), 2.40(\mathrm{t}, J=7.4 \mathrm{~Hz}, 2 \mathrm{H}), 1.74-1.64(\mathrm{~m}, 2 \mathrm{H}), 1.23(\mathrm{~s}, 12 \mathrm{H}), 0.76(\mathrm{t}, J=7.8 \mathrm{~Hz}, 2 \mathrm{H})$ ppm. See spectrum.

${ }^{13}$ C NMR (101 MHz, $\left.\mathrm{CDCl}_{3}\right): \delta_{\mathrm{C}} 210.2,141.2,128.4,128.3,126.0,83.0,45.3,44.2,29.8,24.8,18.5$ $\mathrm{ppm}$. The carbon attached to boron could not be observed due to quadrupolar relaxation. See spectrum. 
${ }^{11} \mathbf{B}$ NMR (128 MHz, $\mathrm{CDCl}_{3}$ ): $\delta_{\mathrm{B}} 33.7$ (br. s, 1B) ppm. See spectrum.

IR (film) $v_{\text {max }}$ : 2981, 2923, 1712(s), 1610, 1469, 1454, 1372, 1320, 1144, 1056, 1033, 904, $727 \mathrm{~cm}^{-1}$.

HRMS (ESI) m/z: [M + Na] $]^{+}$Calcd for $\mathrm{C}_{18} \mathrm{H}_{27} \mathrm{BNaO}_{3}$ 325.1949; Found 325.1948.

6-Oxo-9-(4,4,5,5-tetramethyl-1,3,2-dioxaborolan-2-yl)nonyl 4-methylbenzenesulfonate (11)<smiles>Cc1ccc(S(=O)(=O)OCCCCCC(=O)CCCB2OC(C)(C)C(C)(C)O2)cc1</smiles>

Pinacol boronic ester 11 was prepared following GP3, using $N$-alkoxyphthalimide 11a (92 mg, 0.20 mmol, 1.0 equiv.). Flash chromatography (10\% EtOAc/hexane) gave 11 (53 mg, 0.12 mmol, 60\%) as colourless liquid.

TLC: $\mathrm{R}_{\mathrm{f}}=0.44\left(10 \%\right.$ EtOAc/hexane, $\mathrm{KMnO}_{4}$ stain $)$.

${ }^{1} \mathbf{H}$ NMR $\left(400 \mathrm{MHz}, \mathrm{CDCl}_{3}\right): \delta_{\mathrm{H}} 7.80-7.76(\mathrm{~m}, 2 \mathrm{H}), 7.36-7.32(\mathrm{~m}, 2 \mathrm{H}), 4.01(\mathrm{t}, J=6.4 \mathrm{~Hz}, 2 \mathrm{H}), 2.45$ (s, 3H), 2.37 (t, $J=7.5 \mathrm{~Hz}, 2 \mathrm{H}), 2.34(\mathrm{t}, J=7.3 \mathrm{~Hz}, 2 \mathrm{H}), 1.72-1.60(\mathrm{~m}, 4 \mathrm{H}), 1.54-1.45(\mathrm{~m}, 2 \mathrm{H}), 1.34$ $-1.26(\mathrm{~m}, 2 \mathrm{H}), 1.23(\mathrm{~s}, 12 \mathrm{H}), 0.76(\mathrm{t}, J=7.8 \mathrm{~Hz}, 2 \mathrm{H}) \mathrm{ppm} . \underline{\text { See spectrum. }}$

${ }^{13} \mathrm{C}$ NMR (101 MHz, $\left.\mathrm{CDCl}_{3}\right): \delta_{\mathrm{C}} 210.8,144.7,133.2,129.8,127.9,83.0,70.3,45.1,42.3,28.7,25.0$, 24.8, 23.0, 21.6, $18.6 \mathrm{ppm}$. The carbon attached to boron could not be observed due to quadrupolar relaxation. See spectrum.

${ }^{11} \mathbf{B}$ NMR $\left(128 \mathrm{MHz}, \mathrm{CDCl}_{3}\right): \delta_{\mathrm{B}} 33.8$ (br. s, 1B) ppm. See spectrum.

IR (film) $v_{\max }$ 2974, 2923, 1737, 1710(s), 1598, 1454, 1357, 1322 1188, 1176, 1149, 1056, $905 \mathrm{~cm}^{-1}$.

HRMS (ESI) m/z: [M + Na ${ }^{+}$Calcd for $\mathrm{C}_{22} \mathrm{H}_{35} \mathrm{BNaO}_{6} \mathrm{~S}$ 461.2144; Found 461.2154.

\section{9-Bromo-1-(4,4,5,5-tetramethyl-1,3,2-dioxaborolan-2-yl)nonan-4-one (12)}

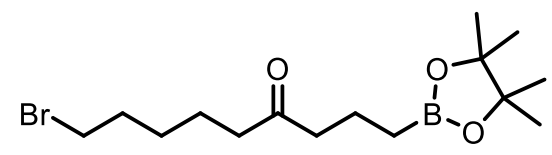

Pinacol boronic ester 12 was prepared following GP3, using $N$-alkoxyphthalimide 12a (73 mg, 0.20 mmol, 1.0 equiv.). Flash chromatography (10\% EtOAc/hexane) gave 12 (42 mg, $0.12 \mathrm{mmol}, 60 \%$ ) as colourless liquid. 
TLC: $\mathrm{R}_{\mathrm{f}}=0.48\left(10 \%\right.$ EtOAc/hexane, $\mathrm{KMnO}_{4}$ stain $)$.

${ }^{1} \mathbf{H}$ NMR $\left(400 \mathrm{MHz}, \mathrm{CDCl}_{3}\right): \delta_{\mathrm{H}} 3.40(\mathrm{t}, J=6.8 \mathrm{~Hz}, 2 \mathrm{H}), 2.41(\mathrm{t}, J=7.3 \mathrm{~Hz}, 2 \mathrm{H}), 2.40(\mathrm{t}, J=7.5 \mathrm{~Hz}$, $2 \mathrm{H}), 1.91-1.81(\mathrm{~m}, 2 \mathrm{H}), 1.73-1.64(\mathrm{~m}, 2 \mathrm{H}), 1.63-1.53(\mathrm{~m}, 2 \mathrm{H}), 1.47-1.38(\mathrm{~m}, 2 \mathrm{H}), 1.24(\mathrm{~s}, 12 \mathrm{H})$, $0.77(\mathrm{t}, J=7.8 \mathrm{~Hz}, 2 \mathrm{H}) \mathrm{ppm}$. See spectrum.

${ }^{13}$ C NMR (101 MHz, $\left.\mathrm{CDCl}_{3}\right): \delta_{\mathrm{C}} 211.0,83.0,45.1,42.4,33.6,32.6,27.8,24.8,22.8,18.6 \mathrm{ppm}$. The carbon attached to boron could not be observed due to quadrupolar relaxation. See spectrum.

${ }^{11} \mathbf{B}$ NMR (128 MHz, $\left.\mathrm{CDCl}_{3}\right): \delta_{\mathrm{B}} 33.8$ (br. s, 1B) ppm. See spectrum.

IR (film) $v_{\max }: 2974,2902,1710(\mathrm{~s}), 1454,1047,1372,1319,1143,1056,905,728 \mathrm{~cm}^{-1}$.

HRMS (ESI) m/z: [M + Na $]^{+}$Calcd for $\mathrm{C}_{15} \mathrm{H}_{28} \mathrm{BBrNaO}_{3} 369.1210$; Found 369.1216.

4-Oxo-7-(4,4,5,5-tetramethyl-1,3,2-dioxaborolan-2-yl)heptyl benzoate (13)<smiles>CC1(C)OB(CCCC(=O)CCCOC(=O)c2ccccc2)OC1(C)C</smiles>

Pinacol boronic ester 13 was prepared following GP3, using $N$-alkoxyphthalimide 13a (76 mg, 0.20 mmol, 1.0 equiv.). Flash chromatography (20\% EtOAc/hexane) gave 13 (51 mg, 0.14 mmol, 71\%) as colourless liquid.

TLC: $\mathrm{R}_{\mathrm{f}}=0.34\left(10 \%\right.$ EtOAc/hexane, $\mathrm{KMnO}_{4}$ stain $)$.

${ }^{1} \mathbf{H}$ NMR $\left(400 \mathrm{MHz}, \mathrm{CDCl}_{3}\right): \delta_{\mathrm{H}} 8.04-8.00(\mathrm{~m}, 2 \mathrm{H}), 7.58-7.53(\mathrm{~m}, 1 \mathrm{H}), 7.46-7.41(\mathrm{~m}, 2 \mathrm{H}), 4.32(\mathrm{t}$, $J=6.4 \mathrm{~Hz}, 2 \mathrm{H}), 2.56(\mathrm{t}, J=7.3 \mathrm{~Hz}, 2 \mathrm{H}), 2.43(\mathrm{t}, J=7.4 \mathrm{~Hz}, 2 \mathrm{H}), 2.10-2.00(\mathrm{~m}, 2 \mathrm{H}), 1.75-1.65(\mathrm{~m}$, 2H), $1.22(\mathrm{~s}, 12 \mathrm{H}), 0.77$ (t, $J=7.8 \mathrm{~Hz}, 2 \mathrm{H}) \mathrm{ppm}$. See spectrum.

${ }^{13} \mathrm{C}$ NMR $\left(101 \mathrm{MHz}, \mathrm{CDCl}_{3}\right): \delta_{\mathrm{C}} 209.9,166.5,132.9,130.2,129.5,128.3,83.0,64.2,45.2,39.0,24.8$, $22.9,18.5 \mathrm{ppm}$. The carbon attached to boron could not be observed due to quadrupolar relaxation. See spectrum.

${ }^{11}$ B NMR $\left(128 \mathrm{MHz}, \mathrm{CDCl}_{3}\right): \delta_{\mathrm{B}} 33.8$ (br. s, 1B) ppm. See spectrum.

IR (film) $v_{\max }: 2980,2936,1713(\mathrm{~s}), 1602,1585,1452,1410,1373,1274,1144,905,725 \mathrm{~cm}^{-1}$.

HRMS (ESI) m/z: $[\mathrm{M}+\mathrm{H}]^{+}$Calcd for $\mathrm{C}_{20} \mathrm{H}_{30} \mathrm{BO}_{5}[\mathrm{M}+\mathrm{H}]^{+}$361.2184; Found 361.2200. 


\section{4-Oxo-7-(4,4,5,5-tetramethyl-1,3,2-dioxaborolan-2-yl)heptyl 4-ethylbenzoate (14)}

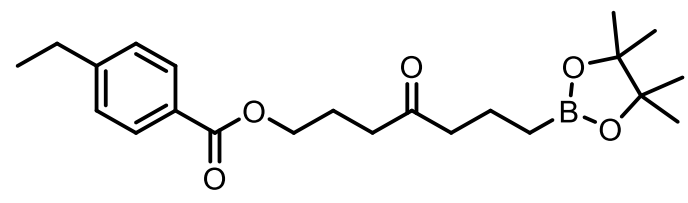

Pinacol boronic ester 14 was prepared following GP3, using $N$-alkoxyphthalimide 14a (81 mg, 0.20 mmol, 1.0 equiv.). Flash chromatography (10\% EtOAc/hexane) gave 14 (54 mg, 0.14 mmol, 69\%) as colourless liquid.

TLC: $\mathrm{R}_{\mathrm{f}}=0.45\left(10 \%\right.$ EtOAc/hexane, $\mathrm{KMnO}_{4}$ stain $)$.

${ }^{1} \mathrm{H}$ NMR $\left(400 \mathrm{MHz}, \mathrm{CDCl}_{3}\right): \delta_{\mathrm{H}} 7.97-7.90(\mathrm{~m}, 2 \mathrm{H}), 7.29-7.22(\mathrm{~m}, 2 \mathrm{H}), 4.30(\mathrm{t}, J=6.4 \mathrm{~Hz}, 2 \mathrm{H}), 2.70$ (q, $J=7.6 \mathrm{~Hz}, 2 \mathrm{H}), 2.55$ (t, $J=7.3 \mathrm{~Hz}, 2 \mathrm{H}), 2.45$ (t, $J=7.4 \mathrm{~Hz}, 2 \mathrm{H}), 2.10-1.98(\mathrm{~m}, 2 \mathrm{H}), 1.76-1.64$ $(\mathrm{m}, 2 \mathrm{H}), 1.25(\mathrm{~d}, J=7.6 \mathrm{~Hz}, 3 \mathrm{H}), 1.23(\mathrm{~s}, 12 \mathrm{H}), 0.77(\mathrm{t}, J=7.8 \mathrm{~Hz}, 2 \mathrm{H}) \mathrm{ppm}$. See spectrum.

${ }^{13} \mathrm{C}$ NMR $\left(101 \mathrm{MHz}, \mathrm{CDCl}_{3}\right): \delta_{\mathrm{C}} 210.0,166.6,149.8,129.7,127.9,127.7,83.0,64.0,45.2,39.0,28.9$, $24.8,22.9,18.5,15.2 \mathrm{ppm}$. The carbon attached to boron could not be observed due to quadrupolar relaxation. See spectrum.

${ }^{11} \mathbf{B}$ NMR (128 MHz, $\left.\mathrm{CDCl}_{3}\right): \delta_{\mathrm{B}} 33.9$ (br. s, 1B) ppm. See spectrum. IR (film) $v_{\max }$ : 2987, 2901, 1731(s), 1714(s), 1609, 1453, 1407, 1373, 1271, 1242, 1048, $907 \mathrm{~cm}^{-1}$. HRMS (ESI) m/z: $[\mathrm{M}+\mathrm{H}]^{+}$Calcd for $\mathrm{C}_{22} \mathrm{H}_{34} \mathrm{BO}_{5} 389.2499$; Found 389.2514.

\section{4-Oxo-7-(4,4,5,5-tetramethyl-1,3,2-dioxaborolan-2-yl)heptyl 4-chlorobenzoate (15)}<smiles>CC1(C)OB(CCCC(=O)CCCOC(=O)c2ccc(Cl)cc2)OC1(C)C</smiles>

Pinacol borane 15 was prepared following GP3, using $N$-alkoxyphthalimide 15 ( $83 \mathrm{mg}, 0.20 \mathrm{mmol}, 1.0$ equiv.). Flash chromatography (10\% EtOAc/hexane) gave 15 (55 mg, $0.14 \mathrm{mmol}, 70 \%)$ as colourless liquid.

TLC: $\mathrm{R}_{\mathrm{f}}=0.40\left(10 \% \mathrm{EtOAc} /\right.$ hexane, $\mathrm{KMnO}_{4}$ stain $)$.

${ }^{1} \mathbf{H}$ NMR $\left(400 \mathrm{MHz}, \mathrm{CDCl}_{3}\right): \delta_{\mathrm{H}} 7.97-7.93(\mathrm{~m}, 2 \mathrm{H}), 7.43-7.39(\mathrm{~m}, 2 \mathrm{H}), 4.31(\mathrm{t}, J=6.4 \mathrm{~Hz}, 2 \mathrm{H}), 2.55$ (t, $J=7.2 \mathrm{~Hz}, 2 \mathrm{H}), 2.44$ (t, $J=7.4 \mathrm{~Hz}, 2 \mathrm{H}), 2.09-2.00(\mathrm{~m}, 2 \mathrm{H}), 1.76-1.64(\mathrm{~m}, 2 \mathrm{H}), 1.23(\mathrm{~s}, 12 \mathrm{H})$, $0.77(\mathrm{t}, J=7.8 \mathrm{~Hz}, 2 \mathrm{H}) \mathrm{ppm} . \underline{\text { See spectrum. }}$. 
${ }^{13} \mathrm{C} \mathrm{NMR}\left(101 \mathrm{MHz}, \mathrm{CDCl}_{3}\right): \delta_{\mathrm{C}} 209.8,165.7,139.4,130.9,128.7,128.7,83.0,64.5,45.2,38.9,24.8$, $22.8,18.6 \mathrm{ppm}$. The carbon attached to boron could not be observed due to quadrupolar relaxation. See spectrum.

${ }^{11} \mathbf{B}$ NMR (128 MHz, $\left.\mathrm{CDCl}_{3}\right): \delta_{\mathrm{B}} 33.9$ (br. s, 1B) ppm. See spectrum.

IR (film) $v_{\max }: 2974,2923,1715(\mathrm{~s}), 1595,1454,1403,1372,1272,1144,1091,1055,906,727 \mathrm{~cm}^{-1}$.

HRMS (ESI) m/z: [M + Na $]^{+}$Calcd for $\mathrm{C}_{20} \mathrm{H}_{28} \mathrm{BClNaO}_{5}$ 417.1614; Found 417.1622.

4-Oxo-7-(4,4,5,5-tetramethyl-1,3,2-dioxaborolan-2-yl)heptyl 3-bromobenzoate (16)<smiles>CC1(C)OB(CCCC(=O)CCCOC(=O)c2cccc(Br)c2)OC1(C)C</smiles>

Pinacol boronic ester 16 was prepared following GP3, using $N$-alkoxyphthalimide 16 (92 $\mathrm{mg}, 0.20$ mmol, 1.0 equiv.). Flash chromatography (10\% EtOAc/hexane) gave 16 (58 mg, $0.66 \mathrm{mmol}, 66 \%)$ as colourless liquid.

TLC: $\mathrm{R}_{\mathrm{f}}=0.42\left(10 \%\right.$ EtOAc/hexane, $\mathrm{KMnO}_{4}$ stain).

${ }^{1} \mathrm{H}$ NMR $\left(400 \mathrm{MHz}, \mathrm{CDCl}_{3}\right): \delta_{\mathrm{H}} 8.14(\mathrm{t}, J=1.7 \mathrm{~Hz}, 1 \mathrm{H}), 7.95(\mathrm{ddd}, J=7.8,1.6,1.1 \mathrm{~Hz}, 1 \mathrm{H}), 7.68(\mathrm{ddd}$, $J=8.0,2.1,1.1 \mathrm{~Hz}, 1 \mathrm{H}), 7.32(\mathrm{t}, J=7.8 \mathrm{~Hz}, 1 \mathrm{H}), 4.32(\mathrm{t}, J=6.4 \mathrm{~Hz}, 2 \mathrm{H}), 2.55(\mathrm{t}, J=7.2 \mathrm{~Hz}, 2 \mathrm{H}), 2.45$ $(\mathrm{t}, J=7.4 \mathrm{~Hz}, 2 \mathrm{H}), 2.09-2.01(\mathrm{~m}, 2 \mathrm{H}), 1.75-1.66(\mathrm{~m}, 2 \mathrm{H}), 1.23(\mathrm{~s}, 12 \mathrm{H}), 0.78(\mathrm{t}, J=7.8 \mathrm{~Hz}, 2 \mathrm{H})$ ppm. $\underline{\text { See spectrum. }}$.

${ }^{13} \mathrm{C}$ NMR (101 MHz, $\left.\mathrm{CDCl}_{3}\right): \delta_{\mathrm{C}} 209.8,165.2,135.9,132.5,132.2,129.9,128.1,122.4,83.0,64.7$, $45.2,38.9,24.8,22.8,18.5 \mathrm{ppm}$. The carbon attached to boron could not be observed due to quadrupolar relaxation. See spectrum.

${ }^{11} \mathbf{B}$ NMR (128 MHz, $\left.\mathrm{CDCl}_{3}\right)$ : $\delta_{\mathrm{B}} 33.8$ (br. s, 1B) ppm. See spectrum.

IR (film) $v_{\max }: 2974,2923,1716(\mathrm{~s}), 1570,147,1407,1373,1280,1254,1143,1056,1033,906 \mathrm{~cm}^{-1}$.

HRMS (ESI) m/z: [M + Na $]^{+}$Calcd for $\mathrm{C}_{20} \mathrm{H}_{28} \mathrm{BBrNaO}_{5} 461.1109$; Found 461.1104. 


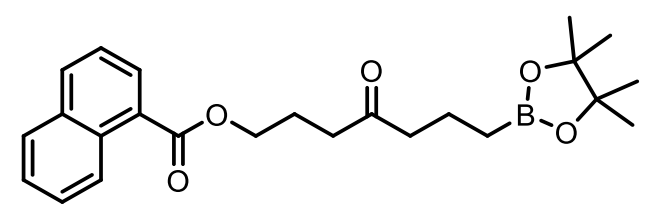

Pinacol boronic ester 17 was prepared following GP3, using $N$-alkoxyphthalimide 17a (64 mg, 0.15 mmol, 1.0 equiv.). Flash chromatography (10\% EtOAc/hexane) gave 17 (42 mg, 0.10 mmol, 67\%) as colourless liquid.

TLC: $\mathrm{R}_{\mathrm{f}}=0.45\left(10 \% \mathrm{EtOAc} / \mathrm{hexane}, \mathrm{KMnO}_{4}\right.$ stain $)$.

${ }^{1} \mathbf{H}$ NMR $\left(400 \mathrm{MHz}, \mathrm{CDCl}_{3}\right): \delta_{\mathrm{H}} 8.93-8.86(\mathrm{~m}, 1 \mathrm{H}), 8.16(\mathrm{dd}, J=7.3,1.3 \mathrm{~Hz}, 1 \mathrm{H}), 8.02(\mathrm{~d}, J=8.2 \mathrm{~Hz}$, $1 \mathrm{H}), 7.90-7.87(\mathrm{~m}, 1 \mathrm{H}), 7.64-7.59(\mathrm{~m}, 1 \mathrm{H}), 7.56-7.48(\mathrm{~m}, 2 \mathrm{H}), 4.42(\mathrm{t}, J=6.4 \mathrm{~Hz}, 2 \mathrm{H}), 2.61(\mathrm{t}, J=$ $7.3 \mathrm{~Hz}, 2 \mathrm{H}), 2.45(\mathrm{t}, J=7.4 \mathrm{~Hz}, 2 \mathrm{H}), 2.16-2.07(\mathrm{~m}, 2 \mathrm{H}), 1.78-1.65(\mathrm{~m}, 2 \mathrm{H}), 1.22(\mathrm{~s}, 12 \mathrm{H}), 0.78(\mathrm{t}, J$ $=7.8 \mathrm{~Hz}, 2 \mathrm{H})$ ppm. See spectrum.

${ }^{13} \mathrm{C}$ NMR (101 MHz, $\left.\mathrm{CDCl}_{3}\right): \delta_{\mathrm{C}} 210.0,167.5,133.8,133.3,131.4,130.1,128.5,127.8,127.2,126.2$, $125.8,124.5,83.0,64.3,45.2,39.0,24.8,22.9,18.6 \mathrm{ppm}$. The carbon attached to boron could not be observed due to quadrupolar relaxation. See spectrum.

${ }^{11} \mathbf{B}$ NMR (128 MHz, $\left.\mathrm{CDCl}_{3}\right): \delta_{\mathrm{B}} 33.7$ (br. s, 1B) ppm. See spectrum.

IR (film) $v_{\max }: 2988,2901,1732(\mathrm{~s}), 1478,1449,1373,1241,1047,909,728 \mathrm{~cm}^{-1}$.

HRMS (ESI) m/z: $[\mathrm{M}+\mathrm{Na}]^{+}$Calcd for $\mathrm{C}_{24} \mathrm{H}_{31} \mathrm{BNaO}_{5} 433.2161$; Found 433.2177.

\section{4-Oxo-7-(4,4,5,5-tetramethyl-1,3,2-dioxaborolan-2-yl)heptyl furan-2-carboxylate (18)}

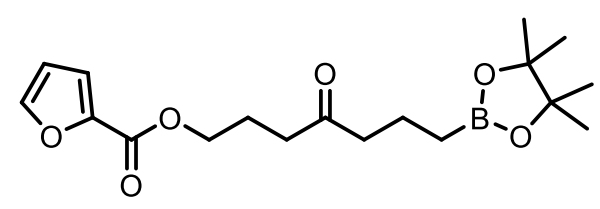

Pinacol boronic ester 18 was prepared following GP3, using $N$-alkoxyphthalimide 18a (55 mg, 0.15 mmol, 1.0 equiv.). Flash chromatography (10\% EtOAc/hexane) gave 18 (34 mg, $0.10 \mathrm{mmol}, 65 \%)$ as colourless liquid.

TLC: $\mathrm{R}_{\mathrm{f}}=0.40\left(10 \%\right.$ EtOAc/hexane, $\mathrm{KMnO}_{4}$ stain $)$.

${ }^{1} \mathbf{H}$ NMR $\left(400 \mathrm{MHz}, \mathrm{CDCl}_{3}\right): \delta_{\mathrm{H}} 7.57(\mathrm{dd}, J=1.8,0.9 \mathrm{~Hz}, 1 \mathrm{H}), 7.16(\mathrm{dd}, J=3.5,0.9 \mathrm{~Hz}, 1 \mathrm{H}), 6.50(\mathrm{dd}$, $J=3.5,1.7 \mathrm{~Hz}, 1 \mathrm{H}), 4.30(\mathrm{t}, J=6.4 \mathrm{~Hz}, 2 \mathrm{H}), 2.54(\mathrm{t}, J=7.2 \mathrm{~Hz}, 2 \mathrm{H}), 2.44(\mathrm{t}, J=7.4 \mathrm{~Hz}, 2 \mathrm{H}), 2.06-$ $1.97(\mathrm{~m}, 2 \mathrm{H}), 1.75-1.65(\mathrm{~m}, 2 \mathrm{H}), 1.23(\mathrm{~s}, 12 \mathrm{H}), 0.77(\mathrm{t}, J=7.8 \mathrm{~Hz}, 2 \mathrm{H})$ ppm. See spectrum. 
${ }^{13} \mathrm{C}$ NMR $\left(101 \mathrm{MHz}, \mathrm{CDCl}_{3}\right): \delta_{\mathrm{C}} 209.8,158.7,146.3,144.7,117.9,111.8,83.0,64.2,45.2,38.8,24.8$, $22.8,18.5 \mathrm{ppm}$. The carbon attached to boron could not be observed due to quadrupolar relaxation. See spectrum.

${ }^{11} \mathbf{B}$ NMR (128 MHz, $\left.\mathrm{CDCl}_{3}\right): \delta_{\mathrm{B}} 33.8$ (br. s, 1B) ppm. See spectrum.

IR (film) $v_{\max }: 2974$ 2923, 1714(s), 1581, 1475, 1372, 1320, 1295, 1231, 1144, 1056, $764 \mathrm{~cm}^{-1}$.

HRMS (ESI) m/z: [M + Na $]^{+}$Calcd for $\mathrm{C}_{18} \mathrm{H}_{27} \mathrm{BNaO}_{6}$ 373.1796; Found 373.1795.

\section{4-Oxo-7-(4,4,5,5-tetramethyl-1,3,2-dioxaborolan-2-yl)heptyl thiophene-3-carboxylate (19)}

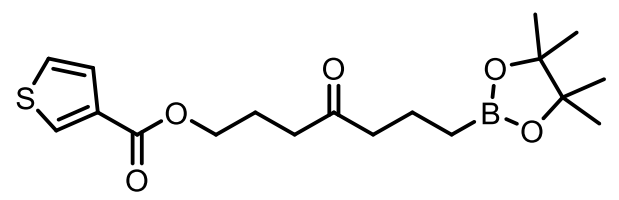

Pinacol boronic ester 19 was prepared following GP3, using $N$-alkoxyphthalimide 19a (116 mg, 0.30 mmol, 1.0 equiv.). Flash chromatography (10\% EtOAc/hexane) gave 19 (78 mg, $0.21 \mathrm{mmol}, 71 \%$ ) as colourless liquid.

TLC: $\mathrm{R}_{\mathrm{f}}=0.40\left(10 \%\right.$ EtOAc/hexane, $\mathrm{KMnO}_{4}$ stain $)$.

${ }^{1} \mathbf{H}$ NMR $\left(400 \mathrm{MHz}, \mathrm{CDCl}_{3}\right): \delta_{\mathrm{H}} 8.09(\mathrm{dd}, J=3.1,1.2 \mathrm{~Hz}, 1 \mathrm{H}), 7.51(\mathrm{dd}, J=5.1,1.2 \mathrm{~Hz}, 1 \mathrm{H}), 7.30(\mathrm{dd}$, $J=5.1,3.1 \mathrm{~Hz}, 1 \mathrm{H}), 4.27(\mathrm{t}, J=6.4 \mathrm{~Hz}, 2 \mathrm{H}), 2.54(\mathrm{t}, J=7.2 \mathrm{~Hz}, 2 \mathrm{H}), 2.43(\mathrm{t}, J=7.4 \mathrm{~Hz}, 2 \mathrm{H}), 2.07-$ $1.92(\mathrm{~m}, 2 \mathrm{H}), 1.75-1.64(\mathrm{~m}, 2 \mathrm{H}), 1.23(\mathrm{~s}, 12 \mathrm{H}), 0.77(\mathrm{t}, J=7.8 \mathrm{~Hz}, 2 \mathrm{H}) \mathrm{ppm}$. See spectrum.

${ }^{13} \mathbf{C}$ NMR $\left(101 \mathrm{MHz}, \mathrm{CDCl}_{3}\right): \delta_{\mathrm{C}} 210.0,162.7,133.7,132.6,127.9,126.0,83.0,64.0,45.2,38.9,24.8$, $22.9,18.6 \mathrm{ppm}$. The carbon attached to boron could not be observed due to quadrupolar relaxation. See spectrum.

${ }^{11} \mathbf{B}$ NMR (128 MHz, $\left.\mathrm{CDCl}_{3}\right): \delta_{\mathrm{B}} 33.7$ (br. s, 1B) ppm. See spectrum.

IR (film) $v_{\max }: 2979,2933,1711(\mathrm{~s}), 1523,1468,1411,1372,1319,1263,1144,1103,968,733 \mathrm{~cm}^{-1}$.

HRMS (ESI) m/z: [M + Na] ${ }^{+}$Calcd for $\mathrm{C}_{18} \mathrm{H}_{27} \mathrm{BNaSO}_{5}$ 389.1568; Found 389.1569. 


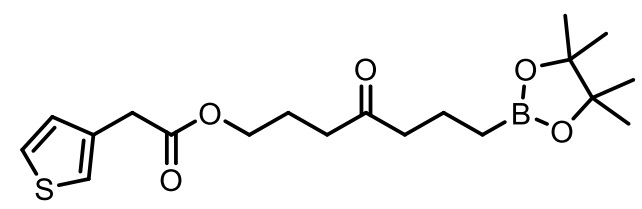

Pinacol boronic ester 20 was prepared following GP3, using $N$-alkoxyphthalimide 20a (60 mg, 0.15 mmol, 1.0 equiv.). Flash chromatography (10\% EtOAc/hexane) gave 20 (38 mg, 0.10 mmol, 67\%) as colourless liquid.

TLC: $\mathrm{R}_{\mathrm{f}}=0.40\left(20 \% \mathrm{EtOAc} / \mathrm{h}\right.$ exane, $\mathrm{KMnO}_{4}$ stain $)$.

${ }^{1} \mathbf{H}$ NMR $\left(400 \mathrm{MHz}, \mathrm{CDCl}_{3}\right): \delta_{\mathrm{H}} 7.21(\mathrm{dd}, J=5.0,1.4 \mathrm{~Hz}, 1 \mathrm{H}), 6.99-6.90(\mathrm{~m}, 2 \mathrm{H}), 4.12(\mathrm{t}, J=6.4 \mathrm{~Hz}$, 2H), $3.82(\mathrm{~s}, 2 \mathrm{H}), 2.44(\mathrm{t}, J=7.3 \mathrm{~Hz}, 2 \mathrm{H}), 2.39$ (t, $J=7.4 \mathrm{~Hz}, 2 \mathrm{H}), 1.95-1.86(\mathrm{~m}, 2 \mathrm{H}), 1.73-1.62(\mathrm{~m}$, 2H), $1.24(\mathrm{~s}, 12 \mathrm{H}), 0.77(\mathrm{t}, J=7.8 \mathrm{~Hz}, 2 \mathrm{H}) \mathrm{ppm}$. See spectrum.

${ }^{13} \mathrm{C}$ NMR (101 MHz, $\left.\mathrm{CDCl}_{3}\right): \delta_{\mathrm{C}} 209.9,170.3,135.1,126.8,126.8,125.0,83.1,64.4,45.2,38.7,35.5$, 24.8, 22.6, $18.6 \mathrm{ppm}$. The carbon attached to boron could not be observed due to quadrupolar relaxation. See spectrum.

${ }^{11} \mathbf{B}$ NMR $\left(128 \mathrm{MHz}, \mathrm{CDCl}_{3}\right): \delta_{\mathrm{B}} 33.8$ (br. s, 1B) ppm. See spectrum.

IR (film) $v_{\max }: 2977,2933,1736(\mathrm{~s}), 1712(\mathrm{~s}), 1535,1439,1410,1371,1319,1265,1144,968 \mathrm{~cm}^{-1}$.

HRMS (ESI) m/z: [M + Na ${ }^{+}$Calcd for $\mathrm{C}_{19} \mathrm{H}_{29} \mathrm{BNaSO}_{5} 403.1725$; Found 403.1724 .

4-Oxo-7-(4,4,5,5-tetramethyl-1,3,2-dioxaborolan-2-yl)heptyl cyclopropanecarboxylate (21)

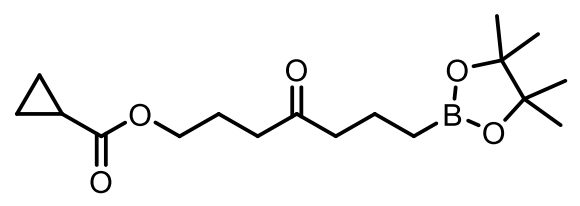

Pinacol boronic ester 21 was prepared following GP3, using $N$-alkoxyphthalimide 21a (69 mg, 0.20 mmol, 1.0 equiv.). Flash chromatography (10\% EtOAc/hexane) gave 21 (44 mg, 0.14 mmol, 68\%) as colourless liquid.

TLC: $\mathrm{R}_{\mathrm{f}}=0.45\left(10 \%\right.$ EtOAc/hexane, $\mathrm{KMnO}_{4}$ stain $)$.

${ }^{1} \mathbf{H}$ NMR $\left(400 \mathrm{MHz}, \mathrm{CDCl}_{3}\right): \delta_{\mathrm{H}} 4.06(\mathrm{t}, J=6.4 \mathrm{~Hz}, 2 \mathrm{H}), 2.47(\mathrm{t}, J=7.3 \mathrm{~Hz}, 2 \mathrm{H}), 2.43(\mathrm{t}, J=7.4 \mathrm{~Hz}$, 2H), $1.94-1.86(\mathrm{~m}, 2 \mathrm{H}), 1.73-1.65(\mathrm{~m}, 2 \mathrm{H}), 1.58(\mathrm{tt}, J=8.0,4.7 \mathrm{~Hz}, 1 \mathrm{H}), 1.23(\mathrm{~s}, 12 \mathrm{H}), 1.01-0.94$ $(\mathrm{m}, 2 \mathrm{H}), 0.87-0.81(\mathrm{~m}, 2 \mathrm{H}), 0.77(\mathrm{t}, J=7.8 \mathrm{~Hz}, 2 \mathrm{H}) \mathrm{ppm}$. See spectrum. 
${ }^{13} \mathrm{C}$ NMR $\left(101 \mathrm{MHz}, \mathrm{CDCl}_{3}\right): \delta_{\mathrm{C}} 210.0,174.8,83.0,63.7,45.1,38.9,24.8,22.8,18.6,12.8,8.4 \mathrm{ppm}$. The carbon attached to boron could not be observed due to quadrupolar relaxation. See spectrum.

${ }^{11} \mathbf{B}$ NMR (128 MHz, $\left.\mathrm{CDCl}_{3}\right): \delta_{\mathrm{B}} 34.0$ (br. s, 1B) ppm. See spectrum.

IR (film) $v_{\max }: 2974,2923,1727(\mathrm{~s}), 1454,1404,1372,1319,1265,1172,1050,732,703 \mathrm{~cm}^{-1}$.

HRMS (ESI) m/z: [M + Na $]^{+}$Calcd for $\mathrm{C}_{17} \mathrm{H}_{29} \mathrm{BNaO}_{5} 347.2003$; Found 347.2002

6-Oxo-9-(4,4,5,5-tetramethyl-1,3,2-dioxaborolan-2-yl)nonyl $\left(1 R^{*}, 2 R^{*}\right)$-2phenylcyclopropane-1-carboxylate (22)

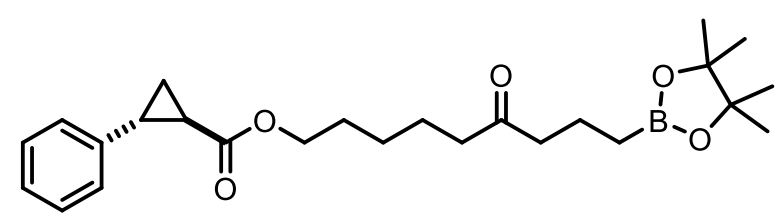

Pinacol boronic ester 22 was prepared following GP3, using $N$-alkoxyphthalimide 22a (90 mg, 0.20 mmol, 1.0 equiv.). Flash chromatography (20\% EtOAc/hexane) gave 22 (51 mg, 0.12 mmol, 60\%) as colourless liquid.

TLC: $\mathrm{R}_{\mathrm{f}}=0.40$ (20\% EtOAc/hexane, $\mathrm{KMnO}_{4}$ stain).

${ }^{1} \mathbf{H}$ NMR $\left(400 \mathrm{MHz}, \mathrm{CDCl}_{3}\right): \delta_{\mathrm{H}} 7.30-7.25(\mathrm{~m}, 2 \mathrm{H}), 7.22-7.17(\mathrm{~m}, 1 \mathrm{H}), 7.11-7.07(\mathrm{~m}, 2 \mathrm{H}), 4.09(\mathrm{t}$, $J=6.6 \mathrm{~Hz}, 2 \mathrm{H}), 2.51(\mathrm{ddd}, J=9.2,6.5,4.1 \mathrm{~Hz}, 1 \mathrm{H}), 2.40(\mathrm{t}, J=7.4 \mathrm{~Hz}, 4 \mathrm{H}), 1.89(\mathrm{ddd}, J=8.4,5.3,4.2$ $\mathrm{Hz}, 1 \mathrm{H}), 1.73-1.55(\mathrm{~m}, 7 \mathrm{H}), 1.39-1.31(\mathrm{~m}, 2 \mathrm{H}), 1.31(\mathrm{ddd}, J=8.4,6.5,4.5 \mathrm{~Hz}, 1 \mathrm{H}), 1.23(\mathrm{~s}, 12 \mathrm{H})$, $0.77(\mathrm{t}, J=7.8 \mathrm{~Hz}, 2 \mathrm{H}) \mathrm{ppm}$. See spectrum.

${ }^{13} \mathrm{C}$ NMR $\left(101 \mathrm{MHz}, \mathrm{CDCl}_{3}\right): \delta_{\mathrm{C}} 211.1,173.4,140.1,128.5,126.5,126.2,83.0,64.6,45.1,42.5,28.5$, $26.2,25.6,24.8,24.1,23.4,18.6,17.1 \mathrm{ppm}$. The carbon attached to boron could not be observed due to quadrupolar relaxation. See spectrum.

${ }^{11} \mathbf{B}$ NMR (128 MHz, $\mathrm{CDCl}_{3}$ ): $\delta_{\mathrm{B}} 33.8$ (br. s, 1B) ppm. See spectrum.

IR (film) $v_{\max }: 2984,2932,1713(\mathrm{~s}), 1505,1498,1410,1336,1323,1176,1144,905,725 \mathrm{~cm}^{-1}$.

HRMS (ESI) m/z: $[\mathrm{M}+\mathrm{Na}]^{+}$Calcd for $\mathrm{C}_{25} \mathrm{H}_{37} \mathrm{BNaO}_{5}$ 451.2631; Found 451.2634. 


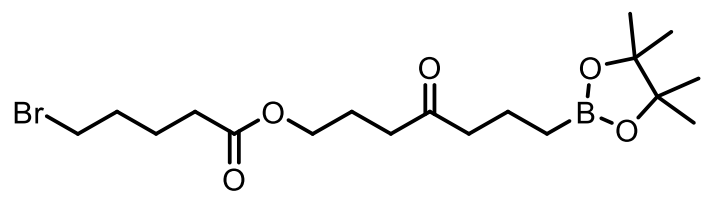

Pinacol boronic ester 23 was prepared following GP3, using $N$-alkoxyphthalimide 23a (66 mg, 0.15 mmol, 1.0 equiv.). Flash chromatography (10\% EtOAc/hexane) gave 23 (38 mg, 0.09 mmol, 60\%) as colourless liquid.

TLC: $\mathrm{R}_{\mathrm{f}}=0.48\left(10 \% \mathrm{EtOAc} /\right.$ hexane, $\mathrm{KMnO}_{4}$ stain $)$.

${ }^{1} \mathbf{H}$ NMR $\left(400 \mathrm{MHz}, \mathrm{CDCl}_{3}\right): \delta_{\mathrm{H}} 4.07(\mathrm{t}, J=6.5 \mathrm{~Hz}, 2 \mathrm{H}), 3.41(\mathrm{t}, J=6.6 \mathrm{~Hz}, 2 \mathrm{H}), 2.47(\mathrm{t}, J=7.3 \mathrm{~Hz}$, $2 \mathrm{H}), 2.42(\mathrm{t}, J=7.4 \mathrm{~Hz}, 2 \mathrm{H}), 2.33(\mathrm{t}, J=7.3 \mathrm{~Hz}, 2 \mathrm{H}), 1.95-1.86(\mathrm{~m}, 4 \mathrm{H}), 1.82-1.74(\mathrm{~m}, 2 \mathrm{H}), 1.74-$ $1.65(\mathrm{~m}, 2 \mathrm{H}), 1.24(\mathrm{~s}, 12 \mathrm{H}), 0.78(\mathrm{t}, J=7.8 \mathrm{~Hz}, 2 \mathrm{H}) \mathrm{ppm}$. See spectrum.

${ }^{13}$ C NMR $\left(101 \mathrm{MHz}, \mathrm{CDCl}_{3}\right): \delta_{\mathrm{C}} 209.9,173.1,83.1,63.8,45.2,38.9,33.2,33.0,32.0,24.8,23.5,22.8$, $18.6 \mathrm{ppm}$. The carbon attached to boron could not be observed due to quadrupolar relaxation. See spectrum.

${ }^{11} \mathbf{B}$ NMR (128 MHz, $\left.\mathrm{CDCl}_{3}\right): \delta_{\mathrm{B}} 33.8$ (br. s, 1B) ppm. $\underline{\text { See spectrum. }}$ IR (film) $v_{\max }: 2973,2901,1733(\mathrm{~s}), 1714(\mathrm{~s}), 1453,1379,1264,1144,1055,907,729 \mathrm{~cm}^{-1}$.

HRMS (ESI) m/z: [M + Na $]^{+}$Calcd for $\mathrm{C}_{18} \mathrm{H}_{32} \mathrm{BBrNaO}_{5}$ 441.1422; Found 441.1433.

\section{4-Oxo-7-(4,4,5,5-tetramethyl-1,3,2-dioxaborolan-2-yl)heptyl $(3 r, 5 r, 7 r)$-adamantane-1-} carboxylate (24)

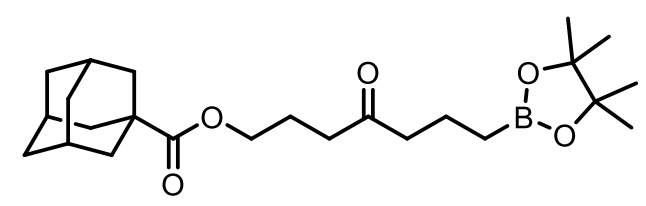

Pinacol boronic ester 24 was prepared following GP3, using $N$-alkoxyphthalimide $24 a$ (44 $\mathrm{mg}, 0.10$ mmol, 1.0 equiv.). Flash chromatography (10\% EtOAc/hexane) gave 24 (25 mg, $0.06 \mathrm{mmol}, 60 \%)$ as colourless liquid.

TLC: $\mathrm{R}_{\mathrm{f}}=0.42\left(10 \%\right.$ EtOAc/hexane, $\mathrm{KMnO}_{4}$ stain $)$.

${ }^{1} \mathbf{H}$ NMR $\left(400 \mathrm{MHz}, \mathrm{CDCl}_{3}\right): \delta_{\mathrm{H}} 4.02(\mathrm{t}, J=6.4 \mathrm{~Hz}, 2 \mathrm{H}), 2.45(\mathrm{t}, J=7.3 \mathrm{~Hz}, 2 \mathrm{H}), 2.45(\mathrm{t}, J=7.6 \mathrm{~Hz}$, 2H), $2.03-1.97(\mathrm{~m}, 3 \mathrm{H}), 1.92-1.84(\mathrm{~m}, 8 \mathrm{H}), 1.75-1.64(\mathrm{~m}, 8 \mathrm{H}), 1.22(\mathrm{~s}, 12 \mathrm{H}), 0.77(\mathrm{t}, J=7.8 \mathrm{~Hz}$, 2H) ppm. See spectrum. 
${ }^{13} \mathrm{C}$ NMR $\left(101 \mathrm{MHz}, \mathrm{CDCl}_{3}\right): \delta_{\mathrm{C}} 210.0,177.6,83.0,63.3,45.1,40.7,38.9,38.8,36.5,27.9,24.8,22.8$, $18.5 \mathrm{ppm}$. The carbon attached to boron could not be observed due to quadrupolar relaxation. See spectrum.

${ }^{11} \mathbf{B}$ NMR (128 MHz, $\left.\mathrm{CDCl}_{3}\right): \delta_{\mathrm{B}} 33.8$ (br. s, 1B) ppm. See spectrum.

IR (film) $v_{\max }$ 2974, 2909, 1714(s), 1454, 1372, 1323, 1143, 1057, 1033, 906, $729 \mathrm{~cm}^{-1}$.

HRMS (ESI) m/z: [M + H] $]^{+}$Calcd for $\mathrm{C}_{24} \mathrm{H}_{40} \mathrm{BO}_{5} 419.2963$; Found 419.3004 .

4-Oxo-7-(4,4,5,5-tetramethyl-1,3,2-dioxaborolan-2-yl)heptyl $(1 R, 4 S)$-1,7,7-trimethyl-3-oxo-2oxabicyclo[2.2.1]heptane-4-carboxylate (25)

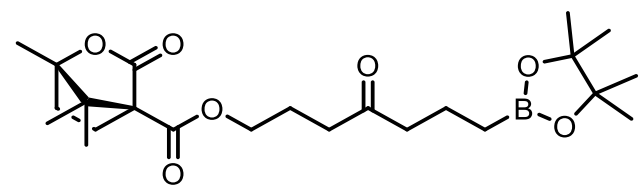

Pinacol boronic ester 25 was prepared following GP3, using $N$-alkoxyphthalimide 25a (68 mg, 0.15 mmol, 1.0 equiv.). Flash chromatography (10\% EtOAc/hexane) gave 25 (43 mg, $0.10 \mathrm{mmol}, 65 \%$ ) as colourless liquid.

TLC: $\mathrm{R}_{\mathrm{f}}=0.28\left(50 \%\right.$ EtOAc/hexane, $\mathrm{KMnO}_{4}$ stain $)$.

${ }^{1} \mathbf{H}$ NMR (400 MHz, CDCl$): \delta_{\mathrm{H}} 4.23(\mathrm{td}, J=6.5,1.3 \mathrm{~Hz}, 2 \mathrm{H}), 2.50(\mathrm{t}, J=7.2 \mathrm{~Hz}, 2 \mathrm{H}), 2.46-2.37(\mathrm{~m}$, $3 \mathrm{H}), 2.02(\mathrm{ddd}, J=13.5,9.3,4.5 \mathrm{~Hz}, 1 \mathrm{H}), 2.00-1.88(\mathrm{~m}, 3 \mathrm{H}), 1.73-1.65(\mathrm{~m}, 3 \mathrm{H}), 1.23(\mathrm{~s}, 12 \mathrm{H}), 1.11$ (s, 3H), 1.05 (s, 3H), 0.95 (s, 3H), 0.77 (t, $J=7.8 \mathrm{~Hz}, 2 \mathrm{H}) \mathrm{ppm}$. See spectrum.

${ }^{13}$ C NMR $\left(101 \mathrm{MHz}, \mathrm{CDCl}_{3}\right): \delta_{\mathrm{C}} 209.6,178.1,167.5,91.1,83.1,64.9,54.7,54.1,45.2,38.5,30.6,28.9$, $24.8,22.6,18.5,16.8,9.7 \mathrm{ppm}$. The carbon attached to boron could not be observed due to quadrupolar relaxation. See spectrum.

${ }^{11} \mathbf{B} \mathbf{N M R}\left(128 \mathrm{MHz}, \mathrm{CDCl}_{3}\right): \delta_{\mathrm{B}} 33.8$ (br. s, 1B) ppm. See spectrum.

IR (film) $v_{\max }: 2977,2936,1788(\mathrm{~s}), 1732(\mathrm{~s}), 1469,1373,1316,1245,1144,1104,907,727 \mathrm{~cm}^{-1}$.

HRMS (ESI) m/z: [M + H] ${ }^{+}$Calcd for $\mathrm{C}_{23} \mathrm{H}_{38} \mathrm{BO}_{7}$ 437.2709; Found 437.2714. 


\section{7-(4,4,5,5-Tetramethyl-1,3,2-dioxaborolan-2-yl)heptan-3-one (26)}

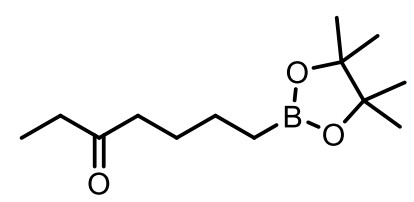

Pinacol boronic ester 26 was prepared following GP3, using $N$-alkoxyphthalimide 26a (52 $\mathrm{mg}, 0.20$ mmol, 1.0 equiv.). Flash chromatography (10\% EtOAc/hexane) gave 26 (43 mg, 0.18 mmol, 88\%) as colourless liquid. All recorded spectroscopic data matched those previously reported in the literature. ${ }^{13}$

${ }^{1} \mathbf{H}$ NMR (400 MHz, CDCl $): \delta_{\mathrm{H}} 2.40(\mathrm{q}, J=7.3 \mathrm{~Hz}, 2 \mathrm{H}), 2.38(\mathrm{t}, J=7.5 \mathrm{~Hz}, 2 \mathrm{H}), 1.61-1.53(\mathrm{~m}, 2 \mathrm{H})$, $1.43-1.35(\mathrm{~m}, 2 \mathrm{H}), 1.22(\mathrm{~s}, 12 \mathrm{H}), 1.03(\mathrm{t}, J=7.3 \mathrm{~Hz}, 3 \mathrm{H}), 0.77(\mathrm{t}, J=7.8 \mathrm{~Hz}, 2 \mathrm{H}) \mathrm{ppm}$. See spectrum. ${ }^{13} \mathbf{C}$ NMR (101 MHz, $\left.\mathrm{CDCl}_{3}\right): \delta_{\mathrm{C}} 211.9,82.9,42.3,35.7,26.5,24.8,23.7,7.8 \mathrm{ppm}$. The carbon attached to boron could not be observed due to quadrupolar relaxation. See spectrum.

\section{1-(4,4,5,5-Tetramethyl-1,3,2-dioxaborolan-2-yl)undecan-5-one (27)}

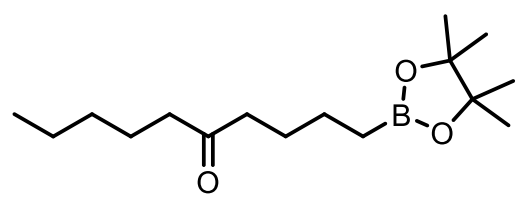

Pinacol boronic ester 27 was prepared following GP3, using $N$-alkoxyphthalimide 27a (60 mg, 0.20 mmol, 1.0 equiv.). Flash chromatography (10\% EtOAc/hexane) gave 27 (47 mg, $0.17 \mathrm{mmol}, 83 \%$ ) as colourless liquid.

TLC: $\mathrm{R}_{\mathrm{f}}=0.45$ (10\% EtOAc/hexane, $\mathrm{KMnO}_{4}$ stain).

${ }^{1} \mathbf{H}$ NMR (400 MHz, CDCl $)$ ): $\delta_{\mathrm{H}} 2.37(\mathrm{t}, J=7.4 \mathrm{~Hz}, 2 \mathrm{H}), 2.36(\mathrm{t}, J=7.5 \mathrm{~Hz}, 2 \mathrm{H}), 1.60-1.50(\mathrm{~m}, 4 \mathrm{H})$, $1.44-1.35(\mathrm{~m}, 2 \mathrm{H}), 1.33-1.20(\mathrm{~m}, 4 \mathrm{H}), 1.23(\mathrm{~s}, 12 \mathrm{H}), 0.87(\mathrm{t}, J=7.0 \mathrm{~Hz}, 3 \mathrm{H}), 0.77(\mathrm{t}, J=7.8 \mathrm{~Hz}, 2 \mathrm{H})$ ppm. See spectrum.

${ }^{13}$ C NMR (101 MHz, $\left.\mathrm{CDCl}_{3}\right): \delta_{\mathrm{C}} 211.6,82.9,42.7,42.6,31.4,26.5,24.8,23.7,23.5,22.4,13.9$ ppm. The carbon attached to boron could not be observed due to quadrupolar relaxation. See spectrum.

${ }^{11}$ B NMR (128 MHz, $\left.\mathrm{CDCl}_{3}\right): \delta_{\mathrm{B}} 34.0$ (br. s, 1B) ppm. $\underline{\text { See spectrum. }}$

IR (film) $v_{\max }: 2977,2901,1711(\mathrm{~s}), 1461,1372,1250,1144,1056,905,728 \mathrm{~cm}^{-1}$.

HRMS (ESI) m/z: [M + Na $]^{+}$Calcd for $\mathrm{C}_{16} \mathrm{H}_{31} \mathrm{BNaO}_{3}$ 305.2261; Found 305.2272. 
4-Methyl-6-(4,4,5,5-tetramethyl-1,3,2-dioxaborolan-2-yl)hexan-2-one (28-1) and 5-methyl-6(4,4,5,5-tetramethyl-1,3,2-dioxaborolan-2-yl)hexan-2-one (28-2)

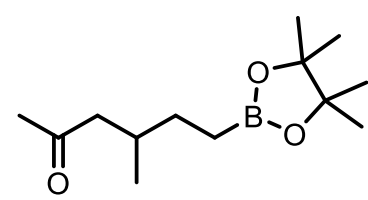

28-1

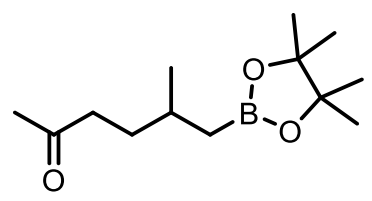

28-2

Pinacol boronic ester 28 was prepared following GP3, using $N$-alkoxyphthalimide $28 \mathbf{a}$ (78 $\mathrm{mg}, 0.30$ mmol, 1.0 equiv.). Flash chromatography (10\% EtOAc/hexane) gave a mixture of 28-1 and 28-2 (58 mg, 0.24 mmol, $80 \%, 63: 37$ r.r. in favor of $\mathbf{2 8 - 1}$ ) as colourless liquid. The r.r. was determined by ${ }^{1} \mathrm{H}$ NMR analysis of the purified product.

TLC: $\mathrm{R}_{\mathrm{f}}=0.45\left(10 \%\right.$ EtOAc/hexane, $\mathrm{KMnO}_{4}$ stain $)$.

${ }^{1} \mathbf{H}$ NMR (400 MHz, $\left.\mathrm{CDCl}_{3}\right)$ : (63:37 mixture of regioisomers) $\delta_{\mathrm{H}} 2.40(\mathrm{dd}, J=15.7,5.5 \mathrm{~Hz}, 0.63 \mathrm{H})$, $2.39(\mathrm{t}, J=7.8 \mathrm{~Hz}, 0.74 \mathrm{H}), 2.16(\mathrm{dd}, J=15.7,8.4 \mathrm{~Hz}, 0.63 \mathrm{H}), 2.11(\mathrm{~s}, 1.89 \mathrm{H}), 2.09$ (s, 1.11H), $1.97-$ $1.85(\mathrm{~m}, 0.63), 1.73-1.60(\mathrm{~m}, 0.37 \mathrm{H}), 1.59-1.27(\mathrm{~m}, 2 \mathrm{H}), 1.22(\mathrm{~s}, 12 \mathrm{H}), 0.86(\mathrm{~d}, J=6.7 \mathrm{~Hz}, 1.89 \mathrm{H})$, $0.90(\mathrm{~d}, J=6.6 \mathrm{~Hz}, 1.11 \mathrm{H}), 0.83-0.61(\mathrm{~m}, 2 \mathrm{H}) \mathrm{ppm} . \underline{\text { See spectrum. }}$

${ }^{13} \mathrm{C}$ NMR (101 MHz, $\left.\mathrm{CDCl}_{3}\right)$ : 63:37 mixture of regioisomers: Major regioisomer: $\delta_{\mathrm{C}} 209.1,82.9,50.9$, 31.3, 30.9, 29.1, 24.8, 19.3 ppm; Minor regioisomer: $\delta_{\mathrm{C}} 209.4,82.9,41.8,33.2,30.2,29.7,24.8,24.7$, $22.0 \mathrm{ppm}$. The carbons attached to boron could not be observed due to quadrupolar relaxation. See spectrum.

${ }^{11}$ B NMR (128 MHz, $\left.\mathrm{CDCl}_{3}\right): \delta_{\mathrm{B}} 33.8$ (br. s, 1B) ppm. See spectrum. IR (film) $v_{\max }: 2979,2901,1711(\mathrm{~s}), 1449,1372,1317,1254,1144,1056,908,728 \mathrm{~cm}^{-1}$.

HRMS (ESI) m/z: [M + Na] ${ }^{+}$Calcd for $\mathrm{C}_{13} \mathrm{H}_{25} \mathrm{BNaO}_{3} 263.1791$; Found 263.1792.

4-Phenyl-6-(4,4,5,5-tetramethyl-1,3,2-dioxaborolan-2-yl)hexan-2-one (29-1) and 5-phenyl-6$(4,4,5,5$-tetramethyl-1,3,2-dioxaborolan-2-yl)hexan-2-one (29-2)

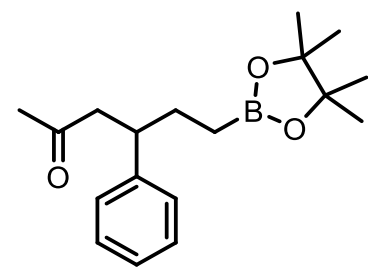

29-1

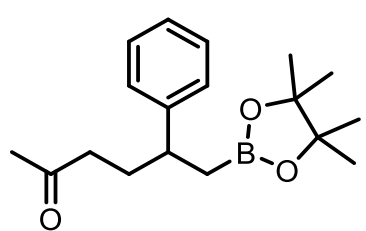

29-2

Pinacol boronic ester 29 was prepared following GP3, using $N$-alkoxyphthalimide 29a (96 mg, 0.30 mmol, 1.0 equiv.). Flash chromatography (10\% EtOAc/hexane) gave a mixture of 29-1 and 29-2 (59 
mg, $0.20 \mathrm{mmol}, 65 \%, 67: 33$ r.r.in favor of 29-1) as colourless liquid. The r.r. was determined by ${ }^{1} \mathrm{H}$ NMR analysis of the purified product.

TLC: $\mathrm{R}_{\mathrm{f}}=0.40\left(10 \% \mathrm{EtOAc} / \mathrm{h}\right.$ exane, $\mathrm{KMnO}_{4}$ stain $)$.

${ }^{1} \mathbf{H}$ NMR (400 MHz, $\left.\mathrm{CDCl}_{3}\right)$ : 67:33 mixture of regioisomers: Major regioisomer: $\delta_{\mathrm{H}} 7.29-7.23(\mathrm{~m}$, $2 \mathrm{H}), 7.19-7.13(\mathrm{~m}, 3 \mathrm{H}), 2.72(\mathrm{~d}, J=7.2 \mathrm{~Hz}, 2 \mathrm{H}), 2.35-2.15(\mathrm{~m}, 1 \mathrm{H}), 2.01$ (s, 3H), $1.79-1.60$ (m, $2 \mathrm{H}), 1.21(\mathrm{~s}, 12 \mathrm{H}), 0.71-0.55(\mathrm{~m}, 2 \mathrm{H}) \mathrm{ppm}$; Minor regioisomer: $\delta_{\mathrm{H}} 7.29-7.23(\mathrm{~m}, 2 \mathrm{H}), 7.19-7.13$ $(\mathrm{m}, 3 \mathrm{H}), 3.06(\mathrm{dtd}, J=9.3,7.2,5.4 \mathrm{~Hz}, 2 \mathrm{H}), 2.84-2.76(\mathrm{~m}, 1 \mathrm{H}), 2.03(\mathrm{~s}, 3 \mathrm{H}), 2.00-1.90(\mathrm{~m}, 1 \mathrm{H}), 1.87$ $-1.77(\mathrm{~m}, 1 \mathrm{H}), 1.19-1.14(\mathrm{~m}, 2 \mathrm{H}), 1.09(\mathrm{~s}, 12 \mathrm{H}) \mathrm{ppm}$. See spectrum.

${ }^{13} \mathrm{C}$ NMR (101 MHz, $\left.\mathrm{CDCl}_{3}\right)$ : 67:33 mixture of regioisomers: Major regioisomer: $\delta_{\mathrm{C}} 208.0,144.2$, $128.4,127.7,126.3,83.0,50.5,43.5,30.9,30.5,24.8,24.8$ ppm; Minor regioisomer: $\delta_{\mathrm{C}} 208.9,146.2$, 128.2, 127.4, 126.1, 83.0, 42.0, 40.9, 32.9, 29.8, 24.7, 24.6 ppm. The carbons attached to boron could not be observed due to quadrupolar relaxation. See spectrum.

${ }^{11} \mathbf{B}$ NMR (128 MHz, $\mathrm{CDCl}_{3}$ ): $\delta_{\mathrm{B}} 33.8$ (br. s, 1B) ppm. See spectrum.

IR (film) $v_{\max }: 2978,2930,1714(\mathrm{~s}), 1602,1494,1452,1370,1319,1144,967,847,731,700 \mathrm{~cm}^{-1}$.

HRMS (ESI) m/z: [M + Na] $]^{+}$Calcd for $\mathrm{C}_{18} \mathrm{H}_{27} \mathrm{BNaO}_{3}$ 325.1949; Found 325.1955.

1-(3-(4,4,5,5-Tetramethyl-1,3,2-dioxaborolan-2-yl)cyclopentyl)propan-2-one (30-1) and 1-(3((4,4,5,5-tetramethyl-1,3,2-dioxaborolan-2-yl)methyl)cyclopentyl)ethan-1-one (30-2)

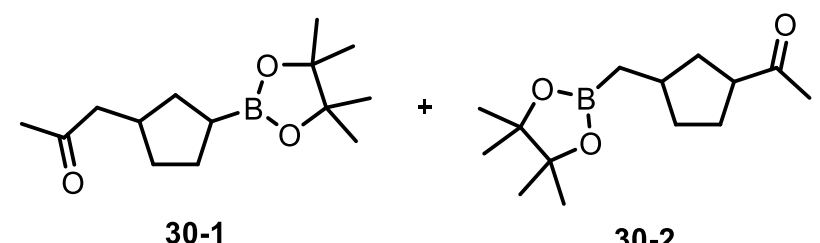

Pinacol boronic ester 30 was prepared following GP3, using $N$-alkoxyphthalimide 30a (135 mg, 0.50 mmol, 1.0 equiv.). Flash chromatography (10\% EtOAc/hexane) gave a mixture of 30-1 and 30-2 (87 $\mathrm{mg}, 0.35 \mathrm{mmol}, 70 \%, 87: 13$ r.r.) as colourless liquid. The r.r. was determined by ${ }^{1} \mathrm{H}$ NMR analysis of the purified product. The d.r. for both regioisomers were determined by averaging of the ${ }^{13} \mathrm{C}$ NMR signals of the purified product: Major regioisomer (30-1) $=50: 50$; Minor regioisomer (30-2) $=53: 47$. Although the d.r. of 50:50 for 30-1 is expected because a stereocentre is formed by unselective borylation of a cyclopentyl radical, the d.r. of 53:47 for 30-2 is unexpected as the two stereocentres should be unaffected by the fragmentation borylation process and the starting material (30a) is a single diastereomer. Therefore, the d.r. of 53:47 for 30-2 is indicative of epimerization of the $\alpha$-ketone stereocentre under the reaction conditions. 
${ }^{1} \mathbf{H}$ NMR (400 MHz, $\mathrm{CDCl}_{3}$ ): 87:13 mixture of regioisomers: Major regioisomer (50:50 ratio of diastereomers) $\delta_{\mathrm{H}} 2.50-2.34(\mathrm{~m}, 2 \mathrm{H}), 2.34-2.13(\mathrm{~m}, 1 \mathrm{H}), 2.09(2 \times \mathrm{s}, 3 \mathrm{H}), 1.98-1.90(\mathrm{~m}, 0.5 \mathrm{H})$, $1.89-1.67(\mathrm{~m}, 2.5 \mathrm{H}), 1.61-1.41(\mathrm{~m}, 1 \mathrm{H}), 1.37-1.24(\mathrm{~m}, 1 \mathrm{H}), 1.20(2 \times \mathrm{s}, 12 \mathrm{H}), 1.17-0.97(\mathrm{~m}, 2 \mathrm{H})$ ppm; Minor regioisomer (53:47 ratio of diastereomers) $\delta_{\mathrm{H}} 2.99-2.81(\mathrm{~m}, 1 \mathrm{H}), 2.10(\mathrm{~s}, 3 \mathrm{H}), 1.21(2 \times$ s, $12 \mathrm{H}), 0.85(\mathrm{~d}, J=6.9 \mathrm{~Hz}, 1 \mathrm{H}), 0.81(\mathrm{~d}, J=7.0 \mathrm{~Hz}, 0.5 \mathrm{H}), 0.80$ (d, $J=7.3 \mathrm{~Hz}, 0.5 \mathrm{H}) \mathrm{ppm}$. The remaining signals could not be identified due to signal overlap. $\underline{\text { See spectrum. }}$

${ }^{13} \mathrm{C}$ NMR (101 MHz, $\left.\mathrm{CDCl}_{3}\right)$ : 87:13 mixture of regioisomers: Major regioisomer (50:50 ratio of diastereomers) $\delta_{\mathrm{C}} 209.04,209.01,82.85,82.83,50.1,49.8,37.1,35.73,35.68,34.3,33.9,32.8,30.1$, 30.0, 28.0, 27.0, 24.7 ppm; Minor regioisomer (53:47 ratio of diastereomers) $\delta_{\mathrm{C}} 211.0,210.9,82.9$, 52.3, 51.4, 38.2, 36.9, 36.7, 35.4, 35.2, 34.3, 28.6, 28.5, 28.3, 27.3, $24.74 \mathrm{ppm}$. The carbons attached to boron could not be observed due to quadrupolar relaxation. See spectrum.

\section{1-(3-Hydroxycyclopentyl)propan-2-one (30p-1) and 1-(3-(hydroxymethyl)cyclopentyl)ethan-1-one (30p-2)}

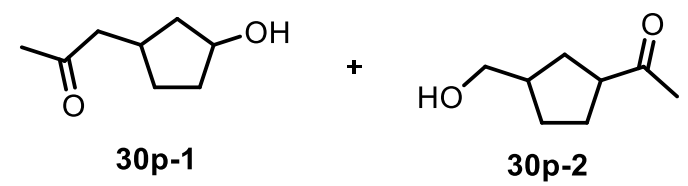

A round bottomed flask containing boronic ester $30(0.30 \mathrm{mmol})$ in THF $(6 \mathrm{~mL})$ was cooled to $0{ }^{\circ} \mathrm{C}$. A solution of $2 \mathrm{M} \mathrm{NaOH} / 30 \%$ aqueous $\mathrm{H}_{2} \mathrm{O}_{2}(3.0 \mathrm{~mL}, 2: 1 \mathrm{v} / \mathrm{v})$ was added dropwise. The mixture was stirred at $0{ }^{\circ} \mathrm{C}$ for $1 \mathrm{~h}$ and then at room temperature for $1 \mathrm{~h}$. After this time, water $(5 \mathrm{~mL})$ and EtOAc $(10 \mathrm{~mL})$ were added and the phases separated. The aqueous phase was extracted with EtOAc $(3 \times 20$ $\mathrm{mL}$ ) and the combined organic phases were washed with brine, dried over $\mathrm{MgSO}_{4}$, filtered, and concentrated under reduced pressure. Purification by flash column chromatography (40\% EtOAc/hexane) gave 30p (34 mg, $0.24 \mathrm{mmol}, 80 \%$, 91:9 r.r.) as a colorless oil. The r.r. was determined by ${ }^{1} \mathrm{H}$ NMR analysis of the purified product. The d.r. for both regioisomers was determined by ${ }^{1} \mathrm{H}$ NMR analysis of the purified product: Major regioisomer $(\mathbf{3 0 p - 1})=50: 50$; Minor regioisomer $(\mathbf{3 0 p}-\mathbf{2})=55: 45$.

${ }^{1} \mathbf{H}$ NMR (400 MHz, $\mathrm{CDCl}_{3}$ ): $91: 9$ mixture of regioisomers: Major regioisomer (50:50 ratio of diastereomers) $\delta_{\mathrm{H}} 4.37-4.27(\mathrm{~m}, 1 \mathrm{H}), 2.62-2.48(\mathrm{~m}, 0.5 \mathrm{H}), 2.57(\mathrm{~d}, J=6.9 \mathrm{~Hz}, 1 \mathrm{H}), 2.43(\mathrm{~d}, J=7.3$ $\mathrm{Hz}, 1 \mathrm{H}), 2.33-2.21(\mathrm{~m}, 0.5 \mathrm{H}), 2.22-2.13(\mathrm{~m}, 0.5 \mathrm{H}), 2.12+2.11(2 \times \mathrm{s}, 3 \mathrm{H}), 2.06-1.90(\mathrm{~m}, 1 \mathrm{H}), 1.88$ $-1.69(\mathrm{~m}, 2.5 \mathrm{H}), 1.67-1.52(\mathrm{~m}, 1 \mathrm{H}), 1.45-1.28(\mathrm{~m}, 1 \mathrm{H}), 1.22-1.08(\mathrm{~m}, 1 \mathrm{H}) \mathrm{ppm}$; Minor regioisomer ( $55: 45$ ratio of diastereomers) $\delta_{\mathrm{H}} 3.56(\mathrm{dd}, J=10.5,6.6 \mathrm{~Hz}, 0.45 \mathrm{H}), 3.52(\mathrm{dd}, J=10.7,6.6 \mathrm{~Hz}, 0.55 \mathrm{H})$, $3.51(\mathrm{dd}, J=10.5,6.6 \mathrm{~Hz}, 0.45 \mathrm{H}), 3.49(\mathrm{dd}, J=10.6,6.9 \mathrm{~Hz}, 0.55 \mathrm{H}), 2.98-2.88(\mathrm{~m}, 1 \mathrm{H}), 2.15+2.14$ $(2 \times \mathrm{s}, 3 \mathrm{H}) \mathrm{ppm}$. The remaining signals could not be identified due to signal overlap. See spectrum. 
${ }^{13} \mathrm{C}$ NMR (101 MHz, $\mathrm{CDCl}_{3}$ ): $91: 9$ mixture of regioisomers: Major regioisomer (50:50 ratio of diastereomers) $\delta_{\mathrm{C}} 208.9,208.7,73.5,73.3,50.2,49.9,42.3,41.7,35.4,35.0,33.5,32.9,30.3,30.2,30.0$, 29.9 ppm; Minor regioisomer (55:45 ratio of diastereomers) $\delta_{\mathrm{C}} 211.0,210.8,66.5,66.4,52.1,51.4$, 42.3, 41.6, 31.6, 31.0, 29.1, 28.8, 28.7, 28.6, 28.4, 28.0 ppm. See spectrum.

IR (film) $v_{\max }: 3455$ (br), 2987, 2901, 1732(s), 1374, 1243, 1045, 907, $727 \mathrm{~cm}^{-1}$.

HRMS (ESI) m/z: [M + Na $]^{+}$Calcd for $\mathrm{C}_{8} \mathrm{H}_{14} \mathrm{BNaO}_{2}$ 165.0886; Found 165.0890.

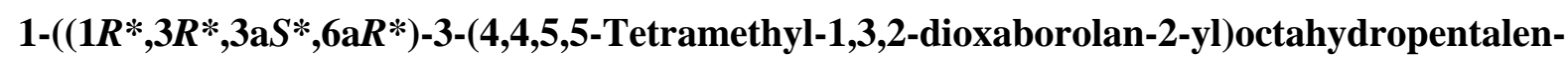
1-yl)propan-2-one $(31-1)$ and $1-\left(\left(1 R^{*}, 3 S^{*}, 3 a R^{*}, 6 a R^{*}\right)-3-((4,4,5,5-\right.$ tetramethyl-1,3,2dioxaborolan-2-yl)methyl)octahydropentalen-1-yl)ethan-1-one (31-2)

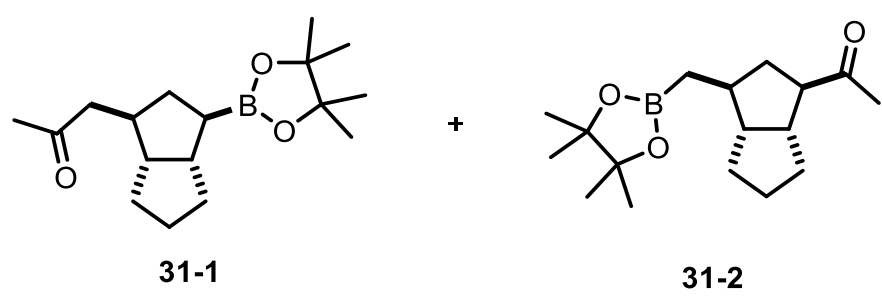

Pinacol boronic ester 31 was prepared following GP3, using $N$-alkoxyphthalimide 31a (156 mg, 0.50 mmol, 1.0 equiv.). Flash chromatography (10\% EtOAc/hexane) gave a mixture of 31-1 and 31-2 (97 mg, 0.33 mmol, 66\%, 75:25 r.r., >97:3 d.r.) as colourless liquid. The r.r. and d.r. (>97:3 for both regioisomers) were determined by ${ }^{1} \mathrm{H}$ NMR analysis of the purified product.

${ }^{1} \mathbf{H}$ NMR (400 MHz, $\left.\mathrm{CDCl}_{3}\right):$ 75:25 mixture of regioisomers: Major regioisomer: $\delta_{\mathrm{H}} 2.52(\mathrm{dd}, J=15.3$, $5.5 \mathrm{~Hz}, 1 \mathrm{H}), 2.37(\mathrm{dd}, J=15.2,8.5 \mathrm{~Hz}, 1 \mathrm{H}), 2.10(\mathrm{~s}, 3 \mathrm{H}), 2.03-1.85(\mathrm{~m}, 2 \mathrm{H}), 1.81-1.69(\mathrm{~m}, 1 \mathrm{H}), 1.64$ $-1.30(\mathrm{~m}, 7 \mathrm{H}), 1.21(\mathrm{~s}, 12 \mathrm{H}), 1.04(\mathrm{dt}, J=13.1,11.4 \mathrm{~Hz}, 1 \mathrm{H}), 0.84(\mathrm{ddd}, J=13.0,9.9,5.7 \mathrm{~Hz}, 1 \mathrm{H})$ ppm; Minor regioisomer: $\delta_{\mathrm{H}} 2.61-2.51(\mathrm{~m}, 1 \mathrm{H}), 2.47-2.35(\mathrm{~m}, 2 \mathrm{H}), 2.12(\mathrm{~s}, 3 \mathrm{H}), 1.22(2 \times \mathrm{s}, 12 \mathrm{H})$, $0.98(\mathrm{dd}, J=15.5,5.7 \mathrm{~Hz}, 1 \mathrm{H}), 0.75(\mathrm{dd}, J=15.4,8.8 \mathrm{~Hz}, 1 \mathrm{H}) \mathrm{ppm}$. The remaining signals could not be identified due to signal overlap. See spectrum.

1-((1R*,3R*,3a $\left.\left.R^{*}, 6 \mathrm{a} R^{*}\right)-3-H y d r o x y o c t a h y d r o p e n t a l e n-1-y l\right) p r o p a n-2-o n e ~(31 \mathrm{p})$<smiles>CC(=O)CC1CC(O)C2CCCCC12</smiles>

A round bottomed flask containing the boronic ester $(0.33 \mathrm{mmol})$ in THF $(6 \mathrm{~mL})$ was cooled to $0{ }^{\circ} \mathrm{C}$. A solution of $2 \mathrm{M} \mathrm{NaOH} / 30 \%$ aqueous $\mathrm{H}_{2} \mathrm{O}_{2}(3.0 \mathrm{~mL}, 2: 1 \mathrm{v} / \mathrm{v})$ was added dropwise. The mixture was stirred at $0{ }^{\circ} \mathrm{C}$ for $1 \mathrm{~h}$ and then at room temperature for $1 \mathrm{~h}$. After this time, water $(5 \mathrm{~mL})$ and EtOAc 
$(10 \mathrm{~mL})$ were added and the phases separated. The aqueous phase was extracted with EtOAc $(3 \times 20$ $\mathrm{mL}$ ) and the combined organic phases were washed with brine, dried over $\mathrm{MgSO}_{4}$, filtered, and concentrated under reduced pressure. Purification by flash column chromatography (40\% EtOAc/hexane) gave 31 p (40 mg, $0.22 \mathrm{mmol}, 66 \%$ ) as colorless oil and a single regioisomer and diastereomer.

TLC: $\mathrm{R}_{\mathrm{f}}=0.25\left(40 \% \mathrm{EtOAc} /\right.$ hexane, $\mathrm{KMnO}_{4}$ stain $)$.

${ }^{1} \mathbf{H}$ NMR $\left(400 \mathrm{MHz}, \mathrm{CDCl}_{3}\right): \delta_{\mathrm{H}} 3.79(\mathrm{dt}, J=8.4,5.7 \mathrm{~Hz}, 1 \mathrm{H}), 2.61(\mathrm{dd}, J=16.3,5.8 \mathrm{~Hz}, 1 \mathrm{H}), 2.48(\mathrm{dd}$, $J=16.3,8.4 \mathrm{~Hz}, 1 \mathrm{H}), 2.30-2.20(\mathrm{~m}, 1 \mathrm{H}), 2.20-2.12(\mathrm{~m}, 1 \mathrm{H}), 2.12(\mathrm{~s}, 3 \mathrm{H}), 2.11-2.06(\mathrm{~m}, 1 \mathrm{H}), 1.89$ -1.77 (m, 1H), 1.74 (br. s, 1H), $1.64-1.42$ (m, 5H), $1.40-1.33$ (m, 1H), 1.23 (ddd, $J=12.0,10.2,8.5$ $\mathrm{Hz}, 1 \mathrm{H}) \mathrm{ppm}$. See spectrum.

${ }^{13} \mathrm{C}$ NMR $\left(101 \mathrm{MHz}, \mathrm{CDCl}_{3}\right): \delta_{\mathrm{C}} 208.9,79.7,52.2,49.8,48.8,42.0,39.4,32.3,31.3,30.4,25.5$ ppm. See spectrum.

IR (film) $v_{\max }: 3455(\mathrm{br}), 2987,2901,1732(\mathrm{~s}), 1374,1243,1045,907,728 \mathrm{~cm}^{-1}$.

HRMS (ESI) m/z: [M + Na $]^{+}$Calcd for $\mathrm{C}_{11} \mathrm{H}_{18} \mathrm{BNaO}_{2}$ 205.1199; Found 205.1202.

\section{5-(4,4,5,5-Tetramethyl-1,3,2-dioxaborolan-2-yl)pentanal (32)}

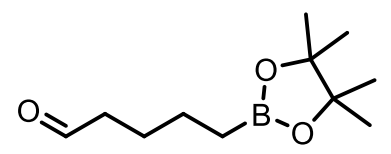

Pinacol boronic ester 32 was prepared following GP3, using $N$-alkoxyphthalimide 32a (69 mg, 0.30 mmol, 1.0 equiv.). Flash chromatography (10\% EtOAc/hexane) gave 32 (13 mg, 0.06 mmol, 20\%) as colourless liquid.

TLC: $\mathrm{R}_{\mathrm{f}}=0.40$ (10\% EtOAc/hexane, $\mathrm{KMnO}_{4}$ stain).

${ }^{1} \mathbf{H}$ NMR $\left(400 \mathrm{MHz}, \mathrm{CDCl}_{3}\right): \delta_{\mathrm{H}} 9.75(\mathrm{t}, J=2.0 \mathrm{~Hz}, 1 \mathrm{H}), 2.41(\mathrm{td}, J=7.3,1.9 \mathrm{~Hz}, 2 \mathrm{H}), 1.68-1.60(\mathrm{~m}$, 2H), $1.49-1.40$ (m, 2H), 1.24 (s, 12H), 0.80 (t, $J=7.8 \mathrm{~Hz}, 2 \mathrm{H}) \mathrm{ppm}$. See spectrum.

${ }^{13} \mathrm{C} \mathrm{NMR}\left(101 \mathrm{MHz}, \mathrm{CDCl}_{3}\right): \delta_{\mathrm{C}} 202.9,83.0,43.7,25.0,24.8,23.7 \mathrm{ppm}$. The carbon attached to boron could not be observed due to quadrupolar relaxation. See spectrum.

${ }^{11} \mathbf{B}$ NMR (128 MHz, $\mathrm{CDCl}_{3}$ ): $\delta_{\mathrm{B}} 33.9$ (br. s, 1B) ppm. See spectrum. IR (film) $v_{\max }: 2980,2932,1724(\mathrm{~s}), 1468,1372,1265,1144,906,727 \mathrm{~cm}^{-1}$.

HRMS (ESI) m/z: [M + Na] $]^{+}$Calcd for $\mathrm{C}_{11} \mathrm{H}_{21} \mathrm{BNaO}_{3} 235.1478$; Found 235.1484. 


\section{7-(4,4,5,5-Tetramethyl-1,3,2-dioxaborolan-2-yl)heptan-2-one (33)}

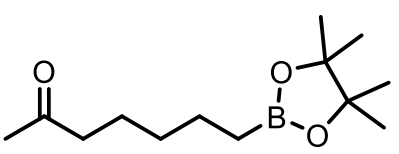

Pinacol boronic ester 33 was prepared following GP3, using $N$-alkoxyphthalimide 33a (51 mg, 0.20 mmol, 1.0 equiv.). Flash chromatography (10\% EtOAc/hexane) gave 33 (24 mg, $0.10 \mathrm{mmol}, 50 \%$ ) as colourless liquid. All recorded spectroscopic data matched those previously reported in the literature. ${ }^{14}$

${ }^{1} \mathbf{H}$ NMR $\left(400 \mathrm{MHz}, \mathrm{CDCl}_{3}\right): \delta_{\mathrm{H}} 2.4(\mathrm{t}, J=7.5 \mathrm{~Hz}, 2 \mathrm{H}), 2.11(\mathrm{~s}, 3 \mathrm{H}), 1.60-1.52(\mathrm{~m}, 2 \mathrm{H}), 1.46-1.37$ $(\mathrm{m}, 2 \mathrm{H}), 1.32-1.25(\mathrm{~m}, 2 \mathrm{H}), 1.23(\mathrm{~s}, 12 \mathrm{H}), 0.76(\mathrm{t}, J=7.7 \mathrm{~Hz}, 2 \mathrm{H}) \mathrm{ppm}$. See spectrum.

${ }^{13} \mathrm{C}$ NMR (101 MHz, $\left.\mathrm{CDCl}_{3}\right): \delta_{\mathrm{C}} 209.3,82.9,43.7,39.4,31.8,29.8,24.8,23.7 \mathrm{ppm}$. The carbon attached to boron could not be observed due to quadrupolar relaxation. See spectrum.

\section{5-Methyl-7-(4,4,5,5-tetramethyl-1,3,2-dioxaborolan-2-yl)heptan-2-one (34)}

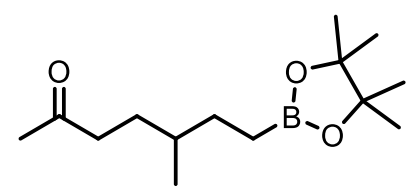

Pinacol boronic ester 34 was prepared following GP3, using $N$-alkoxyphthalimide 34a (41 mg, 0.15 mmol, 1.0 equiv.). Flash chromatography (10\% EtOAc/hexane) gave 34 (16 mg, 0.06 mmol, 41\%) as colourless liquid.

TLC: $\mathrm{R}_{\mathrm{f}}=0.45\left(10 \%\right.$ EtOAc/hexane, $\mathrm{KMnO}_{4}$ stain $)$.

${ }^{1} \mathbf{H}$ NMR $\left(400 \mathrm{MHz}, \mathrm{CDCl}_{3}\right): \delta_{\mathrm{H}} 2.49-2.34(\mathrm{~m}, 2 \mathrm{H}), 2.13(\mathrm{~s}, 3 \mathrm{H}), 1.64-1.57(\mathrm{~m}, 1 \mathrm{H}), 1.49-1.18(\mathrm{~m}$, 4H), $1.24(\mathrm{~s}, 12 \mathrm{H}), 0.85(\mathrm{~d}, J=6.3 \mathrm{~Hz}, 3 \mathrm{H}), 0.82-0.67(\mathrm{~m}, 2 \mathrm{H}) \mathrm{ppm}$. See spectrum.

${ }^{13} \mathrm{C}$ NMR $\left(101 \mathrm{MHz}, \mathrm{CDCl}_{3}\right): \delta_{\mathrm{C}} 209.6,82.9,41.6,34.5,30.6,30.4,29.8,24.8,24.8,19.0 \mathrm{ppm}$. The carbon attached to boron could not be observed due to quadrupolar relaxation. See spectrum.

${ }^{11} \mathbf{B}$ NMR (128 MHz, $\left.\mathrm{CDCl}_{3}\right): \delta_{\mathrm{B}} 34.1$ (br. s, 1B) ppm. See spectrum.

IR (film) $v_{\max }: 2974,2923,1715(\mathrm{~s}), 1454,1371,1320,1144,1056,904,728 \mathrm{~cm}^{-1}$.

HRMS (ESI) m/z: [M + Na $]^{+}$Calcd for $\mathrm{C}_{14} \mathrm{H}_{27} \mathrm{BNaO}_{3} 277.1948$; Found 277.1958. 


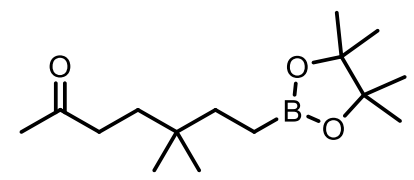

Pinacol boronic ester 35 was prepared following GP3, using $N$-alkoxyphthalimide 35a (86 mg, 0.30 mmol, 1.0 equiv.). Flash chromatography (10\% EtOAc/hexane) gave 35 (33 mg, 0.12 mmol, 41\%) as colourless liquid.

TLC: $\mathrm{R}_{\mathrm{f}}=0.45\left(10 \%\right.$ EtOAc/hexane, $\mathrm{KMnO}_{4}$ stain $)$.

${ }^{1} \mathbf{H}$ NMR (400 MHz, CDCl $): \delta_{\mathrm{H}} 2.39-2.33(\mathrm{~m}, 2 \mathrm{H}), 2.14(\mathrm{~s}, 3 \mathrm{H}), 1.48-1.43(\mathrm{~m}, 2 \mathrm{H}), 1.32-1.27(\mathrm{~m}$, $2 \mathrm{H}), 1.24(\mathrm{~s}, 12 \mathrm{H}), 0.82(\mathrm{~s}, 6 \mathrm{H}), 0.71-0.65(\mathrm{~m}, 2 \mathrm{H}) \mathrm{ppm}$. See spectrum.

${ }^{13}$ C NMR (101 MHz, $\left.\mathrm{CDCl}_{3}\right): \delta_{\mathrm{C}} 209.7,82.9,39.1,35.3,34.8,32.7,29.9,26.3,24.8 \mathrm{ppm}$. The carbon attached to boron could not be observed due to quadrupolar relaxation. See spectrum.

${ }^{11}$ B NMR $\left(128 \mathrm{MHz}, \mathrm{CDCl}_{3}\right): \delta_{\mathrm{B}} 34.0$ (br. s, 1B) ppm. $\underline{\text { See spectrum. }}$

IR (film) $v_{\max }: 2956,2930,1714(\mathrm{~s}), 1468,1372,1314,1145,966,887,848,733 \mathrm{~cm}^{-1}$.

HRMS (ESI) m/z: [M + Na $]^{+}$Calcd for $\mathrm{C}_{15} \mathrm{H}_{29} \mathrm{BNaO}_{3}$ 291.2105; Found 291.2097.

\section{5-Phenyl-7-(4,4,5,5-tetramethyl-1,3,2-dioxaborolan-2-yl)heptan-2-one (36)}<smiles>CC(=O)CCC(CCB1OC(C)(C)C(C)(C)O1)c1ccccc1</smiles>

Pinacol boronic ester 36 was prepared following GP3, using $N$-alkoxyphthalimide 36a (67 mg, 0.20 mmol, 1.0 equiv.). Flash chromatography (10\% EtOAc/hexane) gave 36 (13 mg, $0.04 \mathrm{mmol}, 21 \%$ ) as colourless liquid.

TLC: $\mathrm{R}_{\mathrm{f}}=0.45$ (10\% EtOAc/hexane, $\mathrm{KMnO}_{4}$ stain $)$.

${ }^{1} \mathbf{H}$ NMR $\left(400 \mathrm{MHz}, \mathrm{CDCl}_{3}\right): \delta_{\mathrm{H}} 7.33-7.23(\mathrm{~m}, 2 \mathrm{H}), 7.20-7.14(\mathrm{~m}, 1 \mathrm{H}), 7.13-7.07(\mathrm{~m}, 2 \mathrm{H}), 2.46-$ $2.37(\mathrm{~m}, 1 \mathrm{H}), 2.32-2.16(\mathrm{~m}, 2 \mathrm{H}), 2.02(\mathrm{~s}, 3 \mathrm{H}), 1.97(\mathrm{ddd}, J=9.2,6.5,4.8 \mathrm{~Hz}, 1 \mathrm{H}), 1.83-1.71(\mathrm{~m}$, 2H), $1.70-1.59(\mathrm{~m}, 1 \mathrm{H}), 1.21(\mathrm{~s}, 12 \mathrm{H}), 0.70-0.55(\mathrm{~m}, 2 \mathrm{H}) \mathrm{ppm}$. See spectrum. 
${ }^{13} \mathrm{C}$ NMR $\left(101 \mathrm{MHz}, \mathrm{CDCl}_{3}\right): \delta_{\mathrm{C}} 209.0,144.6,128.4,127.8,126.1,82.9,47.7,41.9,31.2,30.0,29.9$, 24.8, $24.8 \mathrm{ppm}$. The carbon attached to boron could not be observed due to quadrupolar relaxation. See spectrum.

${ }^{11} \mathbf{B}$ NMR (128 MHz, $\left.\mathrm{CDCl}_{3}\right): \delta_{\mathrm{B}} 34.1$ (br. s, 1B) ppm. See spectrum.

IR (film) $v_{\max }: 2973,2901,1732(\mathrm{~s}), 1477,1452,1406,1375,1249,1065,904,725 \mathrm{~cm}^{-1}$.

HRMS (ESI) m/z: [M + Na $]^{+}$Calcd for $\mathrm{C}_{19} \mathrm{H}_{29} \mathrm{BNaO}_{3}$ 339.2105; Found 339.2098.

8-(4,4,5,5-Tetramethyl-1,3,2-dioxaborolan-2-yl)octan-2-one (37)

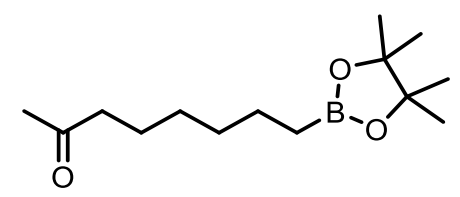

Pinacol boronic ester 37 was prepared following GP3, using $N$-alkoxyphthalimide 37a (82 $\mathrm{mg}, 0.30$ mmol, 1.0 equiv.). Flash chromatography (10\% EtOAc/hexane) gave 37 (22 mg, 0.09 mmol, 29\%) as colourless liquid.

TLC: $\mathrm{R}_{\mathrm{f}}=0.45\left(10 \%\right.$ EtOAc/hexane, $\mathrm{KMnO}_{4}$ stain) .

${ }^{1} \mathbf{H}$ NMR $\left(400 \mathrm{MHz}, \mathrm{CDCl}_{3}\right): \delta_{\mathrm{H}} 2.39(\mathrm{t}, J=7.5 \mathrm{~Hz}, 2 \mathrm{H}), 2.12(\mathrm{~s}, 3 \mathrm{H}), 1.59-1.51(\mathrm{~m}, 2 \mathrm{H}), 1.44-1.35$ $(\mathrm{m}, 2 \mathrm{H}), 1.30-1.26(\mathrm{~m}, 4 \mathrm{H}), 1.23(\mathrm{~s}, 12 \mathrm{H}), 0.75(\mathrm{t}, J=7.7 \mathrm{~Hz}, 2 \mathrm{H}) \mathrm{ppm}$. See spectrum.

${ }^{13}$ C NMR (101 MHz, $\left.\mathrm{CDCl}_{3}\right): \delta_{\mathrm{C}} 209.4,82.9,43.8,32.0,29.8,28.9,24.8,23.8,23.8 \mathrm{ppm}$. The carbon attached to boron could not be observed due to quadrupolar relaxation. See spectrum.

${ }^{11} \mathbf{B}$ NMR (128 MHz, $\mathrm{CDCl}_{3}$ ): $\delta_{\mathrm{B}} 34.2$ (br. s, 1B) ppm. See spectrum.

IR (film) $v_{\max }: 2980,2924,1715(\mathrm{~s}), 1454,1407,1371,1318,1144,1056,907,730 \mathrm{~cm}^{-1}$.

HRMS (ESI) m/z: [M + Na $]^{+}$Calcd for $\mathrm{C}_{14} \mathrm{H}_{27} \mathrm{BNaO}_{3} 277.1945$; Found 277.1940. 


\section{3-(4,4,5,5-Tetramethyl-1,3,2-dioxaborolan-2-yl)propyl formate (38)}

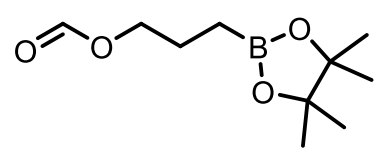

Pinacol boronic ester 38 was prepared following GP3, using $N$-alkoxyphthalimide 38a (47 mg, 0.20 mmol, 1.0 equiv.). Flash chromatography (20\% EtOAc/hexane) gave 38 (14 mg, 0.06 mmol, 32\%) as colourless liquid.

TLC: $\mathrm{R}_{\mathrm{f}}=0.40\left(10 \% \mathrm{EtOAc/hexane,} \mathrm{KMnO}_{4}\right.$ stain $)$.

${ }^{1} \mathbf{H}$ NMR (400 MHz, CDCl $): \delta_{\mathrm{H}} 8.04(\mathrm{t}, J=0.7 \mathrm{~Hz}, 1 \mathrm{H}), 4.14(\mathrm{td}, J=6.8,0.9 \mathrm{~Hz}, 2 \mathrm{H}), 1.82-1.73(\mathrm{~m}$, 2H), 1.24 (s, 12H), 0.82 (t, $J=7.8 \mathrm{~Hz}, 2 \mathrm{H}) \mathrm{ppm}$. See spectrum.

${ }^{13} \mathrm{C}$ NMR $\left(101 \mathrm{MHz}, \mathrm{CDCl}_{3}\right): \delta_{\mathrm{C}} 161.2,83.2,65.7,24.8,23.1 \mathrm{ppm}$. The carbon attached to boron could not be observed due to quadrupolar relaxation. See spectrum.

${ }^{11} \mathbf{B}$ NMR (128 MHz, $\left.\mathrm{CDCl}_{3}\right): \delta_{\mathrm{B}} 33.7$ (br. s, 1B) ppm. See spectrum.

IR (film) $v_{\max }: 2980,2901,1722(\mathrm{~s}), 1469,1372,1320,1142,1056,907,846,728 \mathrm{~cm}^{-1}$

HRMS (ESI) m/z: [M + Na $]^{+}$Calcd for $\mathrm{C}_{10} \mathrm{H}_{19} \mathrm{BNaO}_{4}$ 237.1271; Found 237.1276.

\section{4-(4,4,5,5-Tetramethyl-1,3,2-dioxaborolan-2-yl)butan-2-yl formate (39)}<smiles>CC(CCB1OC(C)(C)C(C)(C)O1)OC=O</smiles>

Pinacol boronic ester 39 was prepared following GP3, using $N$-alkoxyphthalimide 39a (49 mg, 0.20 mmol, 1.0 equiv.). Flash chromatography (10\% EtOAc/hexane) gave 39 (20 mg, 0.09 mmol, 44\%) as colourless liquid.

TLC: $\mathrm{R}_{\mathrm{f}}=0.40\left(10 \% \mathrm{EtOAc} / \mathrm{hexane}, \mathrm{KMnO}_{4}\right.$ stain $)$.

${ }^{1} \mathbf{H}$ NMR $\left(400 \mathrm{MHz}, \mathrm{CDCl}_{3}\right): \delta_{\mathrm{H}} 8.04(\mathrm{~s}, 1 \mathrm{H}), 5.03-4.93(\mathrm{~m}, 1 \mathrm{H}), 1.77-1.62(\mathrm{~m}, 2 \mathrm{H}), 1.24(\mathrm{~d}, J=6.2$ $\mathrm{Hz}, 3 \mathrm{H}), 1.24$ (s, 12H), $0.87-0.71(\mathrm{~m}, 2 \mathrm{H}) \mathrm{ppm} . \underline{\text { See spectrum. }}$

${ }^{13}$ C NMR $\left(101 \mathrm{MHz}, \mathrm{CDCl}_{3}\right): \delta_{\mathrm{C}} 160.9,83.1,72.5,30.1,24.8,19.6 \mathrm{ppm}$. The carbon attached to boron could not be observed due to quadrupolar relaxation. See spectrum.

${ }^{11} \mathbf{B}$ NMR (128 MHz, $\left.\mathrm{CDCl}_{3}\right): \delta_{\mathrm{B}} 33.8$ (br. s, 1B) ppm. See spectrum.

IR (film) $v_{\max }: 2981,2902,1716(\mathrm{~s}), 13800,1325,1198,1144,1050,906,726 \mathrm{~cm}^{-1}$. 
HRMS (ESI) m/z: [M + Na $]^{+}$Calcd for $\mathrm{C}_{11} \mathrm{H}_{21} \mathrm{BNaO}_{4}$ 251.1427; Found 251.1436.

\section{1-Phenyl-3-(4,4,5,5-tetramethyl-1,3,2-dioxaborolan-2-yl)propyl formate (40)}

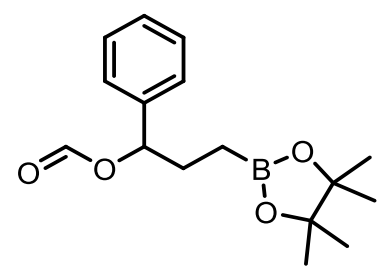

Pinacol boronic ester 40 was prepared following GP3, using $N$-alkoxyphthalimide 40a (62 mg, 0.20 mmol, 1.0 equiv.). Flash chromatography (10\% EtOAc/hexane) gave 40 (20 mg, $0.07 \mathrm{mmol}, 34 \%)$ as colourless liquid.

TLC: $\mathrm{R}_{\mathrm{f}}=0.40\left(10 \%\right.$ EtOAc/hexane, $\mathrm{KMnO}_{4}$ stain $)$.

${ }^{1} \mathbf{H}$ NMR $\left(400 \mathrm{MHz}, \mathrm{CDCl}_{3}\right): \delta_{\mathrm{H}} 8.10(\mathrm{~s}, 1 \mathrm{H}), 7.36-7.25(\mathrm{~m}, 5 \mathrm{H}), 5.81(\mathrm{ddd}, J=7.4,6.1,1.0 \mathrm{~Hz}, 1 \mathrm{H})$, $2.10-1.88(\mathrm{~m}, 2 \mathrm{H}), 1.23(\mathrm{~s}, 12 \mathrm{H}), 0.86-0.69(\mathrm{~m}, 2 \mathrm{H}) \mathrm{ppm} . \underline{\text { See spectrum. }}$

${ }^{13} \mathrm{C}$ NMR $\left(101 \mathrm{MHz}, \mathrm{CDCl}_{3}\right): \delta_{\mathrm{C}} 160.5,139.9,128.4,128.0,126.6,83.2,77.5,30.7,24.8 \mathrm{ppm}$. The carbon attached to boron could not be observed due to quadrupolar relaxation. See spectrum.

${ }^{11}$ B NMR (128 MHz, $\left.\mathrm{CDCl}_{3}\right): \delta_{\mathrm{B}} 33.7$ (br. s, 1B) ppm. See spectrum.

IR (film) $v_{\max }$ : 2980, 2901, 1722(s), 1495, 1454, 1407, 1379, 1318, 1265, 1168, 1143, 909, $731 \mathrm{~cm}^{-1}$.

HRMS (ESI) m/z: [M + Na $]^{+}$Calcd for $\mathrm{C}_{16} \mathrm{H}_{23} \mathrm{BNaO}_{4}$ 313.1585; Found 313.1585.

\section{4-(4,4,5,5-Tetramethyl-1,3,2-dioxaborolan-2-yl)butyl formate (41)}

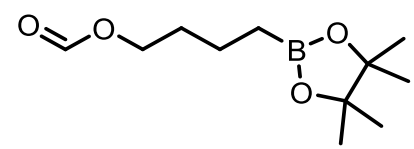

Pinacol boronic ester 41 was prepared following GP3, using $N$-alkoxyphthalimide 41a (74 mg, 0.30 mmol, 1.0 equiv.). Flash chromatography (10\% EtOAc/hexane) gave 41 (35 mg, $0.15 \mathrm{mmol}, 51 \%$ ) as colourless liquid.

TLC: $\mathrm{R}_{\mathrm{f}}=0.40$ (10\% EtOAc/hexane, $\mathrm{KMnO}_{4}$ stain)

${ }^{1} \mathbf{H}$ NMR $\left(400 \mathrm{MHz}, \mathrm{CDCl}_{3}\right): \delta_{\mathrm{H}} 8.04(\mathrm{t}, J=0.7 \mathrm{~Hz}, 1 \mathrm{H}), 4.15(\mathrm{td}, J=6.6,0.9 \mathrm{~Hz}, 2 \mathrm{H}), 1.71-1.62(\mathrm{~m}$, 2H), $1.53-1.45$ (m, 2H), 1.23 (s, 12H), 0.80 (t, $J=7.8 \mathrm{~Hz}, 2 \mathrm{H}) \mathrm{ppm}$. See spectrum. 
${ }^{13} \mathrm{C} \mathrm{NMR}\left(101 \mathrm{MHz}, \mathrm{CDCl}_{3}\right): \delta_{\mathrm{C}} 161.2,83.0,63.9,30.9,24.8,20.3 \mathrm{ppm}$. The carbon attached to boron could not be observed due to quadrupolar relaxation. See spectrum.

${ }^{11} \mathbf{B}$ NMR $\left(128 \mathrm{MHz}, \mathrm{CDCl}_{3}\right): \delta_{\mathrm{B}} 33.9$ (br. s, 1B) ppm. See spectrum.

IR (film) $v_{\max }: 2987,2901,1721(\mathrm{~s}), 1380,1321,1144,1066,882,731,703 \mathrm{~cm}^{-1}$.

HRMS (ESI) m/z: [M + Na $]^{+}$Calcd for $\mathrm{C}_{11} \mathrm{H}_{21} \mathrm{BNaO}_{4}$ 251.1427; Found 251.1435.

\section{1-Phenyl-4-(4,4,5,5-tetramethyl-1,3,2-dioxaborolan-2-yl)butyl formate (42)}

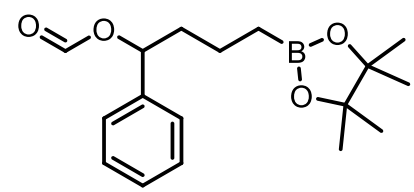

Pinacol boronic ester 42 was prepared following GP3, using $N$-alkoxyphthalimide 42a (65 mg, 0.20 mmol, 1.0 equiv.). Flash chromatography (10\% EtOAc/hexane) gave 42 (28 mg, 0.09 mmol, 45\%) as colourless liquid.

TLC: $\mathrm{R}_{\mathrm{f}}=0.40\left(10 \% \mathrm{EtOAc} /\right.$ hexane, $\mathrm{KMnO}_{4}$ stain $)$.

${ }^{1} \mathbf{H}$ NMR $\left(400 \mathrm{MHz}, \mathrm{CDCl}_{3}\right): \delta_{\mathrm{H}} 8.09(\mathrm{~d}, J=1.0 \mathrm{~Hz}, 1 \mathrm{H}), 7.36-7.26(\mathrm{~m}, 5 \mathrm{H}), 5.84$ (ddd, $J=7.5,6.0$, $1.1 \mathrm{~Hz}, 1 \mathrm{H}), 1.97$ (dddd, $J=13.2,10.4,7.8,5.2 \mathrm{~Hz}, 1 \mathrm{H}$ ), 1.83 (ddt, $J=13.7,11.0,5.8 \mathrm{~Hz}, 1 \mathrm{H}$ ), $1.56-$ $1.44(\mathrm{~m}, 1 \mathrm{H}), 1.42-1.32(\mathrm{~m}, 1 \mathrm{H}), 1.23(\mathrm{~s}, 12 \mathrm{H}), 0.79(\mathrm{t}, J=7.8 \mathrm{~Hz}, 2 \mathrm{H}) \mathrm{ppm}$. See spectrum.

${ }^{13} \mathrm{C}$ NMR (101 MHz, $\left.\mathrm{CDCl}_{3}\right): \delta_{\mathrm{C}} 160.5,140.1,128.5,128.0,126.6,83.0,76.0,38.6,24.8,24.8,19.9$ ppm. The carbon attached to boron could not be observed due to quadrupolar relaxation. See spectrum.

${ }^{11} \mathbf{B}$ NMR (128 MHz, $\left.\mathrm{CDCl}_{3}\right): \delta_{\mathrm{B}} 33.8$ (br. s, 1B) ppm. See spectrum.

IR (film) $v_{\max }$ : 2987, 2901, 1721(s), 1587, 1495, 1453, 1380, 1325, 1265, 1144, 1066, 904, $725 \mathrm{~cm}^{-1}$.

HRMS (ESI) m/z: [M + Na $]^{+}$Calcd for $\mathrm{C}_{17} \mathrm{H}_{25} \mathrm{BNaO}_{4}$ 327.1741; Found 327.1750. 


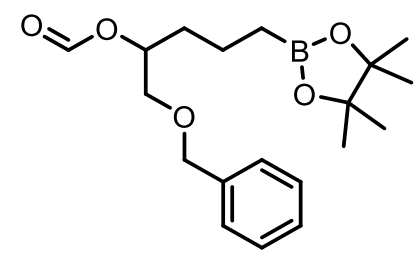

Pinacol boronic ester 43 was prepared following GP3, using $N$-alkoxyphthalimide 43a (73 mg, 0.20 mmol, 1.0 equiv.). Flash chromatography (10\% EtOAc/hexane) gave 43 (40 mg, $0.11 \mathrm{mmol}, 57 \%$ ) as colourless liquid.

TLC: $\mathrm{R}_{\mathrm{f}}=0.34\left(10 \%\right.$ EtOAc/hexane, $\mathrm{KMnO}_{4}$ stain $)$.

${ }^{1} \mathbf{H}$ NMR $\left(400 \mathrm{MHz}, \mathrm{CDCl}_{3}\right): \delta_{\mathrm{H}} 8.11(\mathrm{~d}, J=0.8 \mathrm{~Hz}, 1 \mathrm{H}), 7.36-7.25(\mathrm{~m}, 5 \mathrm{H}), 5.16-5.10(\mathrm{~m}, 1 \mathrm{H})$, $4.57(\mathrm{~d}, J=12.1 \mathrm{~Hz}, 1 \mathrm{H}), 4.50(\mathrm{~d}, J=12.1 \mathrm{~Hz}, 1 \mathrm{H}), 3.54(\mathrm{~d}, J=5.0 \mathrm{~Hz}, 2 \mathrm{H}), 1.68-1.62(\mathrm{~m}, 2 \mathrm{H}), 1.54$ $-1.37(\mathrm{~m}, 2 \mathrm{H}), 1.23(\mathrm{~s}, 12 \mathrm{H}), 0.82-0.75(\mathrm{~m}, 2 \mathrm{H}) \mathrm{ppm}$. See spectrum.

${ }^{13} \mathrm{C}$ NMR (101 MHz, $\left.\mathrm{CDCl}_{3}\right): \delta_{\mathrm{C}} 161.0,137.9,128.4,127.7,127.6,83.0,73.2,72.9,71.0,33.2,25.0$, $24.8,19.7 \mathrm{ppm}$. The carbon attached to boron could not be observed due to quadrupolar relaxation. See spectrum.

${ }^{11} \mathbf{B}$ NMR (128 MHz, $\left.\mathrm{CDCl}_{3}\right): \delta_{\mathrm{B}} 33.8$ (br. s, 1B) ppm. See spectrum.

IR (film) $v_{\max }$ 2979, 2901, 1721(s), 1496, 1453, 1372, 1318, 1265, 1184, 1144, 847, 732, $700 \mathrm{~cm}^{-1}$.

HRMS (ESI) m/z: [M + Na $]^{+}$Calcd for $\mathrm{C}_{19} \mathrm{H}_{29} \mathrm{BNaO}_{5}$ 371.2004; Found 371.2004.

2-(Formyloxy)-5-(4,4,5,5-tetramethyl-1,3,2-dioxaborolan-2-yl)pentyl acetate (44)<smiles>CC(=O)OCC(CCCB1OC(C)(C)C(C)(C)O1)OC=O</smiles>

Pinacol boronic ester 44 was prepared following GP3, using $N$-alkoxyphthalimide 44a (64 mg, 0.20 mmol, 1.0 equiv.). Flash chromatography (10\% EtOAc/hexane) gave 44 (39 mg, 0.13 mmol, 65\%) as colourless liquid.

TLC: $\mathrm{R}_{\mathrm{f}}=0.38\left(10 \%\right.$ EtOAc/hexane, $\mathrm{KMnO}_{4}$ stain $)$.

${ }^{1} \mathbf{H}$ NMR $\left(400 \mathrm{MHz}, \mathrm{CDCl}_{3}\right): \delta_{\mathrm{H}} 8.08(\mathrm{~s}, 1 \mathrm{H}), 5.22-5.16(\mathrm{~m}, 1 \mathrm{H}), 4.24(\mathrm{dd}, J=12.0,3.1 \mathrm{~Hz}, 1 \mathrm{H}), 4.06$ (dd, $J=12.0,7.0 \mathrm{~Hz}, 1 \mathrm{H}), 2.05$ (s, 3H), $1.69-1.57$ (m, 2H), $1.54-1.37$ (m, 2H), $1.23(\mathrm{~s}, 12 \mathrm{H}), 0.79$ (t, $J=7.6 \mathrm{~Hz}, 2 \mathrm{H}) \mathrm{ppm}$. See spectrum. 
${ }^{13} \mathrm{C} \mathrm{NMR}\left(101 \mathrm{MHz}, \mathrm{CDCl}_{3}\right): \delta_{\mathrm{C}} 170.7,160.5,83.1,71.4,64.9,33.1,24.8,20.7,19.5 \mathrm{ppm}$. The carbon attached to boron could not be observed due to quadrupolar relaxation. See spectrum.

${ }^{11} \mathbf{B}$ NMR (128 MHz, $\left.\mathrm{CDCl}_{3}\right)$ : $\delta_{\mathrm{B}} 33.7$ (br. s, 1B) ppm. See spectrum.

IR (film) $v_{\max }: 2981,2943,1724(\mathrm{~s}), 1372,1819,1233,1144,1050,904,724 \mathrm{~cm}^{-1}$.

HRMS (ESI) m/z: [M + Na $]^{+}$Calcd for $\mathrm{C}_{14} \mathrm{H}_{25} \mathrm{BNaO}_{6}$ 323.1639; Found 323.1639.

\section{2-(Formyloxy)-5-(4,4,5,5-tetramethyl-1,3,2-dioxaborolan-2-yl)pentyl benzoate (45)}

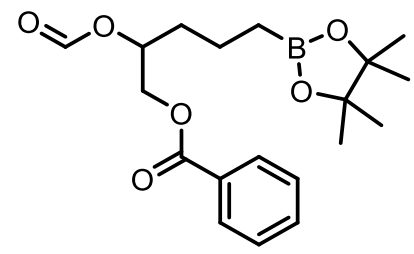

Pinacol boronic ester 45 was prepared following GP3, using $N$-alkoxyphthalimide 45a (76 mg, 0.20 mmol, 1.0 equiv.). Flash chromatography (10\% EtOAc/hexane) gave 45 (49 mg, $0.14 \mathrm{mmol}, 68 \%$ ) as colourless liquid.

TLC: $\mathrm{R}_{\mathrm{f}}=0.35\left(10 \%\right.$ EtOAc/hexane, $\mathrm{KMnO}_{4}$ stain $)$.

${ }^{1} \mathbf{H}$ NMR $\left(400 \mathrm{MHz}, \mathrm{CDCl}_{3}\right): \delta_{\mathrm{H}} 8.12(\mathrm{~d}, J=1.0 \mathrm{~Hz}, 1 \mathrm{H}), 8.04-8.00(\mathrm{~m}, 2 \mathrm{H}), 7.59-7.53(\mathrm{~m}, 1 \mathrm{H})$, $7.46-7.41(\mathrm{~m}, 2 \mathrm{H}), 5.40-5.33(\mathrm{~m}, 1 \mathrm{H}), 4.47(\mathrm{dd}, J=12.0,3.2 \mathrm{~Hz}, 1 \mathrm{H}), 4.33(\mathrm{dd}, J=12.0,6.9 \mathrm{~Hz}$, 1H), $1.78-1.68(\mathrm{~m}, 2 \mathrm{H}), 1.60-1.46(\mathrm{~m}, 2 \mathrm{H}), 1.23(\mathrm{~s}, 12 \mathrm{H}), 0.82(\mathrm{t}, J=8.0 \mathrm{~Hz}, 2 \mathrm{H}) \mathrm{ppm}$. See spectrum. ${ }^{13}$ C NMR (101 MHz, $\left.\mathrm{CDCl}_{3}\right): \delta_{\mathrm{C}} 166.2,160.6,133.1,129.7,129.7,128.4,83.1,71.4,65.5,33.1,24.8$, $19.6 \mathrm{ppm}$. The carbon attached to boron could not be observed due to quadrupolar relaxation. See spectrum.

${ }^{11}$ B NMR (128 MHz, $\left.\mathrm{CDCl}_{3}\right): \delta_{\mathrm{B}} 33.7$ (br. s, 1B) ppm. See spectrum.

IR (film) $v_{\max }: 2987,2901,1722(\mathrm{~s}), 1585,1452,1406,1381,1265,1177,1144,1065,904,725 \mathrm{~cm}^{-1}$. HRMS (ESI) m/z: [M + Na $]^{+}$Calcd for $\mathrm{C}_{19} \mathrm{H}_{27} \mathrm{BNaO}_{6}$ 385.1796; Found 385.1795. 


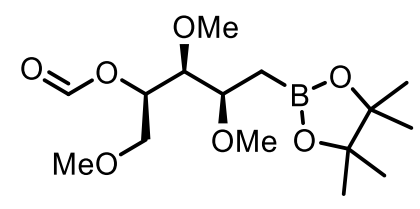

Pinacol boronic ester 46 was prepared following GP3, using $N$-alkoxyphthalimide $46 a$ (70 mg, 0.20 mmol, 1.0 equiv.). Flash chromatography (20\% EtOAc/hexane) gave 46 (42.0 mg, $0.13 \mathrm{mmol}, 63 \%)$ as colourless liquid.

TLC: $\mathrm{R}_{\mathrm{f}}=0.25$ (20\% EtOAc/hexane, PMA stain).

$[\alpha]^{20}{ }_{\mathrm{D}}:+16.0\left(c 1.0, \mathrm{CHCl}_{3}\right)$.

${ }^{1} \mathbf{H}$ NMR $\left(400 \mathrm{MHz}, \mathrm{CDCl}_{3}\right): \delta_{\mathrm{H}} 8.13(\mathrm{~s}, 1 \mathrm{H}), 5.24(\mathrm{td}, J=5.9,2.7 \mathrm{~Hz}, 1 \mathrm{H}), 3.71(\mathrm{dd}, J=11.0,2.6 \mathrm{~Hz}$, $1 \mathrm{H}), 3.66(\mathrm{dd}, J=11.1,5.6 \mathrm{~Hz}, 1 \mathrm{H}), 3.54(\mathrm{ddd}, J=9.2,6.0,2.9 \mathrm{~Hz}, 1 \mathrm{H}), 3.51$ (s, 3H), 3.46 (dd, $J=6.6$, $2.9 \mathrm{~Hz}, 1 \mathrm{H}), 3.38$ (s, 3H), 3.31 (s, 3H), 1.32 (dd, $J=15.3,6.0 \mathrm{~Hz}, 1 \mathrm{H}), 1.26$ (s, 12H), 1.17 (dd, $J=15.4$, $9.0 \mathrm{~Hz}, 1 \mathrm{H})$. See spectrum.

${ }^{13} \mathrm{C}$ NMR $\left(101 \mathrm{MHz}, \mathrm{CDCl}_{3}\right): \delta_{\mathrm{C}} 160.5,83.3,81.9,77.4,72.8,71.0,60.9,58.9,57.2,24.8,24.8,12.8$ (br.) ppm. See spectrum.

IR (film) $v_{\max }$ : 2979, 2930, 2829, 1724(s), 1456, 1371, 1319, 1167, 1144, 1091, 967, 847, $731 \mathrm{~cm}^{-1}$.

HRMS (ESI) m/z: [M + Na $]^{+}$Calcd for $\mathrm{C}_{15} \mathrm{H}_{29} \mathrm{BNaO}_{7} 355.1904$; Found 355.1916. 


\section{Mechanistic Studies}

\subsection{Control Experiments}

The following competition experiments with various radical inhibitors suggest that the fragmentation borylation reaction proceeds through a radical pathway.

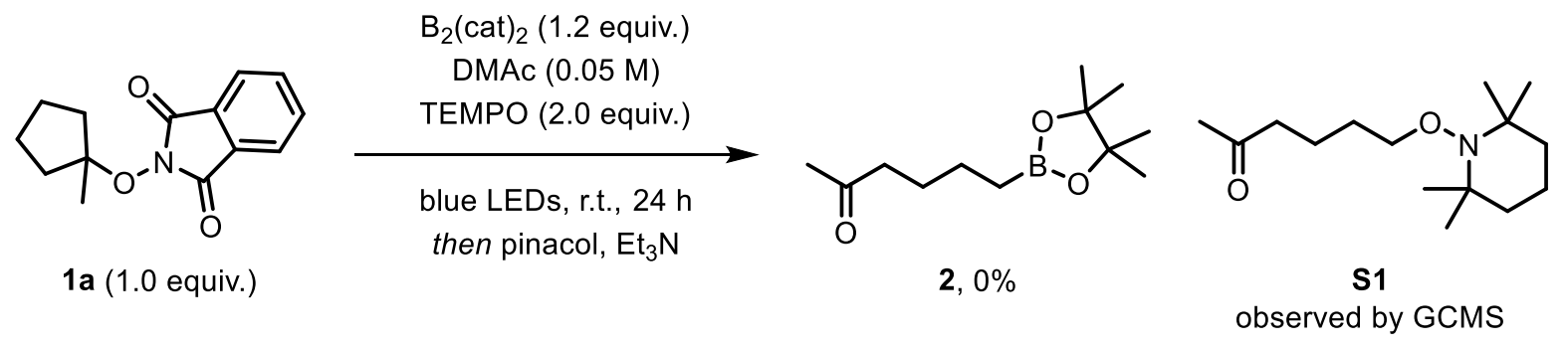

$N$-Alkoxyphthalimide $1 \mathbf{a}(25 \mathrm{mg}, 0.10 \mathrm{mmol}, 1.0$ equiv.), TEMPO (31 mg, $0.20 \mathrm{mmol}, 2.0$ equiv.) and $\mathrm{B}_{2}$ (cat) $)_{2}\left(29 \mathrm{mg}, 0.12 \mathrm{mmol}, 1.2\right.$ equiv.) were carefully weighed into an $\mathrm{N}_{2}$-flushed $7 \mathrm{~mL}$ vial containing a small magnetic stirrer bar. Dry DMAc $(0.05 \mathrm{M})$ was added and the headspace of the vial was purged with a gentle stream of $\mathrm{N}_{2}$ for approximately $10 \mathrm{~min}$. The vial was tightly sealed and stirred under blue LED irradiation for $24 \mathrm{~h}$. Irradiation was stopped and a solution of pinacol (3.0 equiv.) in $\mathrm{Et}_{3} \mathrm{~N}$ (20.0 equiv.) was added to the reaction mixture, which was stirred at room temperature for a further $1 \mathrm{~h}$. The reaction mixture was diluted with DCM $(50 \mathrm{~mL})$ and washed with water $(3 \times 30 \mathrm{~mL})$ and brine $(20 \mathrm{~mL})$, dried over $\mathrm{MgSO}_{4}$, filtered, and concentrated under reduced pressure. The crude product was analysed by GC-MS. Product $\mathbf{2}$ was not observed, however, the corresponding TEMPO-adduct $\mathbf{S 1}$ was observed.

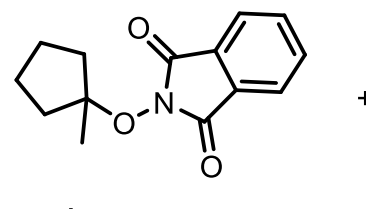

1 a (1.0 equiv.)

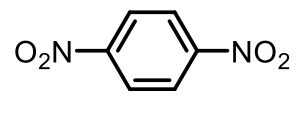

(2.0 equiv.)

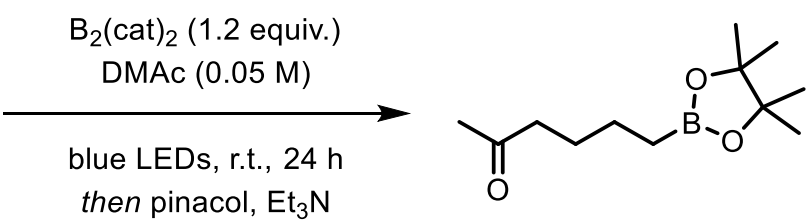

2, $0 \%$

$N$-alkoxyphthalimide 1a (25 mg, $0.10 \mathrm{mmol}, 1.0$ equiv.), 1,4-dinitrobenzene (34 mg, $0.20 \mathrm{mmol}, 2.0$ equiv.) and $\mathrm{B}_{2}$ (cat) $)_{2}\left(29 \mathrm{mg}, 0.12 \mathrm{mmol}, 1.2\right.$ equiv.) were carefully weighed into an $\mathrm{N}_{2}$-flushed $7 \mathrm{~mL}$ vial containing a small magnetic stirrer bar. Dry DMAc $(0.05 \mathrm{M})$ was added and the headspace of the vial was purged with a gentle stream of $\mathrm{N}_{2}$ for approximately $10 \mathrm{~min}$. The vial was tightly sealed and stirred under blue LED irradiation for $24 \mathrm{~h}$. Irradiation was stopped and a solution of pinacol (3.0 equiv.) in $\mathrm{Et}_{3} \mathrm{~N}$ (20.0 equiv.) was added to the reaction mixture, which was stirred at room temperature for a further $1 \mathrm{~h}$. The reaction mixture was diluted with DCM $(50 \mathrm{~mL})$ and washed with water $(3 \times 30 \mathrm{~mL})$ and brine $(20 \mathrm{~mL})$, dried over $\mathrm{MgSO}_{4}$, filtered, and concentrated under reduced pressure. The crude product was analysed by GC-MS. Product 2 was not observed. 


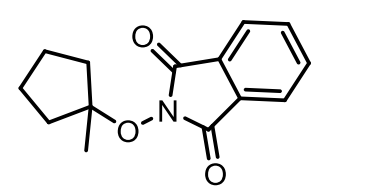

1a (1.0 equiv.)

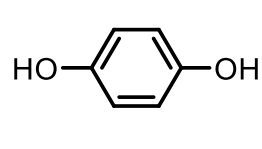

(2.0 equiv.)

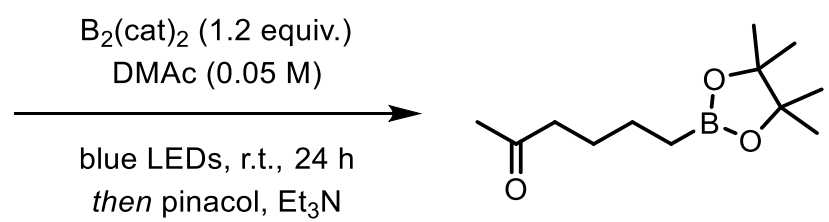

2, $45 \%$

$N$-alkoxyphthalimide $1 \mathbf{a}$ ( $25 \mathrm{mg}, 0.10 \mathrm{mmol}, 1.0$ equiv.), hydroquinone (22 mg, $0.20 \mathrm{mmol}, 2.0$ equiv.) and $\mathrm{B}_{2}$ (cat) $)_{2}\left(29 \mathrm{mg}, 0.12 \mathrm{mmol}, 1.2\right.$ equiv.) were carefully weighed into an $\mathrm{N}_{2}$-flushed $7 \mathrm{~mL}$ vial containing a small magnetic stirrer bar. Dry DMAc $(0.05 \mathrm{M})$ was added and the headspace of the vial was purged with a gentle stream of $\mathrm{N}_{2}$ for approximately $10 \mathrm{~min}$. The vial was tightly sealed and stirred under blue LED irradiation for $24 \mathrm{~h}$. Irradiation was stopped and a solution of pinacol (3.0 equiv.) in $\mathrm{Et}_{3} \mathrm{~N}$ (20.0 equiv.) was added to the reaction mixture, which was stirred at room temperature for a further $1 \mathrm{~h}$. The reaction mixture was diluted with DCM $(50 \mathrm{~mL})$ and washed with water $(3 \times 30 \mathrm{~mL})$ and brine (20 mL), dried over $\mathrm{MgSO}_{4}$, filtered, and concentrated under reduced pressure. The crude mixture was analysed by ${ }^{1} \mathrm{H}$ NMR with diethyl phthalate an internal standard. Product 2 was formed in $45 \%$ yield. 


\subsection{UV/Vis Absorption Spectroscopy}

The reaction of $O$-phthalimido cyclopentanol $1 \mathbf{a}$ and $\mathrm{B}_{2}(\text { cat })_{2}$ was investigated by UV/Vis spectroscopy to determine whether EDA complex formation occurred under the reaction conditions. From the absorption spectra of DMAc solutions of $1 \mathbf{a}(0.05 \mathrm{M}), \mathrm{B}_{2}(\mathrm{cat})_{2}(0.06 \mathrm{M})$, and a 1:1.2 mixture of 1a $(0.05$ $\mathrm{M})$ and $\mathrm{B}_{2}(\mathrm{cat})_{2}(0.06 \mathrm{M})$ [spectrum recorded $10 \mathrm{~min}$ after mixing], it can be seen that no significant complex formation occurs (Figure S2). However, the spectra for both $\mathbf{1 a}$ and $\mathrm{B}_{2}(\mathrm{cat})_{2}$ have absorption tails that extend to $450 \mathrm{~nm}$, which means that both substrates can potentially be excited by blue light ( $\lambda_{\max }$ of the LEDs used for the reactions is $450 \mathrm{~nm}$ ).

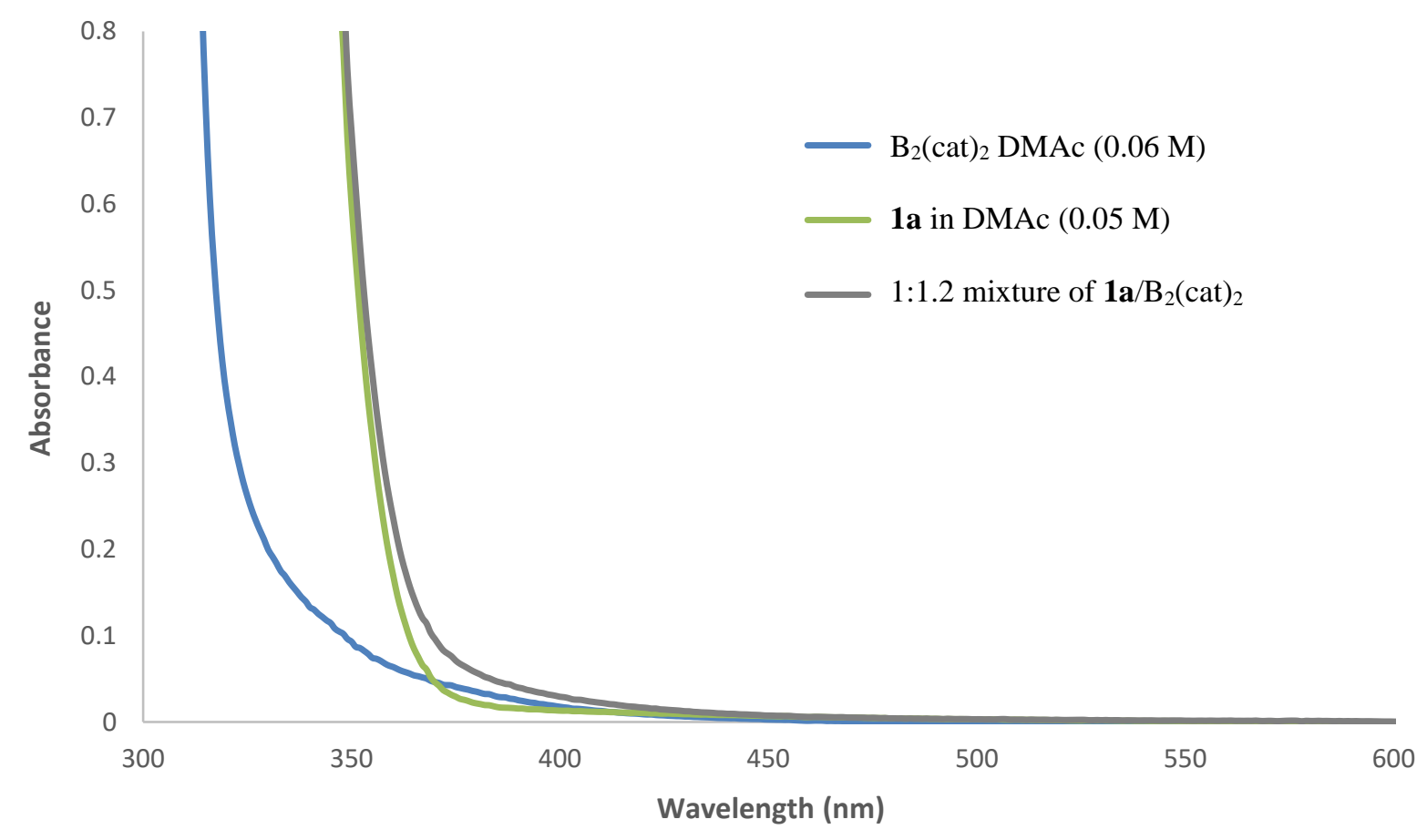

Figure S2. UV/Vis absorption spectra of $\mathbf{1 a}$ and $\mathrm{B}_{2}(\mathrm{cat})_{2}$.

To further investigate the potential formation of EDA complexes between $1 \mathbf{a}$ and $\mathrm{B}_{2}(\mathrm{cat})_{2}$, we analysed the change in absorption spectra with time, while keeping the DMAc solution of the $1 \mathbf{a} / \mathrm{B}_{2}(\mathrm{cat})_{2}$ mixture in the dark (Figure S3). We observed a bathochromic shift that became more pronounced with time, and a new absorbance peak appeared at $383 \mathrm{~nm}$. The intensity of this signal continued to increase with time, and after $30 \mathrm{~h}$ in the dark new absorbance peaks appeared at $454 \mathrm{~nm}$ and $484 \mathrm{~nm}$ (Figure S4). However, upon measuring the absorption spectrum of the reaction mixture after $20 \mathrm{~h}$ of irradiation (complete conversion to product), these signals persist and become more intense (Figure S4). This suggests that the bathochromic shift observed in Figure S3 is due to the formation of a by-product in a thermallyinduced fragmentation borylation reaction, rather than the an EDA complex between $\mathbf{1 a}$ and $\mathrm{B}_{2}(\mathrm{cat})_{2}$. 


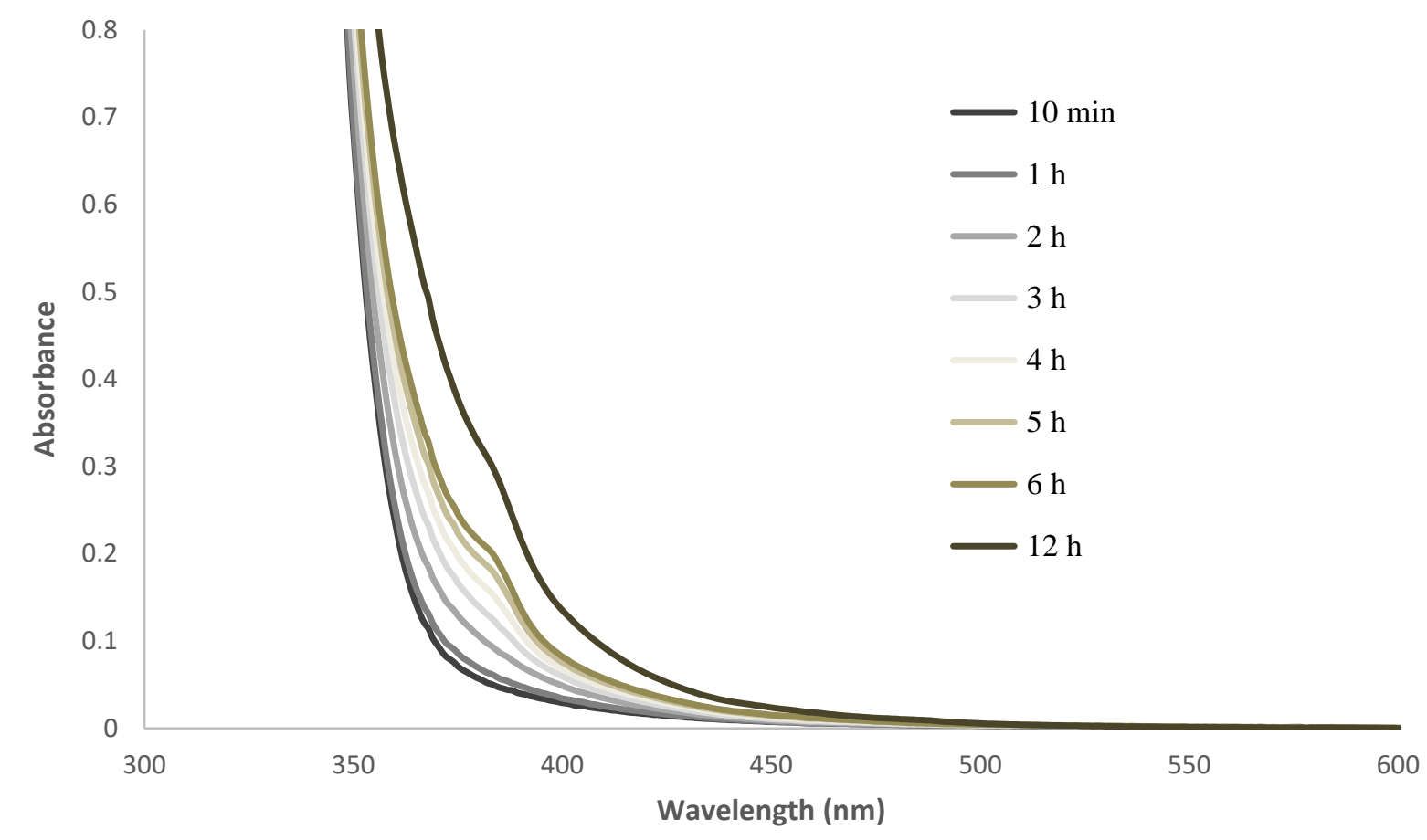

Figure S3. UV/Vis absorption spectra of a mixture of $1 \mathbf{a}(0.05 \mathrm{M})$ and $\mathrm{B}_{2}(\mathrm{cat})_{2}(0.06 \mathrm{M})$ in DMAc after extended mixing periods in the dark.

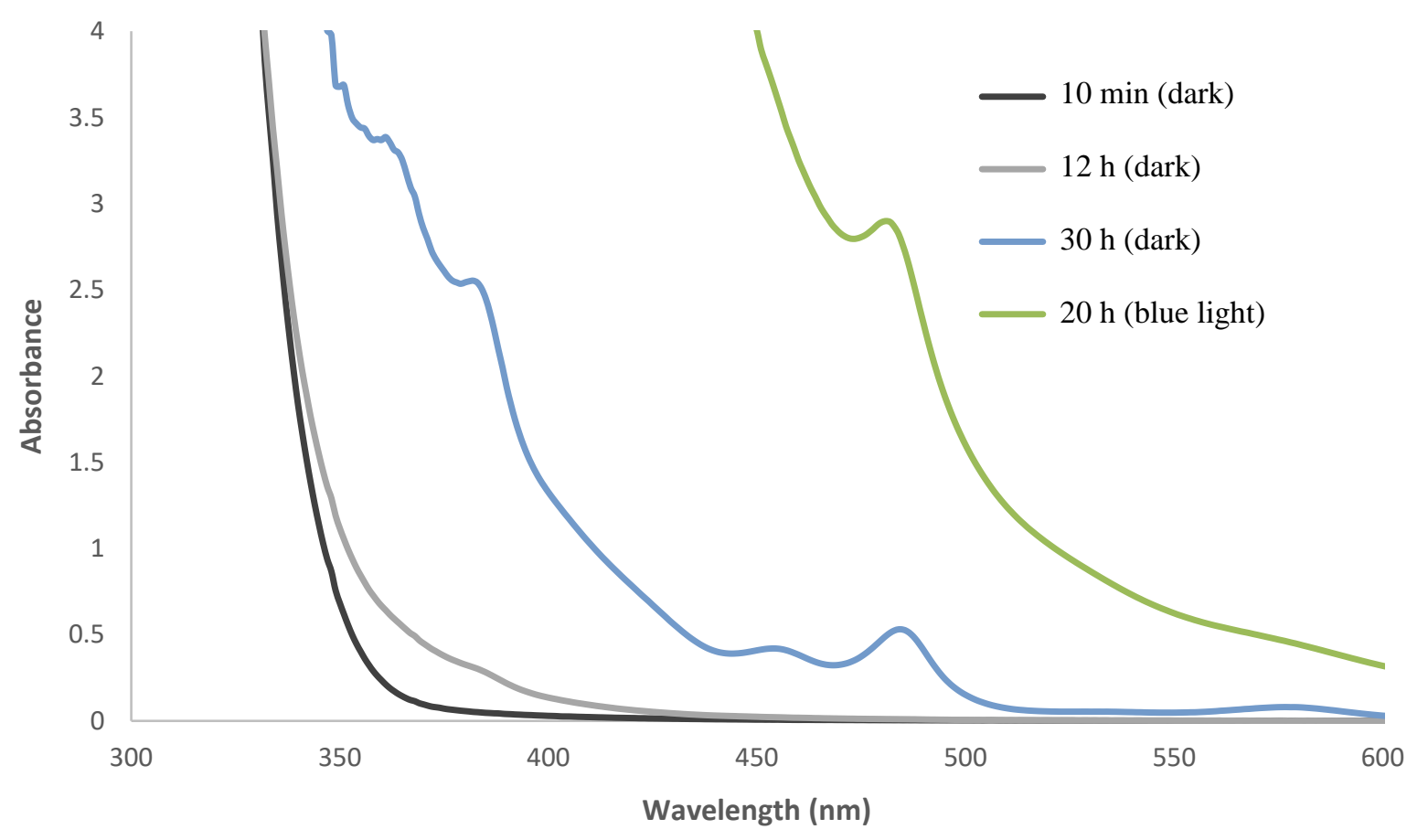

Figure S4. UV/Vis absorption spectra of a mixture of $1 \mathbf{a}(0.05 \mathrm{M})$ and $\mathrm{B}_{2}(\mathrm{cat})_{2}(0.06 \mathrm{M})$ in DMAc after extended mixing periods in the dark, and after $20 \mathrm{~h}$ irradiation with blue LEDs. 


\subsection{Quantum Yield}

The measurement of the quantum yield was complicated by the formation of a blue-light absorbing byproduct (see Figures S3 and S4), which caused the light absorbed by the reaction mixture to vary with time. However, as the absorbance of the reaction mixture at $450 \mathrm{~nm}\left(\lambda_{\max }\right.$ of the LEDs used for the measurement) only varied from 0.0074 to 0.011 for the first $3 \mathrm{~h}$ of the reaction when using low power LEDs, this enabled us to determine an approximate value for the quantum yield.

The quantum yield was measured for the reaction of $N$-alkoxyphthalimide $1 \mathrm{a}$ with $\mathrm{B}_{2}(\mathrm{cat})_{2}$, generating boronic ester 2 . The reaction was performed in a quartz cuvette (path length: $1=1.0 \mathrm{~cm}$ ) positioned 5 $\mathrm{cm}$ away from a single $0.1 \mathrm{~W}$ blue LED $\left(\lambda_{\max }=450 \mathrm{~nm}\right)$.
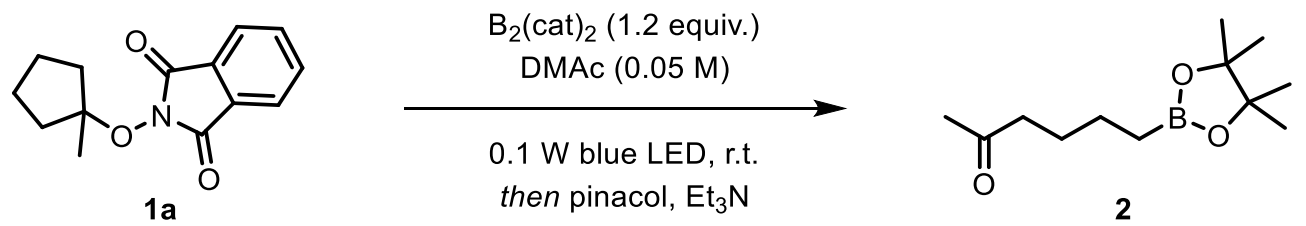

To a quartz cuvette (path length, $1=1 \mathrm{~cm}$ ) equipped with a magnetic stir bar was added $1 \mathbf{a}(24.5 \mathrm{mg}$, 0.10 mmol, 1.0 equiv.) and $\mathrm{B}_{2}(\mathrm{cat})_{2}\left(28.5 \mathrm{mg}, 0.12 \mathrm{mmol}, 1.2\right.$ equiv.). The cuvette was purged with $\mathrm{N}_{2}$ before being sealed with a septum. Dry DMAc $(0.05 \mathrm{M}, 2 \mathrm{~mL})$ was added and the headspace of the cuvette was purged with a gentle stream of $\mathrm{N}_{2}$ for approximately $10 \mathrm{~min}$. The cuvette was tightly sealed and positioned $5 \mathrm{~cm}$ away from a single $0.1 \mathrm{~W}$ blue LED and the reaction was stirred and irradiated for $3 \mathrm{~h}$. Irradiation was stopped and a solution of pinacol (3.0 equiv.) in $\mathrm{Et}_{3} \mathrm{~N}$ (20.0 equiv.) was added to the reaction mixture, which was stirred at room temperature for a further $1 \mathrm{~h}$. The reaction was diluted with DCM $(30 \mathrm{~mL})$ and washed with water $(3 \times 30 \mathrm{~mL})$ and brine $(20 \mathrm{~mL})$, dried over $\mathrm{MgSO}_{4}$, filtered, and concentrated under reduced pressure. The yield was determined by ${ }^{1} \mathrm{H}$ NMR using diethyl phthalate as an internal standard to be $16 \%$.

In parallel, a reaction was run in the dark to obtain the yield of the thermal background reaction, which found to be $11 \%$. Therefore, the photochemical yield after $3 \mathrm{~h}$ was $5 \%\left(5.0 \times 10^{-6} \mathrm{~mol}\right)$.

The quantum yield $(\Phi)$ was then calculated using:

$$
\Phi=\frac{\text { moles of product }}{\text { photon flux } \bullet \mathrm{t} \cdot \mathrm{f}}
$$

Where $t$ is the time (10800 s) and $\mathrm{f}$ is the fraction of light absorbed by the reaction mixture at $\lambda=450$ $\mathrm{nm}$, where $\mathrm{f}=1-10^{-\mathrm{A}}$ (the absorbance of the reaction mixture (A) was determined by UV/Vis 
spectroscopy to be 0.011 , thus $\mathrm{f}=0.025$ ). The photon flux of the LED setup was previously determined using ferrioxalate actinometry to be $4.31 \times 10^{-10}$ einstein $\mathrm{s}^{-1} .^{15}$

$$
\Phi=\frac{5.0 \times 10^{-6}}{4.31 \times 10^{-10} \cdot 10800 \cdot 0.025}=43.0
$$

As measured quantum yield after $3 \mathrm{~h}$ of irradiation is $>1$, this suggests that the fragmentation borylation reaction of $1 \mathbf{a}$ with $\mathrm{B}_{2}(\mathrm{cat})_{2}$ proceeds through a radical chain mechanism. 


\section{Synthetic Utility}

\subsection{Gram-Scale Reaction}

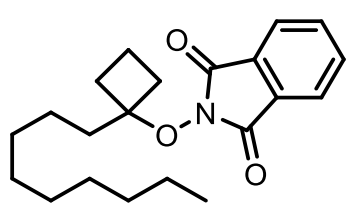

$6 a$

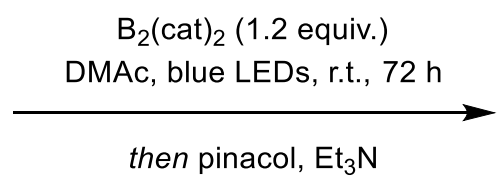

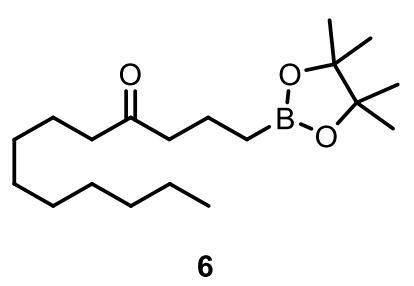

$68 \%(2.39 \mathrm{mmol})$

According to a modified GP3, a solution of $N$-alkoxyphthalimide $6 \mathbf{a}(1.2 \mathrm{~g}, 3.5 \mathrm{mmol}, 1.0$ equiv.) and $\mathrm{B}_{2}(\mathrm{cat})_{2}(1.0 \mathrm{~g}, 4.2 \mathrm{mmol}, 1.2$ equiv.) in dry DMAc $(60 \mathrm{~mL})$ in a $100 \mathrm{~mL}$ Schlenk flask was stirred under blue LED irradiation for 3 days. Irradiation was stopped and a solution of pinacol (1.24 g, $10.5 \mathrm{mmol}$, 3.0 equiv.) in $\mathrm{Et}_{3} \mathrm{~N}$ (7.0 mL, $50 \mathrm{mmol}, 14$ equiv.) was added to the reaction mixture, which was stirred at room temperature for a further $1 \mathrm{~h}$. The reaction mixture was diluted with DCM $(100 \mathrm{~mL})$ and washed with water $(3 \times 60 \mathrm{~mL})$ and brine $(30 \mathrm{~mL})$, dried over $\mathrm{MgSO}_{4}$, filtered, and concentrated under reduced pressure. The crude product was purified by column chromatography ( $20 \%$ EtOAc/hexane) to give $\mathbf{6}$ (776 mg, $2.39 \mathrm{mmol}, 68 \%$ ) as a pale-yellow oil. 


\subsection{Synthetic Transformations}

(4-Oxotridecyl)boronic acid (6b):

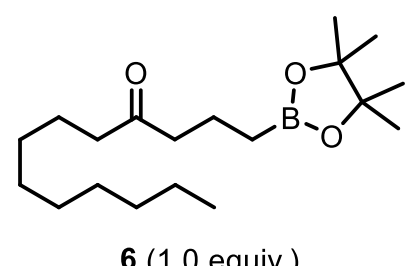

6 (1.0 equiv.)

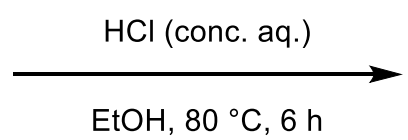

$80^{\circ} \mathrm{C}, 6 \mathrm{~h}$

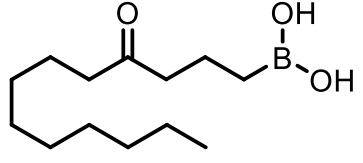

6b, $70 \%$

Prepared following a modified literature procedure: ${ }^{16}$ Compound 6 (65 mg, $\left.0.20 \mathrm{mmol}\right)$ was dissolved in EtOH $(1.0 \mathrm{~mL})$, and concentrated aqueous hydrochloric acid $(0.5 \mathrm{~mL})$ was added. The mixture was stirred for $5 \mathrm{~min}$ at room temperature. The reaction mixture was then heated at $80{ }^{\circ} \mathrm{C}$ for $8 \mathrm{~h}$ before allowing to cool to room temperature and concentrating under reduced pressure. The resulting crude product was purified by column chromatography (50\% EtOAc/hexane) to give $\mathbf{6 b}$ ( $34 \mathrm{mg}, 0.14 \mathrm{mmol}$, $70 \%)$ as a colourless oil.

TLC: $\mathrm{R}_{\mathrm{f}}=0.28\left(50 \% \mathrm{EtOAc} / \mathrm{hexane}, \mathrm{KMnO}_{4}\right.$ stain $)$

${ }^{1} \mathbf{H}$ NMR $\left(400 \mathrm{MHz}, \mathrm{CDCl}_{3}\right): \delta_{\mathrm{H}} 4.76(\mathrm{~s}, 2 \mathrm{H}), 2.49(\mathrm{t}, J=6.7 \mathrm{~Hz}, 2 \mathrm{H}), 2.40(\mathrm{t}, J=7.5 \mathrm{~Hz}, 2 \mathrm{H}), 1.74-$ $1.65(\mathrm{~m}, 2 \mathrm{H}), 1.61-1.50(\mathrm{~m}, 2 \mathrm{H}), 1.34-1.21(\mathrm{~m}, 12 \mathrm{H}), 0.88(\mathrm{t}, J=6.7 \mathrm{~Hz}, 3 \mathrm{H}), 0.81(\mathrm{t}, J=7.5 \mathrm{~Hz}$, 2H) ppm. See spectrum.

${ }^{13}$ C NMR (101 MHz, $\mathrm{CDCl}_{3}$ ): $\delta_{\mathrm{C}} 213.2,44.7,43.0,31.9,29.4,29.4,29.3,29.2,23.9,22.7,18.0,14.1$. ppm. The carbon attached to boron could not be observed due to quadrupolar relaxation. See spectrum.

${ }^{11}$ B NMR $\left(128 \mathrm{MHz}, \mathrm{CDCl}_{3}\right): \delta_{\mathrm{B}} 32.8$. See spectrum.

IR (film) $v_{\max }$ : 3393(br), 2987, 2919, 1702(s), 1378, 1264, 1066, 892, 734, $703 \mathrm{~cm}^{-1}$.

HRMS (ESI) m/z: [M + Na] ${ }^{+}$Calcd for $\mathrm{C}_{13} \mathrm{H}_{27} \mathrm{BNaO}_{3}$ 265.1948; Found 265.1945.

\section{1-Fluorotridecan-4-one (6c):}

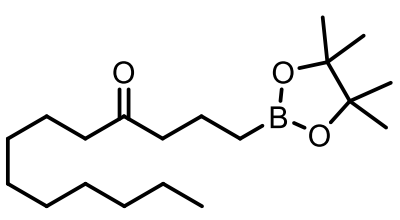

6 (1.0 equiv.)

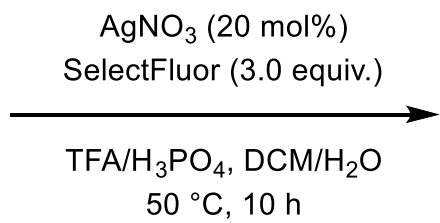

$50{ }^{\circ} \mathrm{C}, 10 \mathrm{~h}$

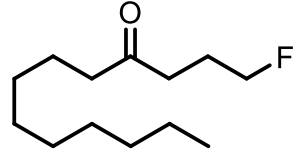

6c, $71 \%$

Prepared following a modified literature procedure: ${ }^{17}$ To a Schenk flask was added boronic ester 6 (65 $\mathrm{mg}, 0.20 \mathrm{mmol}, 1.0$ equiv.), $\mathrm{AgNO}_{3}(6.8 \mathrm{mg}, 0.040 \mathrm{mmol}, 20 \mathrm{~mol} \%)$ and Selectfluor (212 mg, 0.600 mmol, 3.0 equiv.). The reaction vessel was evacuated and filled with $\mathrm{N}_{2}$ three times before dry 
dichloromethane $(0.6 \mathrm{~mL})$, TFA $(0.4 \mathrm{~mL}), \mathrm{H}_{3} \mathrm{PO}_{4}(0.1 \mathrm{~mL})$ and water $(0.9 \mathrm{~mL})$ were added successively at room temperature. The reaction mixture was then heated to $50{ }^{\circ} \mathrm{C}$ and stirred for $10 \mathrm{~h}$. The reaction was allowed to cool to room temperature and extracted with DCM $(3 \times 30 \mathrm{~mL})$. The combined organic phases were dried over $\mathrm{MgSO}_{4}$, filtered, and concentrated under reduced pressure. Purification by column chromatography (5\% EtOAc/hexane) gave $\mathbf{6 c}(31 \mathrm{mg}, 0.14 \mathrm{mmol}, 71 \%)$ as a colourless oil.

TLC: $\mathrm{R}_{\mathrm{f}}=0.43\left(10 \% \mathrm{EtOAc} / \mathrm{hexane}, \mathrm{KMnO}_{4}\right.$ stain $)$.

${ }^{1} \mathbf{H}$ NMR $\left(400 \mathrm{MHz}, \mathrm{CDCl}_{3}\right): \delta_{\mathrm{H}} 4.45(\mathrm{dt}, J=47.3,5.8 \mathrm{~Hz}, 2 \mathrm{H}), 2.56(\mathrm{t}, J=7.2 \mathrm{~Hz}, 2 \mathrm{H}), 2.41(\mathrm{t}, J=7.5$ $\mathrm{Hz}, 2 \mathrm{H}), 2.05-1.87(\mathrm{~m}, 2 \mathrm{H}), 1.62-1.50(\mathrm{~m}, 2 \mathrm{H}), 1.34-1.20(\mathrm{~m}, 12 \mathrm{H}), 0.88(\mathrm{t}, J=6.7 \mathrm{~Hz}, 3 \mathrm{H}) \mathrm{ppm}$. See spectrum.

${ }^{13} \mathrm{C}$ NMR (101 MHz, $\left.\mathrm{CDCl}_{3}\right): \delta_{\mathrm{C}} 210.2,83.3(\mathrm{~d}, J=164.5 \mathrm{~Hz}), 43.0,37.9(\mathrm{~d}, J=4.2 \mathrm{~Hz}), 31.9,29.4$, 29.4, 29.3, 29.2, 24.5 (d, $J=20.0 \mathrm{~Hz}), 23.9,22.7,14.1 \mathrm{ppm}$. See spectrum.

${ }^{19}$ F NMR (377 MHz, CDCl3) $\delta-220.2(\mathrm{tt}, J=47.3,26.7 \mathrm{~Hz}) . \underline{\text { See spectrum. }}$

IR (film) $v_{\max }: 2987,2901,1712(\mathrm{~s}), 1381,1264,1066,905,726 \mathrm{~cm}^{-1}$.

HRMS (ESI) m/z: [M + Na $]^{+}$Calcd for $\mathrm{C}_{13} \mathrm{H}_{25} \mathrm{FNaO} 239.1782$; Found 239.1789.

\section{1-Phenyltridecan-4-one (6d):}
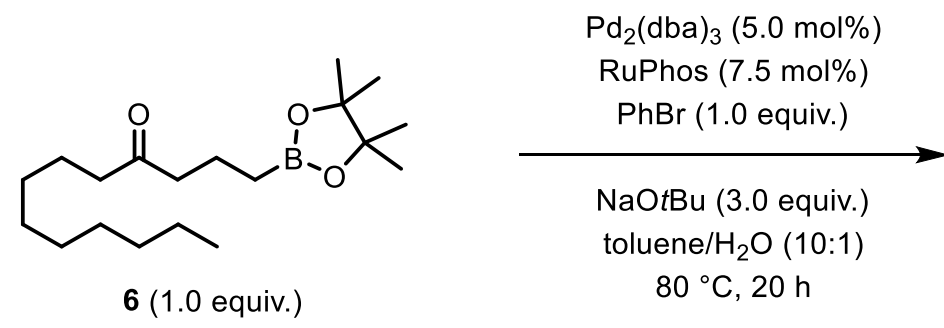

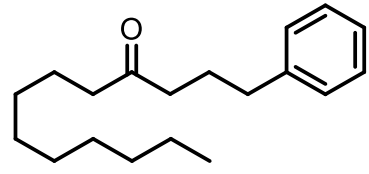

$6 d, 77 \%$

Prepared following a modified literature procedure: ${ }^{18} \mathrm{Pd}_{2}(\mathrm{dba})_{3}(9.2 \mathrm{mg}, 0.010 \mathrm{mmol}, 5 \mathrm{~mol} \%), \mathrm{NaO} t \mathrm{Bu}$ ( $86 \mathrm{mg}, 0.90 \mathrm{mmol}, 4.5$ equiv.), and RuPhos (7.0 mg, $0.015 \mathrm{mmol}, 7.5 \mathrm{~mol} \%$ ) were carefully weighed into a flame-dried, $\mathrm{N}_{2}$-flushed $7 \mathrm{~mL}$ vial containing a magnetic stirrer bar. Toluene $(2.0 \mathrm{~mL})$, bromobenzene ( $31 \mathrm{mg}, 0.20 \mathrm{mmol}, 1.0$ equiv.), boronic ester 6 (97 mg, $0.30 \mathrm{mmol}, 1.5$ equiv.), and water $(0.2 \mathrm{~mL})$ were added. The resulting mixture was heated to $80{ }^{\circ} \mathrm{C}$ and stirred for $24 \mathrm{~h}$. After allowing to cool to room temperature, the reaction was diluted with water and extracted with EtOAc (3 $\times 20 \mathrm{~mL})$. The combined organic extracts were washed with water $(20 \mathrm{~mL})$ and brine $(20 \mathrm{~mL})$, dried over $\mathrm{MgSO}_{4}$, filtered, and concentrated under reduced pressure. Purification by column chromatography (10\% EtOAc/hexane) gave $\mathbf{6 d}(42 \mathrm{mg}, 0.15 \mathrm{mmol}, 77 \%)$ as a colourless oil.

TLC: $\mathrm{R}_{\mathrm{f}}=0.57$ (5\% EtOAc/hexane, $\mathrm{KMnO}_{4}$ stain). 
${ }^{1} \mathbf{H}$ NMR (400 MHz, $\left.\mathrm{CDCl}_{3}\right): \delta_{\mathrm{H}} 7.32-7.24(\mathrm{~m}, 2 \mathrm{H}), 7.22-7.13(\mathrm{~m}, 3 \mathrm{H}), 2.62(\mathrm{t}, J=7.6 \mathrm{~Hz}, 2 \mathrm{H}), 2.40$ $(\mathrm{t}, J=7.4 \mathrm{~Hz}, 2 \mathrm{H}), 2.36(\mathrm{t}, J=7.5 \mathrm{~Hz}, 2 \mathrm{H}), 1.96-1.86(\mathrm{~m}, 2 \mathrm{H}), 1.59-1.50(\mathrm{~m}, 2 \mathrm{H}), 1.32-1.20(\mathrm{~m}$, $12 \mathrm{H}), 0.88(\mathrm{t}, J=6.9 \mathrm{~Hz}, 3 \mathrm{H}) \mathrm{ppm}$. See spectrum.

${ }^{13}$ C NMR $\left(101 \mathrm{MHz}, \mathrm{CDCl}_{3}\right): \delta_{\mathrm{C}} 211.2,141.7,128.5,128.4,125.9,42.9,41.9,35.1,31.9,29.4,29.4$, 29.3, 25.2, 23.9, 22.6, $14.1 \mathrm{ppm}$. See spectrum.

IR (film) $v_{\max }: 2987,2924,1712(\mathrm{~s}), 1603,1496,1453,1377,1264,1066,895,734,699 \mathrm{~cm}^{-1}$.

HRMS (ESI) m/z: [M + Na $]^{+}$Calcd for $\mathrm{C}_{19} \mathrm{H}_{30} \mathrm{NaO}[\mathrm{M}+\mathrm{Na}]^{+}$297.2189; Found 297.2192. 


\subsection{One-Pot Activation/Fragmentation Borylation of Cyclic Enol Ethers}
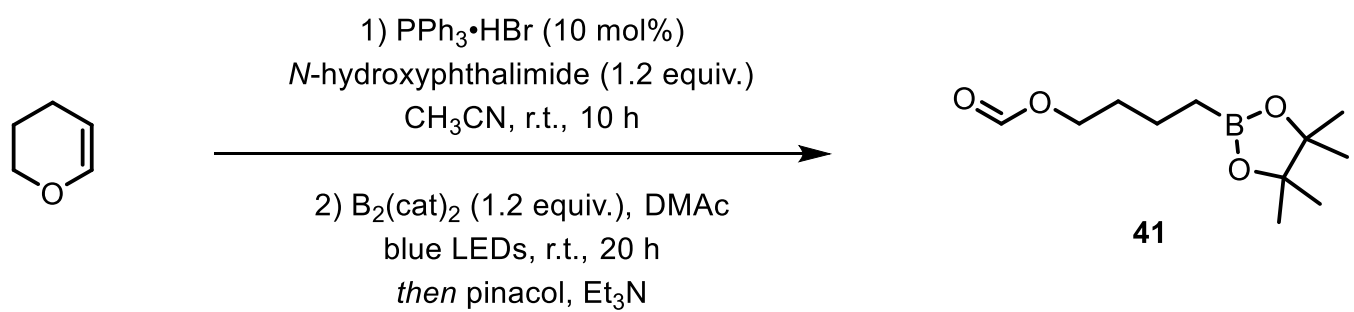

To a stirred solution of 3,4-dihydro- $2 \mathrm{H}$-pyran ( $84 \mathrm{mg}, 1.0 \mathrm{mmol}, 1.0$ equiv.) in acetonitrile $(10 \mathrm{~mL})$ at room temperature were added $N$-hydroxyphthalimide (196 mg, $1.20 \mathrm{mmol}, 1.20$ equiv.) and $\mathrm{Ph}_{3} \mathrm{P} \cdot \mathrm{HBr}$ (34 mg, $0.10 \mathrm{mmol}, 10 \mathrm{~mol} \%$ ). After stirring at room temperature for $10 \mathrm{~h}$, a solution of $\mathrm{B}_{2}(\mathrm{cat})_{2}(285$ $\mathrm{mg}, 1.20 \mathrm{mmol}, 1.20$ equiv.) in dry DMAc $(10 \mathrm{~mL})$ was added. The headspace of the vial was purged with a gentle stream of $\mathrm{N}_{2}$ for approximately $15 \mathrm{~min}$. The vial was tightly sealed and stirred under blue LED irradiation for $20 \mathrm{~h}$ (see Figure S1 for experimental setup). Irradiation was stopped and a solution of pinacol (0.35 g, $3.0 \mathrm{mmol}, 3.0$ equiv.) in $\mathrm{Et}_{3} \mathrm{~N}$ ( $2.0 \mathrm{~g}, 20.0 \mathrm{mmol}, 20.0$ equiv.) was added to the reaction mixture, which was stirred at room temperature for a further $1 \mathrm{~h}$. The reaction mixture was diluted with DCM $(50 \mathrm{~mL})$ and washed with water $(3 \times 50 \mathrm{~mL})$ and brine $(20 \mathrm{~mL})$, dried over $\mathrm{MgSO}_{4}$, filtered, and concentrated under reduced pressure. Purification by flash column chromatography (20\% EtOAc/hexane) gave 41 (100 mg, $0.44 \mathrm{mmol}, 44 \%)$ as a pale-yellow oil. 


\section{References}

${ }^{1}$ Krasovskiy, A.; Kopp, F.; Knochel, P. Angew. Chem., Int. Ed. 2006, 45, 497.

2 (a) Palandoken, H.; Bocian, C. M.; McCombs, M. R.; Nantz, M. H. Tetrahedron Lett. 2005, 46, 6667.

(b) Kang, T.; Kim, H.; Kim, J. G.; Chang, S. Chem. Commun. 2014, 50, 12073.

${ }^{3}$ Martin, N. I.; Woodward, J. J.; Marletta, M. A. Org. Lett. 2006, 8, 4035.

${ }^{4}$ Wu, X.; Cruz, F. A.; Lu, A.; Dong, V. M. J. Am. Chem. Soc. 2018, 140, 10126.

${ }^{5}$ Ren, Z.; Mo, F.; Dong, G. J. Am. Chem. Soc. 2012, 134, 16991.

${ }^{6}$ Ogawa, A.; Tanaka, M.; Sasaki, T.; Matsuda, A. J. Med. Chem. 1998, 41, 5094.

${ }^{7}$ Guo, Z.; Jin, C.; Zhou, J.; Su, W. RSC Adv. 2016, 6, 79016.

${ }^{8}$ Shi, J. L.; Wang, Z.; Zhang, R.; Wang, Y.; Wang, J. Chem. Eur. J. 2019, 25, 8992.

${ }^{9}$ Jiang, H.; Tang, X.; Liu, S.; Wang, L.; Shen, H.; Yang, J.; Wang, H.; Gui, Q.-W. Org. Biomol. Chem. 2019, 17, 10223.

${ }^{10}$ Fuwa, H.; Okamura, Y.; Natsugari, H. Tetrahedron 2004, 60, 5341.

${ }^{11}$ Cheng, Y.; Mück-Lichtenfeld, C.; Studer, A. Angew. Chem., Int. Ed. 2018, 57,16832.

${ }^{12}$ Yang, C.-T.; Zhang, Z.-Q.; Tajuddin, H.; Wu, C.-C.; Liang, J.; Liu, J.-H.; Fu, Y.; Czyzewska, M.;

Steel, P. G.; Marder, T. B.; Liu, L. Angew. Chem., Int. Ed. 2012, 51, 528.

${ }^{13}$ Zhang, L.; Zuo, Z.; Leng, X.; Huang, Z. Angew. Chem., Int. Ed. 2014, 53, 2696.

${ }^{14}$ Pereira, S.; Srebnik, M. J. Org. Chem. 1995, 60, 4316.

${ }^{15}$ Silvi, M.; Schrof, R.; Noble, A.; Aggarwal V. K. Chem. Eur. J. 2018, 24, 4279.

${ }^{16}$ Chen, I.-H.; Yin, L.; Itano, W.; Kanai, M.; Shibasaki, M. J. Am. Chem. Soc. 2009, 131, 11664.

${ }^{17}$ Li, Z.; Wang, Z.; Zhu, L.; Tan, X.; Li, C. J. Am. Chem. Soc. 2014, 136, 16439.

${ }^{18}$ Li, Q.; Liskey, C. W.; Hartwig, J. F. J. Am. Chem. Soc. 2014, 136, 8755. 


\section{NMR Spectra}

${ }^{1} \mathrm{H}$ NMR (400 MHz, $\mathrm{CDCl}_{3}$ ) of compound $1 \mathbf{a}$ (see procedure):

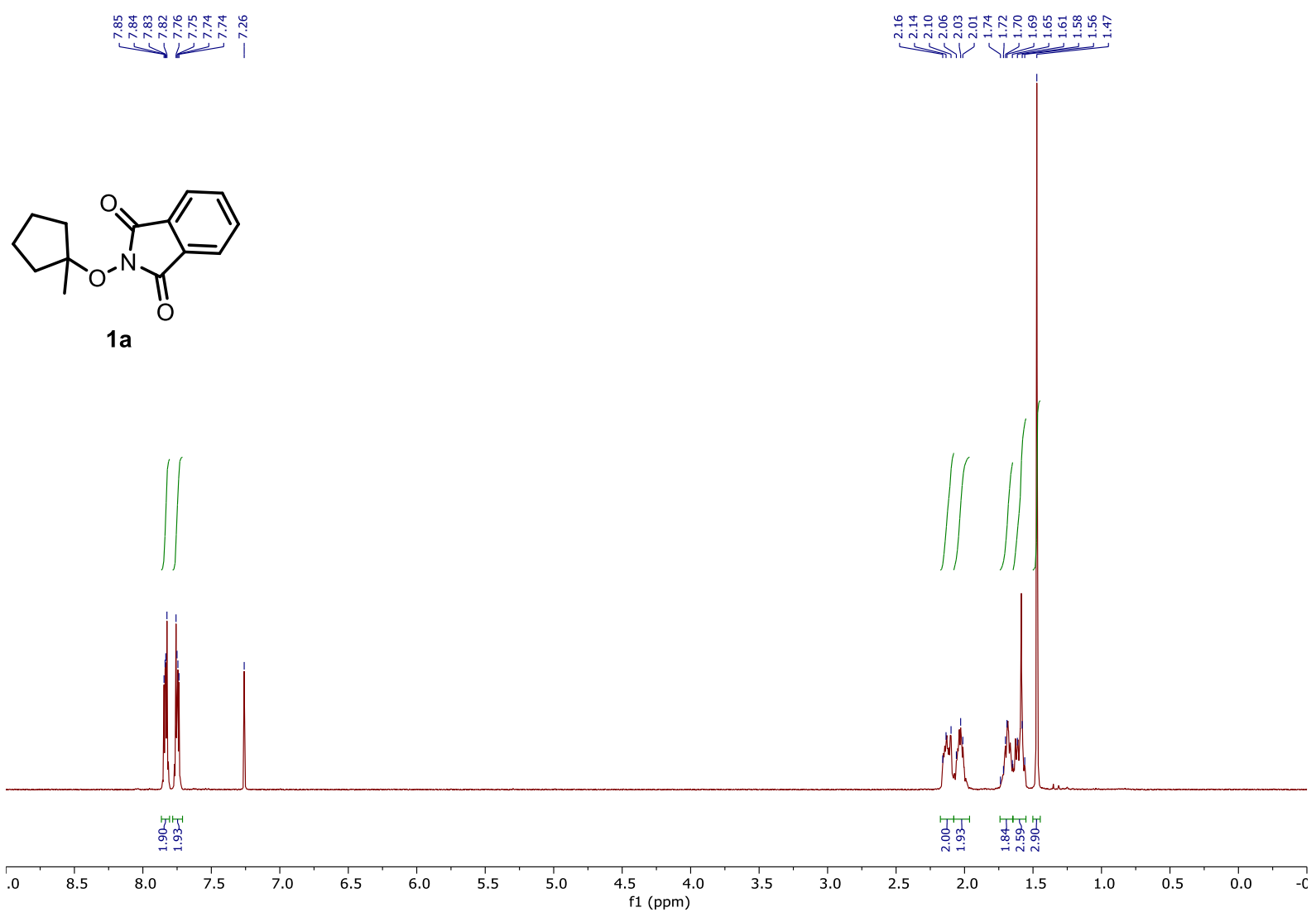

${ }^{13} \mathrm{C} \mathrm{NMR}\left(101 \mathrm{MHz}, \mathrm{CDCl}_{3}\right)$ of compound $\mathbf{1 a}($ see procedure)

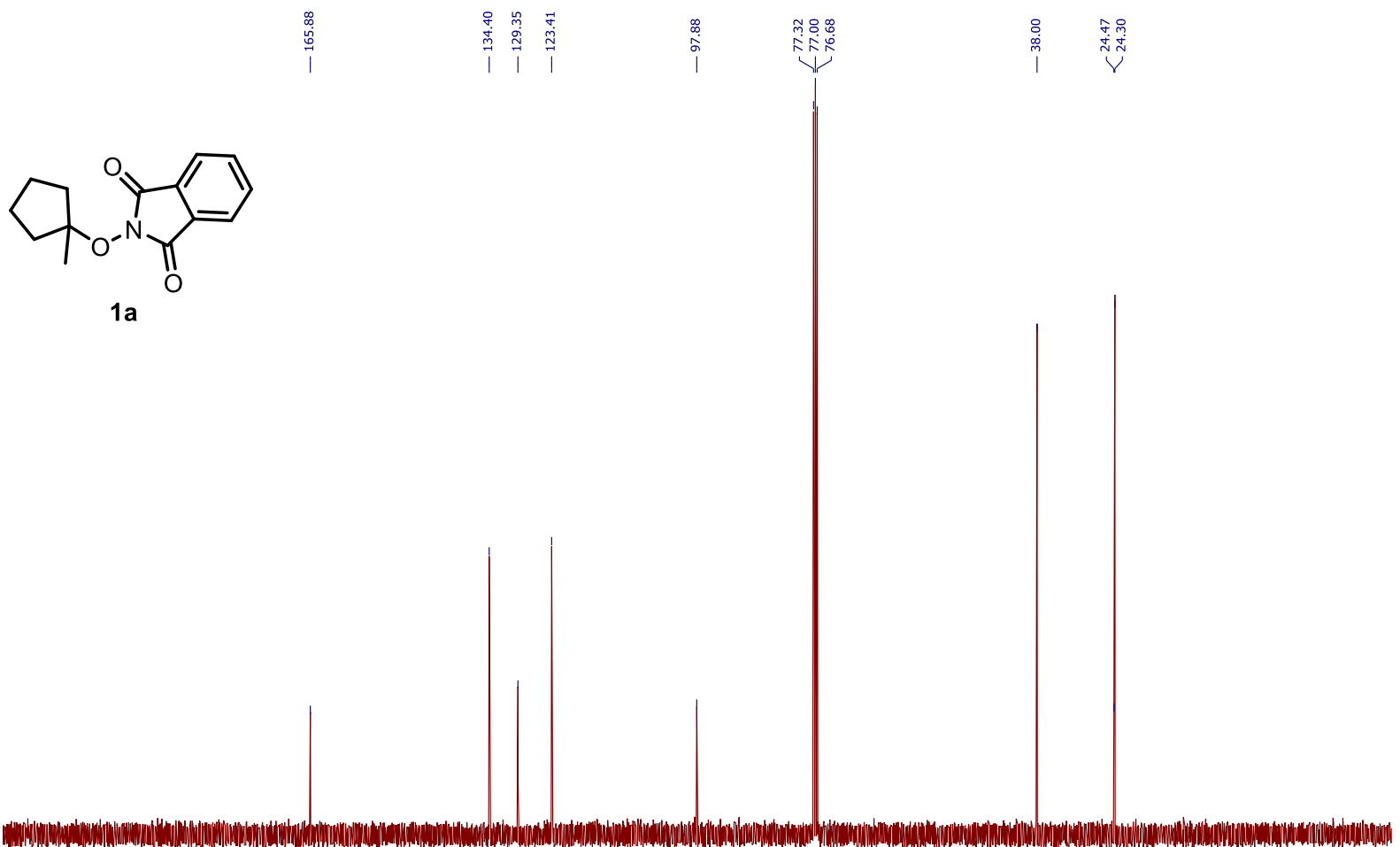

\begin{tabular}{lllllllllllllllllllllllllll}
\hline 20 & 210 & 200 & 190 & 180 & 170 & 160 & 150 & 140 & 130 & 120 & 110 & 100 & 90 & 80 & 70 & 60 & 50 & 40 & 30 & 20 & 10 & 0 & -10 & -20 \\
$f 1(\mathrm{ppm})$ &
\end{tabular} 
${ }^{1} \mathrm{H}$ NMR (400 MHz, $\mathrm{CDCl}_{3}$ ) of compound $\mathbf{1 b}$ (see procedure):

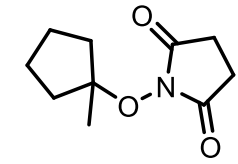

$1 \mathrm{~b}$

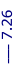

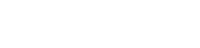

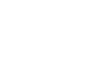

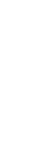

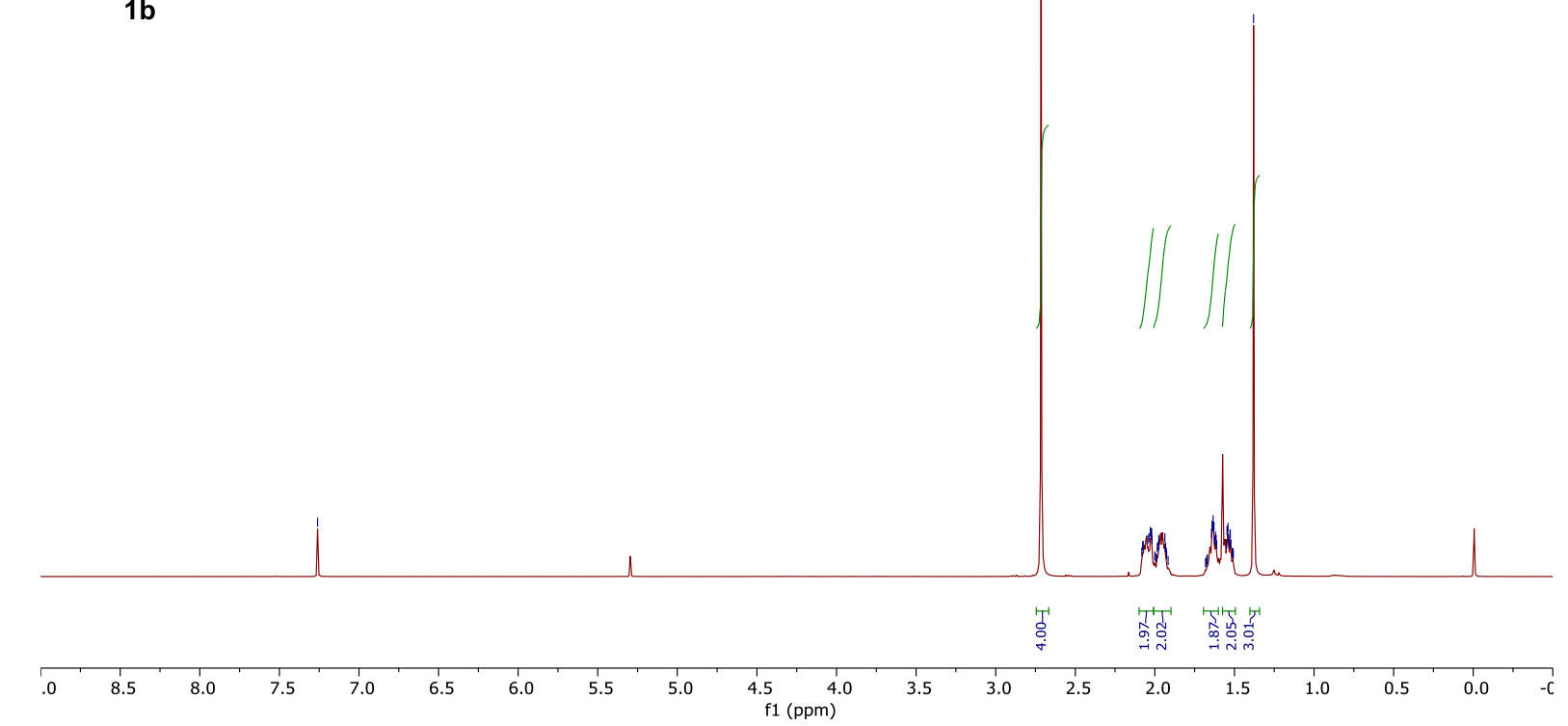

${ }^{13} \mathrm{C}$ NMR (101 MHz, $\mathrm{CDCl}_{3}$ ) of compound $\mathbf{1 b}$ (see procedure):

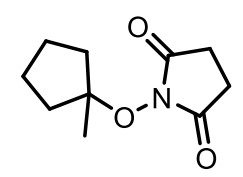

$1 \mathrm{~b}$

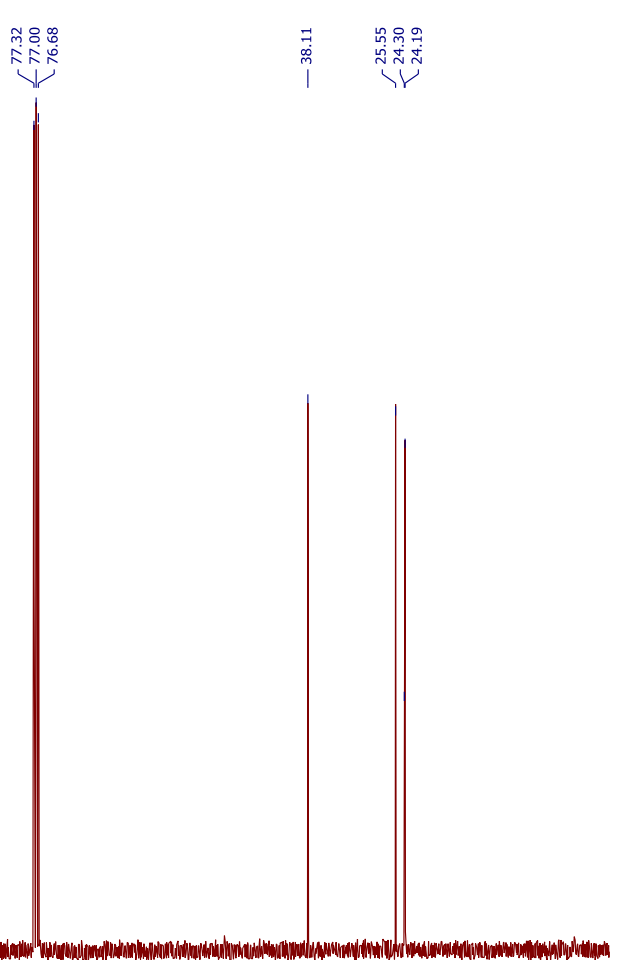


${ }^{1} \mathrm{H}$ NMR (400 MHz, $\mathrm{CDCl}_{3}$ ) of compound $1 \mathrm{c}$ (see procedure):<smiles>CC1(ON2C(=O)c3c(Cl)c(Cl)c(Cl)c(Cl)c3C2=O)CCCC1</smiles>

$1 \mathrm{c}$

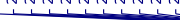

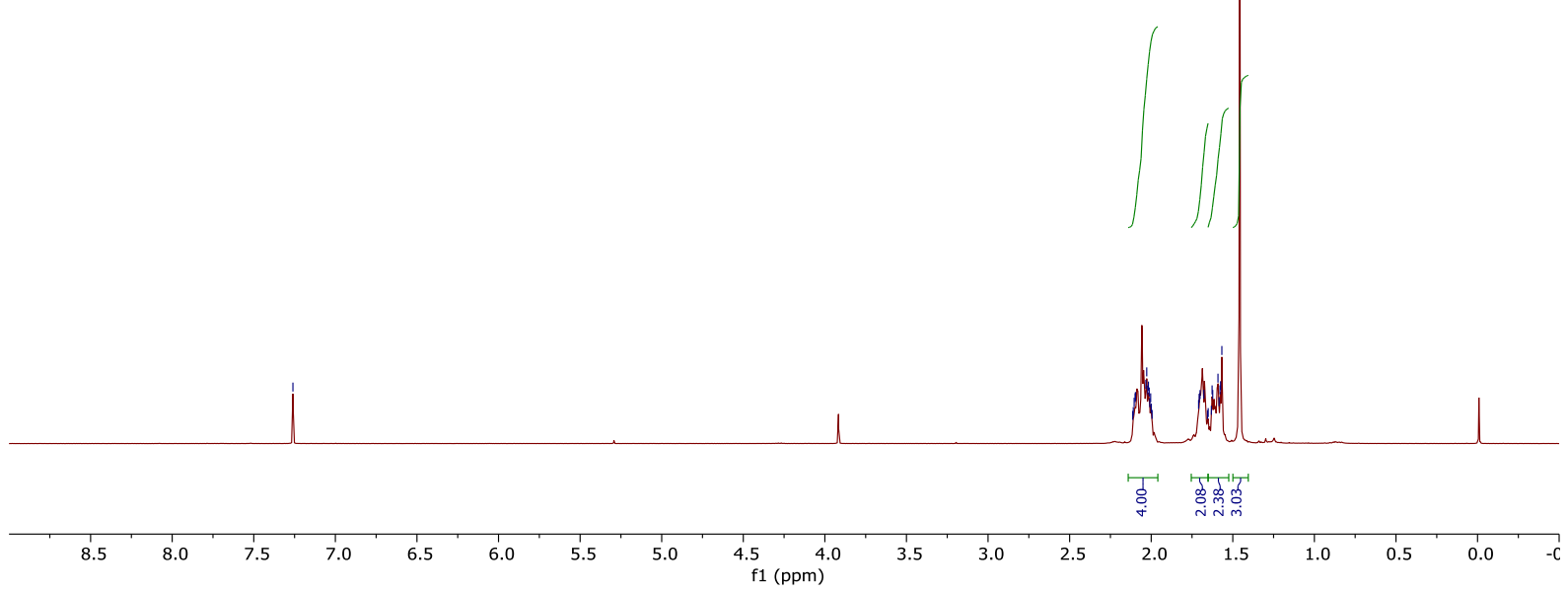

${ }^{13} \mathrm{C}$ NMR (101 MHz, $\mathrm{CDCl}_{3}$ ) of compound $1 \mathrm{c}$ (see procedure):<smiles>CC1(ON2C(=O)c3c(Cl)c(Cl)c(Cl)c(Cl)c3C2=O)CCCC1</smiles>

$1 \mathrm{c}$ 
${ }^{1} \mathrm{H}$ NMR (400 MHz, $\mathrm{CDCl}_{3}$ ) of compound 3a (see procedure):

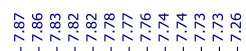

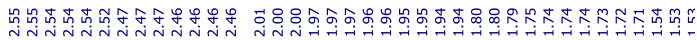

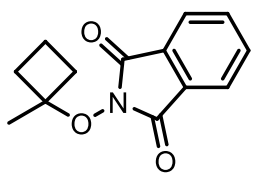

$3 a$
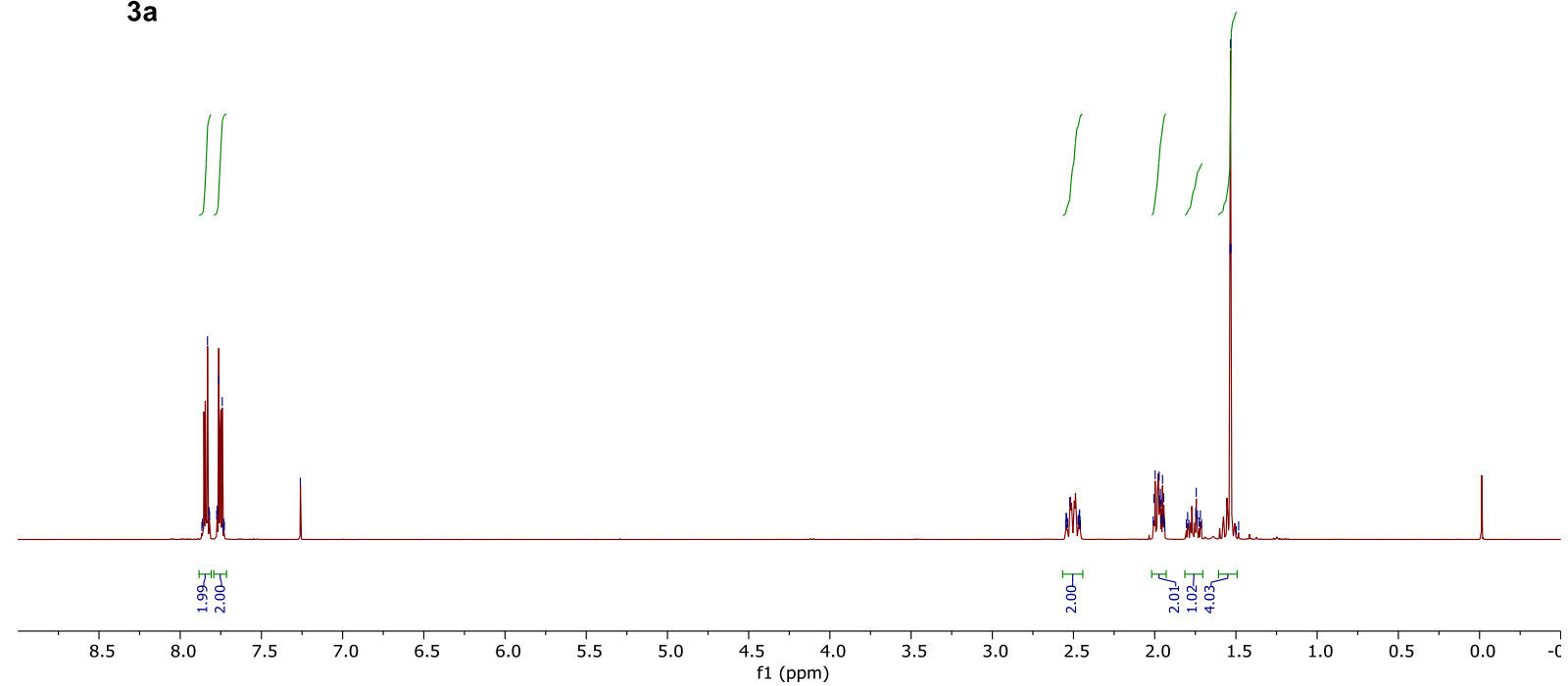

${ }^{13} \mathrm{C} \mathrm{NMR}$ (101 MHz, $\mathrm{CDCl}_{3}$ ) of compound $\mathbf{3 a}$ (see procedure):

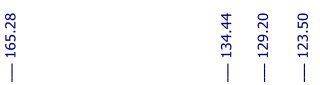

惮

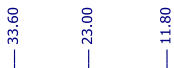

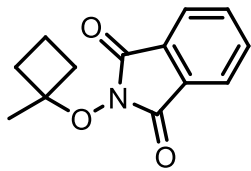

$3 a$

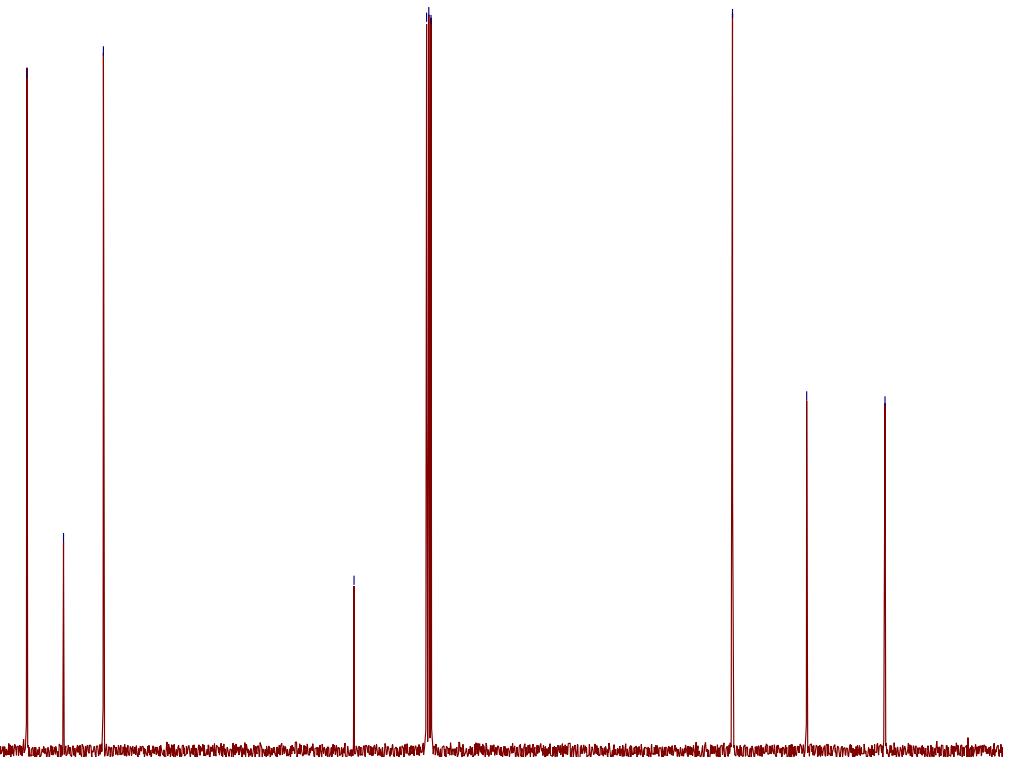
110100 
${ }^{1} \mathrm{H}$ NMR (400 MHz, $\mathrm{CDCl}_{3}$ ) of compound $4 \mathbf{a}$ (see procedure):

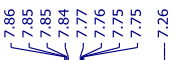

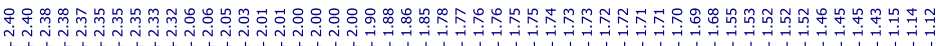

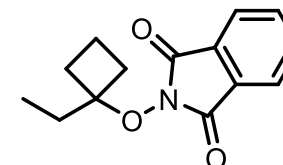

4a
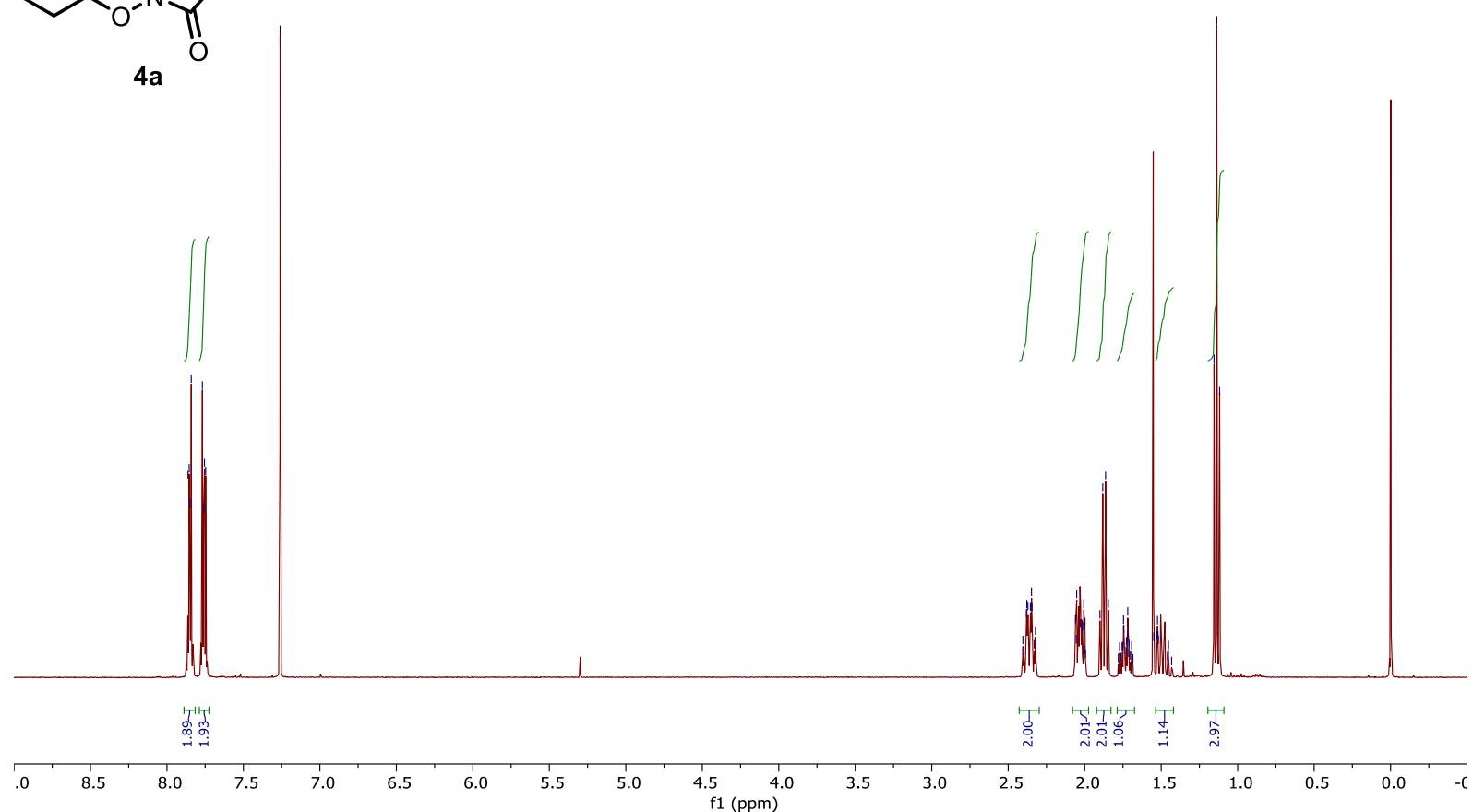

${ }^{13} \mathrm{C}$ NMR (101 MHz, $\left.\mathrm{CDCl}_{3}\right)$ of compound $\mathbf{4 a}$ (see procedure):<smiles>CCC1(ON2C(=O)c3ccccc3C2=O)CCC1</smiles>

4a
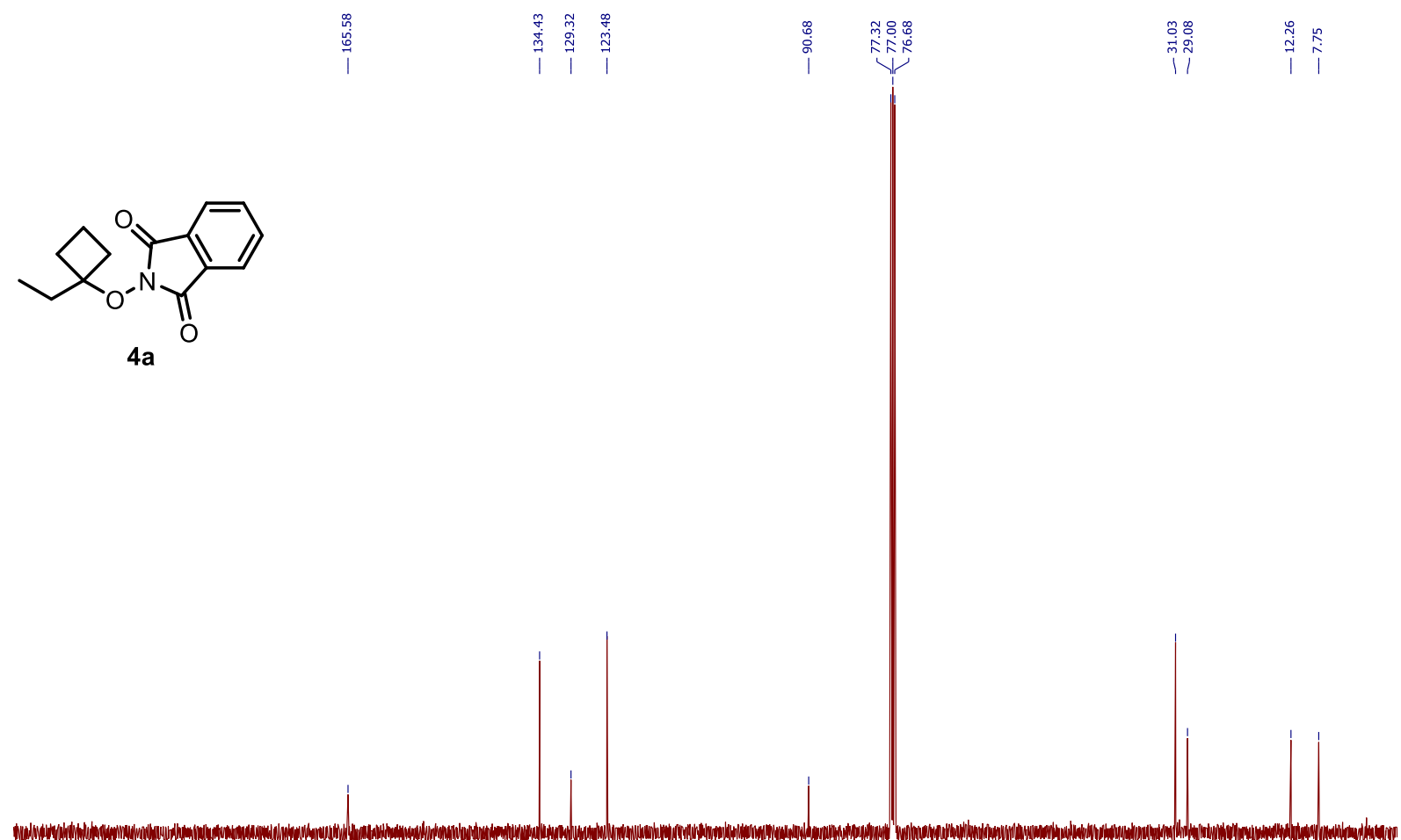

$\begin{array}{llllllllllll}20 & 210 & 200 & 190 & 180 & 170 & 160 & 150 & 140 & 130 & 120 & 110 \\ \mathrm{f} 1(\mathrm{ppm}) & 100\end{array}$ 
${ }^{1} \mathrm{H}$ NMR (400 MHz, $\mathrm{CDCl}_{3}$ ) of compound $\mathbf{5 a}$ (see procedure):

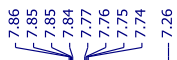

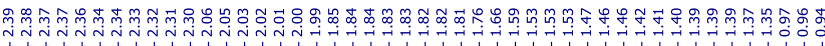

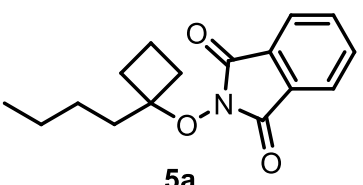

$5 a$

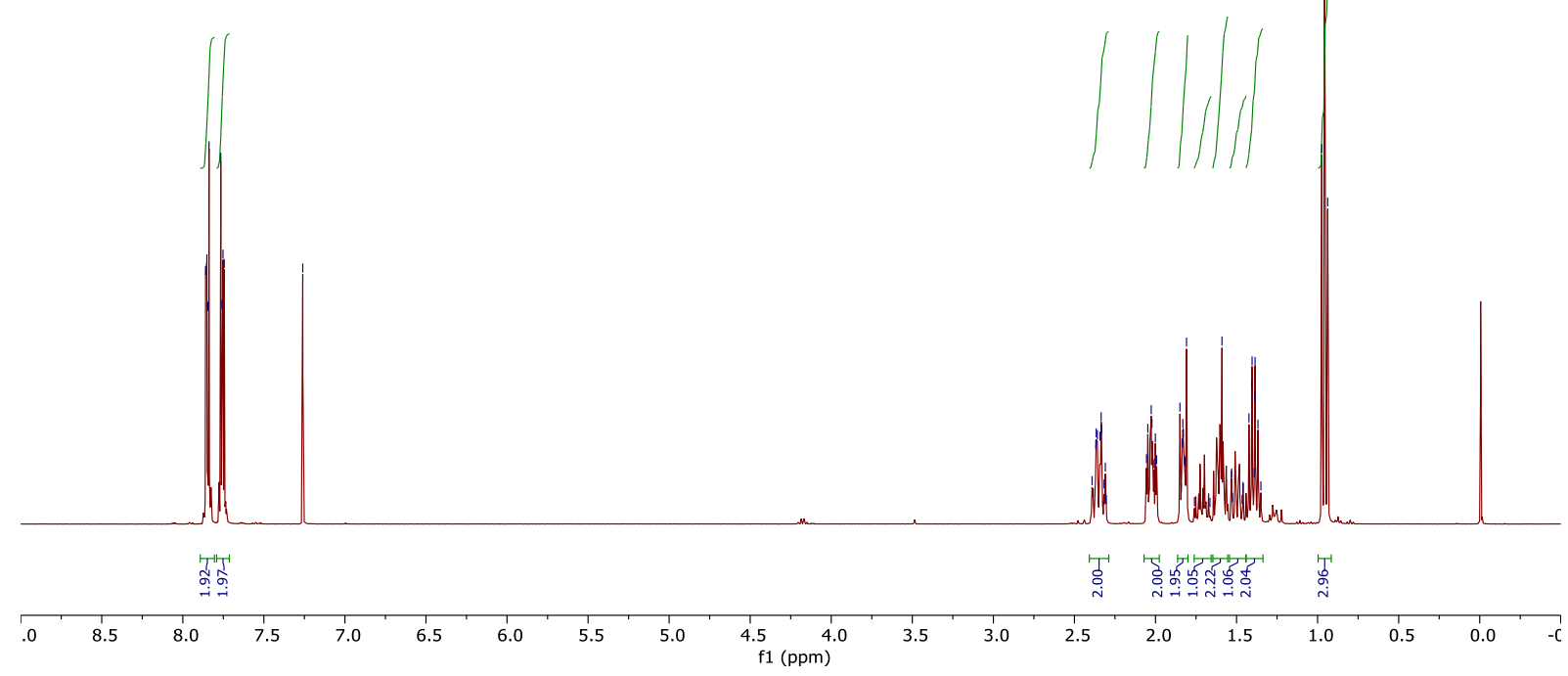

${ }^{13} \mathrm{C}$ NMR (101 MHz, $\mathrm{CDCl}_{3}$ ) of compound $5 \mathbf{a}$ (see procedure):

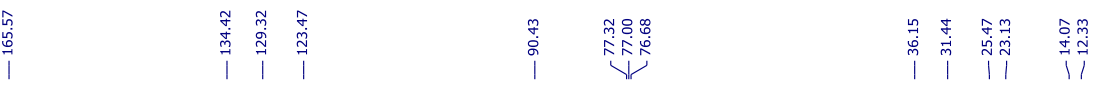<smiles>CCCCC1(ON2C(=O)c3ccccc3C2=O)CCC1</smiles>

$5 a$

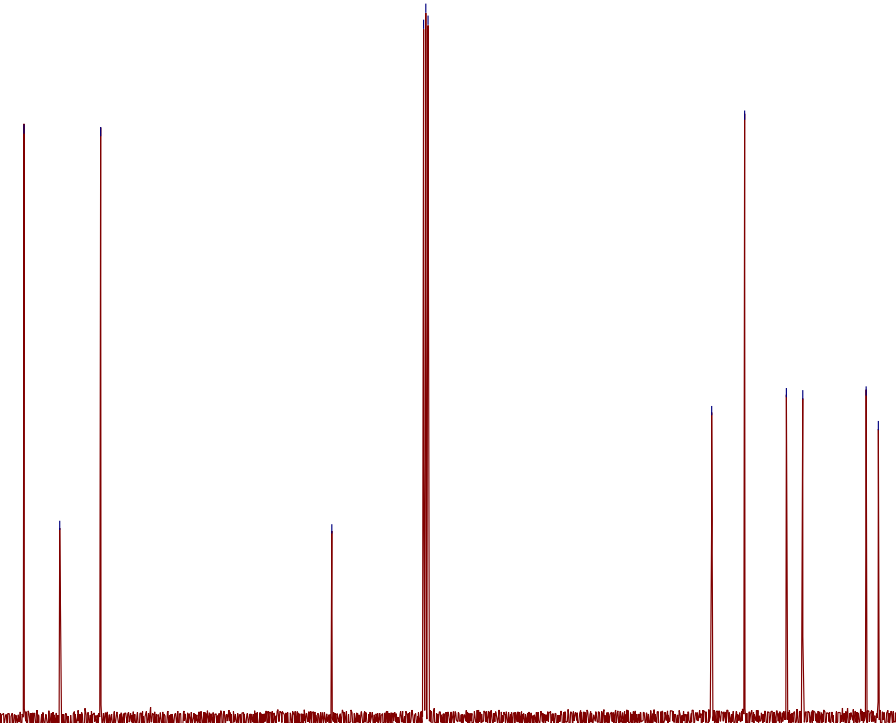

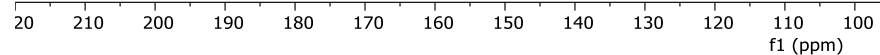

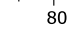

$70 \quad 60$

$\begin{array}{llllll}50 & 40 & 30 & 20 & 10 & 0\end{array}$ 
${ }^{1} \mathrm{H}$ NMR (400 MHz, $\mathrm{CDCl}_{3}$ ) of compound $6 \mathbf{a}$ (see procedure):
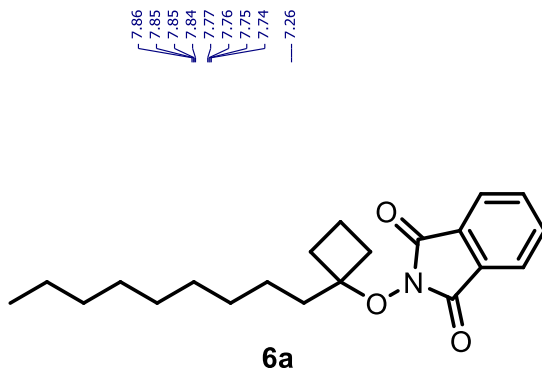

$6 a$
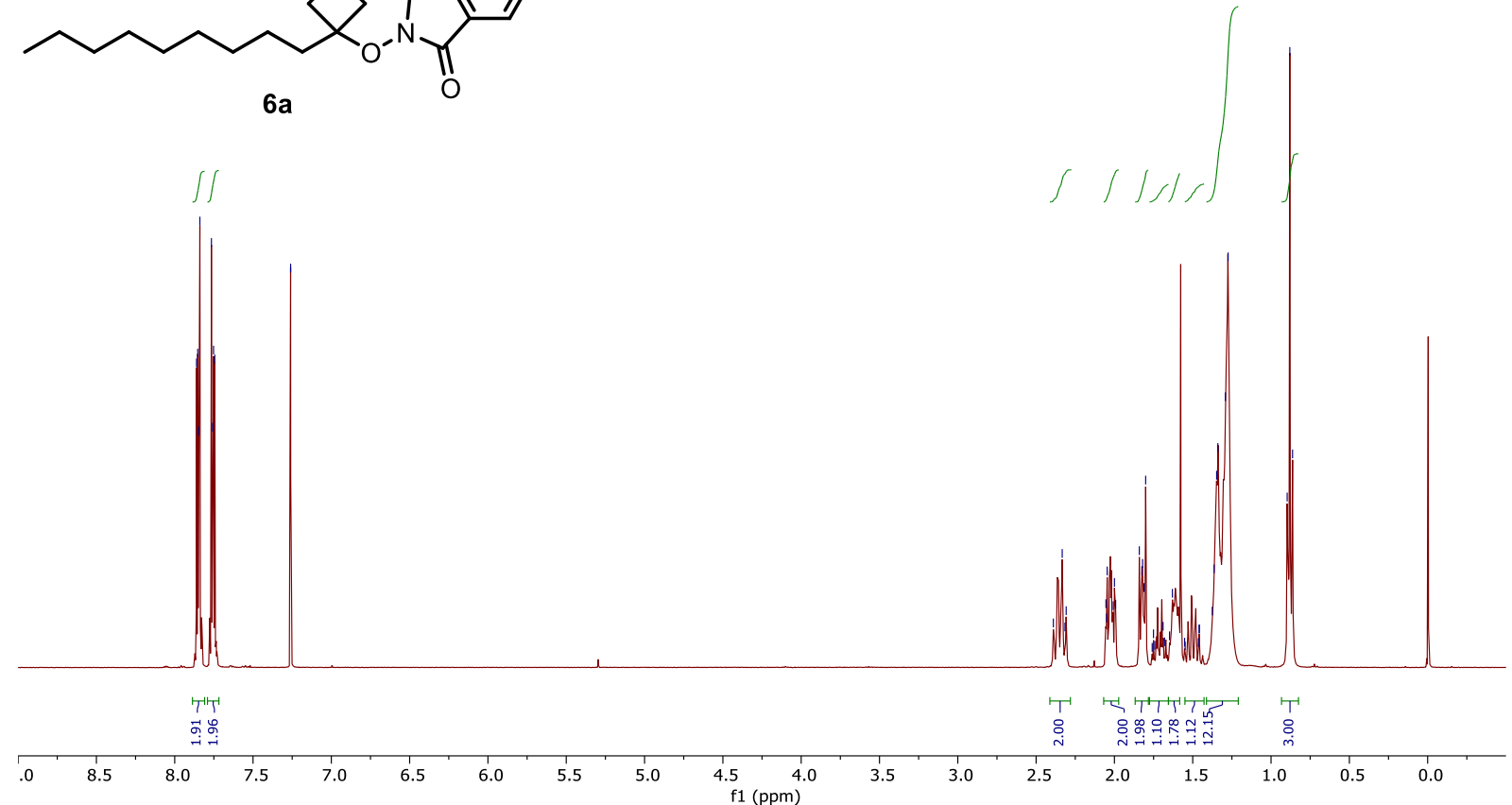

${ }^{13} \mathrm{C}$ NMR (101 MHz, $\mathrm{CDCl}_{3}$ ) of compound $6 \mathbf{a}$ (see procedure):

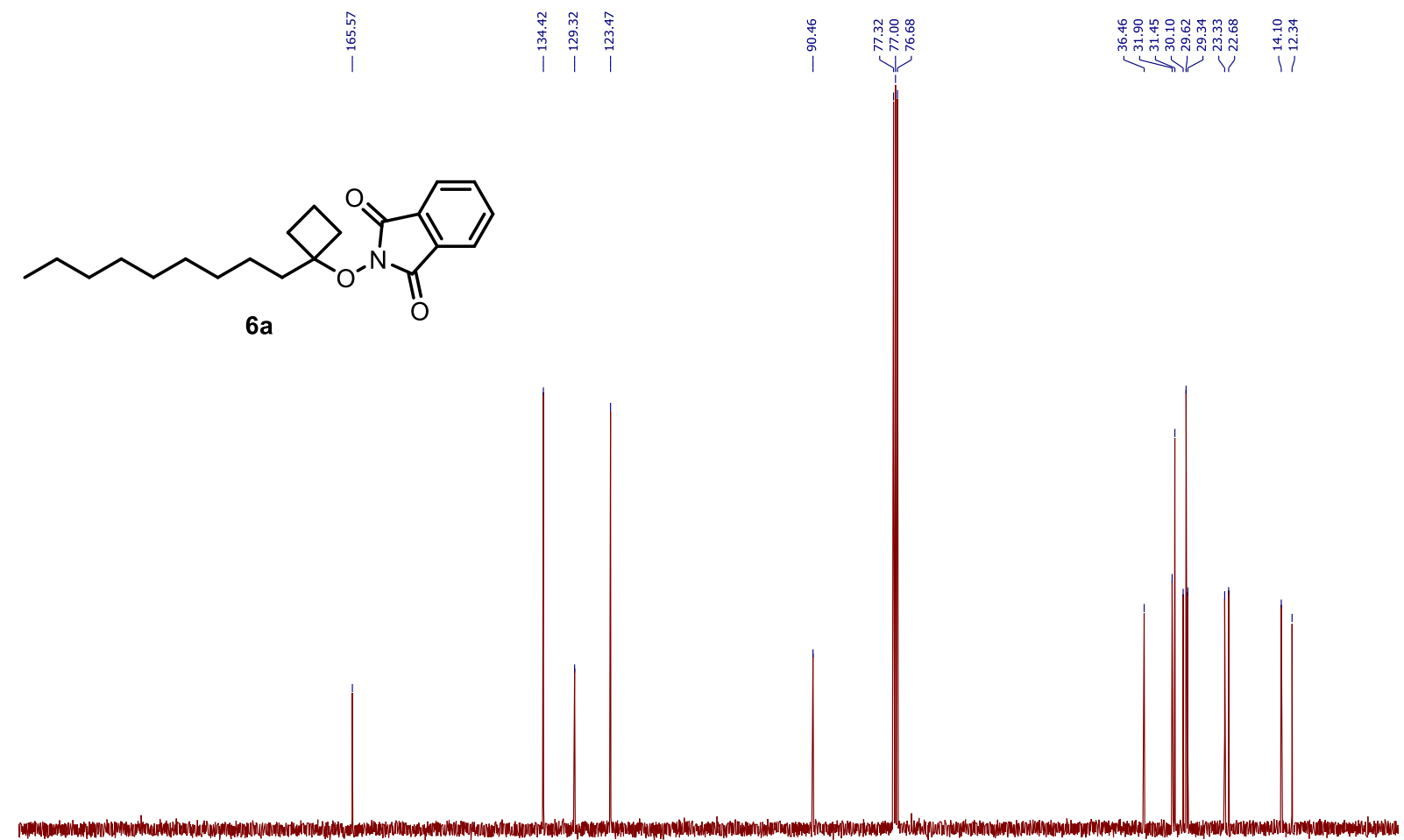

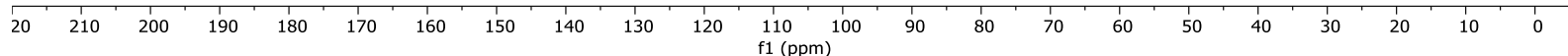


${ }^{1} \mathrm{H}$ NMR (400 MHz, $\mathrm{CDCl}_{3}$ ) of compound $7 \mathbf{a}$ (see procedure):

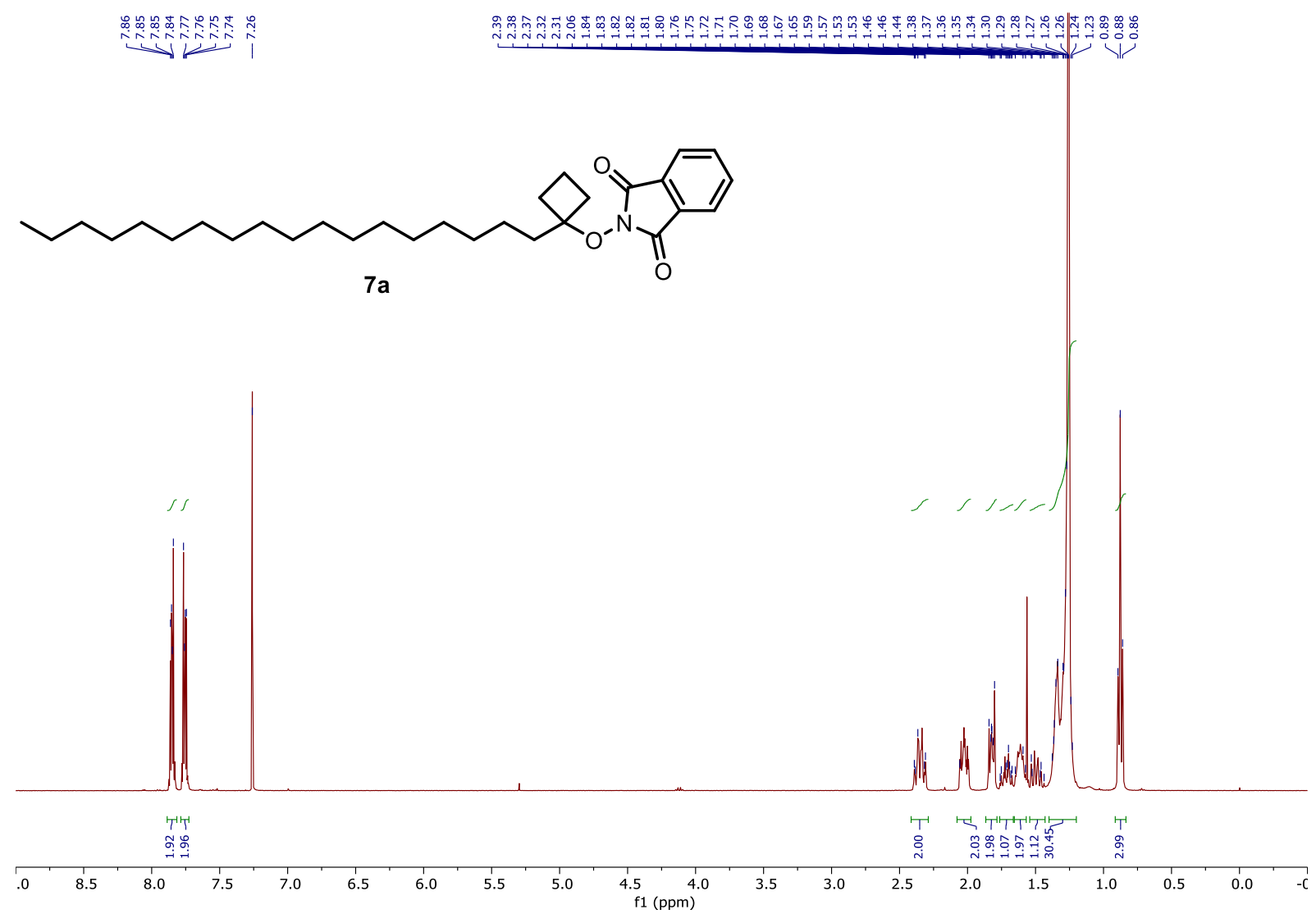

${ }^{13} \mathrm{C}$ NMR (101 MHz, $\left.\mathrm{CDCl}_{3}\right)$ of compound 7a (see procedure):

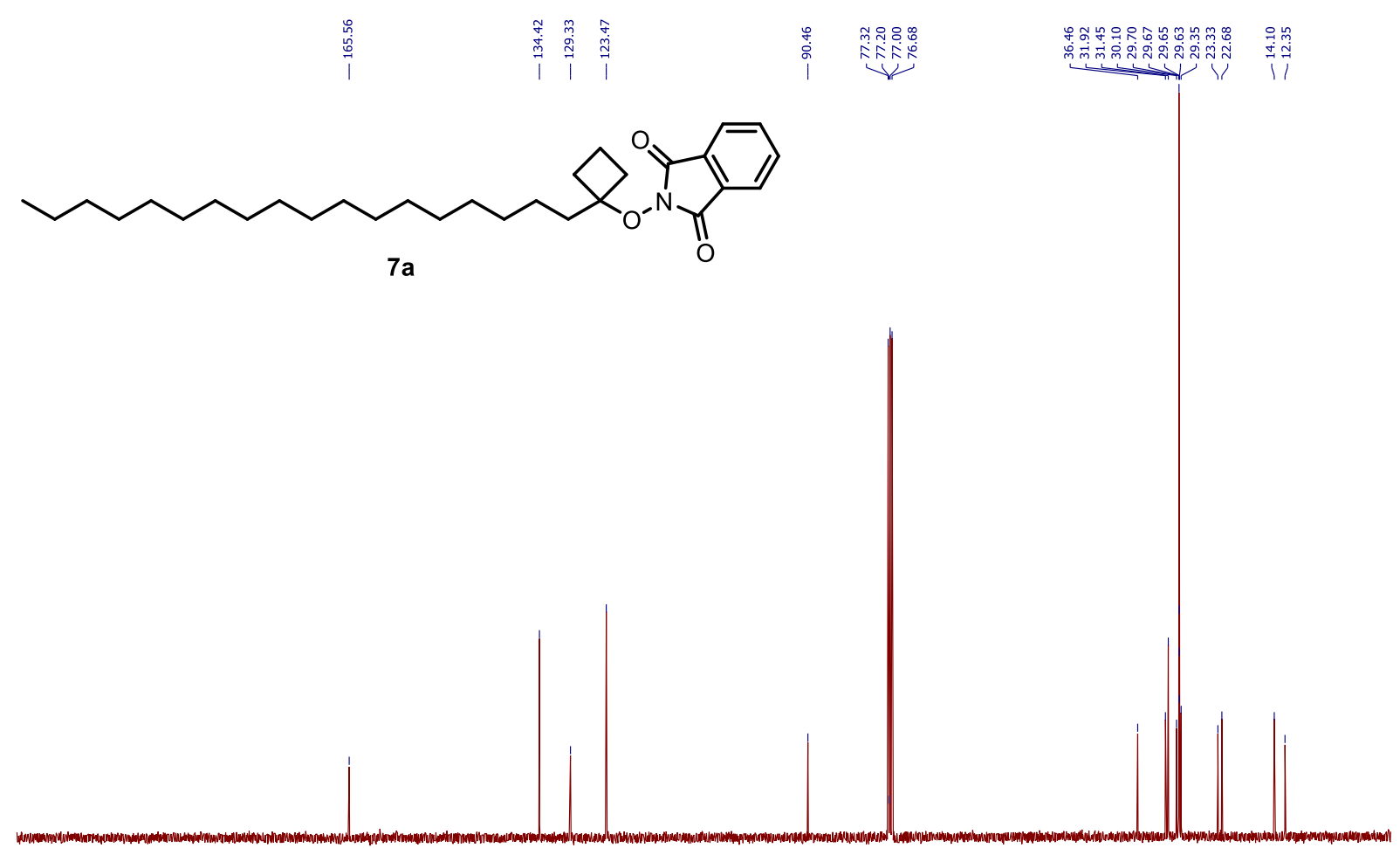

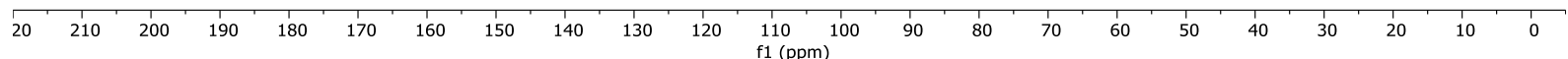


${ }^{1} \mathrm{H}$ NMR (400 MHz, $\mathrm{CDCl}_{3}$ ) of compound 8a (see procedure):

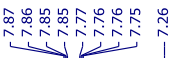

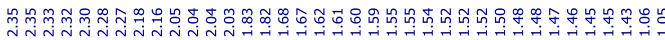

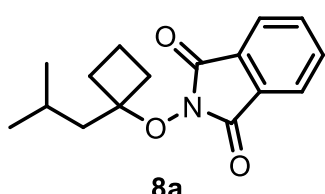

$8 \mathbf{a}$
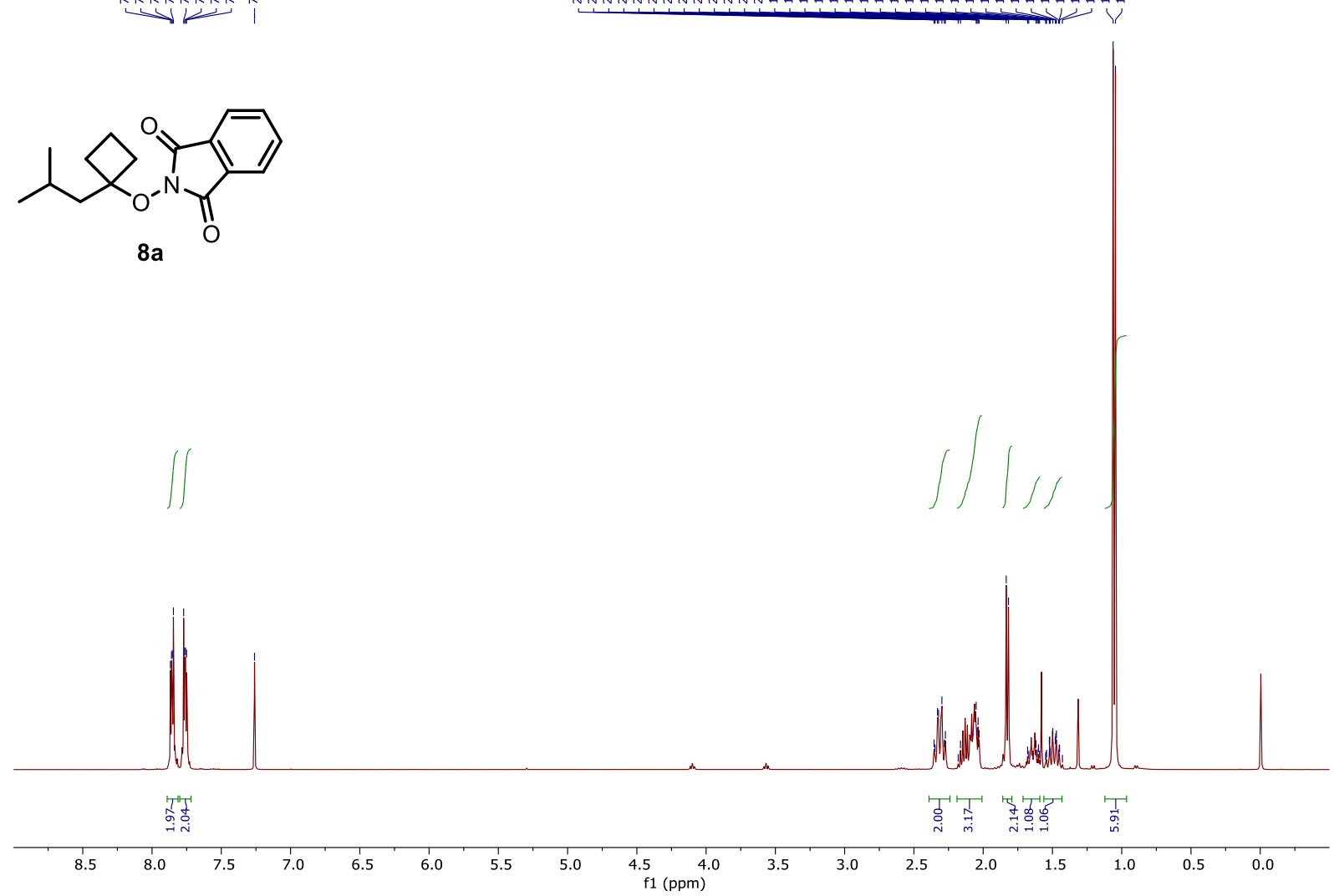

${ }^{13} \mathrm{C}$ NMR (101 MHz, $\mathrm{CDCl}_{3}$ ) of compound $8 \mathbf{a}$ (see procedure):

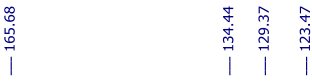

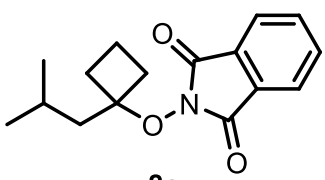

$8 \mathbf{a}$

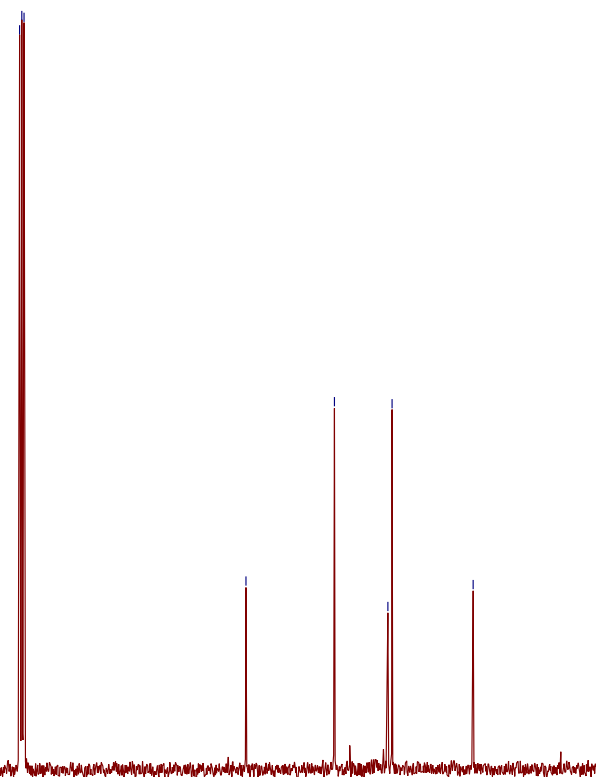

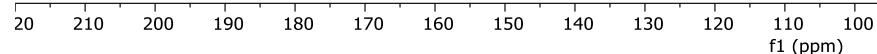


${ }^{1} \mathrm{H}$ NMR (400 MHz, $\mathrm{CDCl}_{3}$ ) of compound 9a (see procedure):

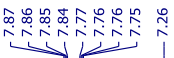

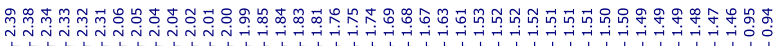<smiles>CC(C)CCC1(ON2C(=O)c3ccccc3C2=O)CCC1</smiles>

9a

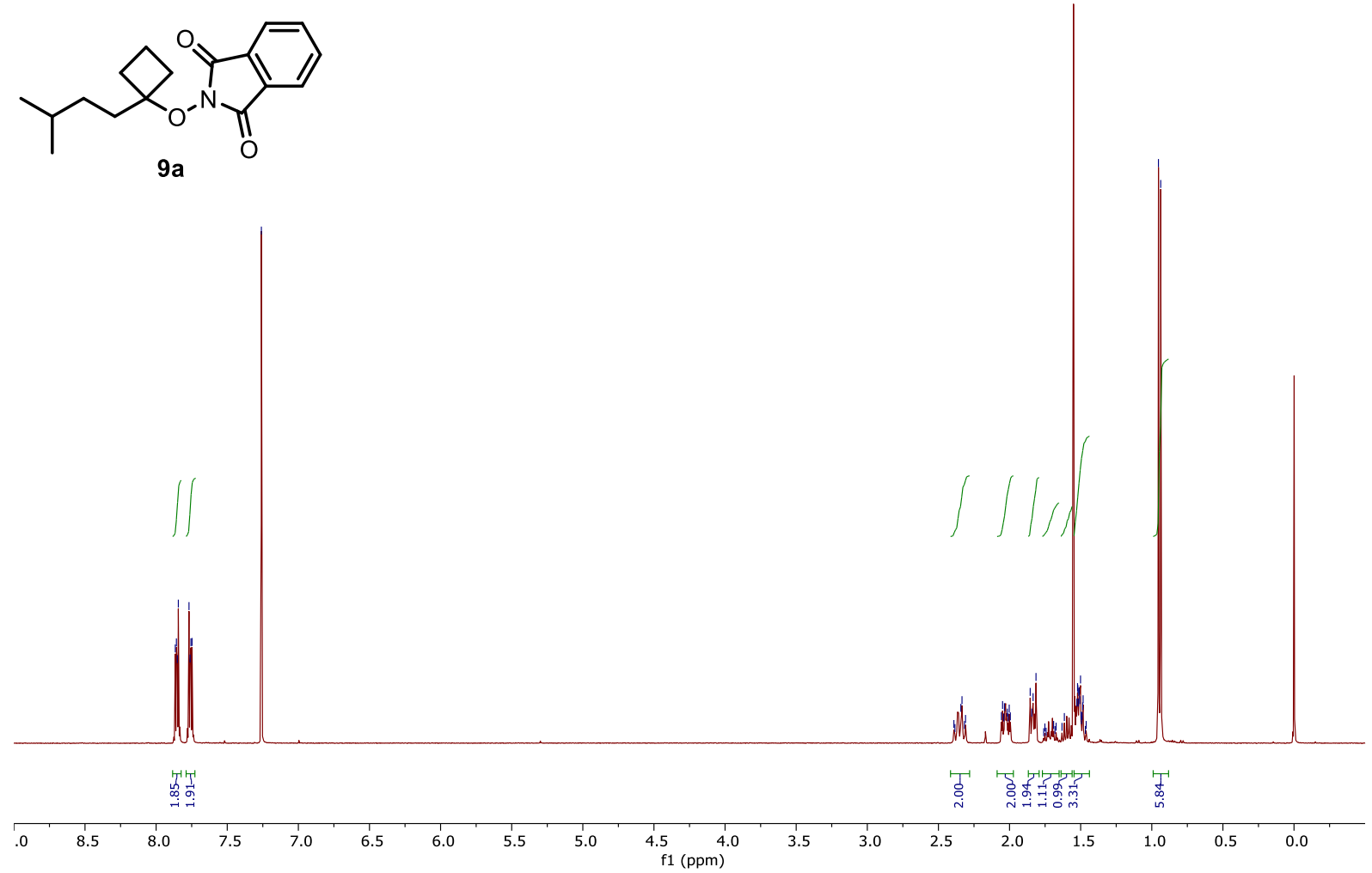

${ }^{13} \mathrm{C}$ NMR (101 MHz, $\mathrm{CDCl}_{3}$ ) of compound 9a (see procedure):

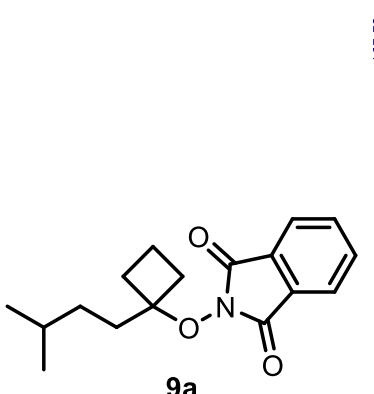

蕉

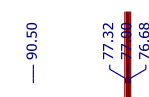

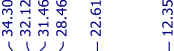

$9 a$ 
${ }^{1} \mathrm{H}$ NMR (400 MHz, $\mathrm{CDCl}_{3}$ ) of compound $\mathbf{1 0 a}$ (see procedure):

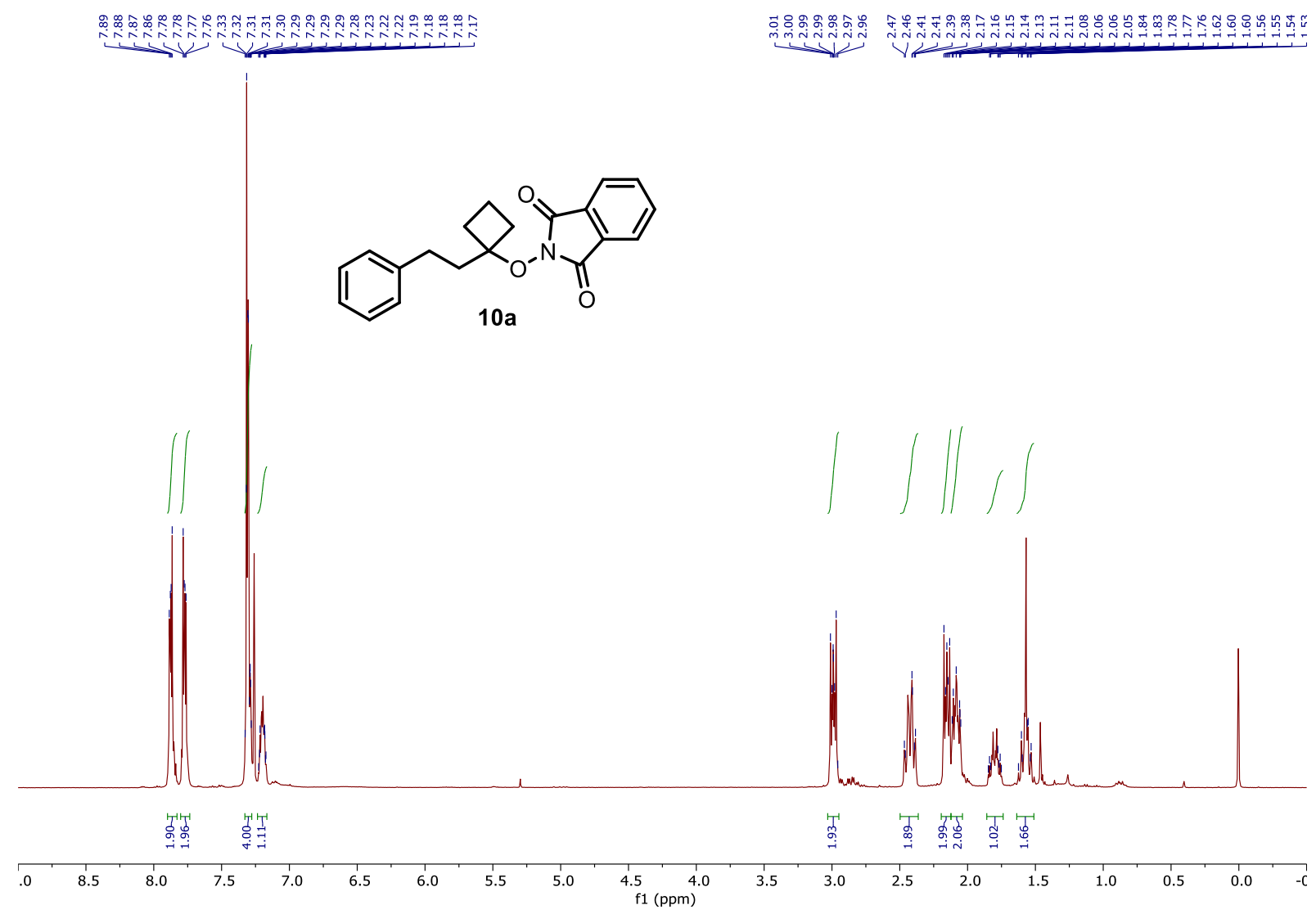

${ }^{13} \mathrm{C}$ NMR (101 MHz, $\left.\mathrm{CDCl}_{3}\right)$ of compound $\mathbf{1 0 a}$ (see procedure):

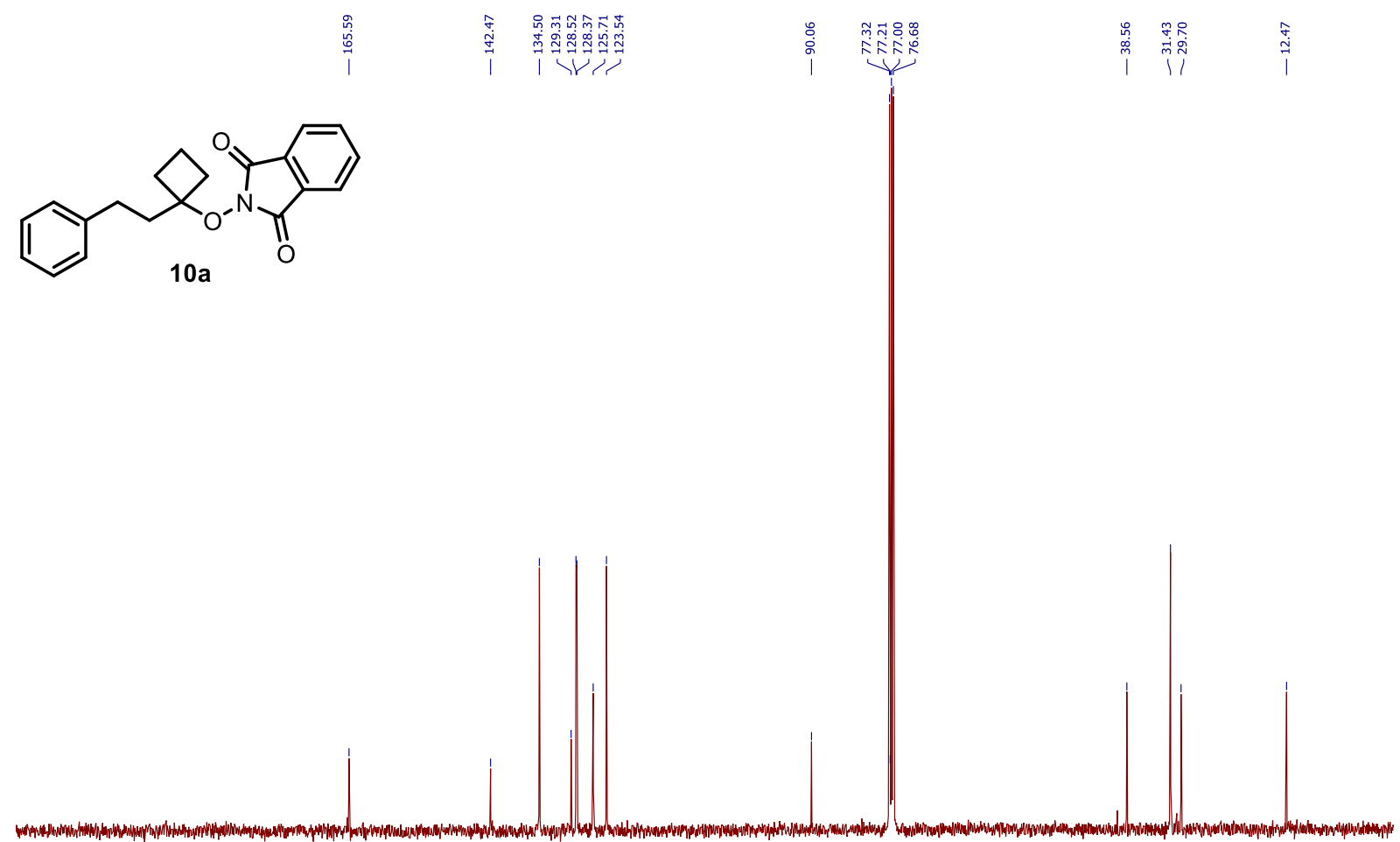

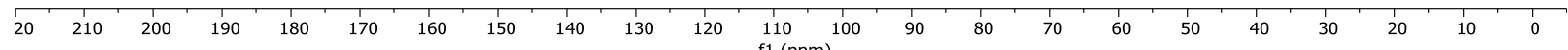


${ }^{1} \mathrm{H}$ NMR (400 MHz, $\mathrm{CDCl}_{3}$ ) of compound 11a (see procedure):

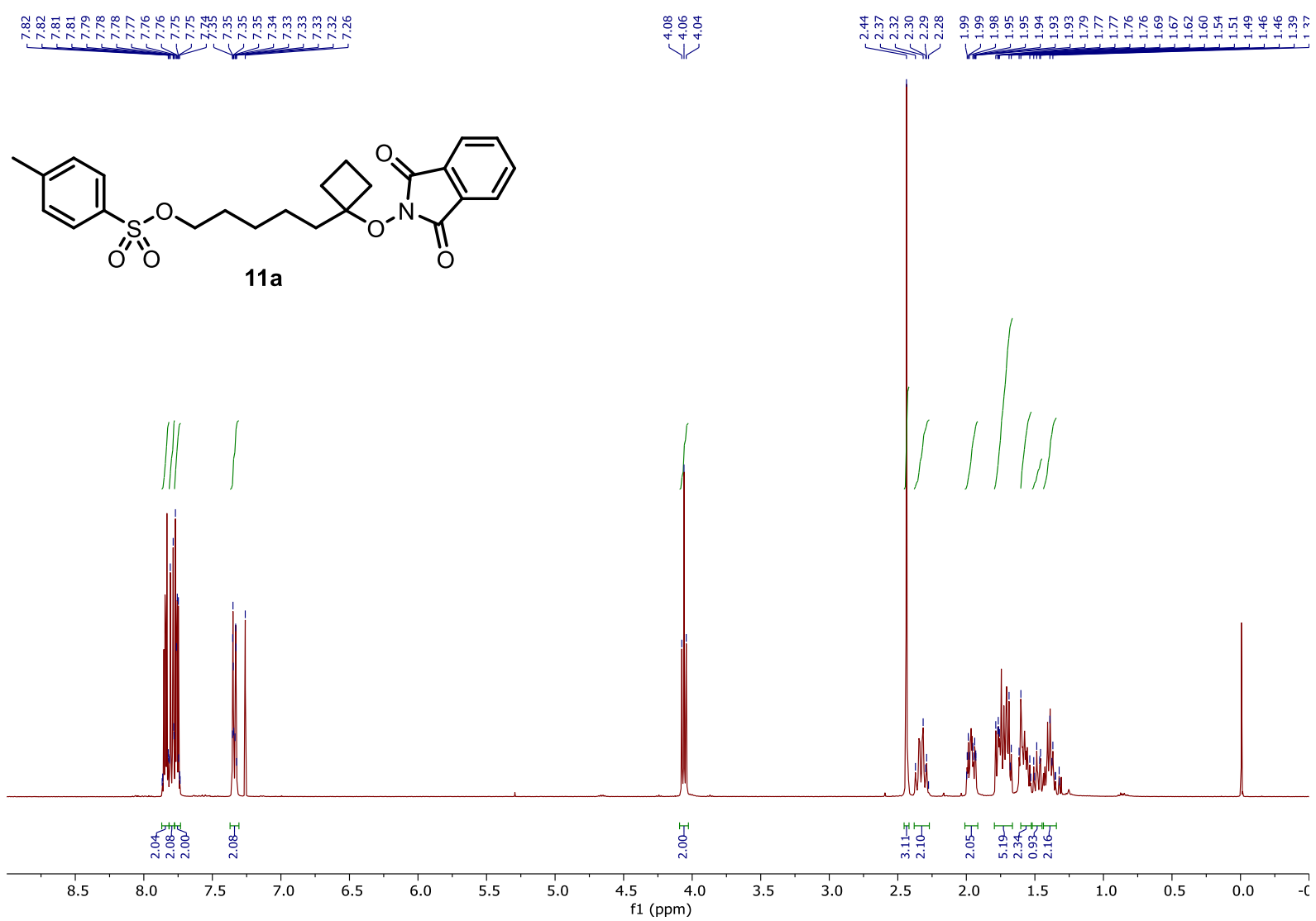

${ }^{13} \mathrm{C}$ NMR (101 MHz, $\mathrm{CDCl}_{3}$ ) of compound $11 \mathrm{a}$ (see procedure):
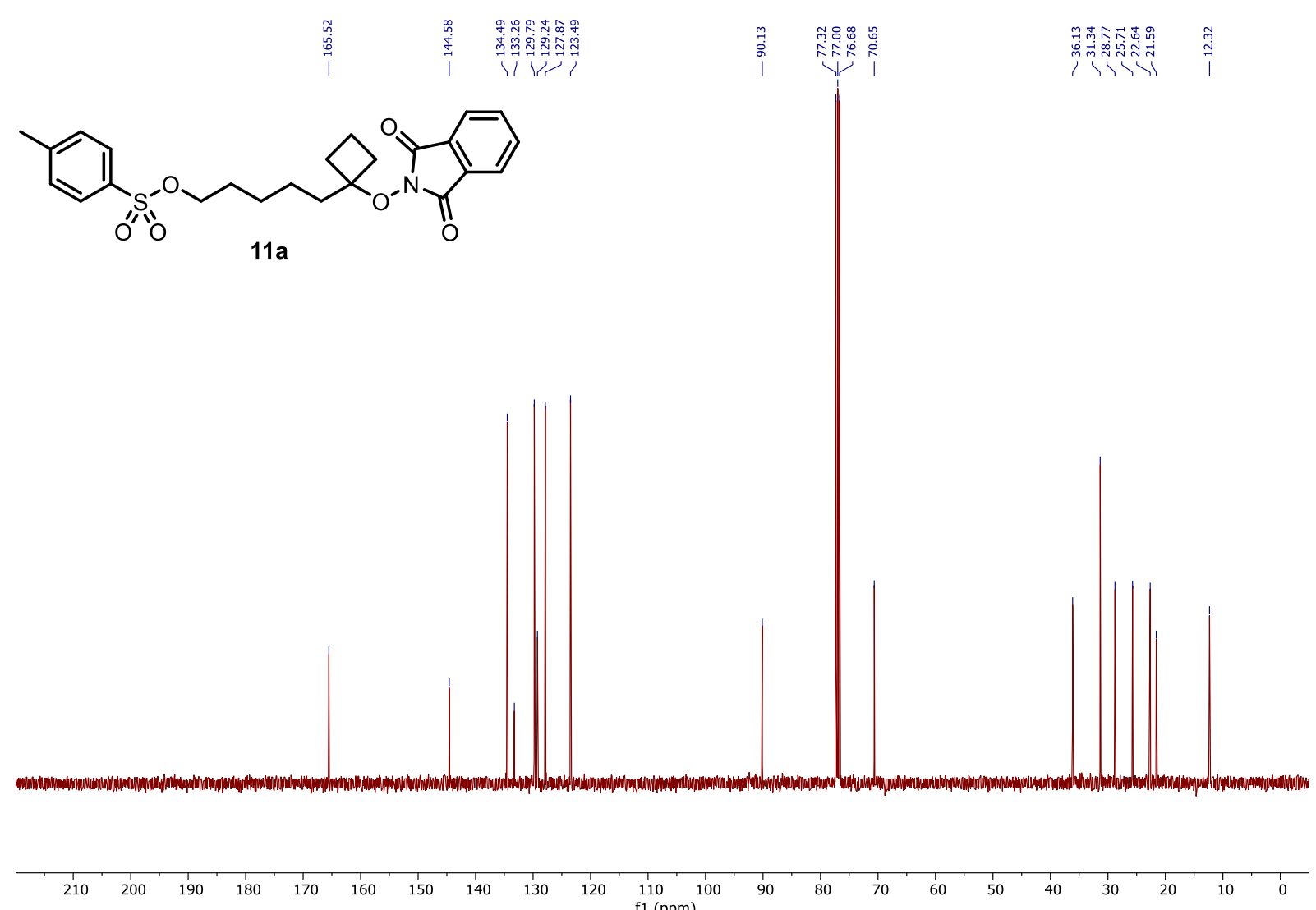
${ }^{1} \mathrm{H}$ NMR (400 MHz, $\mathrm{CDCl}_{3}$ ) of compound 12a (see procedure):

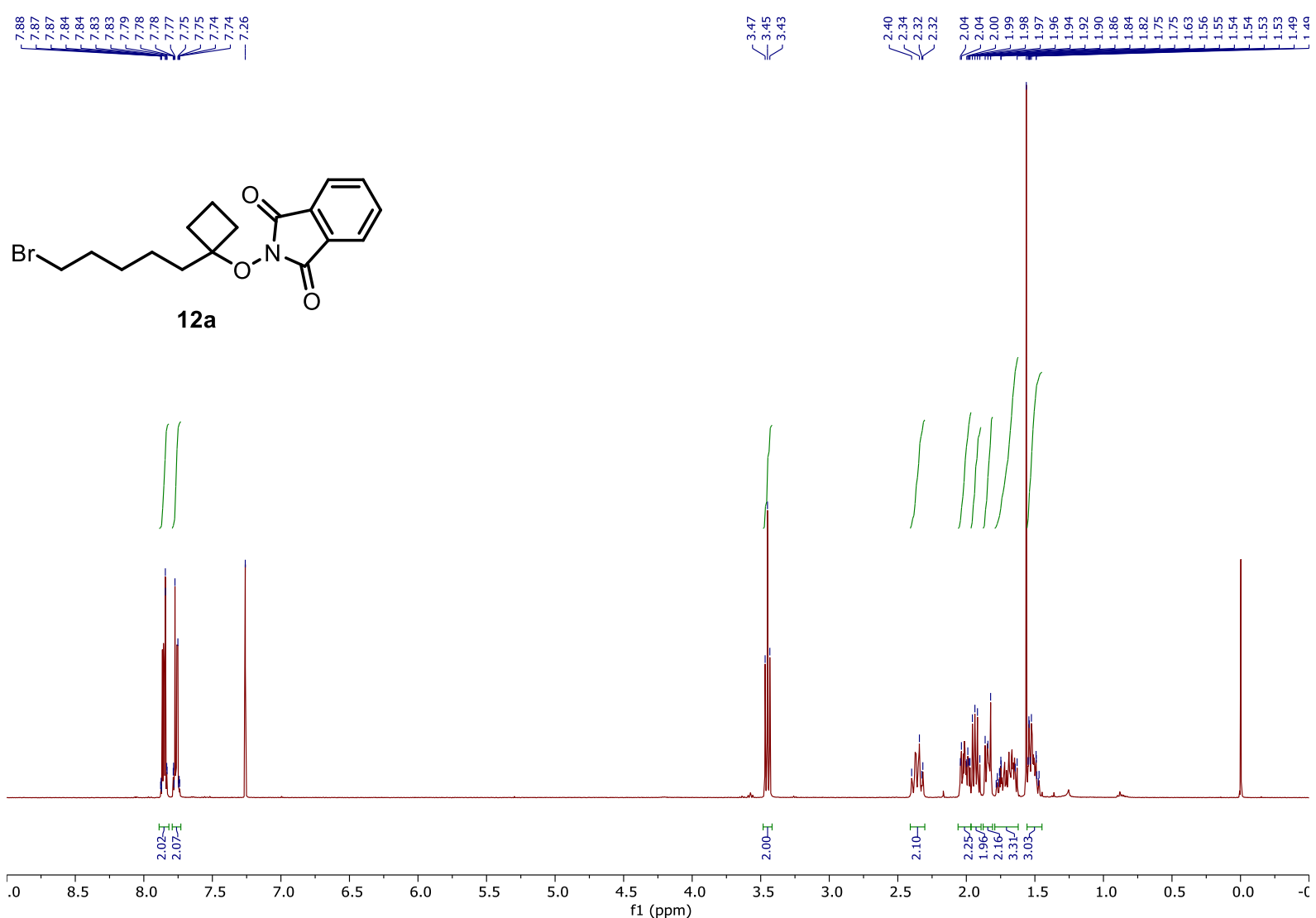

${ }^{13} \mathrm{C}$ NMR (101 MHz, $\mathrm{CDCl}_{3}$ ) of compound 12a (see procedure):

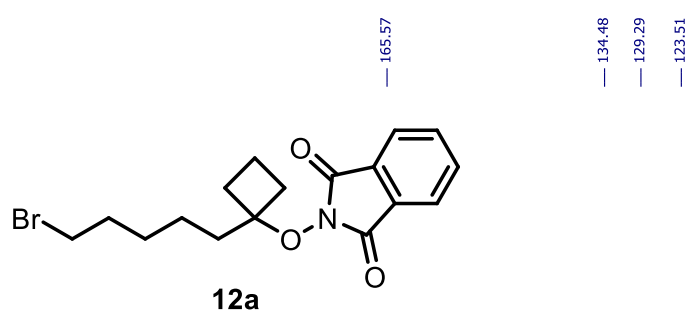

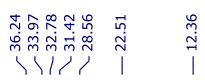

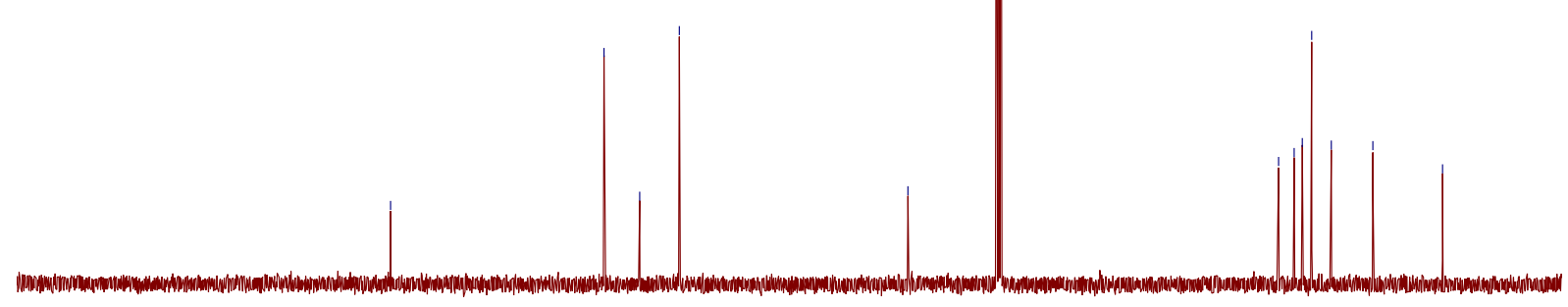

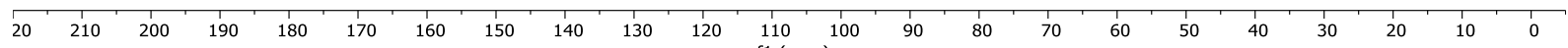


${ }^{1} \mathrm{H}$ NMR (400 MHz, $\mathrm{CDCl}_{3}$ ) of compound 13a (see procedure):

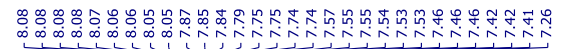<smiles>O=C(OCCCC1(ON2C(=O)c3ccccc3C2=O)CCC1)c1ccccc1</smiles>

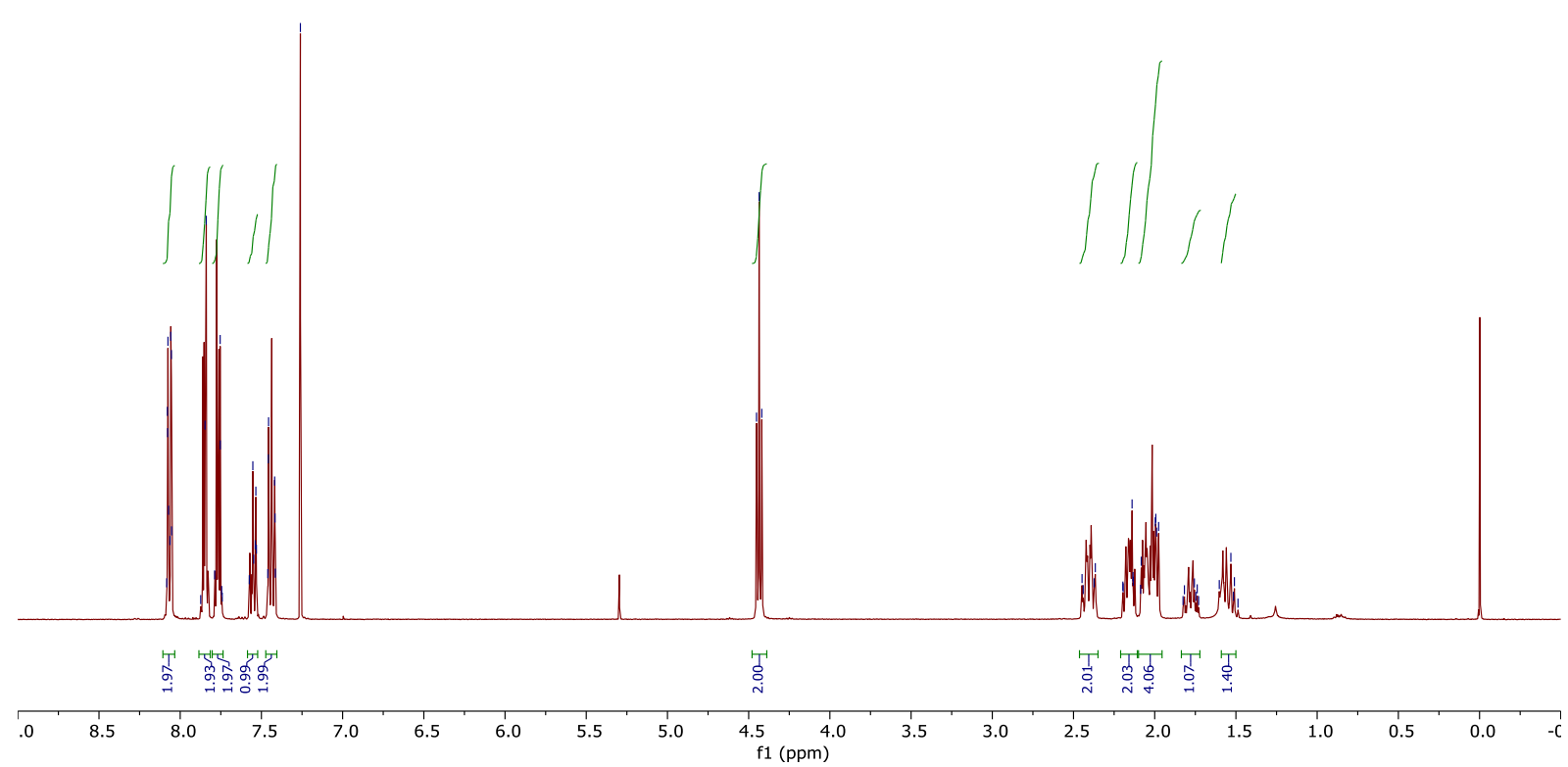

${ }^{13} \mathrm{C}$ NMR (101 MHz, $\mathrm{CDCl}_{3}$ ) of compound 13a (see procedure):

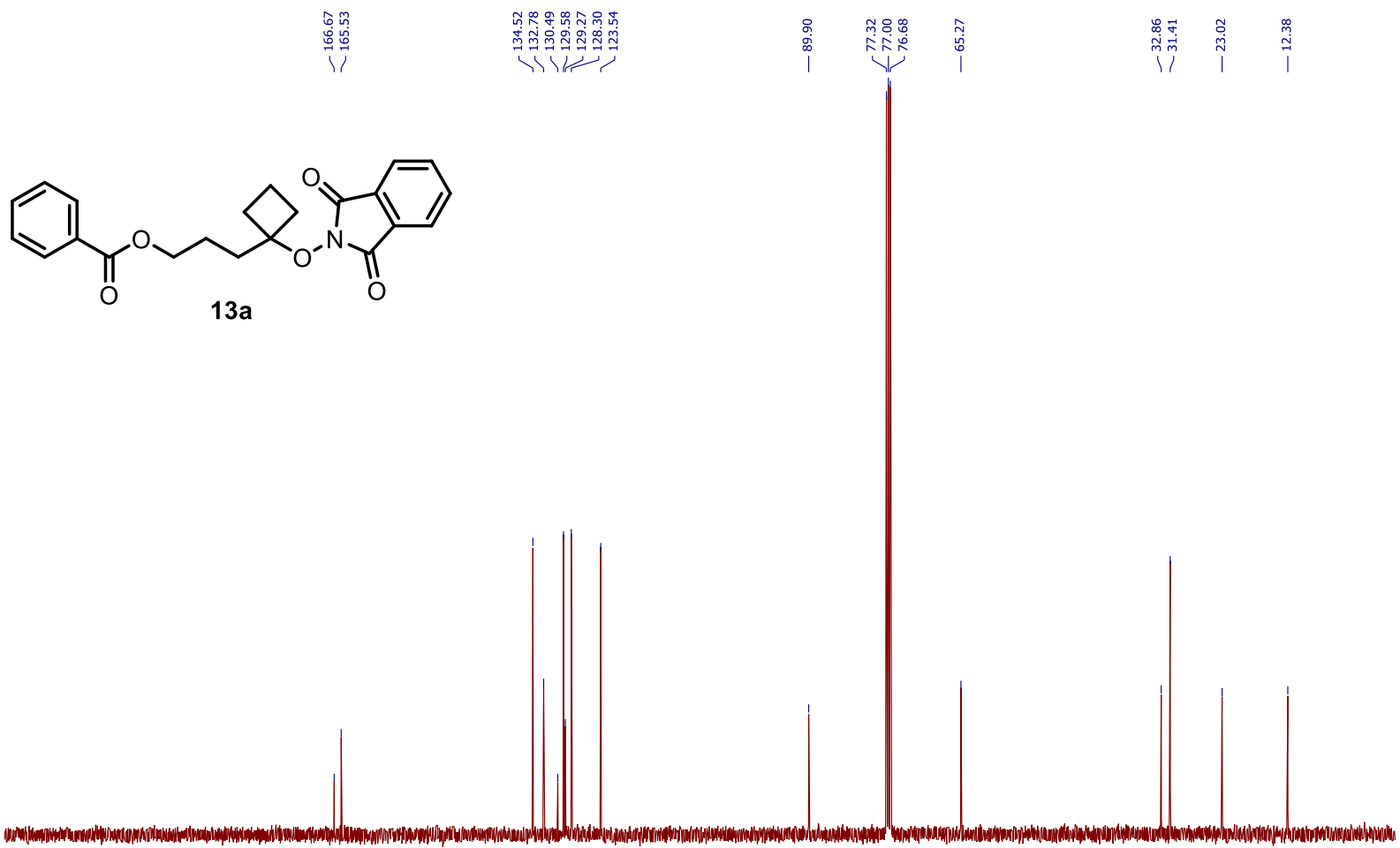

$\begin{array}{llllllllllllllllllllllllll}1 & 210 & 200 & 190 & 180 & 170 & 160 & 150 & 140 & 130 & 120 & 110 & 100 & 90 & 80 & 70 & 60 & 50 & 40 & 30 & 20 & 10 & 0\end{array}$ 
${ }^{1} \mathrm{H}$ NMR (400 MHz, $\mathrm{CDCl}_{3}$ ) of compound $\mathbf{1 4 a}$ (see procedure):

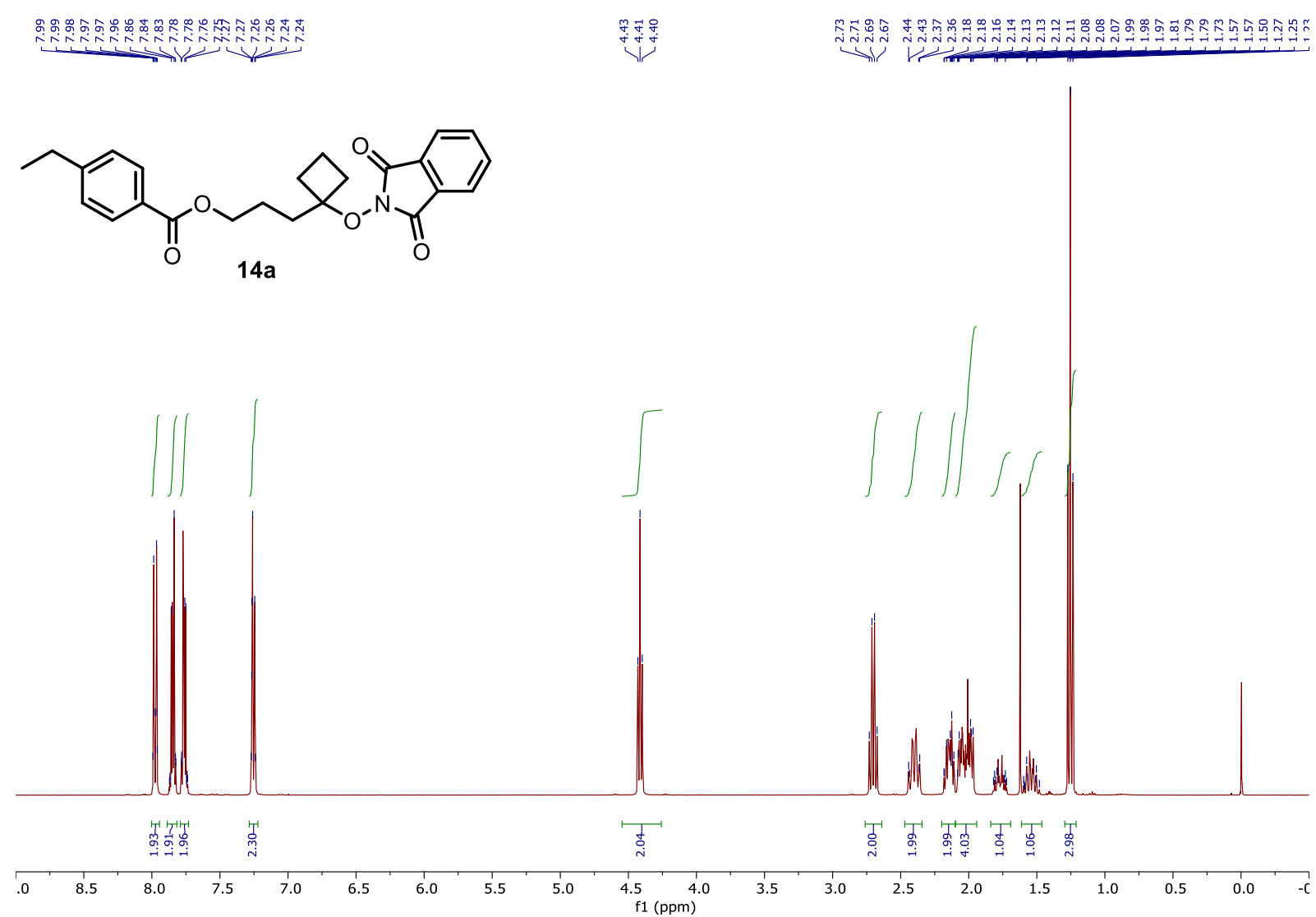

${ }^{13} \mathrm{C}$ NMR (101 MHz, $\mathrm{CDCl}_{3}$ ) of compound $14 \mathbf{a}$ (see procedure):

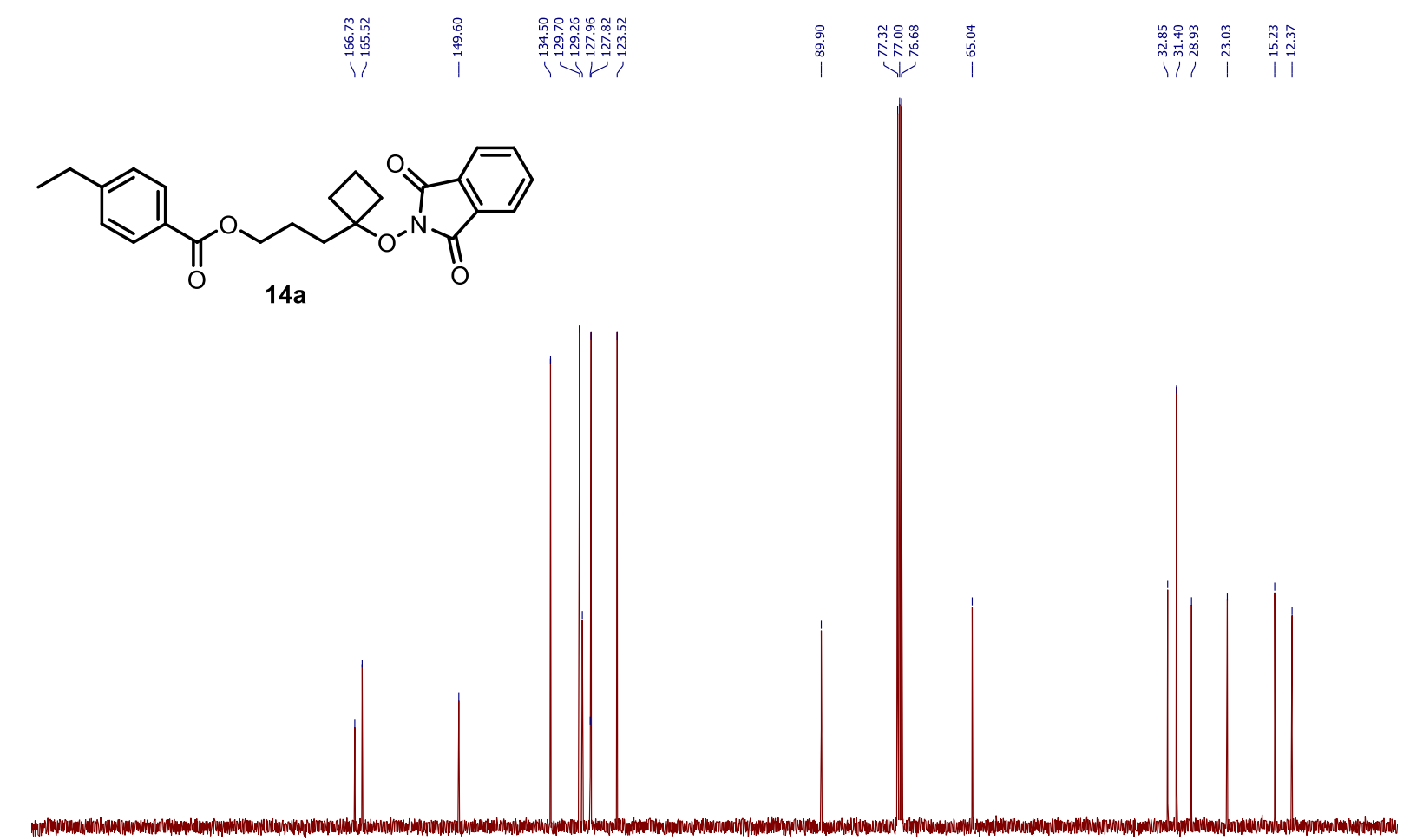

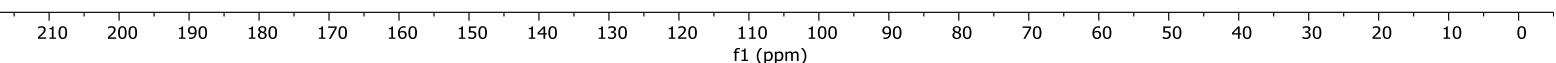


${ }^{1} \mathrm{H}$ NMR (400 MHz, $\mathrm{CDCl}_{3}$ ) of compound $\mathbf{1 5 a}$ (see procedure):

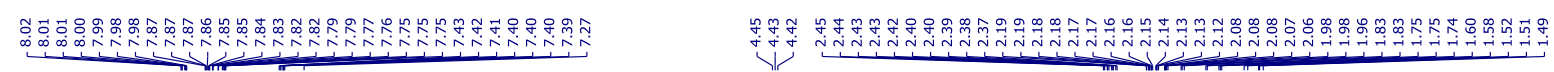<smiles>CC(C)(C)CCCCOC(=O)c1ccc(Cl)cc1</smiles>

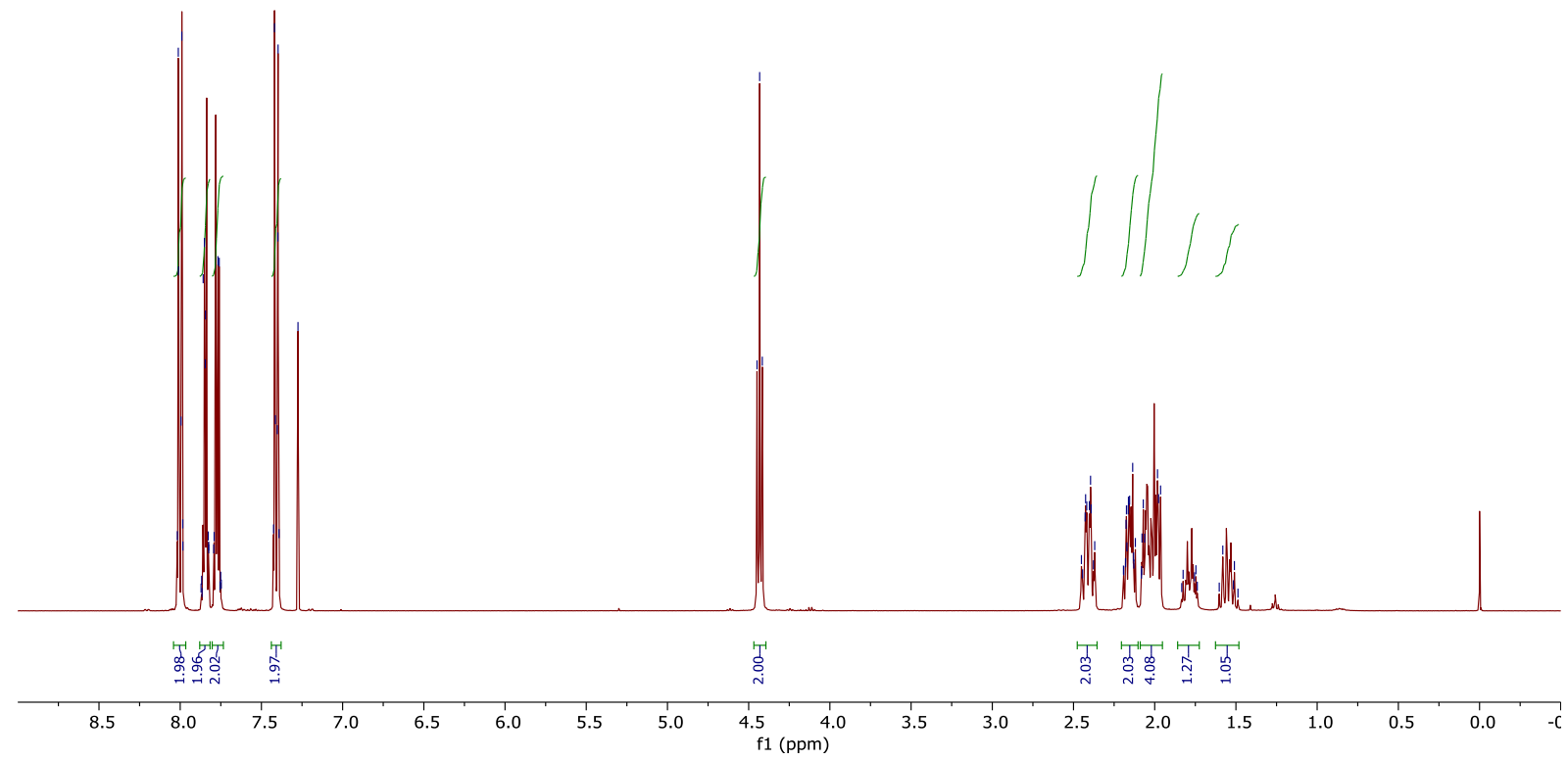

${ }^{13} \mathrm{C}$ NMR (101 MHz, $\mathrm{CDCl}_{3}$ ) of compound $15 \mathbf{a}$ (see procedure):

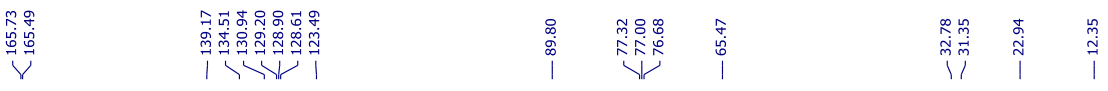<smiles>CC(C)(C)ON1C(=O)c2ccccc2C1=O</smiles>

$15 a$

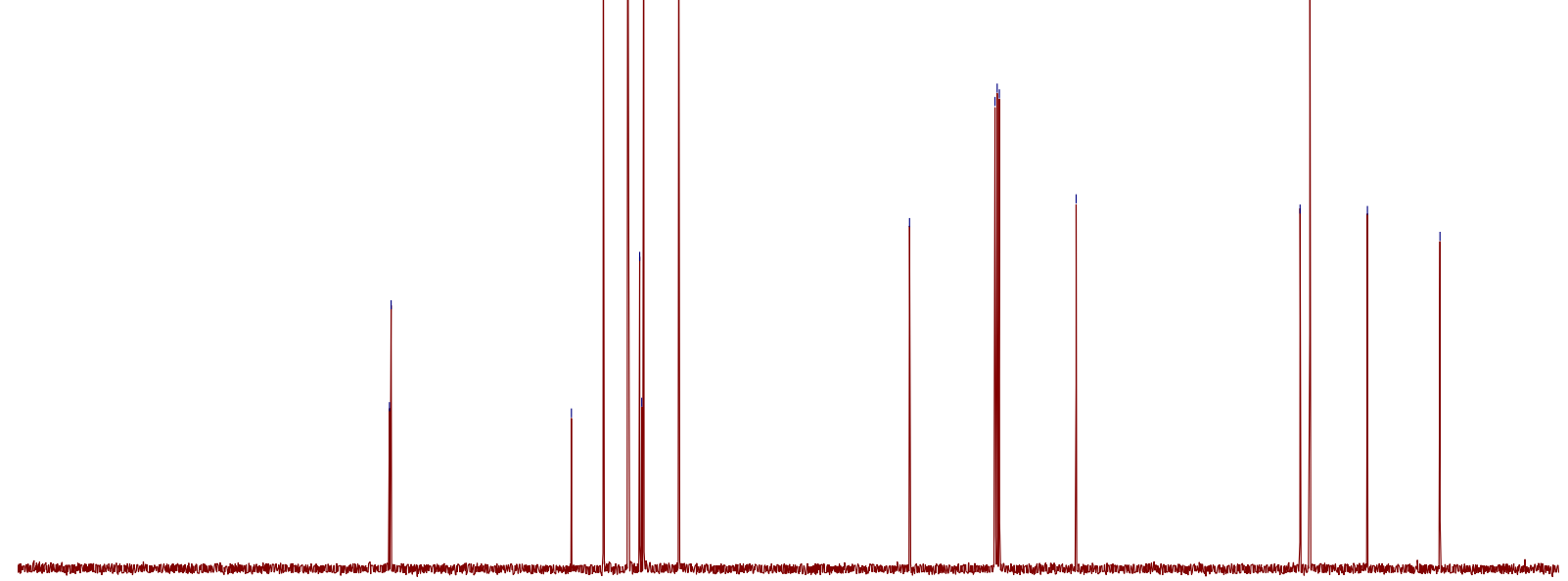

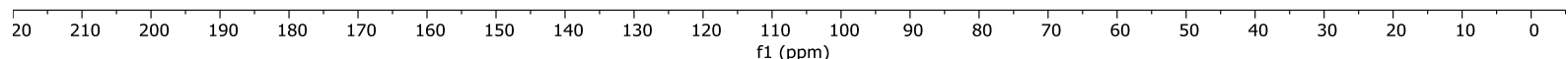


${ }^{1} \mathrm{H}$ NMR (400 MHz, $\mathrm{CDCl}_{3}$ ) of compound $\mathbf{1 6 a}$ (see procedure):

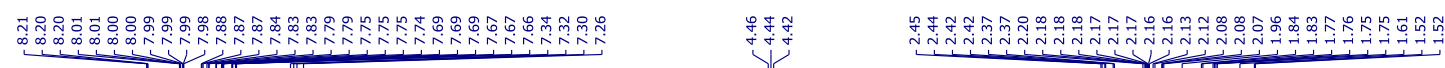

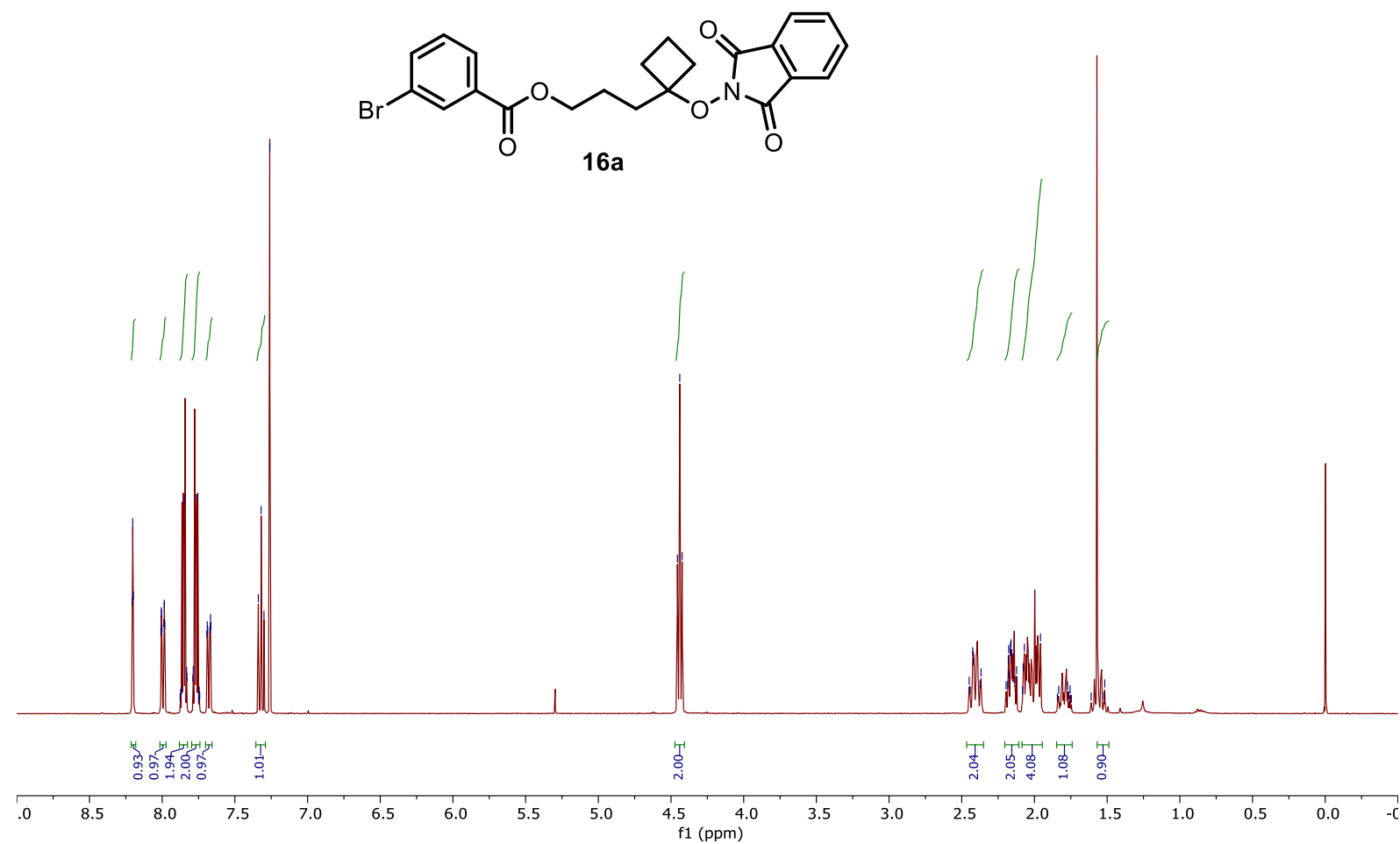

${ }^{13} \mathrm{C}$ NMR (101 MHz, $\mathrm{CDCl}_{3}$ ) of compound 16a (see procedure):

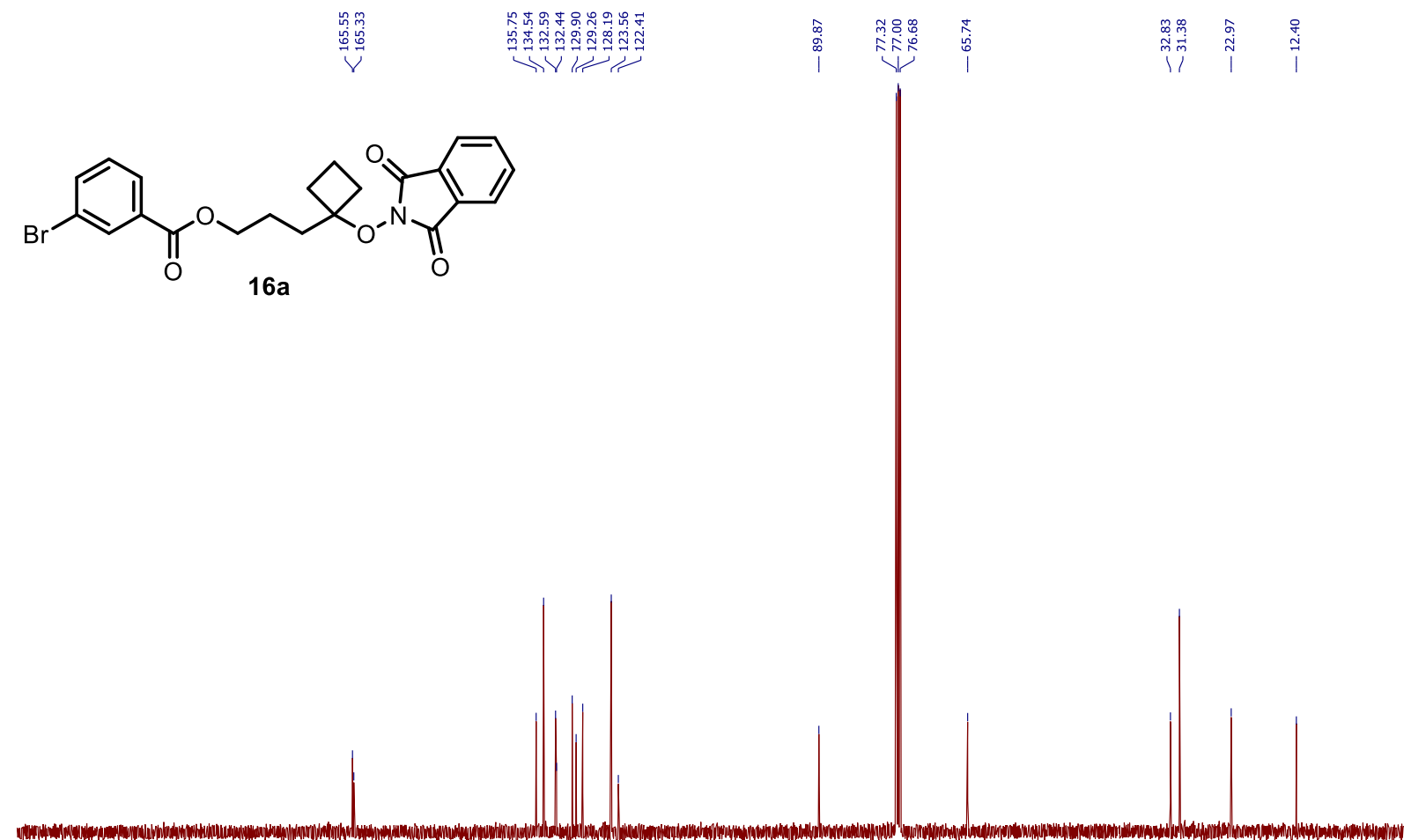

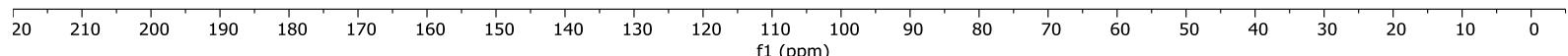


$\mathrm{NMR}\left(400 \mathrm{MHz}, \mathrm{CDCl}_{3}\right)$ of compound 17 a (see procedure

Sh ens?
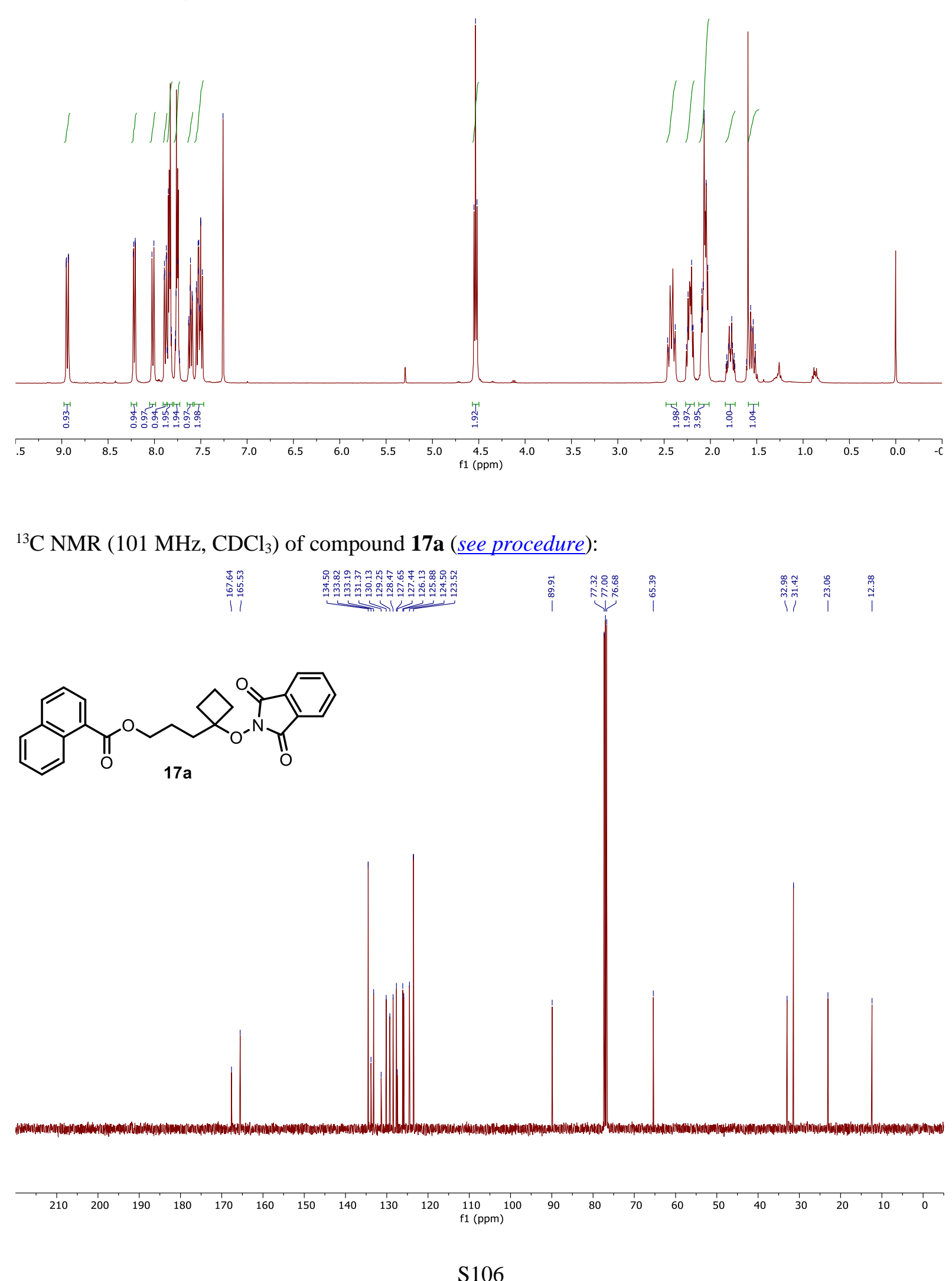
${ }^{1} \mathrm{H}$ NMR (400 MHz, $\mathrm{CDCl}_{3}$ ) of compound $\mathbf{1 8 a}$ (see procedure):

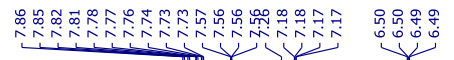

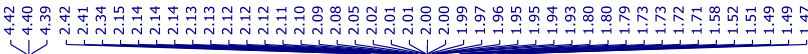<smiles>O=C(OCCCC1(ON2C(=O)c3ccccc3C2=O)CCC1)c1ccco1</smiles>

$18 a$
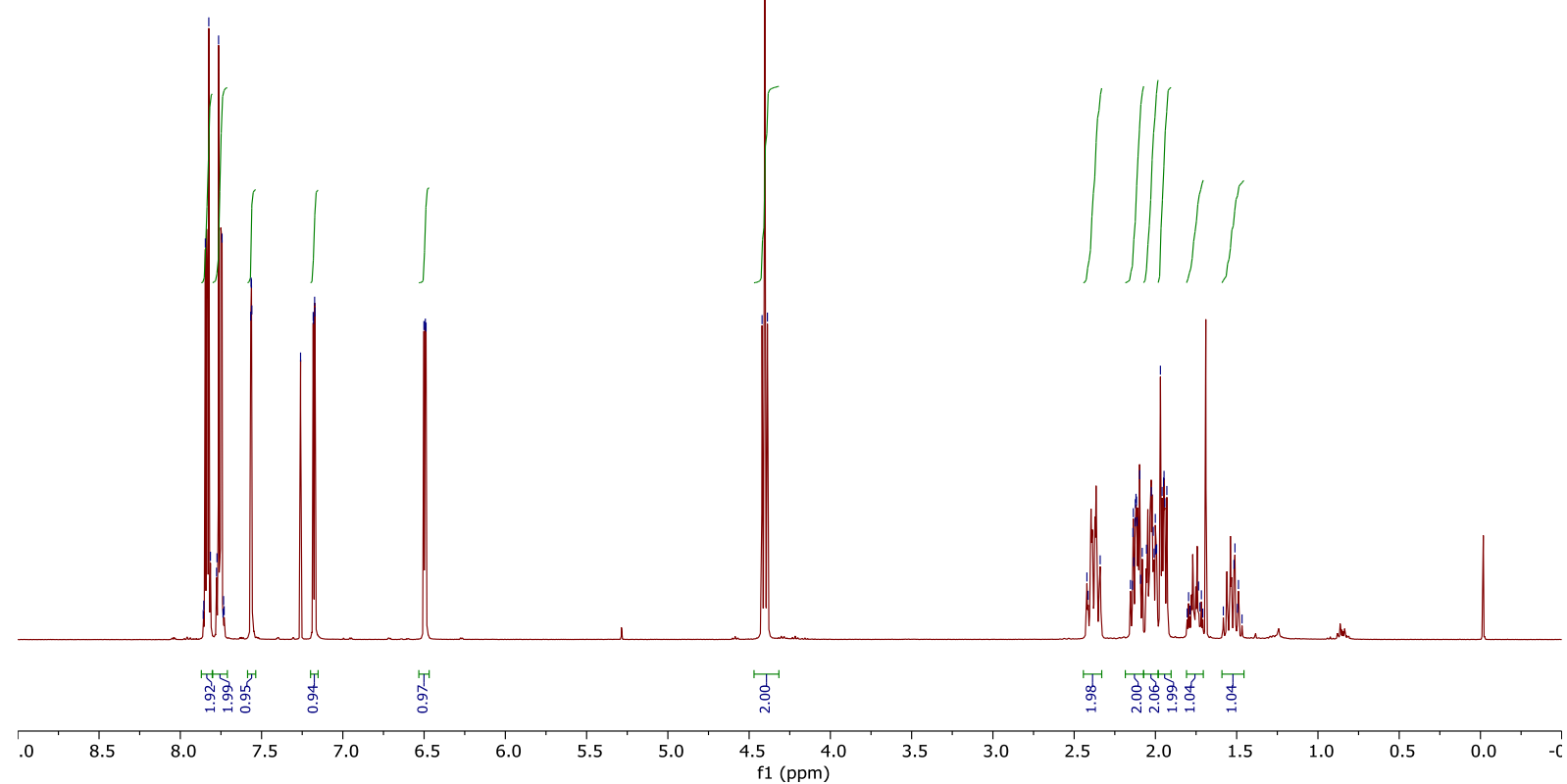

${ }^{13} \mathrm{C}$ NMR (101 MHz, $\mathrm{CDCl}_{3}$ ) of compound 18a (see procedure):

离<smiles>O=C(OCCCC1(ON2C(=O)c3ccccc3C2=O)CCC1)c1ccco1</smiles>

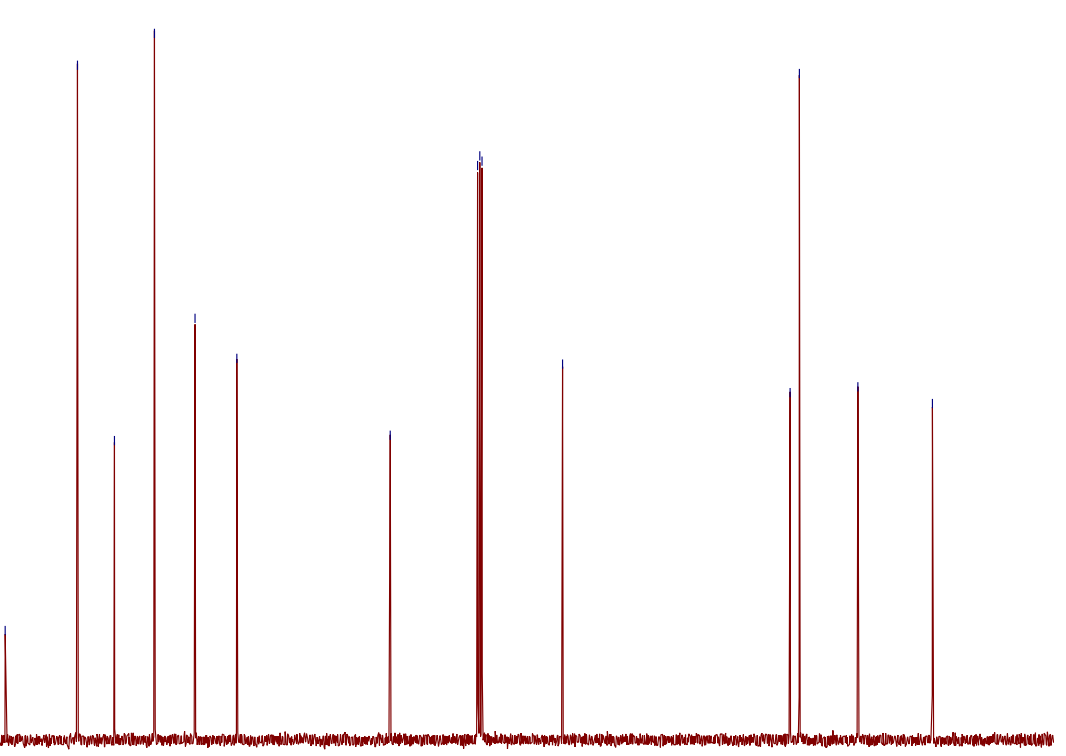

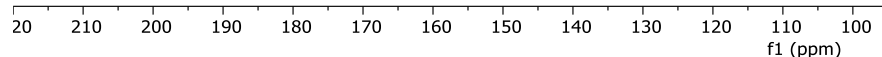

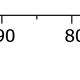

$\begin{array}{lll}70 & 60 & 1 \\ & & \end{array}$

$\begin{array}{lllll}40 & 30 & 20 & 10 & 0\end{array}$ 
${ }^{1} \mathrm{H}$ NMR (400 MHz, $\mathrm{CDCl}_{3}$ ) of compound 19a (see procedure):

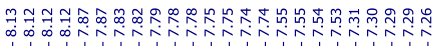

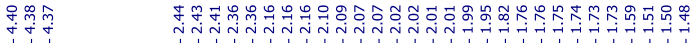<smiles>O=C(OCCCC1(ON2C(=O)c3ccccc3C2=O)CCC1)c1ccsc1</smiles>

$19 a$
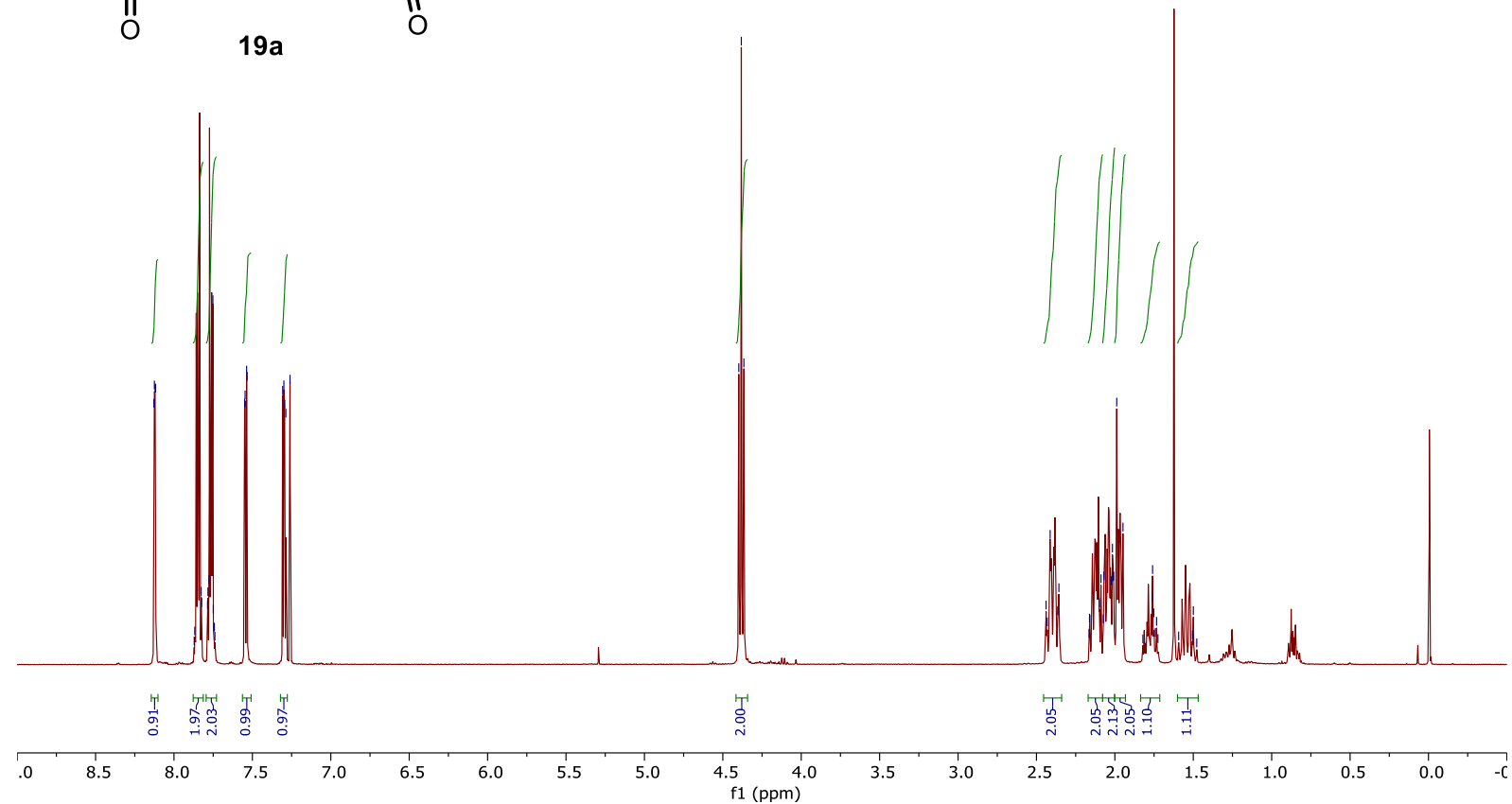

${ }^{13} \mathrm{C}$ NMR (101 MHz, $\mathrm{CDCl}_{3}$ ) of compound 19a (see procedure):

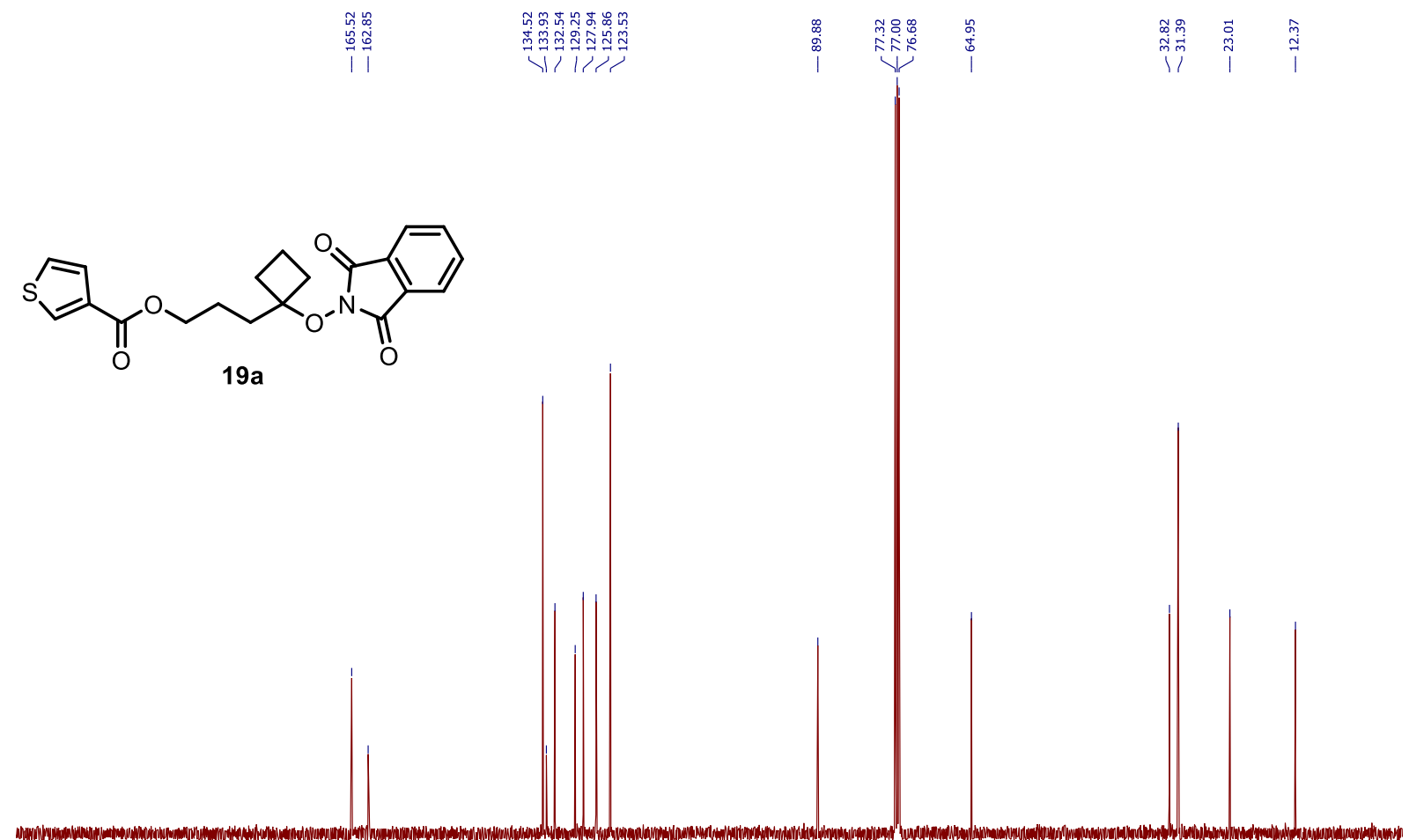

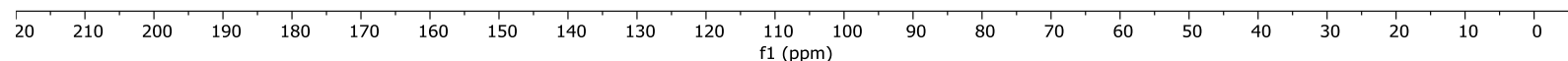


${ }^{1} \mathrm{H}$ NMR (400 MHz, $\mathrm{CDCl}_{3}$ ) of compound $20 \mathrm{a}$ (see procedure):

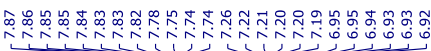<smiles>O=C(Cc1cccs1)OCCCC1(ON2C(=O)c3ccccc3C2=O)CCC1</smiles>

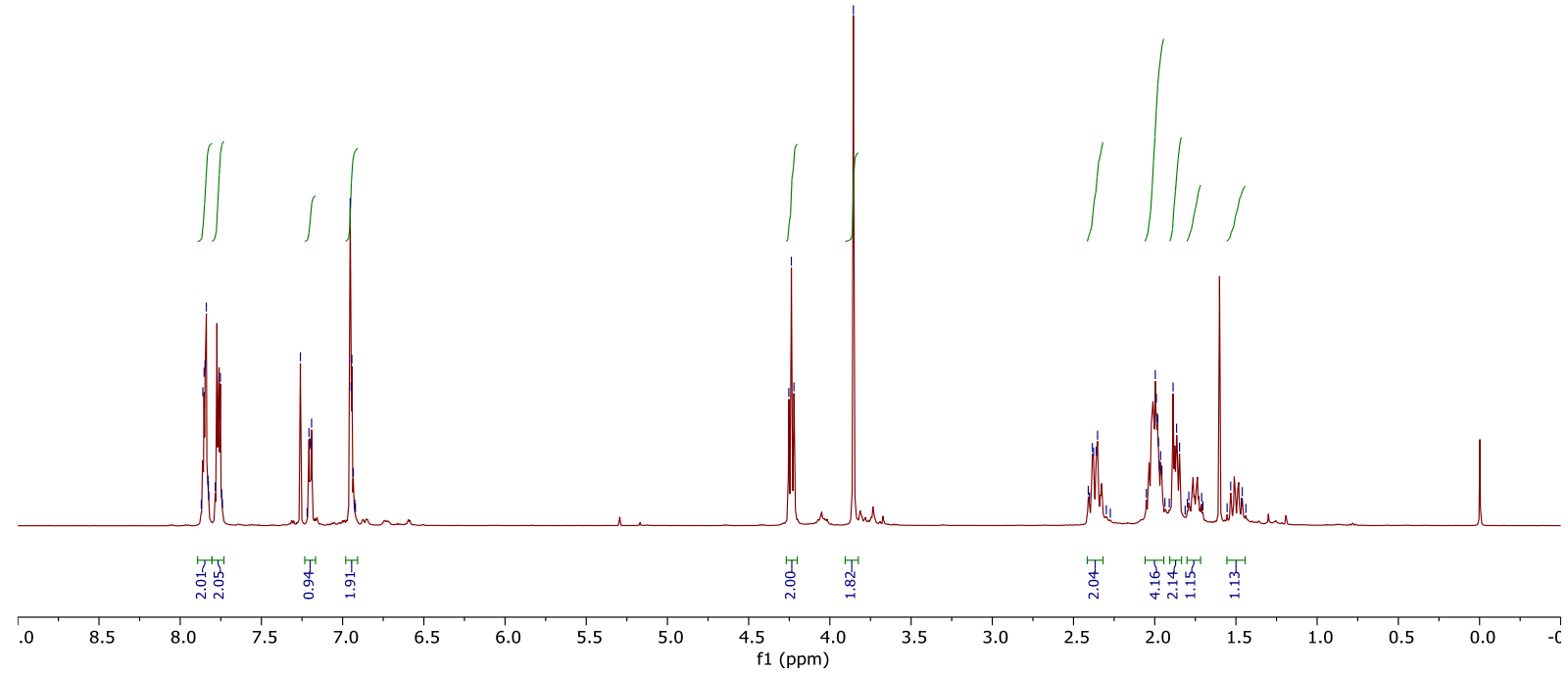

${ }^{13} \mathrm{C}$ NMR (101 MHz, $\mathrm{CDCl}_{3}$ ) of compound 20a (see procedure):

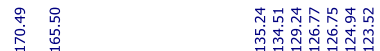<smiles>O=C(Cc1cccs1)OCCCC1(ON2C(=O)c3ccccc3C2=O)CCC1</smiles>

$20 \mathrm{a}$
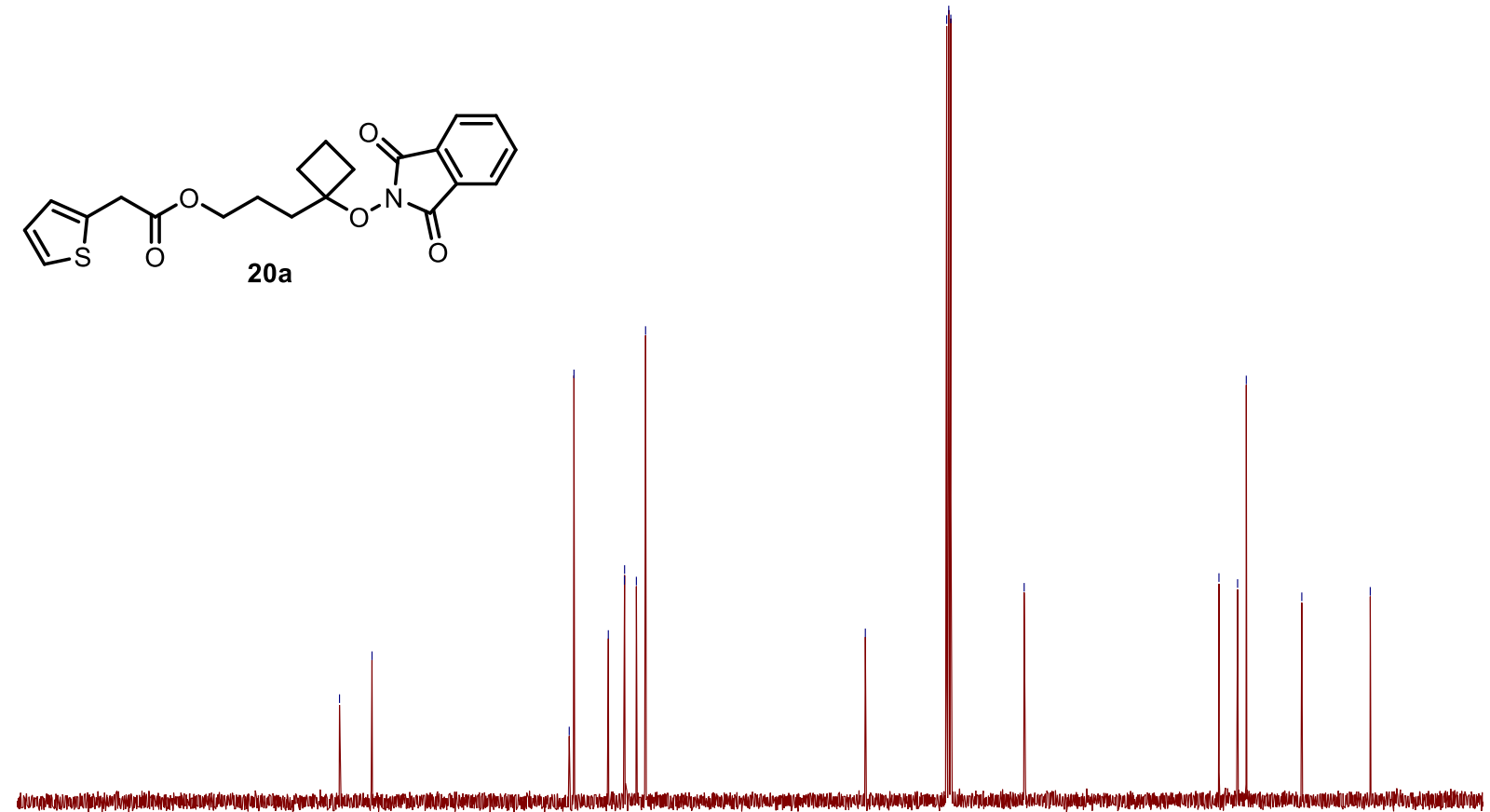

$\begin{array}{lllllllllll}210 & 200 & 190 & 180 & 170 & 160 & 150 & 140 & 130 & 120 & \begin{array}{l}110 \\ f 1(\mathrm{ppm})\end{array}\end{array}$ 
${ }^{1} \mathrm{H}$ NMR (400 MHz, $\mathrm{CDCl}_{3}$ ) of compound 21 a (see procedure):

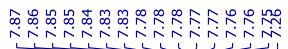

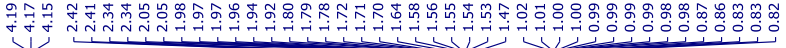<smiles>O=C(OCCCC1(ON2C(=O)c3ccccc3C2=O)CCC1)C1CC1</smiles>

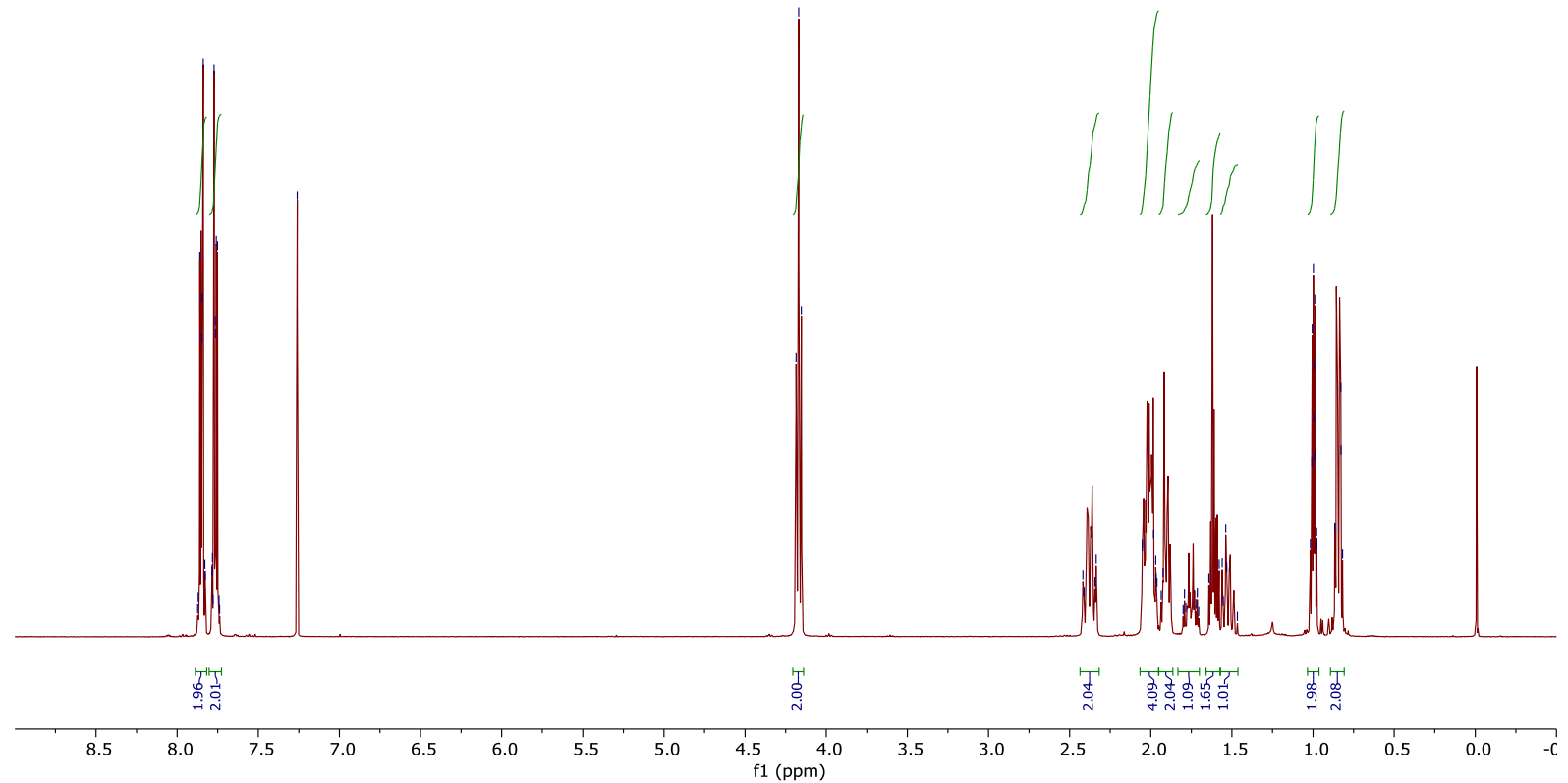

${ }^{13} \mathrm{C}$ NMR (101 MHz, $\mathrm{CDCl}_{3}$ ) of compound 21a (see procedure):

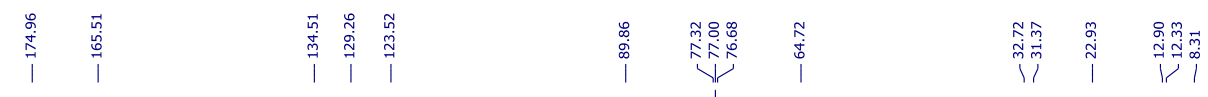<smiles>O=C(OCCCC1(ON2C(=O)c3ccccc3C2=O)CCC1)C1CC1</smiles> 
${ }^{1} \mathrm{H}$ NMR (400 MHz, $\mathrm{CDCl}_{3}$ ) of compound 22a (see procedure):<smiles>O=C(OCCCCC1(ON2C(=O)c3ccccc3C2=O)CCC1)C1C[C@@H]1c1ccccc1</smiles>

22a

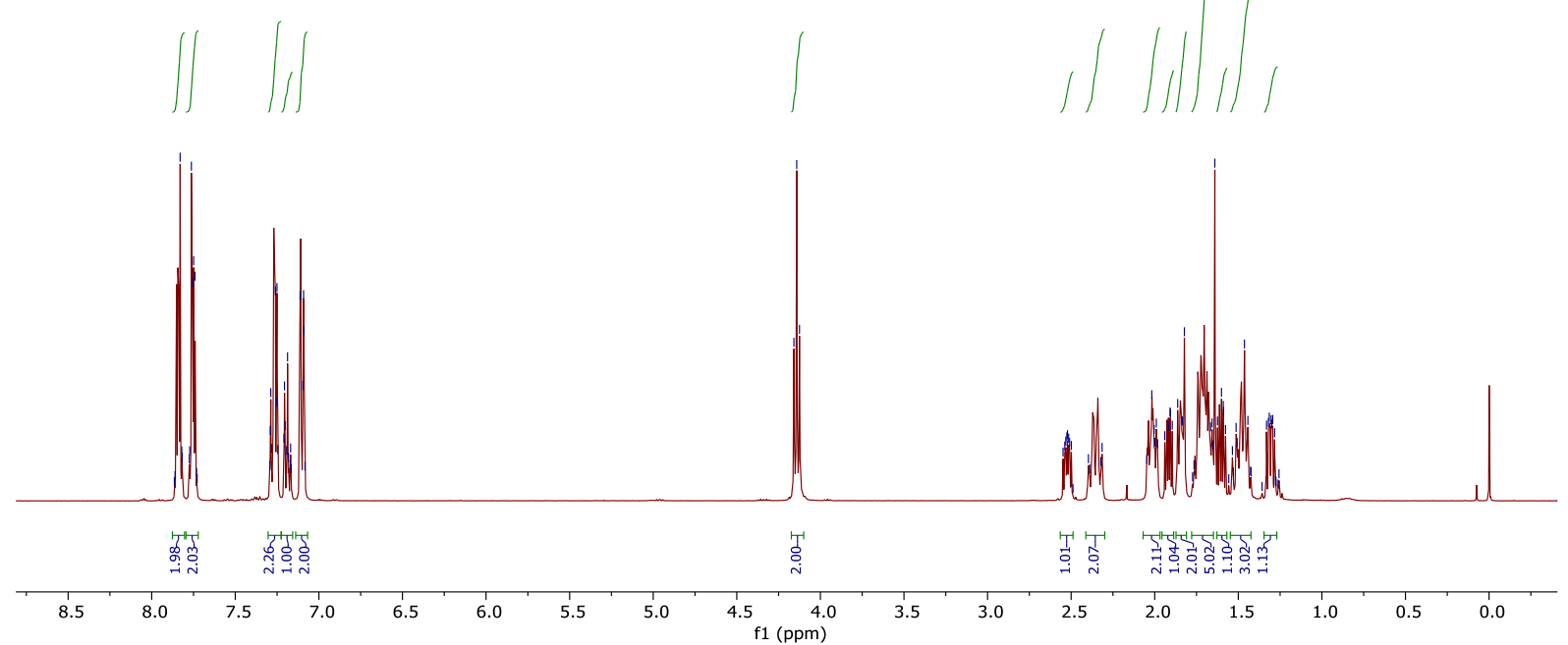

${ }^{13} \mathrm{C}$ NMR (101 MHz, $\left.\mathrm{CDCl}_{3}\right)$ of compound 22a (see procedure):

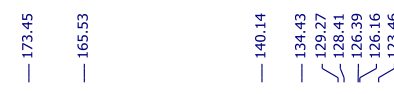<smiles>O=C(OCCCCC1(ON2C(=O)c3ccccc3C2=O)CCC1)C1C[C@@H]1c1ccccc1</smiles>

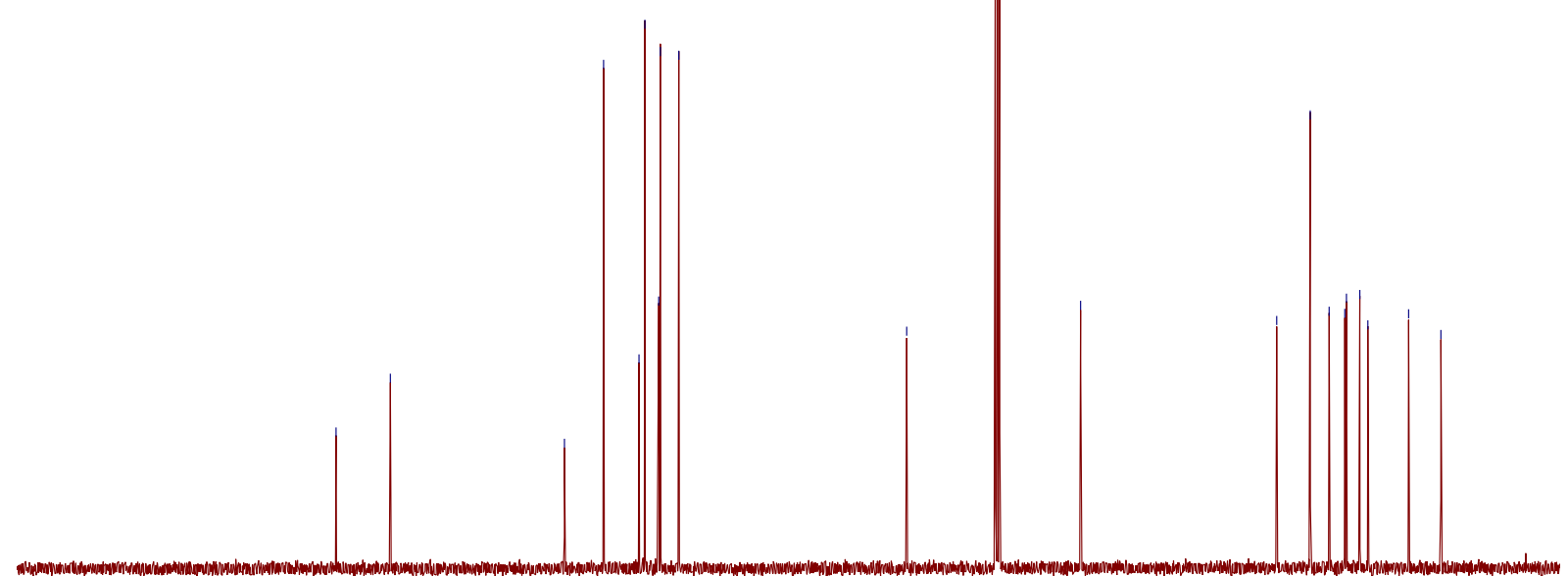

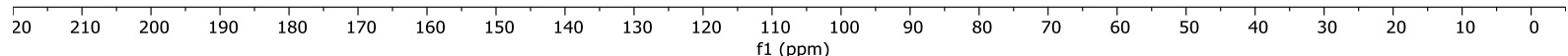


${ }^{1} \mathrm{H}$ NMR (400 MHz, $\mathrm{CDCl}_{3}$ ) of compound 23a (see procedure):

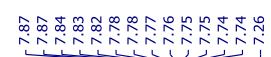<smiles>O=C(CCCCBr)OCCCC1(ON2C(=O)c3ccccc3C2=O)CCC1</smiles>

23a
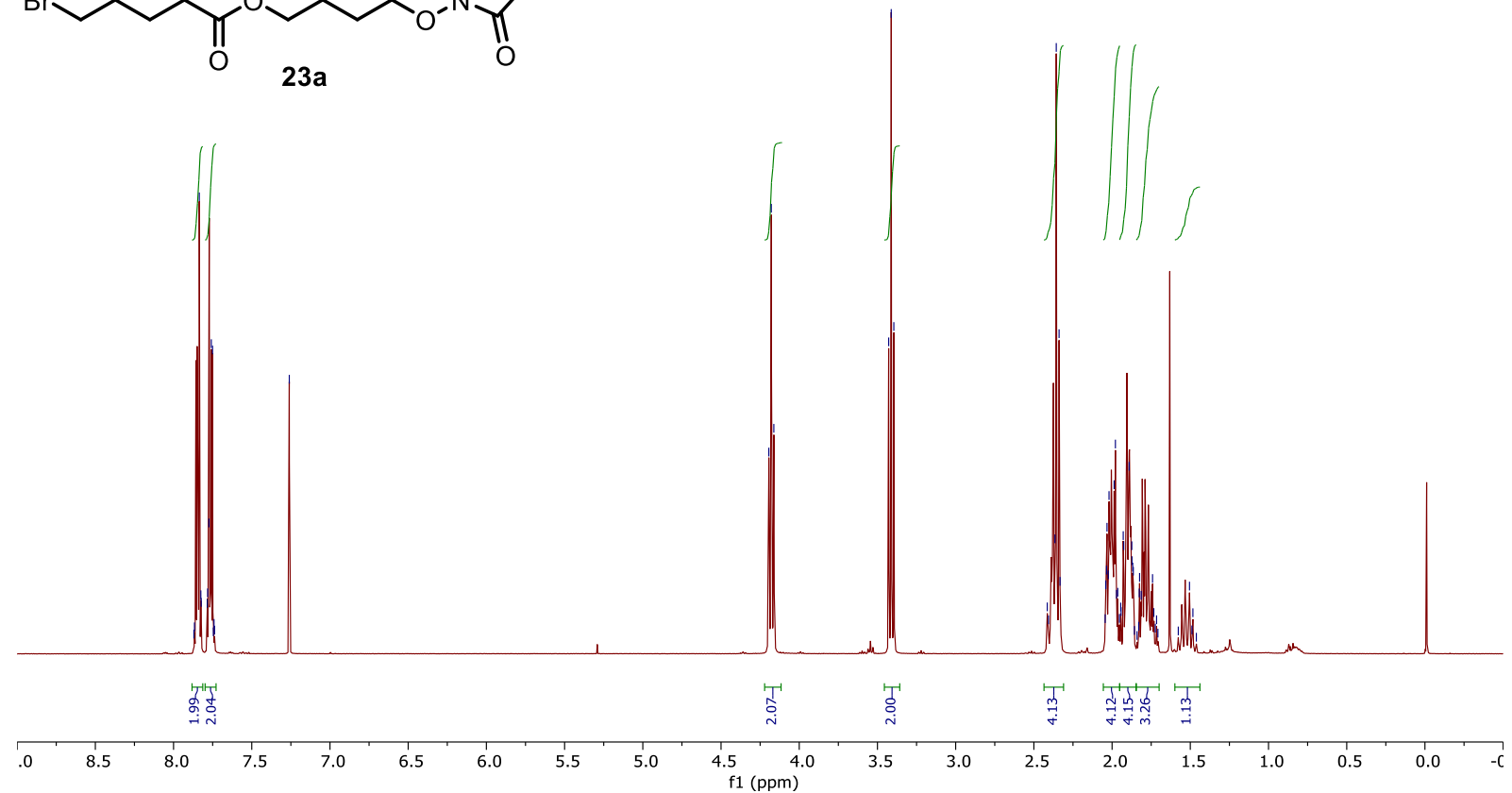

${ }^{13} \mathrm{C}$ NMR (101 MHz, $\mathrm{CDCl}_{3}$ ) of compound 23a (see procedure):

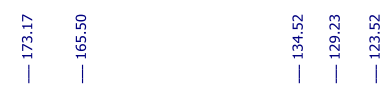<smiles>O=C(CCCCBr)OCCCC1(ON2C(=O)c3ccccc3C2=O)CCC1</smiles>

23a

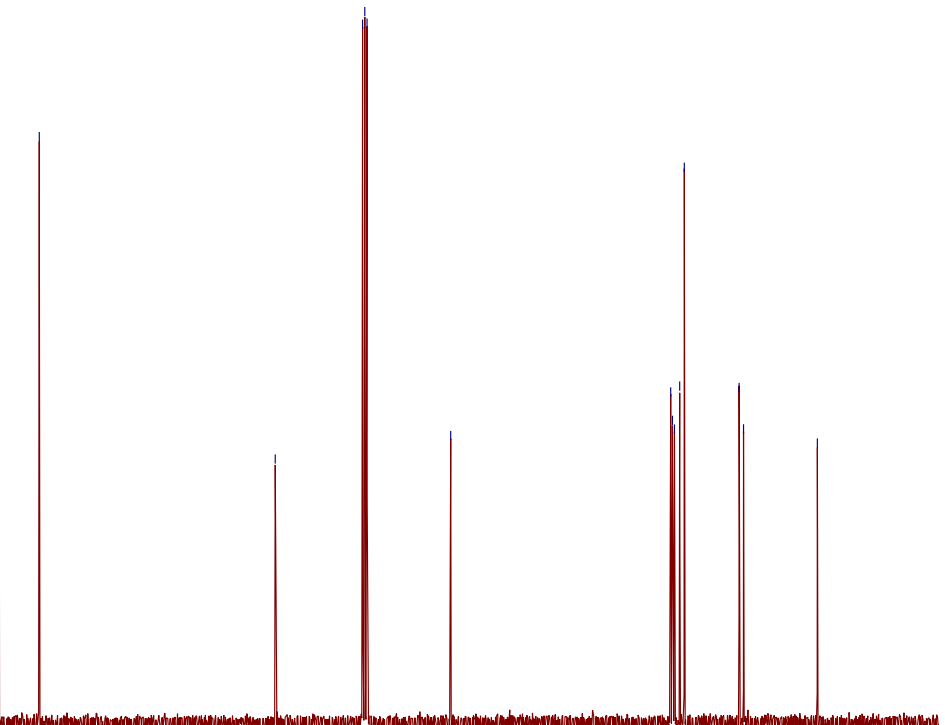

$\begin{array}{lllllllllll}210 & 200 & 190 & 180 & 170 & 160 & 150 & 140 & 130 & 120 & 110 \\ \mathrm{f} 1(\mathrm{ppm})\end{array}$ 
${ }^{1} \mathrm{H}$ NMR (400 MHz, $\mathrm{CDCl}_{3}$ ) of compound $\mathbf{2 4 a}$ (see procedure):

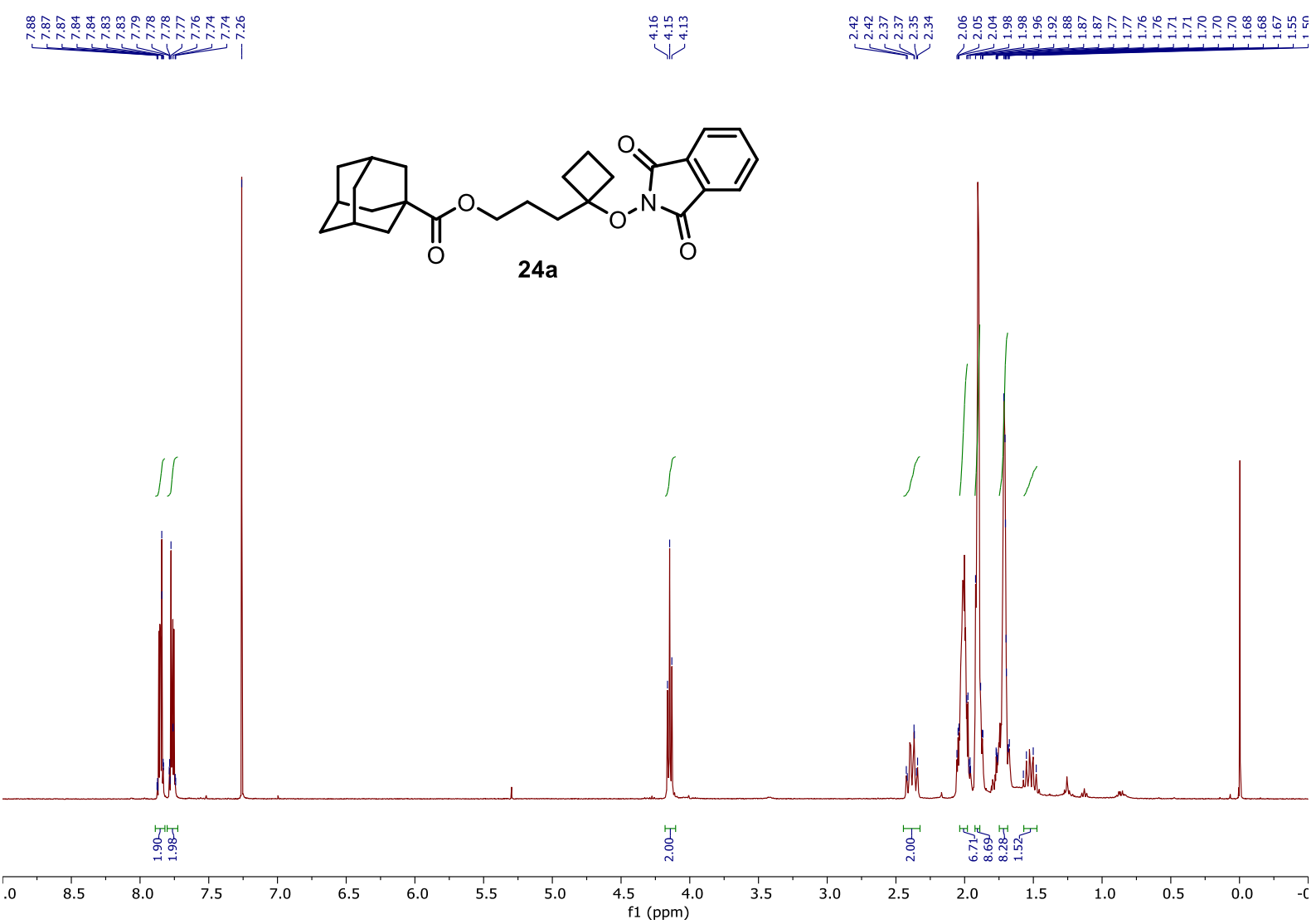

${ }^{13} \mathrm{C}$ NMR (101 MHz, $\mathrm{CDCl}_{3}$ ) of compound $24 \mathbf{a}$ (see procedure):

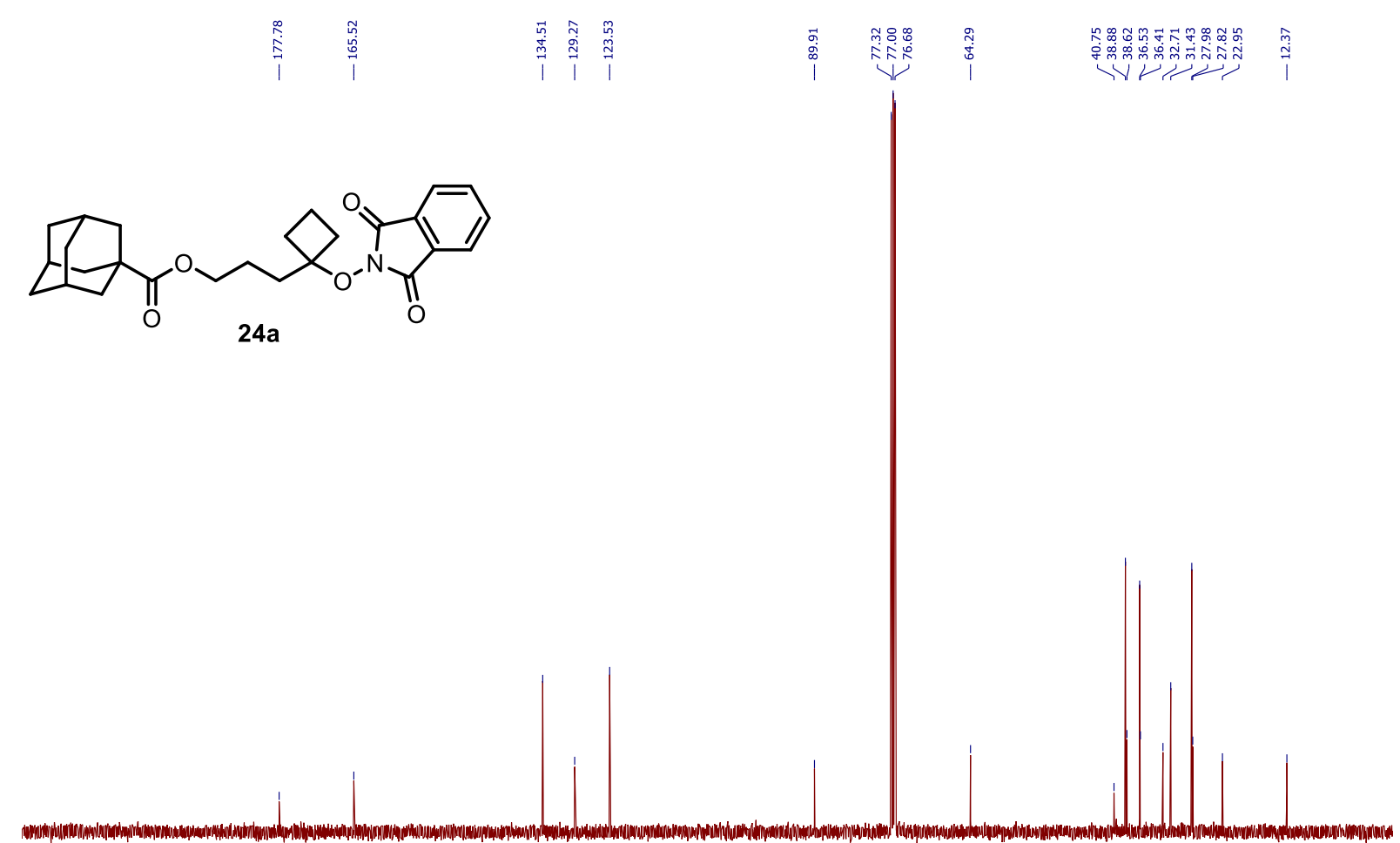

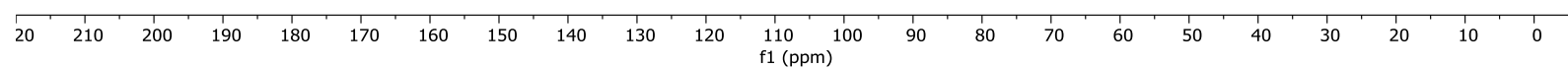


${ }^{1} \mathrm{H}$ NMR (400 MHz, $\mathrm{CDCl}_{3}$ ) of compound 25a (see procedure):

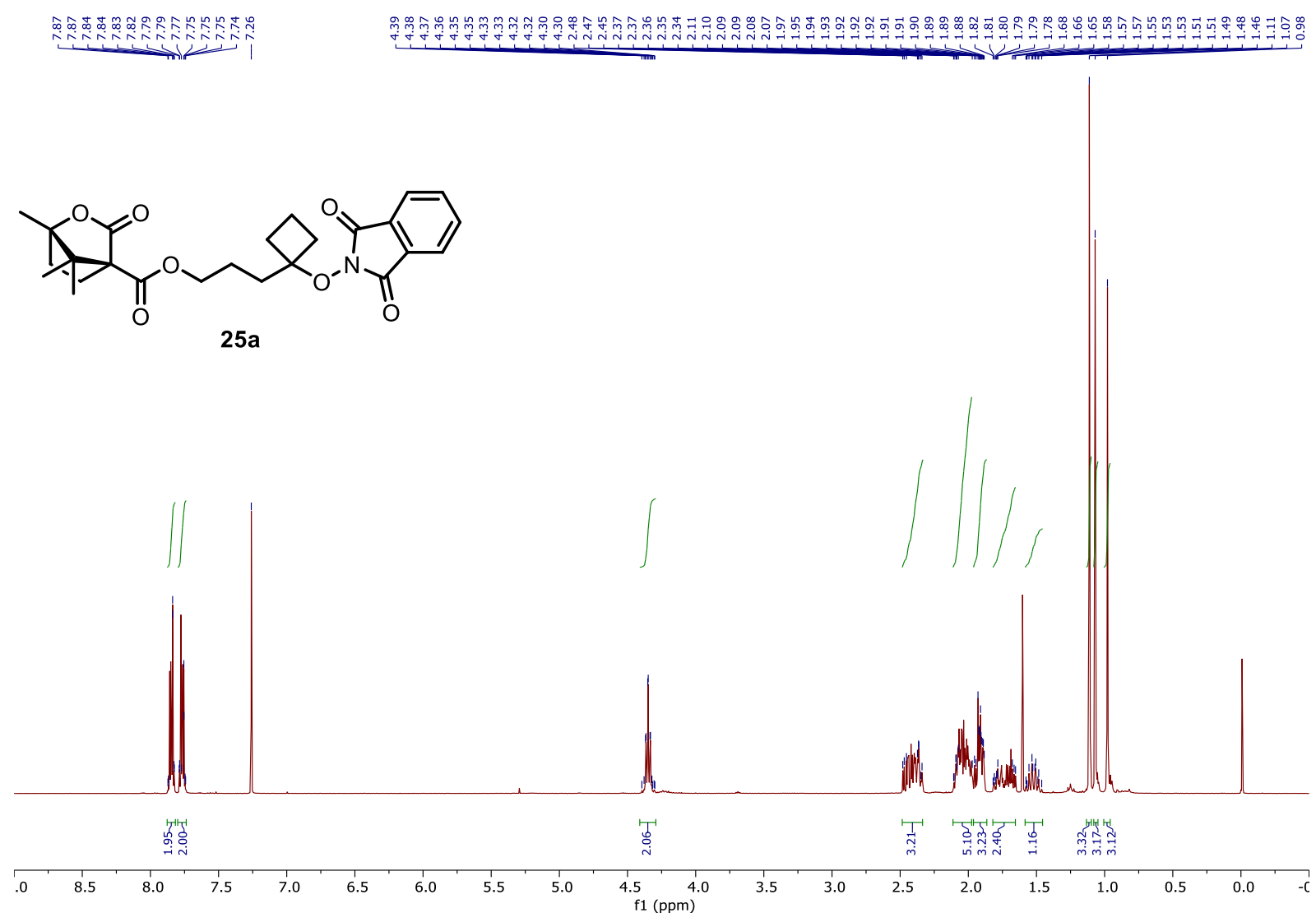

${ }^{13} \mathrm{C}$ NMR (101 MHz, $\mathrm{CDCl}_{3}$ ) of compound 25a (see procedure):<smiles>CC(C)(C)C1(C(=O)OCCCC2(C(=O)ON3C(=O)c4ccccc4C3=O)CCC2)C(=O)OC2(C)C(C)(C)C21C</smiles> 
${ }^{1} \mathrm{H}$ NMR (400 MHz, $\mathrm{CDCl}_{3}$ ) of compound 26a (see procedure):

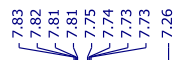

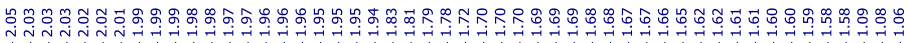<smiles>CCC1(ON2C(=O)c3ccccc3C2=O)CCCC1</smiles>

$26 \mathbf{a}$

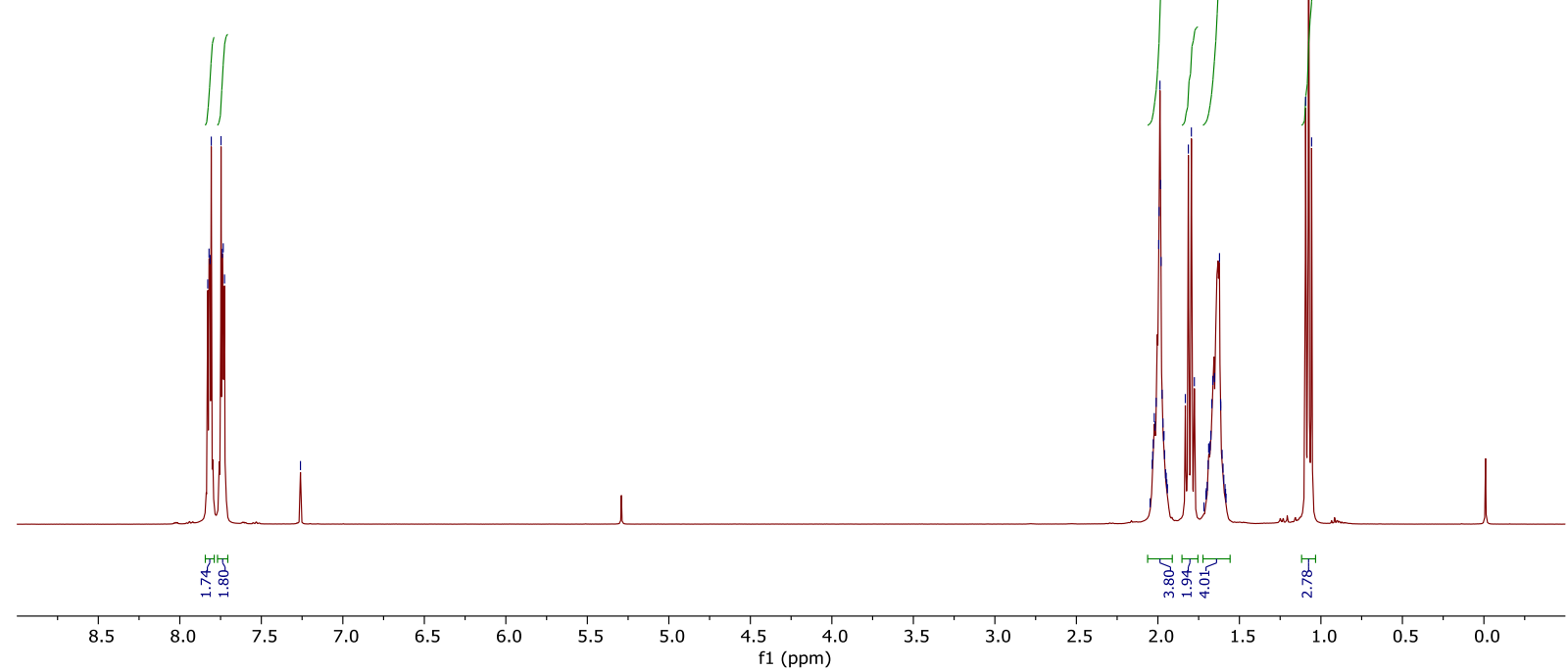

${ }^{13} \mathrm{C}$ NMR (101 MHz, $\mathrm{CDCl}_{3}$ ) of compound $26 \mathbf{a}$ (see procedure):

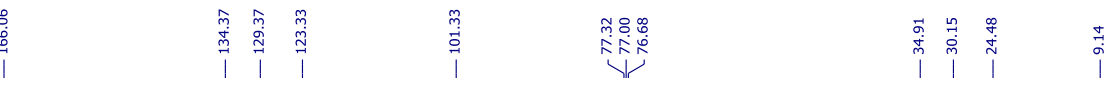<smiles>CCC1(ON2C(=O)c3ccccc3C2=O)CCCC1</smiles>

26a
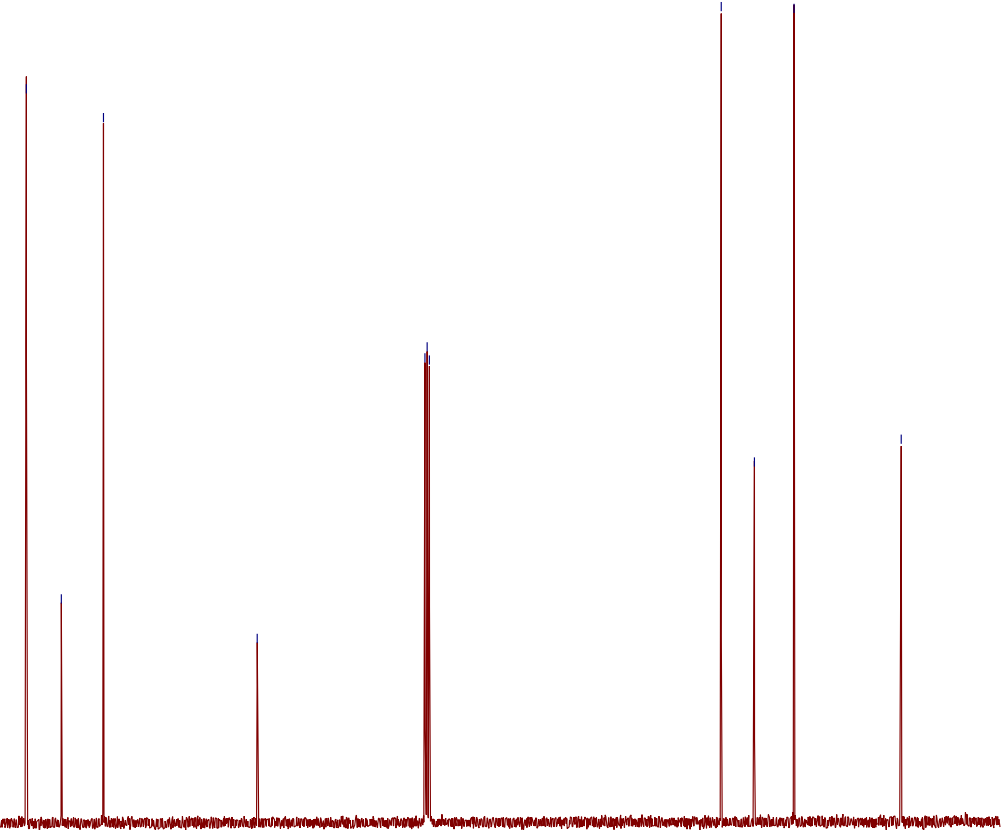

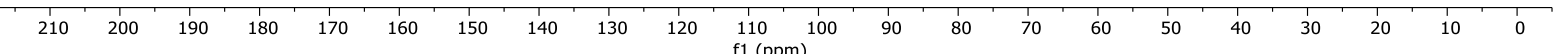


${ }^{1} \mathrm{H}$ NMR (400 MHz, $\mathrm{CDCl}_{3}$ ) of compound $27 \mathbf{a}$ (see procedure):

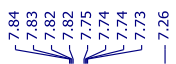

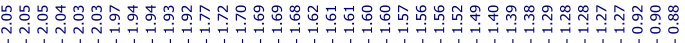

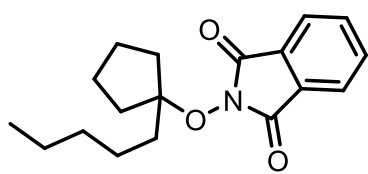

$27 a$
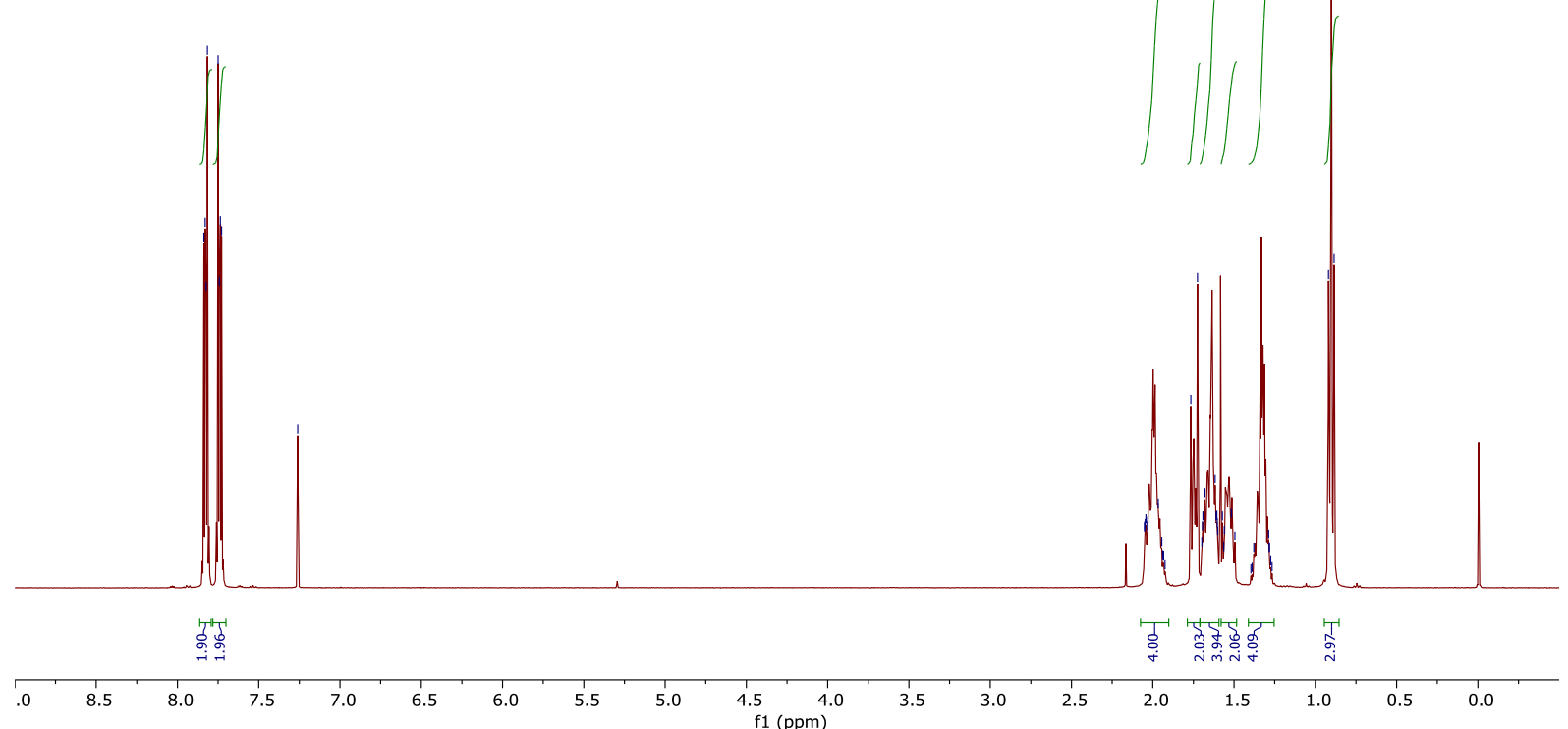

${ }^{13} \mathrm{C}$ NMR (101 MHz, $\mathrm{CDCl}_{3}$ ) of compound $27 \mathbf{a}$ (see procedure):<smiles>CCCCCC1(ON2C(=O)c3ccccc3C2=O)CCCC1</smiles>

$27 a$ 
${ }^{1} \mathrm{H}$ NMR (400 MHz, $\mathrm{CDCl}_{3}$ ) of compound $28 \mathrm{a}$ (see procedure):

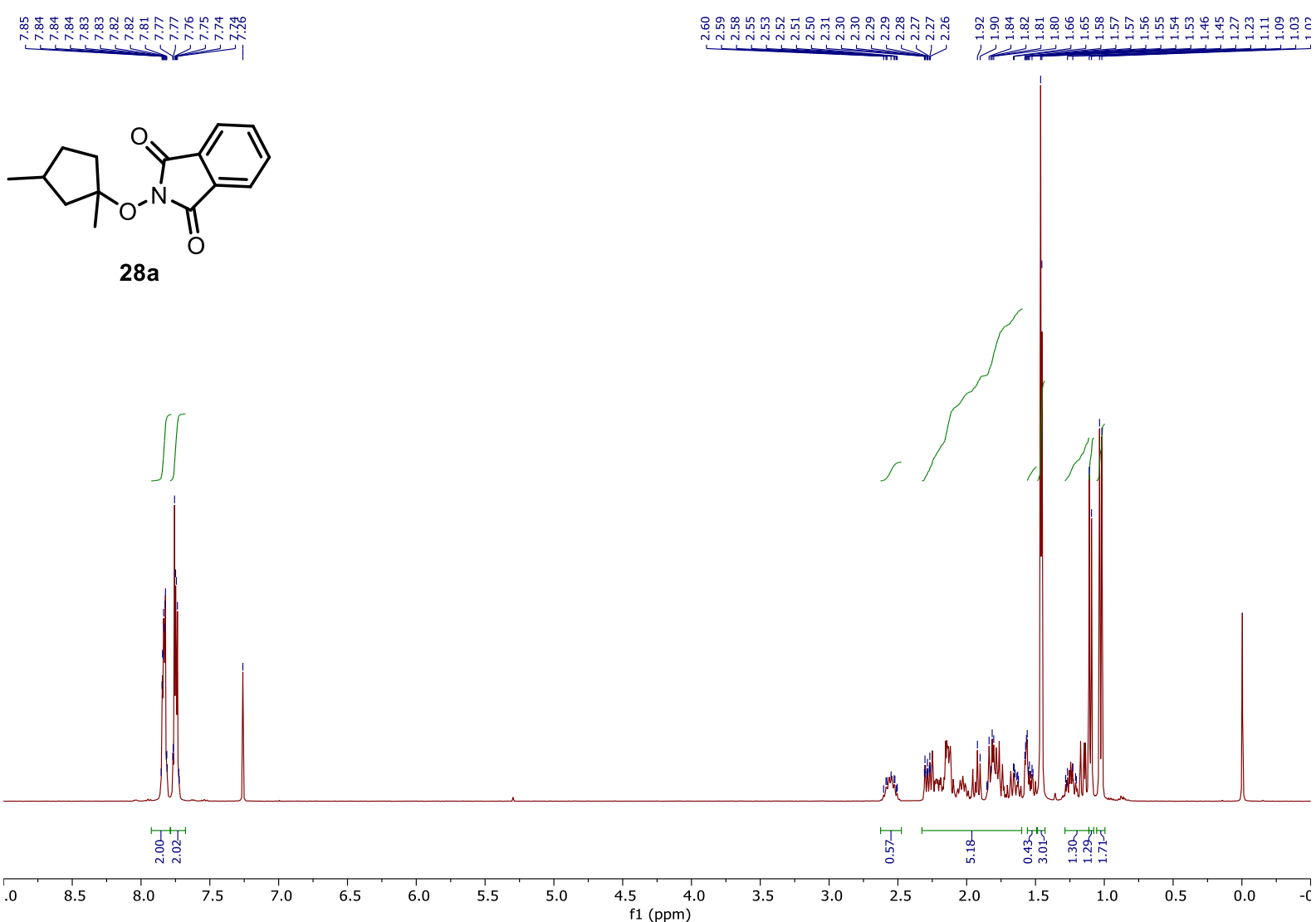

${ }^{13} \mathrm{C}$ NMR (101 MHz, $\mathrm{CDCl}_{3}$ ) of compound $28 \mathbf{a}$ (see procedure):
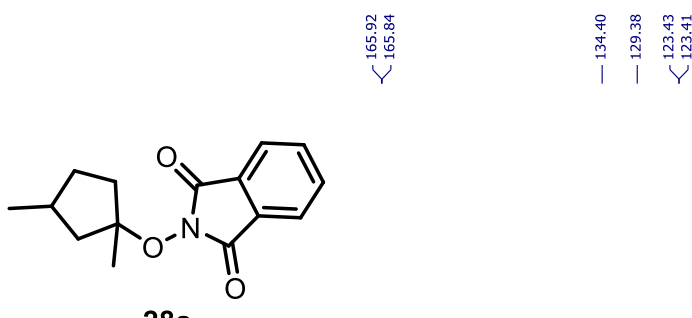

悉哭

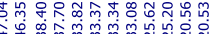

$\checkmark \underbrace{2}$

$28 a$

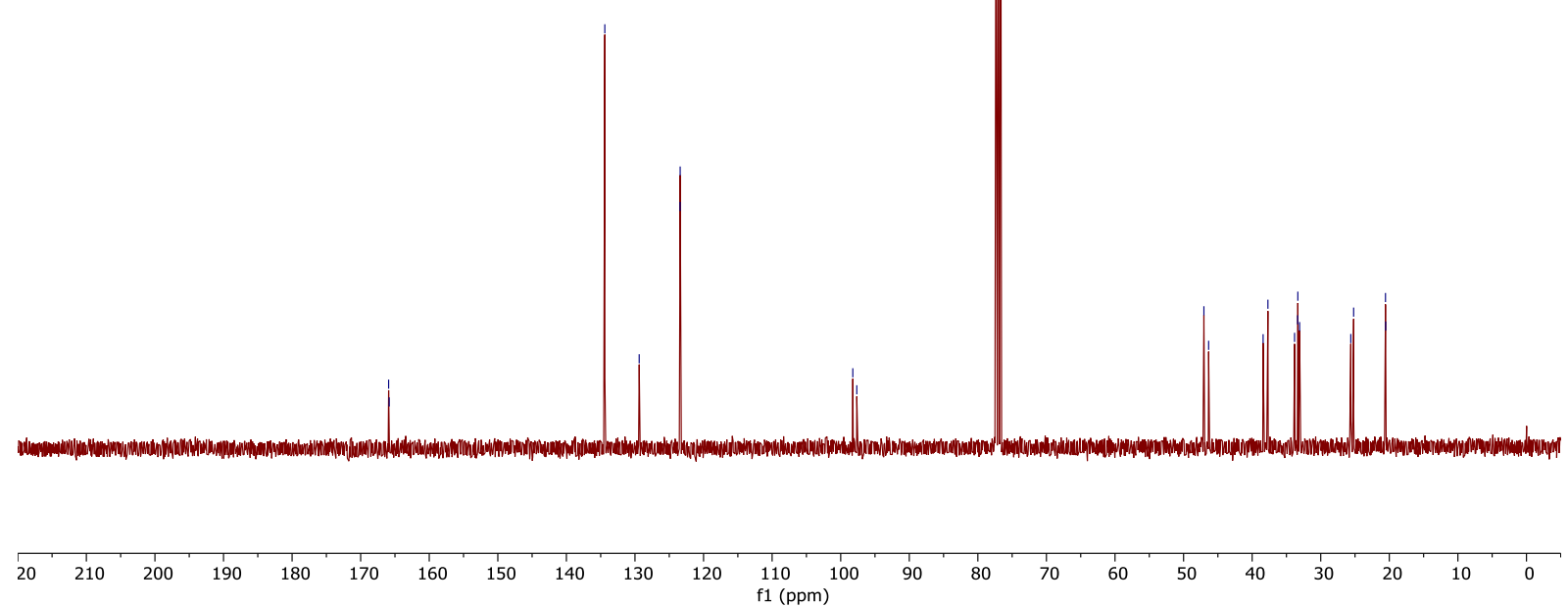


${ }^{1} \mathrm{H}$ NMR (400 MHz, $\mathrm{CDCl}_{3}$ ) of compound 29a (see procedure):

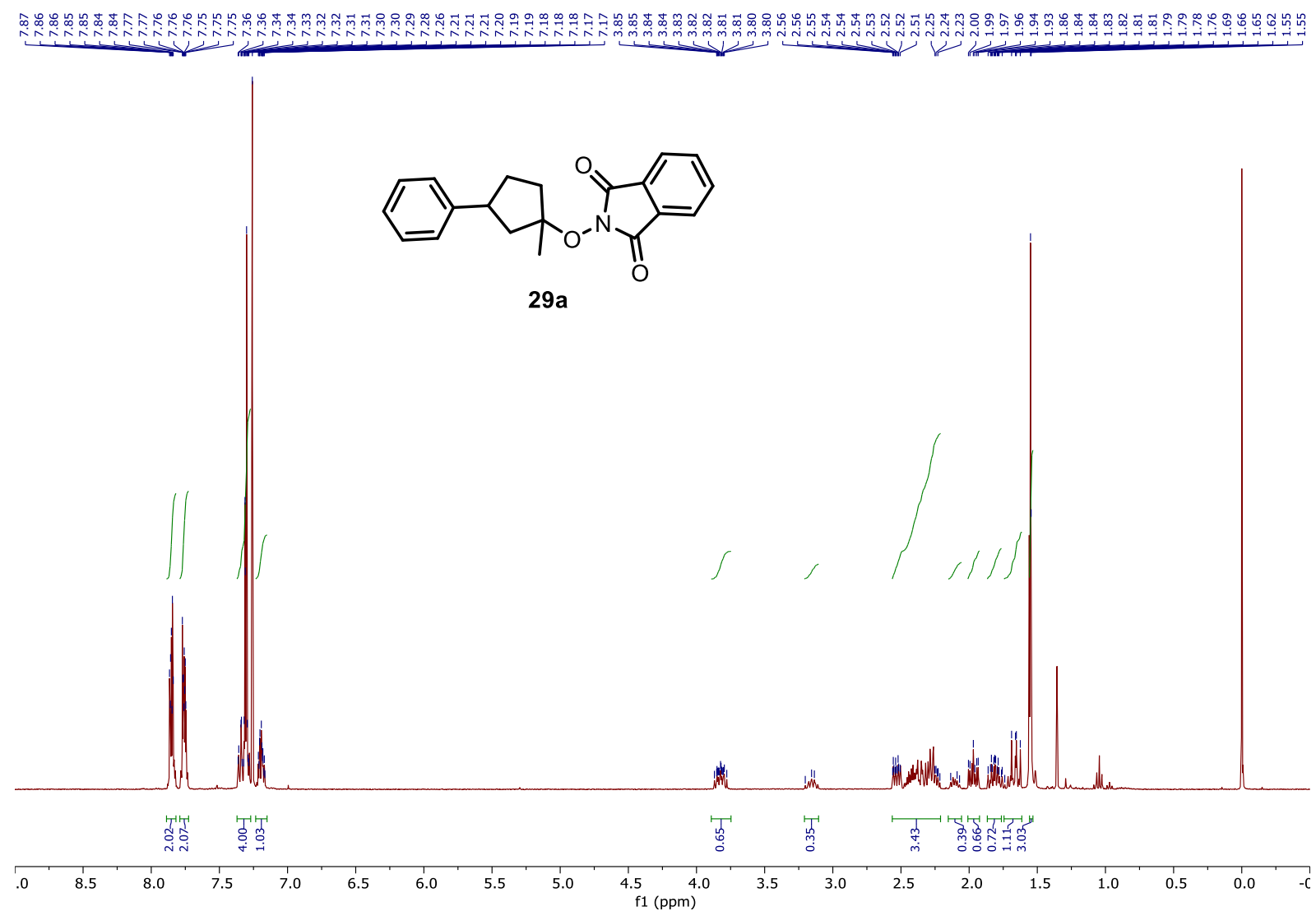

${ }^{13} \mathrm{C}$ NMR (101 MHz, $\mathrm{CDCl}_{3}$ ) of compound 29a (see procedure):<smiles>O=Cc1ccccc1</smiles>

$29 a$

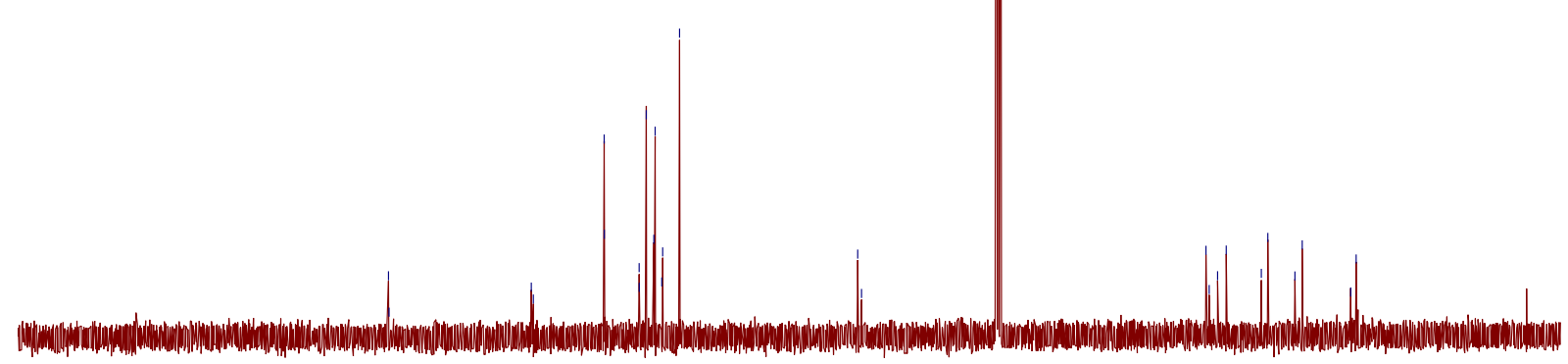

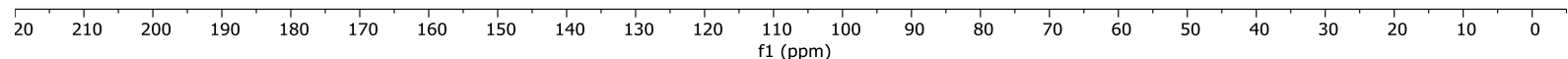


${ }^{1} \mathrm{H}$ NMR (400 MHz, $\mathrm{CDCl}_{3}$ ) of compound $\mathbf{3 0 a}$ (see procedure):

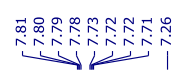

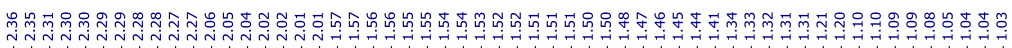
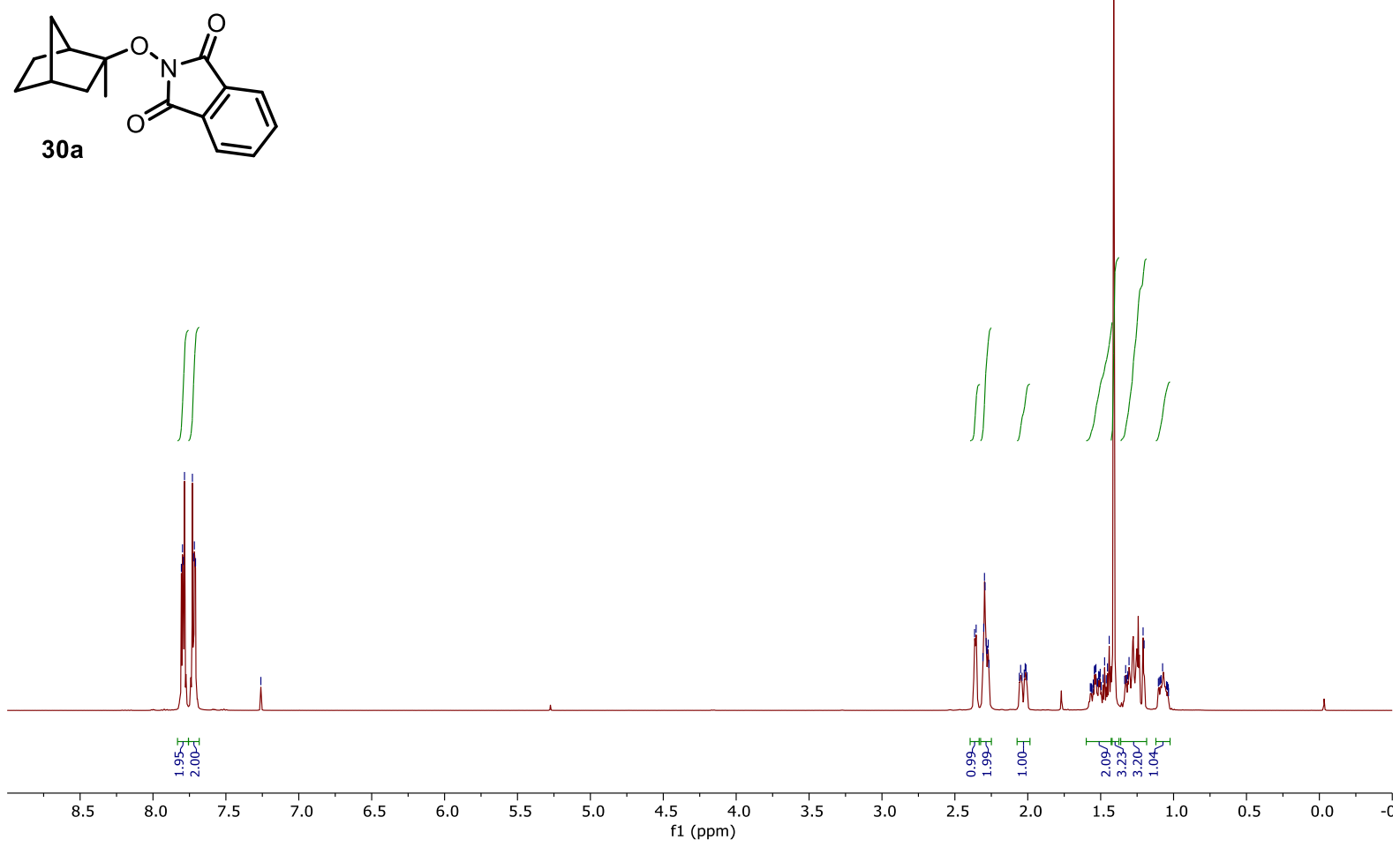

${ }^{13} \mathrm{C}$ NMR (101 MHz, $\mathrm{CDCl}_{3}$ ) of compound 30a (see procedure):

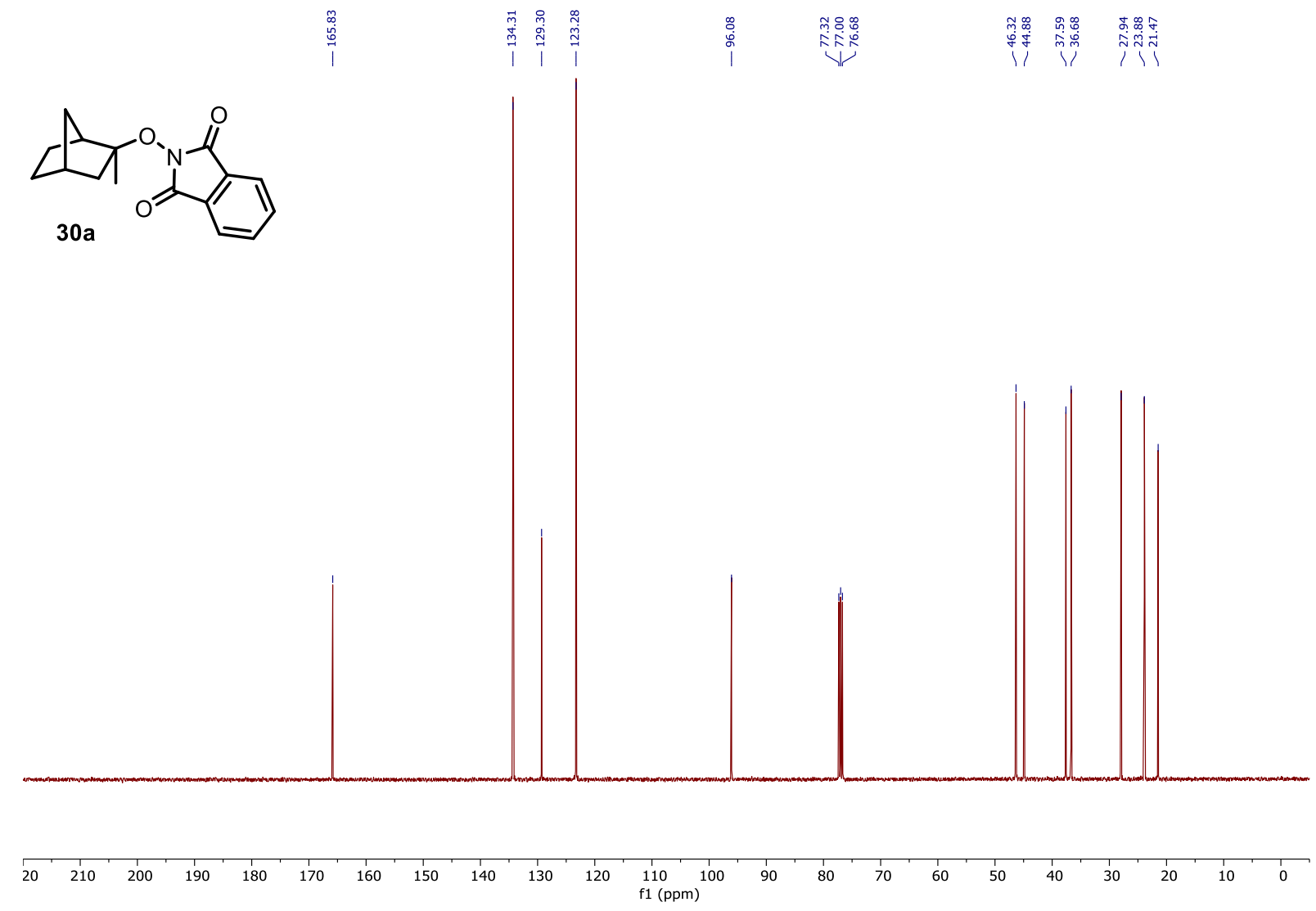

S119 
${ }^{1} \mathrm{H}$ NMR (400 MHz, $\mathrm{CDCl}_{3}$ ) of compound 31a (see procedure):

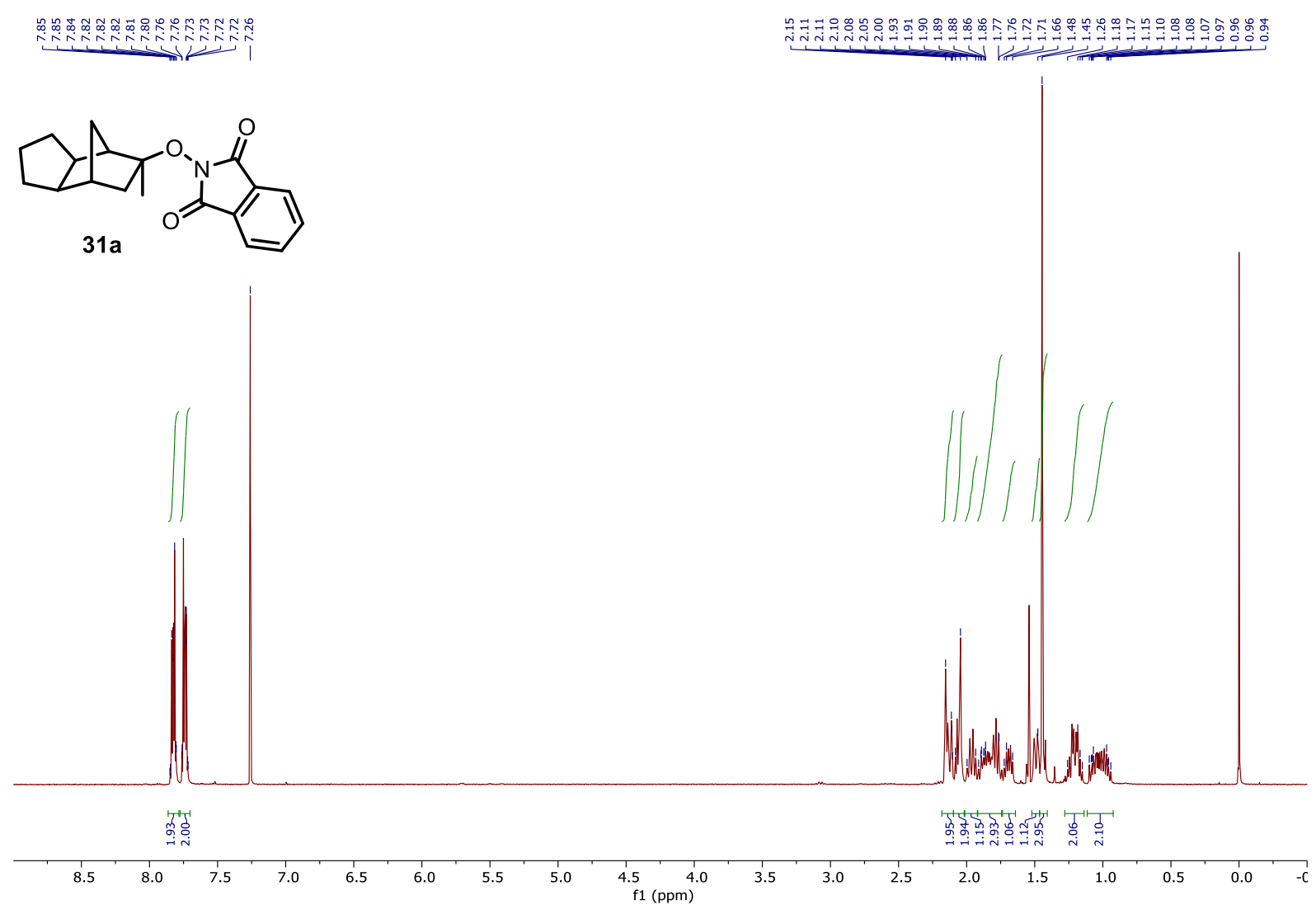

${ }^{13} \mathrm{C}$ NMR (101 MHz, $\mathrm{CDCl}_{3}$ ) of compound 31a (see procedure):
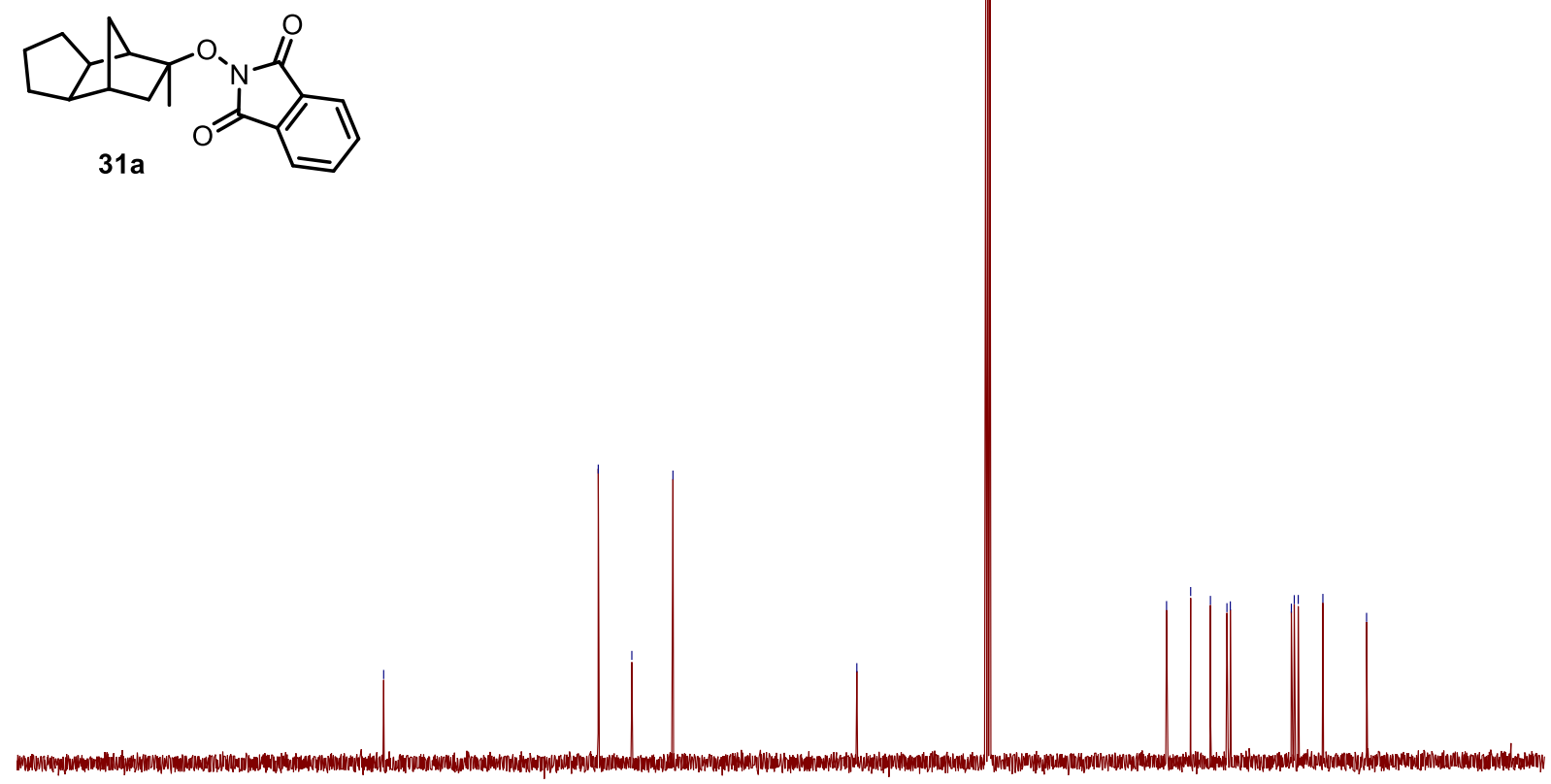

\begin{tabular}{lllllllllllllllllllllll}
\hline 20 & 210 & 200 & 190 & 180 & 170 & 160 & 150 & 140 & 130 & 120 & 110 & 100 & 90 & 80 & 70 & 60 & 50 & 40 & 30 & 20 & 10 & 0
\end{tabular} 
${ }^{1} \mathrm{H}$ NMR (400 MHz, $\mathrm{CDCl}_{3}$ ) of compound 32a (see procedure):
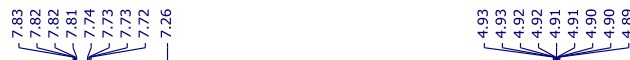

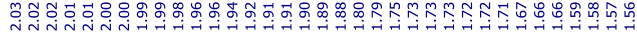

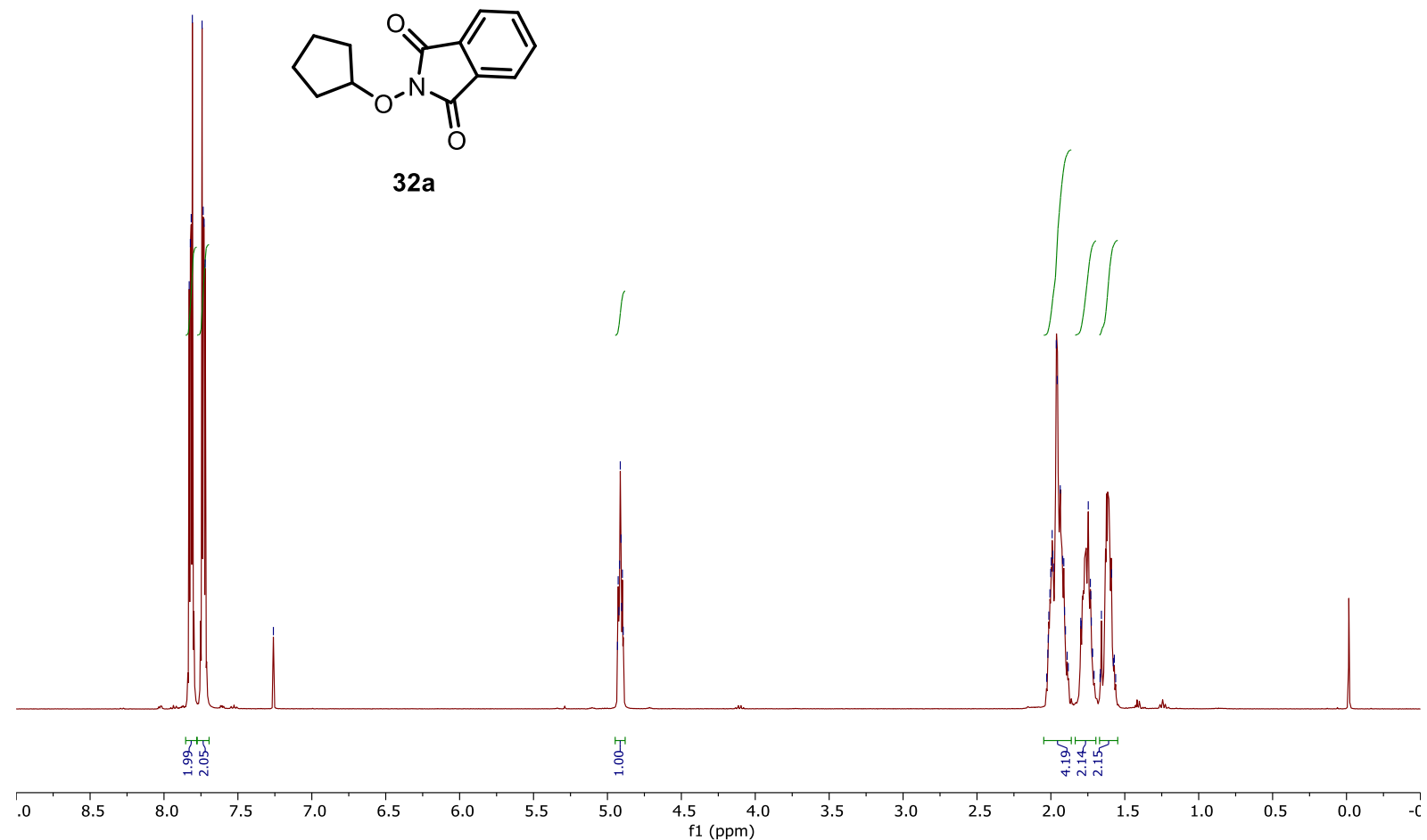

${ }^{13} \mathrm{C}$ NMR (101 MHz, $\mathrm{CDCl}_{3}$ ) of compound 32a (see procedure):

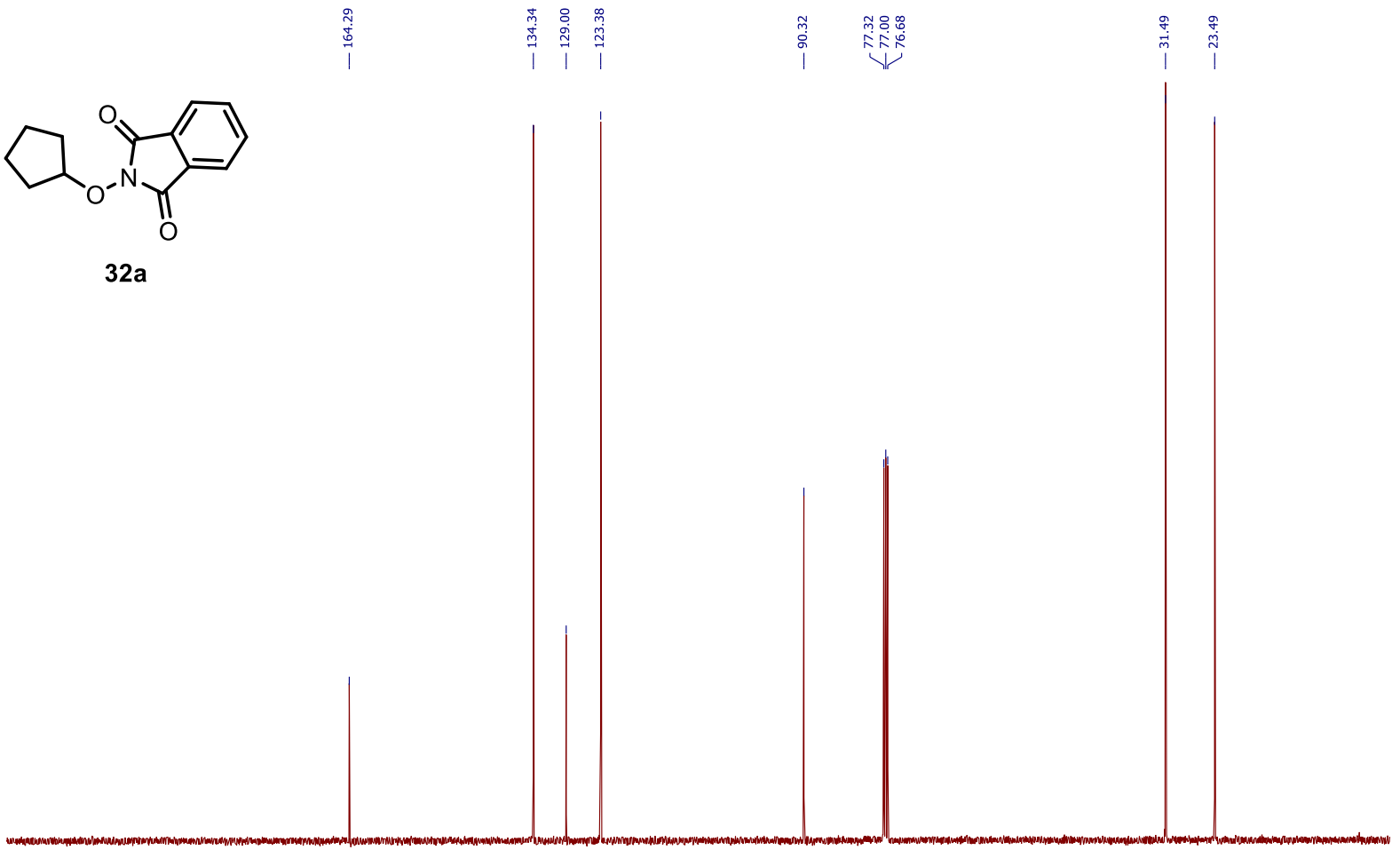

\begin{tabular}{llllllllllllllllllllllll}
\hline 20 & 210 & 200 & 190 & 180 & 170 & 160 & 150 & 140 & 130 & 120 & 110 & 100 & 90 & 80 & 70 & 60 & 50 & 40 & 30 & 20 & 10 & 0
\end{tabular} 
${ }^{1} \mathrm{H}$ NMR (400 MHz, $\mathrm{CDCl}_{3}$ ) of compound 33a (see procedure):

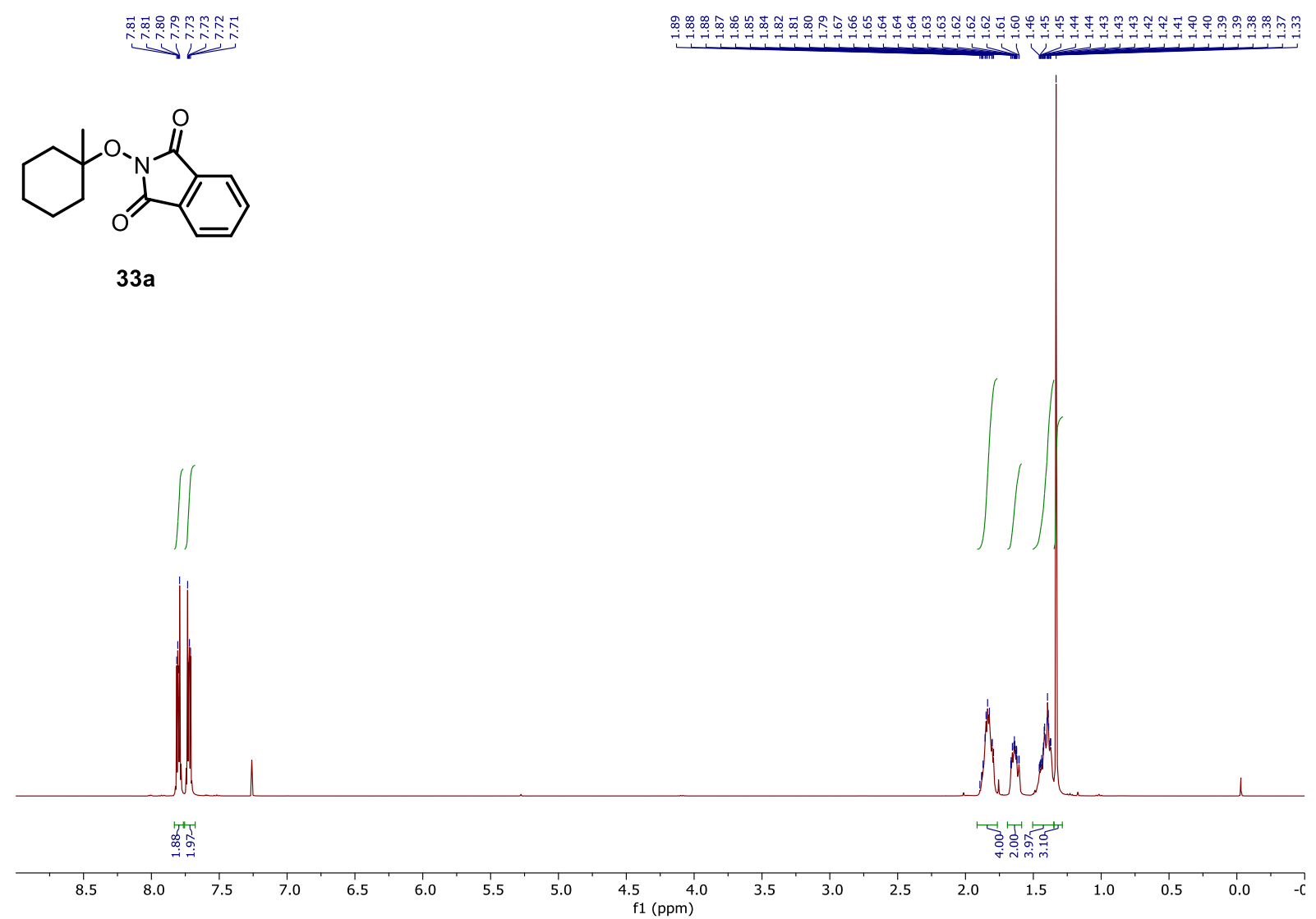

${ }^{13} \mathrm{C}$ NMR (101 MHz, $\mathrm{CDCl}_{3}$ ) of compound 33a (see procedure):

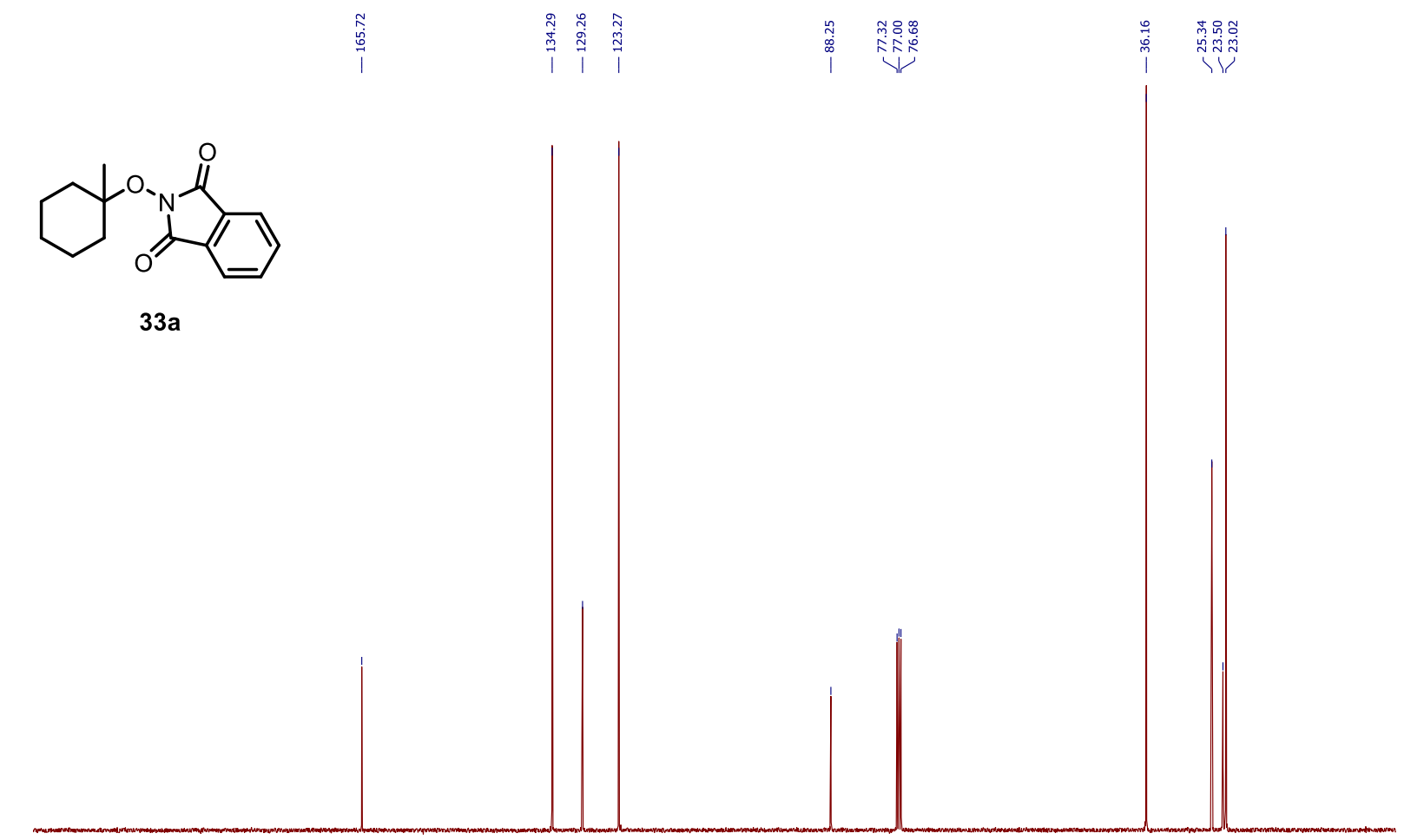

\begin{tabular}{lllllllllllllllllllllllllllll}
\hline & 210 & 200 & 190 & 180 & 170 & 160 & 150 & 140 & 130 & 120 & 110 & 100 & 90 & 80 & 70 & 60 & 50 & 40 & 30 & 20 & 10 & 0
\end{tabular} 
${ }^{1} \mathrm{H}$ NMR (400 MHz, $\mathrm{CDCl}_{3}$ ) of compound $\mathbf{3 4 a}$ (see procedure):

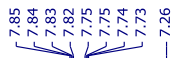<smiles>CC1CCC(C)(ON2C(=O)c3ccccc3C2=O)CC1</smiles>

$34 a$

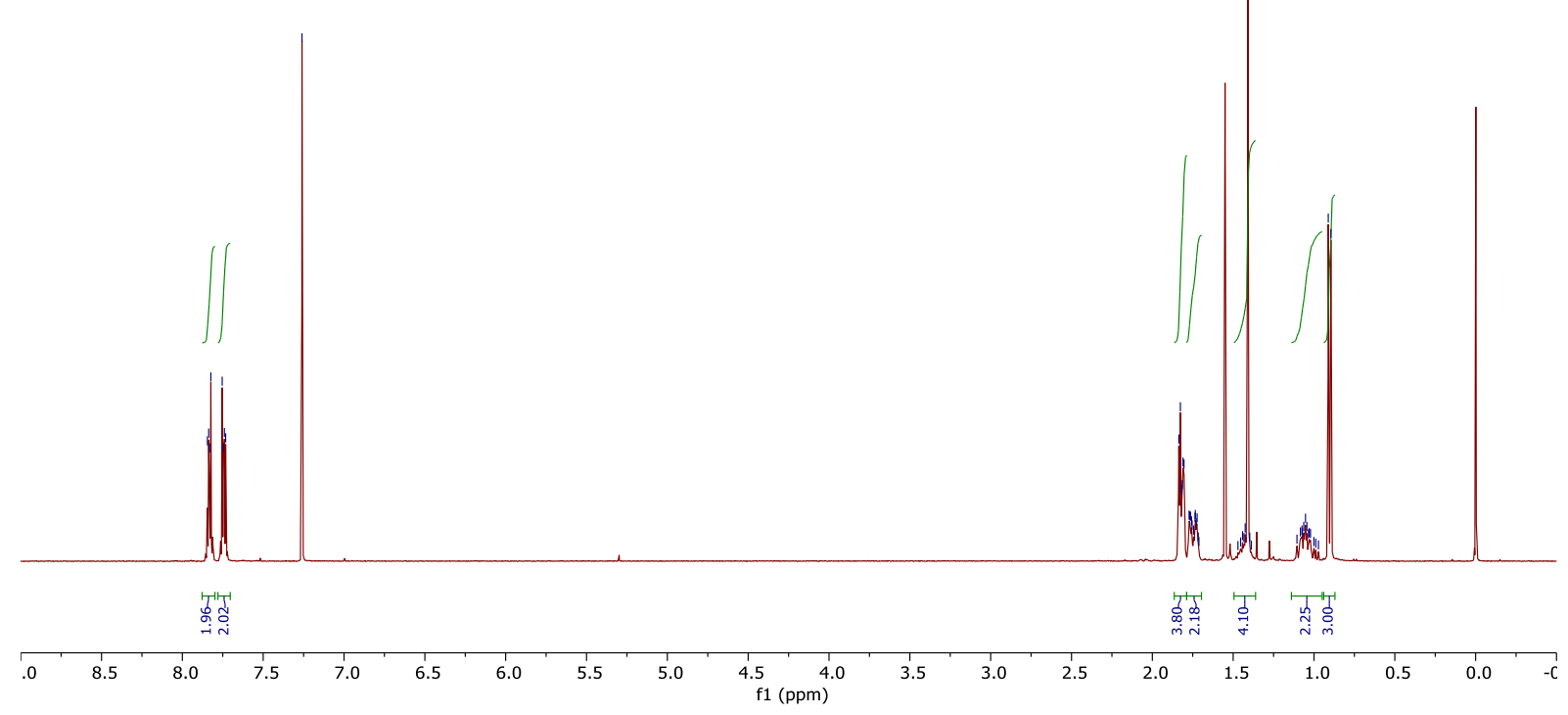

${ }^{13} \mathrm{C}$ NMR (101 MHz, $\mathrm{CDCl}_{3}$ ) of compound 34a (see procedure):

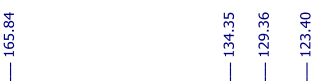<smiles>CC1CCC(C)(ON2C(=O)c3ccccc3C2=O)CC1</smiles>

$34 a$

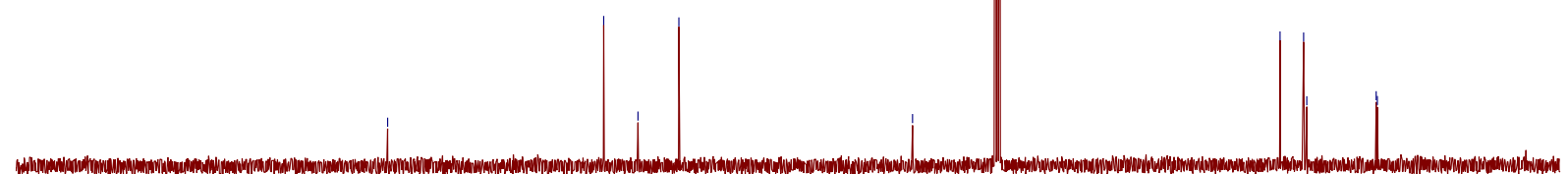

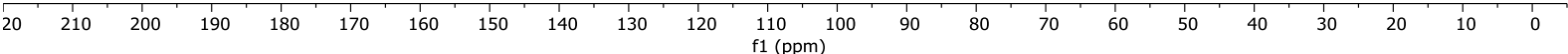


${ }^{1} \mathrm{H}$ NMR (400 MHz, $\mathrm{CDCl}_{3}$ ) of compound 35a (see procedure):

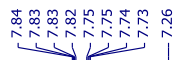

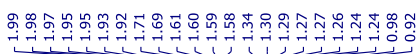

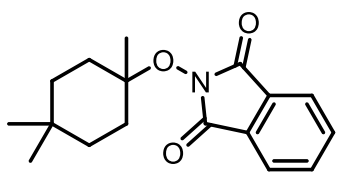

$35 a$
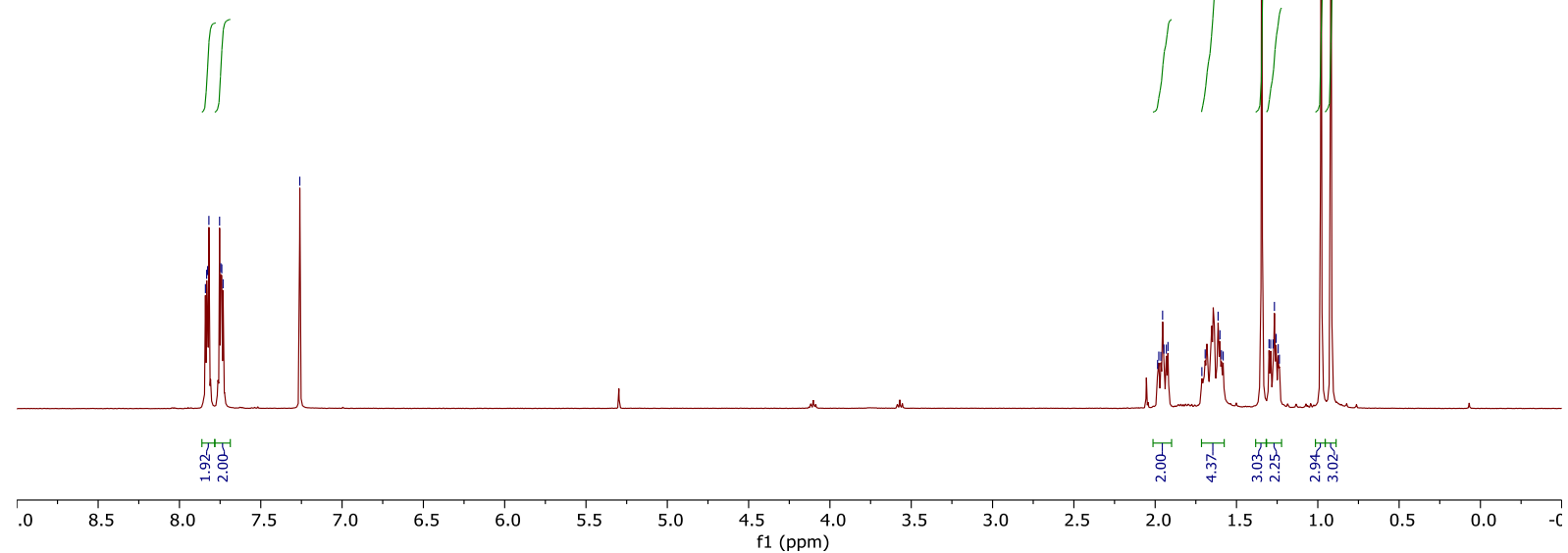

${ }^{13} \mathrm{C}$ NMR (101 MHz, $\mathrm{CDCl}_{3}$ ) of compound 35a (see procedure):
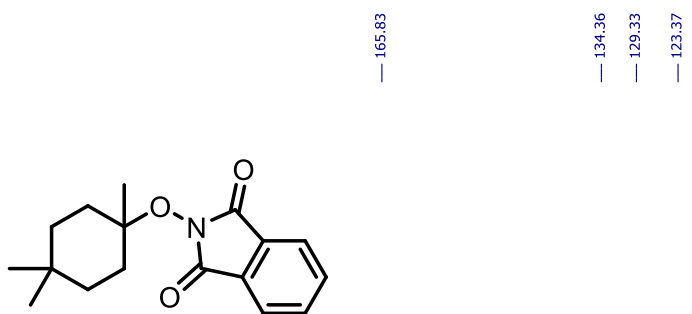

$35 a$

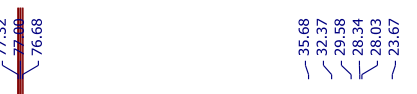

(1)

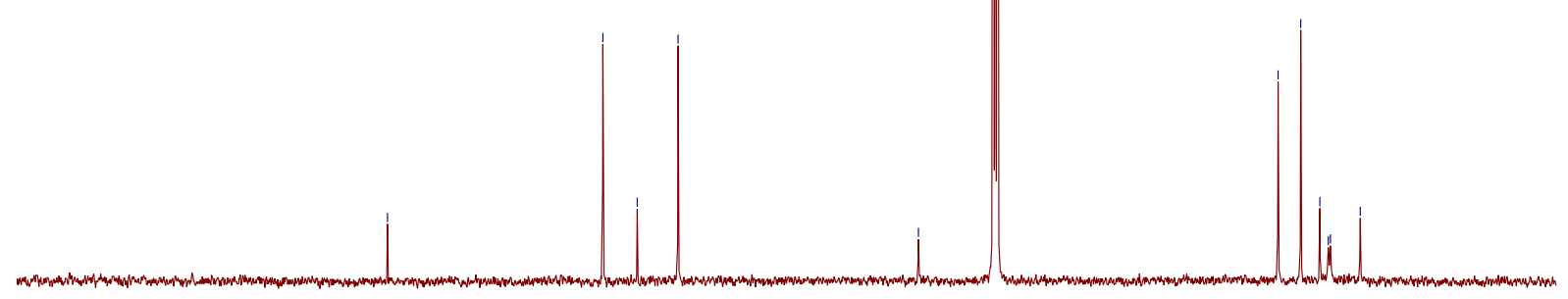

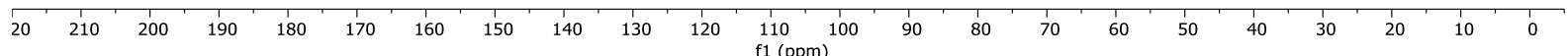


${ }^{1} \mathrm{H}$ NMR (400 MHz, $\mathrm{CDCl}_{3}$ ) of compound 36a (see procedure):

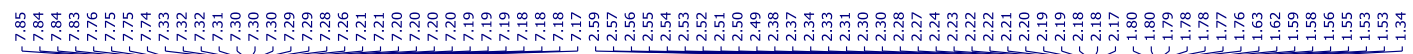

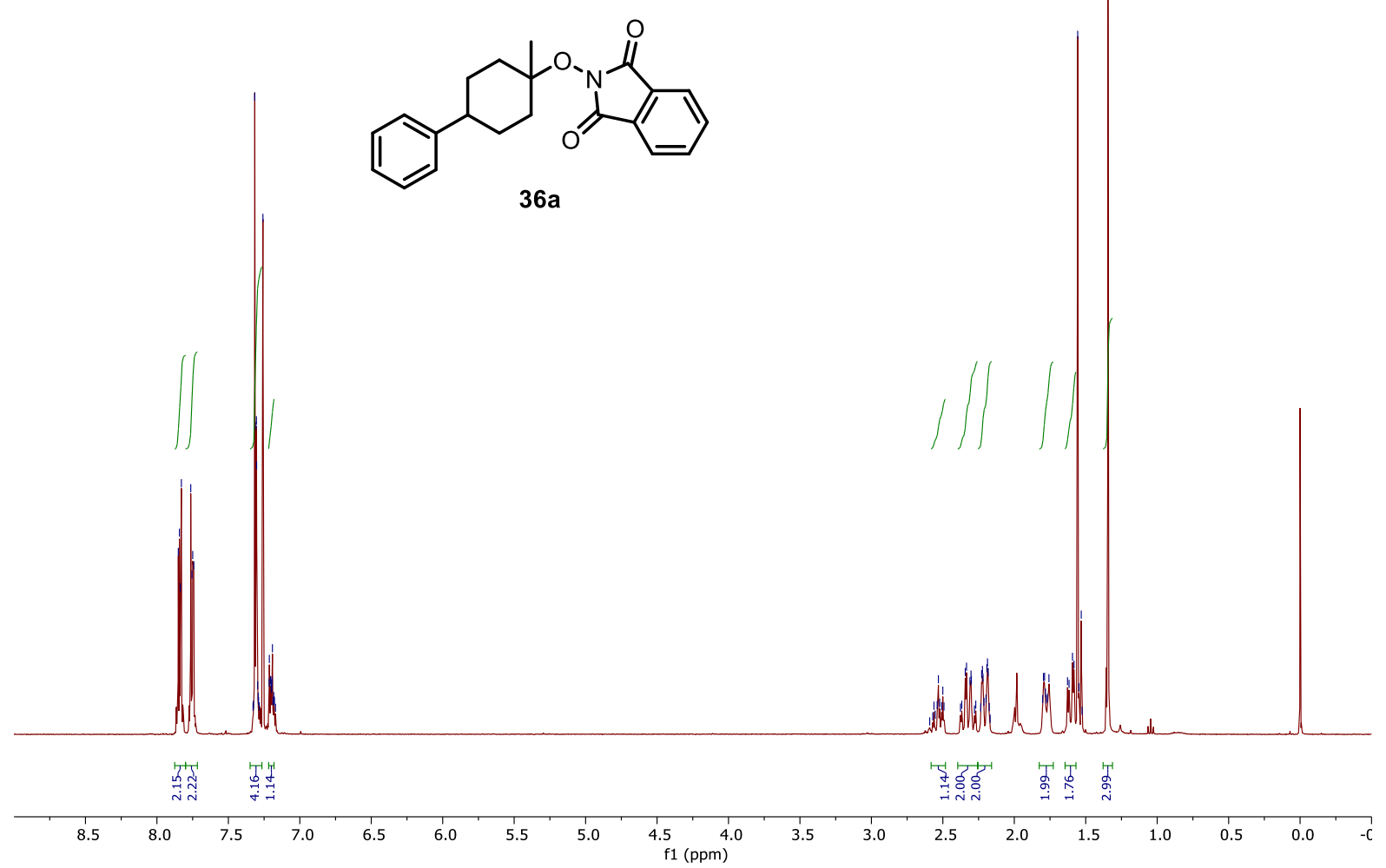

${ }^{13} \mathrm{C}$ NMR (101 MHz, $\mathrm{CDCl}_{3}$ ) of compound 36a (see procedure):<smiles>CC1(ON2C(=O)c3ccccc3C2=O)CCC(c2ccccc2)CC1</smiles>

$36 a$

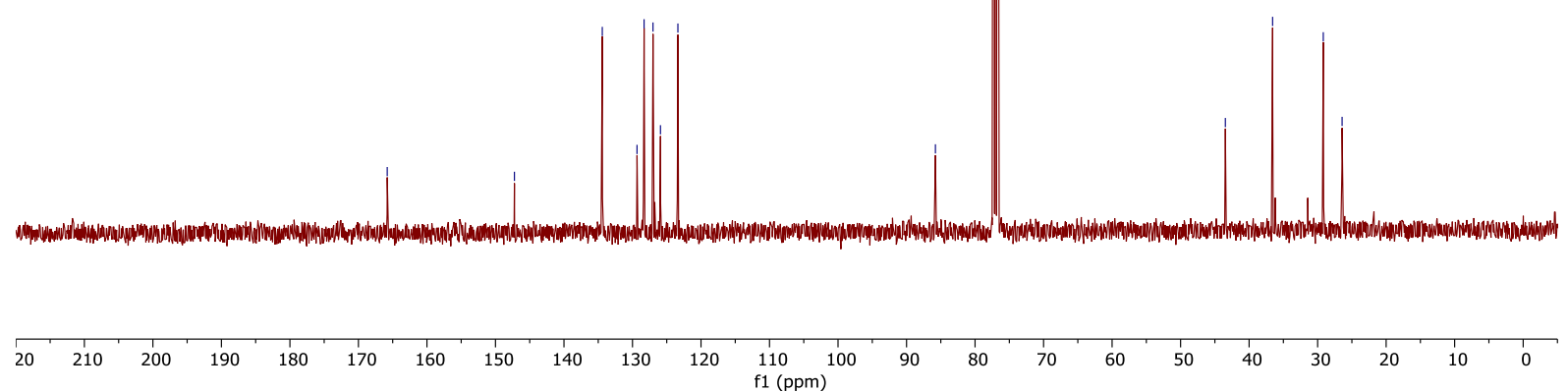


${ }^{1} \mathrm{H}$ NMR (400 MHz, $\mathrm{CDCl}_{3}$ ) of compound 37a (see procedure):

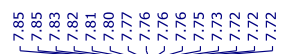<smiles>CC1(ON2C(=O)c3ccccc3C2=O)CCCCCC1</smiles>

$37 a$
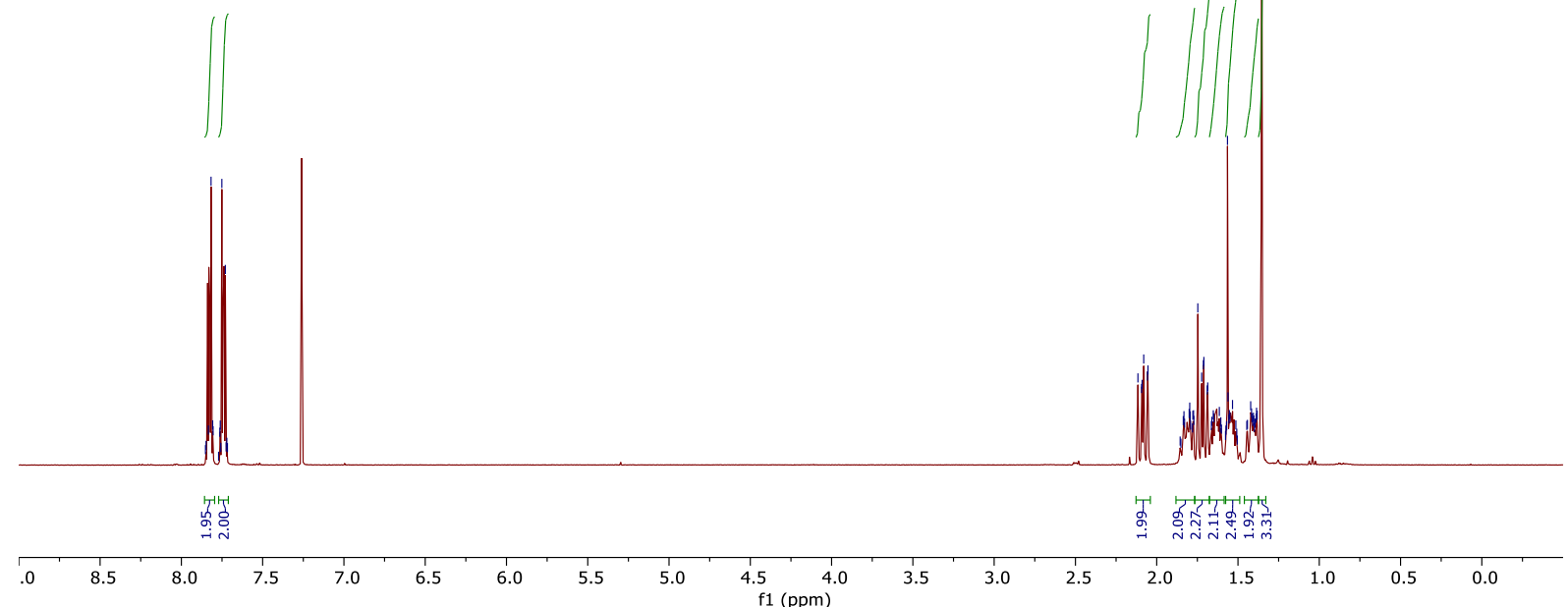

${ }^{13} \mathrm{C} \mathrm{NMR}\left(101 \mathrm{MHz}, \mathrm{CDCl}_{3}\right)$ of compound 37a (see procedure)

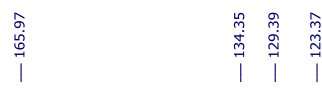<smiles>CC1(ON2C(=O)c3ccccc3C2=O)CCCCC1</smiles>

$37 a$

$\stackrel{\substack{1 \\ \infty}}{\substack{n \\ 0}}$

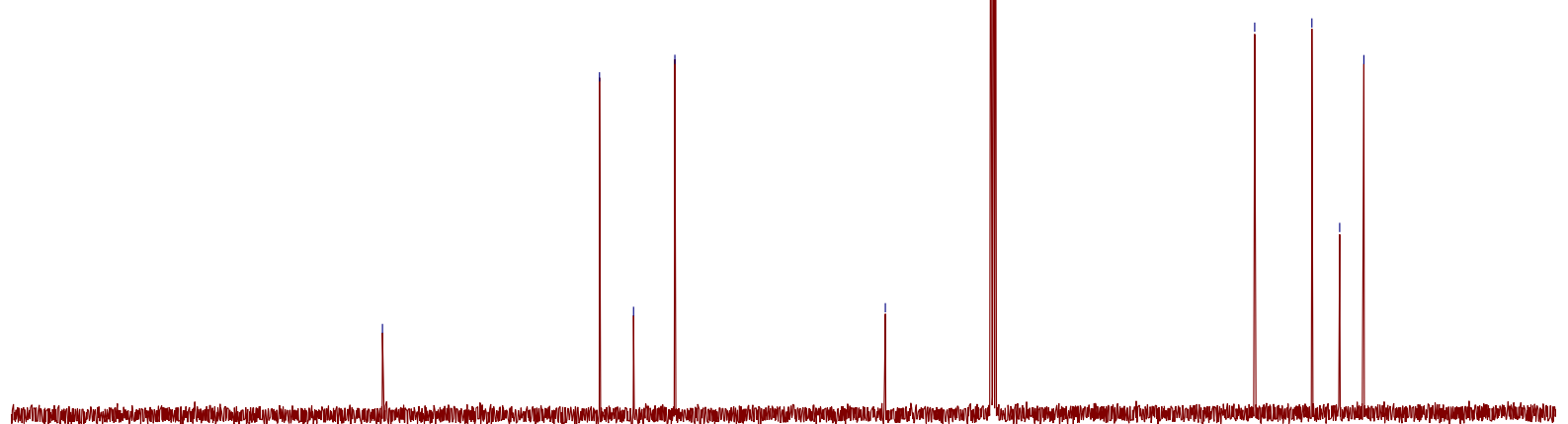

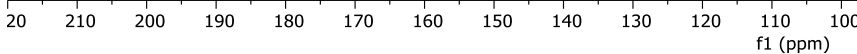


${ }^{1} \mathrm{H}$ NMR (400 MHz, $\mathrm{CDCl}_{3}$ ) of compound 38a (see procedure):

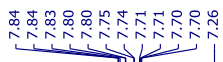

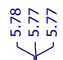

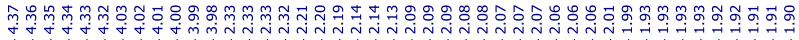<smiles>O=C1c2ccccc2C(=O)N1OC1CCCO1</smiles>

$38 \mathbf{a}$

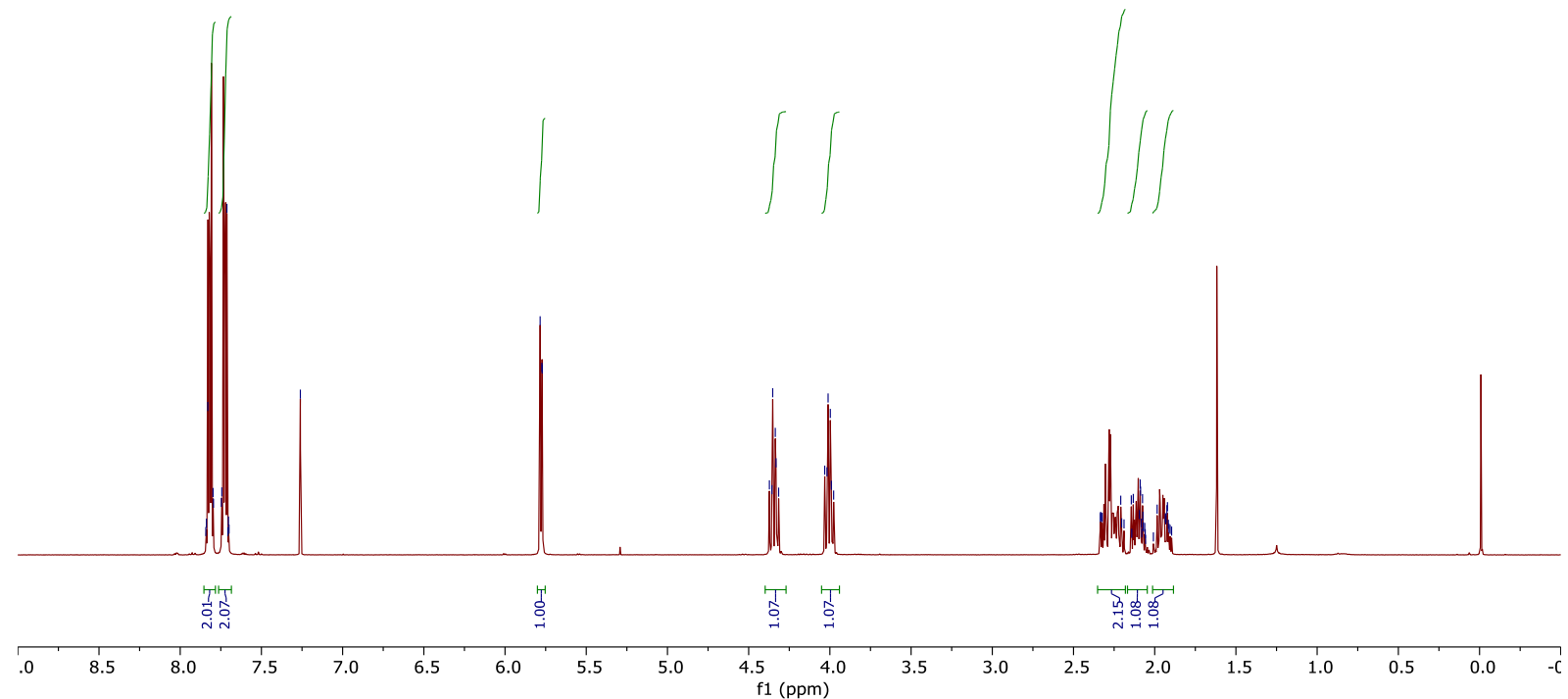

${ }^{13} \mathrm{C}$ NMR (101 MHz, $\mathrm{CDCl}_{3}$ ) of compound 38a (see procedure):

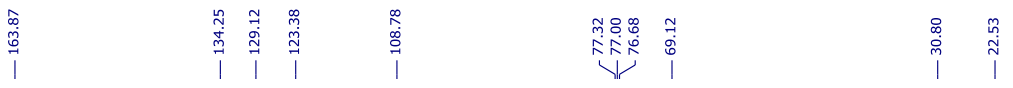<smiles>O=C1c2ccccc2C(=O)N1OC1CCCO1</smiles>

38a

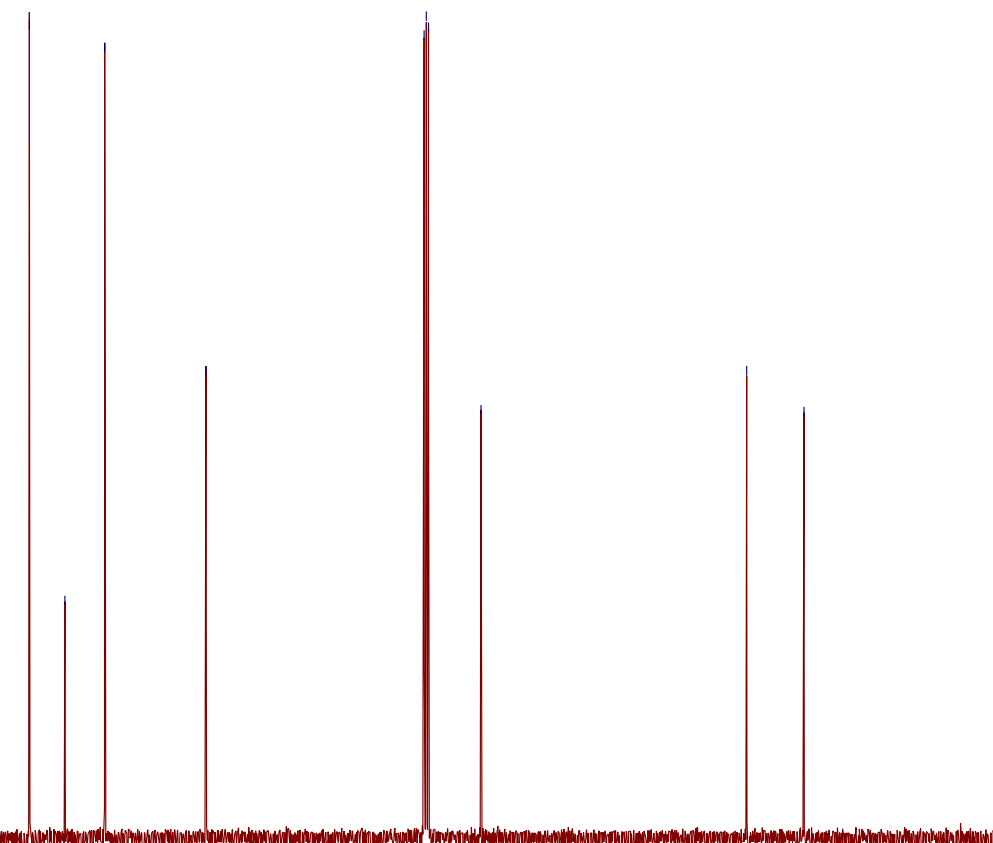

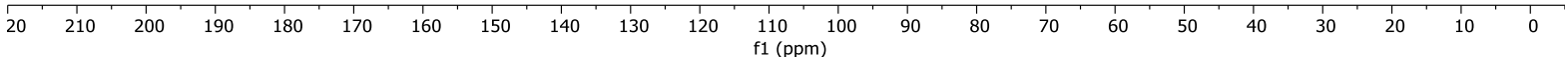


${ }^{1} \mathrm{H}$ NMR (400 MHz, $\mathrm{CDCl}_{3}$ ) of compound 39a (see procedure):

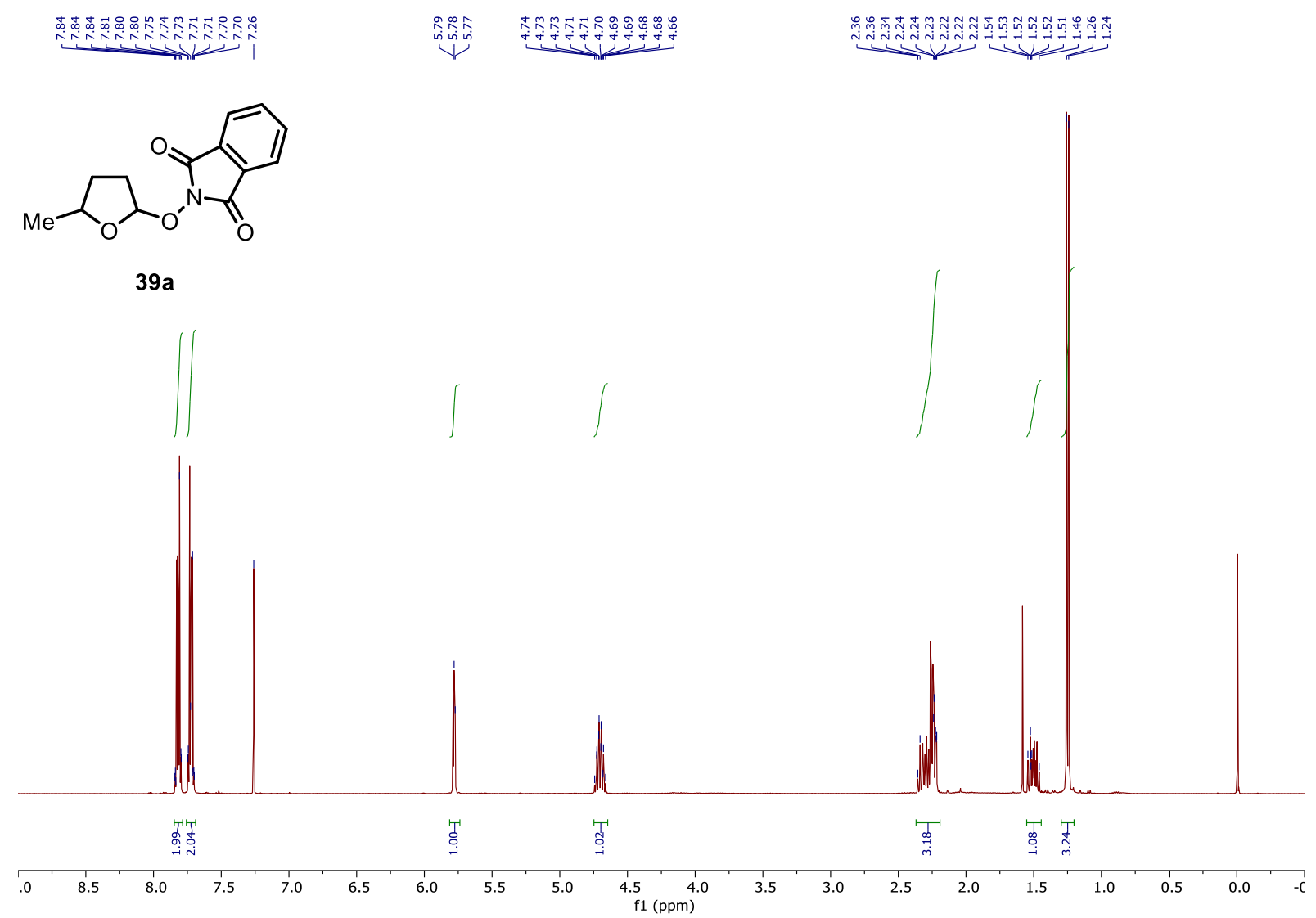

${ }^{13} \mathrm{C}$ NMR (101 MHz, $\mathrm{CDCl}_{3}$ ) of compound 39a (see procedure):

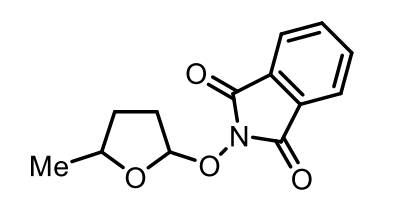

$39 a$

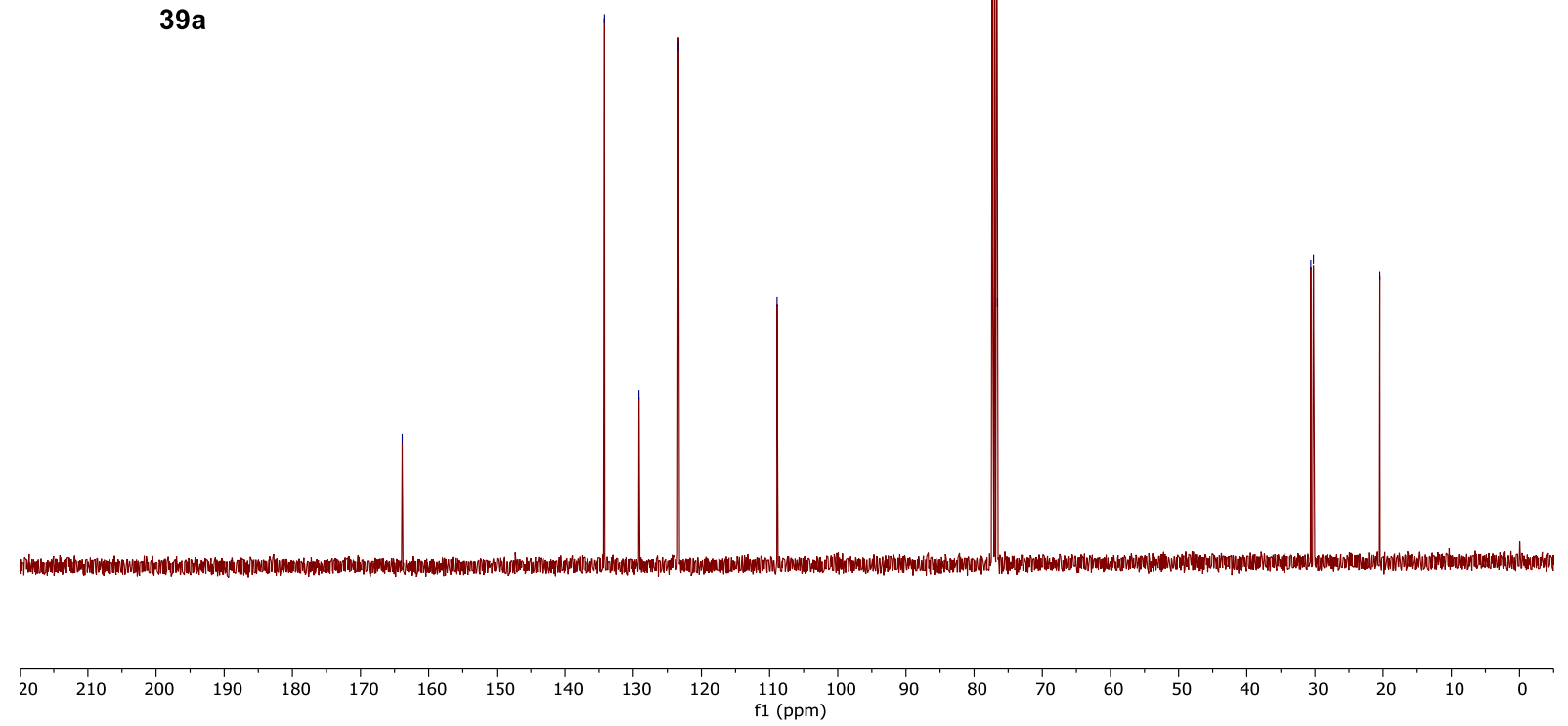


${ }^{1} \mathrm{H}$ NMR (400 MHz, $\mathrm{CDCl}_{3}$ ) of compound 40a (see procedure):

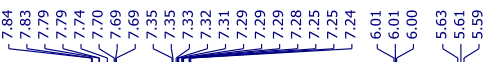

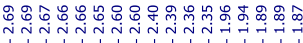

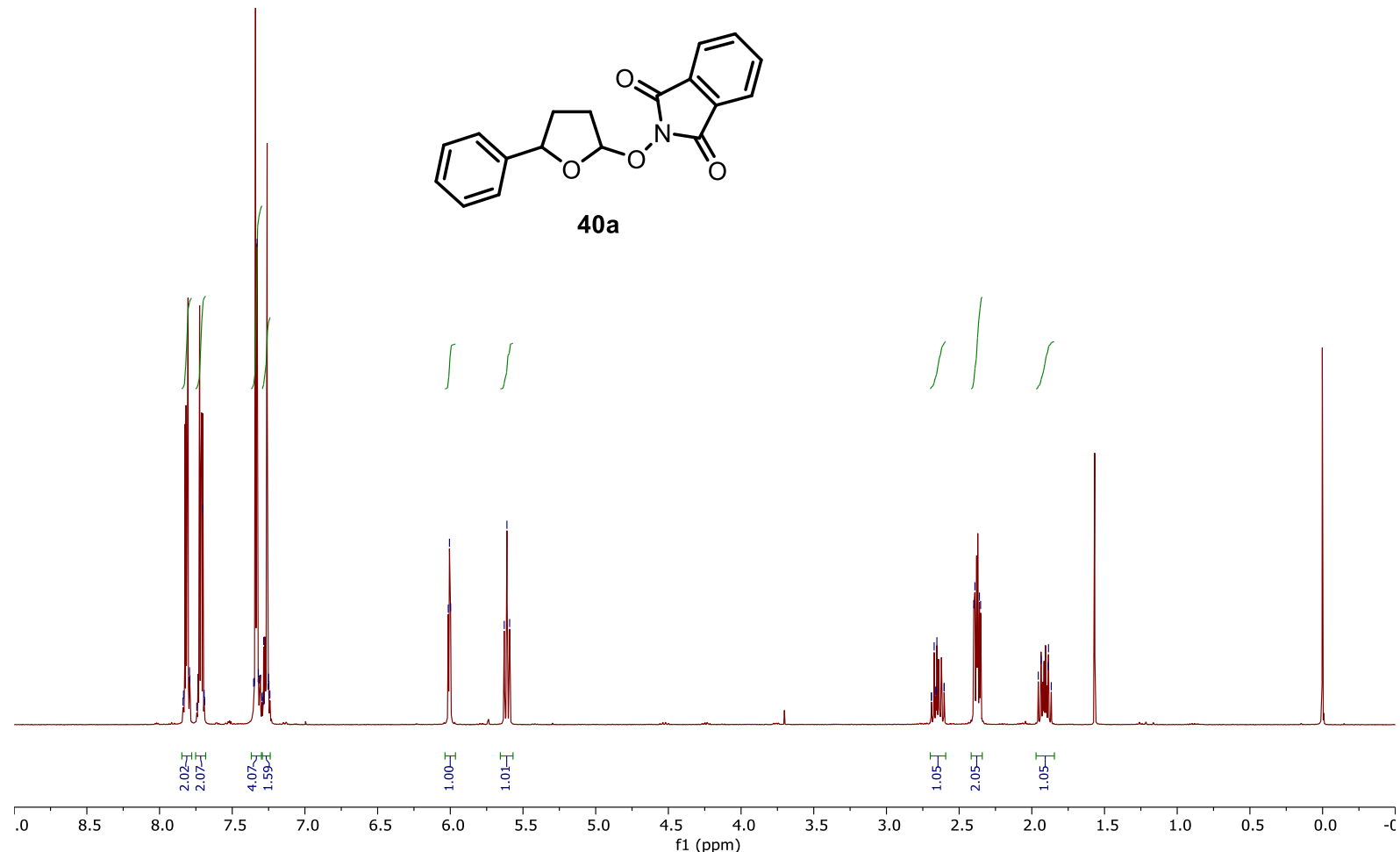

${ }^{13} \mathrm{C}$ NMR (101 MHz, $\mathrm{CDCl}_{3}$ ) of compound 40a (see procedure):

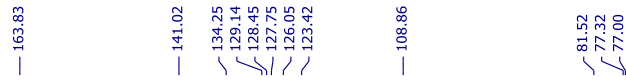

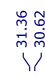<smiles>O=C1c2ccccc2C(=O)N1OC1CCC(c2ccccc2)O1</smiles>

$40 \mathrm{a}$

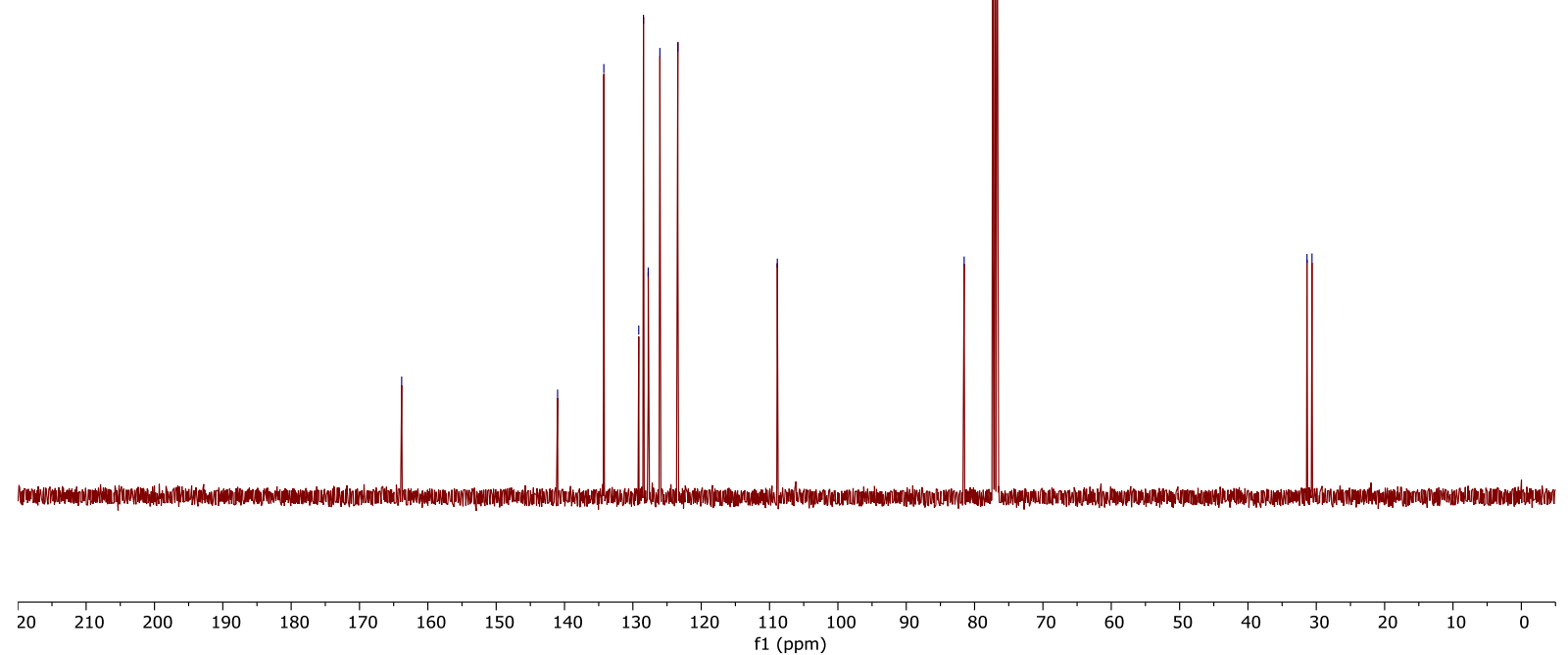


${ }^{1} \mathrm{H}$ NMR (400 MHz, $\mathrm{CDCl}_{3}$ ) of compound 41a (see procedure):

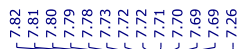<smiles>O=C1c2ccccc2C(=O)N1OC1CCCCO1</smiles>

41a

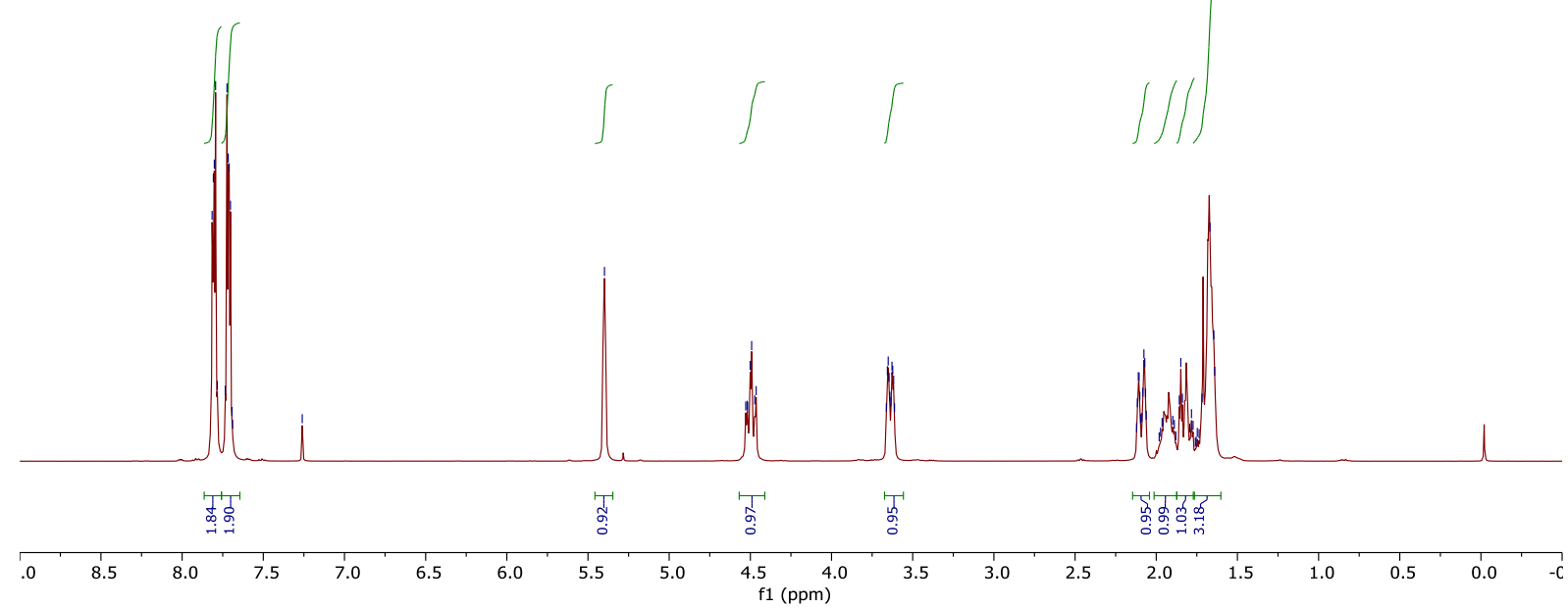

${ }^{13} \mathrm{C}$ NMR (101 MHz, $\mathrm{CDCl}_{3}$ ) of compound $41 \mathrm{a}$ (see procedure):<smiles>CC(C)(C)ON1C(=O)c2ccccc2C1=O</smiles>

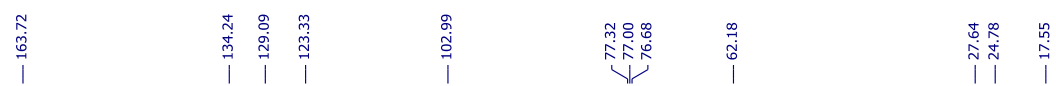

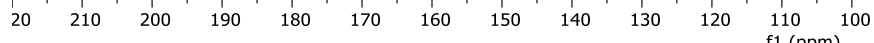


${ }^{1} \mathrm{H}$ NMR (400 MHz, $\mathrm{CDCl}_{3}$ ) of compound 42a (see procedure):

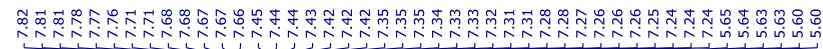<smiles>O=C1c2ccccc2C(=O)N1OC1CCCC(c2ccccc2)O1</smiles>

42a
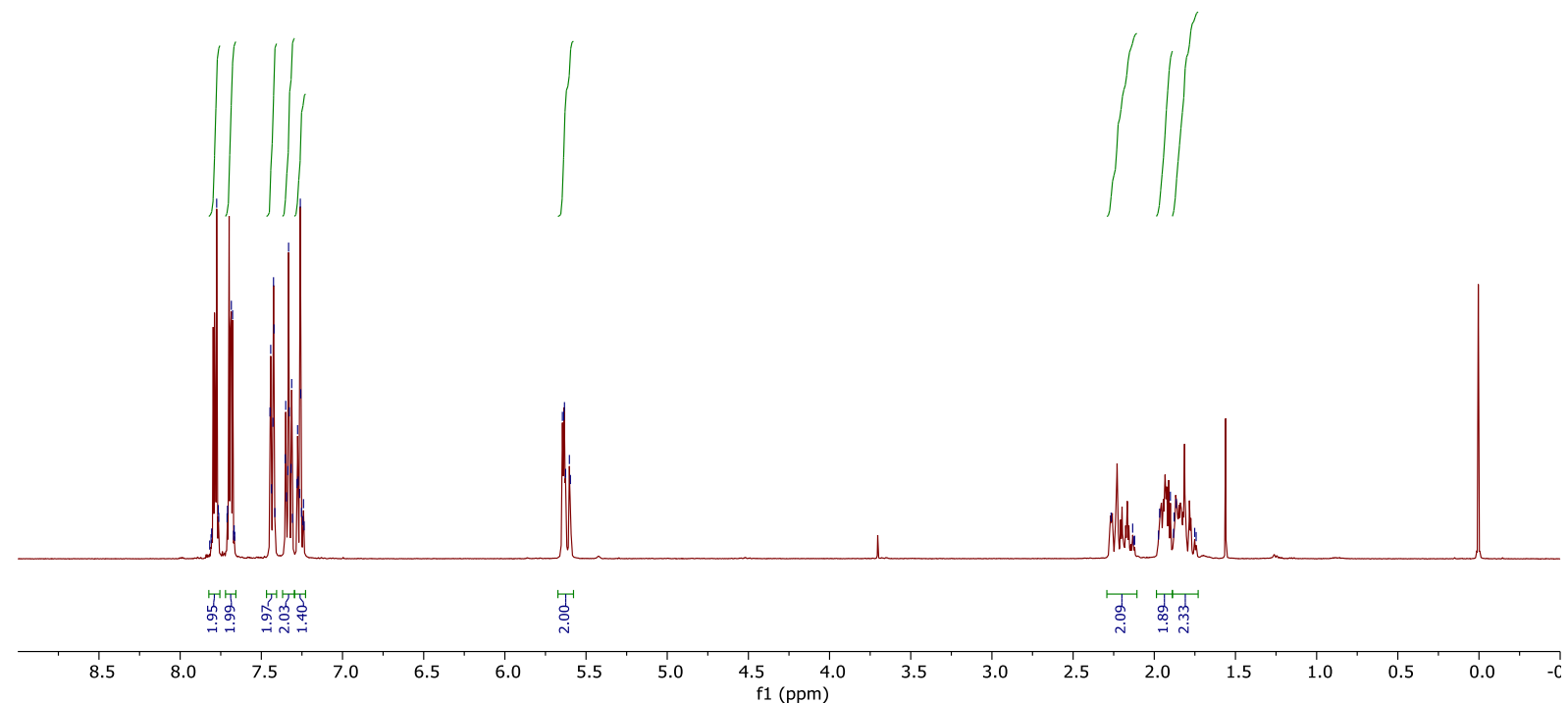

${ }^{13} \mathrm{C} \mathrm{NMR}\left(101 \mathrm{MHz}, \mathrm{CDCl}_{3}\right)$ of compound $42 \mathrm{a}$ (see procedure)

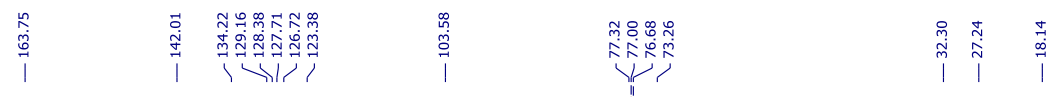<smiles>O=C1c2ccccc2C(=O)N1OC1CCCC(c2ccccc2)O1</smiles>

$42 a$

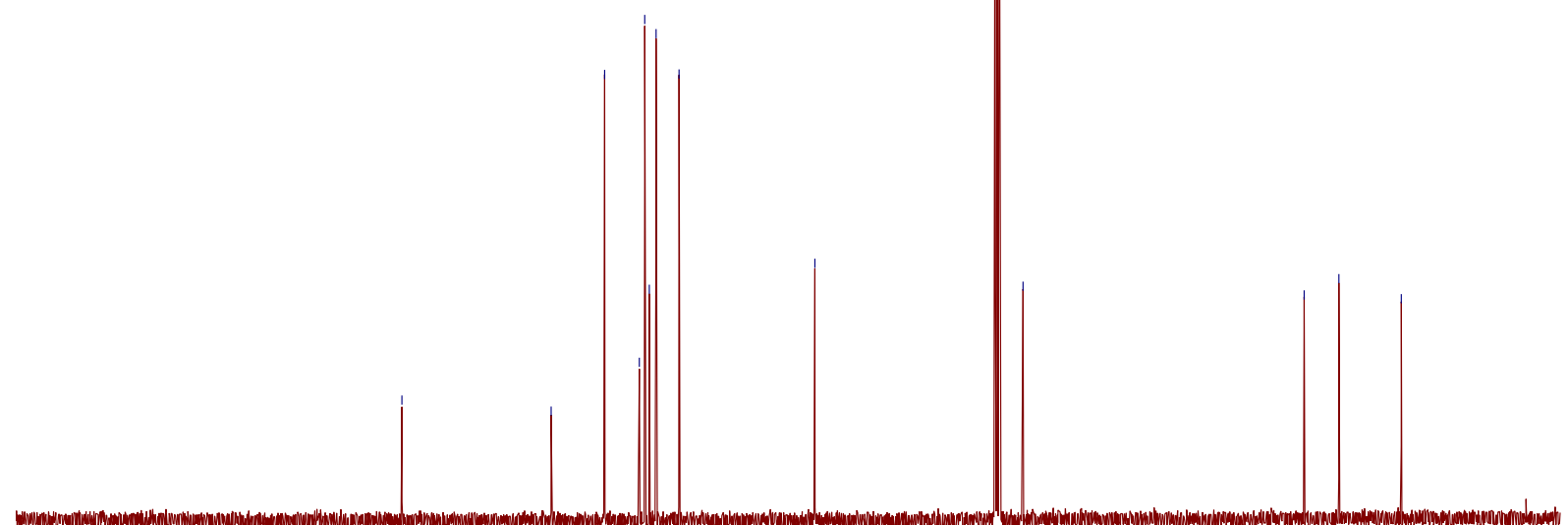

\begin{tabular}{llllllllllllllllllllllllll}
\hline & 20 & 200 & 190 & 180 & 170 & 160 & 150 & 140 & 130 & 120 & $\underset{f 1}{110}$ & 100 & 90 & 80 & 70 & 60 & 50 & 40 & 30 & 20 & 10 & 0
\end{tabular} 
${ }^{1} \mathrm{H}$ NMR (400 MHz, $\mathrm{CDCl}_{3}$ ) of compound 43a (see procedure):

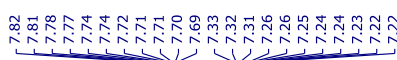

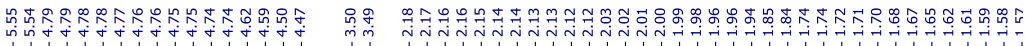

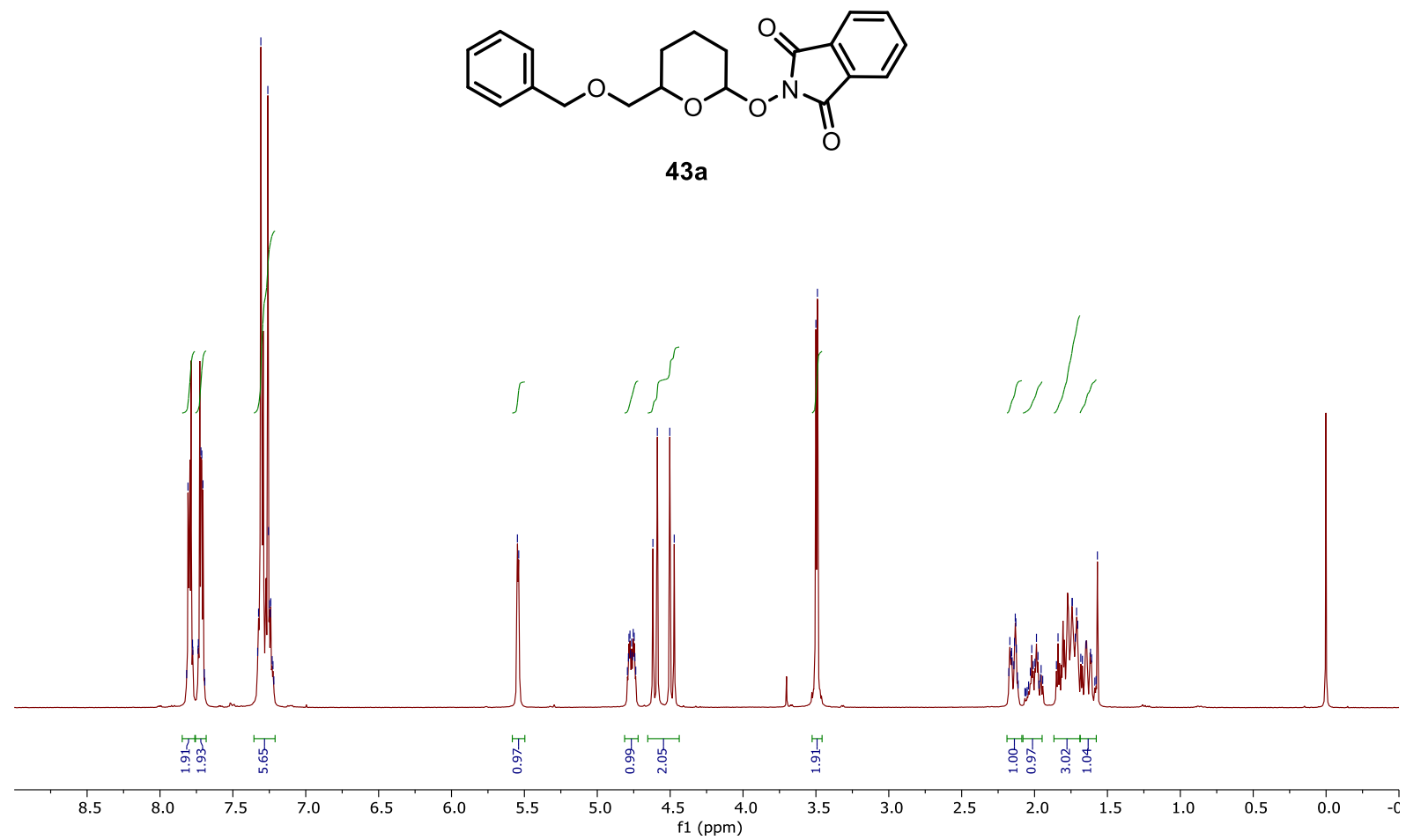

${ }^{13} \mathrm{C}$ NMR (101 MHz, $\mathrm{CDCl}_{3}$ ) of compound 43a (see procedure):<smiles>O=C1c2ccccc2C(=O)N1OC1CCCC(COCc2ccccc2)O1</smiles>

43a

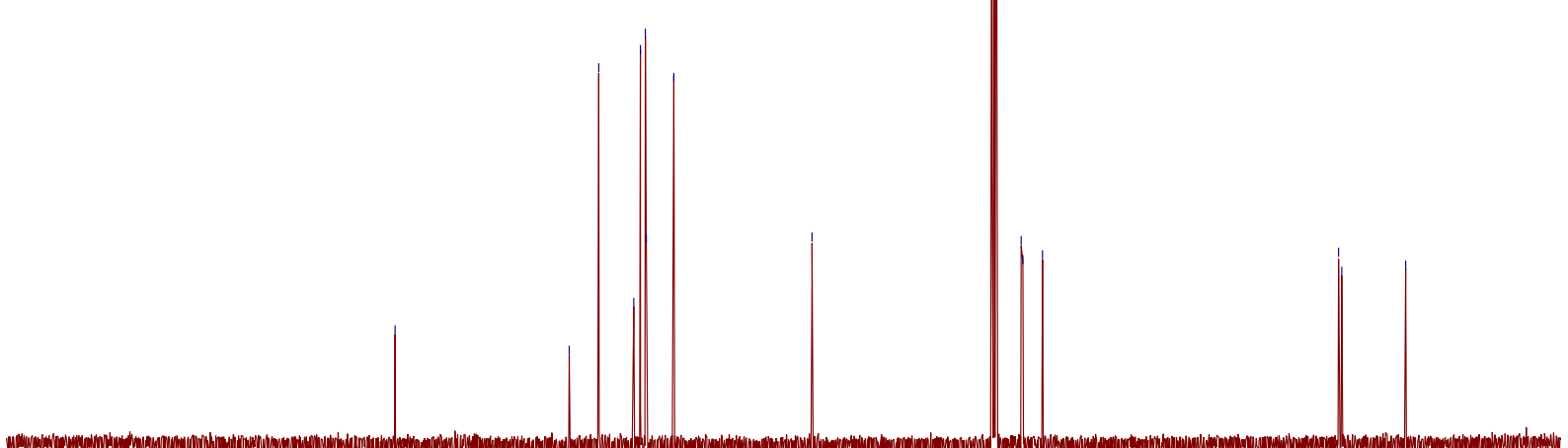

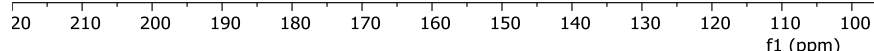


${ }^{1} \mathrm{H}$ NMR (400 MHz, $\mathrm{CDCl}_{3}$ ) of compound 44a (see procedure):

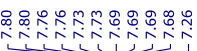

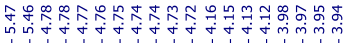

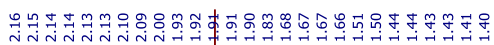<smiles>CC(=O)OCC1CCCC(ON2C(=O)c3ccccc3C2=O)O1</smiles>

$44 a$
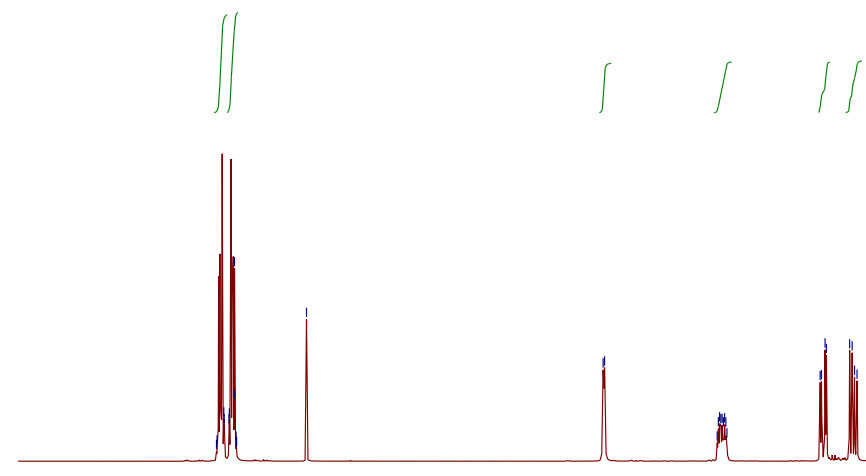

NWW N W

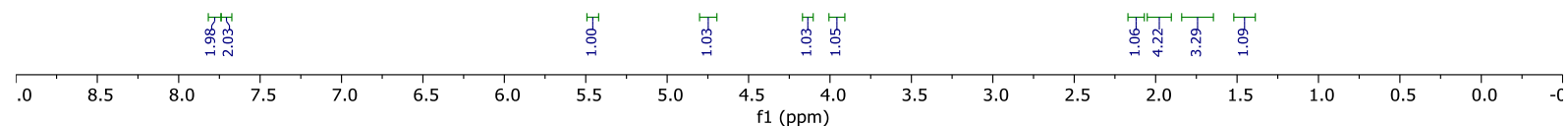

${ }^{13} \mathrm{C}$ NMR (101 MHz, $\mathrm{CDCl}_{3}$ ) of compound $44 \mathbf{a}$ (see procedure):
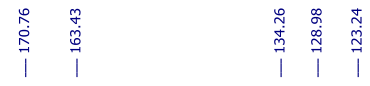

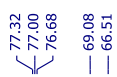

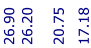<smiles>CC(=O)OCC1CCCC(ON2C(=O)c3ccccc3C2=O)O1</smiles>

$44 a$
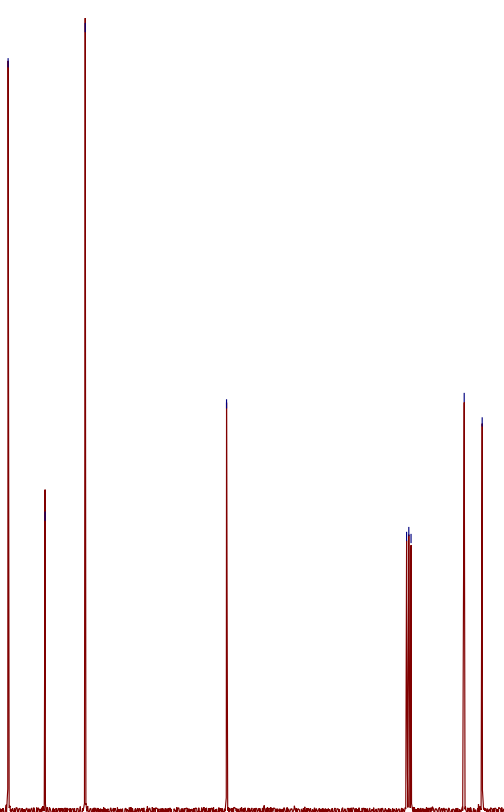

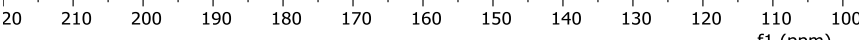


${ }^{1} \mathrm{H}$ NMR (400 MHz, $\mathrm{CDCl}_{3}$ ) of compound 45a (see procedure):

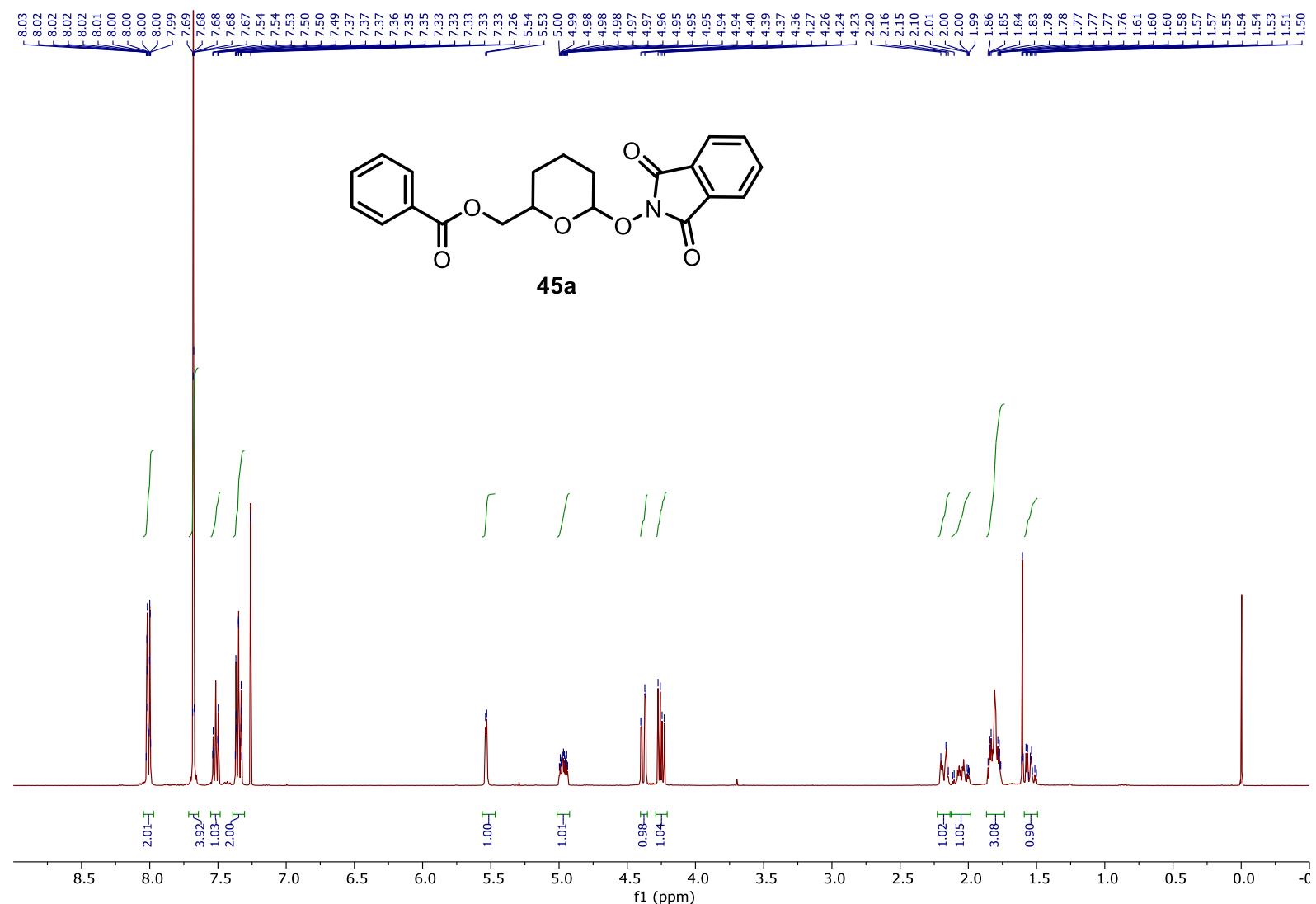

${ }^{13} \mathrm{C}$ NMR (101 MHz, $\mathrm{CDCl}_{3}$ ) of compound $45 \mathrm{a}$ (see procedure):

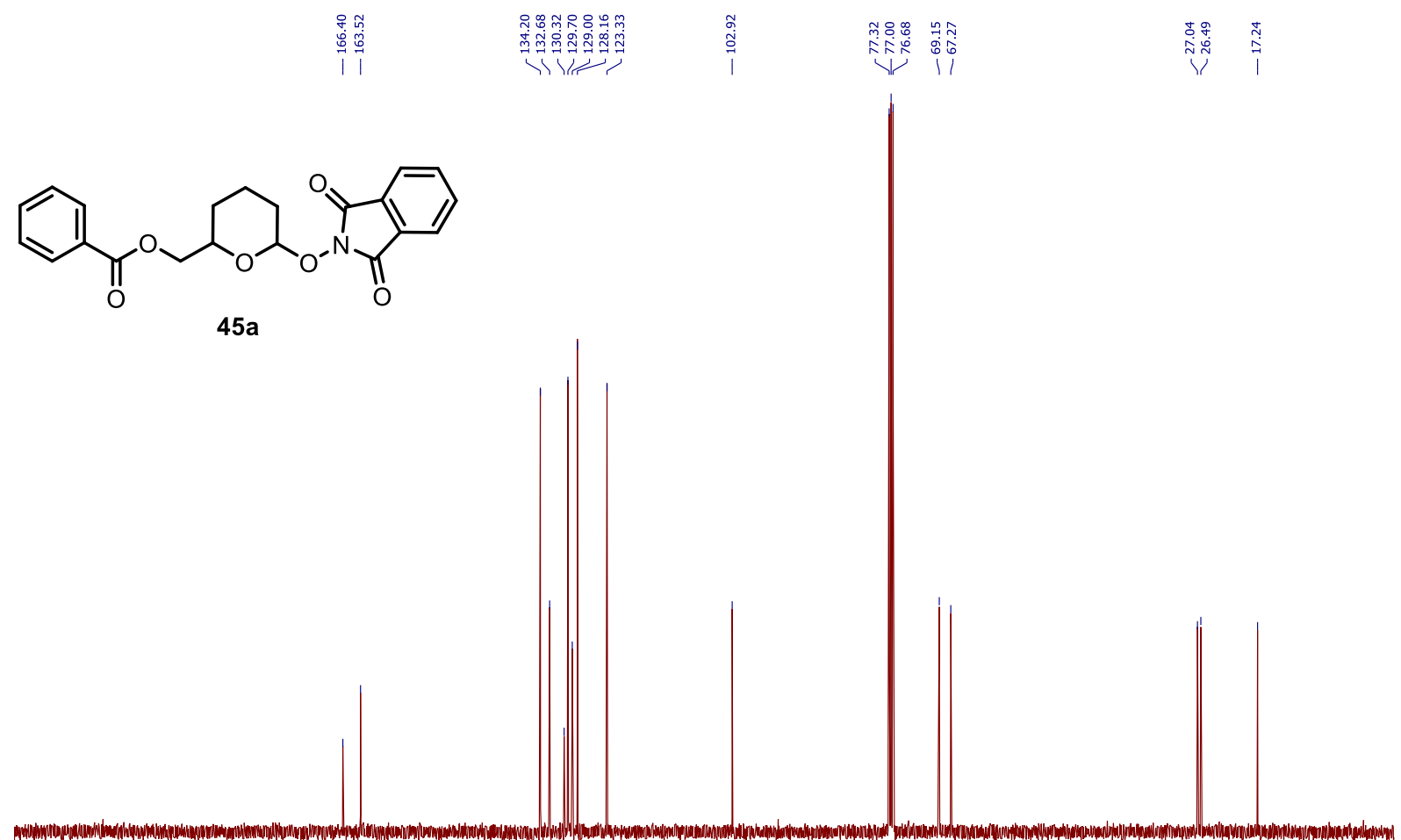

$\begin{array}{llllllllllllllllllllllllllllll} & 20 & 210 & 200 & 190 & 180 & 170 & 160 & 150 & 140 & 130 & 120 & 110 & 100 & 90 & 80 & 70 & 60 & 50 & 40 & 30 & 20 & 10 & 0\end{array}$ 
${ }^{1} \mathrm{H}$ NMR (400 MHz, $\mathrm{CDCl}_{3}$ ) of compound 46a (see procedure):

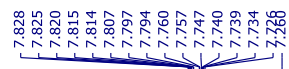

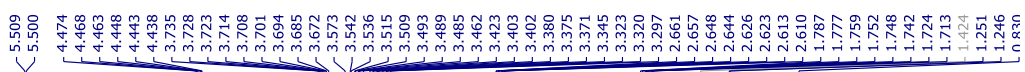<smiles>COC[C@H]1OC(ON2C(=O)c3ccccc3C2=O)C[C@@H](OC)[C@@H]1OC</smiles>

$46 a$
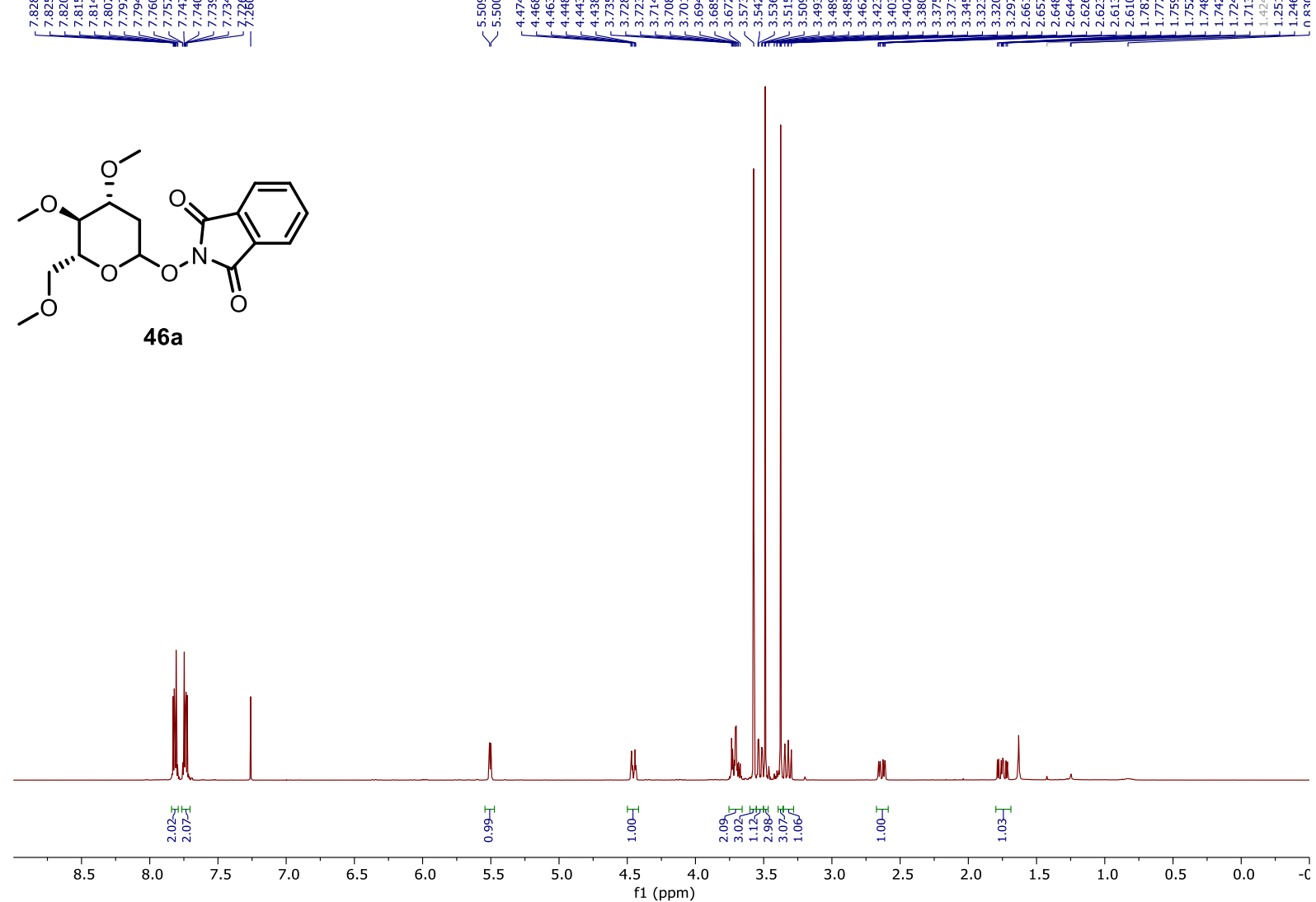

${ }^{13} \mathrm{C}$ NMR (101 MHz, $\mathrm{CDCl}_{3}$ ) of compound $46 \mathrm{a}$ (see procedure):

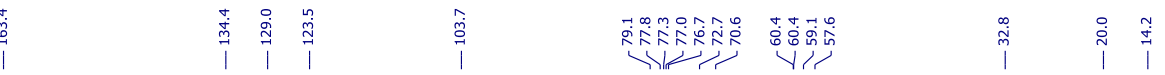<smiles>COC[C@H]1OC(ON2C(=O)c3ccccc3C2=O)C[C@@H](OC)[C@@H]1OC</smiles>

$46 a$

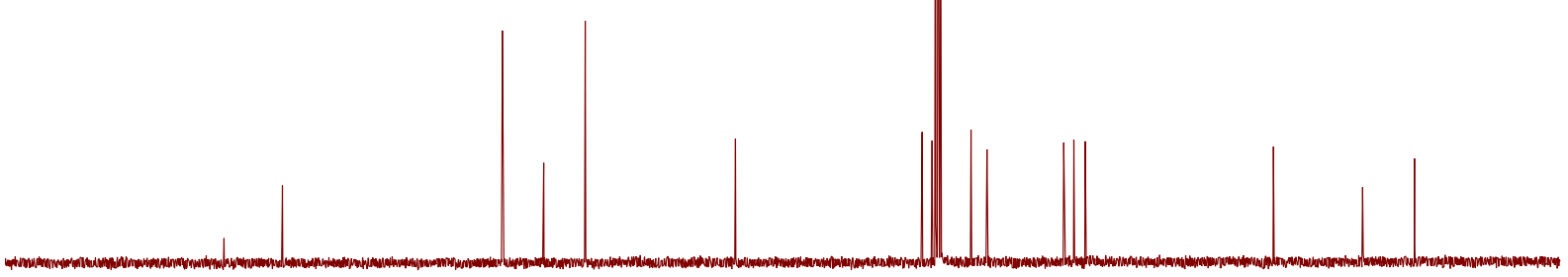

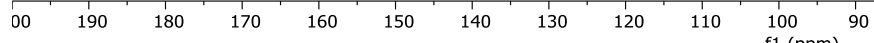


${ }^{1} \mathrm{H}$ NMR (400 MHz, $\mathrm{CDCl}_{3}$ ) of compound $\mathbf{S 1}$ (see procedure):

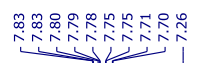

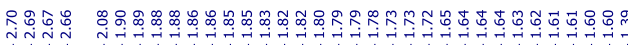

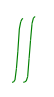<smiles>CC1(ON2C(=O)c3ccccc3C2=O)C2CC3CC(C2)CC1C3</smiles>

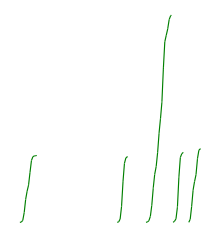

s1
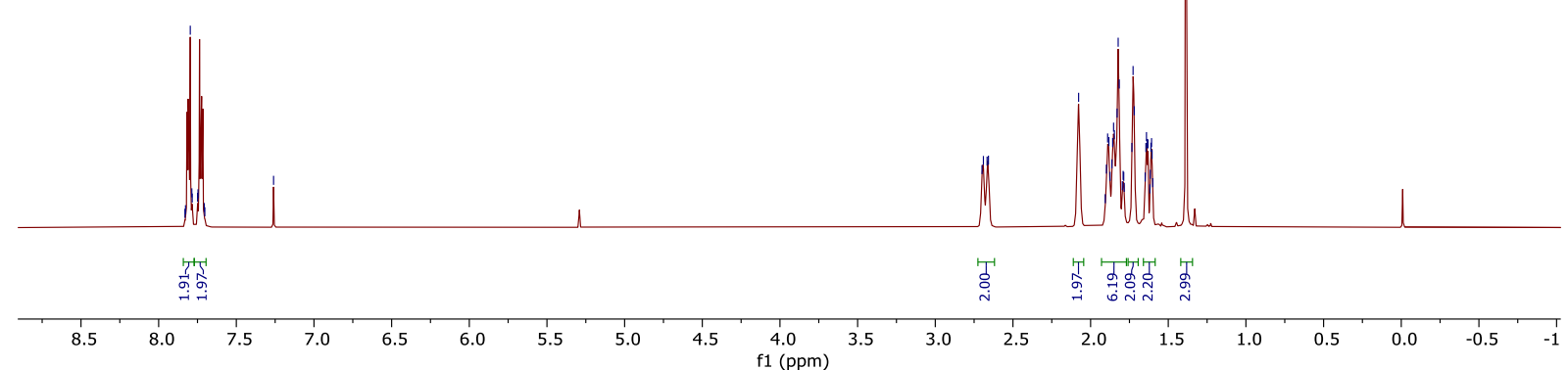

${ }^{13} \mathrm{C}$ NMR (101 MHz, $\mathrm{CDCl}_{3}$ ) of compound $\mathbf{S 1}$ (see procedure):
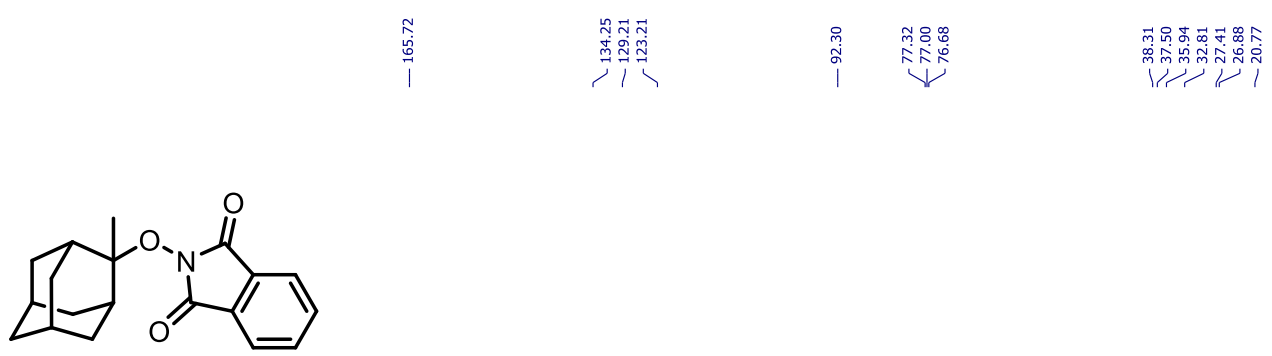

s1

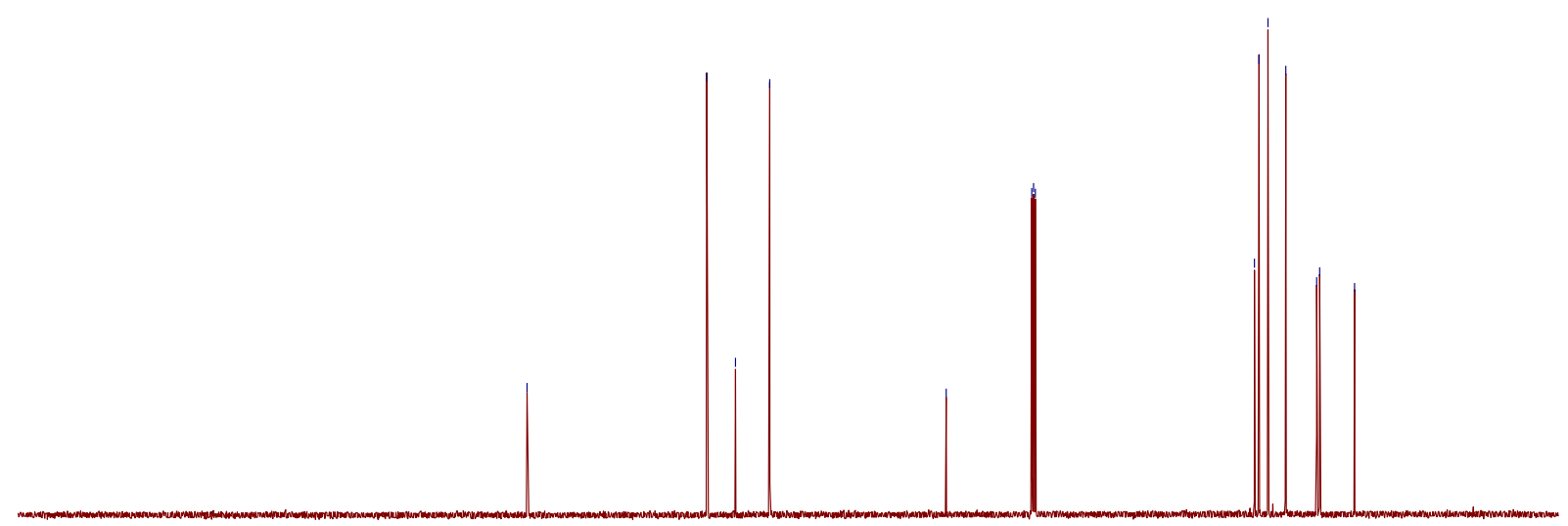

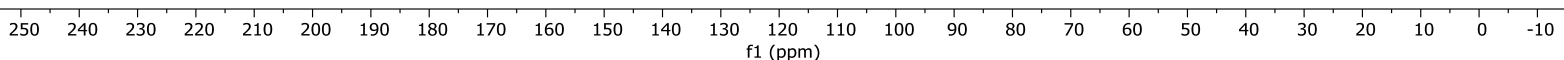


${ }^{1} \mathrm{H}$ NMR (400 MHz, $\mathrm{CDCl}_{3}$ ) of compound $\mathbf{S 2}$ (see procedure):

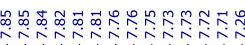

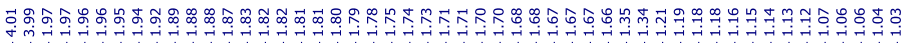

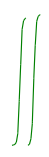<smiles>O=C1c2ccccc2C(=O)N1OCC1CCCCC1</smiles>

s2
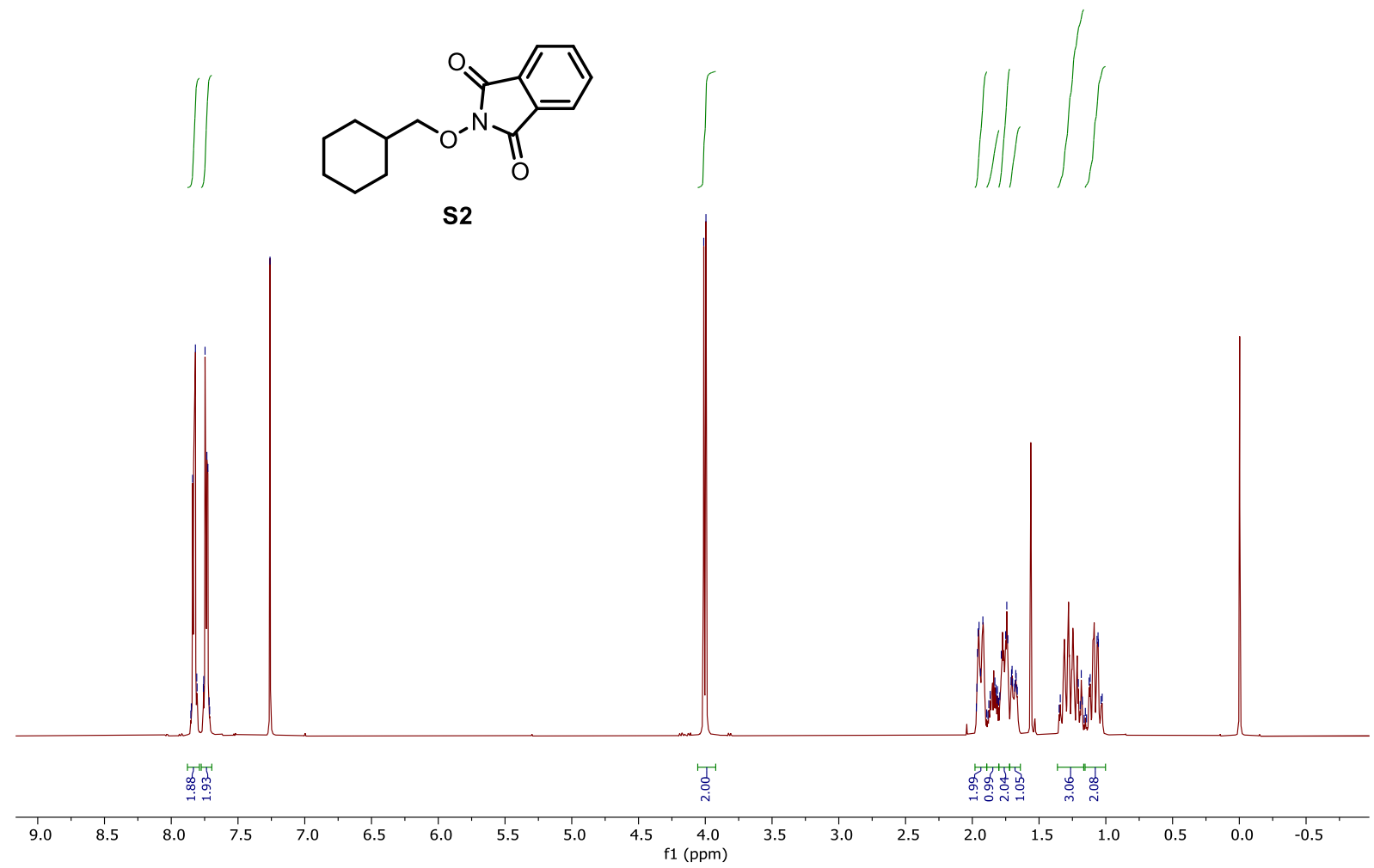

${ }^{13} \mathrm{C}$ NMR (101 MHz, $\mathrm{CDCl}_{3}$ ) of compound $\mathbf{S 2}$ (see procedure):

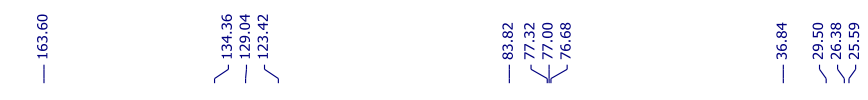<smiles>O=C1c2ccccc2C(=O)N1OCC1CCCCC1</smiles>

s2

$\begin{array}{lllllllllllllllllllllllllllllllll}1 & 1 \\ 250 & 240 & 230 & 220 & 210 & 200 & 190 & 180 & 170 & 160 & 150 & 140 & 130 & 120 & 110 & 100 & 90 & 80 & 70 & 60 & 50 & 40 & 30 & 20 & 10 & 0 & -10\end{array}$ 
${ }^{1} \mathrm{H}$ NMR (400 MHz, $\mathrm{CDCl}_{3}$ ) of compound 2 (see procedure):

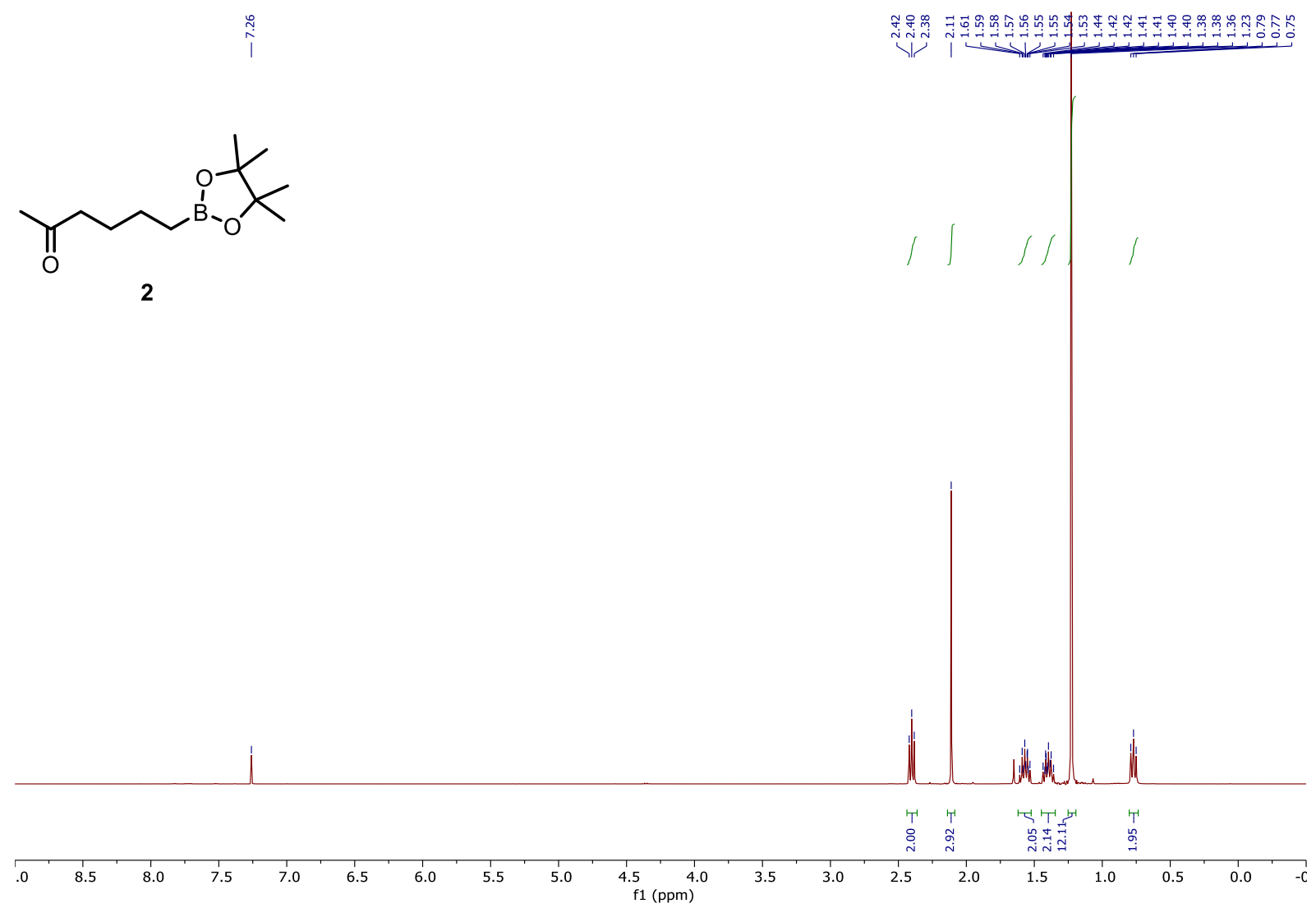

${ }^{13} \mathrm{C} \mathrm{NMR}\left(101 \mathrm{MHz}, \mathrm{CDCl}_{3}\right)$ of compound 2 (see procedure):
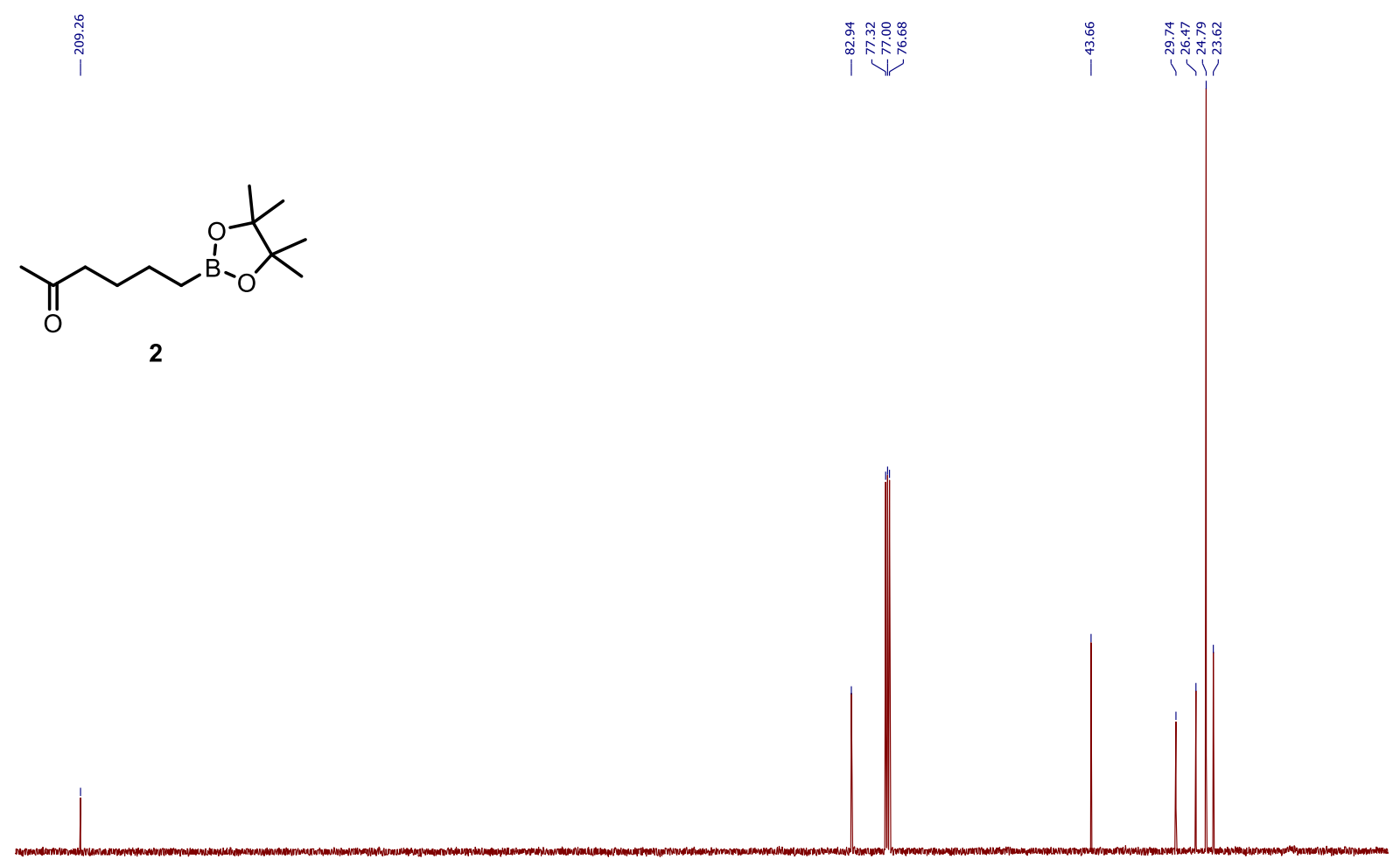

$\begin{array}{llllllllllllllllllllllllll}20 & 210 & 200 & 190 & 180 & 170 & 160 & 150 & 140 & 130 & 120 & 110 & 100 & 90 & 80 & 70 & 60 & 50 & 40 & 30 & 20 & 10 & 0\end{array}$ 
${ }^{1} \mathrm{H}$ NMR (400 MHz, $\mathrm{CDCl}_{3}$ ) of compound $\mathbf{3}$ (see procedure):

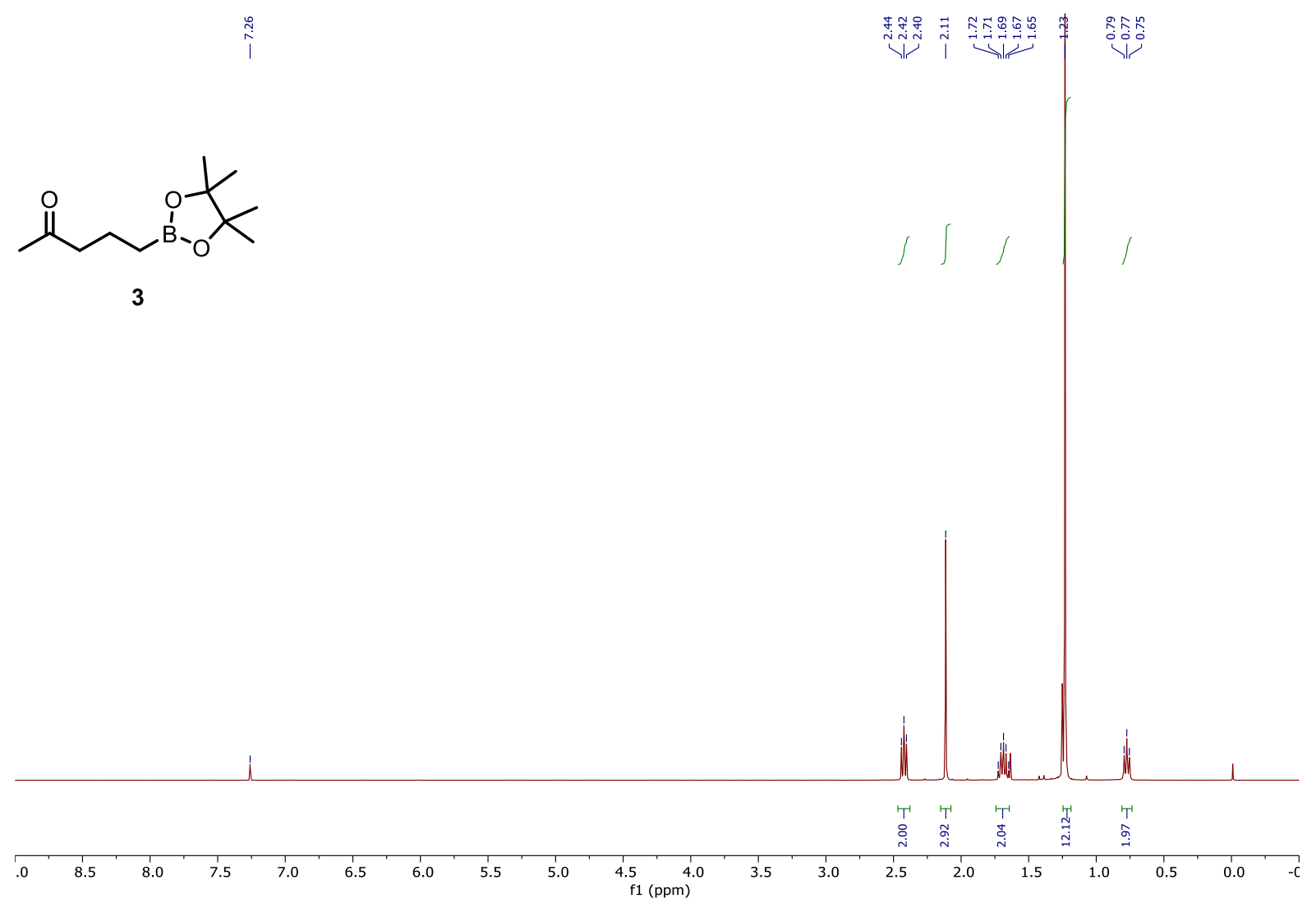

${ }^{13} \mathrm{C}$ NMR (101 MHz, $\mathrm{CDCl}_{3}$ ) of compound $\mathbf{3}$ (see procedure):<smiles>[3H][CH][CH]</smiles>

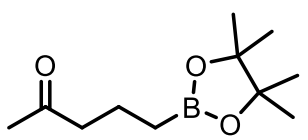

3

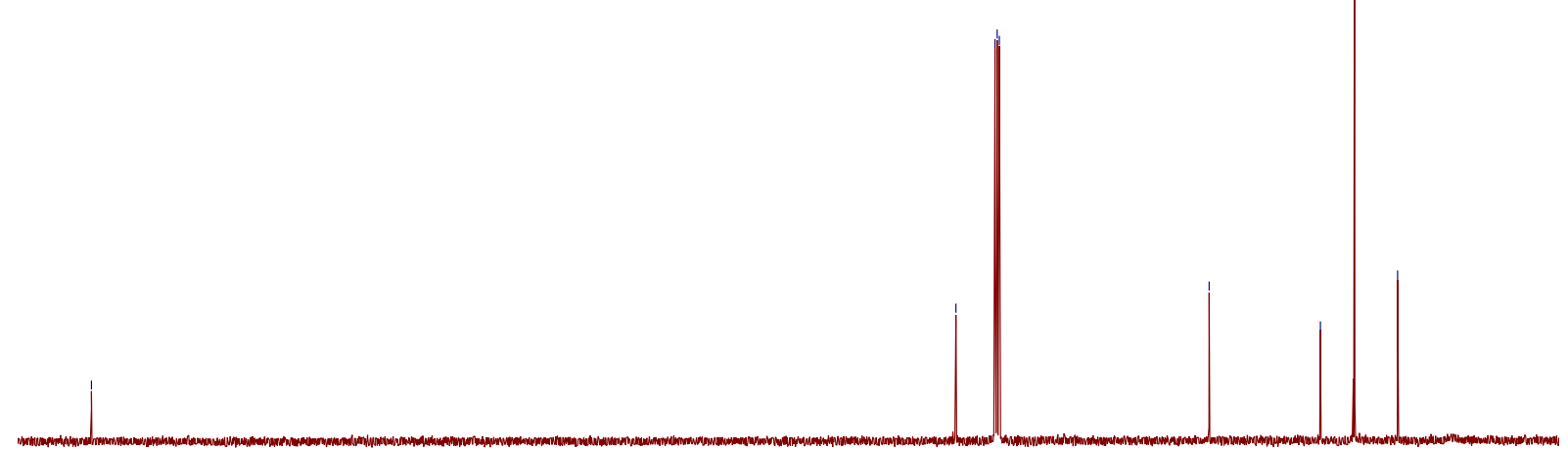

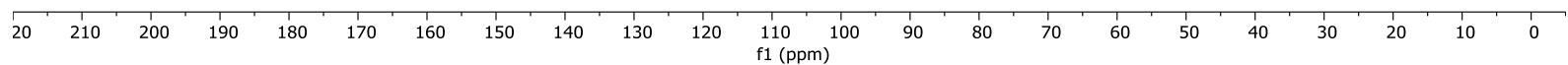


${ }^{1} \mathrm{H}$ NMR (400 MHz, $\mathrm{CDCl}_{3}$ ) of compound 4 (see procedure):
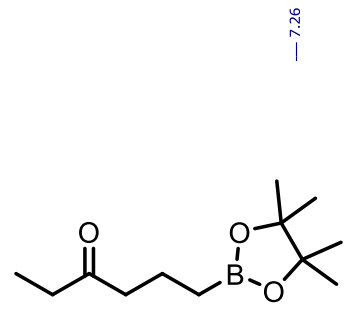

4

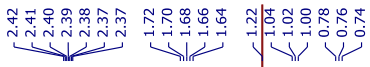
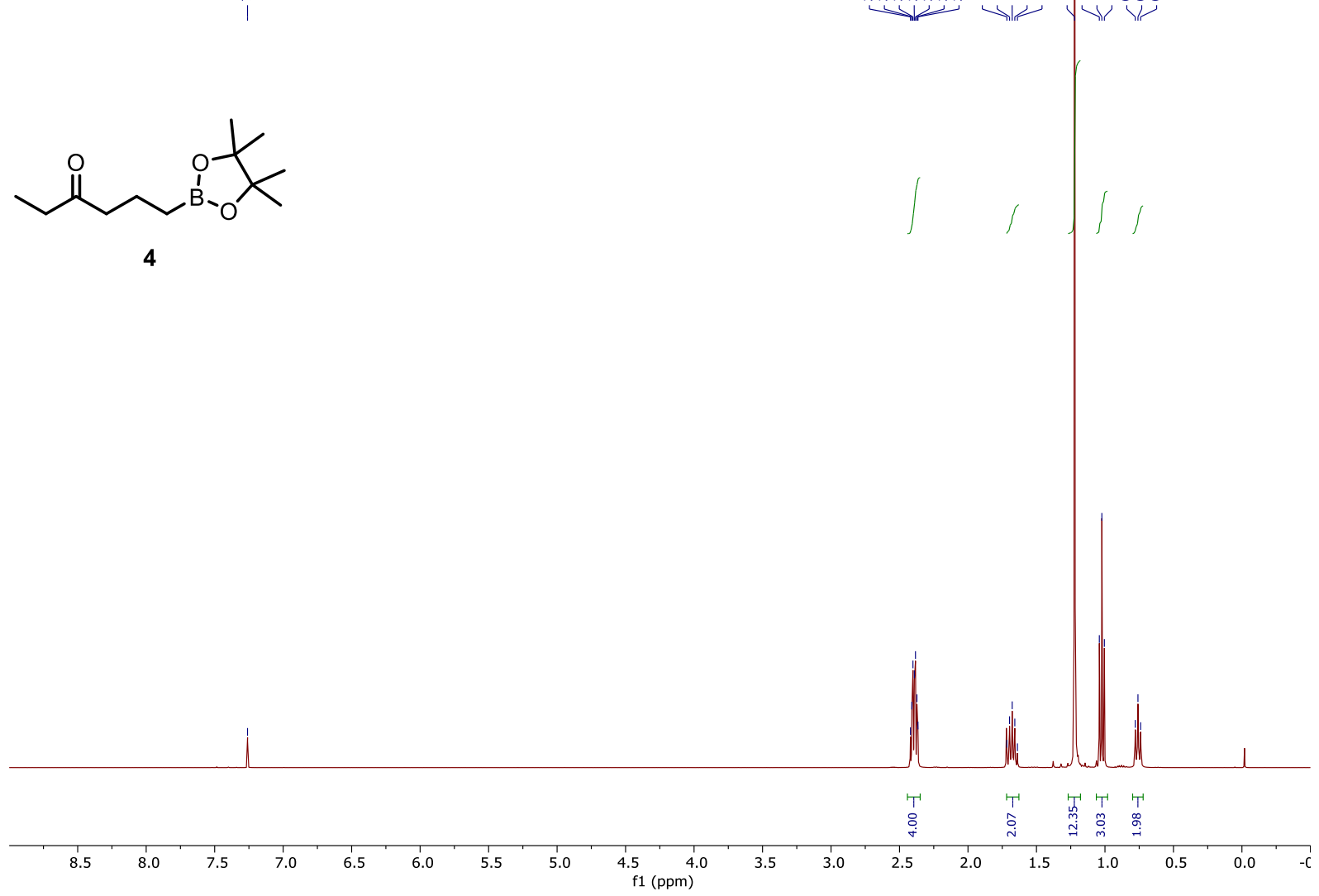

${ }^{13} \mathrm{C}$ NMR $\left(101 \mathrm{MHz}, \mathrm{CDCl}_{3}\right.$ ) of compound 4 (see procedure):

$\stackrel{\vec{\infty}}{\stackrel{\vec{N}}{\sim}}$

解

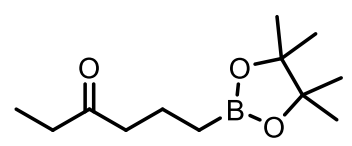

4

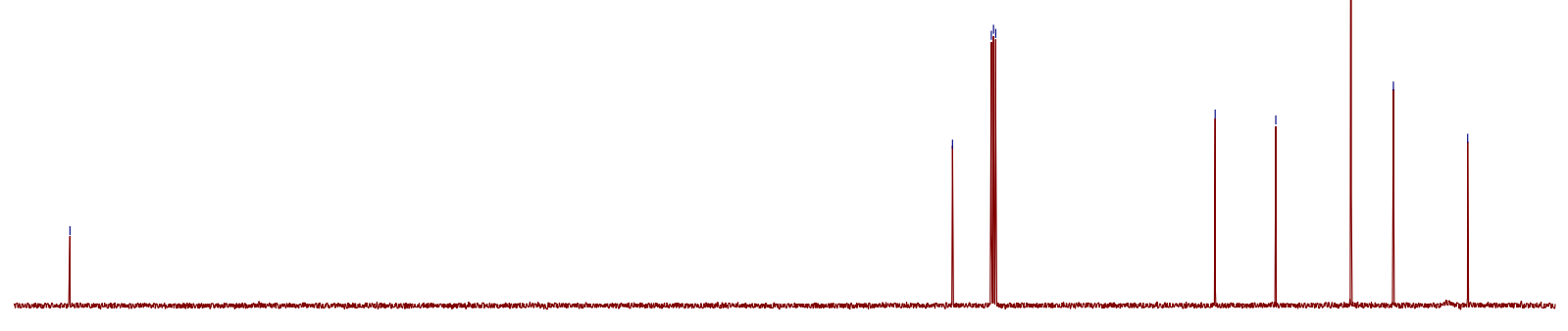

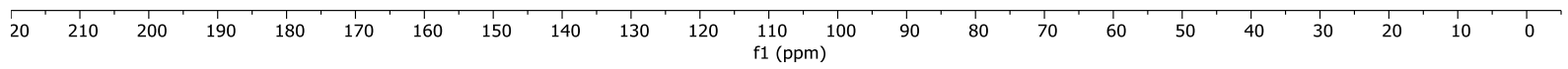

S140 
${ }^{11} \mathrm{~B}$ NMR $\left(128 \mathrm{MHz}, \mathrm{CDCl}_{3}\right)$ of compound 4 (see procedure):

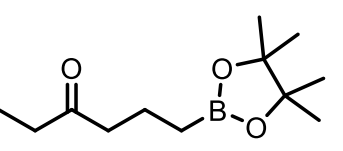

4
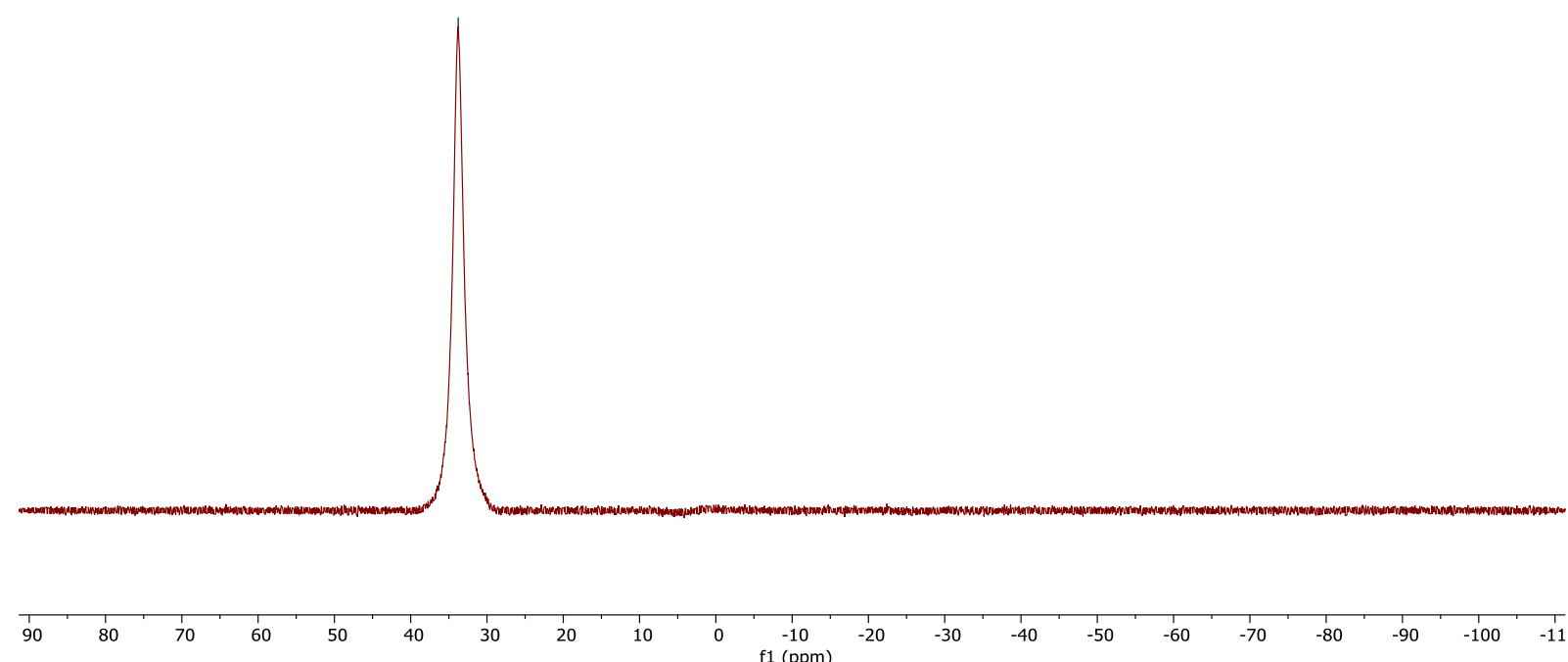
${ }^{1} \mathrm{H}$ NMR (400 MHz, $\mathrm{CDCl}_{3}$ ) of compound 5 (see procedure):

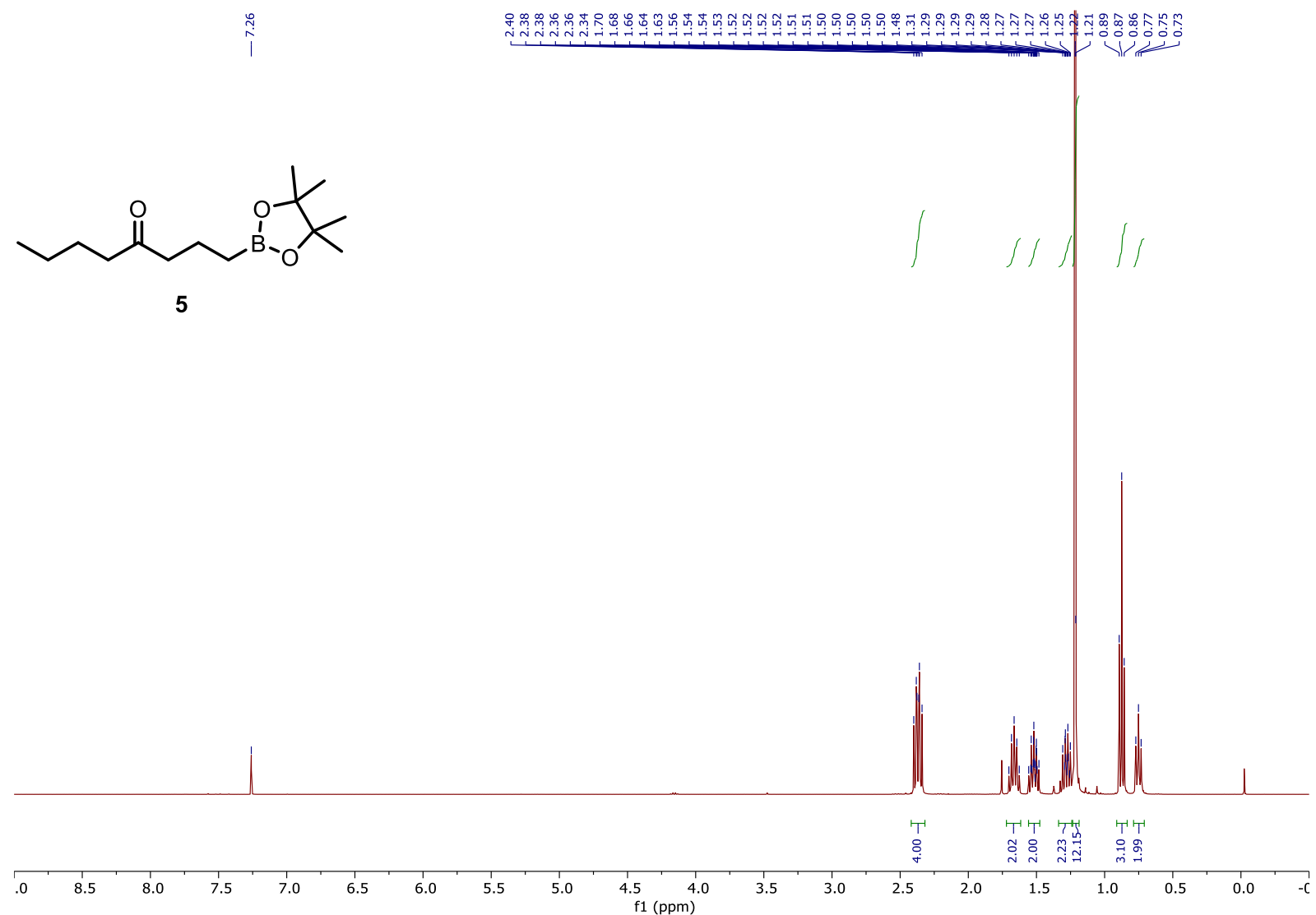

${ }^{13} \mathrm{C}$ NMR $\left(101 \mathrm{MHz}, \mathrm{CDCl}_{3}\right)$ of compound 5 (see procedure):

iำ
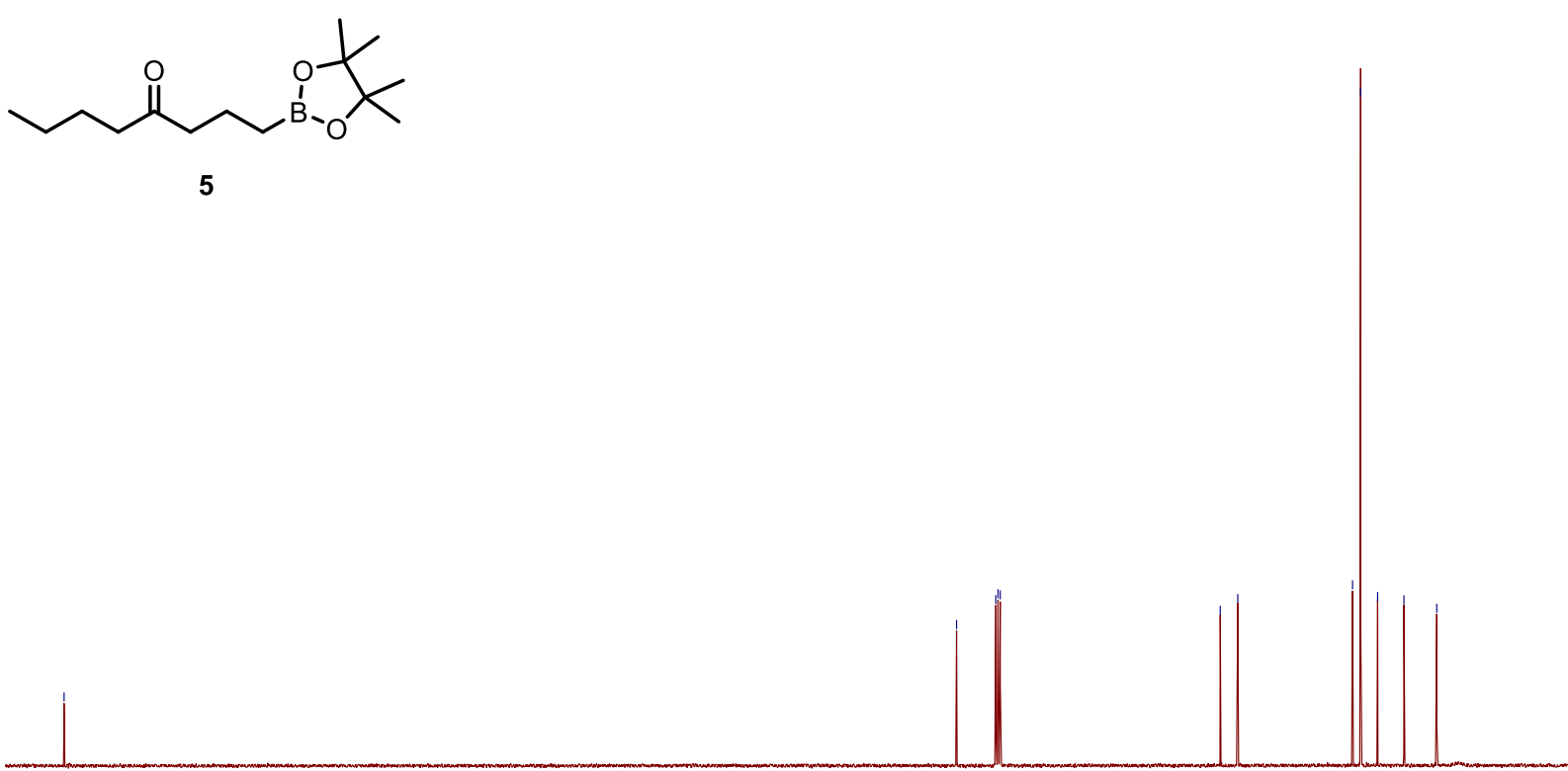

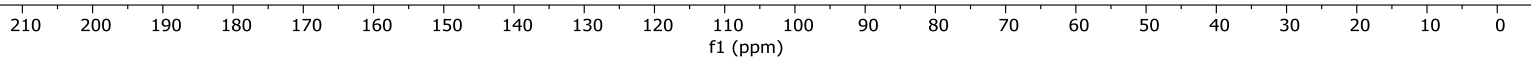


${ }^{11} \mathrm{~B}$ NMR (128 MHz, $\mathrm{CDCl}_{3}$ ) of compound 5 (see procedure):

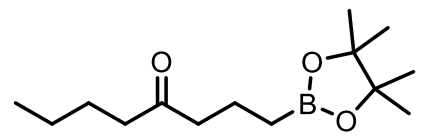

5

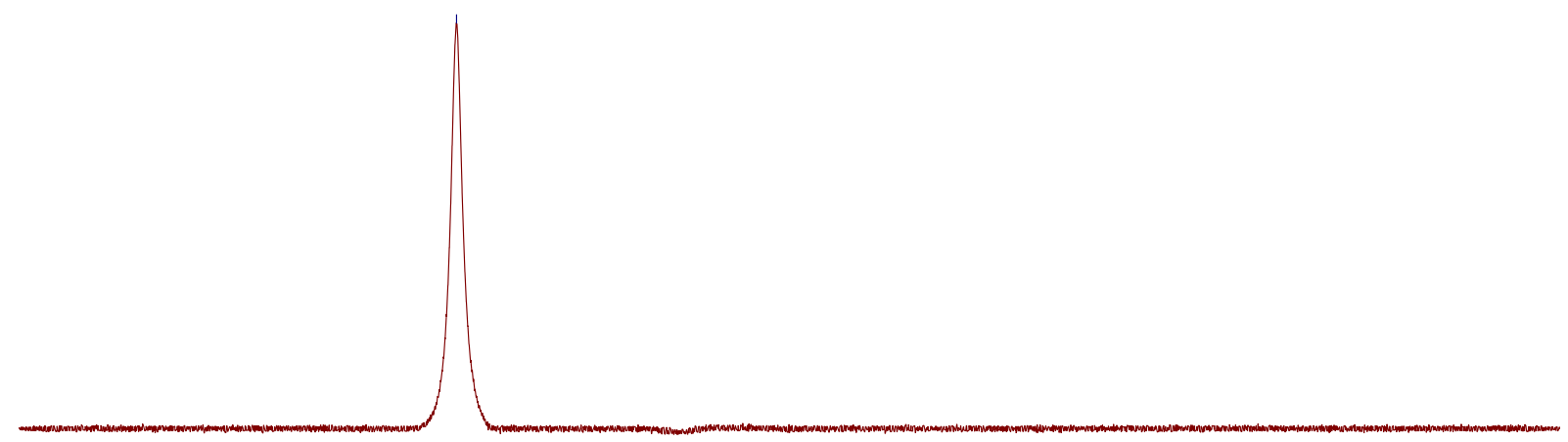

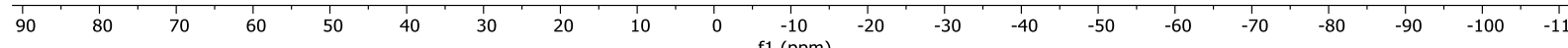


${ }^{1} \mathrm{H} \mathrm{NMR}\left(400 \mathrm{MHz}, \mathrm{CDCl}_{3}\right.$ ) of compound 6 (see procedure):

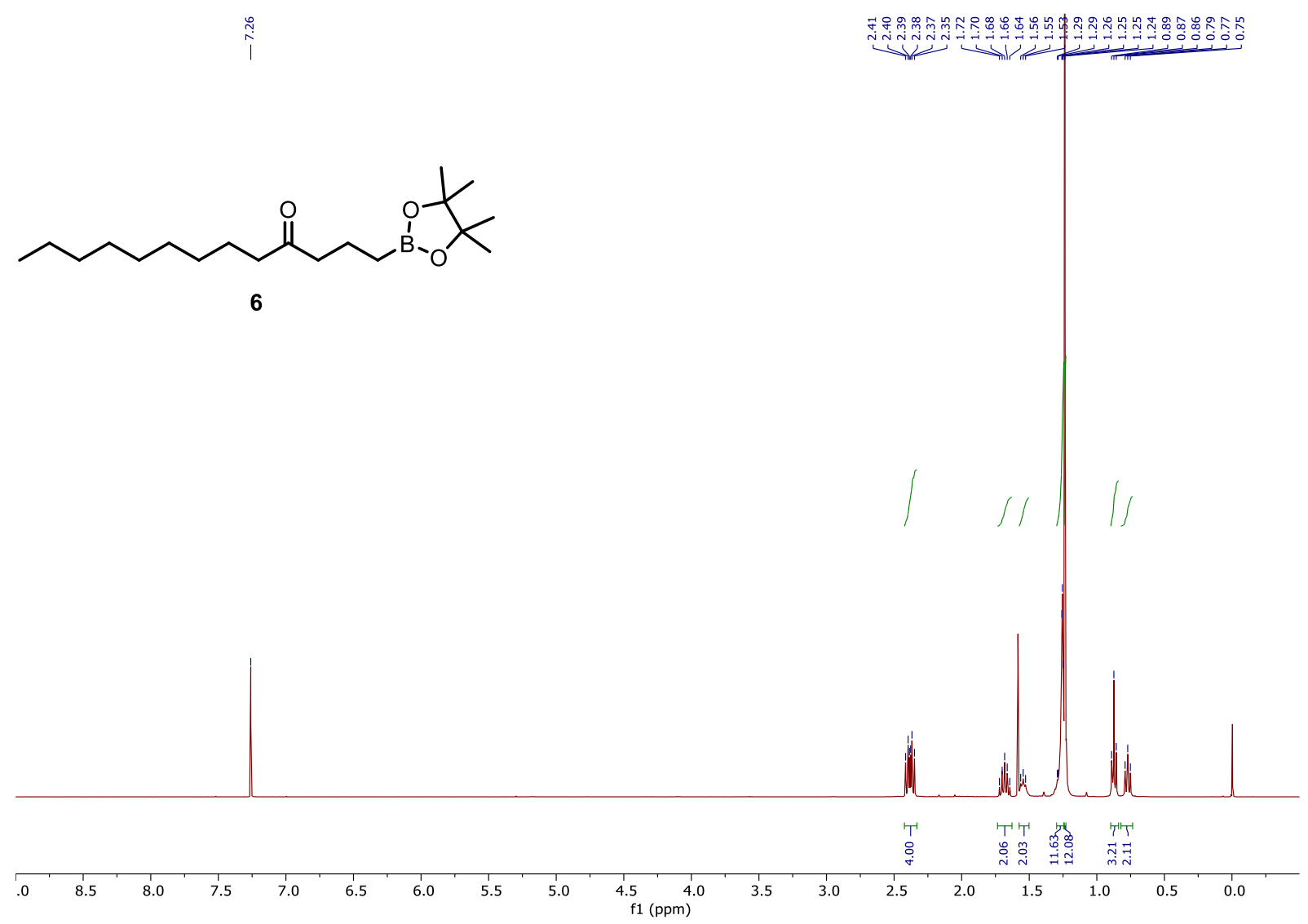

${ }^{13} \mathrm{C}$ NMR (101 MHz, $\mathrm{CDCl}_{3}$ ) of compound 6 (see procedure):
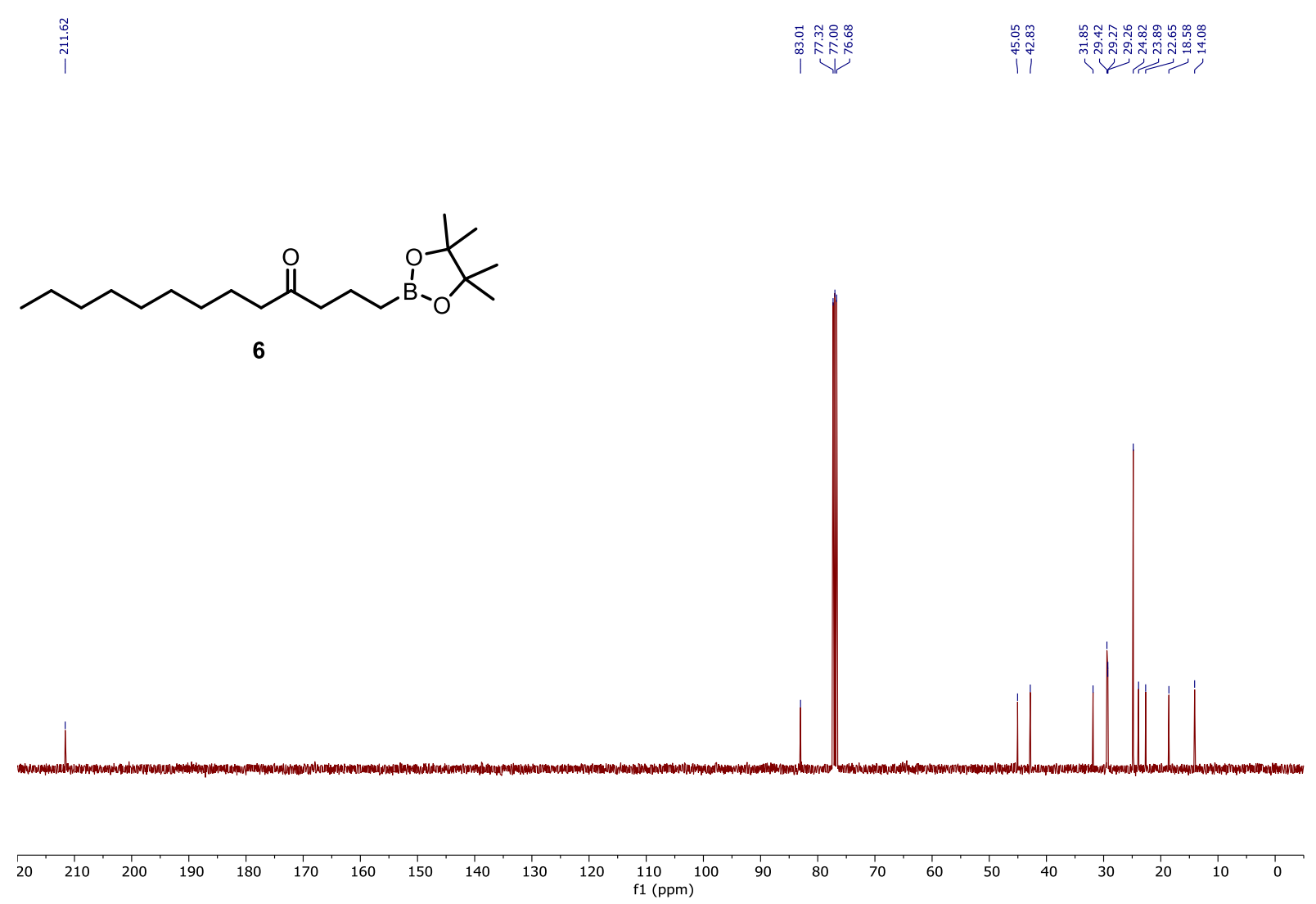
${ }^{11} \mathrm{~B}$ NMR (128 MHz, $\left.\mathrm{CDCl}_{3}\right)$ of compound 6 (see procedure):

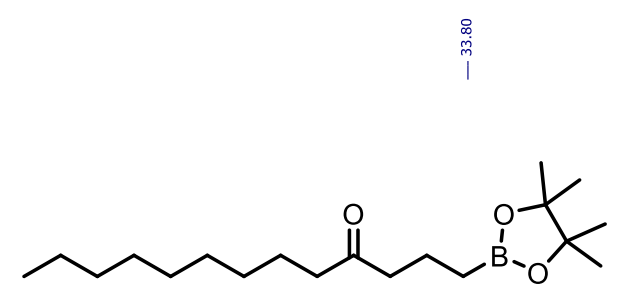

6
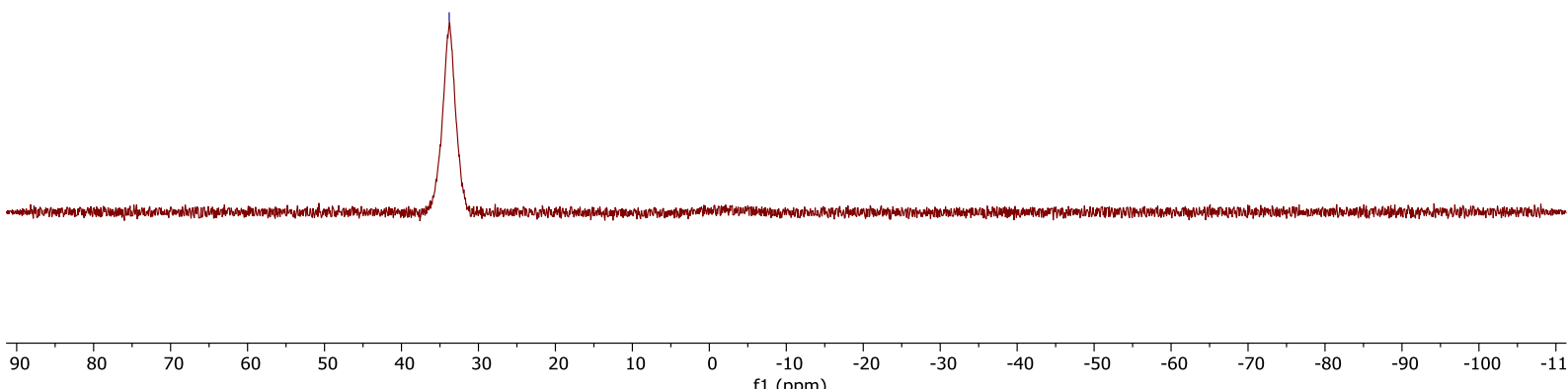
${ }^{1} \mathrm{H}$ NMR (400 MHz, $\mathrm{CDCl}_{3}$ ) of compound 7 (see procedure):

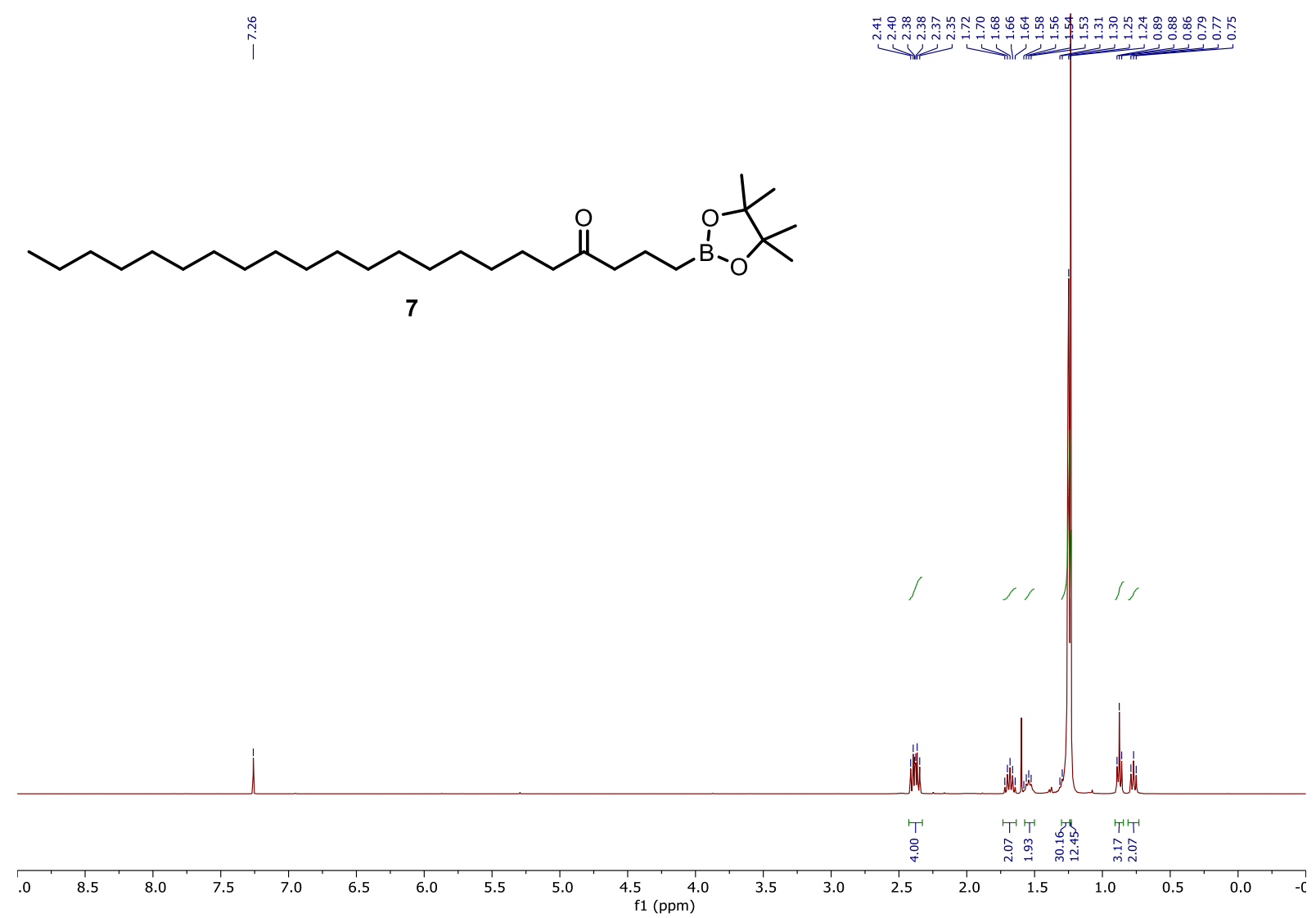

${ }^{13} \mathrm{C}$ NMR (101 MHz, $\mathrm{CDCl}_{3}$ ) of compound 7 (see procedure):
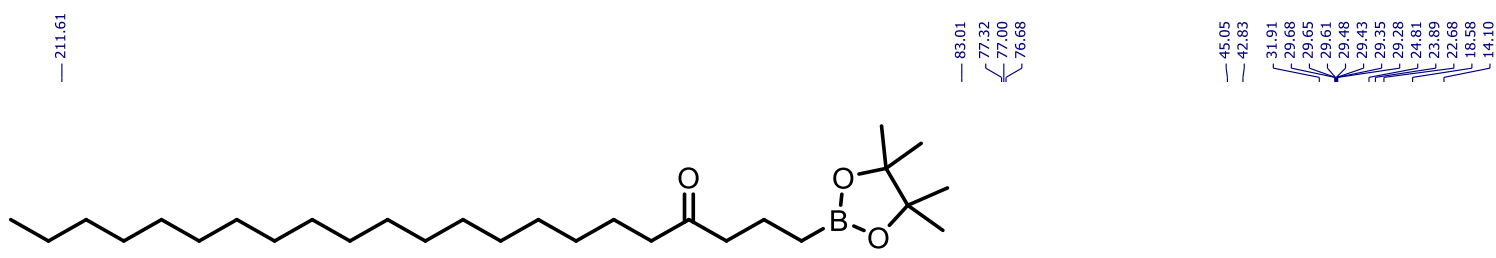

7

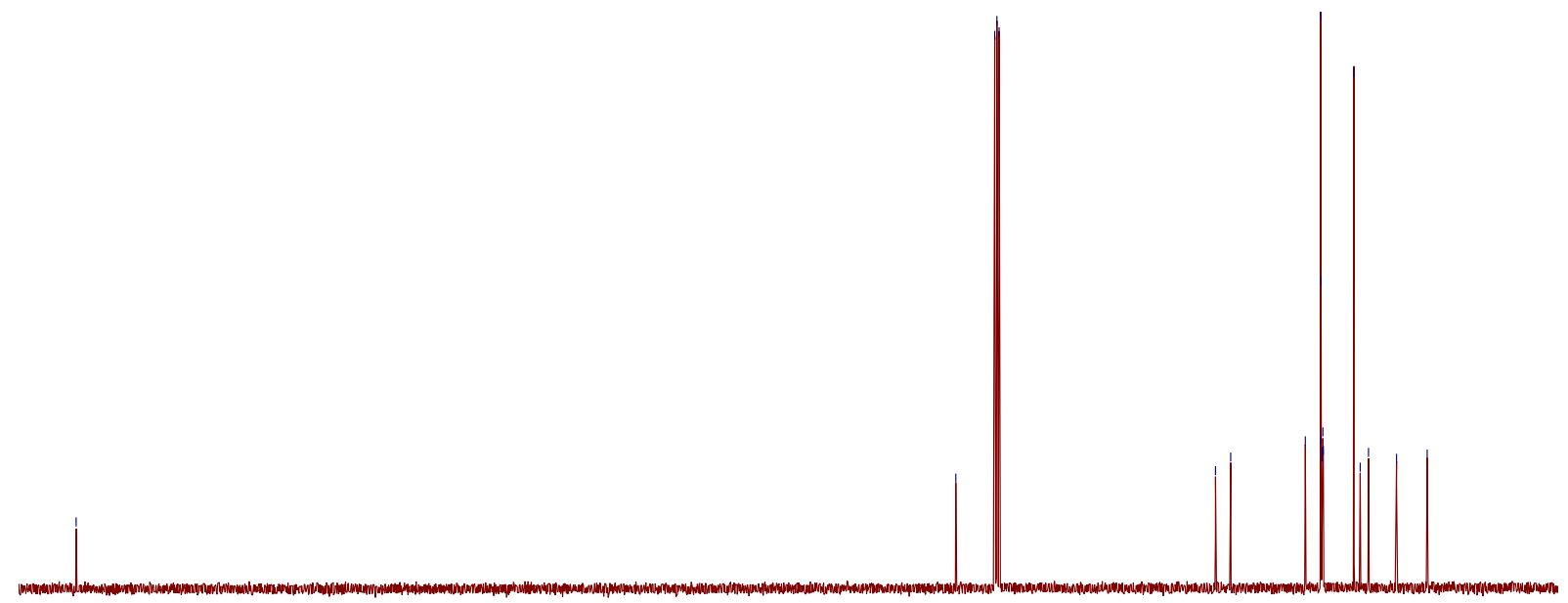

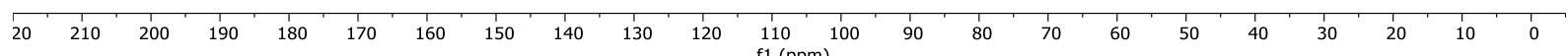


${ }^{11} \mathrm{~B}$ NMR (128 MHz, $\left.\mathrm{CDCl}_{3}\right)$ of compound 7 (see procedure):
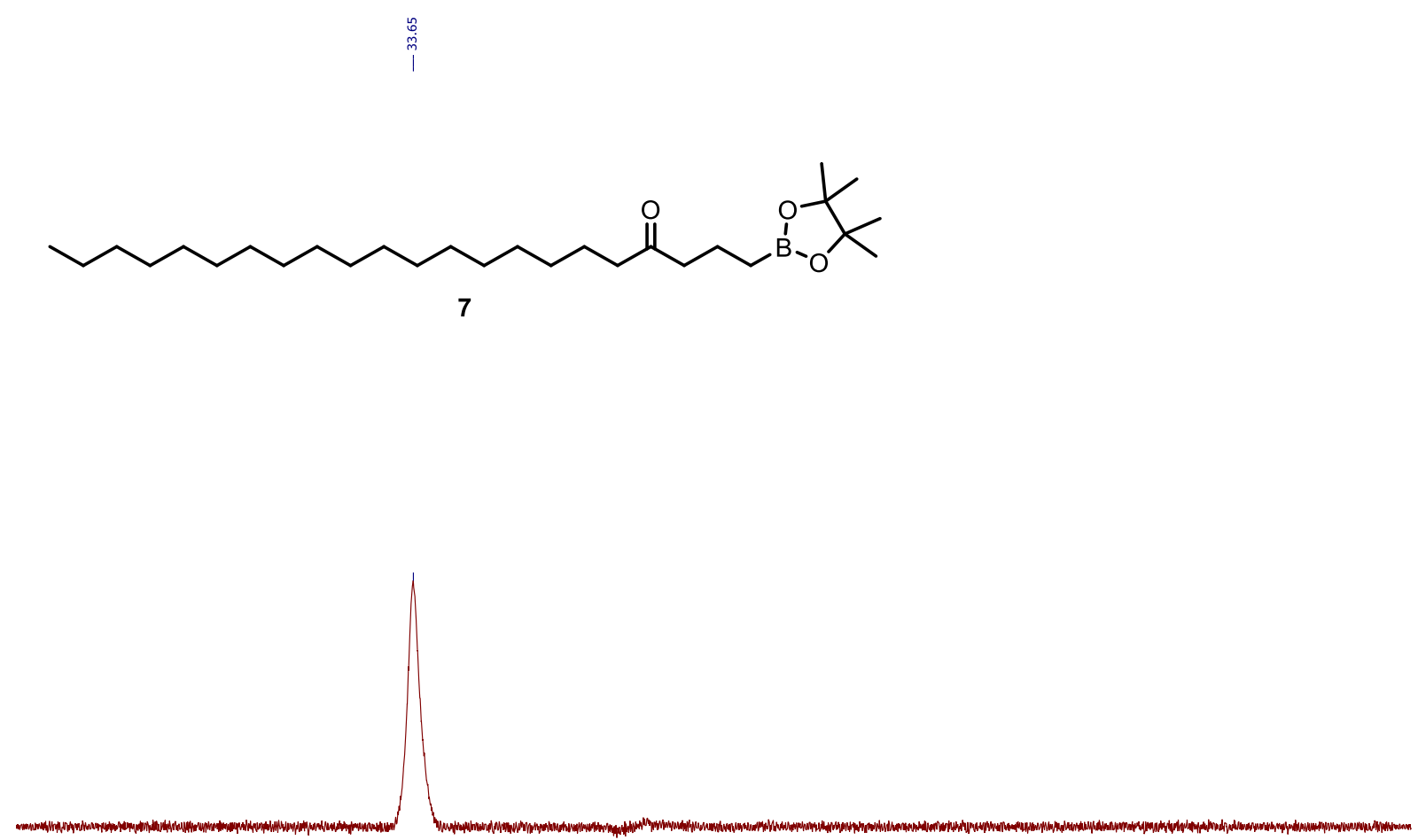

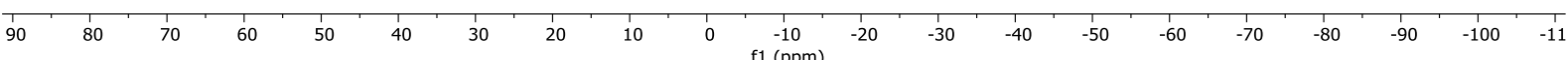


${ }^{1} \mathrm{H}$ NMR (400 MHz, $\mathrm{CDCl}_{3}$ ) of compound 8 (see procedure):

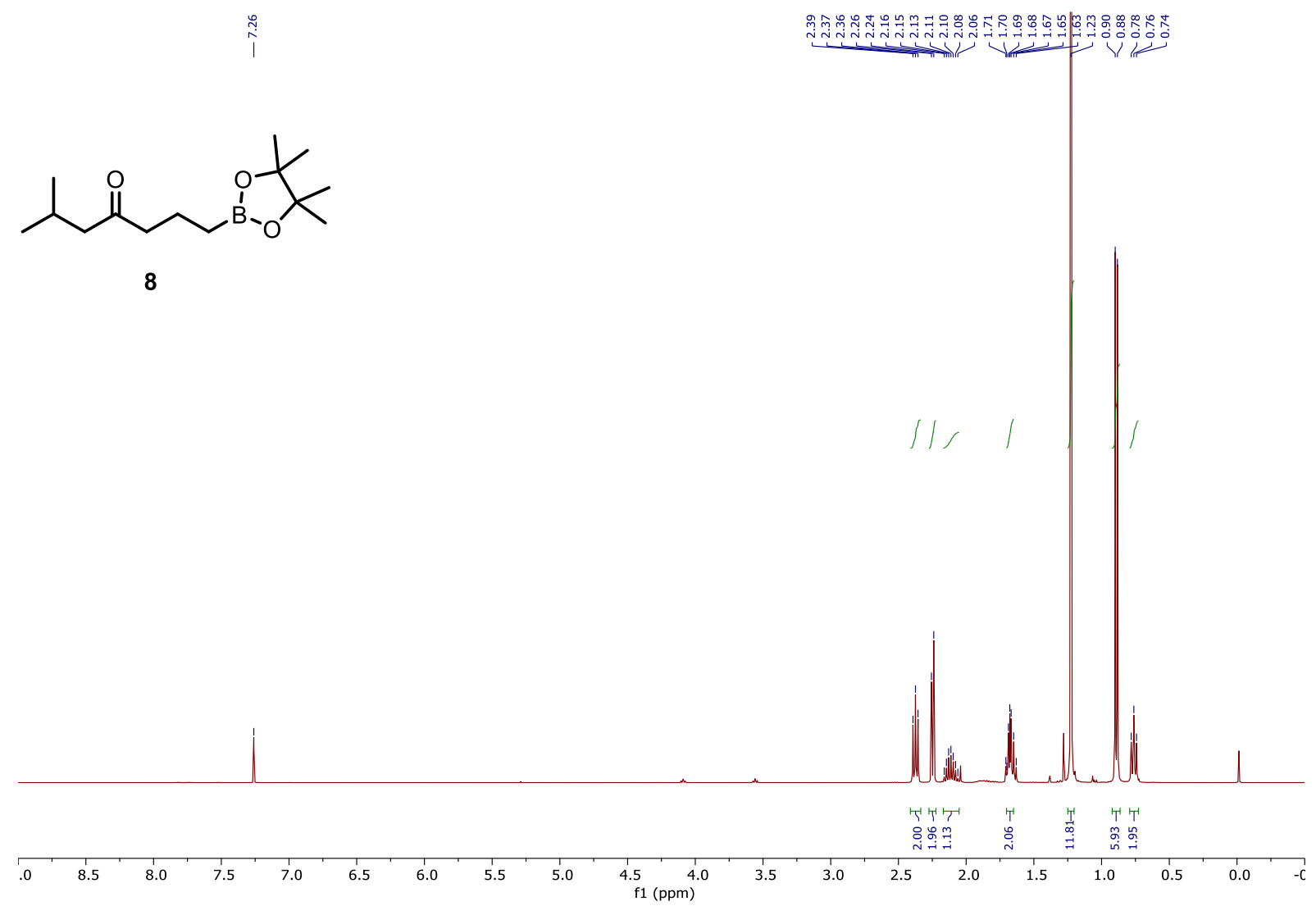

${ }^{13} \mathrm{C}$ NMR (101 MHz, $\mathrm{CDCl}_{3}$ ) of compound 8 (see procedure)

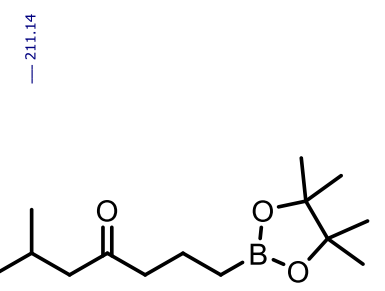

8

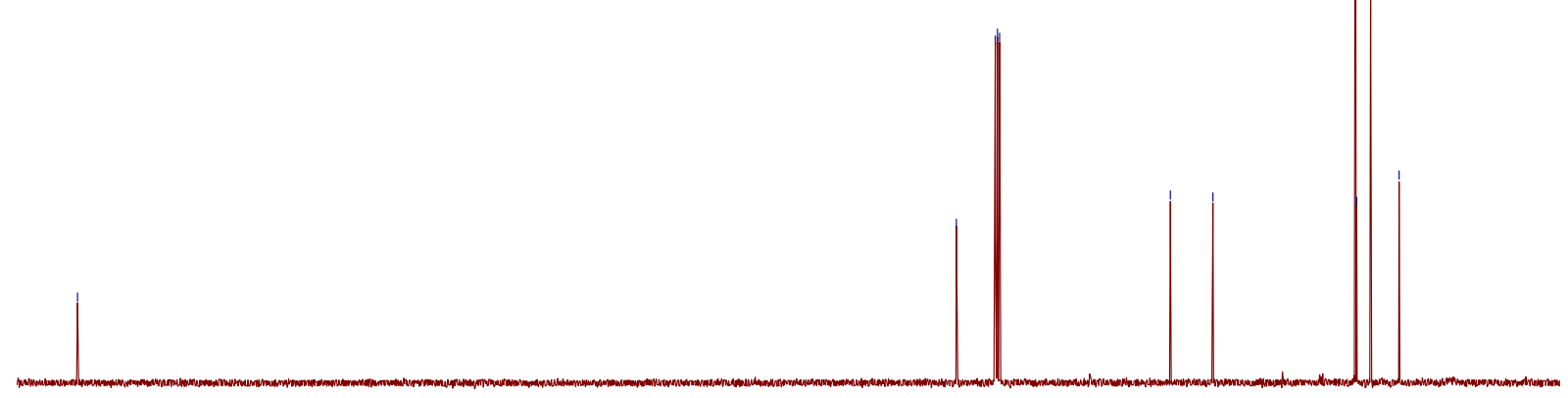

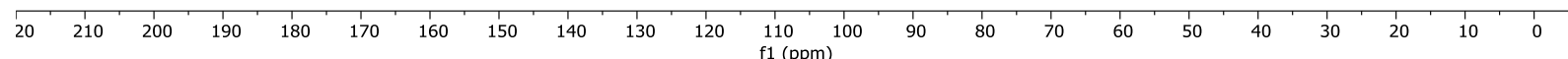


${ }^{11} \mathrm{~B}$ NMR $\left(128 \mathrm{MHz}, \mathrm{CDCl}_{3}\right)$ of compound $\mathbf{8}$ (see procedure):

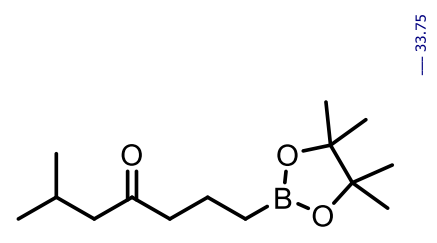

8

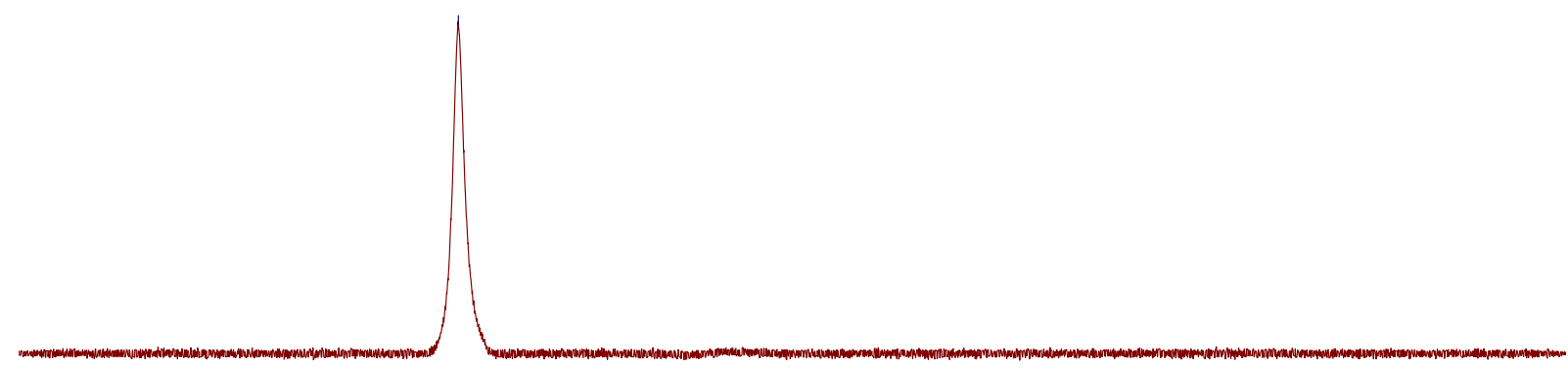

$\begin{array}{lllllllllllllllllllll}1 & 80 & 70 & 60 & 50 & 40 & 30 & 20 & 10 & 0 & \begin{array}{c}1 \\ \mathrm{f} 1(\mathrm{ppm})\end{array} & -20 & -30 & -40 & -50 & -60 & -70 & -80 & -90 & -100 & -11\end{array}$ 
${ }^{1} \mathrm{H} \mathrm{NMR}\left(400 \mathrm{MHz}, \mathrm{CDCl}_{3}\right.$ ) of compound 9 (see procedure):

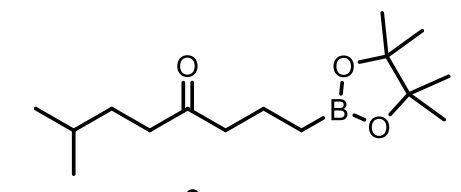

9

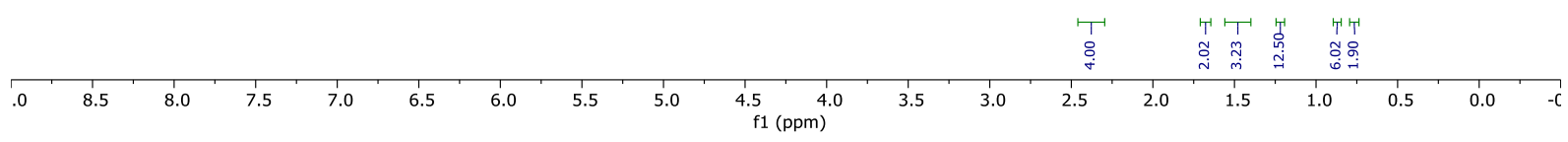

${ }^{13} \mathrm{C}$ NMR (101 MHz, $\mathrm{CDCl}_{3}$ ) of compound 9 (see procedure):

in.

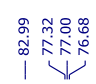

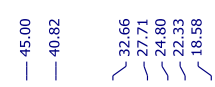

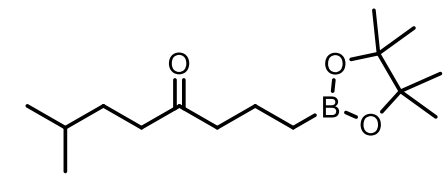

9

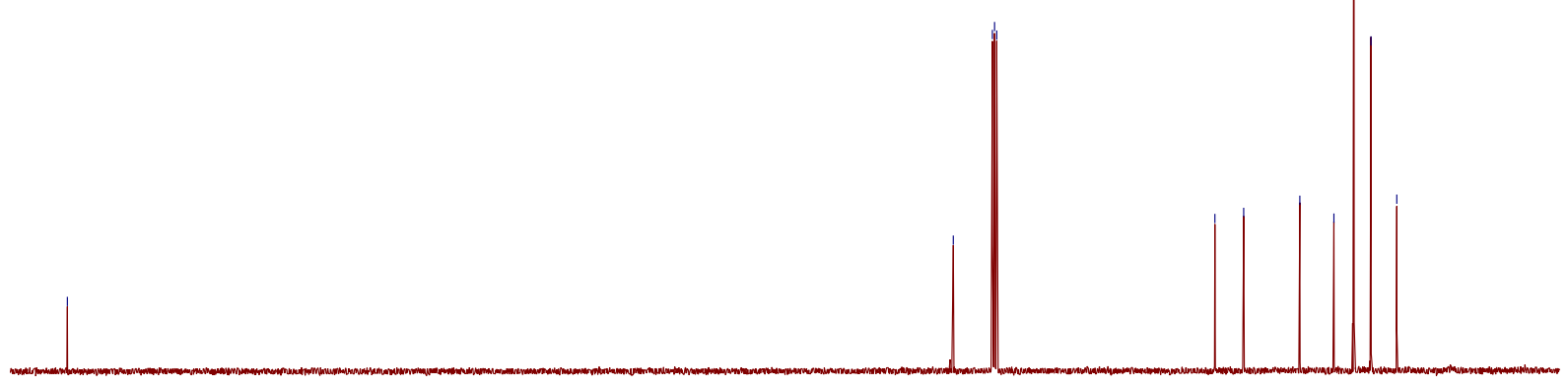

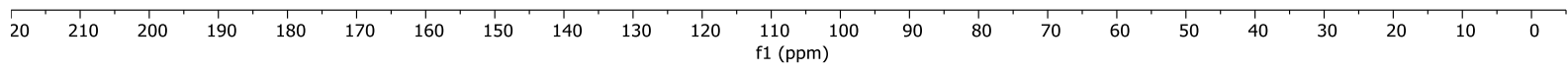

S150 
${ }^{11} \mathrm{~B}$ NMR (128 MHz, $\left.\mathrm{CDCl}_{3}\right)$ of compound 9 (see procedure):

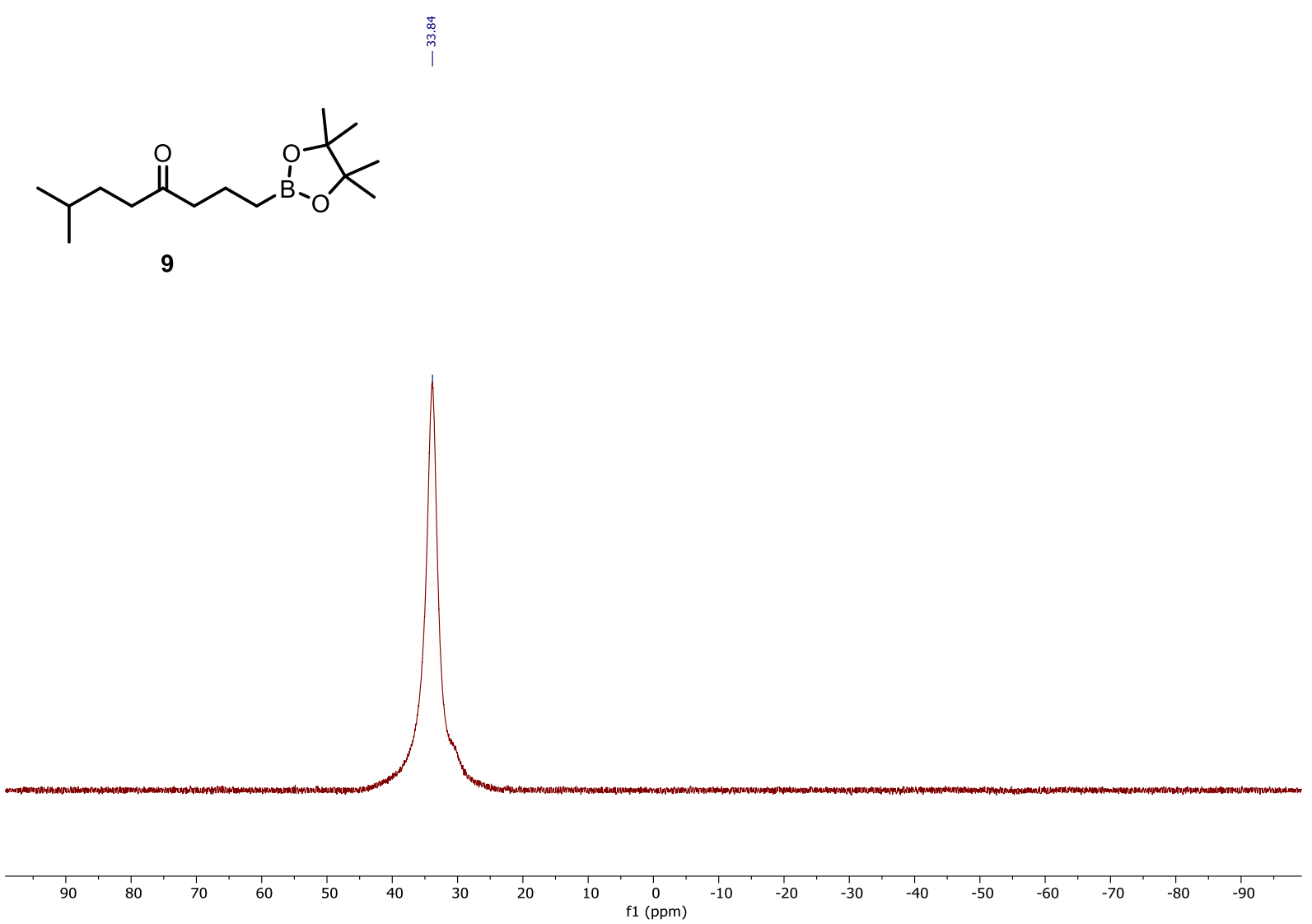


${ }^{1} \mathrm{H}$ NMR (400 MHz, $\mathrm{CDCl}_{3}$ ) of compound 10 (see procedure):

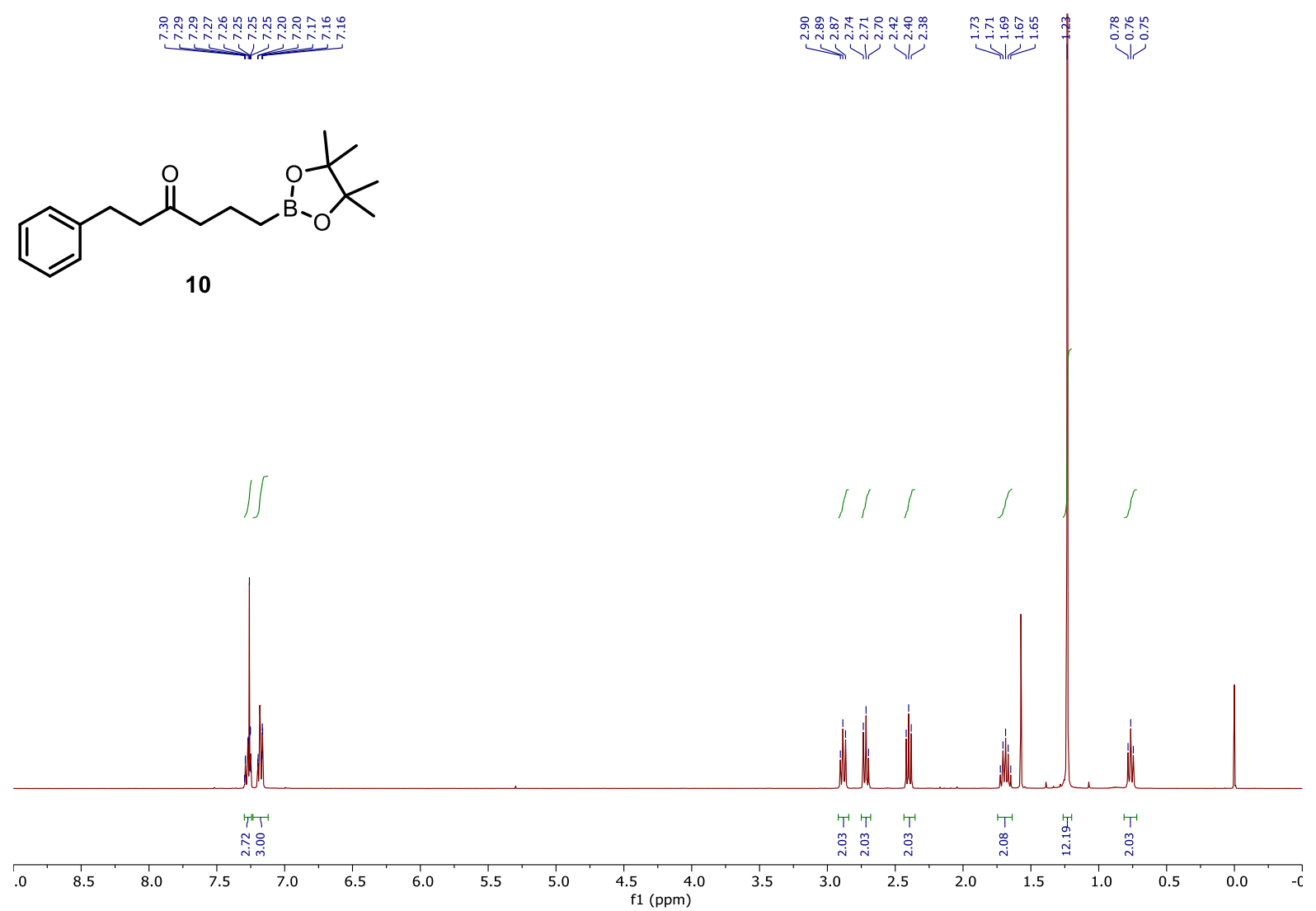

${ }^{13} \mathrm{C}$ NMR (101 MHz, $\mathrm{CDCl}_{3}$ ) of compound 10 (see procedure):

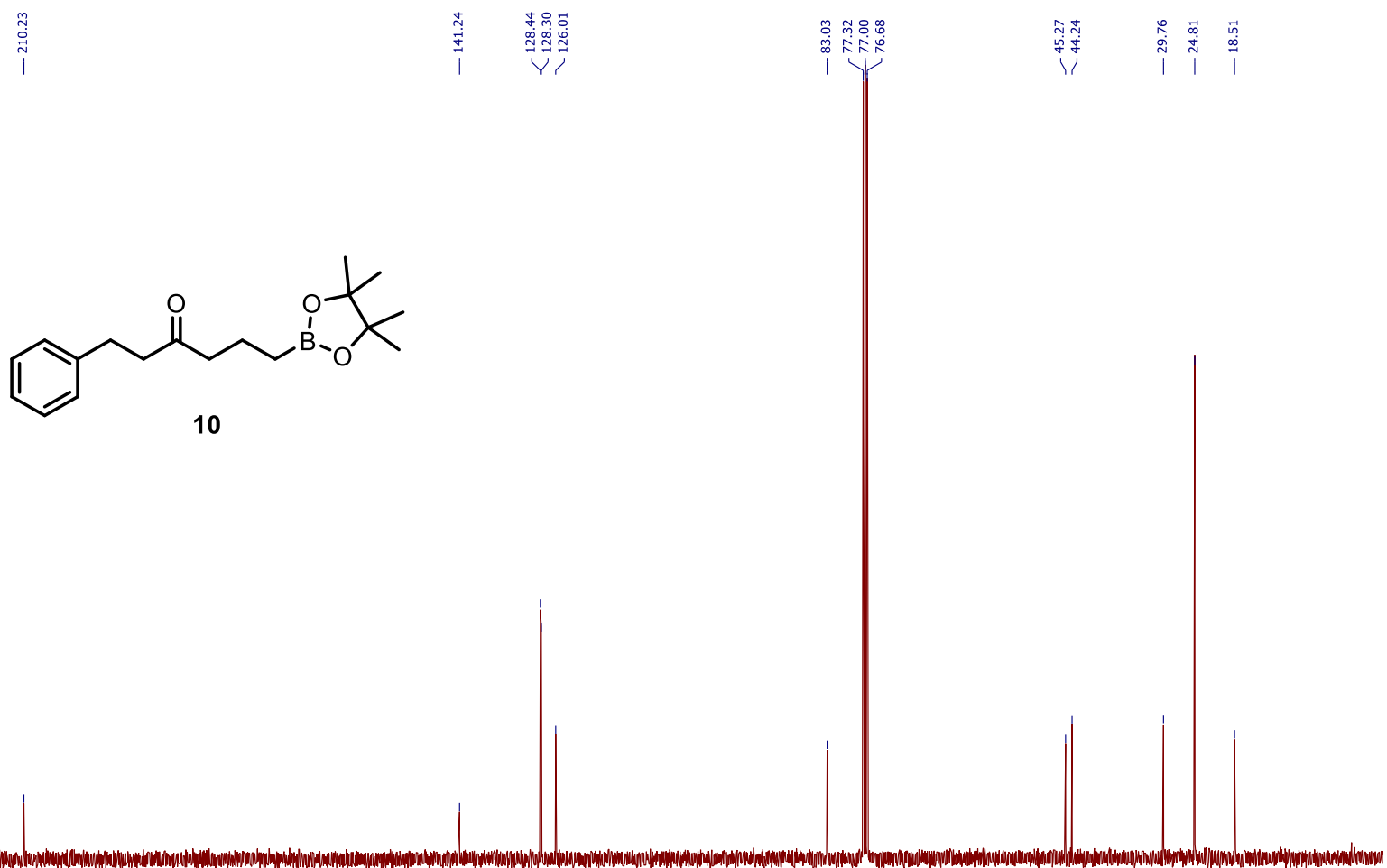

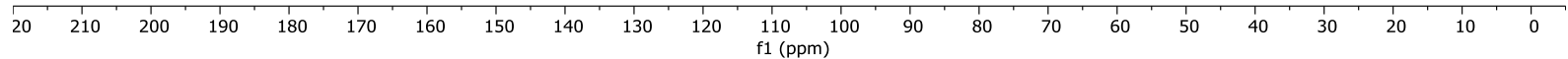


${ }^{11} \mathrm{~B}$ NMR (128 MHz, $\mathrm{CDCl}_{3}$ ) of compound $\mathbf{1 0}$ (see procedure):<smiles>CC1(C)OB(CCCC(=O)CCc2ccccc2)OC1(C)C</smiles>

$\mid$

\begin{tabular}{lllllllllllllllllllll}
\hline 90 & 80 & 70 & 60 & 50 & 40 & 30 & 20 & 10 & 0 & $\begin{array}{c}10 \\
\mathrm{f} 1(\mathrm{ppm})\end{array}$ & -20 & -30 & -40 & -50 & -60 & -70 & -80 & -90 & -100 & -11
\end{tabular}


${ }^{1} \mathrm{H}$ NMR (400 MHz, $\mathrm{CDCl}_{3}$ ) of compound 11 (see procedure):

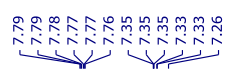

ํㅜㅇㅇㅕ
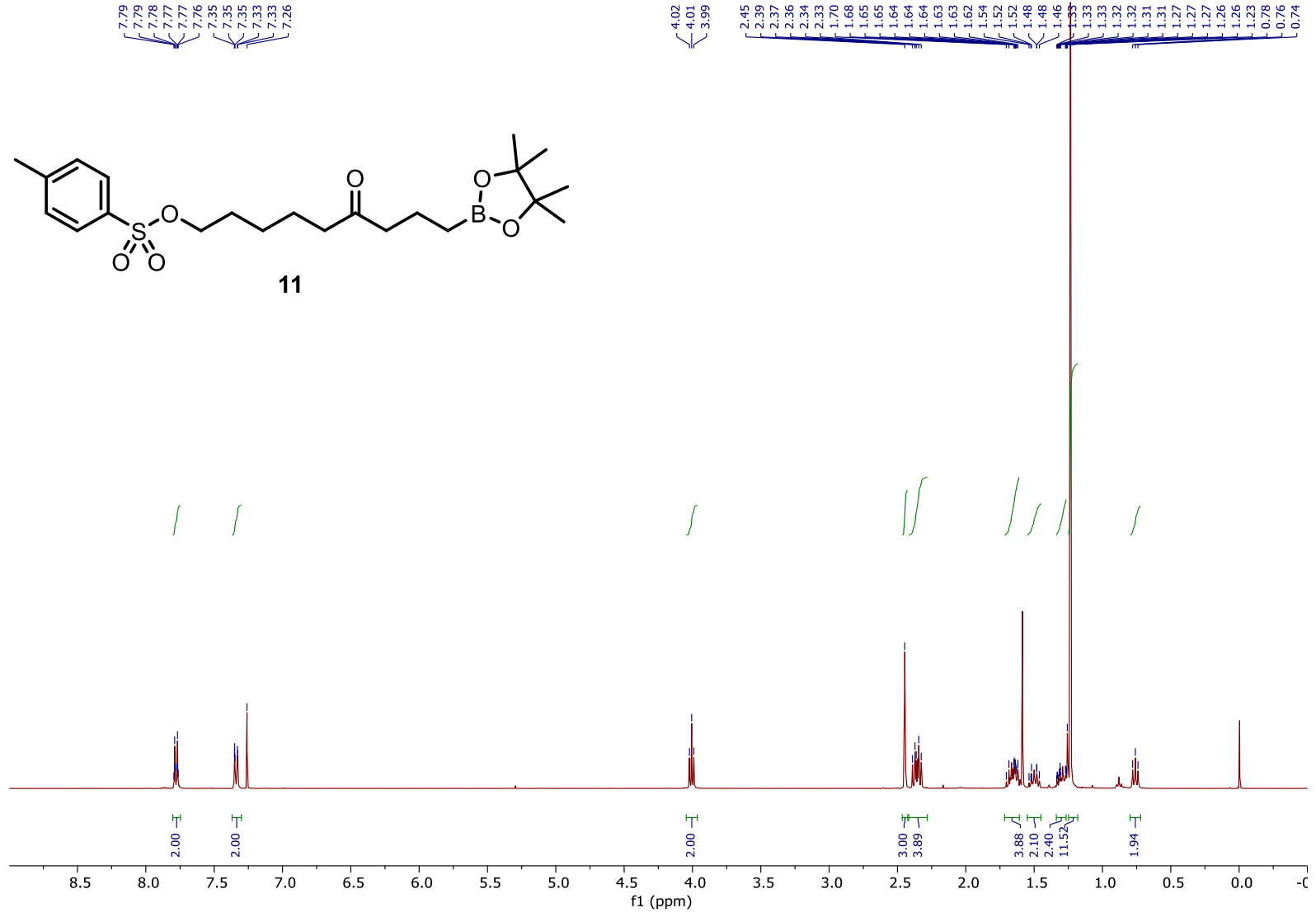

${ }^{13} \mathrm{C}$ NMR (101 MHz, $\mathrm{CDCl}_{3}$ ) of compound 11 (see procedure):
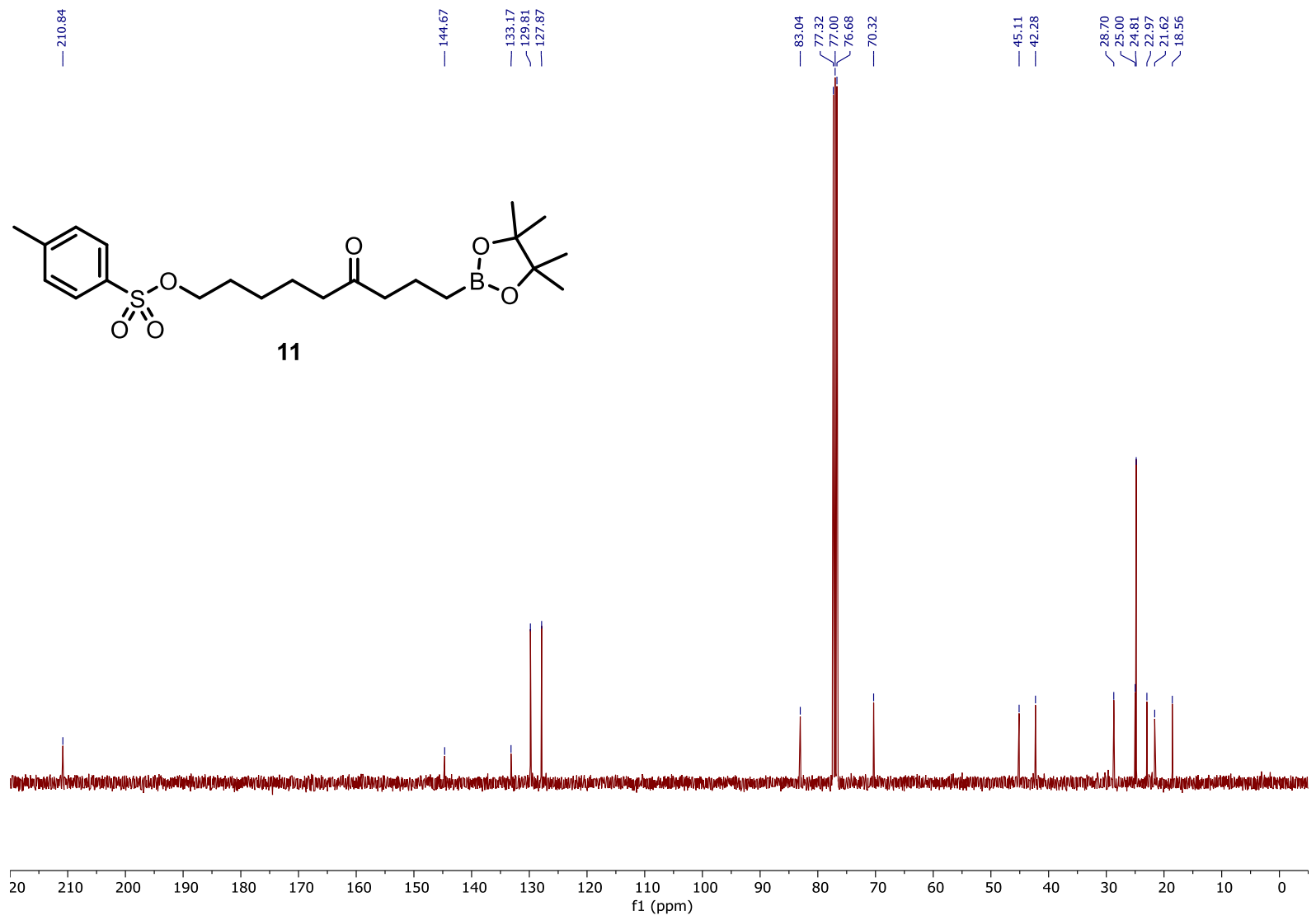

S154 
${ }^{11} \mathrm{~B}$ NMR (128 MHz, $\mathrm{CDCl}_{3}$ ) of compound 11 (see procedure):

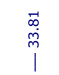

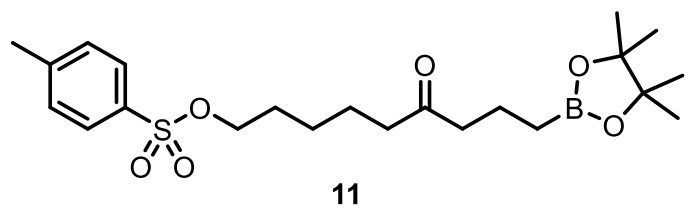

11

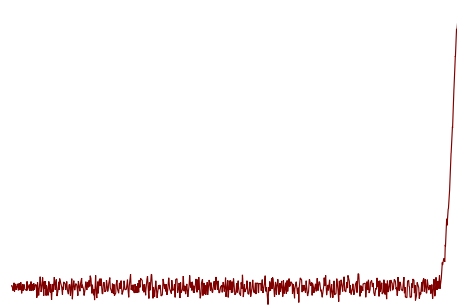

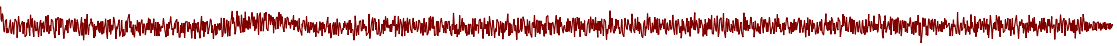

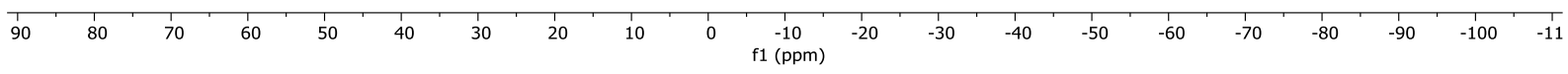

S155 
${ }^{1} \mathrm{H}$ NMR (400 MHz, $\mathrm{CDCl}_{3}$ ) of compound 12 (see procedure):

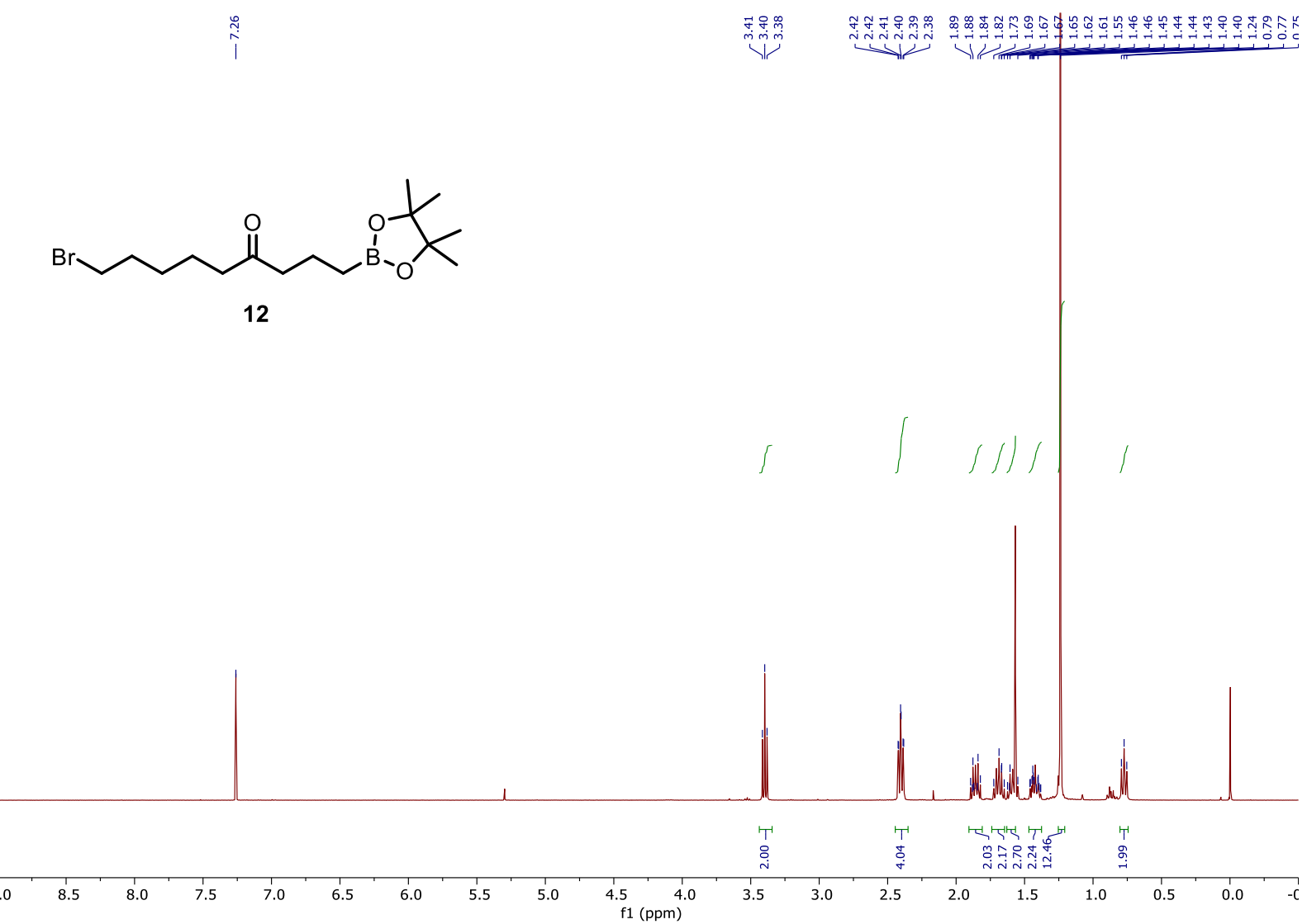

${ }^{13} \mathrm{C} \mathrm{NMR}\left(101 \mathrm{MHz}, \mathrm{CDCl}_{3}\right)$ of compound 12 (see procedure):

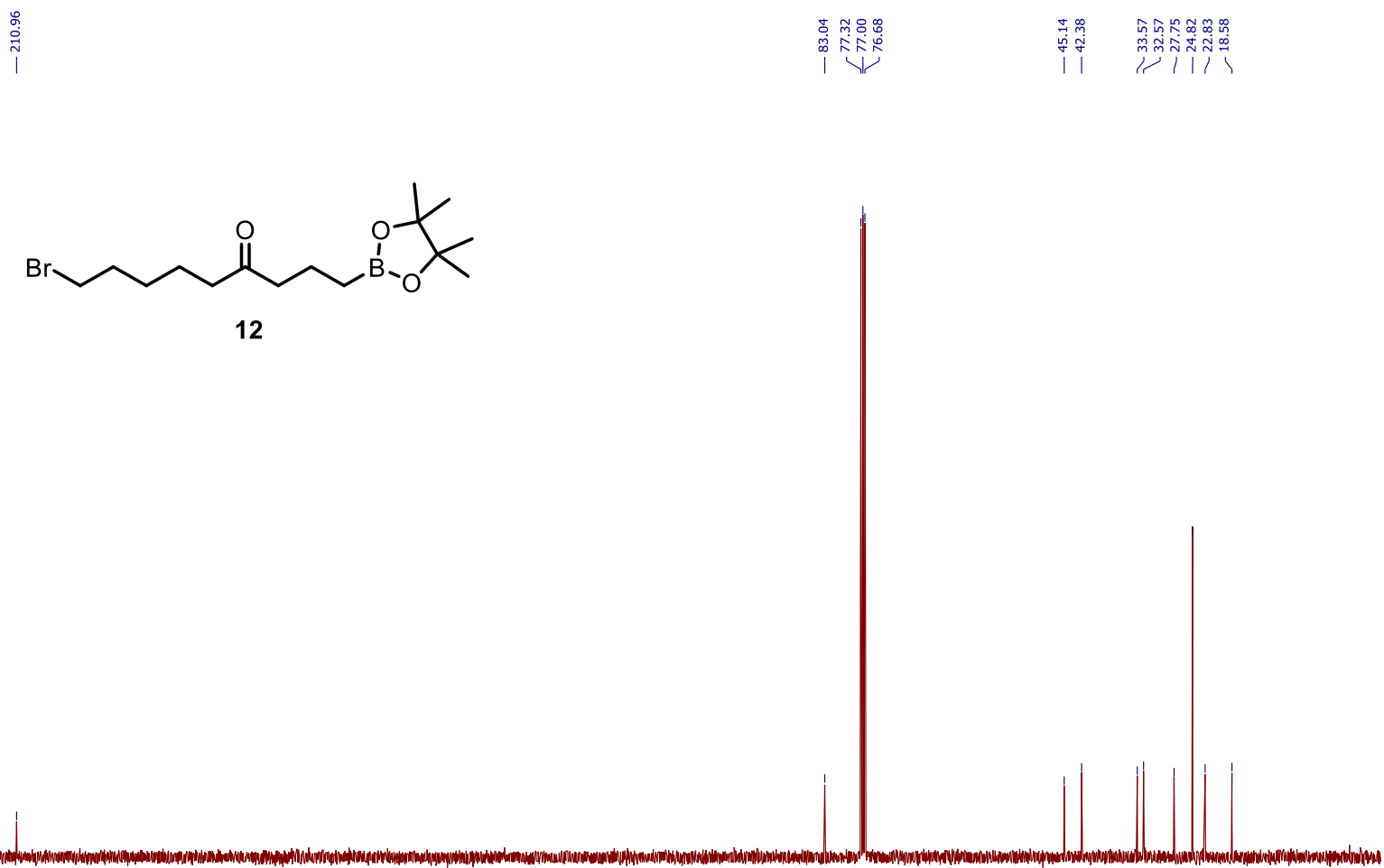

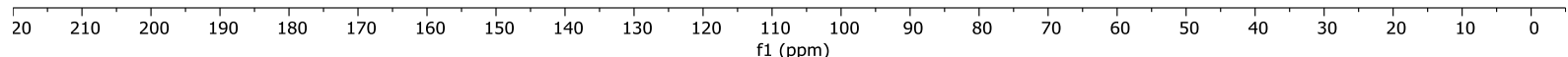


${ }^{11} \mathrm{~B}$ NMR (128 MHz, $\left.\mathrm{CDCl}_{3}\right)$ of compound 12 (see procedure):

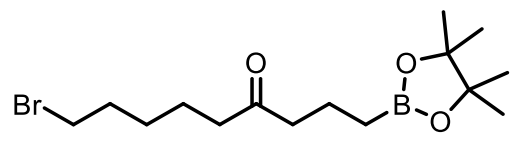

12

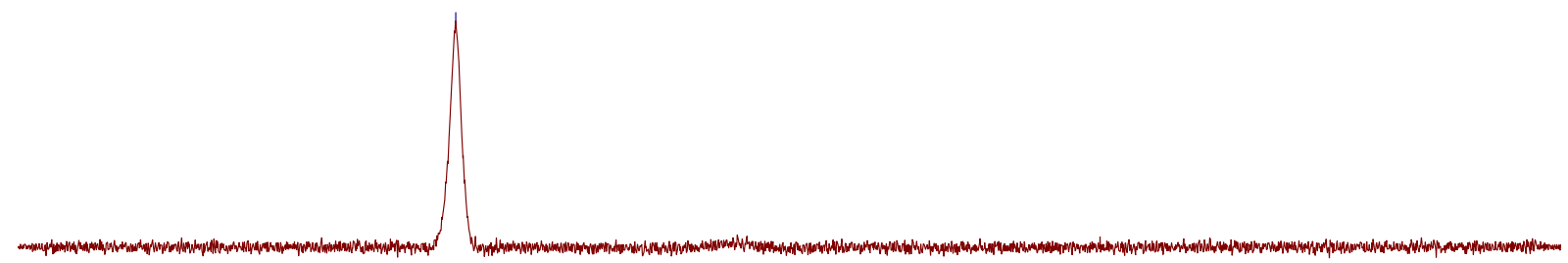

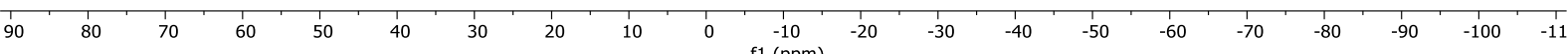


${ }^{1} \mathrm{H}$ NMR (400 MHz, $\mathrm{CDCl}_{3}$ ) of compound 13 (see procedure):
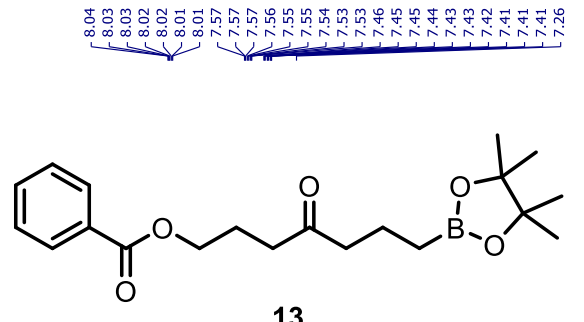

13

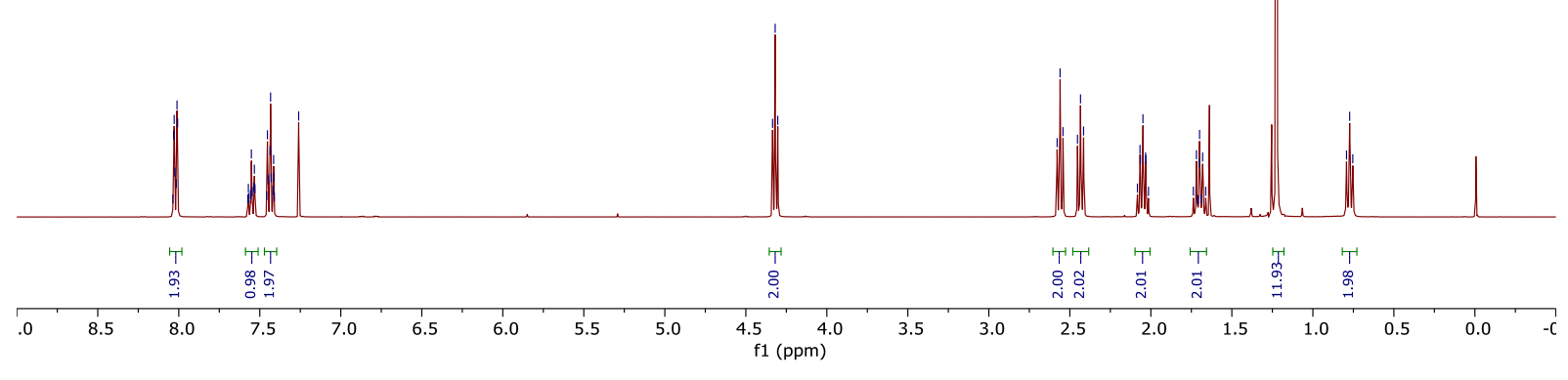

${ }^{13} \mathrm{C}$ NMR (101 MHz, $\mathrm{CDCl}_{3}$ ) of compound 13 (see procedure):

।.

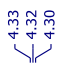

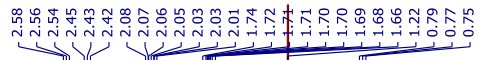

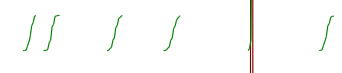

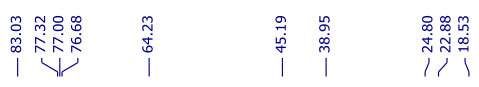

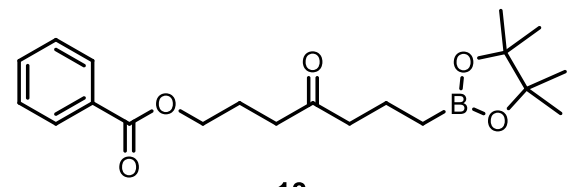

13
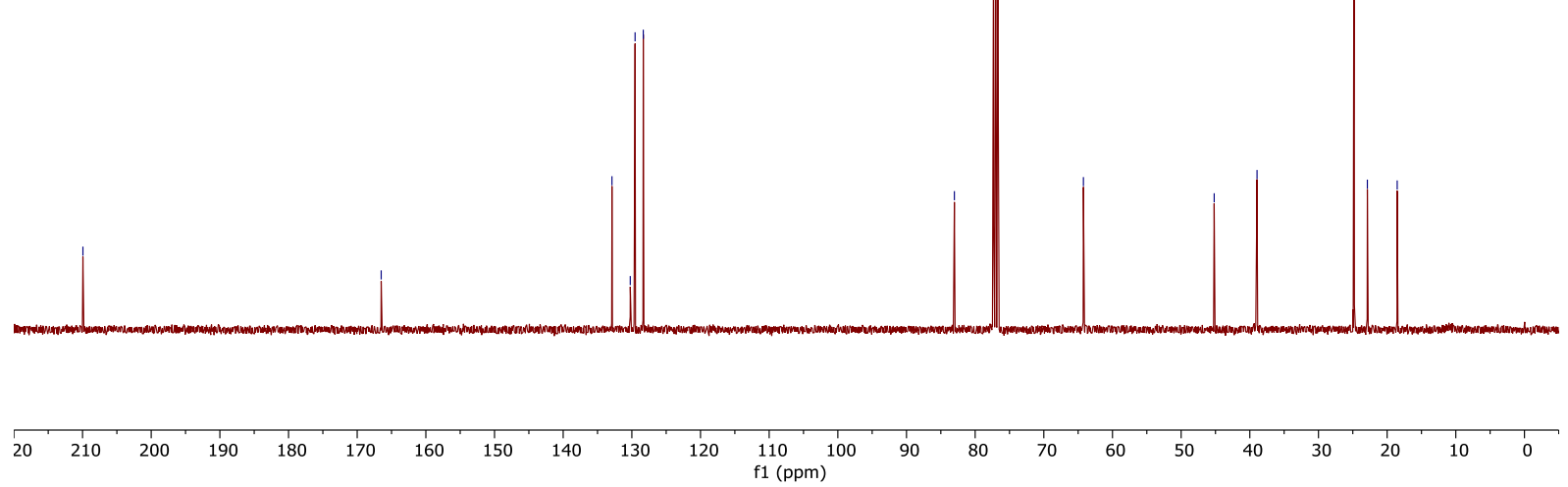

S158 
${ }^{11} \mathrm{~B}$ NMR (128 MHz, $\mathrm{CDCl}_{3}$ ) of compound 13 (see procedure):

ֻ

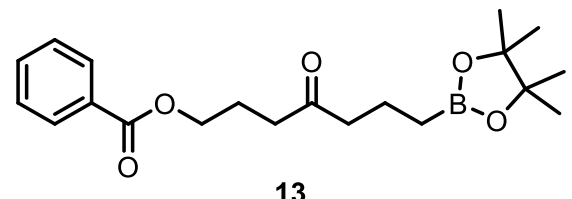

13
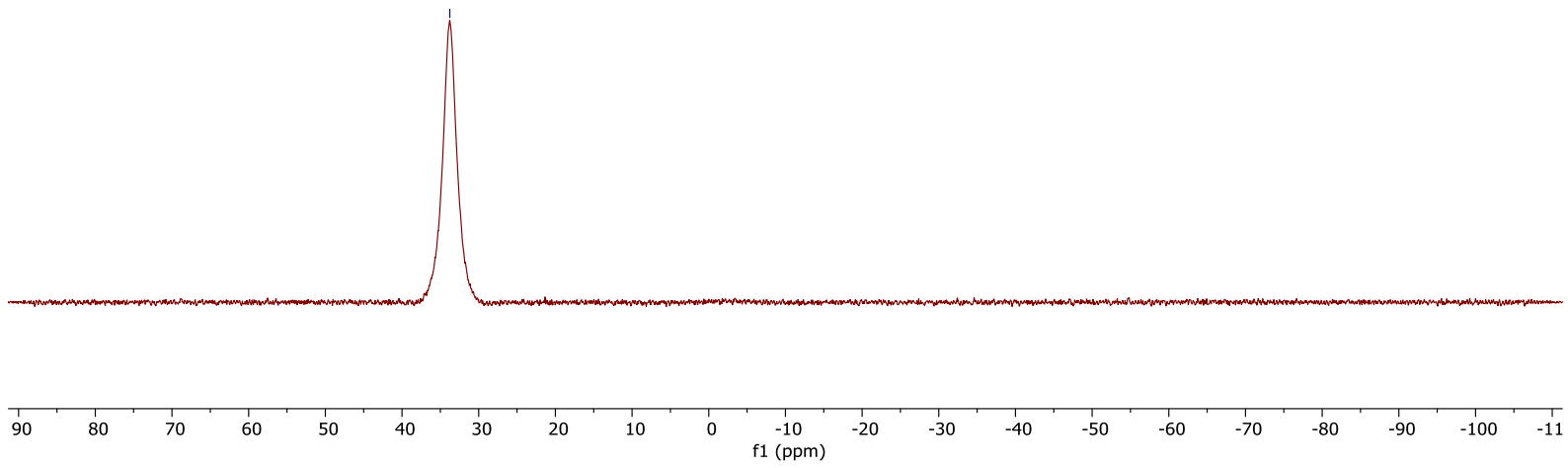

S159 
$\left.{ }^{1} \mathrm{H} \mathrm{NMR} \mathrm{(400} \mathrm{MHz,} \mathrm{CDCl}_{3}\right)$ of compound 14 (see procedure):

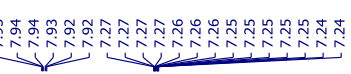

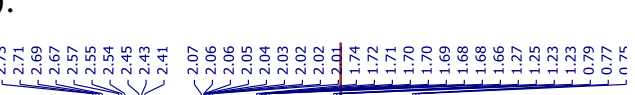

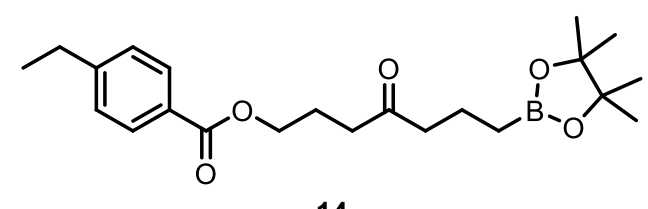
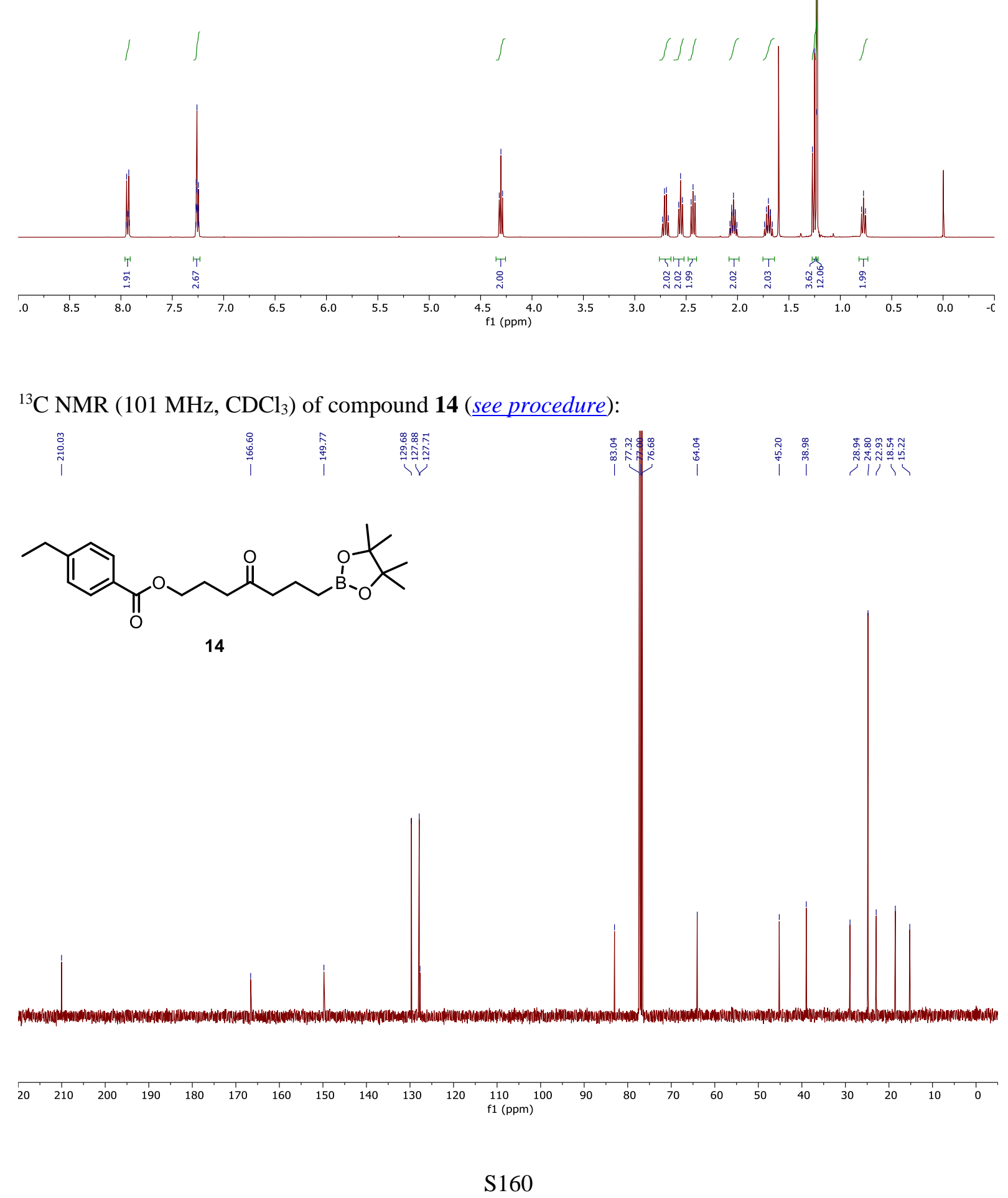
${ }^{11} \mathrm{~B}$ NMR (128 MHz, $\mathrm{CDCl}_{3}$ ) of compound 14 (see procedure):

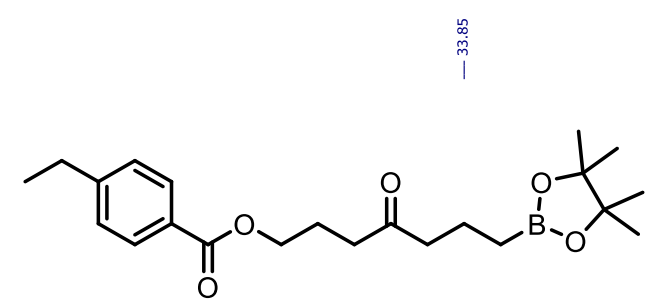

14

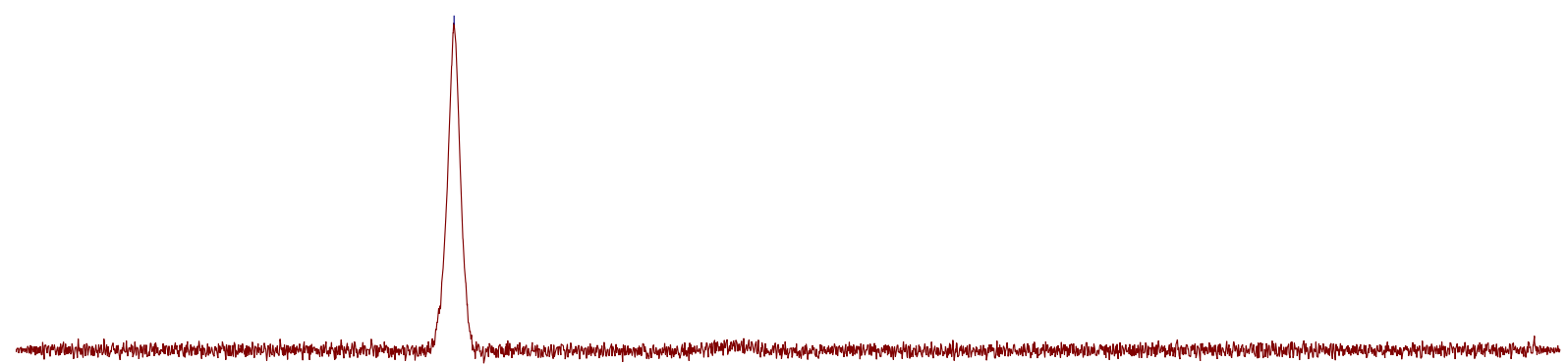

$\begin{array}{lllllllllllllllllllllll}90 & 80 & 70 & 60 & 50 & 40 & 30 & 20 & 10 & 0 & -10 & -20 & -30 & -40 & -50 & -60 & -70 & -80 & -90 & -100 & -11\end{array}$ 
${ }^{1} \mathrm{H}$ NMR (400 MHz, $\mathrm{CDCl}_{3}$ ) of compound 15 (see procedure):

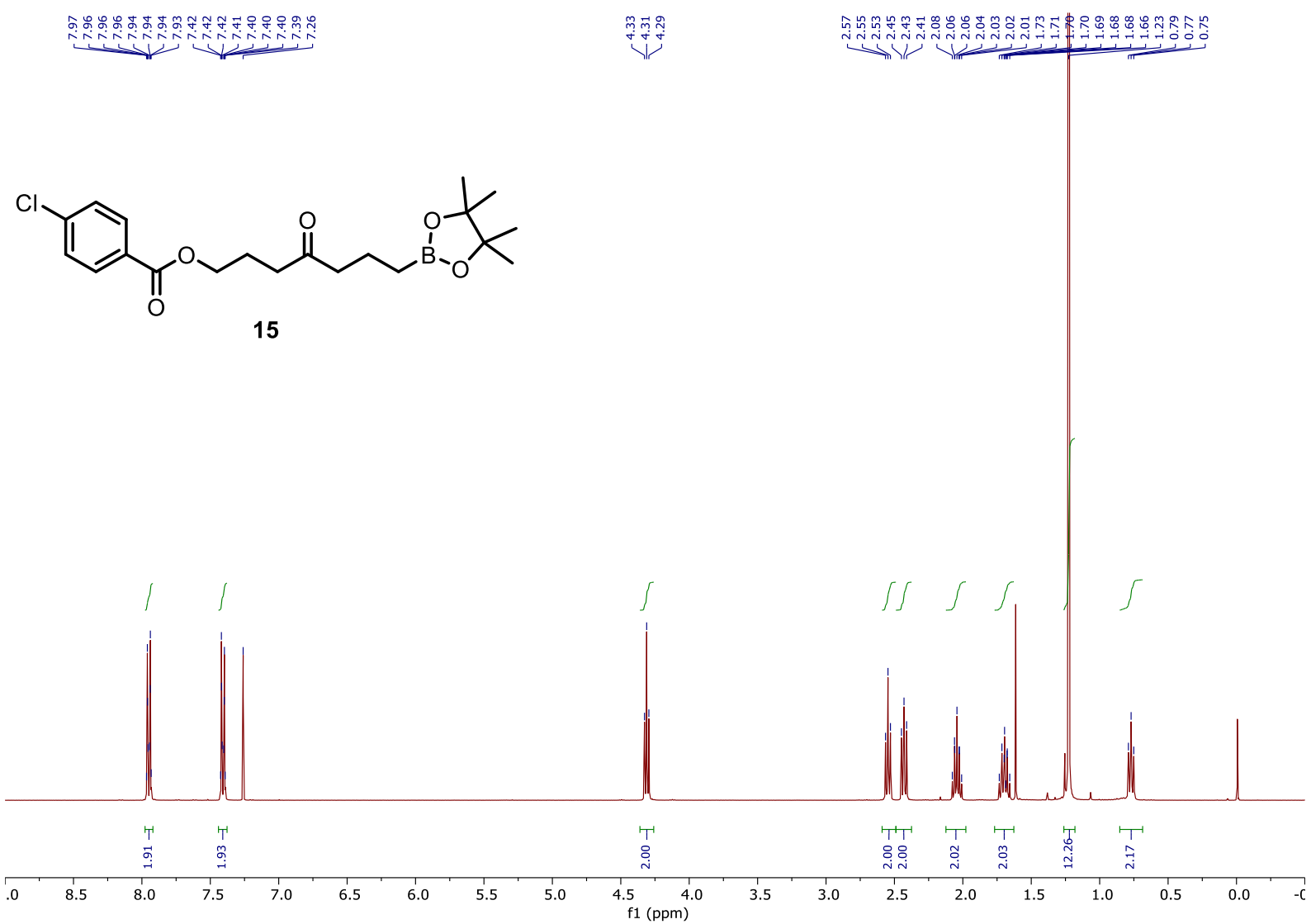

${ }^{13} \mathrm{C} \mathrm{NMR}\left(101 \mathrm{MHz}, \mathrm{CDCl}_{3}\right)$ of compound 15 (see procedure):

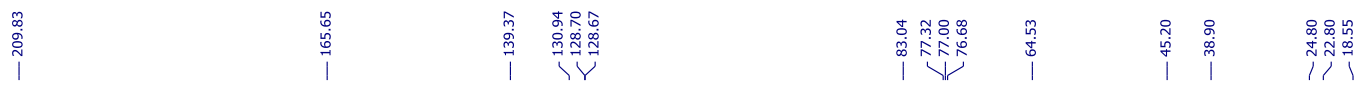<smiles>CC1(C)OB(CCCC(=O)CCCOC(=O)c2ccc(Cl)cc2)OC1(C)C</smiles>

15

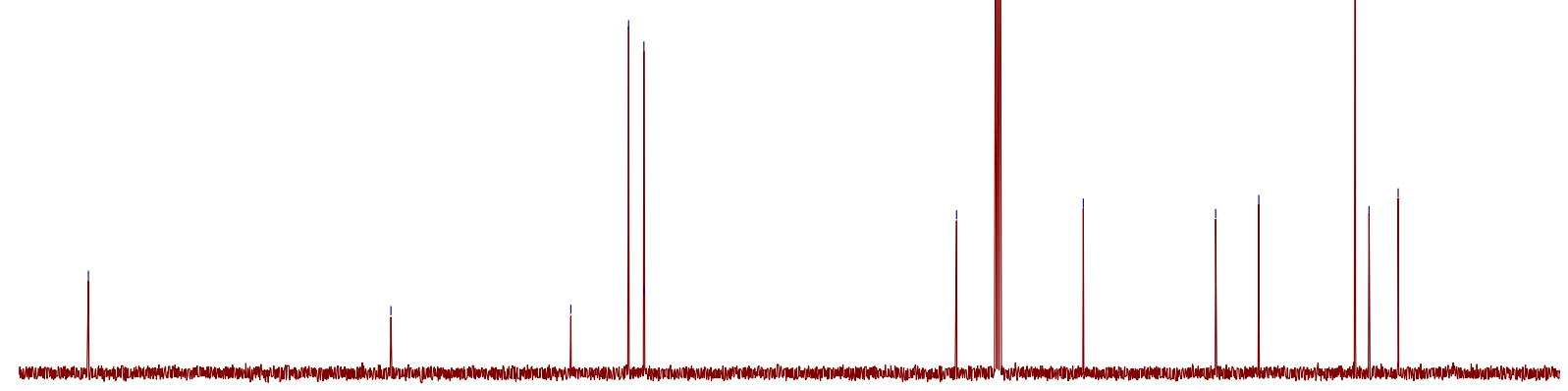

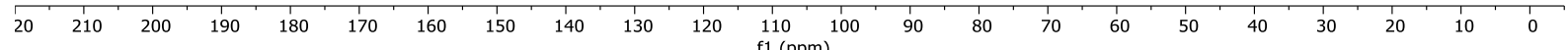


${ }^{11} \mathrm{~B}$ NMR (128 MHz, $\mathrm{CDCl}_{3}$ ) of compound 15 (see procedure):
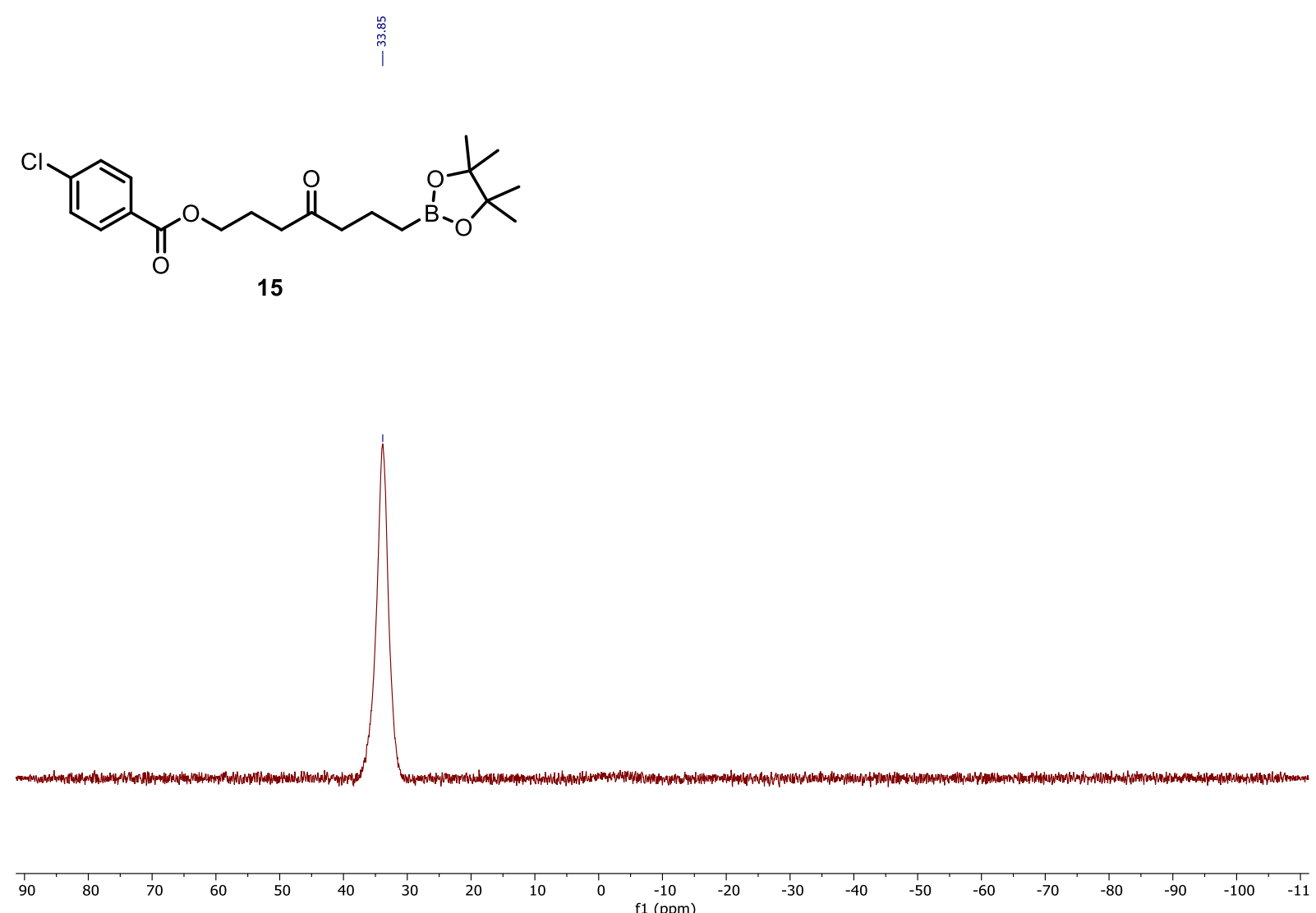
${ }^{1} \mathrm{H}$ NMR (400 MHz, $\mathrm{CDCl}_{3}$ ) of compound 16 (see procedure):

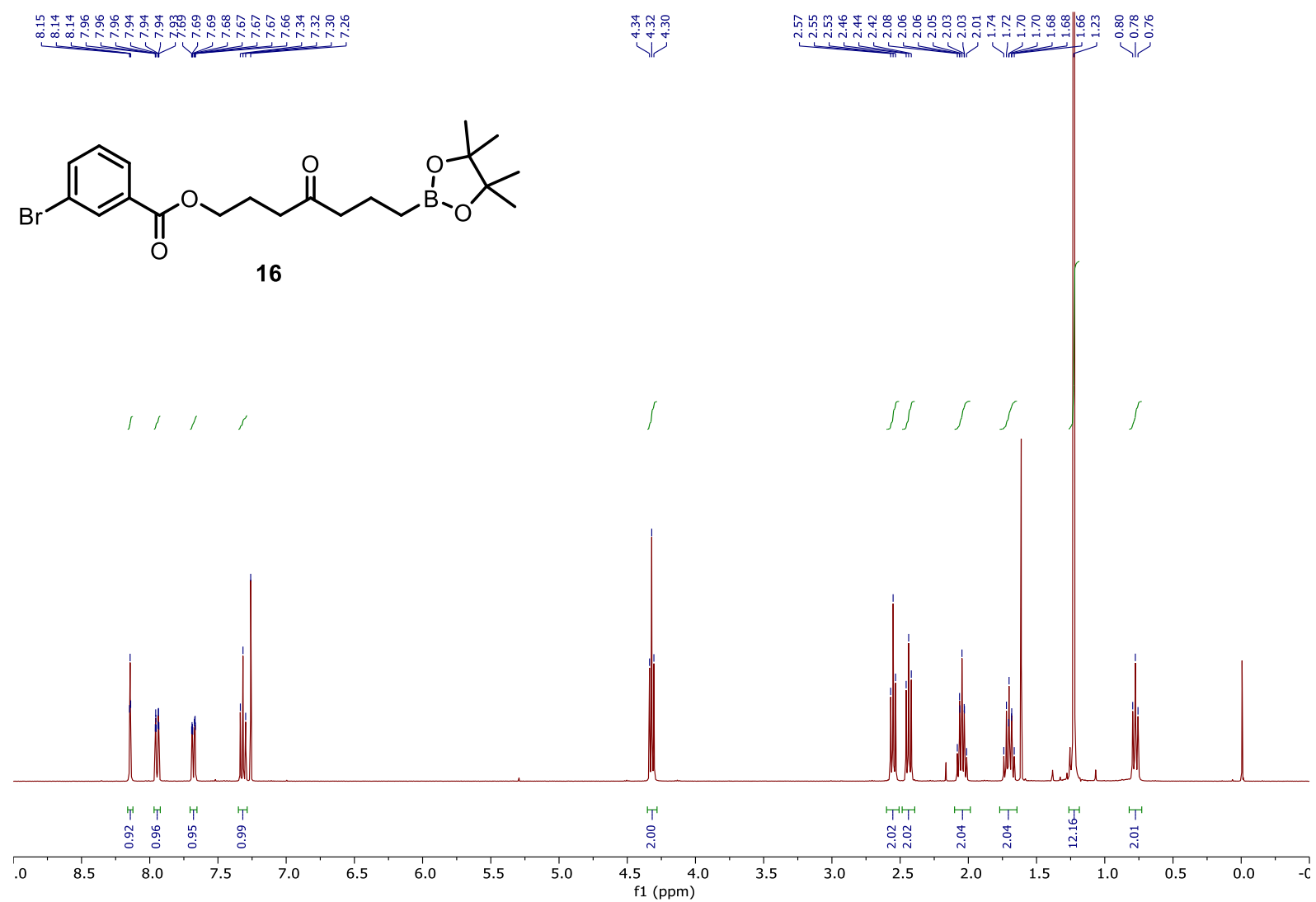

${ }^{13} \mathrm{C}$ NMR (101 MHz, $\mathrm{CDCl}_{3}$ ) of compound 16 (see procedure):

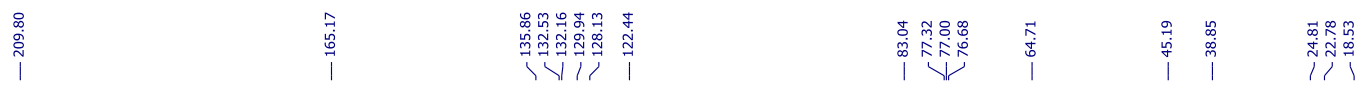<smiles>CC1(C)OB(CCCC(=O)CCCOC(=O)c2cccc(Br)c2)OC1(C)C</smiles>

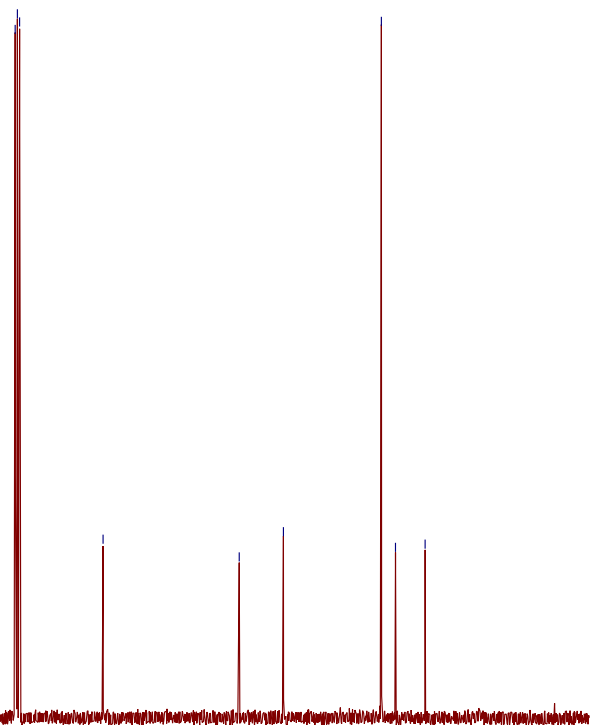

\begin{tabular}{llllllllllllllllllllllllll}
\hline & 20 & 200 & 190 & 180 & 170 & 160 & 150 & 140 & 130 & 120 & 110 & 100 & 90 & 80 & 70 & 60 & 50 & 40 & 30 & 20 & 10 & 0
\end{tabular} 
${ }^{11} \mathrm{~B}$ NMR (128 MHz, $\mathrm{CDCl}_{3}$ ) of compound $\mathbf{1 6}$ (see procedure):

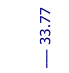
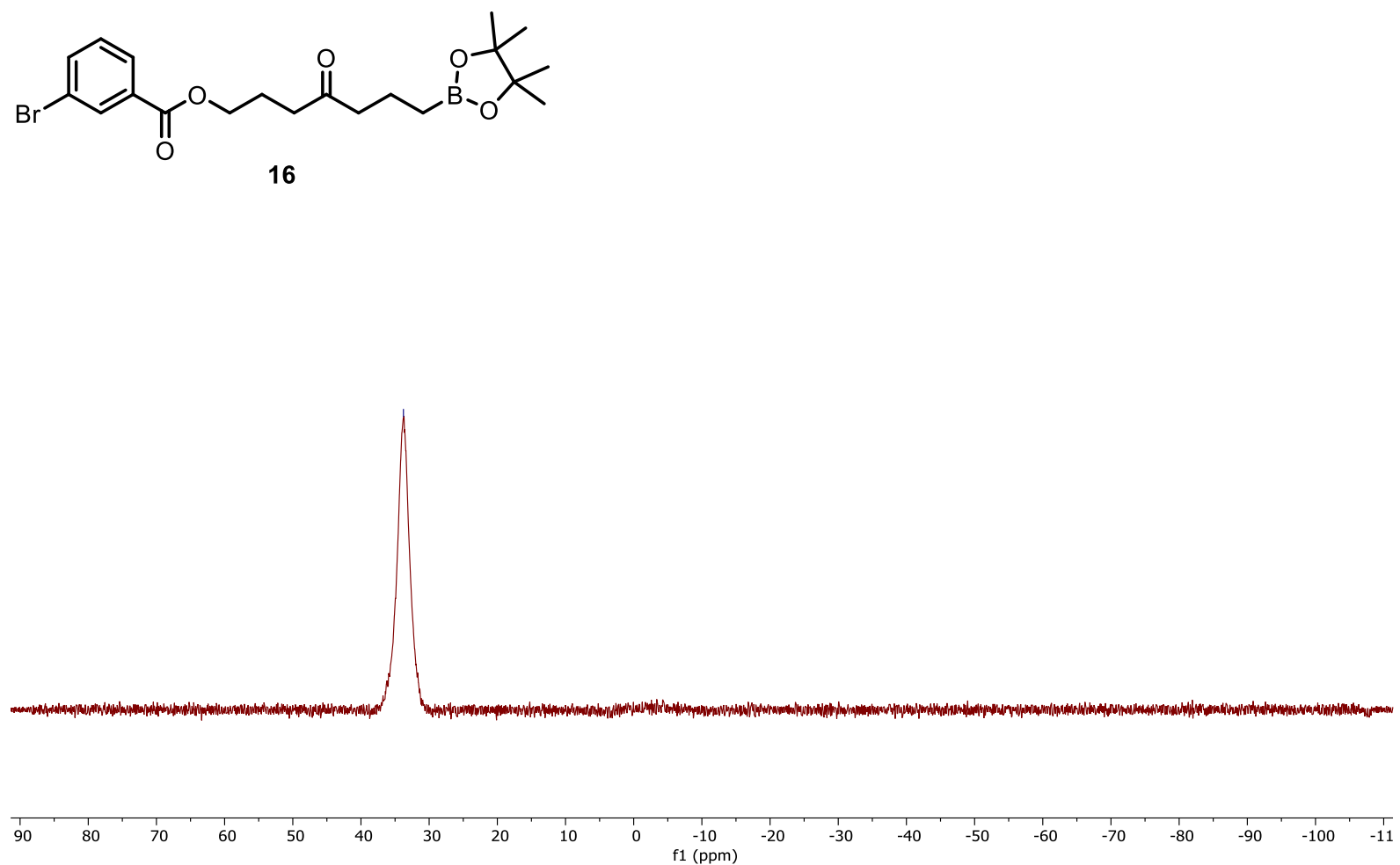

S165 
${ }^{1} \mathrm{H}$ NMR (400 MHz, $\mathrm{CDCl}_{3}$ ) of compound 17 (see procedure):

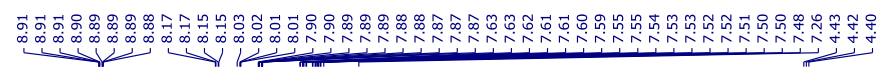<smiles>CC1(C)OB(CCCC(=O)CCCOC(=O)c2cccc3ccccc23)OC1(C)C</smiles>

17
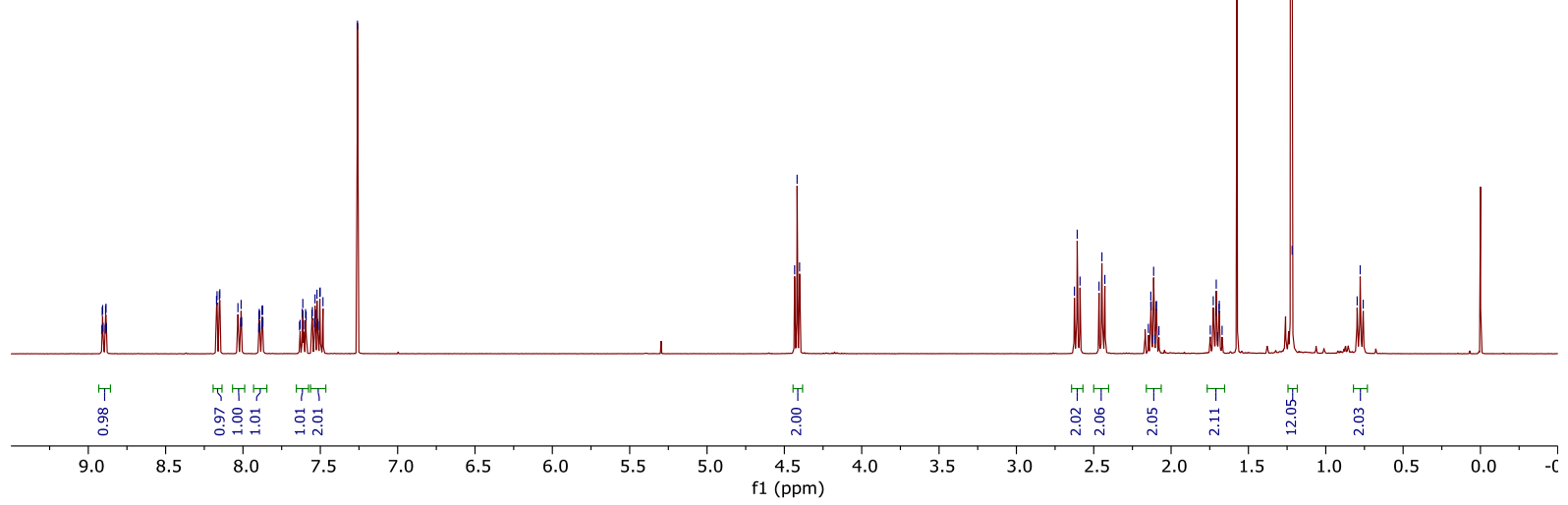

${ }^{13} \mathrm{C}$ NMR (101 MHz, $\mathrm{CDCl}_{3}$ ) of compound 17 (see procedure):
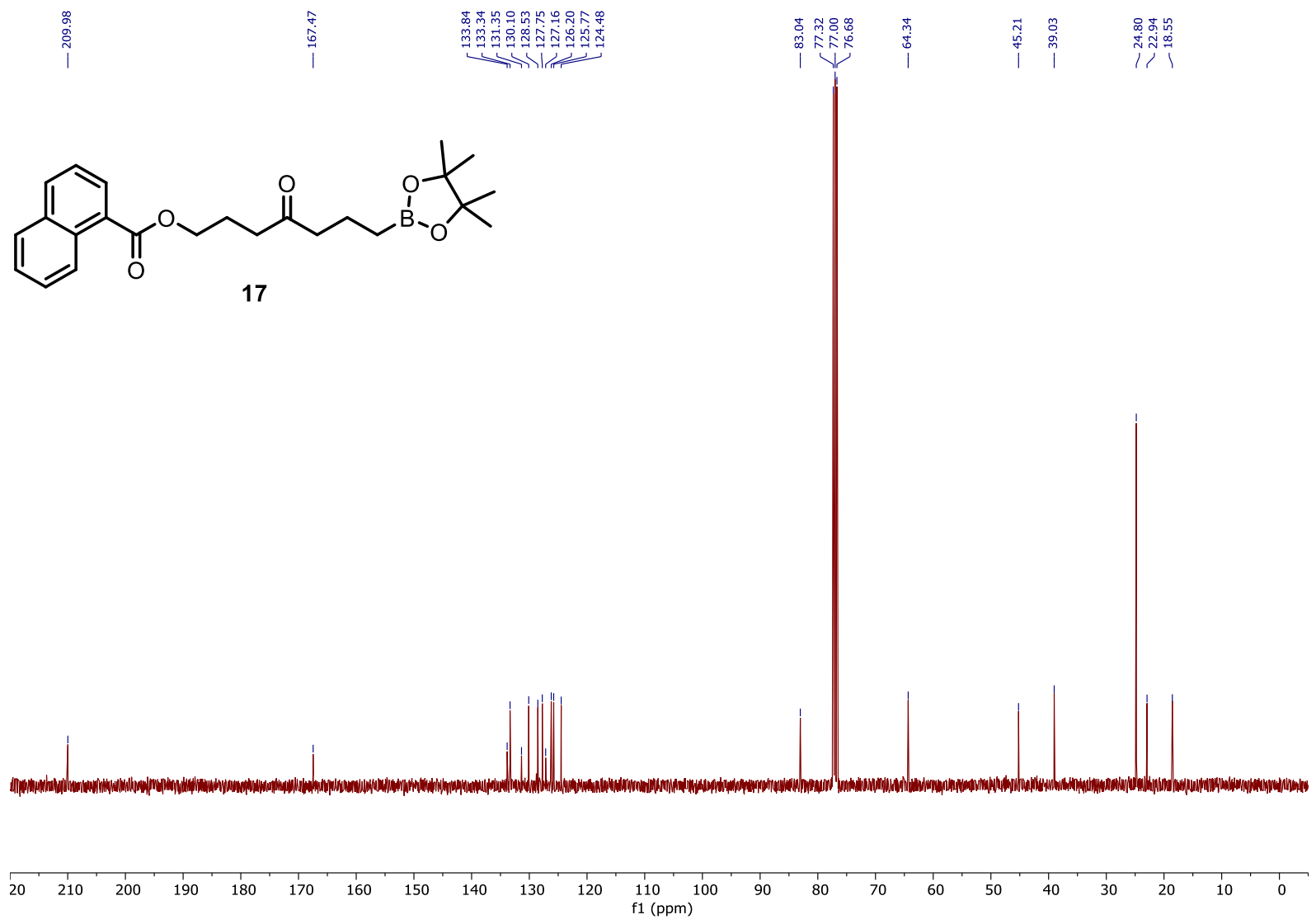

S166 
${ }^{11} \mathrm{~B}$ NMR (128 MHz, $\mathrm{CDCl}_{3}$ ) of compound 17 (see procedure):

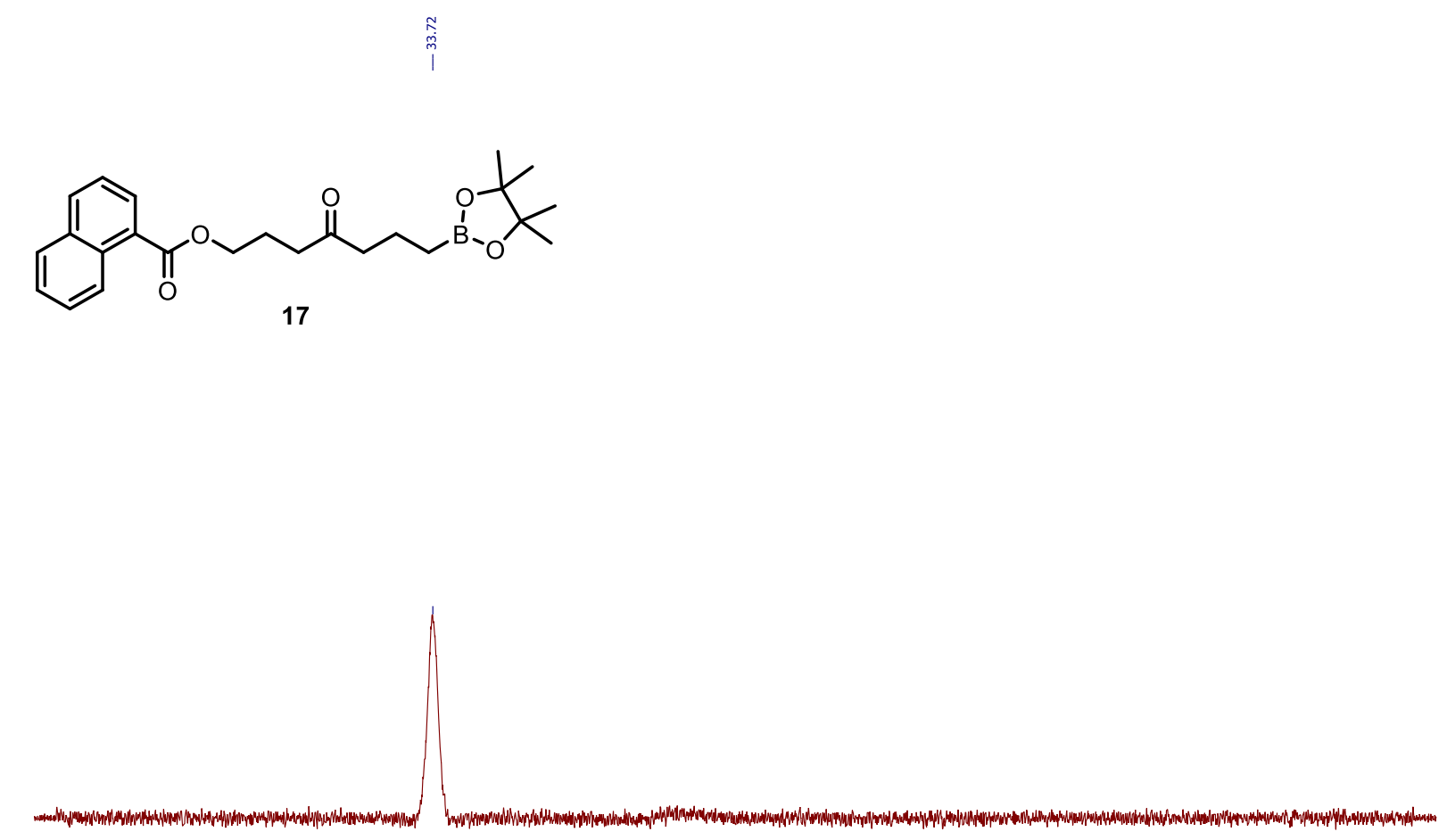

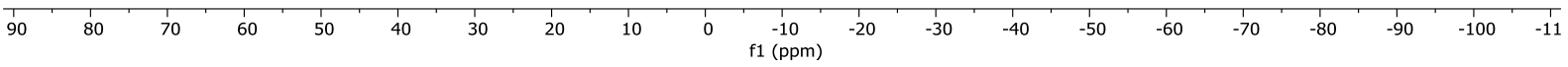


${ }^{1} \mathrm{H}$ NMR (400 MHz, $\mathrm{CDCl}_{3}$ ) of compound 18 (see procedure):

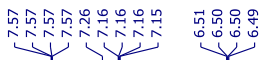

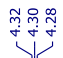

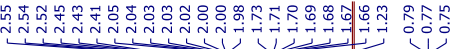<smiles>CC1(C)OB(CCCC(=O)CCCOC(=O)c2ccco2)OC1(C)C</smiles>
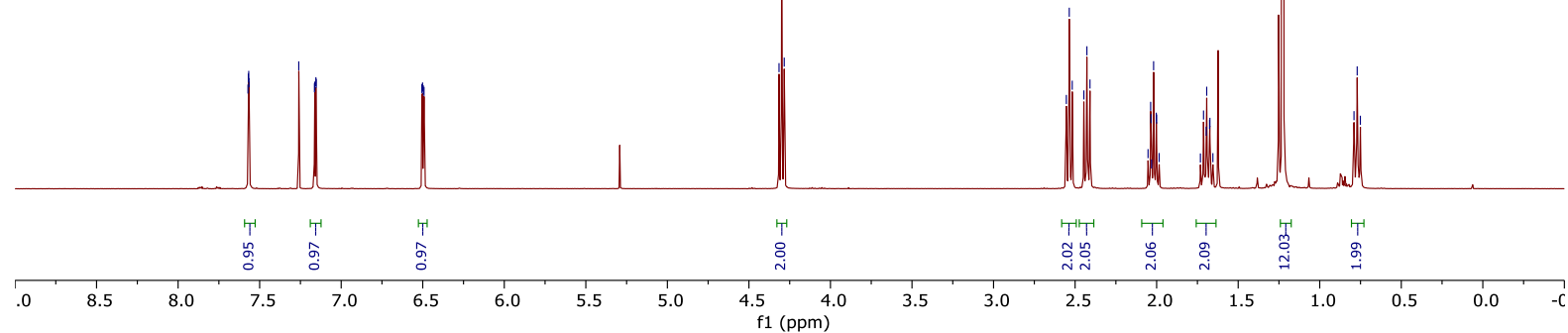

${ }^{13} \mathrm{C}$ NMR (101 MHz, $\mathrm{CDCl}_{3}$ ) of compound $\mathbf{1 8}$ (see procedure):

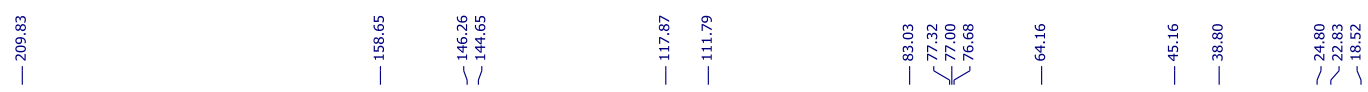<smiles>CC1(C)OB(CCCC(=O)CCCOC(=O)c2ccco2)OC1(C)C</smiles>

18

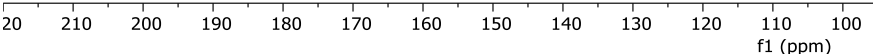


${ }^{11} \mathrm{~B}$ NMR (128 MHz, $\mathrm{CDCl}_{3}$ ) of compound 18 (see procedure):<smiles>CC1(C)OB(CCCC(=O)CCCOC(=O)c2ccco2)OC1(C)C</smiles>

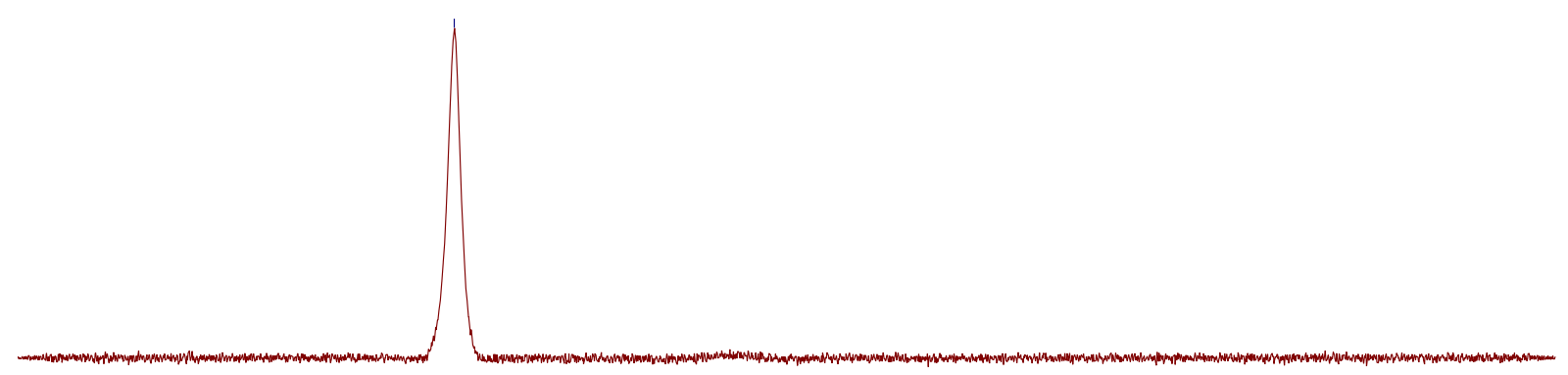

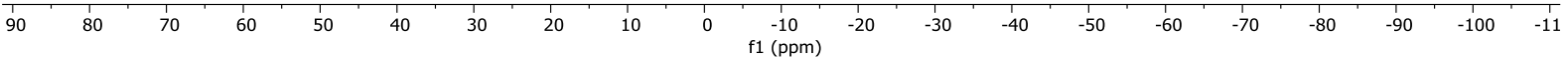


${ }^{1} \mathrm{H}$ NMR (400 MHz, $\mathrm{CDCl}_{3}$ ) of compound 19 (see procedure):
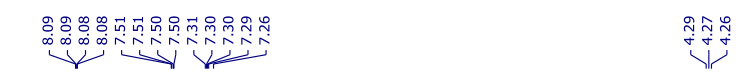

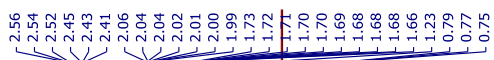<smiles>CC1(C)OB(CCCC(=O)CCCOC(=O)c2ccsc2)OC1(C)C</smiles>

19

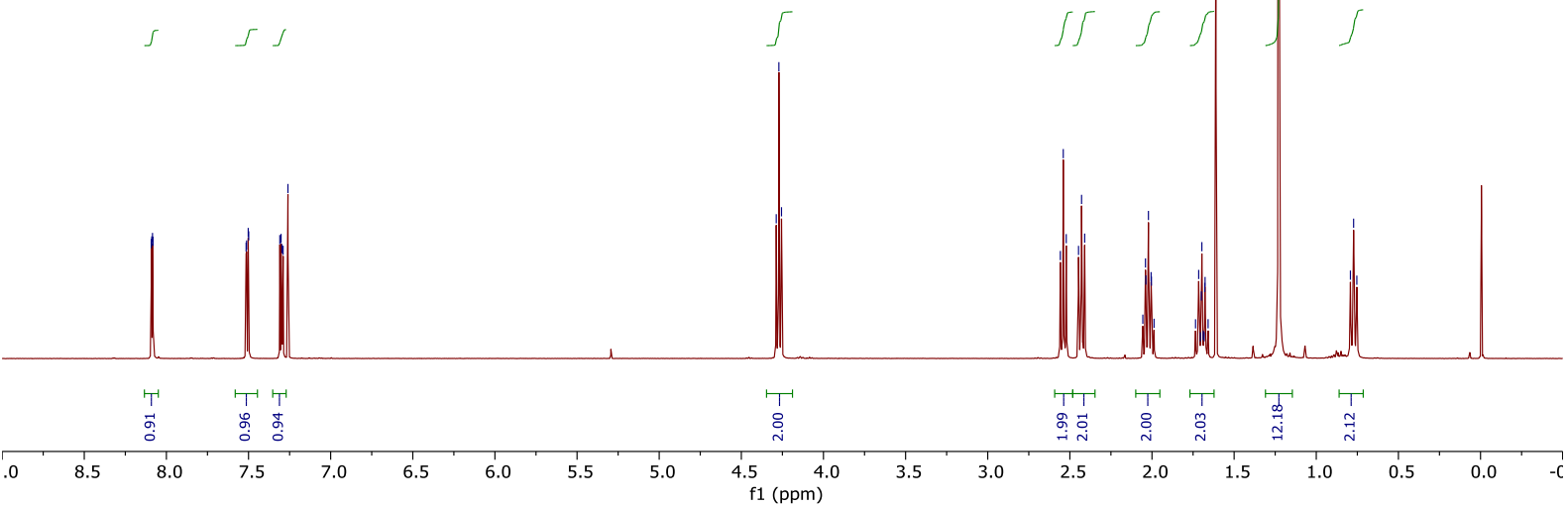

${ }^{13} \mathrm{C}$ NMR (101 MHz, $\mathrm{CDCl}_{3}$ ) of compound $\mathbf{1 9}$ (see procedure):

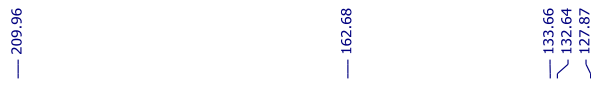

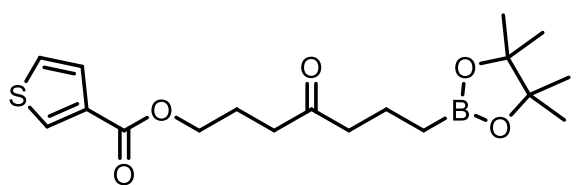

19

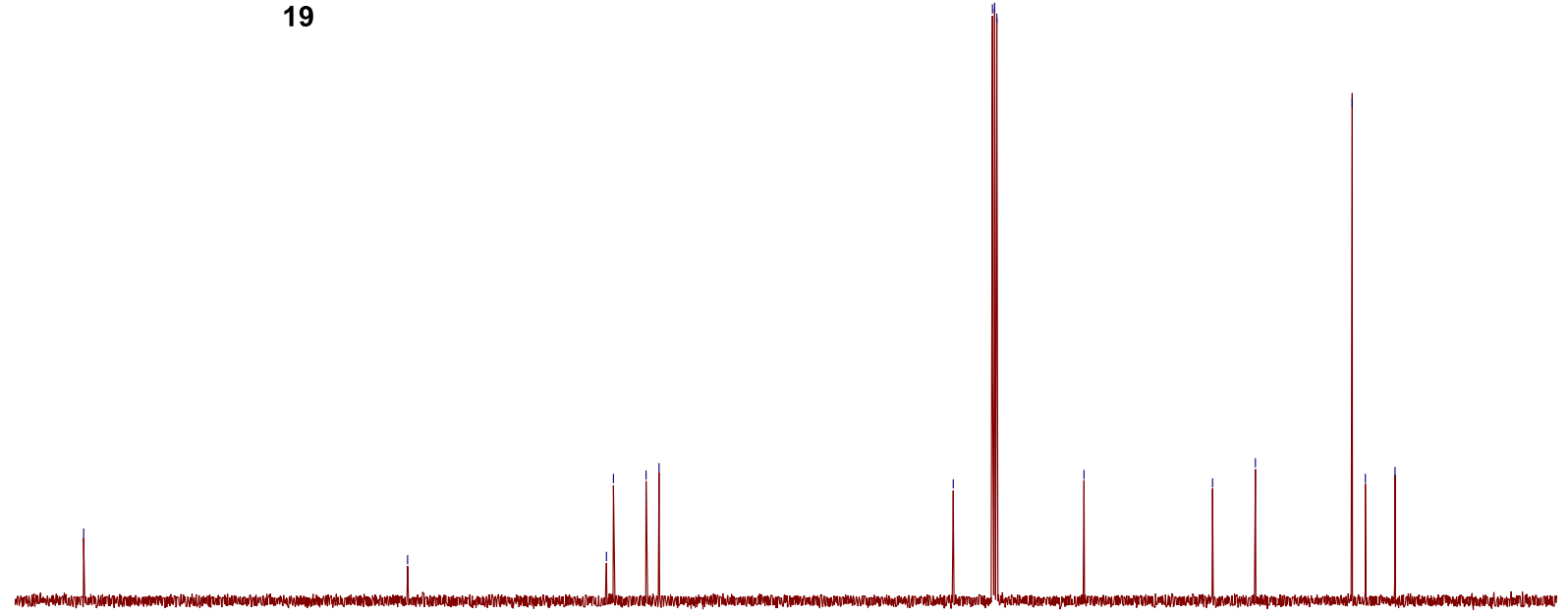

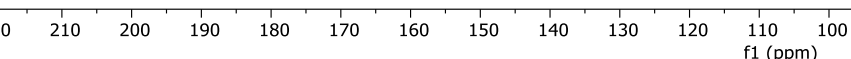


${ }^{11} \mathrm{~B}$ NMR (128 MHz, $\mathrm{CDCl}_{3}$ ) of compound 19 (see procedure):

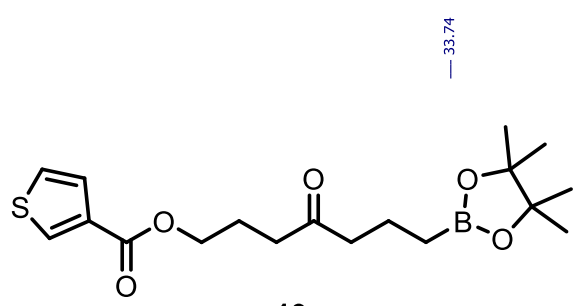

19

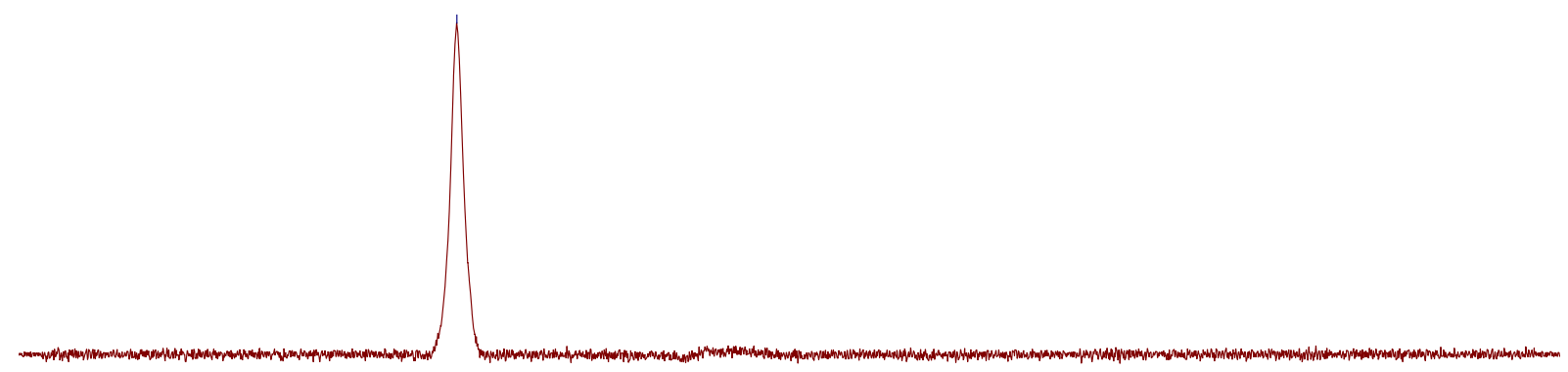

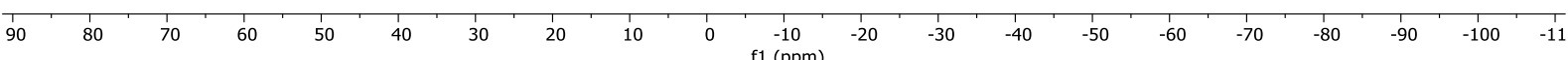


${ }^{1} \mathrm{H}$ NMR (400 MHz, $\mathrm{CDCl}_{3}$ ) of compound 20 (see procedure):

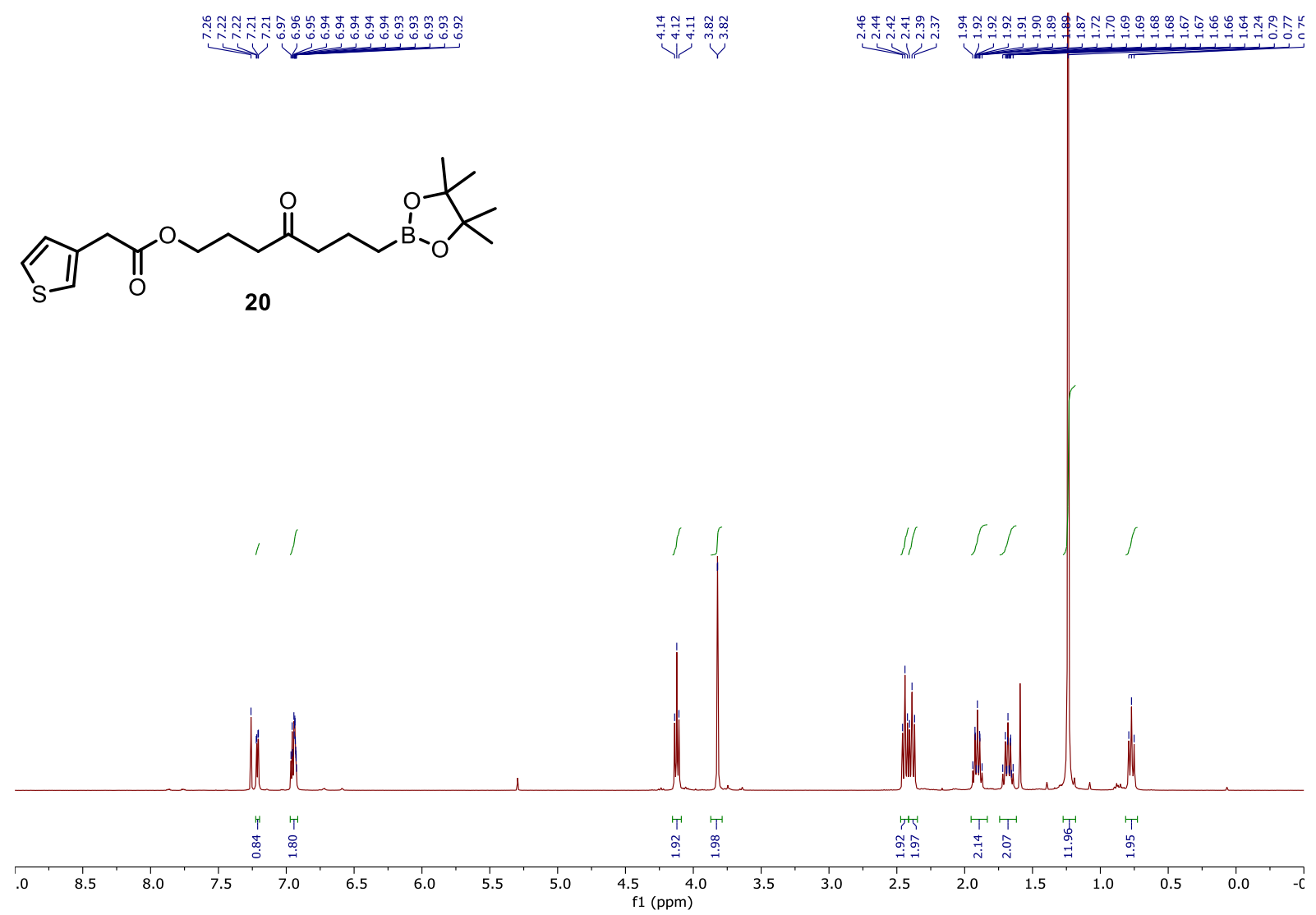

${ }^{13} \mathrm{C}$ NMR (101 MHz, $\mathrm{CDCl}_{3}$ ) of compound 20 (see procedure):

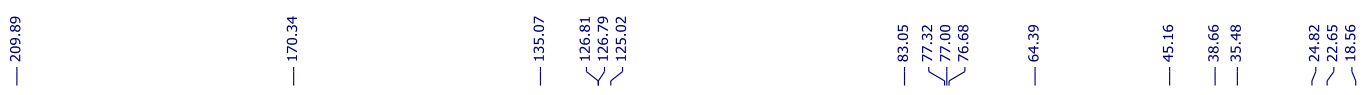<smiles>CC1(C)OB(CCCC(=O)CCCOC(=O)Cc2ccsc2)OC1(C)C</smiles>

20

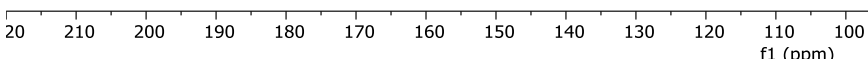


${ }^{11} \mathrm{~B}$ NMR (128 MHz, $\mathrm{CDCl}_{3}$ ) of compound 20 (see procedure):
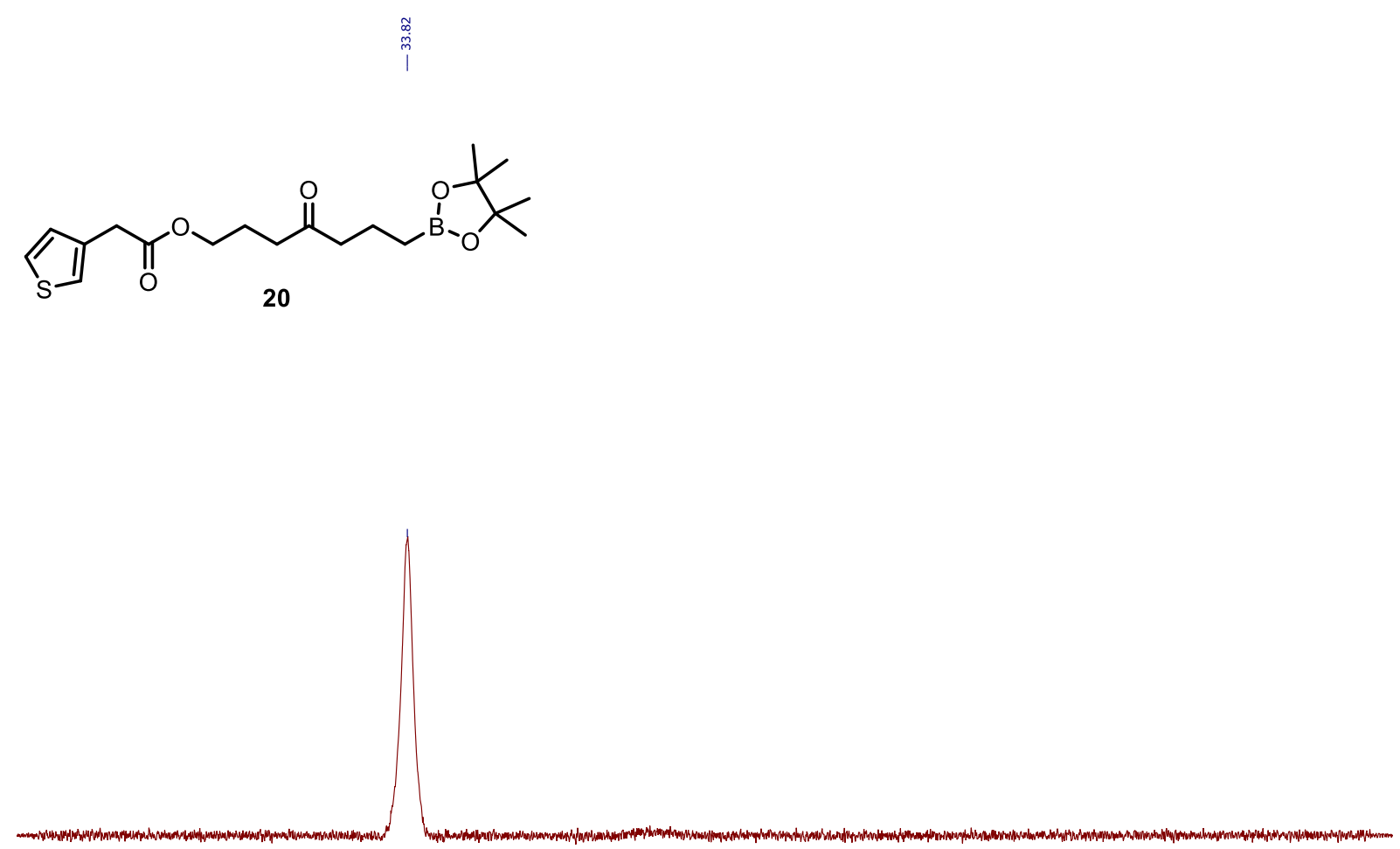

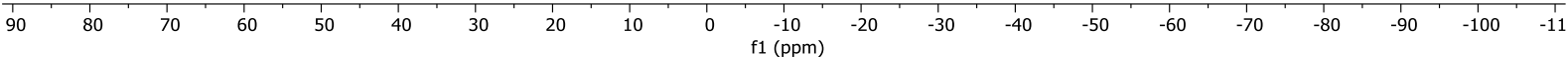


${ }^{1} \mathrm{H}$ NMR (400 MHz, $\mathrm{CDCl}_{3}$ ) of compound 21 (see procedure):

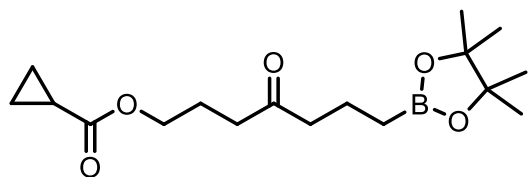

21
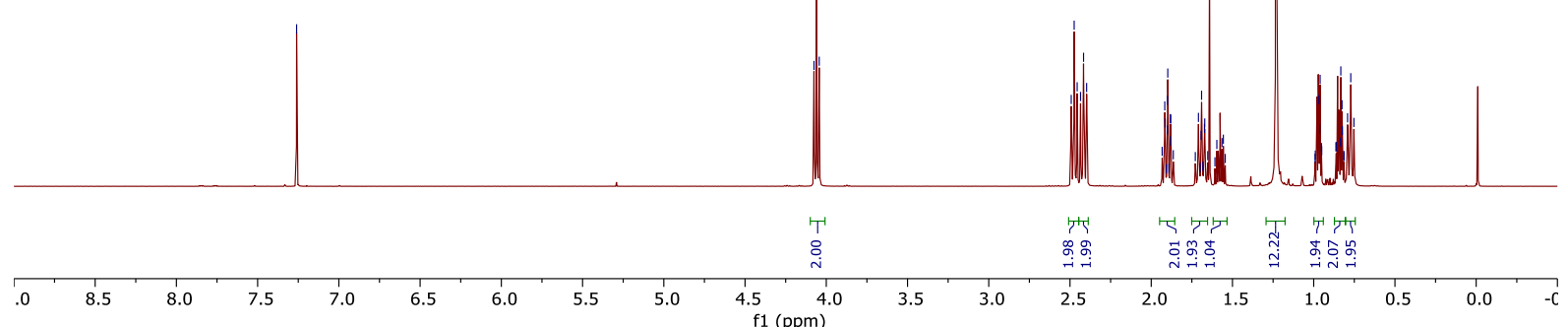

${ }^{13} \mathrm{C}$ NMR (101 MHz, $\mathrm{CDCl}_{3}$ ) of compound 21 (see procedure):

苛

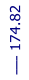<smiles>CC1(C)OB(CCCC(=O)CCCOC(=O)C2CC2)OC1(C)C</smiles>

21

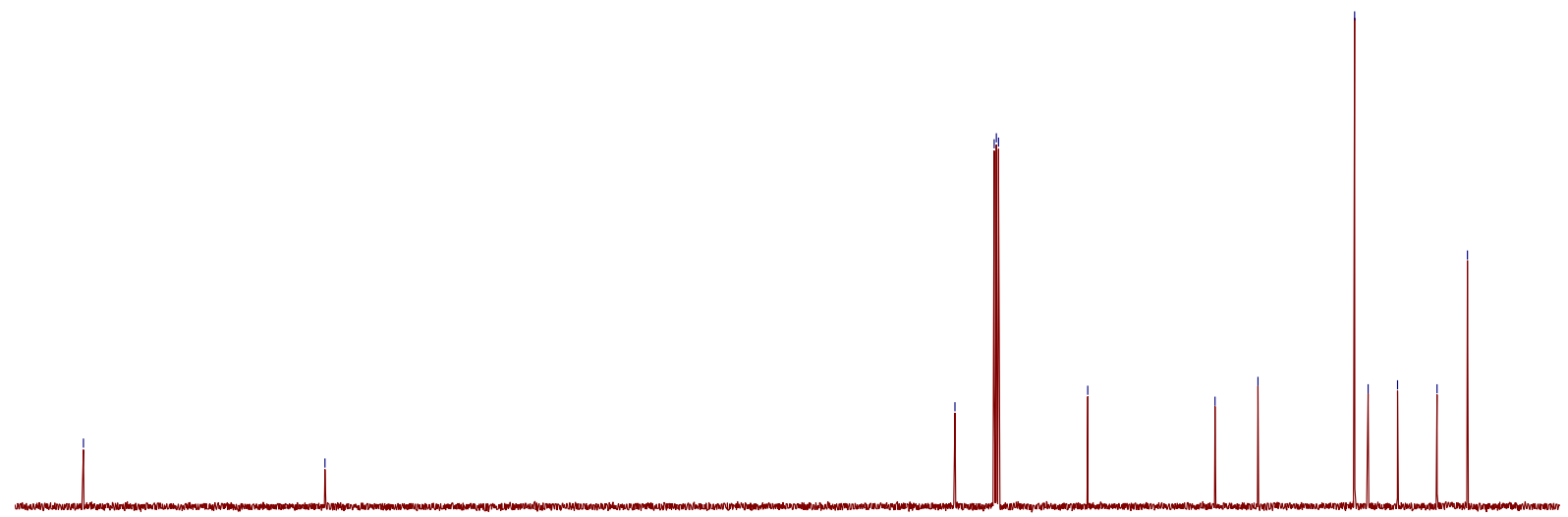

\begin{tabular}{llllllllllllllllllllllll}
\hline & 210 & 200 & 190 & 180 & 170 & 160 & 150 & 140 & 130 & 120 & 110 & 100 & 90 & 80 & 70 & 60 & 50 & 40 & 30 & 20 & 10 & 0
\end{tabular} 
${ }^{11} \mathrm{~B}$ NMR (128 MHz, $\mathrm{CDCl}_{3}$ ) of compound 21 (see procedure):

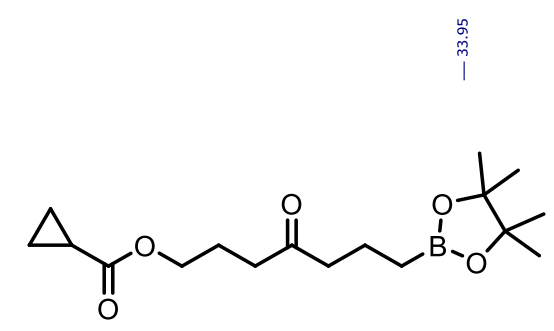

21

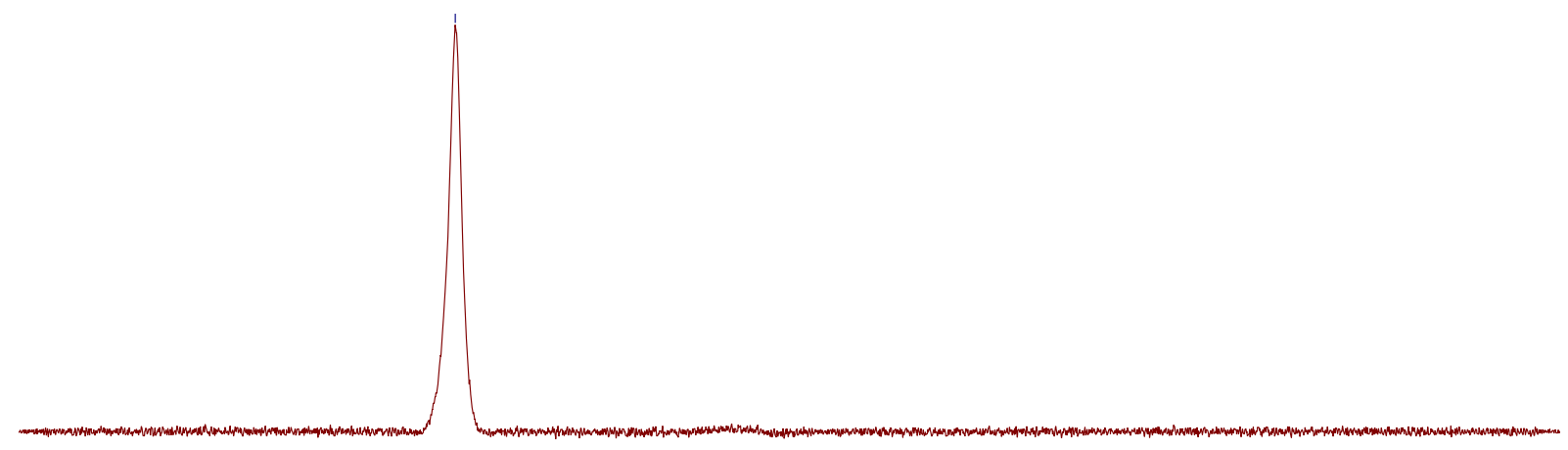

\begin{tabular}{lllllllllllllllllllllll}
70 & 80 & 70 & 60 & 50 & 40 & 30 & 20 & 10 & 0 & -10 & -20 & -30 & -40 & -50 & -60 & -70 & -80 & -90 & -100 & -11 \\
\hline
\end{tabular} 
${ }^{1} \mathrm{H}$ NMR (400 MHz, $\mathrm{CDCl}_{3}$ ) of compound 22 (see procedure):

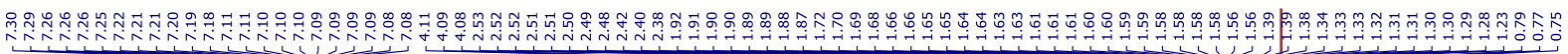<smiles>CC1(C)OB(CCCC(=O)CCCCCOC(=O)C2C[C@H]2c2ccccc2)OC1(C)C</smiles>

22

${ }^{13} \mathrm{C}$ NMR (101 MHz, $\mathrm{CDCl}_{3}$ ) of compound 22 (see procedure):

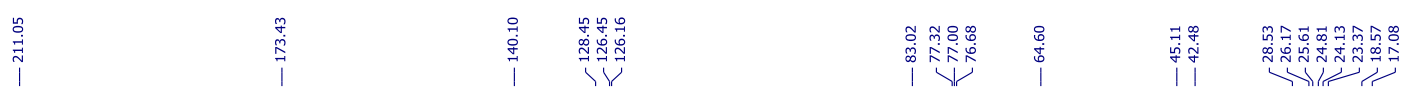<smiles>CC1(C)OB(CCCC(=O)CCCCCOC(=O)C2C[C@H]2c2ccccc2)OC1(C)C</smiles>

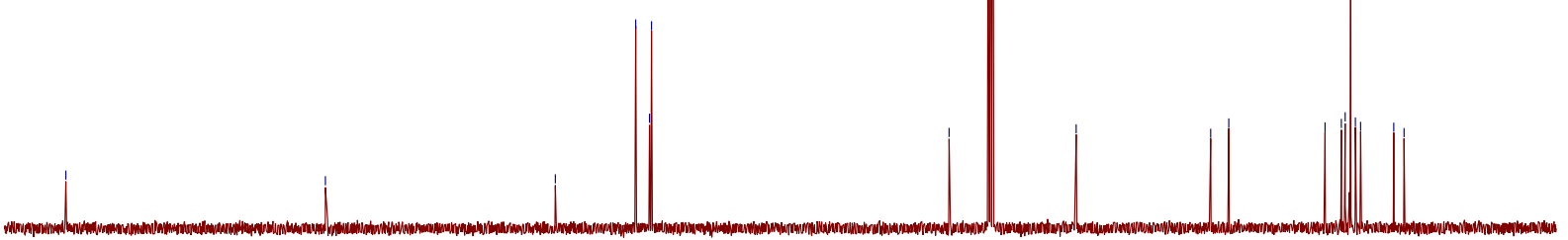

\begin{tabular}{llllllllllllllllllllllllllllll}
\hline 20 & 210 & 200 & 190 & 180 & 170 & 160 & 150 & 140 & 130 & 120 & 110 & 100 & 90 & 80 & 70 & 60 & 50 & 40 & 30 & 20 & 10 & 0
\end{tabular} 
${ }^{11} \mathrm{~B}$ NMR (128 MHz, $\mathrm{CDCl}_{3}$ ) of compound 22 (see procedure):<smiles>CC1(C)OB(CCCC(=O)CCCCCOC(=O)C2C[C@H]2c2ccccc2)OC1(C)C</smiles>

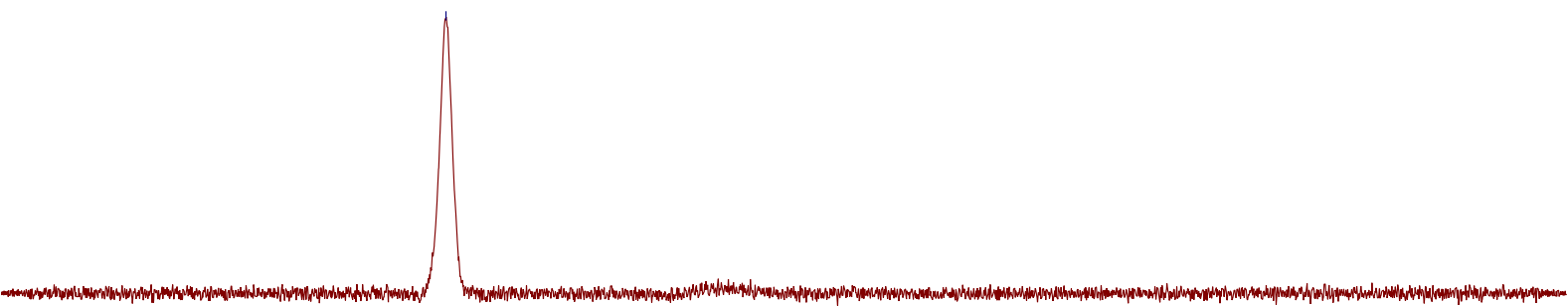

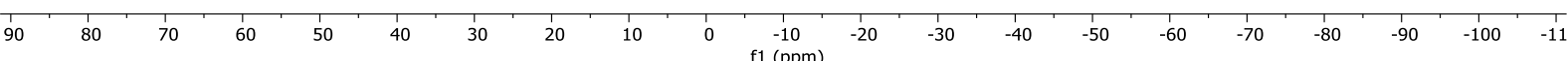


${ }^{1} \mathrm{H}$ NMR (400 MHz, $\mathrm{CDCl}_{3}$ ) of compound 23 (see procedure):

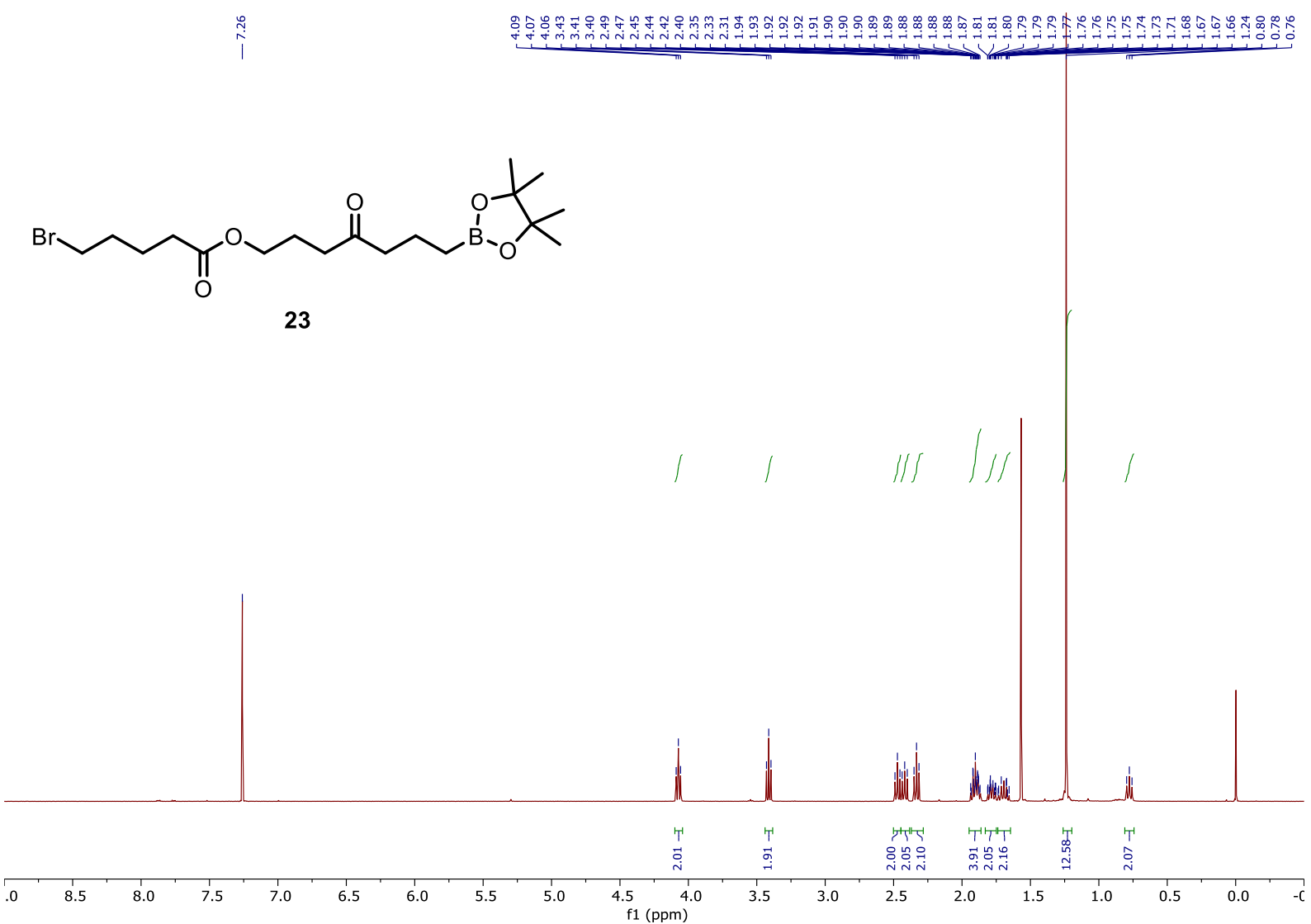

${ }^{13} \mathrm{C} \mathrm{NMR}\left(101 \mathrm{MHz}, \mathrm{CDCl}_{3}\right)$ of compound 23 (see procedure):<smiles>CCCCCC(=O)OCCCC(=O)CCCB1OC(C)(C)C(C)(C)O1</smiles>

23

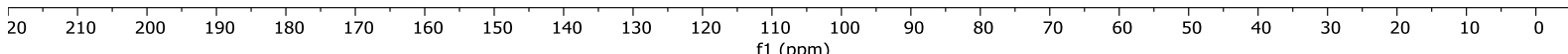


${ }^{11} \mathrm{~B}$ NMR (128 MHz, $\mathrm{CDCl}_{3}$ ) of compound 23 (see procedure):

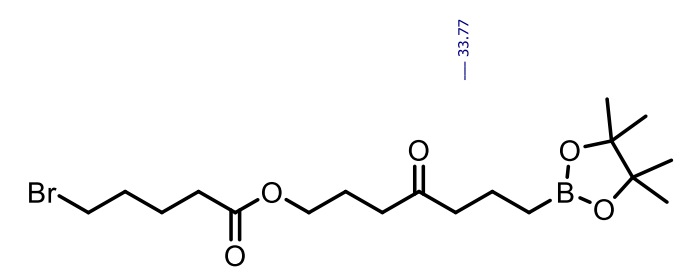

23

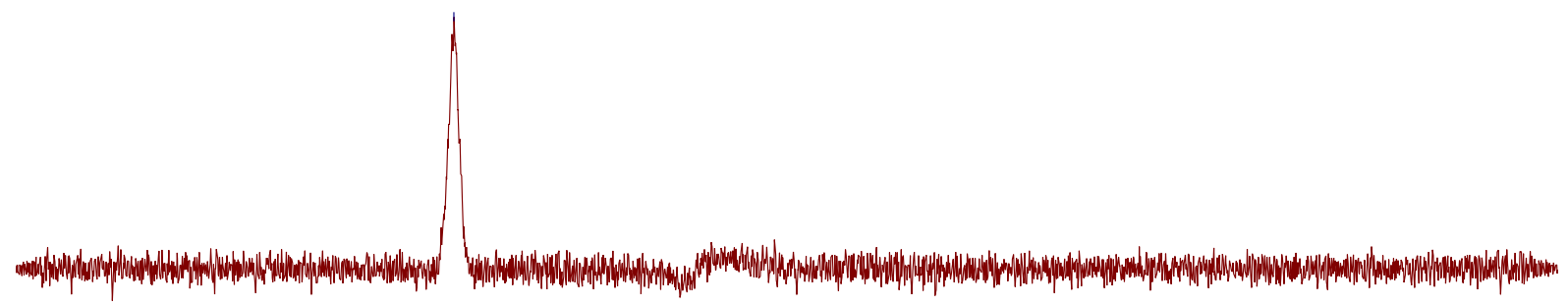

$\begin{array}{lllllllllllllllllllll}90 & 80 & 70 & 60 & 50 & 40 & 30 & 20 & 10 & 0 & \begin{array}{c}10 \\ \mathrm{f} 1(\mathrm{ppm})\end{array} & -20 & -30 & -40 & -50 & -60 & -70 & -80 & -90 & -100 & -11\end{array}$


${ }^{1} \mathrm{H}$ NMR (400 MHz, $\mathrm{CDCl}_{3}$ ) of compound 24 (see procedure):

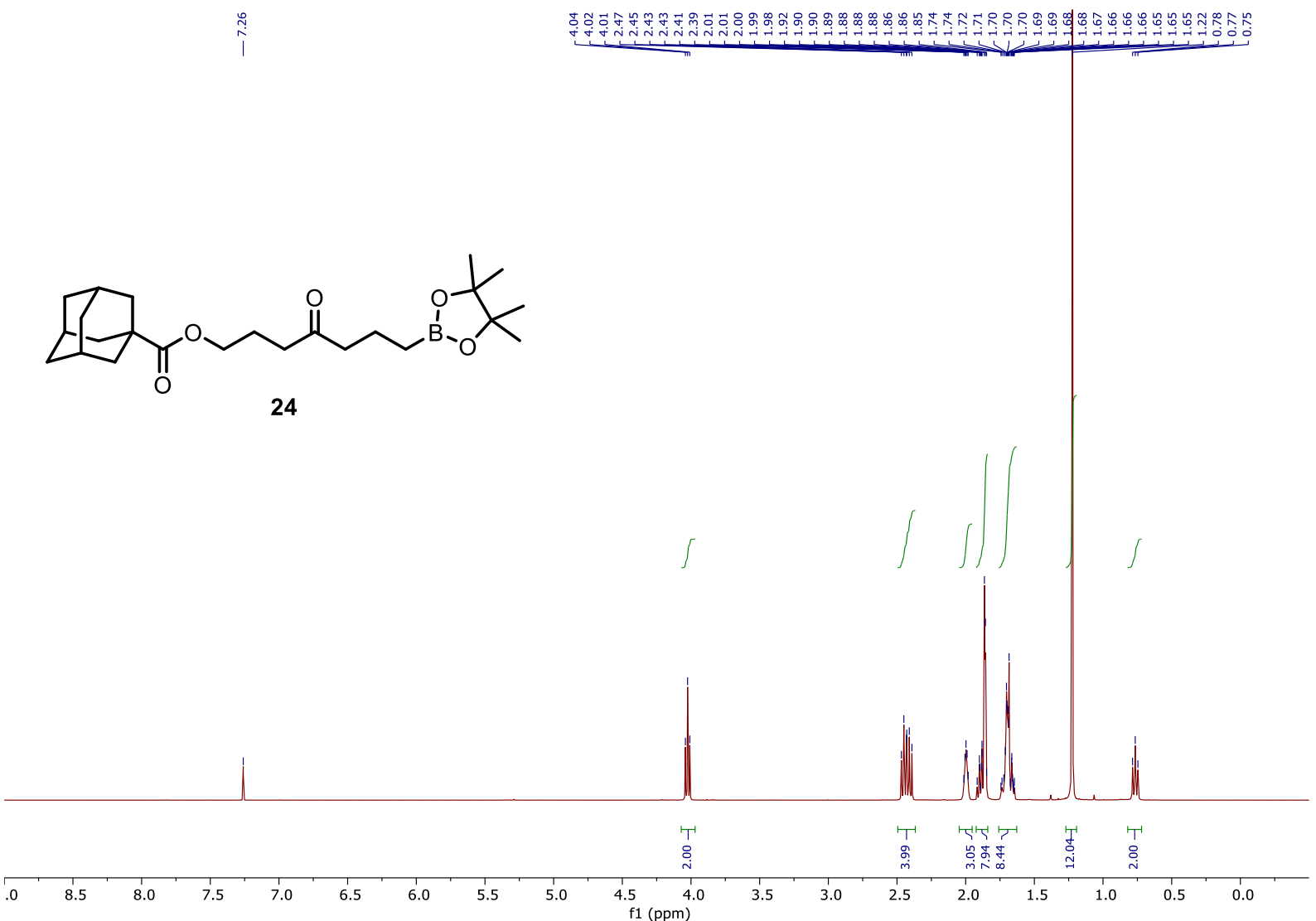

${ }^{13} \mathrm{C}$ NMR (101 MHz, $\mathrm{CDCl}_{3}$ ) of compound 24 (see procedure):<smiles>[3H]</smiles>

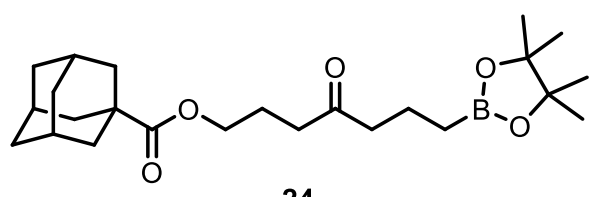

24

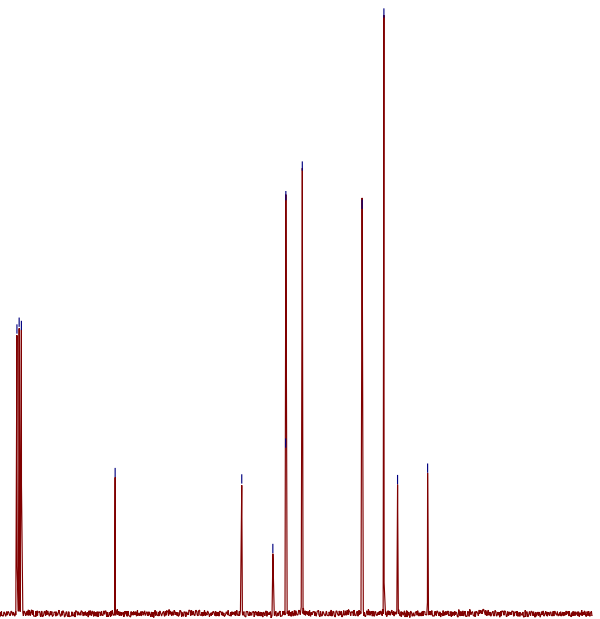

$\begin{array}{llllllllllllllllllllll}210 & 200 & 190 & 180 & 170 & 160 & 150 & 140 & 130 & 120 & 110 & 100 & 90 & 80 & 70 & 60 & 50 & 40 & 30 & 20 & 10 & 0\end{array}$ 
${ }^{11} \mathrm{~B}$ NMR (128 MHz, $\mathrm{CDCl}_{3}$ ) of compound 24 (see procedure):
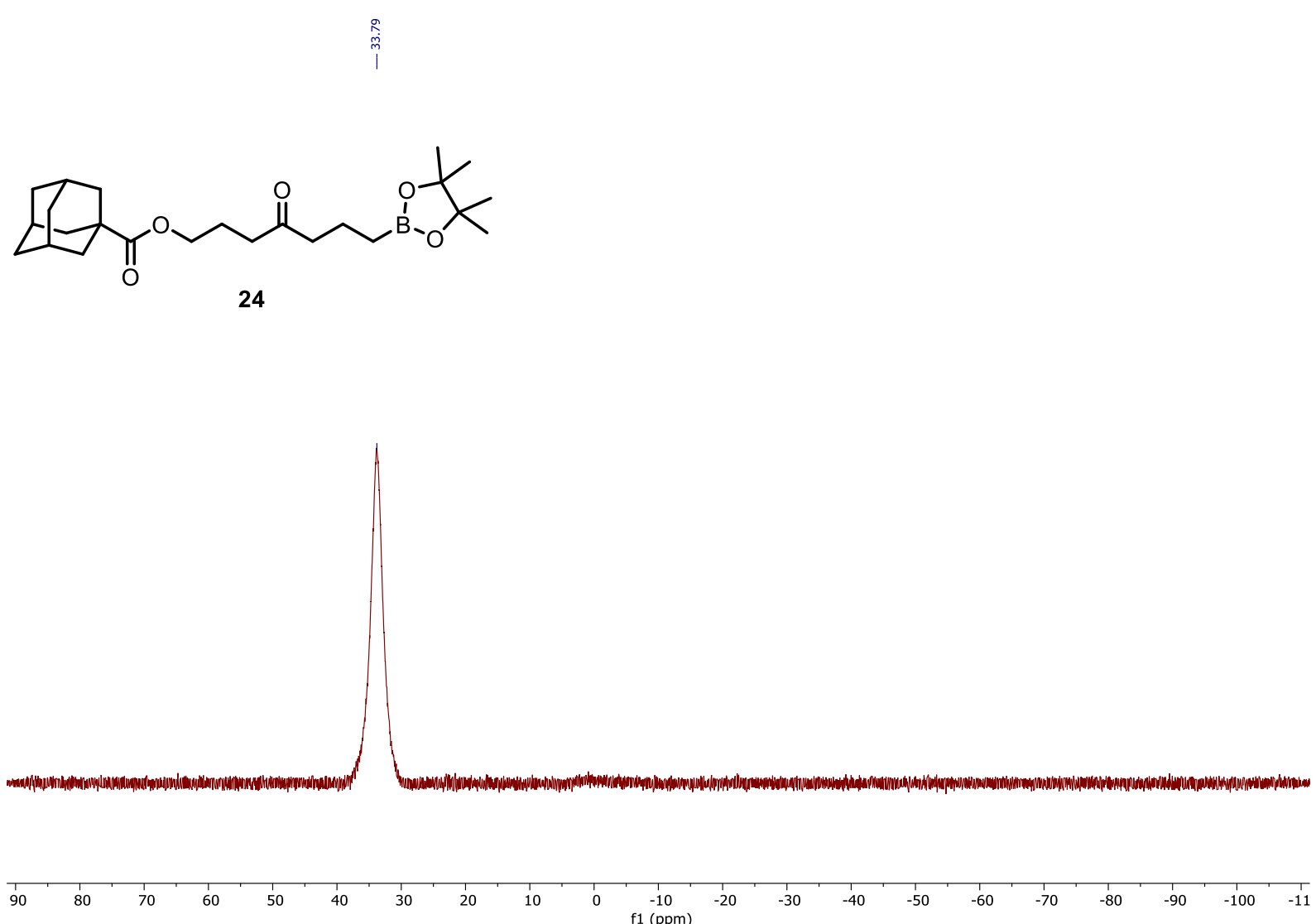
${ }^{1} \mathrm{H}$ NMR (400 MHz, $\mathrm{CDCl}_{3}$ ) of compound 25 (see procedure):<smiles>CC1(C)OB(CCCC(=O)CCCOC(=O)C23C(=O)OC(C)(C2(C)C)C3(C)C)OC1(C)C</smiles>

25

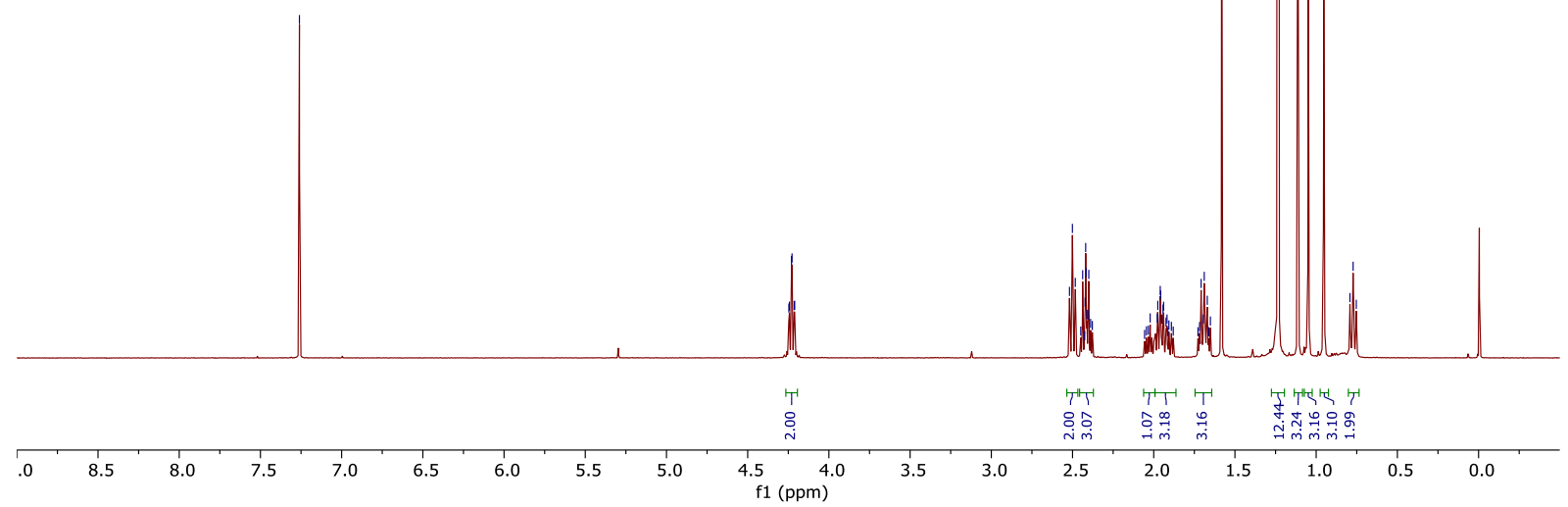

${ }^{13} \mathrm{C}$ NMR (101 MHz, $\mathrm{CDCl}_{3}$ ) of compound 25 (see procedure):

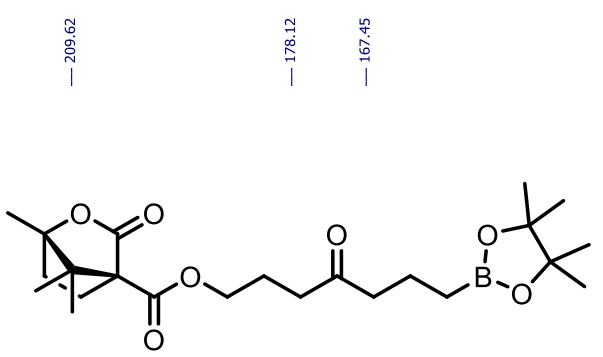

25 
${ }^{11} \mathrm{~B}$ NMR (128 MHz, $\mathrm{CDCl}_{3}$ ) of compound 25 (see procedure):

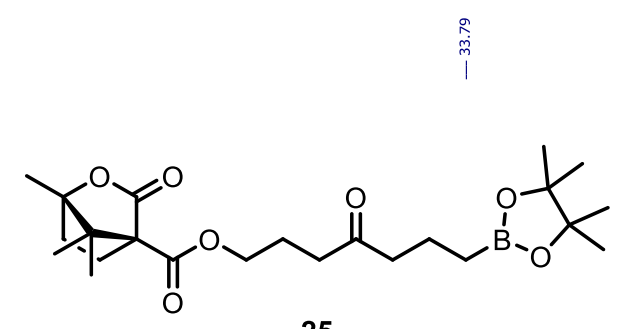

25

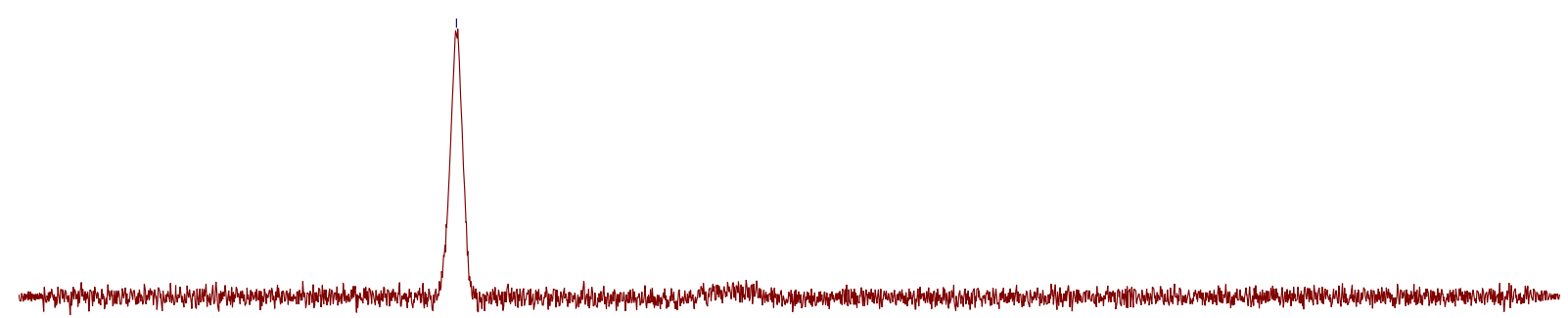

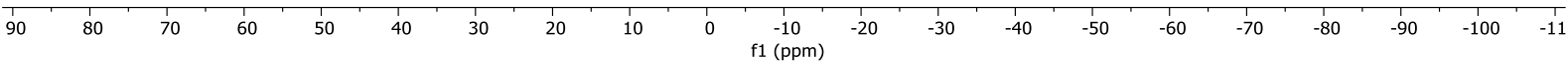


${ }^{1} \mathrm{H}$ NMR (400 MHz, $\mathrm{CDCl}_{3}$ ) of compound 26 (see procedure):

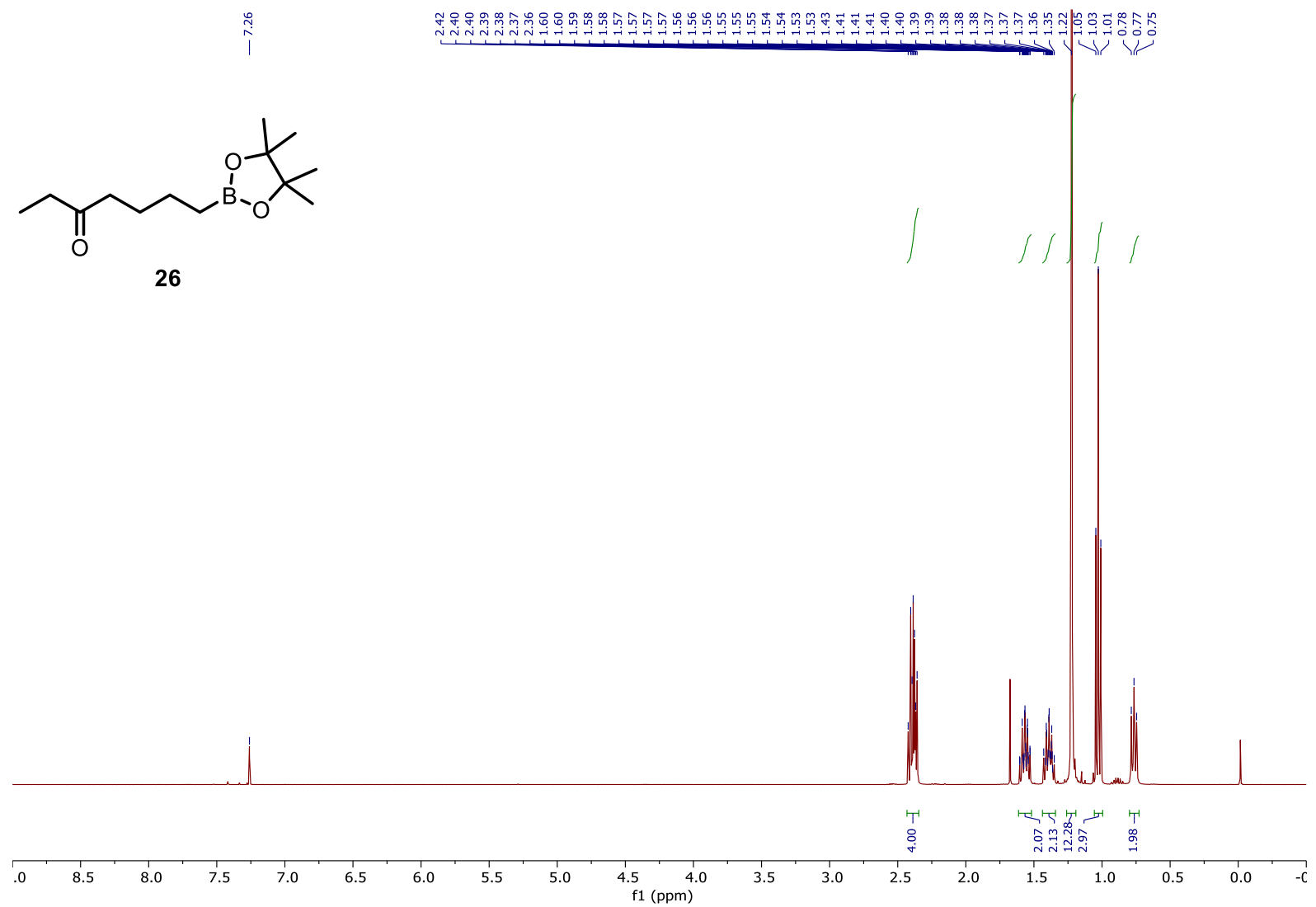

${ }^{13} \mathrm{C}$ NMR (101 MHz, $\mathrm{CDCl}_{3}$ ) of compound 26 (see procedure):

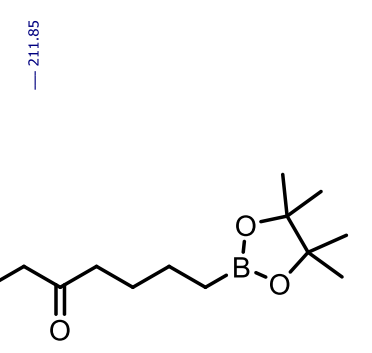

26

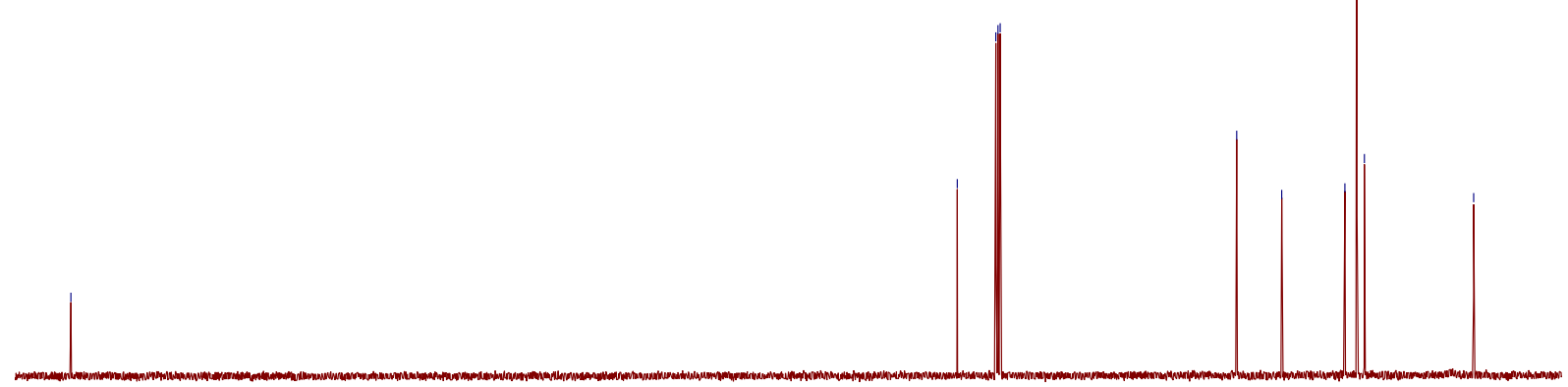

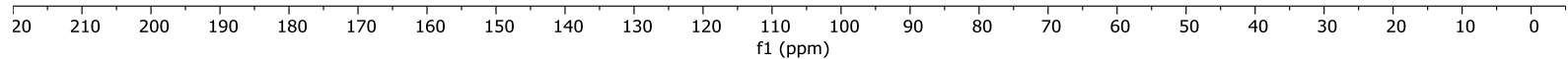


${ }^{1} \mathrm{H}$ NMR (400 MHz, $\mathrm{CDCl}_{3}$ ) of compound 27 (see procedure):

$\stackrel{?}{i}$

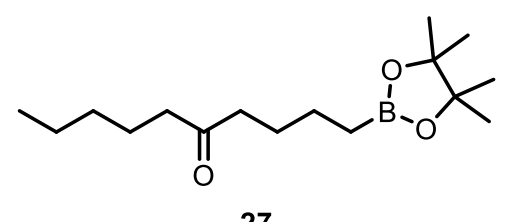

27

${ }^{13} \mathrm{C}$ NMR (101 MHz, $\mathrm{CDCl}_{3}$ ) of compound 27 (see procedure):

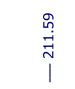

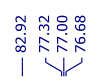

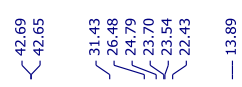
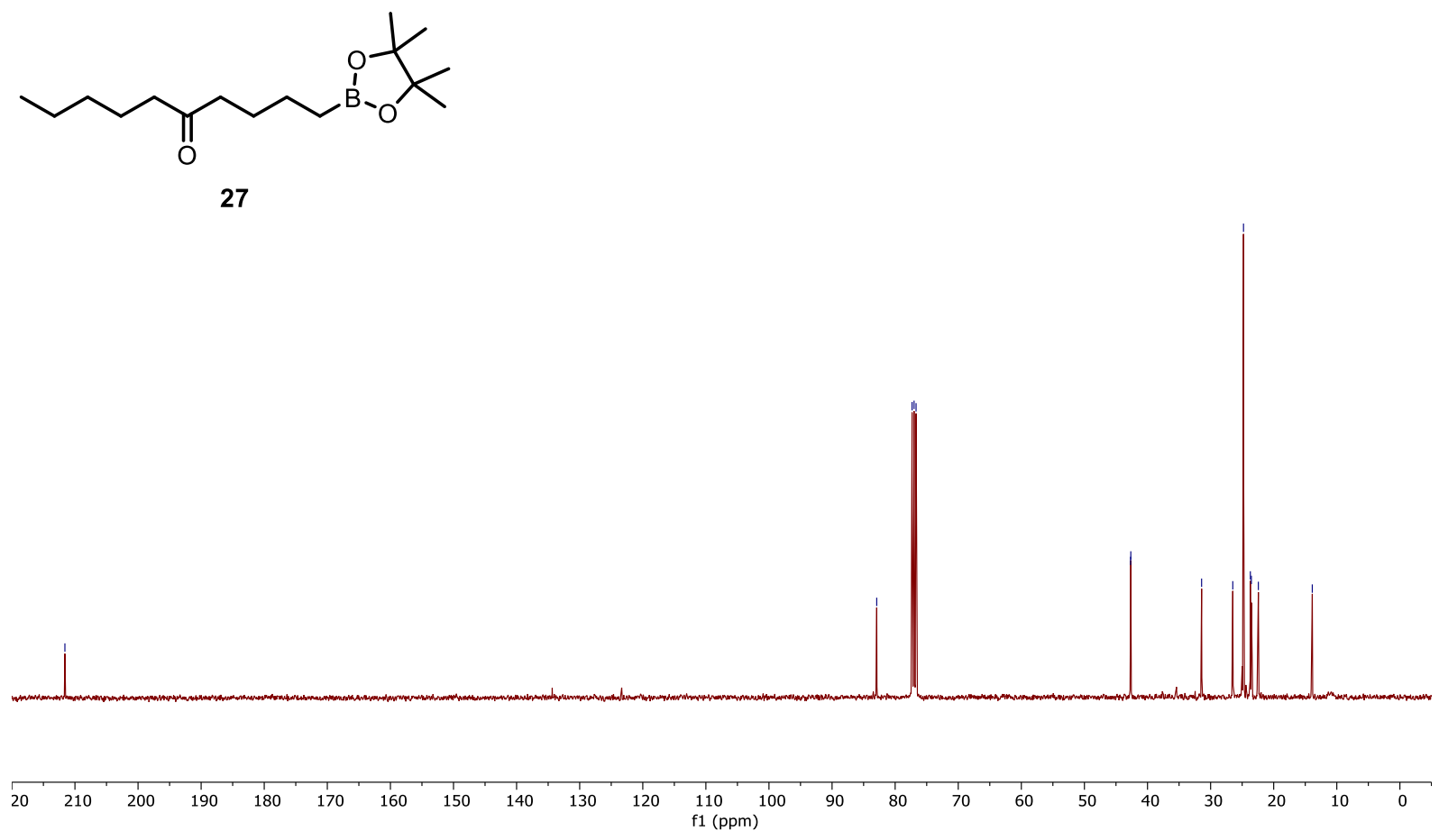

S185 
${ }^{11} \mathrm{~B}$ NMR (128 MHz, $\mathrm{CDCl}_{3}$ ) of compound 27 (see procedure):

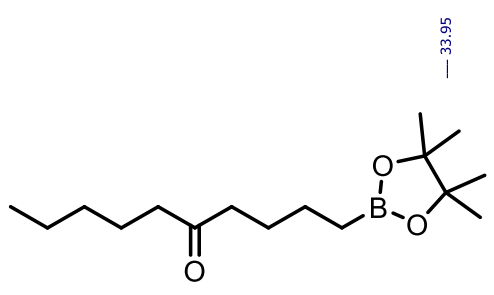

27

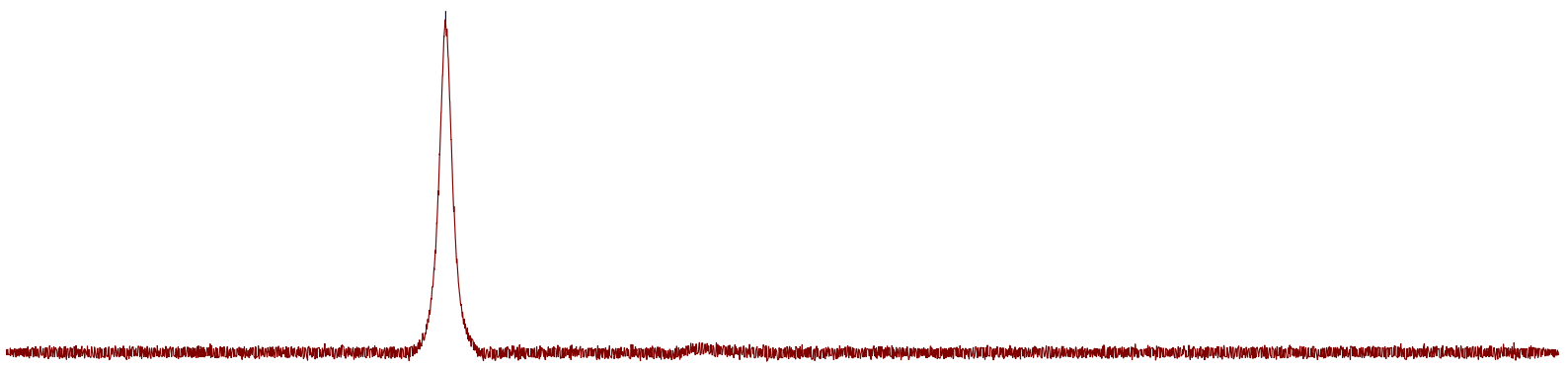

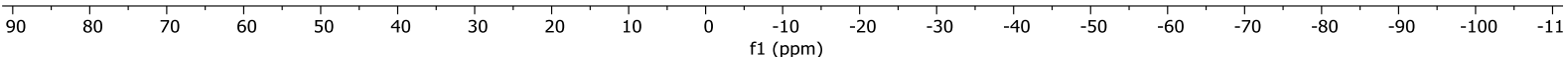


${ }^{1} \mathrm{H}$ NMR (400 MHz, $\mathrm{CDCl}_{3}$ ) of compound 28 (see procedure):

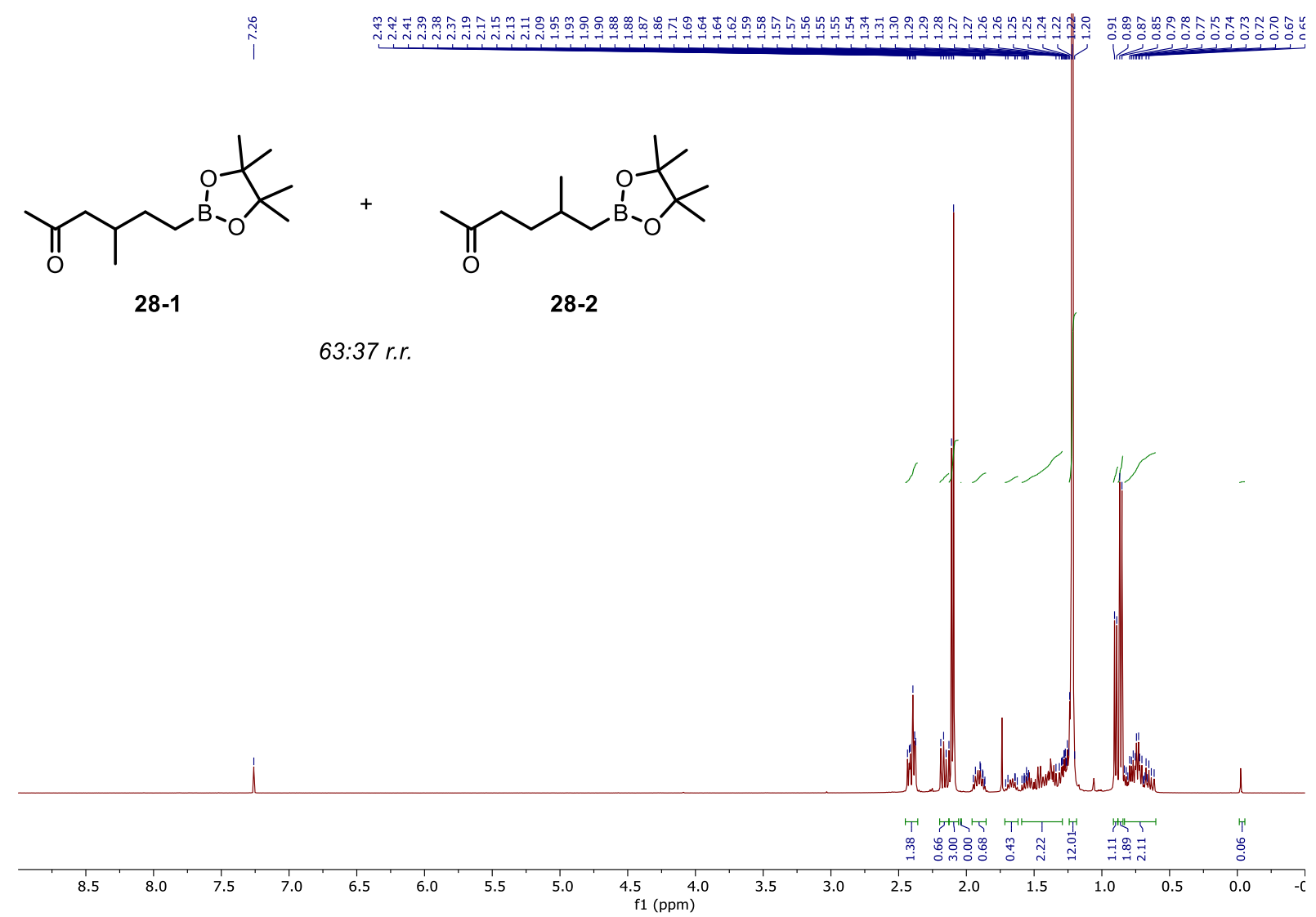

${ }^{13} \mathrm{C}$ NMR (101 MHz, $\mathrm{CDCl}_{3}$ ) of compound 28 (see procedure):

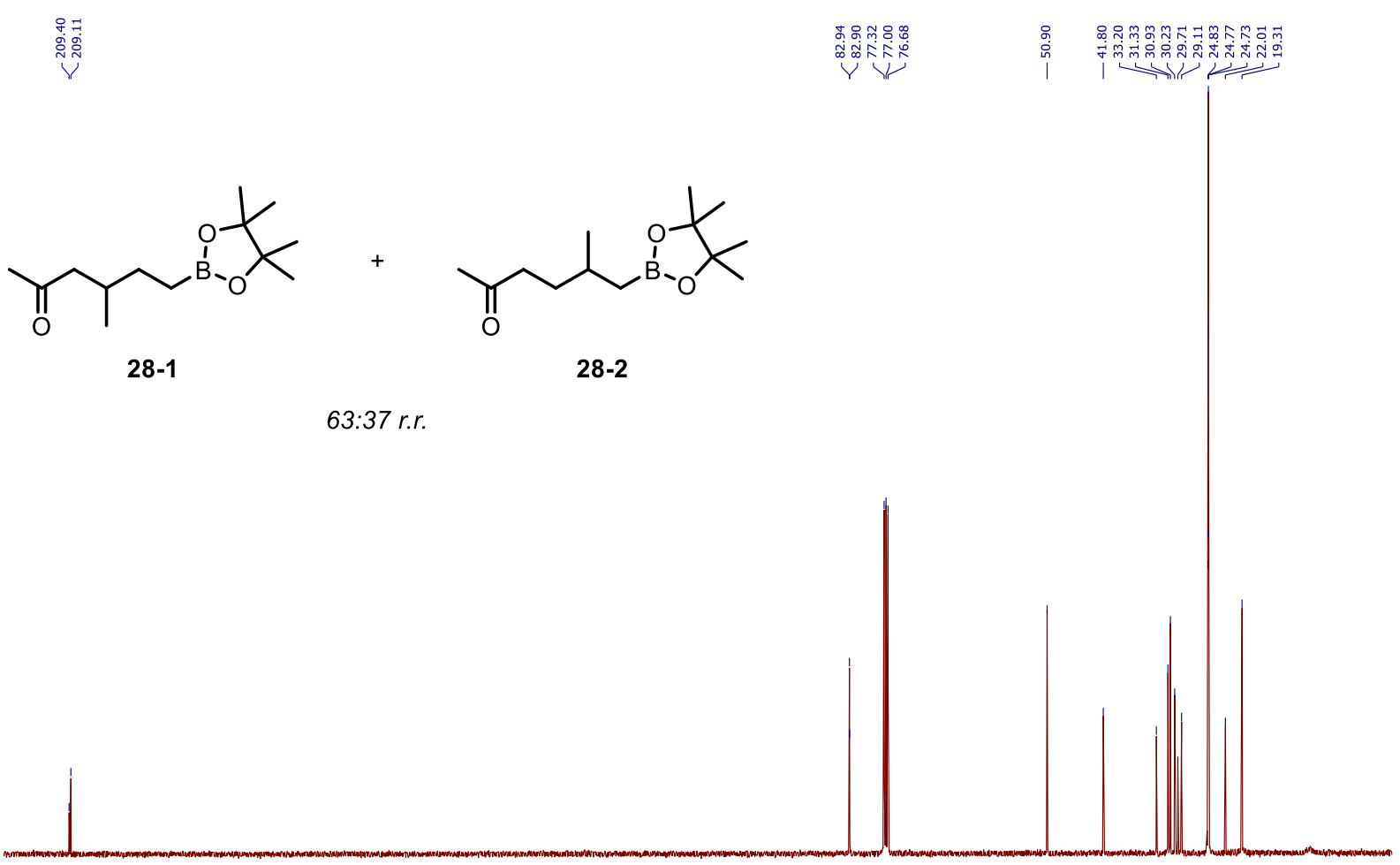

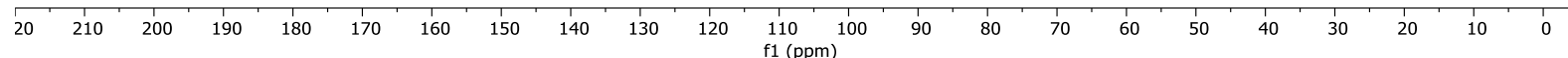


${ }^{11} \mathrm{~B}$ NMR (128 MHz, $\mathrm{CDCl}_{3}$ ) of compound 28 (see procedure):
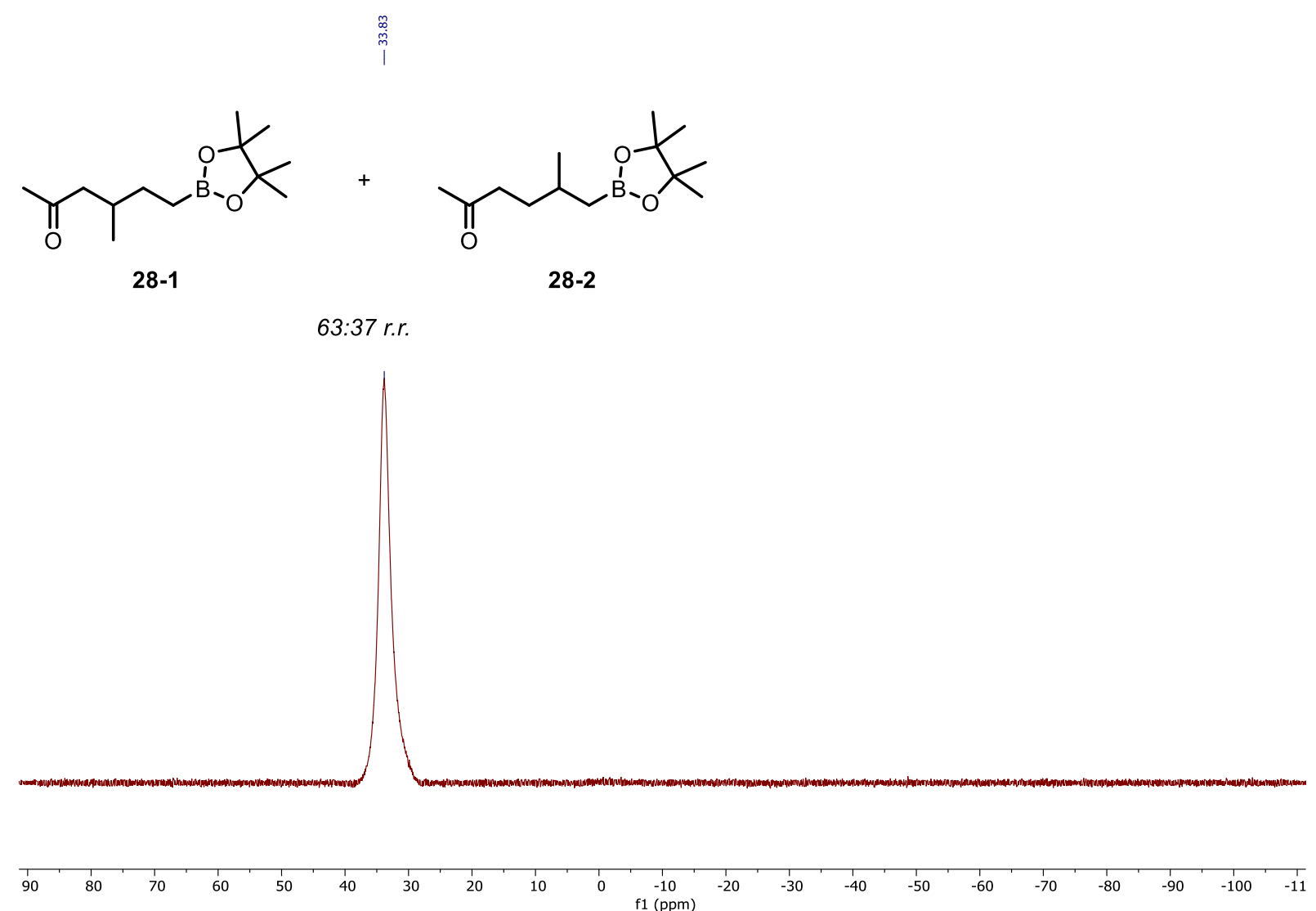
${ }^{1} \mathrm{H}$ NMR (400 MHz, $\mathrm{CDCl}_{3}$ ) of compound 29 (see procedure):

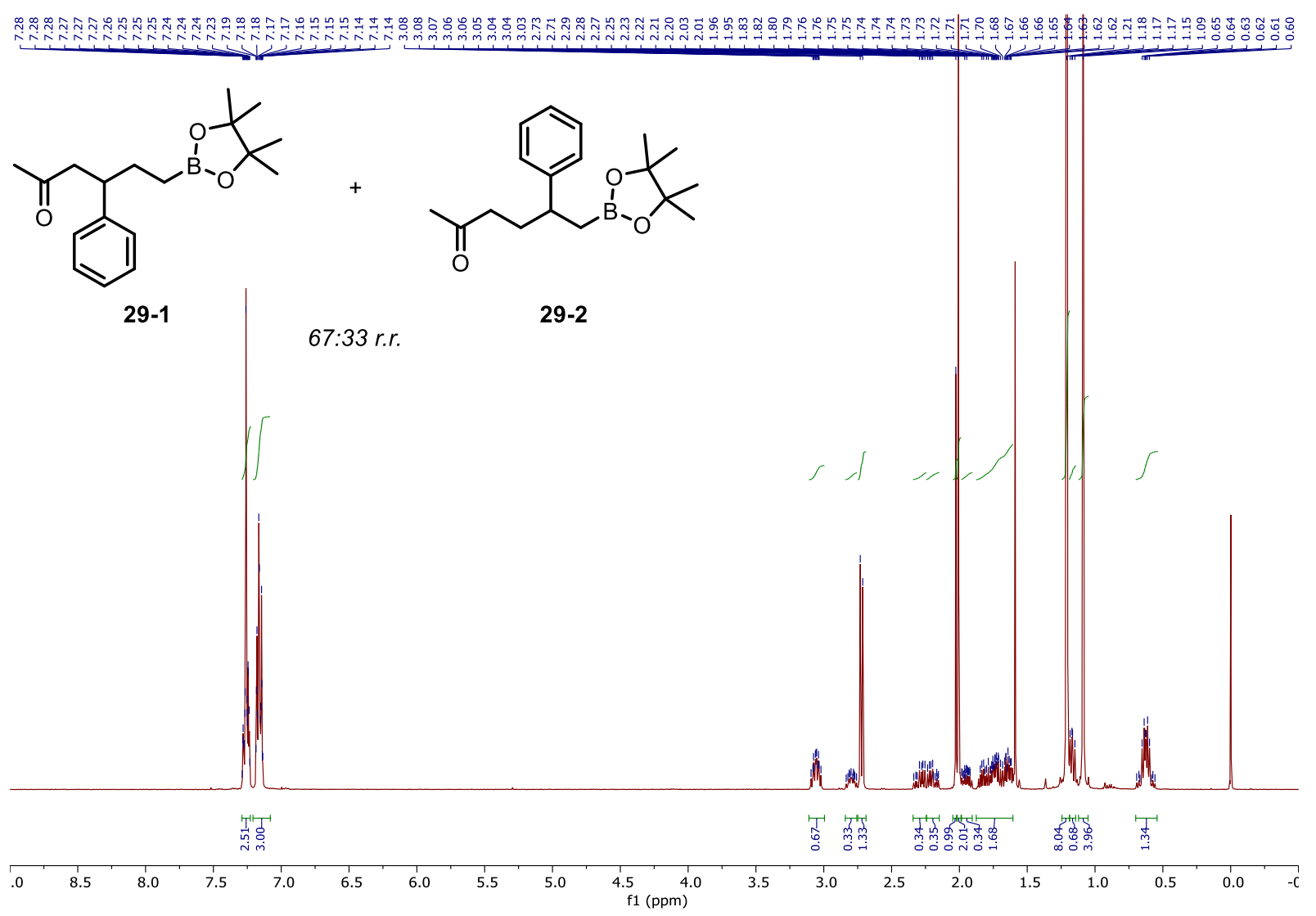

${ }^{13} \mathrm{C}$ NMR (101 MHz, $\mathrm{CDCl}_{3}$ ) of compound 29 (see procedure):

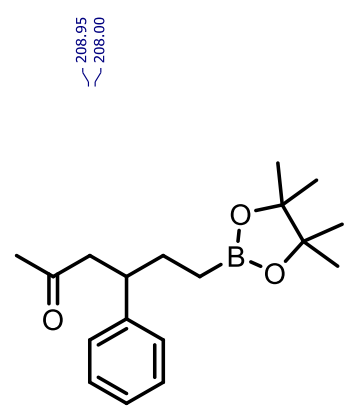

29-1
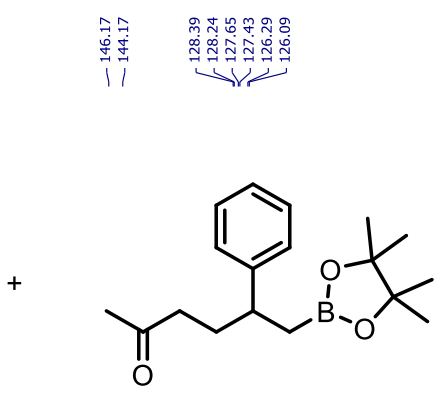

29-2

67:33 rr.
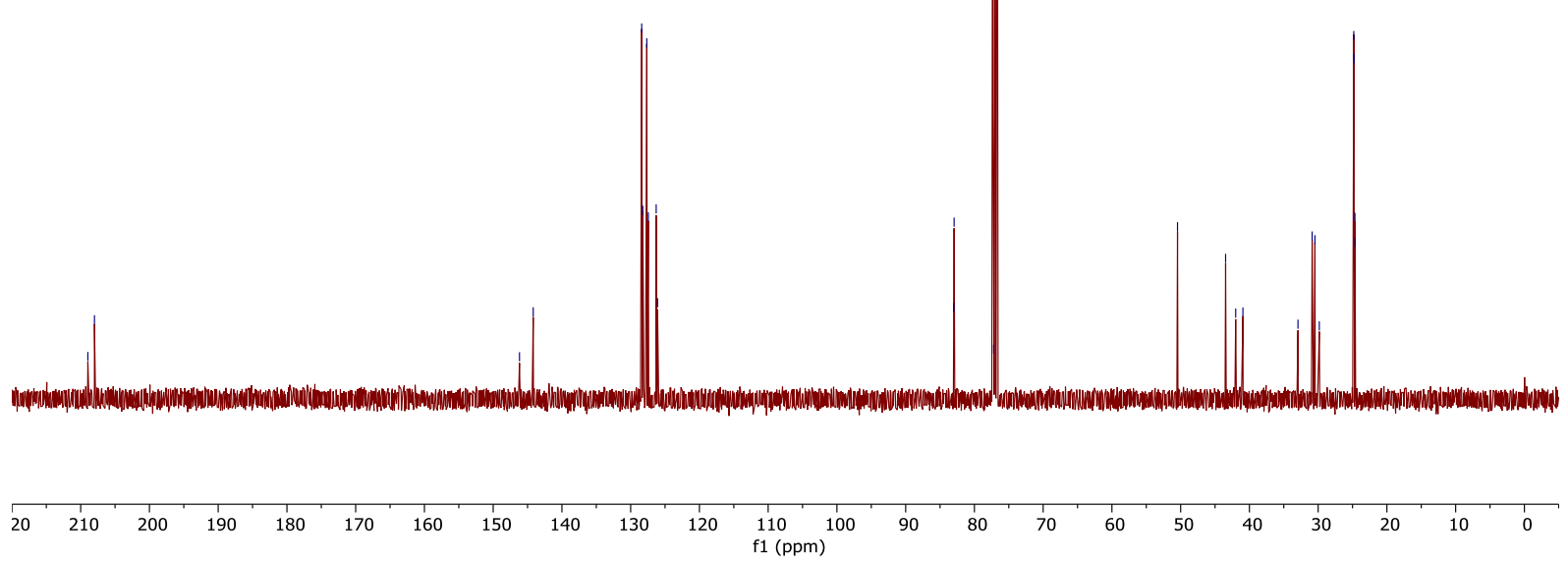

S189 
${ }^{11} \mathrm{~B}$ NMR (128 MHz, $\mathrm{CDCl}_{3}$ ) of compound 29 (see procedure):

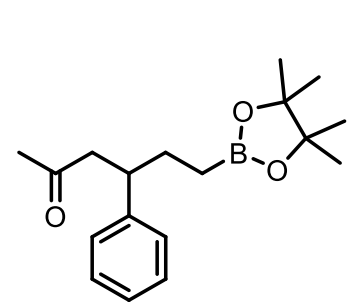

29-1

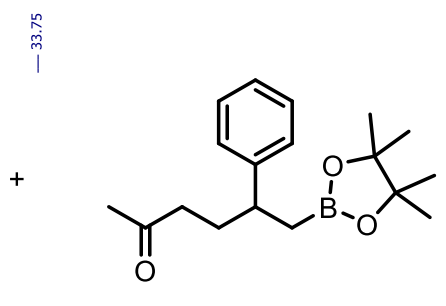

29-2

67:33 r.r.
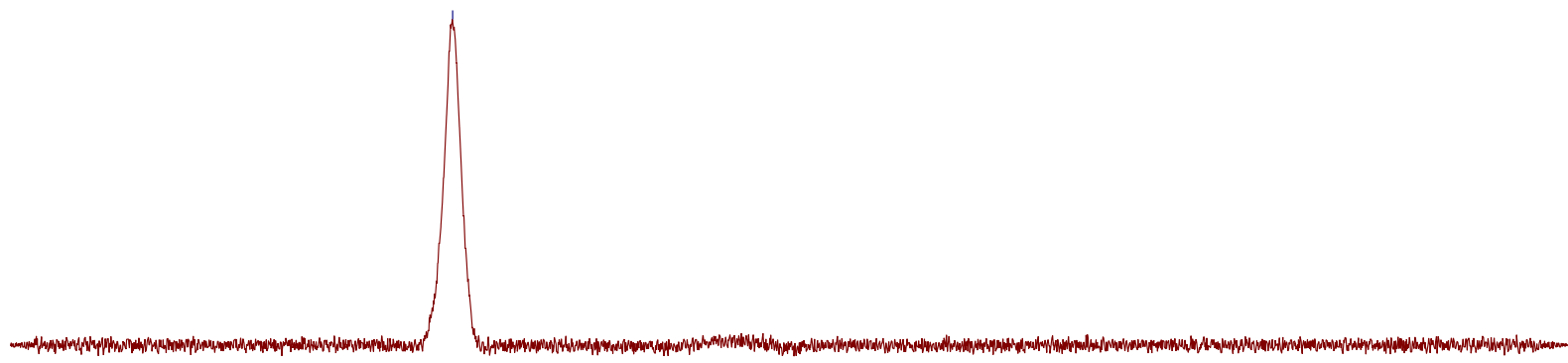

90

80

70

60

50

$40 \quad 30$

20

10

$\begin{array}{cccc}-10 & -20 & -30 & -40 \\ f 1(\mathrm{ppm}) & & & \end{array}$

$-50$

$-60$

$-70$

80

$\begin{array}{lll}-90 \quad-100 & -11\end{array}$

S190 
${ }^{1} \mathrm{H}$ NMR (400 MHz, $\mathrm{CDCl}_{3}$ ) of compound $\mathbf{3 0}$ (see procedure):

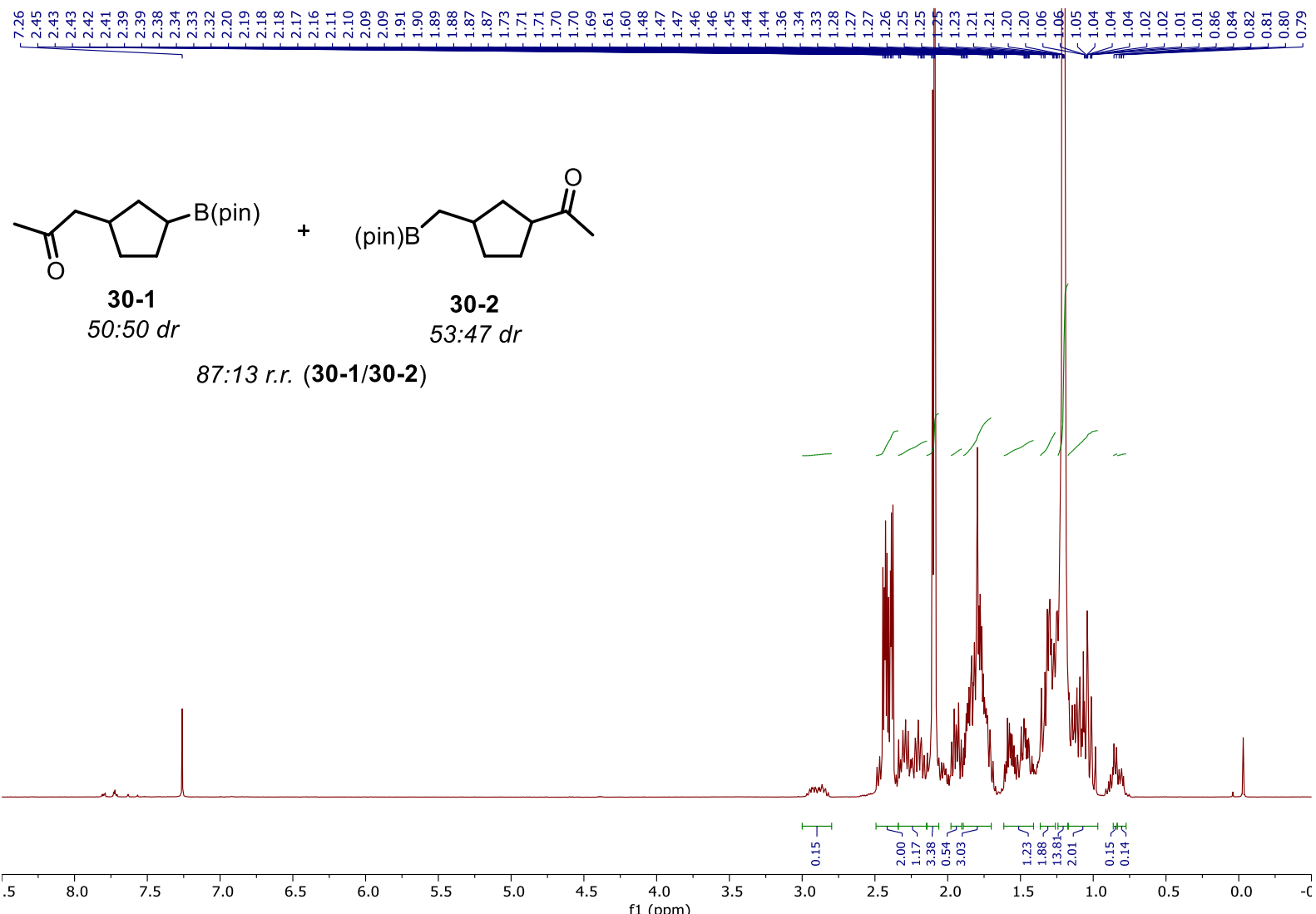

${ }^{13} \mathrm{C}$ NMR (101 MHz, $\mathrm{CDCl}_{3}$ ) of compound $\mathbf{3 0}$ (see procedure):

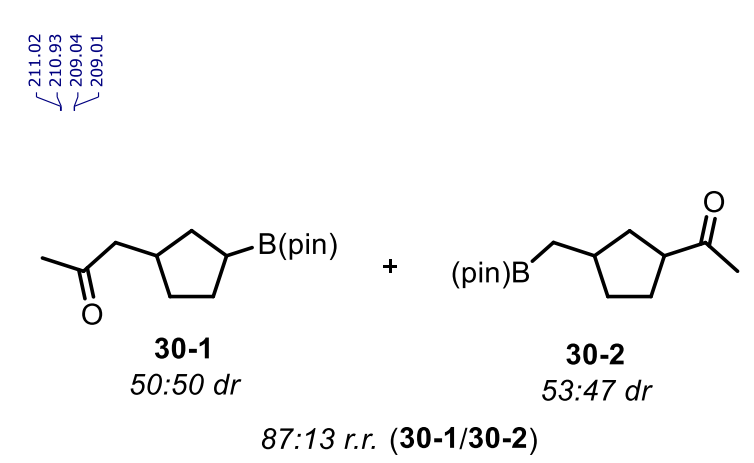

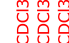

๓ॅฉ

ind

$\sqrt[4]{\infty}$

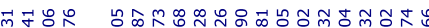

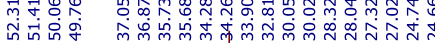

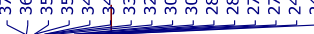

$87: 13$ r.r. $(30-1 / 30-2)$

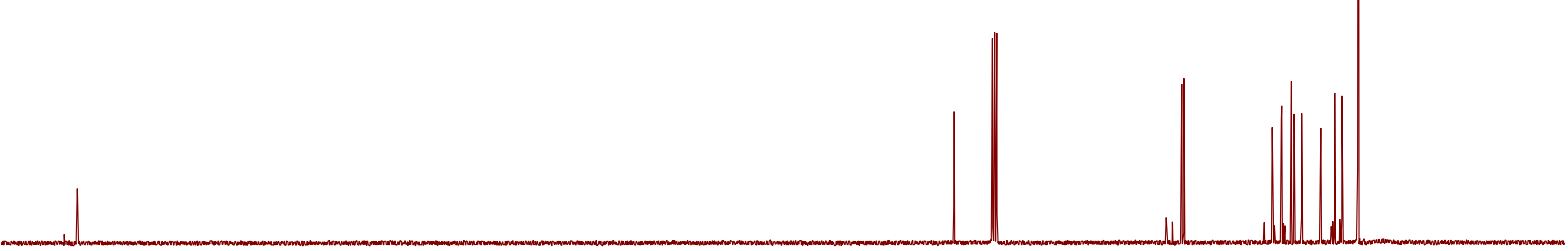

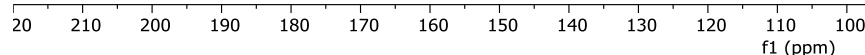

$70 \quad 60$

$\begin{array}{llll}30 & 20 & 10 & 0\end{array}$ 
${ }^{1} \mathrm{H}$ NMR (400 MHz, $\mathrm{CDCl}_{3}$ ) of compound $\mathbf{3 0} \mathbf{p}$ (see procedure):

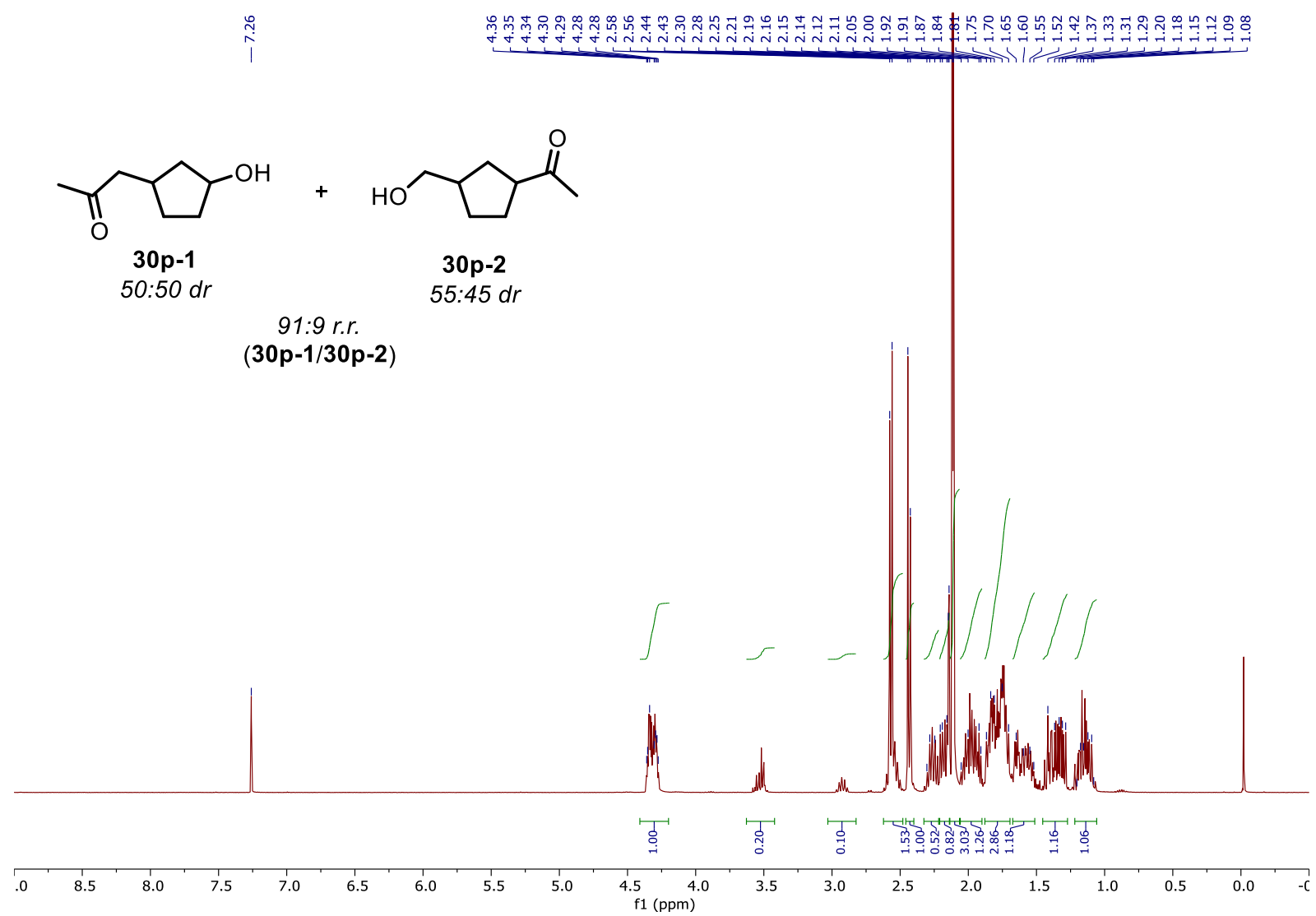

${ }^{13} \mathrm{C}$ NMR (101 MHz, $\mathrm{CDCl}_{3}$ ) of compound 30p (see procedure):
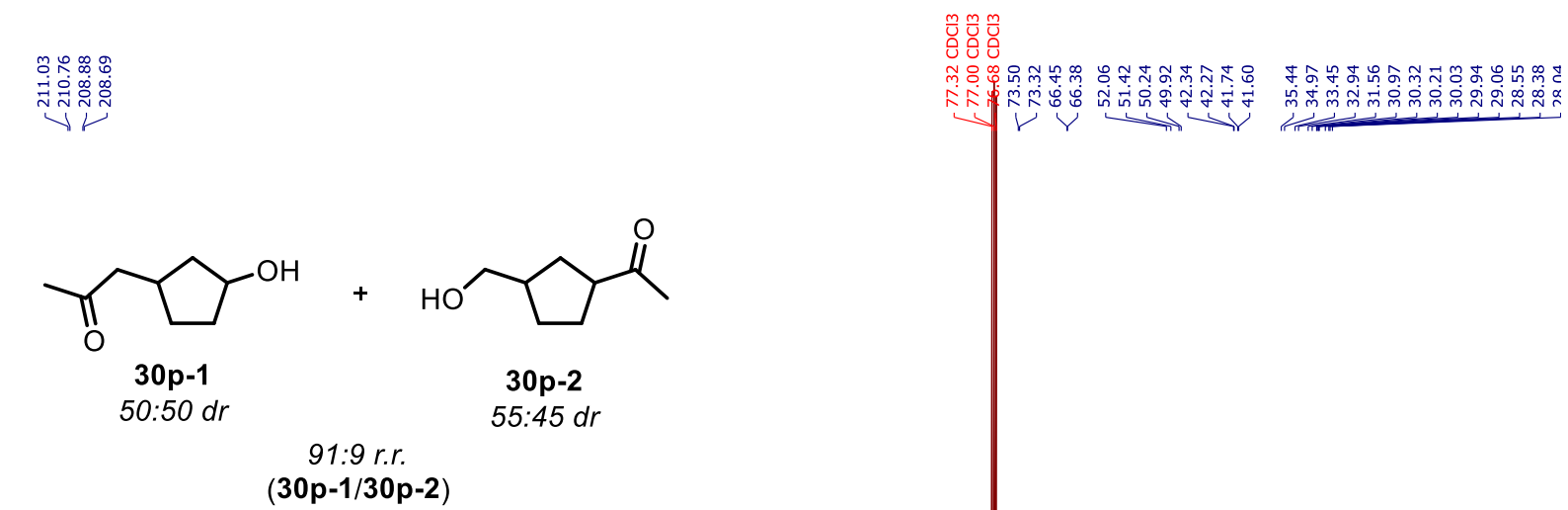

$(30 p-1 / 30 p-2)$

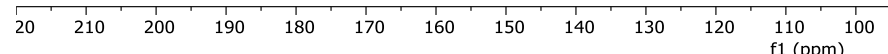


${ }^{1} \mathrm{H}$ NMR (400 MHz, $\mathrm{CDCl}_{3}$ ) of compound 31 (see procedure):

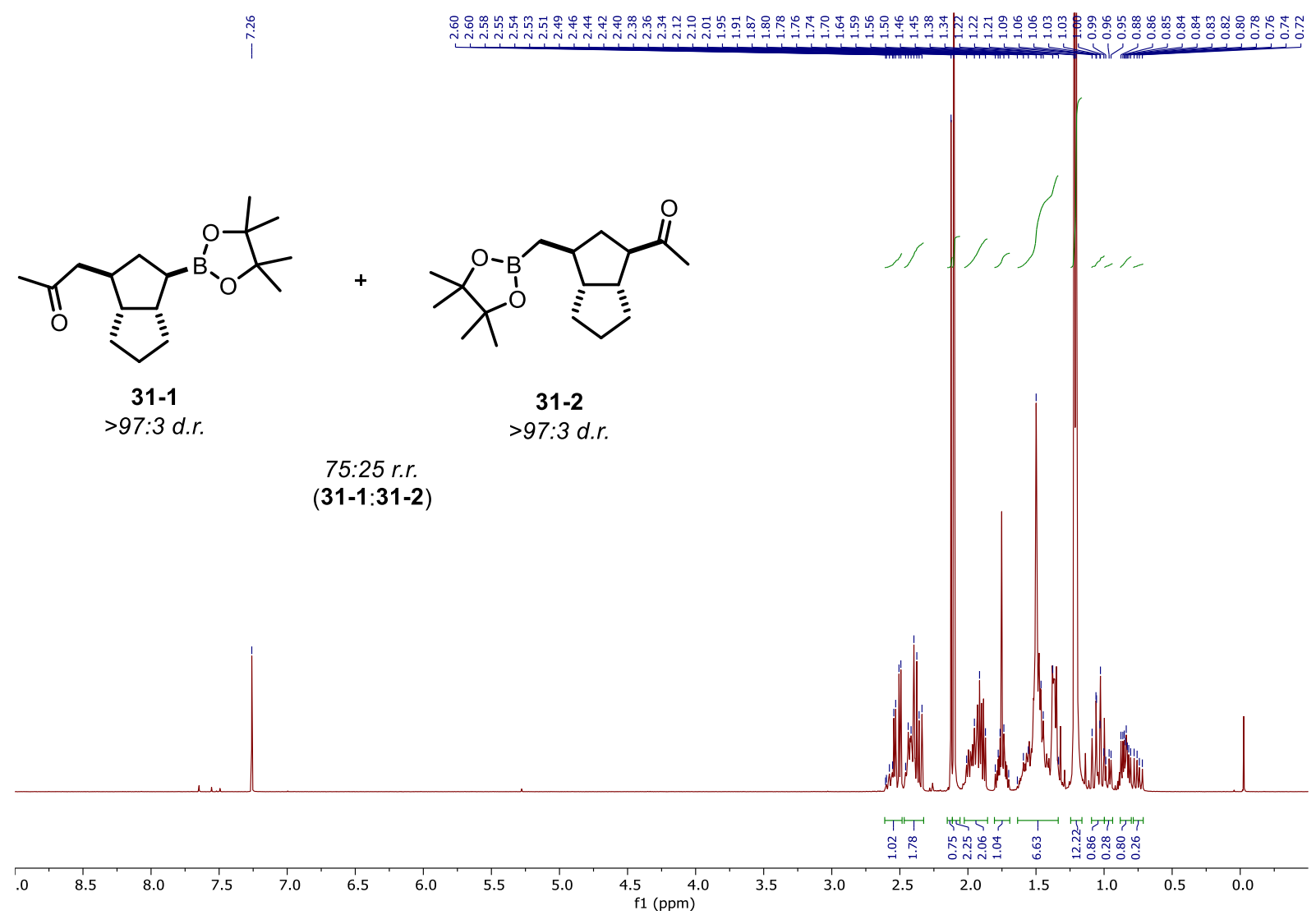


${ }^{1} \mathrm{H}$ NMR (400 MHz, $\mathrm{CDCl}_{3}$ ) of compound $\mathbf{3 1} \mathbf{p}$ (see procedure):

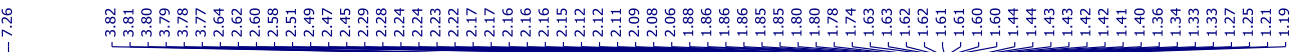<smiles>CC(=O)CC1CC(O)[C@H]2CCC[C@@H]12</smiles>

$31 \mathrm{p}$
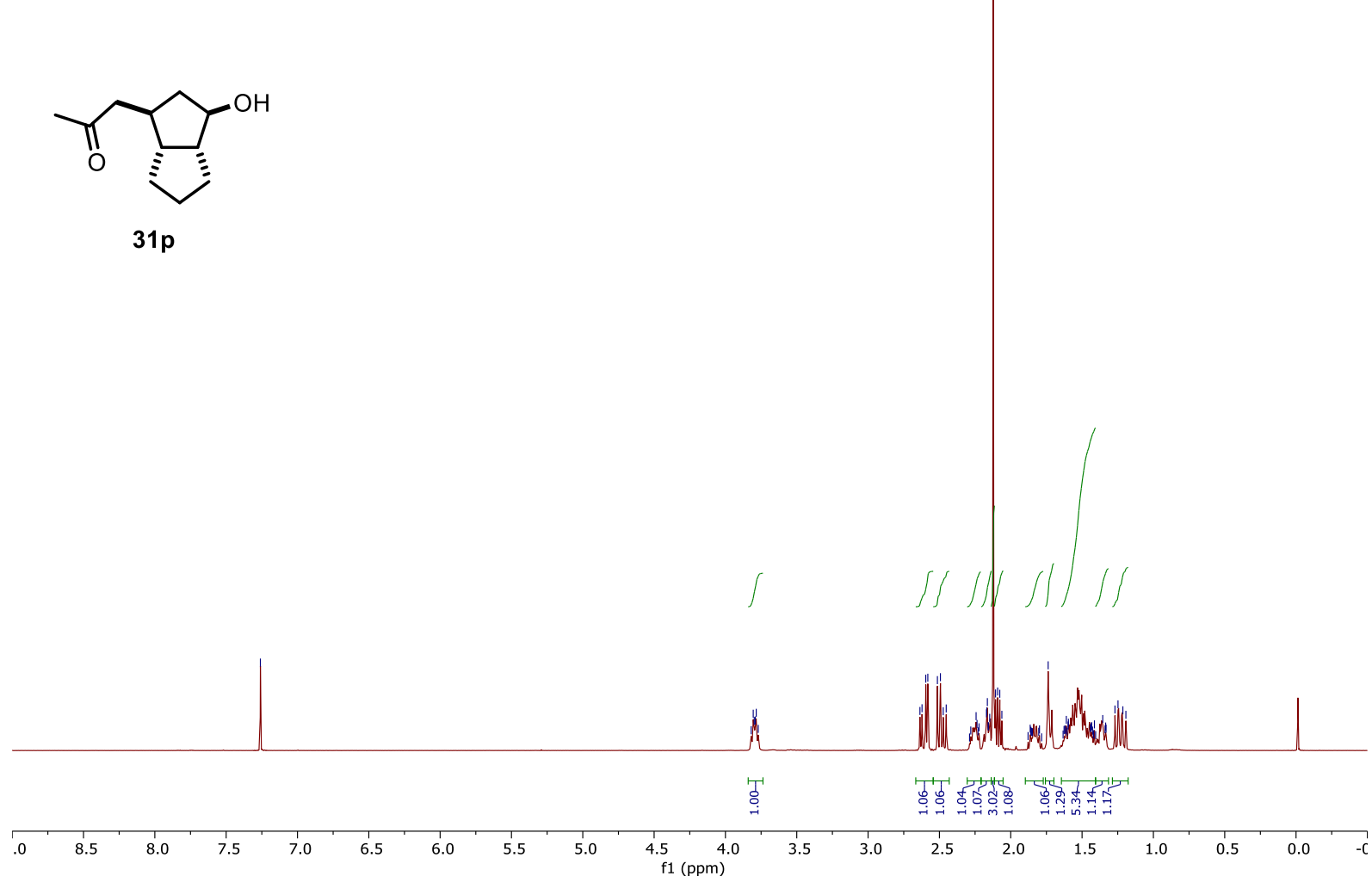

${ }^{13} \mathrm{C}$ NMR (101 MHz, $\mathrm{CDCl}_{3}$ ) of compound $\mathbf{3 1} \mathbf{p}$ (see procedure):<smiles>CC(=O)CC1CC(O)[C@H]2CCC[C@@H]12</smiles>

$31 p$

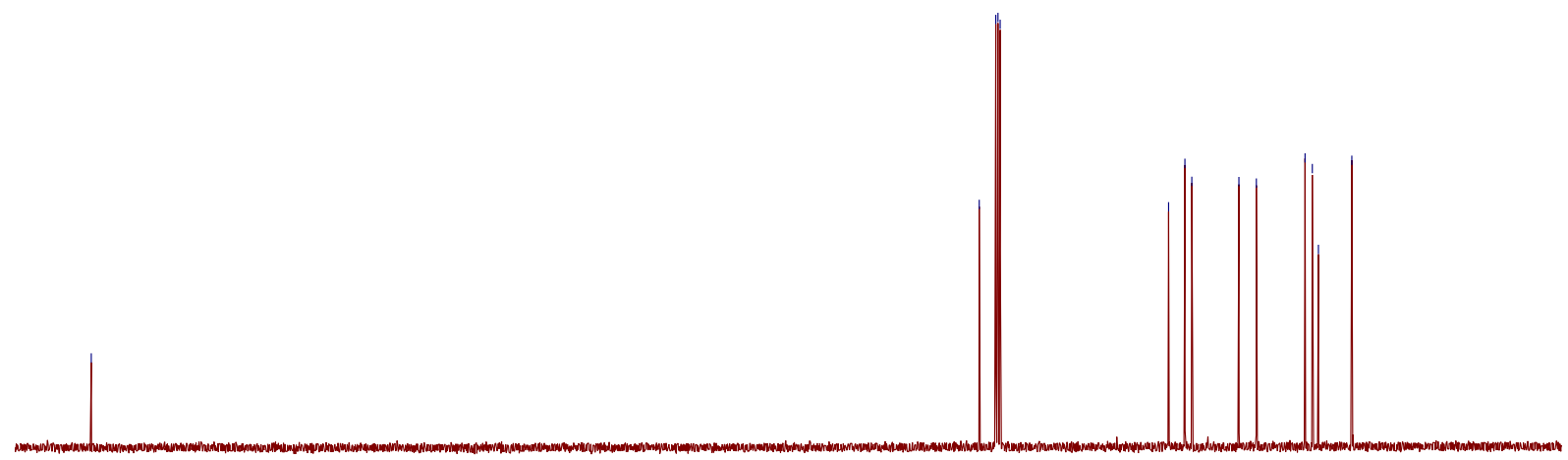

\begin{tabular}{lllllllllllllllllllllll}
\hline 20 & 210 & 200 & 190 & 180 & 170 & 160 & 150 & 140 & 130 & 120 & 110 & 100 & 90 & 80 & 70 & 60 & 50 & 40 & 30 & 20 & 10 & 0
\end{tabular} 
${ }^{1} \mathrm{H}$ NMR (400 MHz, $\mathrm{CDCl}_{3}$ ) of compound 32 (see procedure):

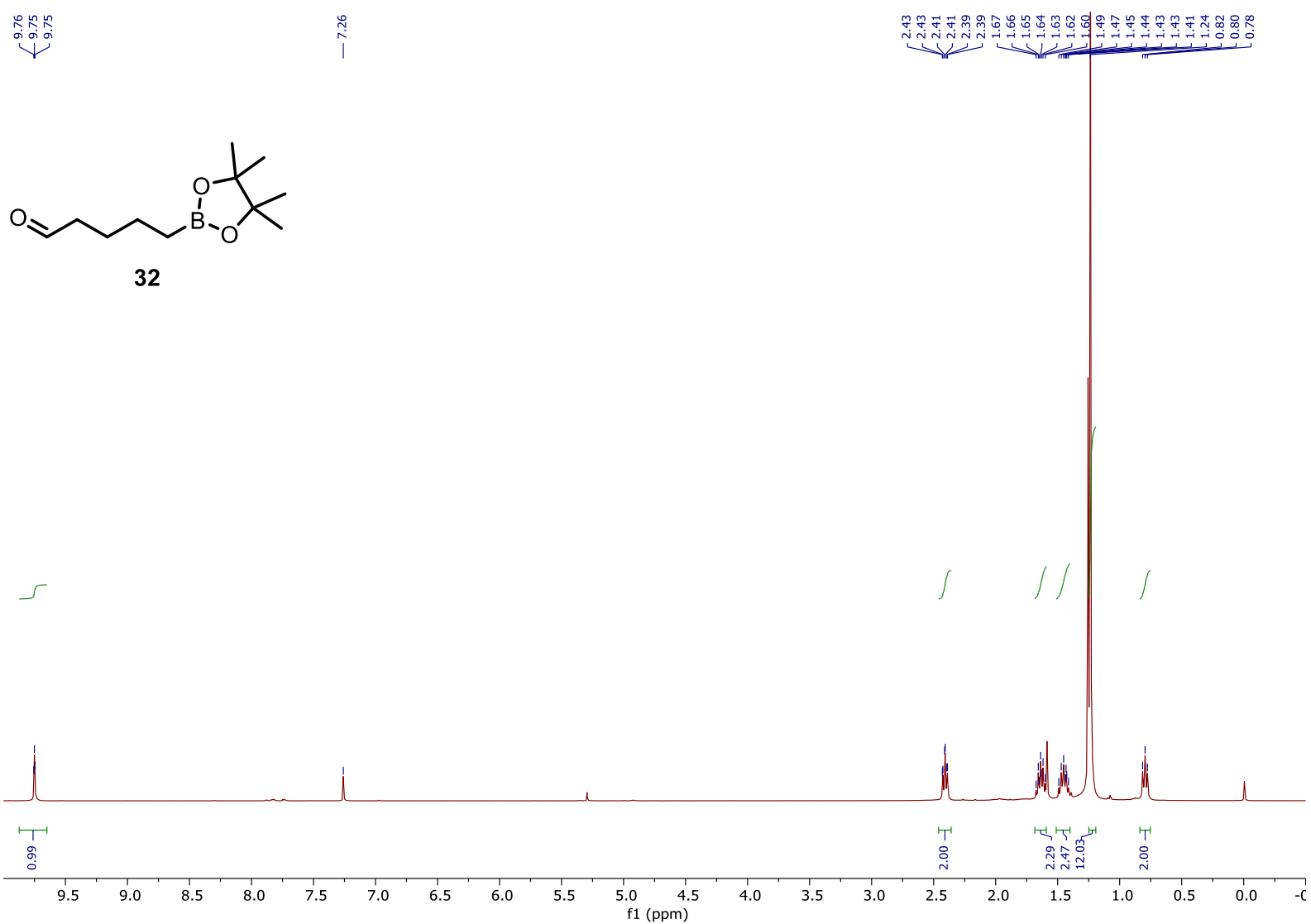

${ }^{13} \mathrm{C}$ NMR (101 MHz, $\mathrm{CDCl}_{3}$ ) of compound 32 (see procedure):

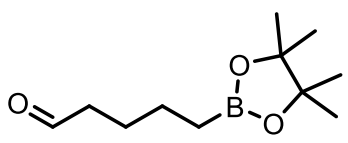

32

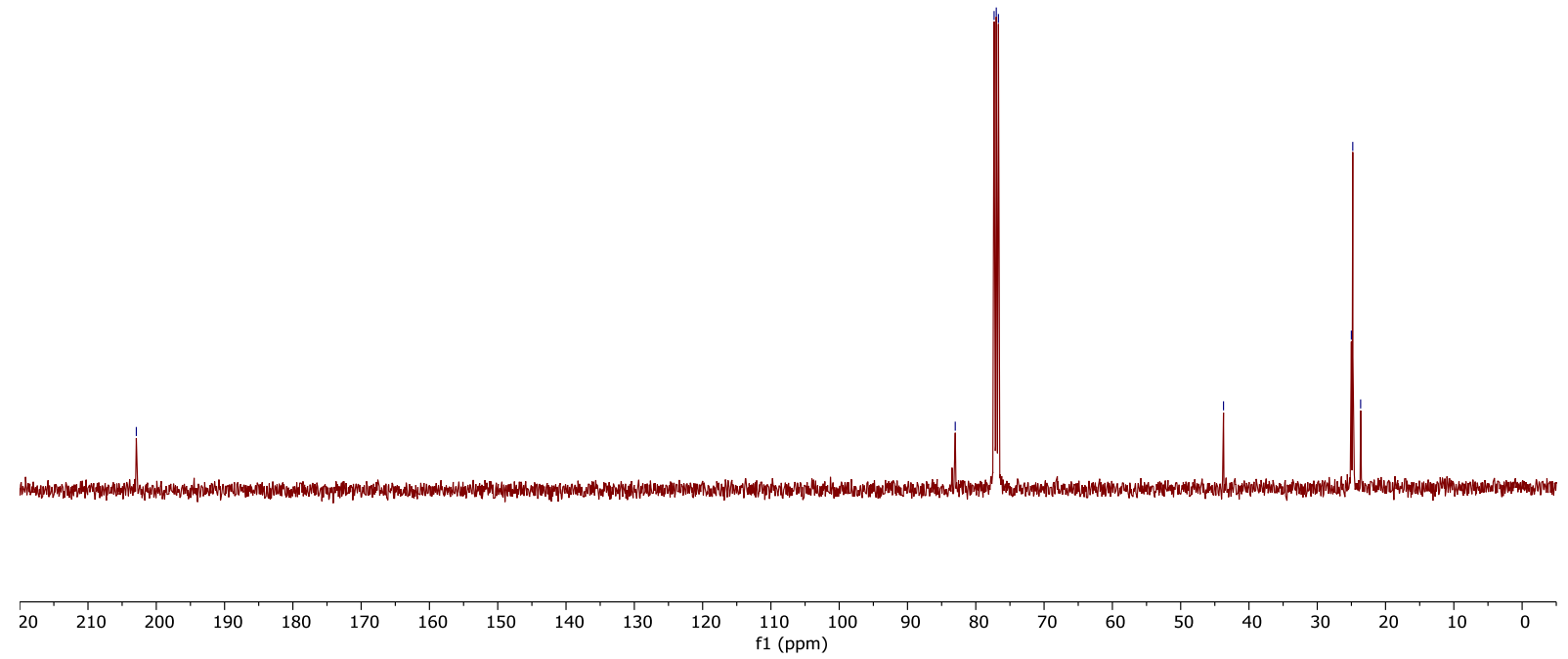


${ }^{11} \mathrm{~B}$ NMR (128 MHz, $\mathrm{CDCl}_{3}$ ) of compound 32 (see procedure):

$\stackrel{\sim}{\sim} \underset{\sim}{\sim}$

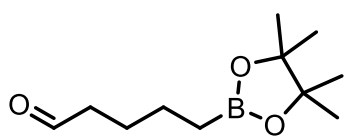

32

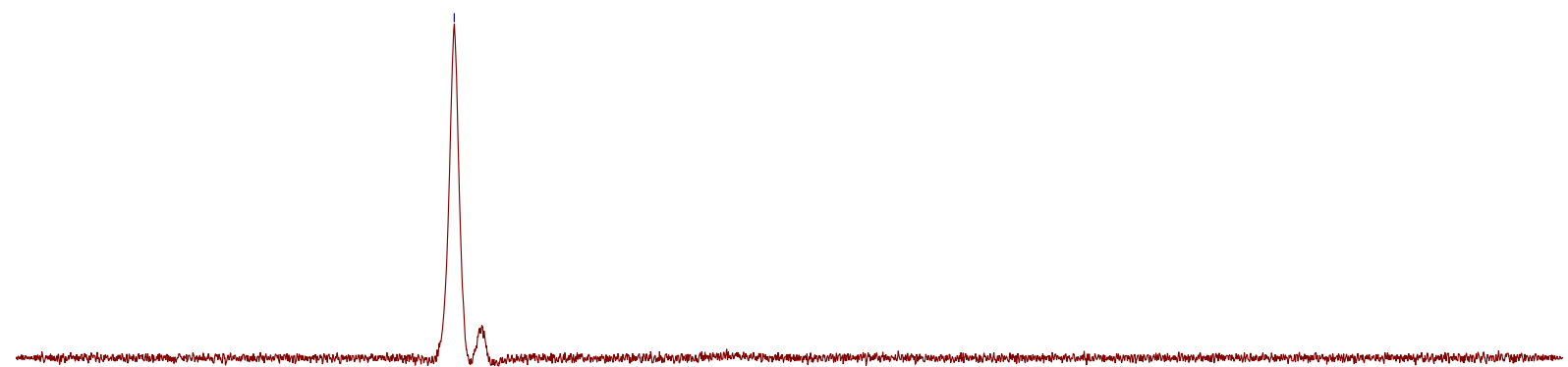

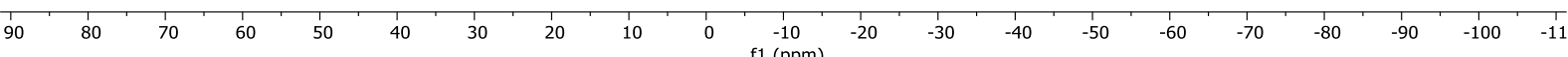


${ }^{1} \mathrm{H}$ NMR (400 MHz, $\mathrm{CDCl}_{3}$ ) of compound 33 (see procedure):

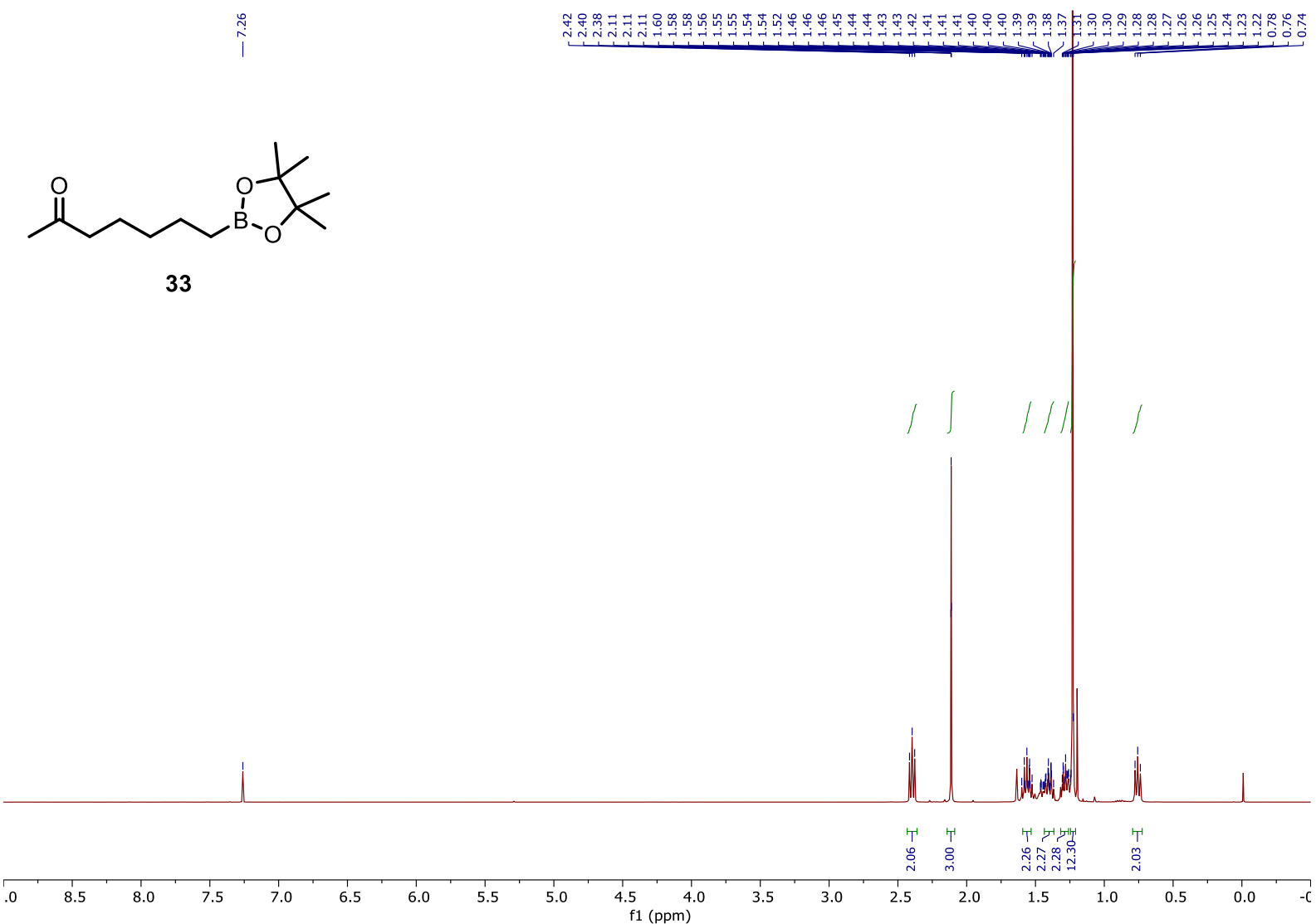

${ }^{13} \mathrm{C}$ NMR (101 MHz, $\mathrm{CDCl}_{3}$ ) of compound $\mathbf{3 3}$ (see procedure):<smiles>[BH2-][BH2-]</smiles>

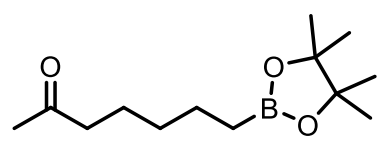

33

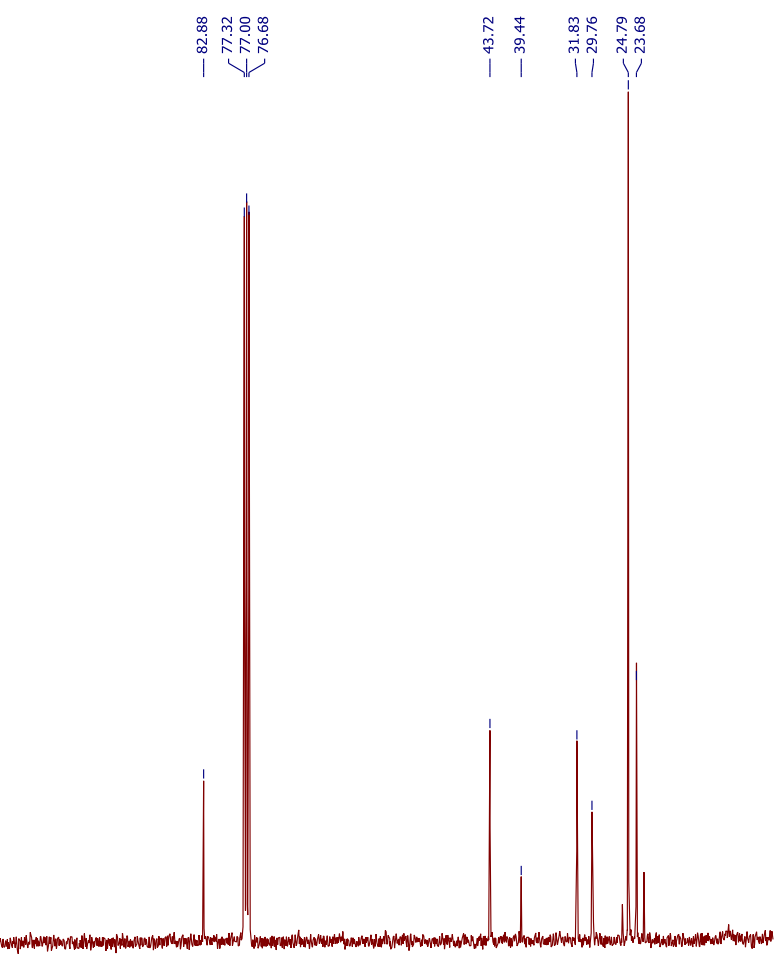

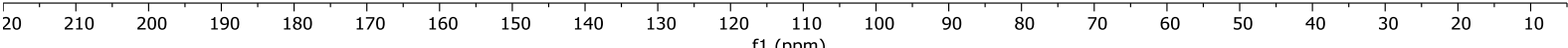


${ }^{1} \mathrm{H}$ NMR (400 MHz, $\mathrm{CDCl}_{3}$ ) of compound 34 (see procedure):

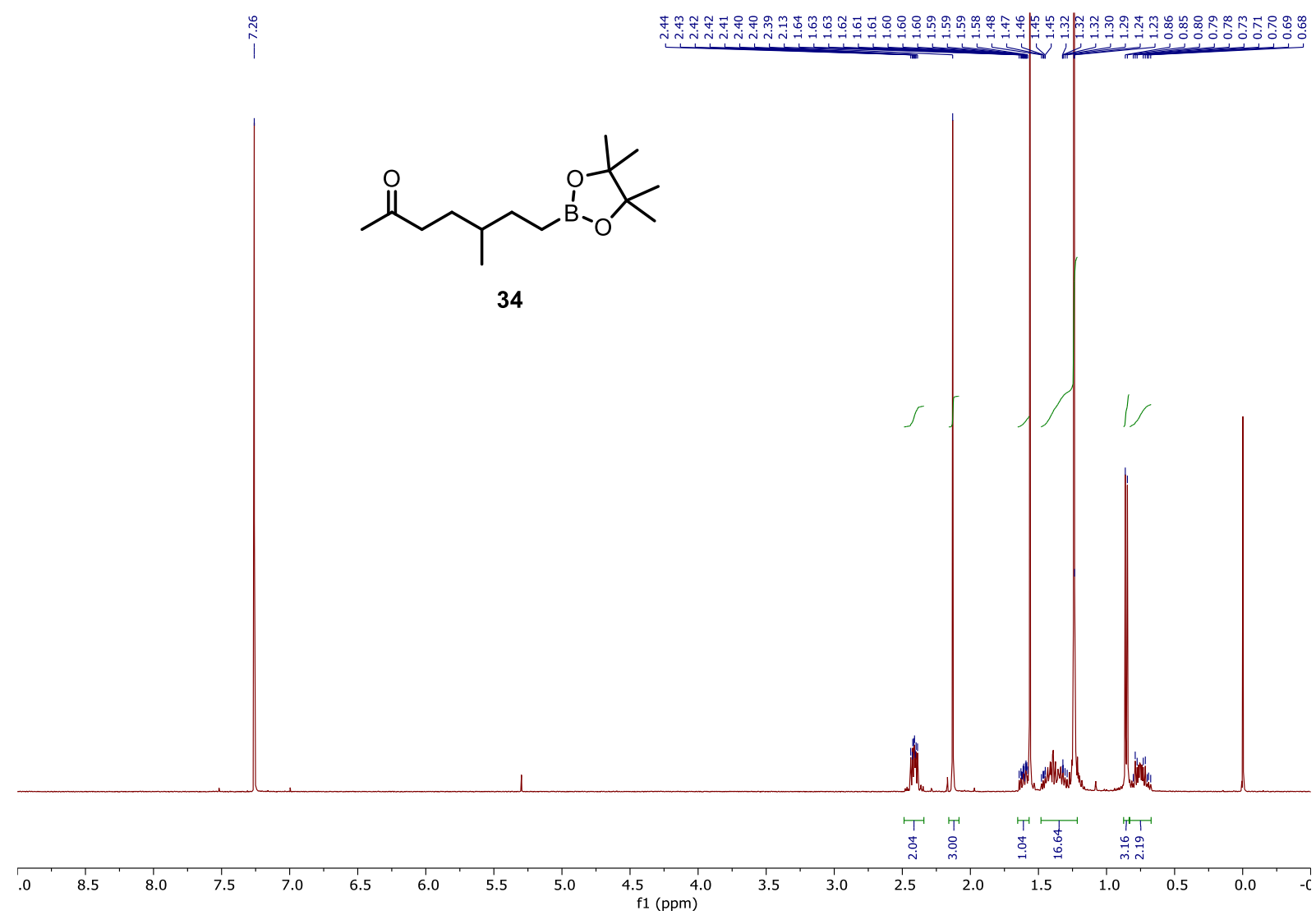

${ }^{13} \mathrm{C} \mathrm{NMR}\left(101 \mathrm{MHz}, \mathrm{CDCl}_{3}\right.$ ) of compound 34 (see procedure):

ind

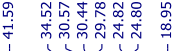<smiles>CC(=O)CCC(C)CC[B]OC(C)(C)C</smiles>

34

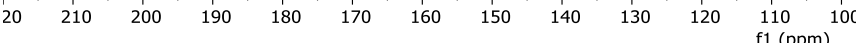


${ }^{11} \mathrm{~B}$ NMR (128 MHz, $\mathrm{CDCl}_{3}$ ) of compound 34 (see procedure):<smiles>CC(=O)CCC(C)CC[B]OC(C)(C)C</smiles>

34

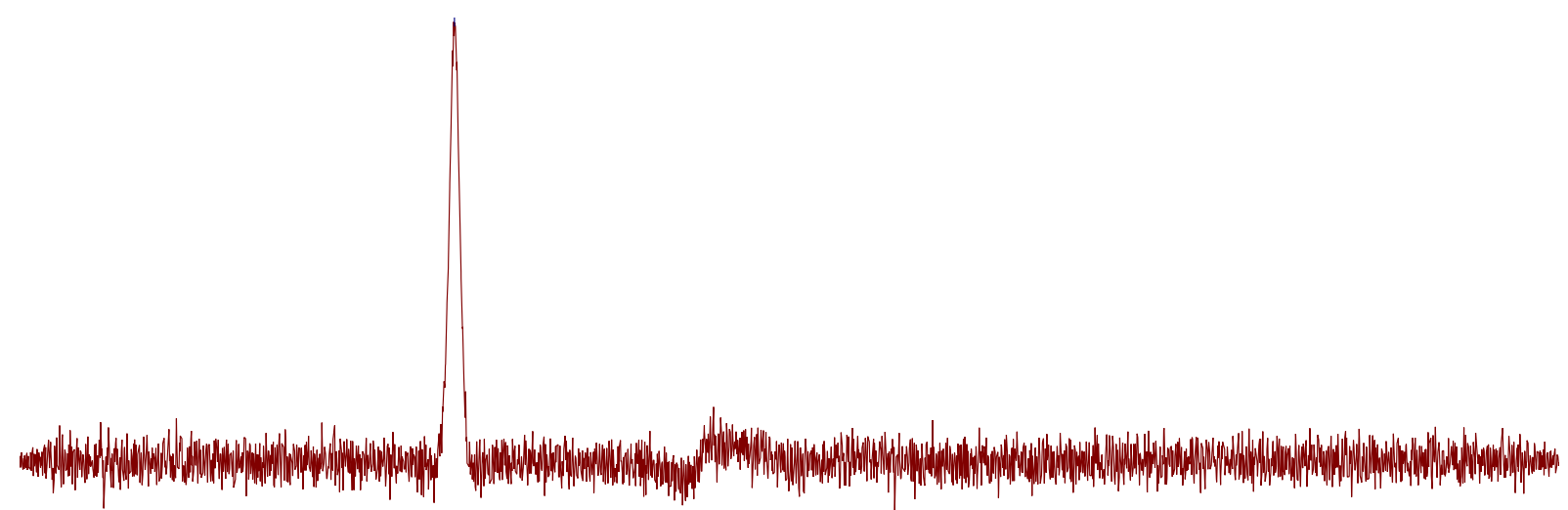

\begin{tabular}{rlllllllllllllllllllllll}
$T$ & $T$ & 80 & 70 & 60 & 50 & 40 & 30 & 20 & 10 & 0 & -10 & -20 & -30 & -40 & -50 & -60 & -70 & -80 & -90 & -100 & -11 \\
\hline
\end{tabular} 
${ }^{1} \mathrm{H}$ NMR (400 MHz, $\mathrm{CDCl}_{3}$ ) of compound 35 (see procedure):

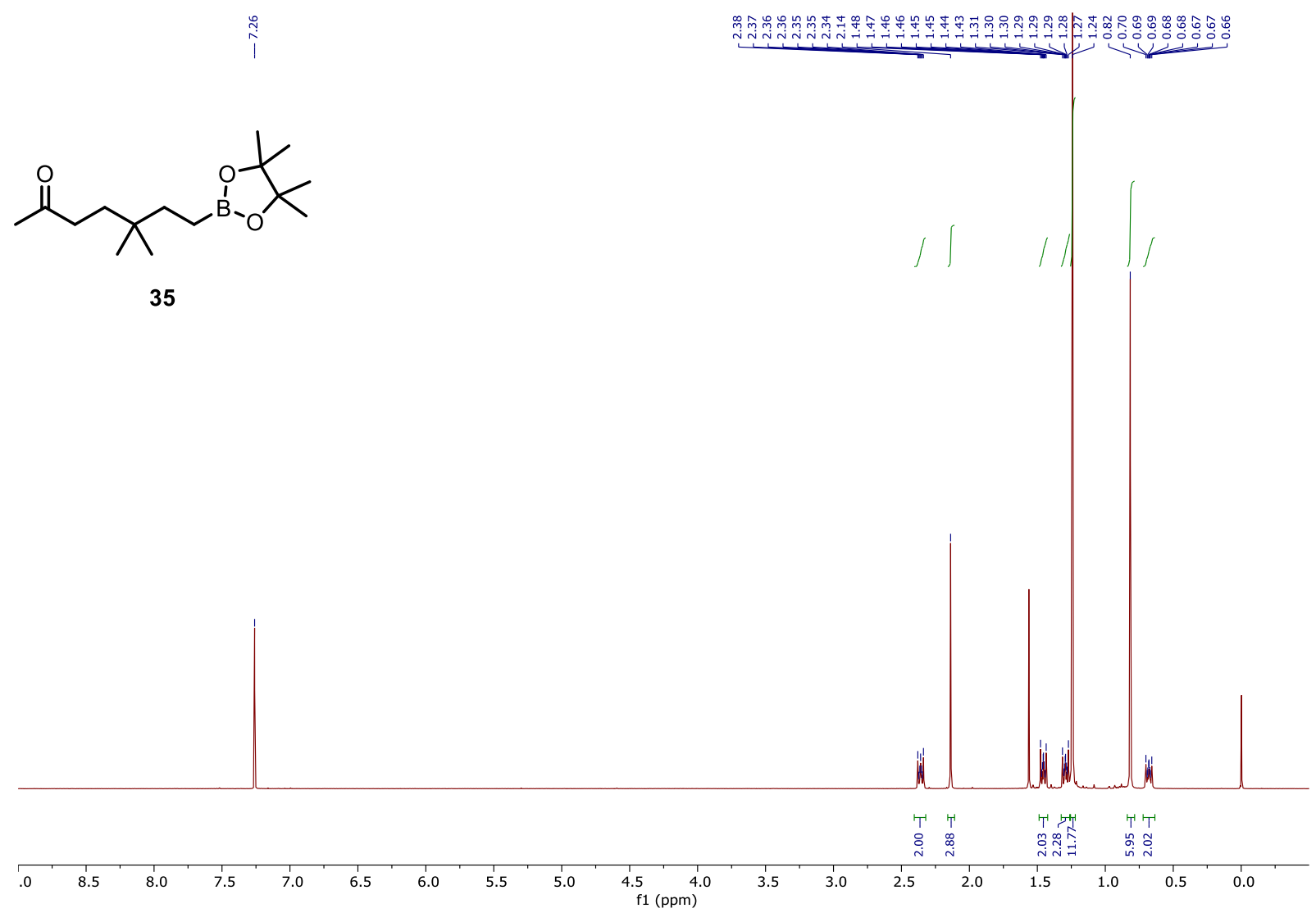

${ }^{13} \mathrm{C}$ NMR (101 MHz, $\mathrm{CDCl}_{3}$ ) of compound 35 (see procedure):

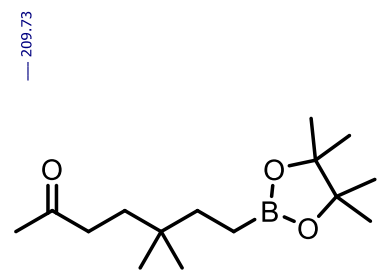

35

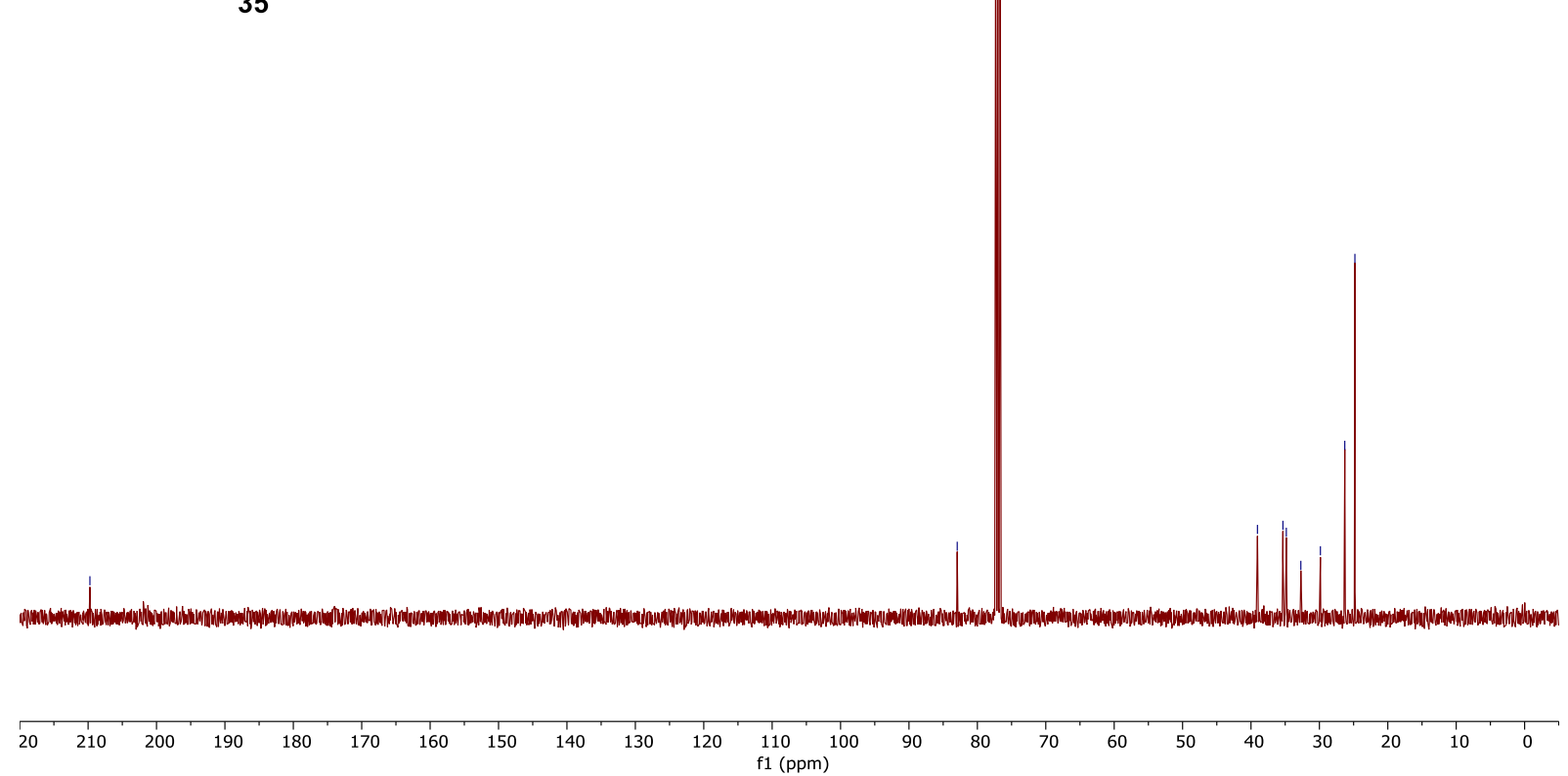


${ }^{11} \mathrm{~B}$ NMR (128 MHz, $\mathrm{CDCl}_{3}$ ) of compound 35 (see procedure):

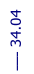<smiles>CC(=O)CCC(C)(C)CC[B]OC(C)(C)C(C)(C)C</smiles>

35

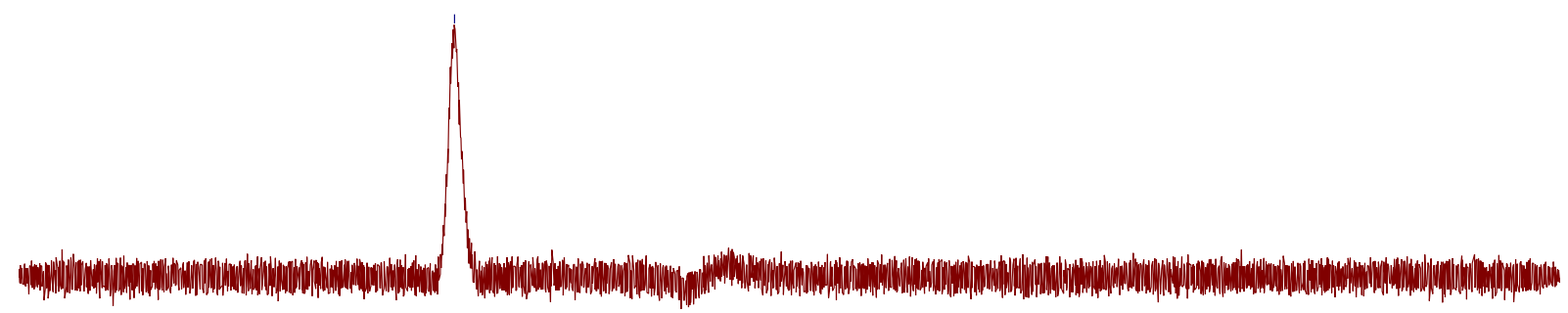

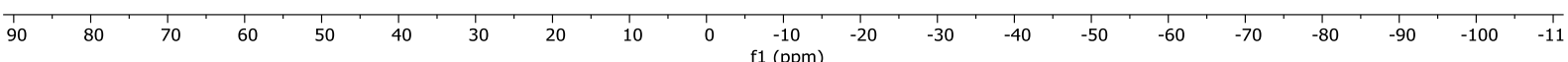


${ }^{1} \mathrm{H}$ NMR (400 MHz, $\mathrm{CDCl}_{3}$ ) of compound 36 (see procedure):

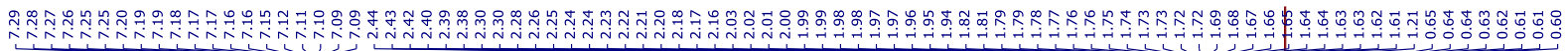<smiles>CC(=O)CCC(CCB1OC(C)(C)C(C)(C)O1)c1ccccc1</smiles>

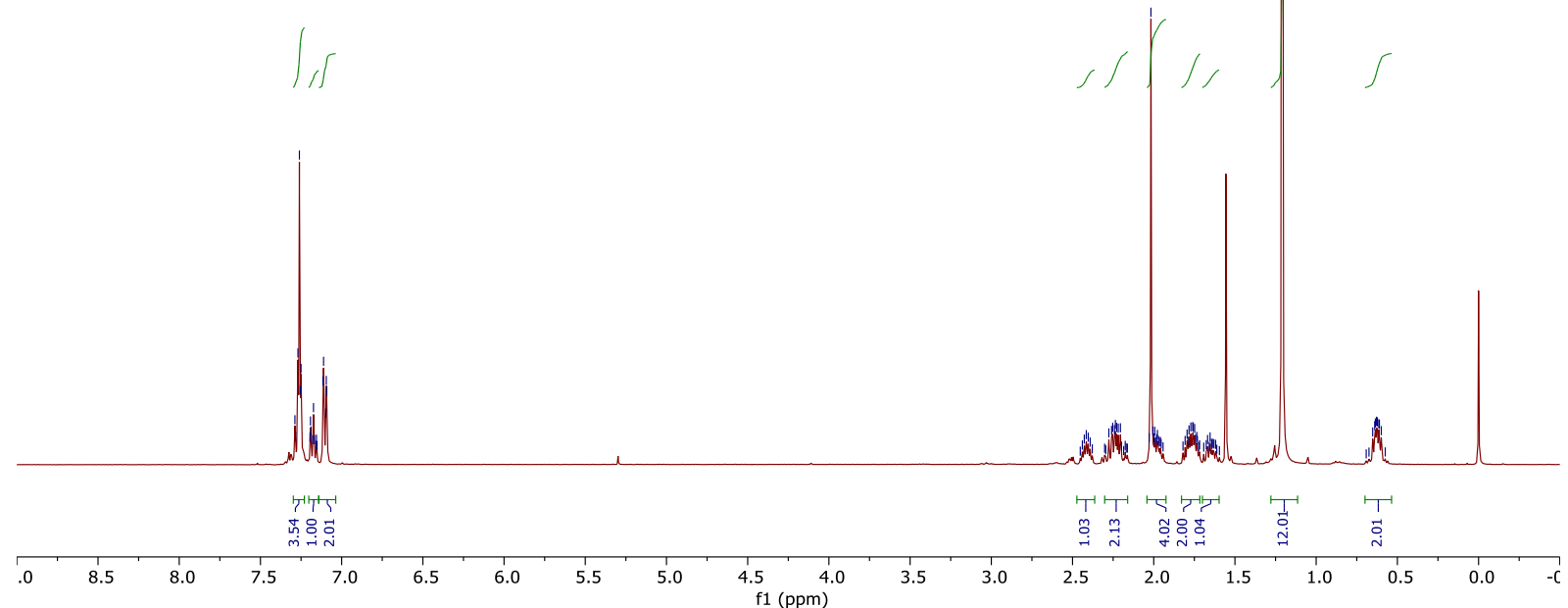

${ }^{13} \mathrm{C}$ NMR (101 MHz, $\mathrm{CDCl}_{3}$ ) of compound $\mathbf{3 6}$ (see procedure):<smiles>[Li][Ca]</smiles><smiles>CC(=O)CCC(CCB1OC(C)(C)C(C)(C)O1)c1ccccc1</smiles>

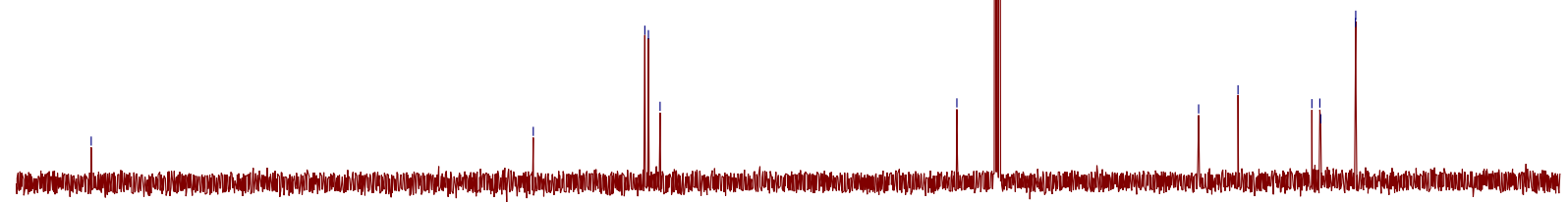

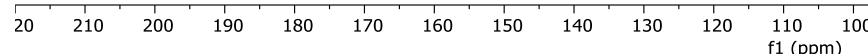


${ }^{11} \mathrm{~B}$ NMR (128 MHz, $\mathrm{CDCl}_{3}$ ) of compound 36 (see procedure):

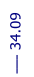<smiles>CC(=O)CCC(CCB1OC(C)(C)C(C)(C)O1)c1ccccc1</smiles>

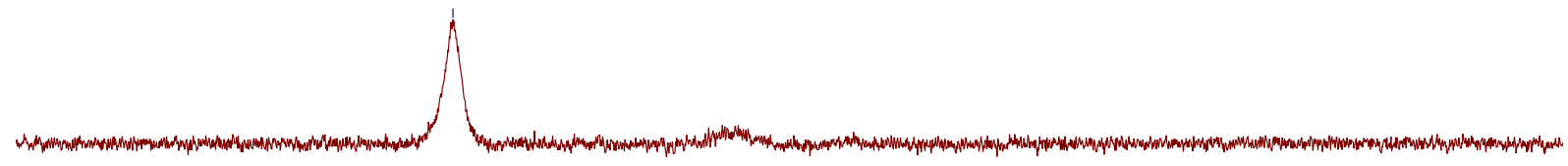

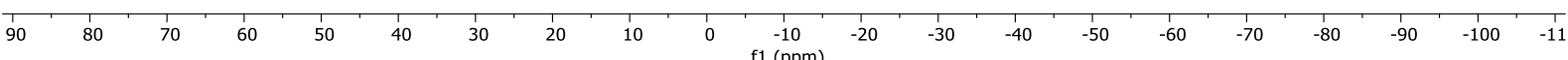


${ }^{1} \mathrm{H}$ NMR (400 MHz, $\mathrm{CDCl}_{3}$ ) of compound 37 (see procedure):

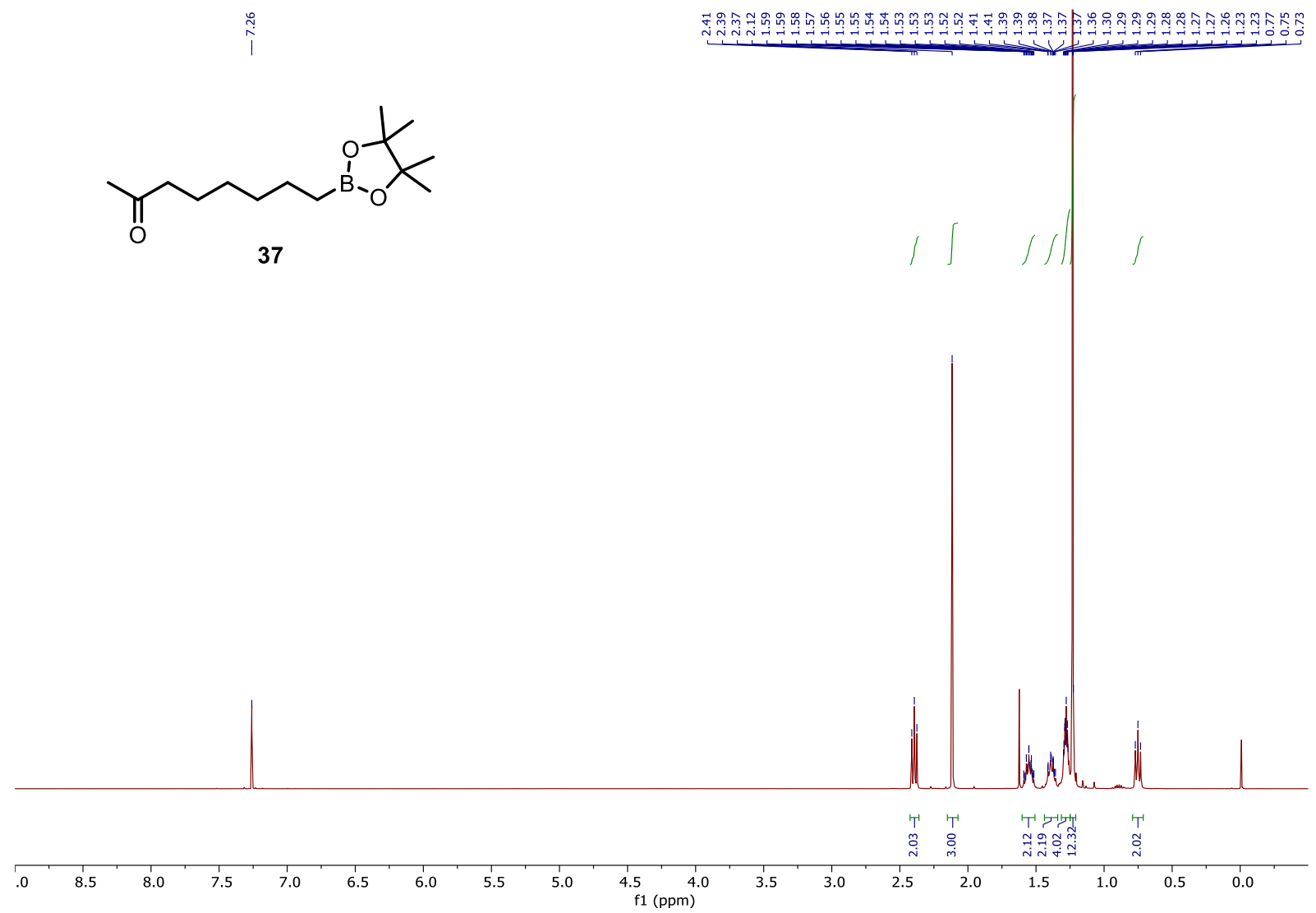

${ }^{13} \mathrm{C} \mathrm{NMR}\left(101 \mathrm{MHz}, \mathrm{CDCl}_{3}\right.$ ) of compound 37 (see procedure):

$\stackrel{\substack{0 \\ \stackrel{0}{0}}}{1}$

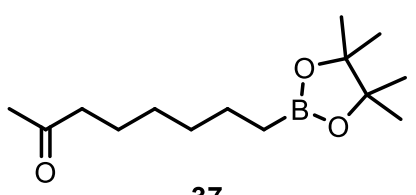

37

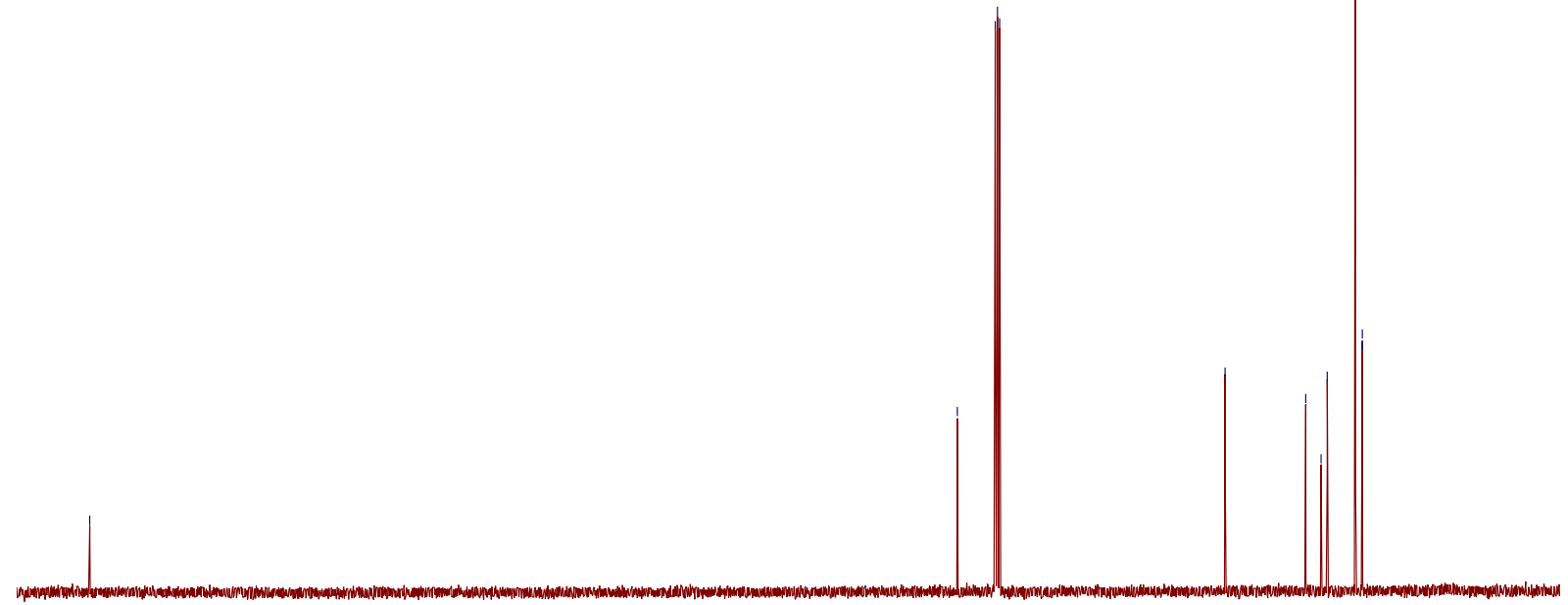

$\begin{array}{llllllllllllllllllllllllll}20 & 210 & 200 & 190 & 180 & 170 & 160 & 150 & 140 & 130 & 120 & 110 & 100 & 90 & 80 & 70 & 60 & 50 & 40 & 30 & 20 & 10 & 0\end{array}$ 
${ }^{11} \mathrm{~B}$ NMR (128 MHz, $\mathrm{CDCl}_{3}$ ) of compound 37 (see procedure):

$\stackrel{\substack{+\stackrel{+}{m}}}{1}$

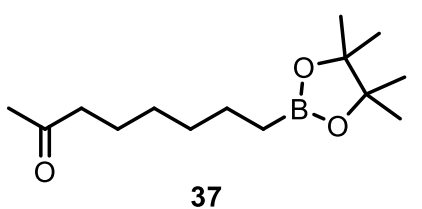

37

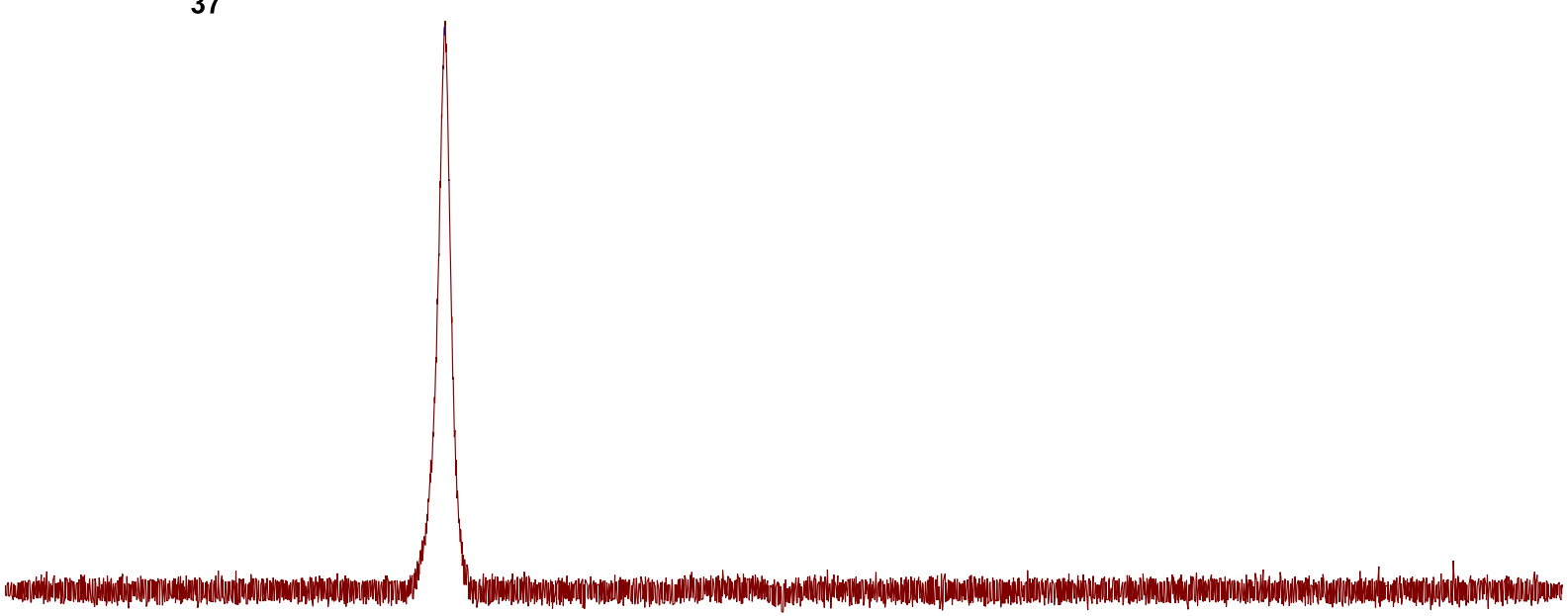

$\begin{array}{lllllllllllllllllllll}90 & 80 & 70 & 60 & 50 & 40 & 30 & 20 & 10 & 0 & \begin{array}{c}1 \\ \mathrm{f} 1(\mathrm{ppm})\end{array} & -20 & -30 & -40 & -50 & -60 & -70 & -80 & -90 & -100 & -11\end{array}$ 
${ }^{1} \mathrm{H}$ NMR (400 MHz, $\mathrm{CDCl}_{3}$ ) of compound 38 (see procedure):

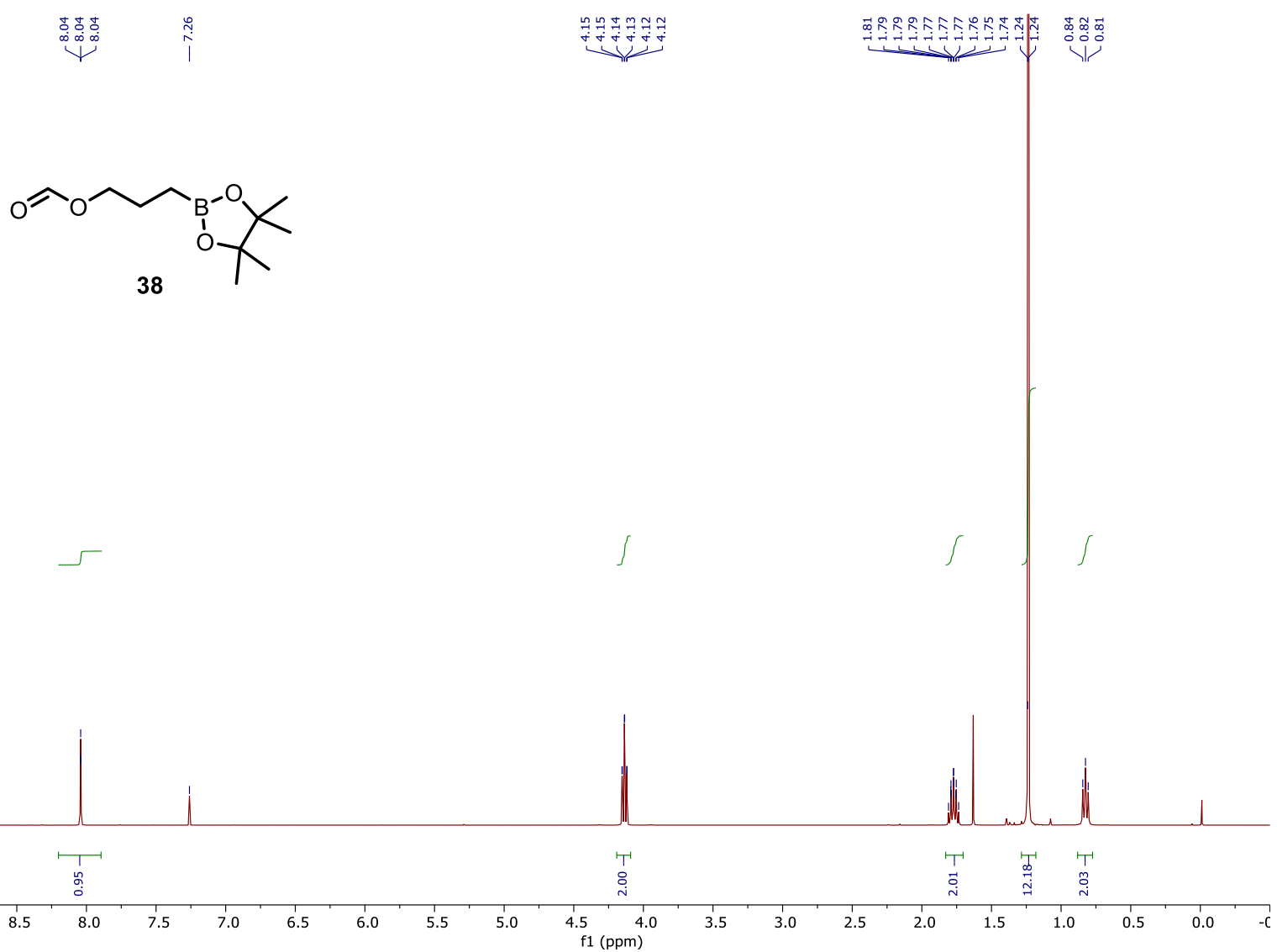

${ }^{13} \mathrm{C}$ NMR (101 MHz, $\mathrm{CDCl}_{3}$ ) of compound 38 (see procedure):

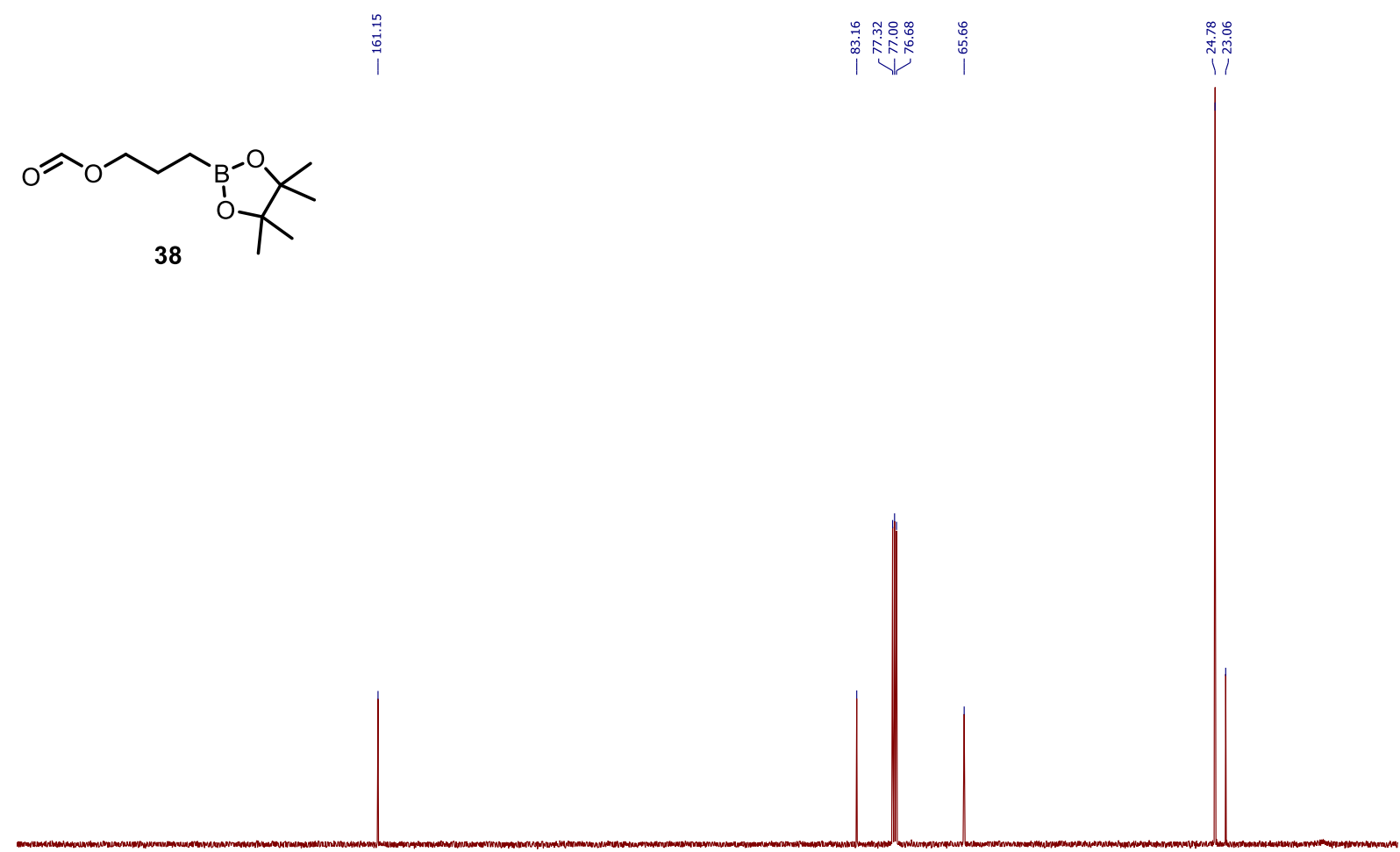

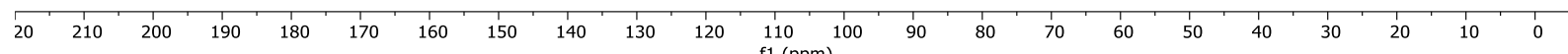


${ }^{11} \mathrm{~B}$ NMR (128 MHz, $\mathrm{CDCl}_{3}$ ) of compound $\mathbf{3 8}$ (see procedure):

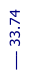<smiles>CC1(C)OB(CCCOC=O)OC1(C)C</smiles>

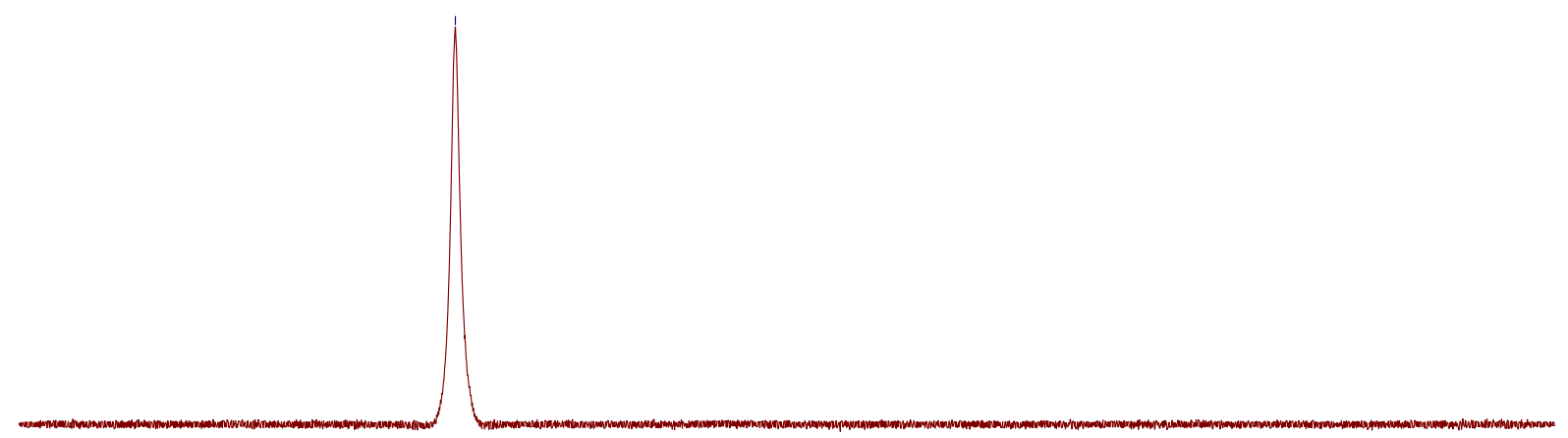

\begin{tabular}{llllllllllllllllllllll}
\hline & 80 & 70 & 60 & 50 & 40 & 30 & 20 & 10 & 0 & -10 & -20 & -30 & -40 & -50 & -60 & -70 & -80 & -90 & -100 & -11
\end{tabular} 
${ }^{1} \mathrm{H}$ NMR (400 MHz, $\mathrm{CDCl}_{3}$ ) of compound 39 (see procedure):
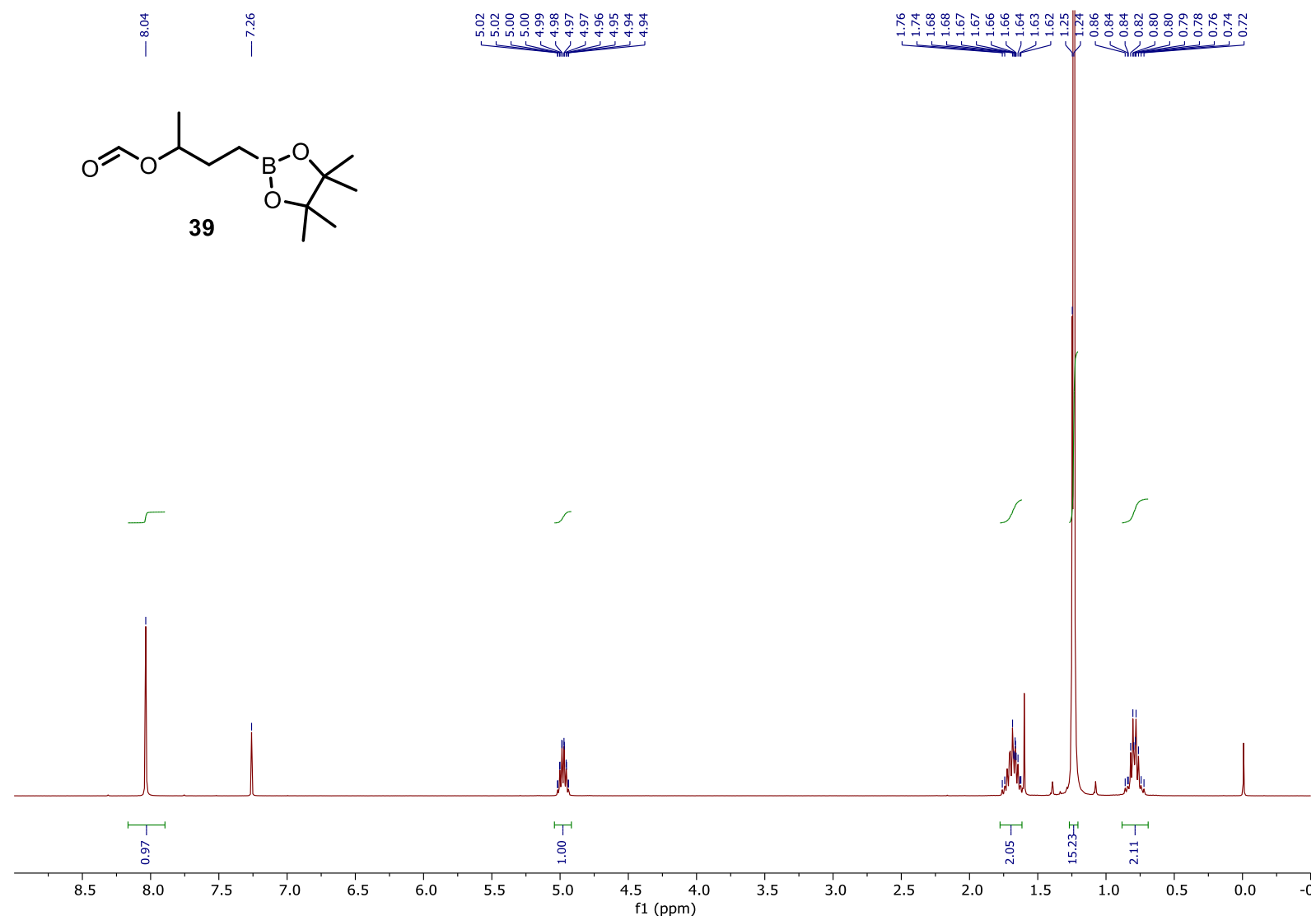

${ }^{13} \mathrm{C} \mathrm{NMR}$ (101 MHz, $\mathrm{CDCl}_{3}$ ) of compound 39 (see procedure):

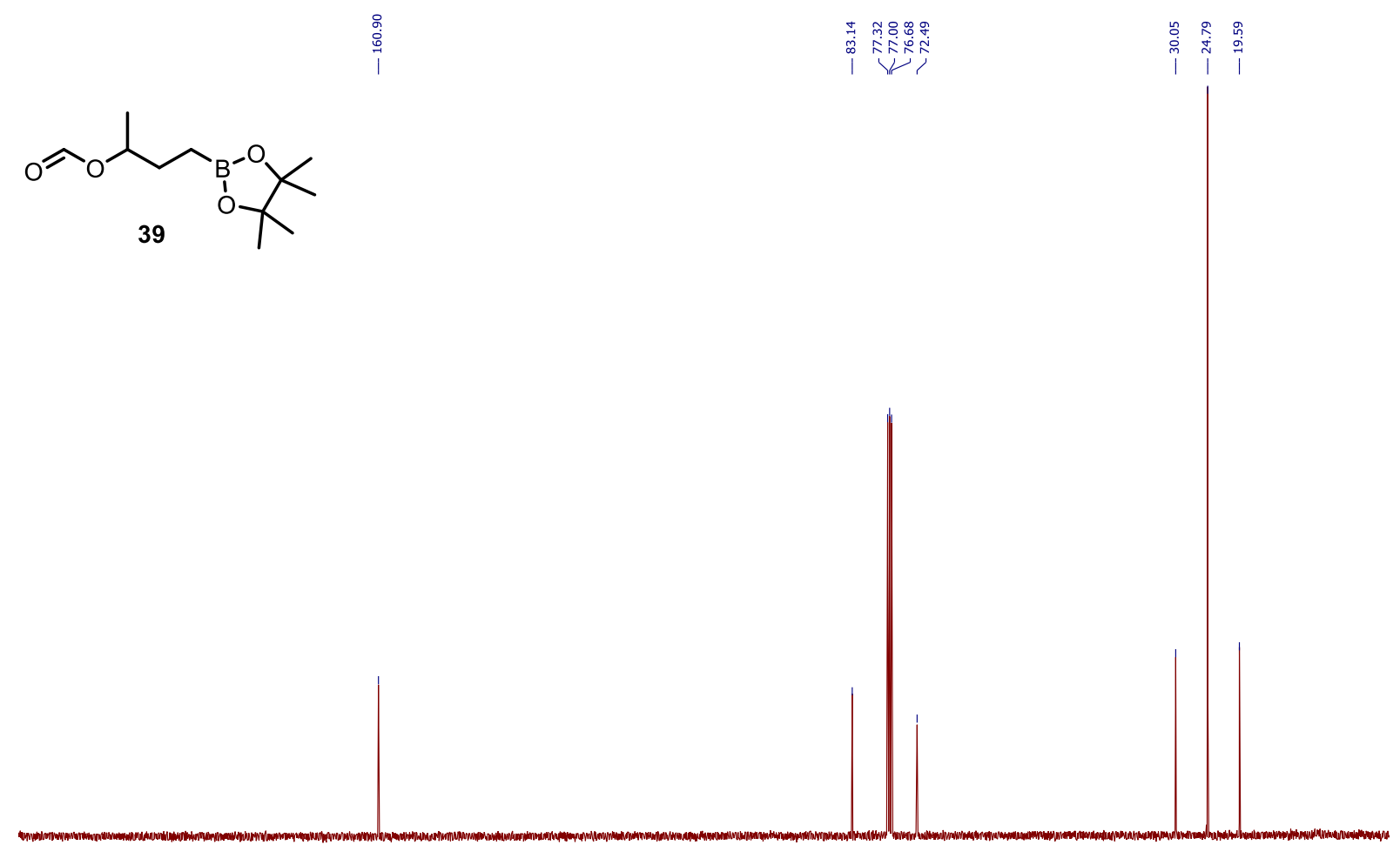

\begin{tabular}{lllllllllllllllllllllllllll}
\hline 20 & 210 & 200 & 190 & 180 & 170 & 160 & 150 & 140 & 130 & 120 & 110 & 100 & 90 & 80 & 70 & 60 & 50 & 40 & 30 & 20 & 10 & 0
\end{tabular} 
${ }^{11} \mathrm{~B}$ NMR (128 MHz, $\mathrm{CDCl}_{3}$ ) of compound 39 (see procedure):

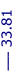<smiles>CC(COB1OC(C)(C)C(C)(C)O1)OC=O</smiles>

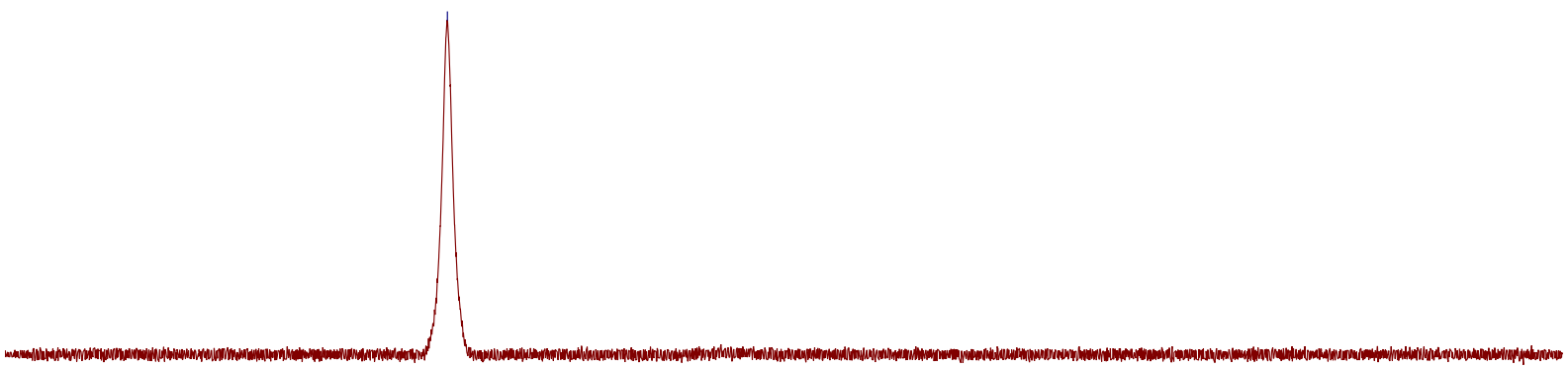

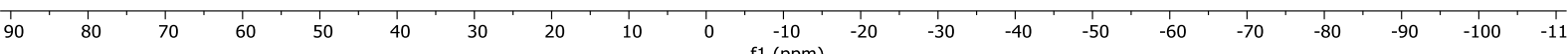


${ }^{1} \mathrm{H}$ NMR (400 MHz, $\mathrm{CDCl}_{3}$ ) of compound 40 (see procedure):

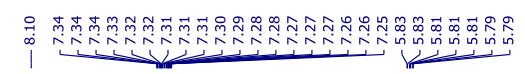

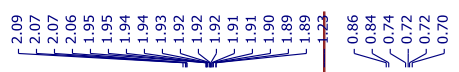<smiles>CC1(C)OB(CCC(OC=O)c2ccccc2)OC1(C)C</smiles>
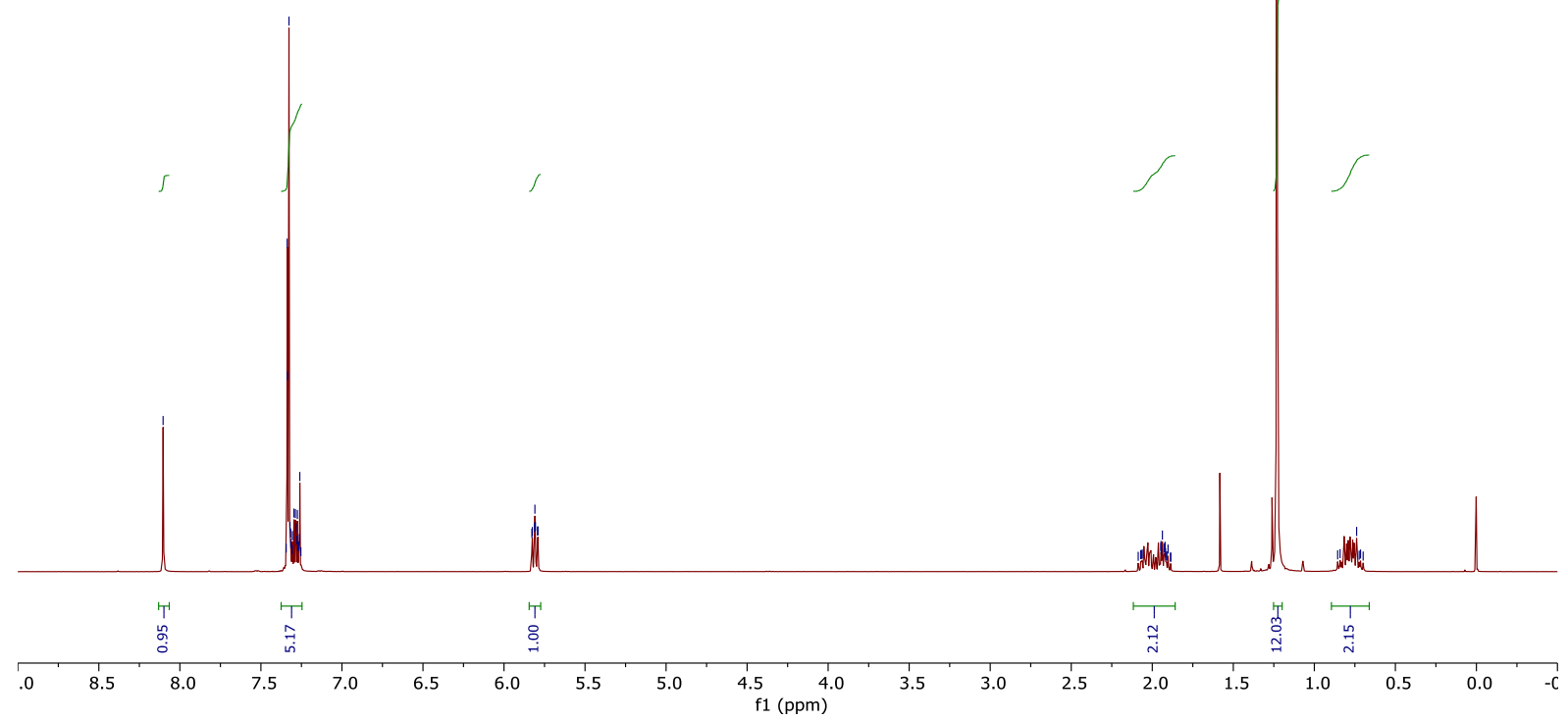

${ }^{13} \mathrm{C}$ NMR (101 MHz, $\mathrm{CDCl}_{3}$ ) of compound 40 (see procedure):<smiles>CC1(C)OB(CCC(OC=O)c2ccccc2)OC1(C)C</smiles>

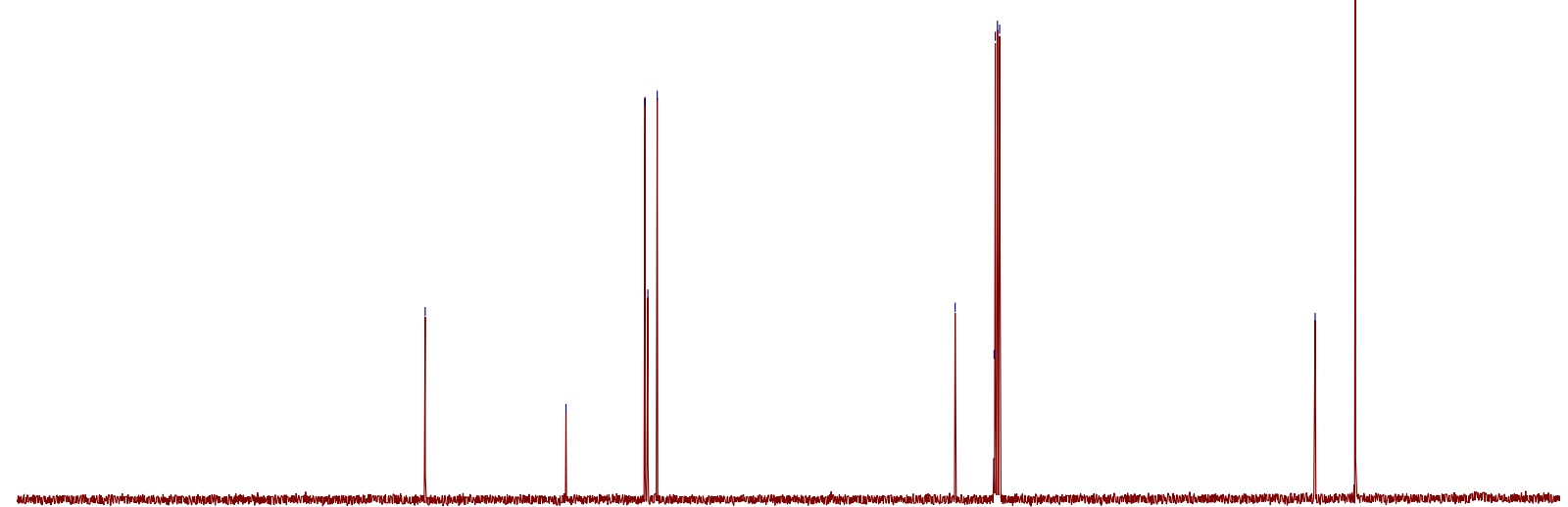

$\begin{array}{lllllllllllll}20 & 210 & 200 & 190 & 180 & 170 & 160 & 150 & 140 & 130 & 120 & 110 & 100\end{array}$ 
${ }^{11} \mathrm{~B}$ NMR (128 MHz, $\mathrm{CDCl}_{3}$ ) of compound 40 (see procedure):

$\stackrel{m}{m}$<smiles>CC1(C)OB(CCC(OC=O)c2ccccc2)OC1(C)C</smiles>

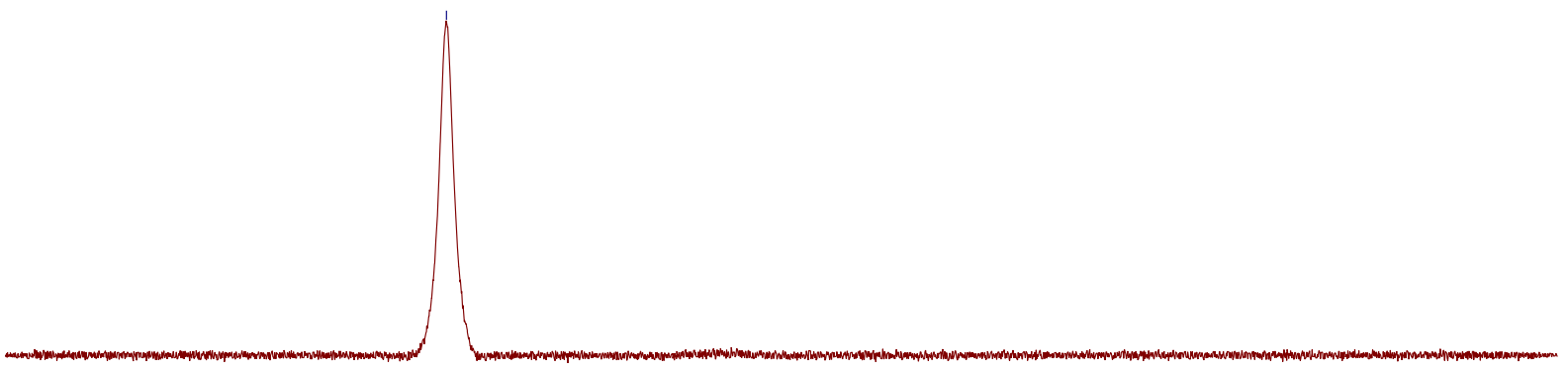

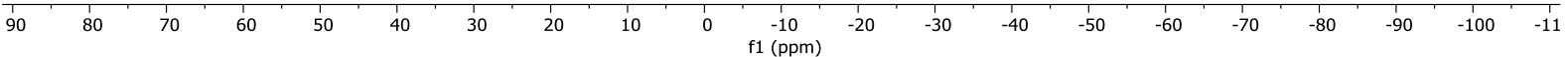


${ }^{1} \mathrm{H}$ NMR (400 MHz, $\mathrm{CDCl}_{3}$ ) of compound 41 (see procedure):

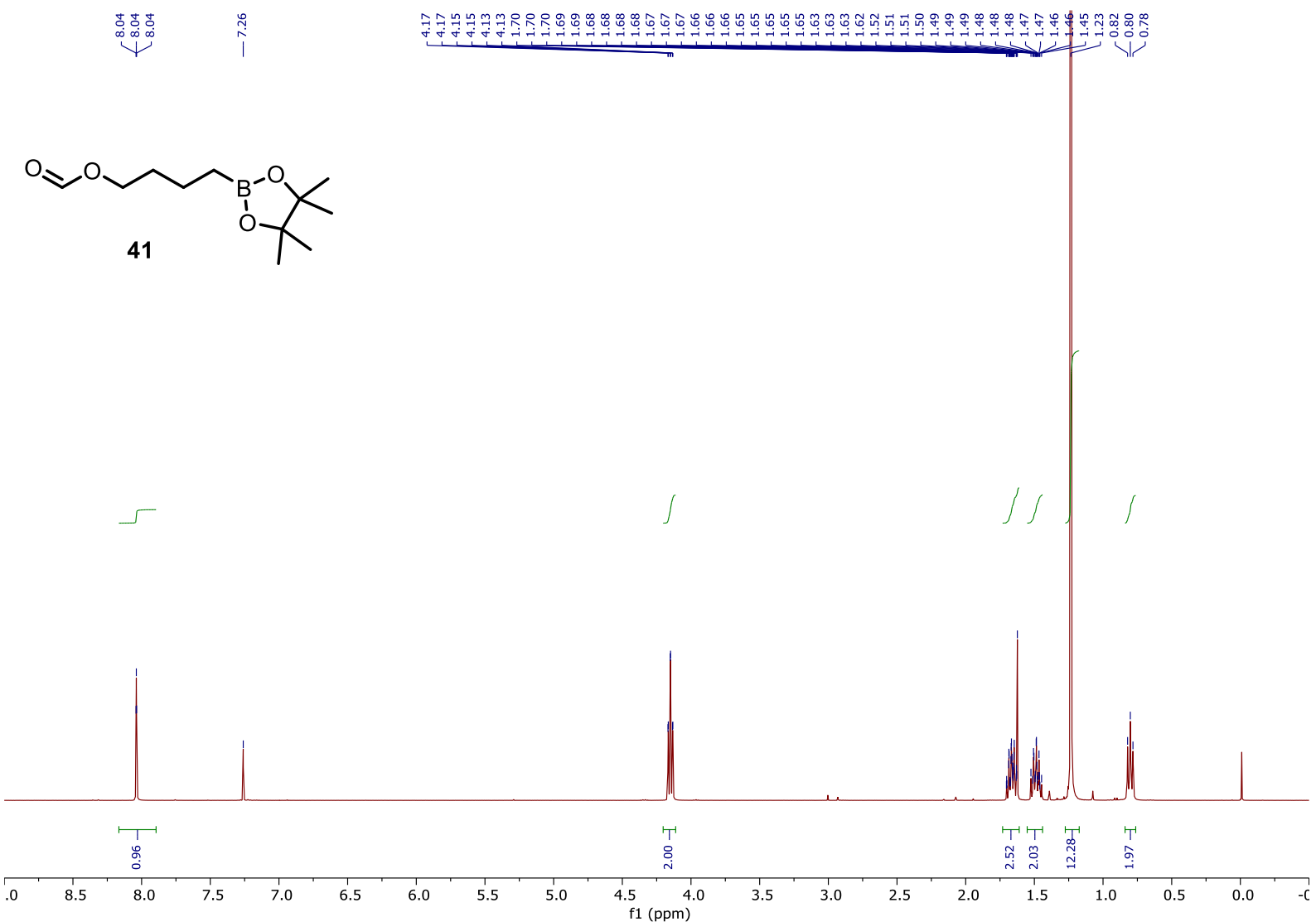

${ }^{13} \mathrm{C}$ NMR (101 MHz, $\mathrm{CDCl}_{3}$ ) of compound 41 (see procedure):

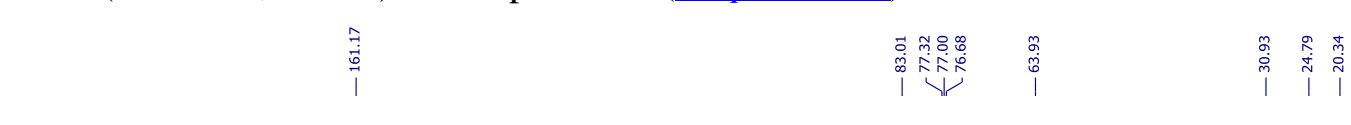<smiles>CC1(C)OB(CCCCOC=O)OC1(C)C</smiles>

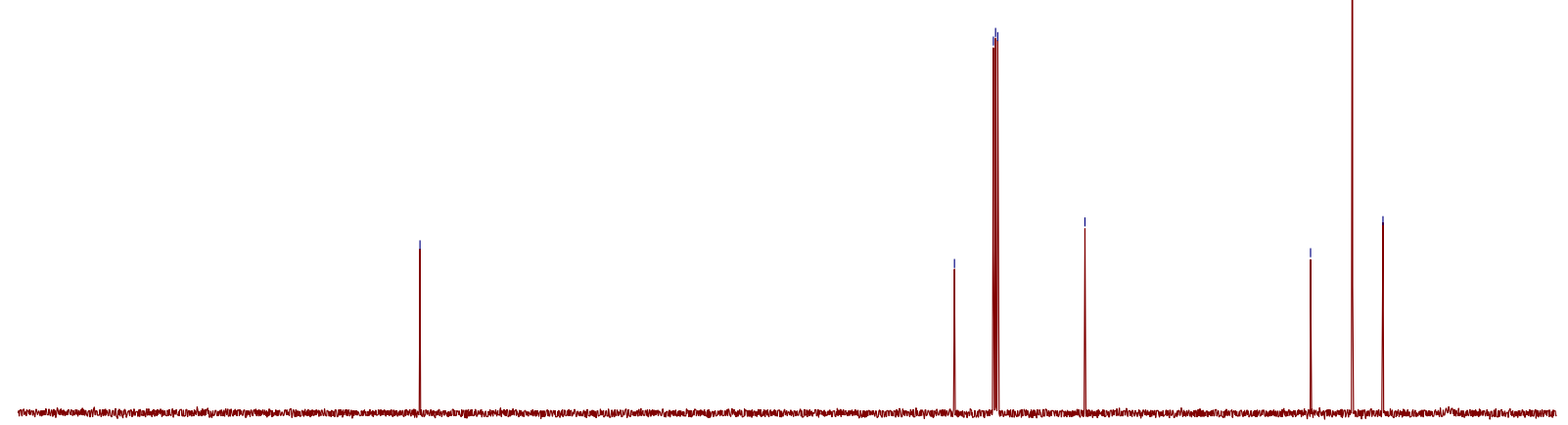

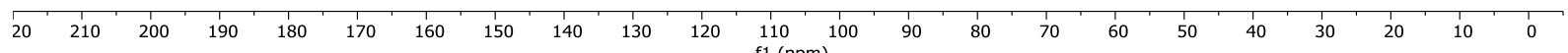


${ }^{11} \mathrm{~B}$ NMR (128 MHz, $\mathrm{CDCl}_{3}$ ) of compound 41 (see procedure):
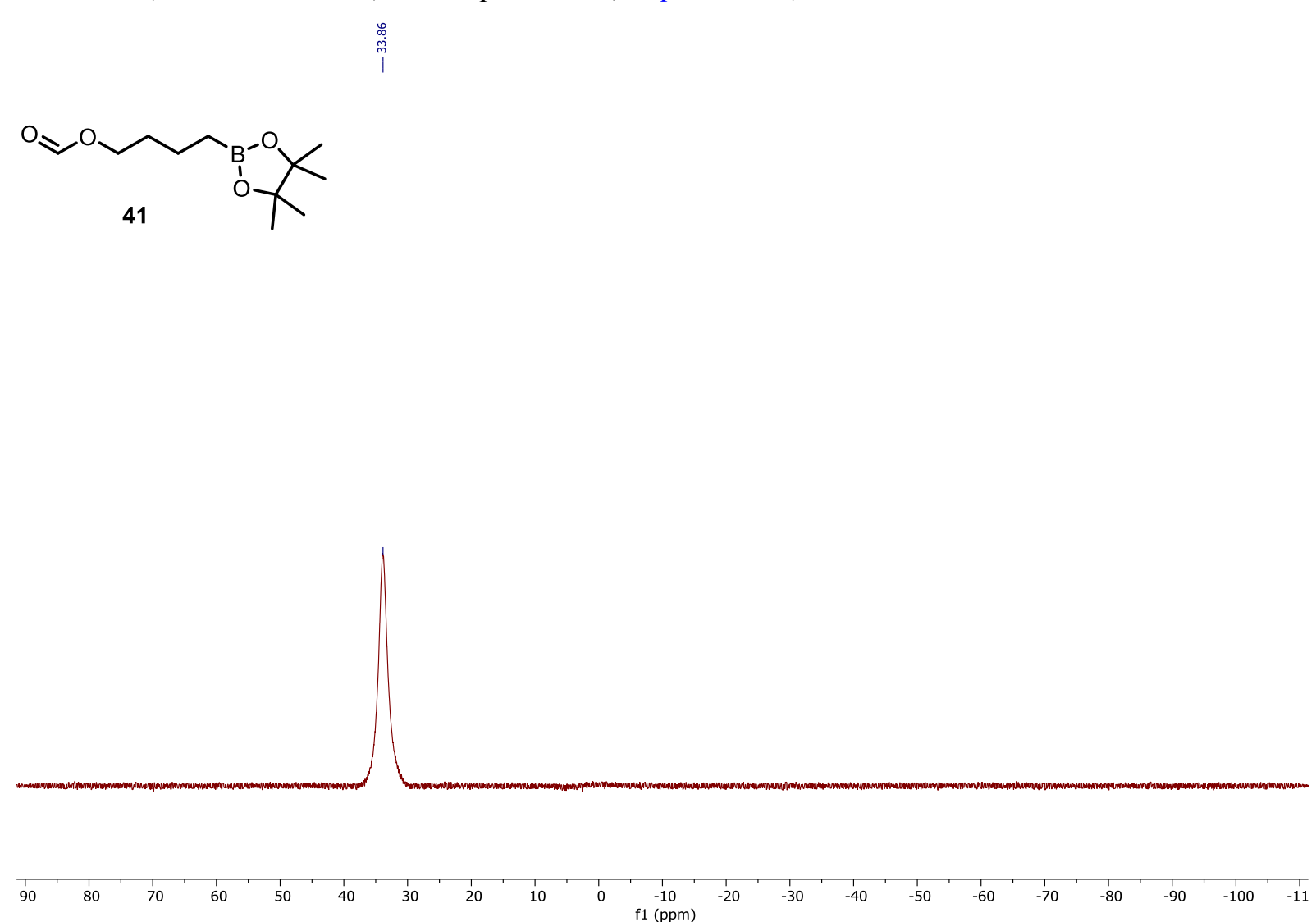
${ }^{1} \mathrm{H}$ NMR (400 MHz, $\mathrm{CDCl}_{3}$ ) of compound 42 (see procedure):

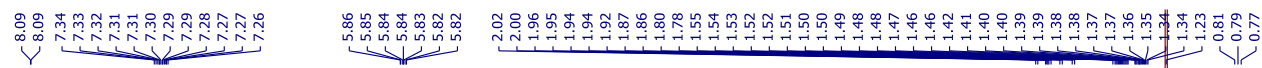

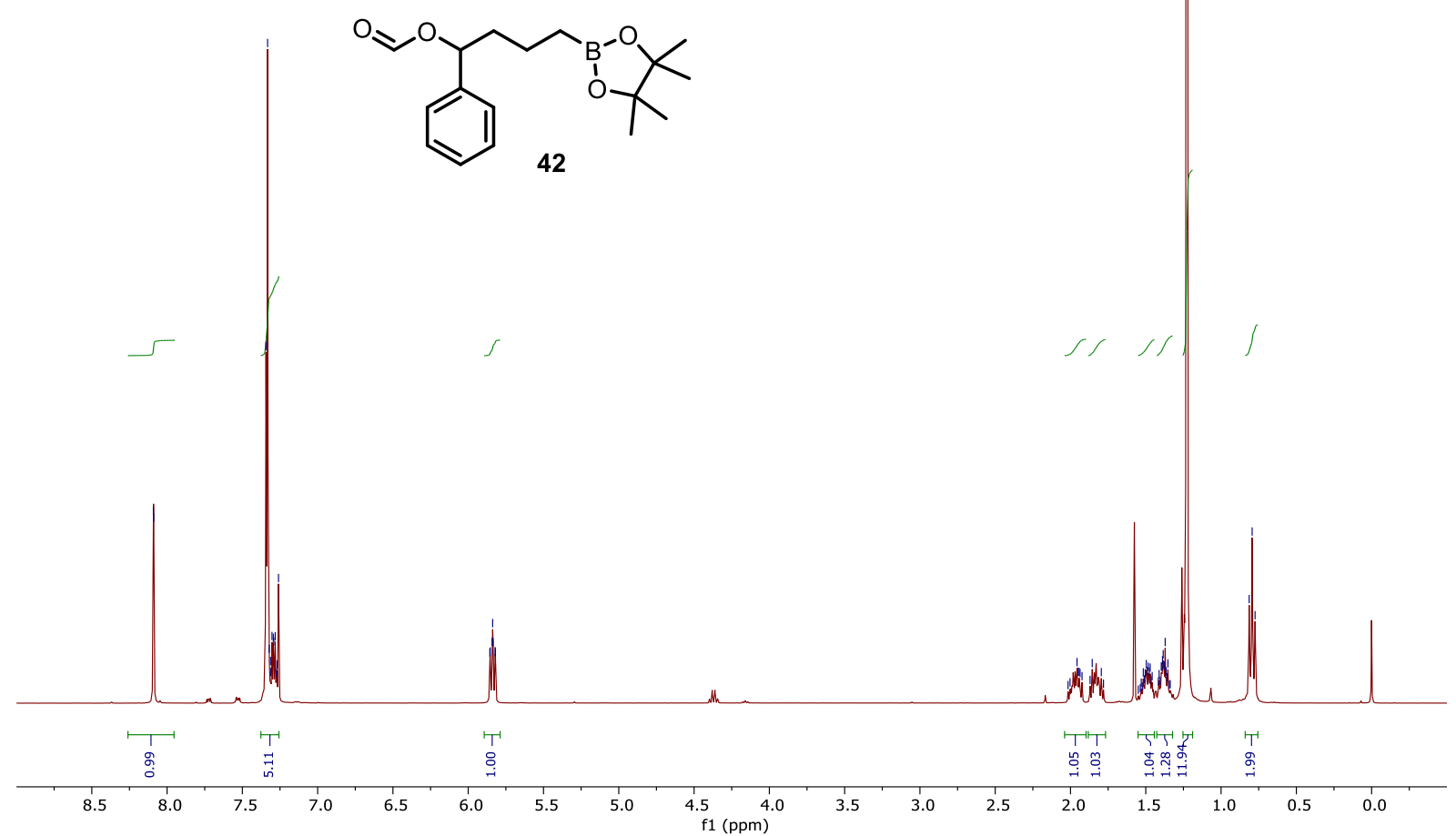

${ }^{13} \mathrm{C}$ NMR (101 MHz, $\mathrm{CDCl}_{3}$ ) of compound 42 (see procedure):<smiles>CC1(C)OB(CCCC(OC=O)c2ccccc2)OC1(C)C</smiles>

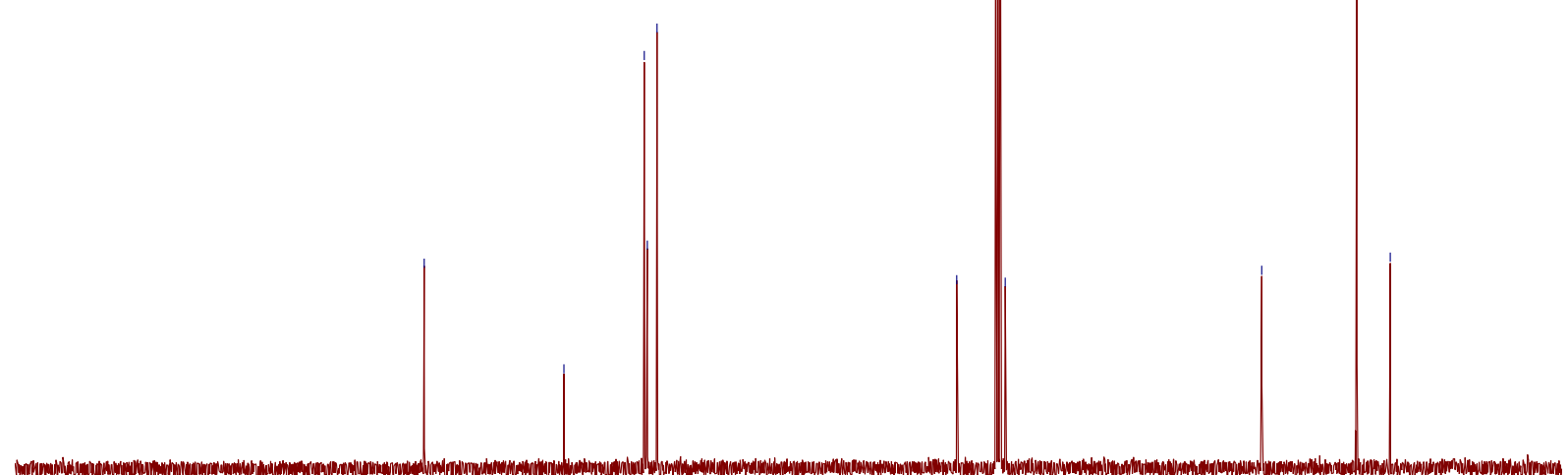

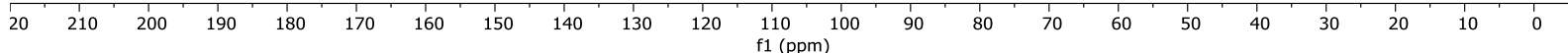


${ }^{11} \mathrm{~B}$ NMR (128 MHz, $\mathrm{CDCl}_{3}$ ) of compound 42 (see procedure):

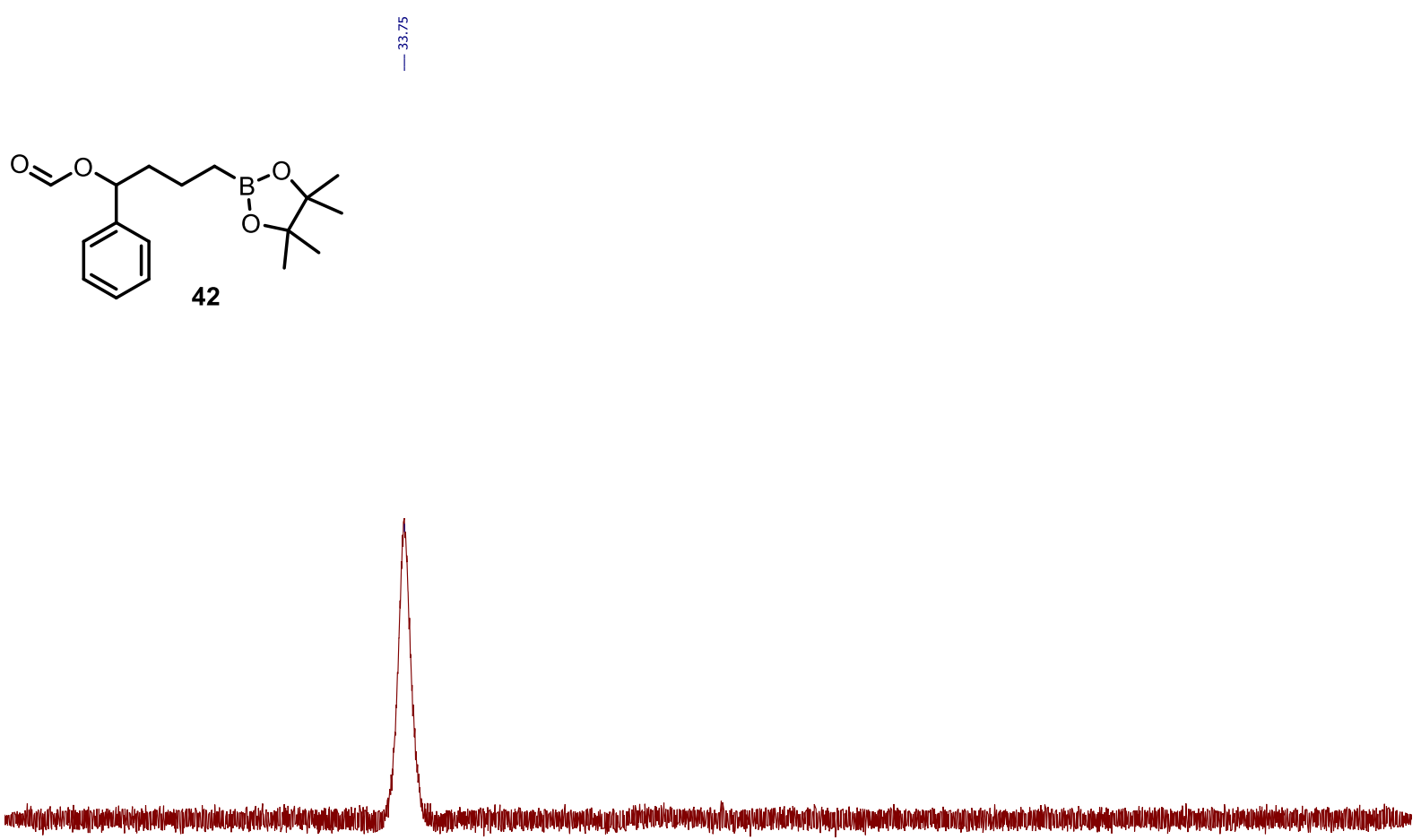

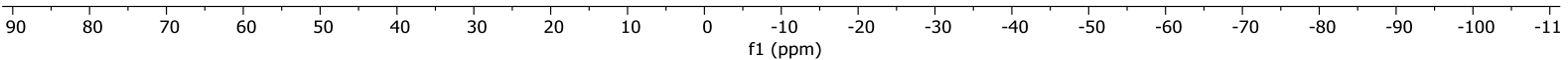


${ }^{1} \mathrm{H}$ NMR (400 MHz, $\mathrm{CDCl}_{3}$ ) of compound 43 (see procedure):

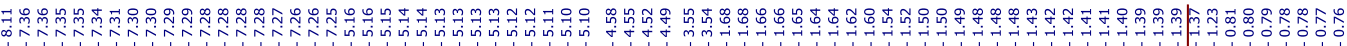<smiles>CC1(C)OB(CCCC(COCc2ccccc2)OC=O)OC1(C)C</smiles>

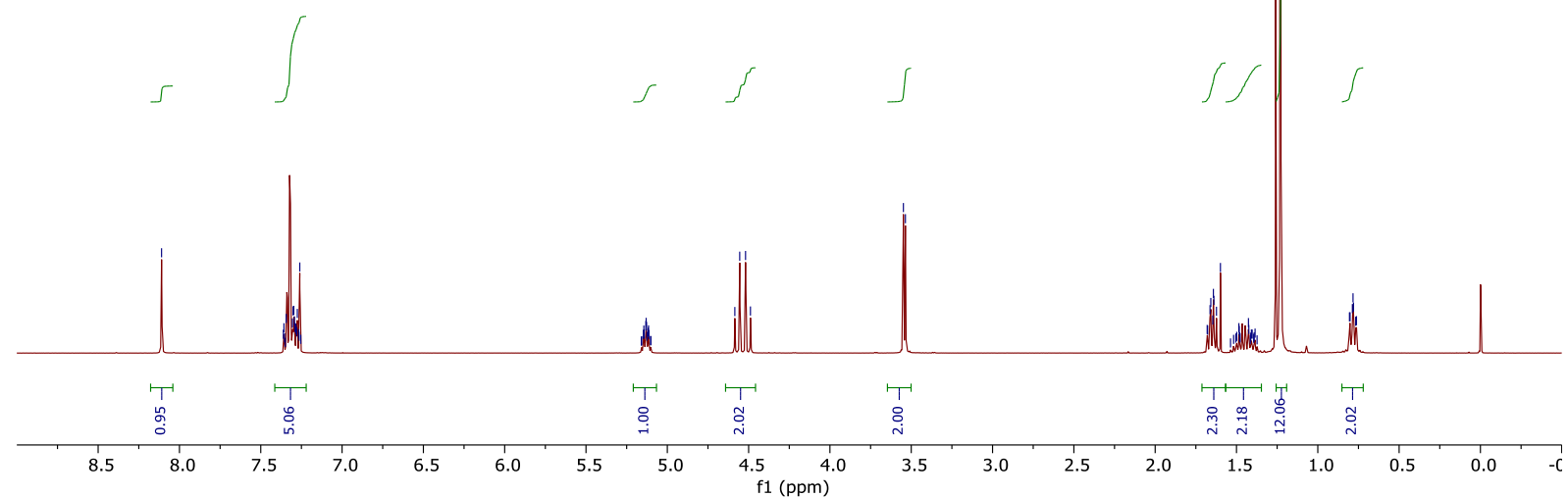

${ }^{13} \mathrm{C}$ NMR (101 MHz, $\mathrm{CDCl}_{3}$ ) of compound 43 (see procedure):

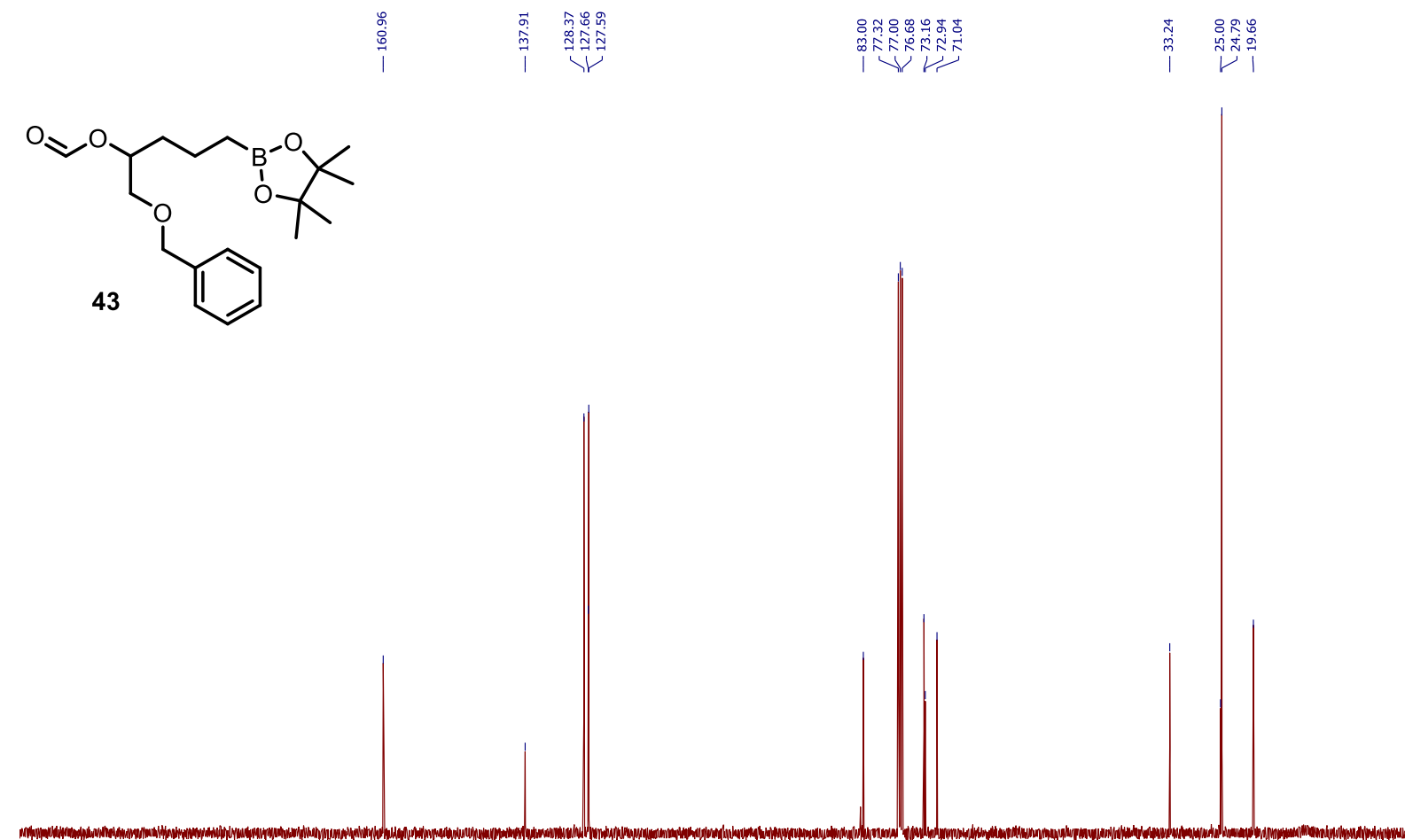

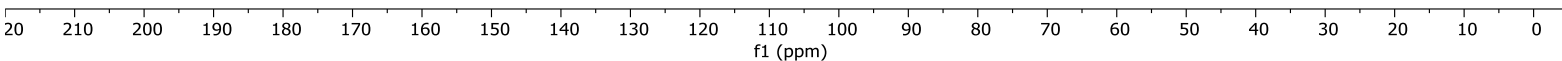


${ }^{11} \mathrm{~B}$ NMR (128 MHz, $\mathrm{CDCl}_{3}$ ) of compound 43 (see procedure):<smiles>CC1(C)OB(CCCC(COCc2ccccc2)OC=O)OC1(C)C</smiles>
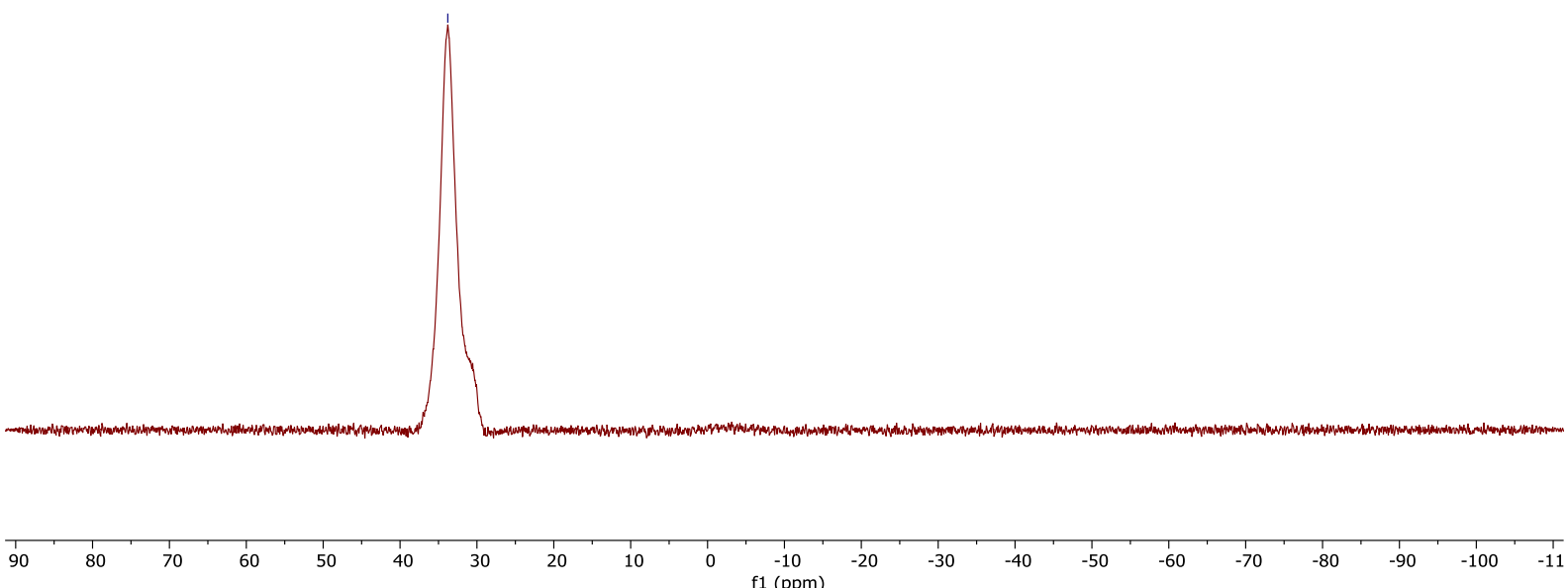
${ }^{1} \mathrm{H}$ NMR (400 MHz, $\mathrm{CDCl}_{3}$ ) of compound 44 (see procedure):

$\infty$
$\infty$
$\infty$
$\infty$

$\stackrel{\stackrel{2}{i}}{i}$

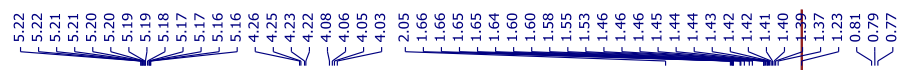

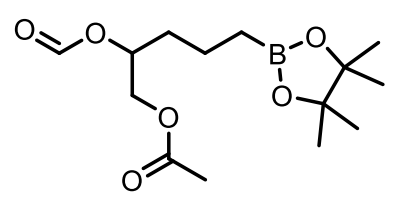

44

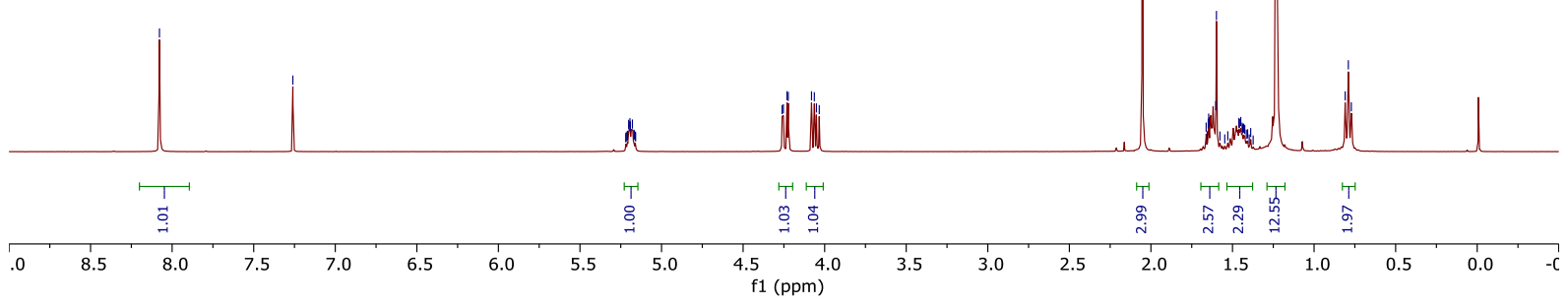

${ }^{13} \mathrm{C}$ NMR (101 MHz, $\mathrm{CDCl}_{3}$ ) of compound 44 (see procedure):

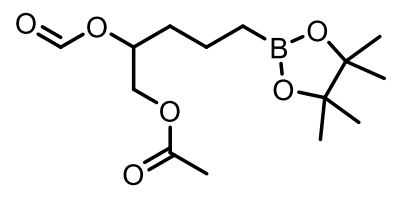

44

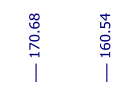

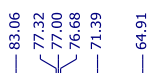

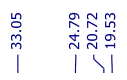
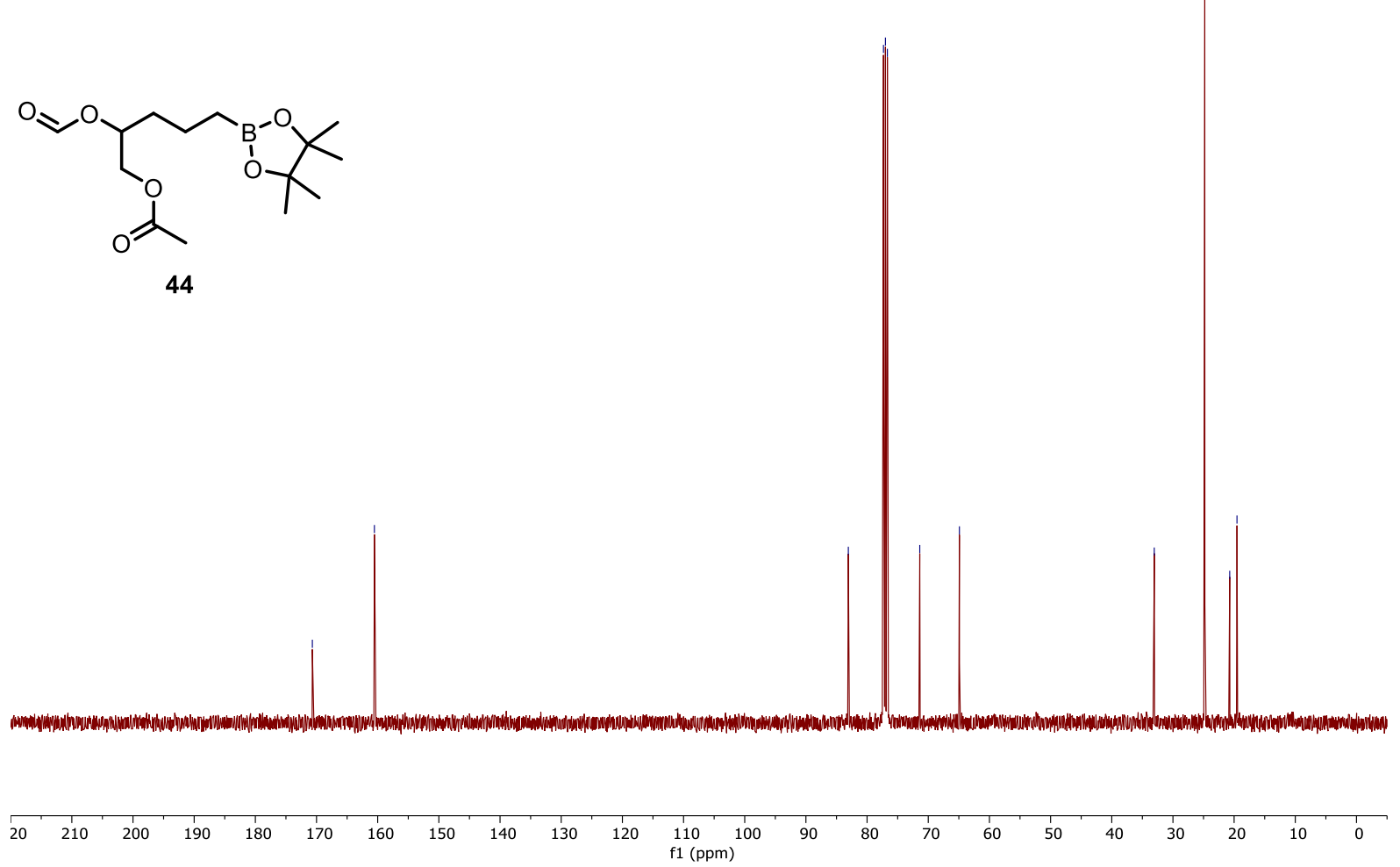

S218 
${ }^{11} \mathrm{~B}$ NMR (128 MHz, $\mathrm{CDCl}_{3}$ ) of compound 44 (see procedure):
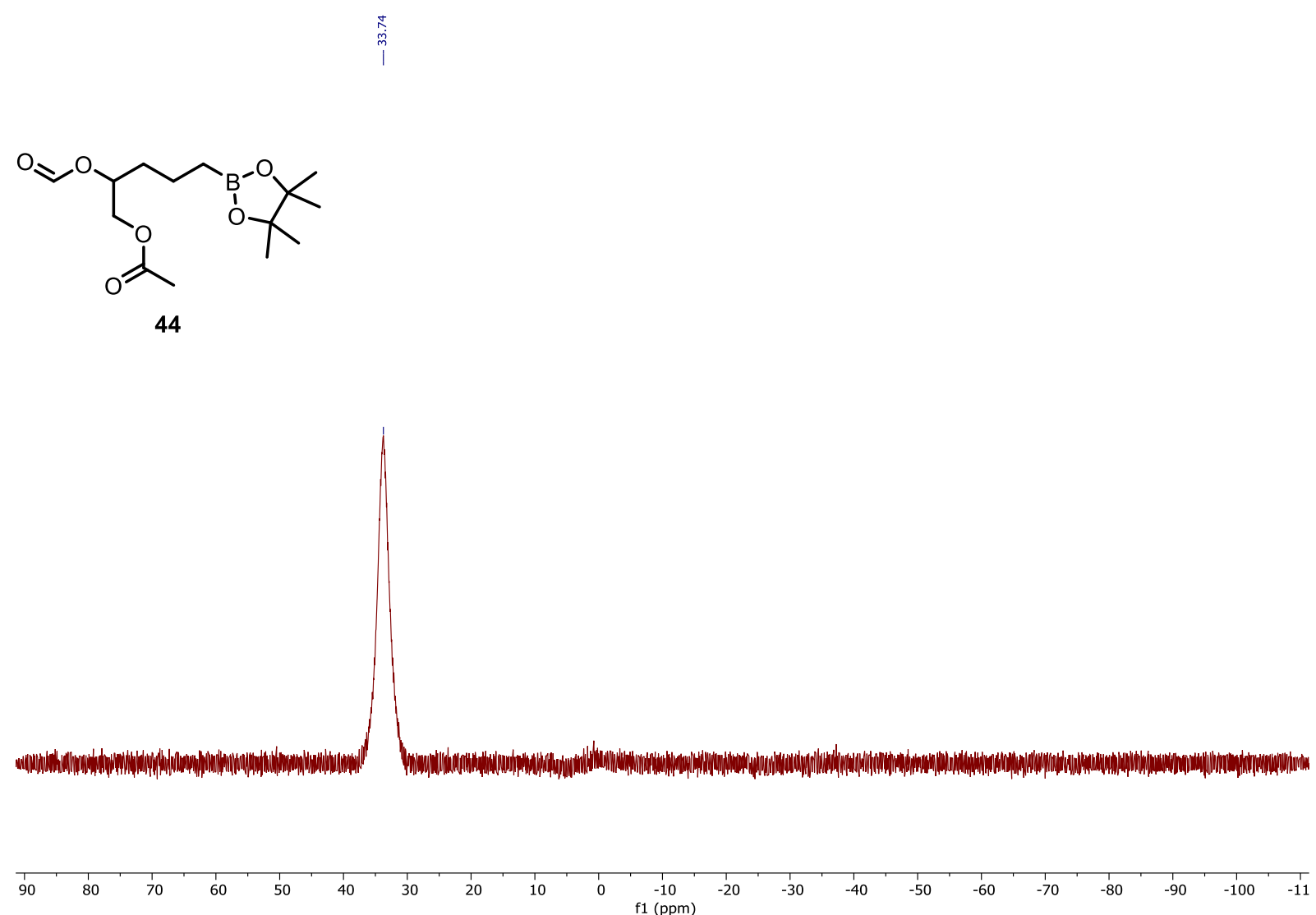
${ }^{1} \mathrm{H}$ NMR (400 MHz, $\mathrm{CDCl}_{3}$ ) of compound 45 (see procedure):

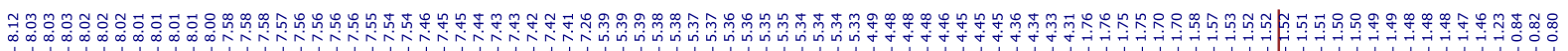<smiles>CC1(C)OB(CCCC(COC=O)OC(=O)c2ccccc2)OC1(C)C</smiles>

45

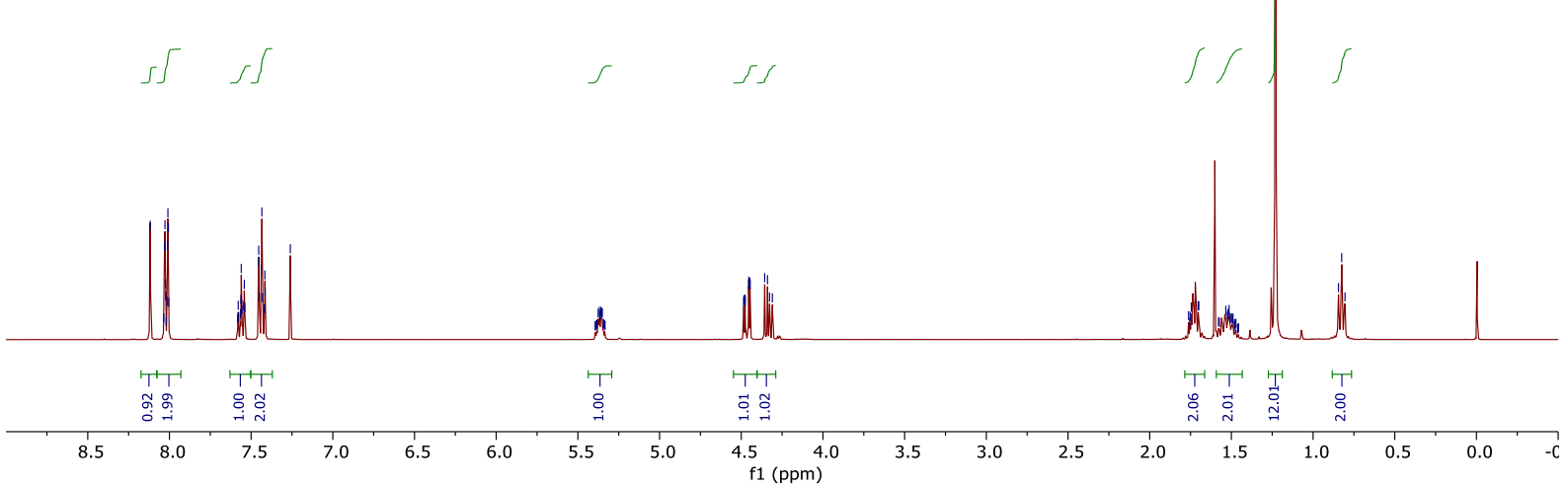

${ }^{13} \mathrm{C}$ NMR (101 MHz, $\mathrm{CDCl}_{3}$ ) of compound 45 (see procedure):

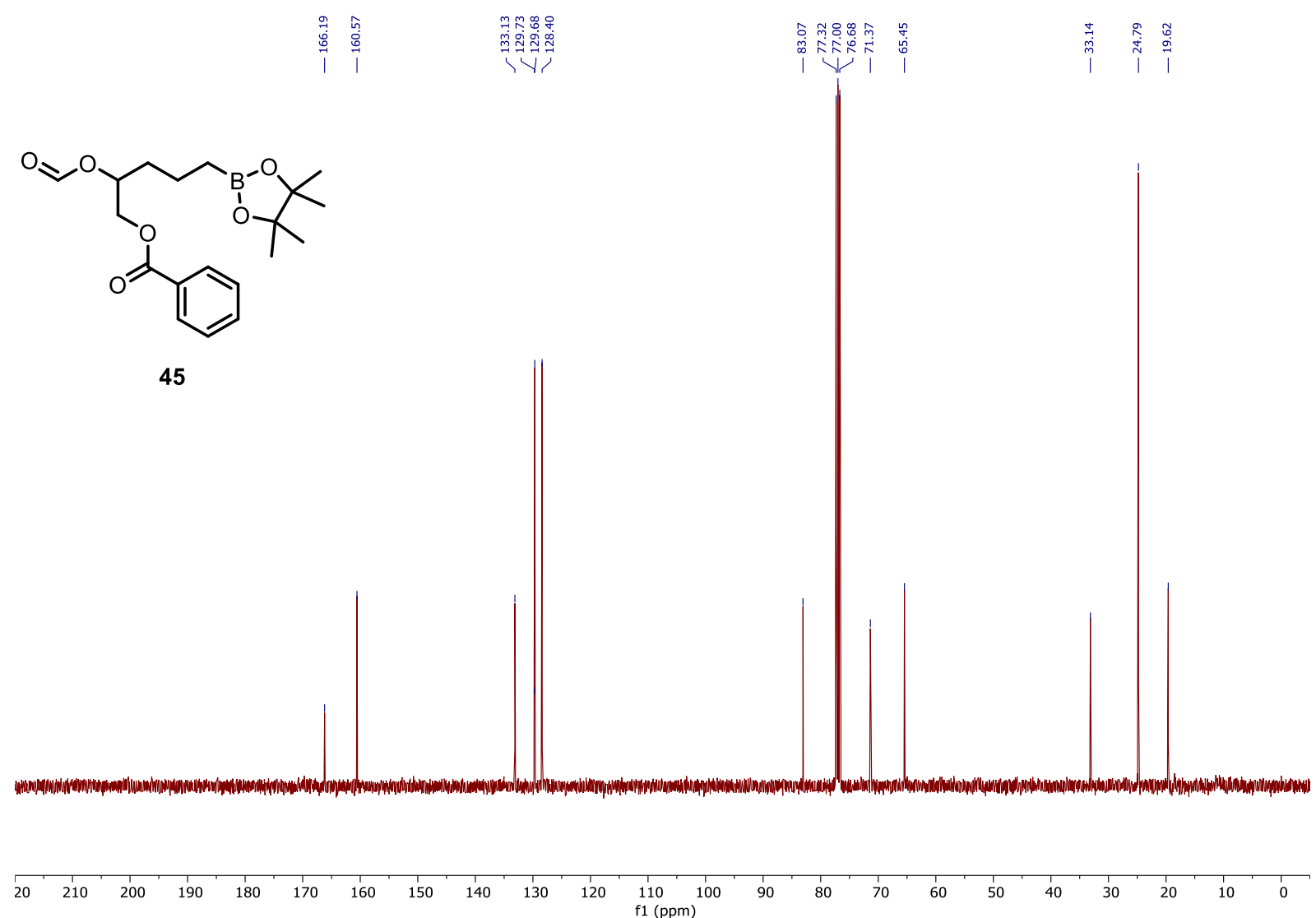


${ }^{11} \mathrm{~B}$ NMR (128 MHz, $\mathrm{CDCl}_{3}$ ) of compound 45 (see procedure):
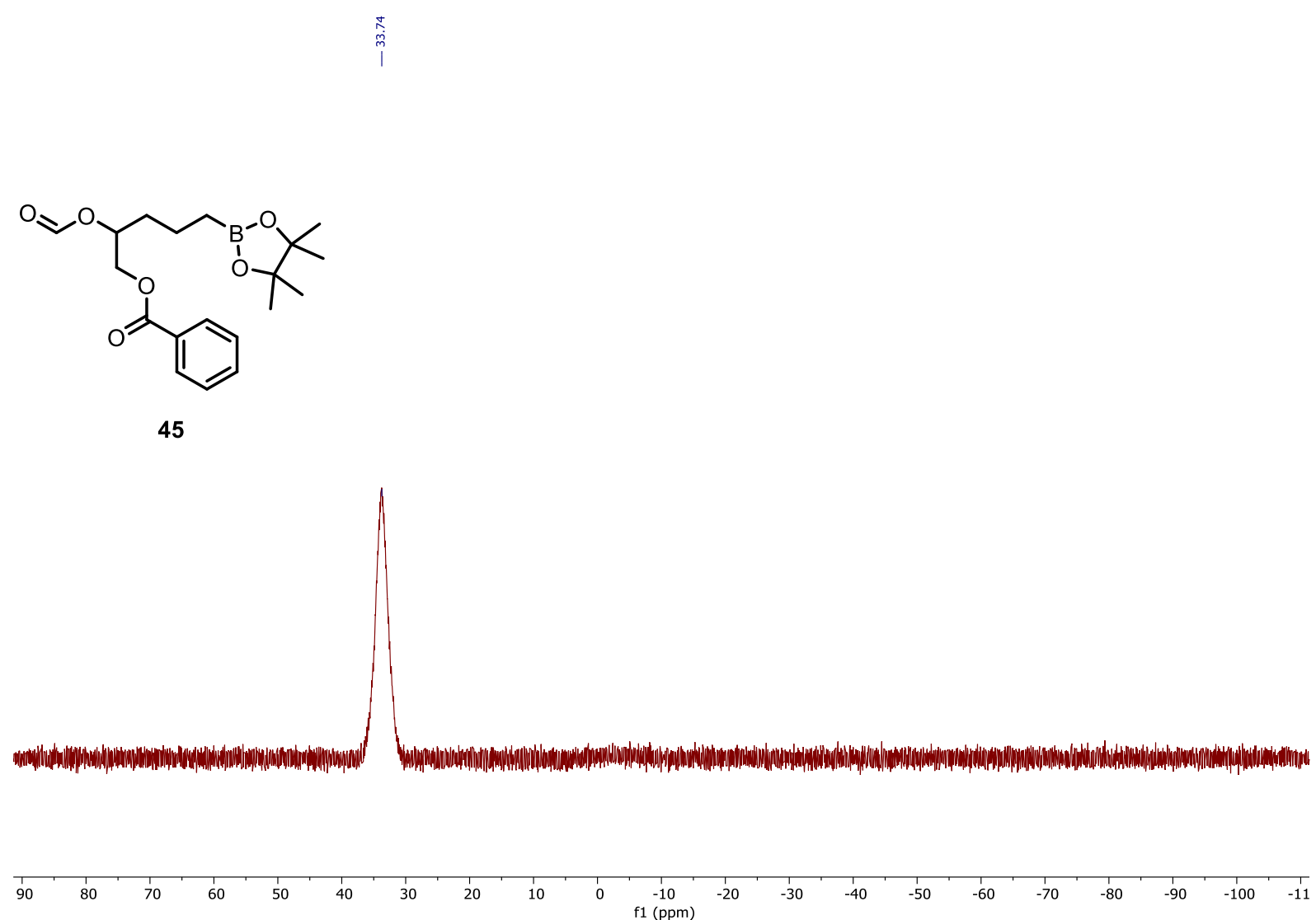
${ }^{1} \mathrm{H}$ NMR (400 MHz, $\mathrm{CDCl}_{3}$ ) of compound 46 (see procedure):

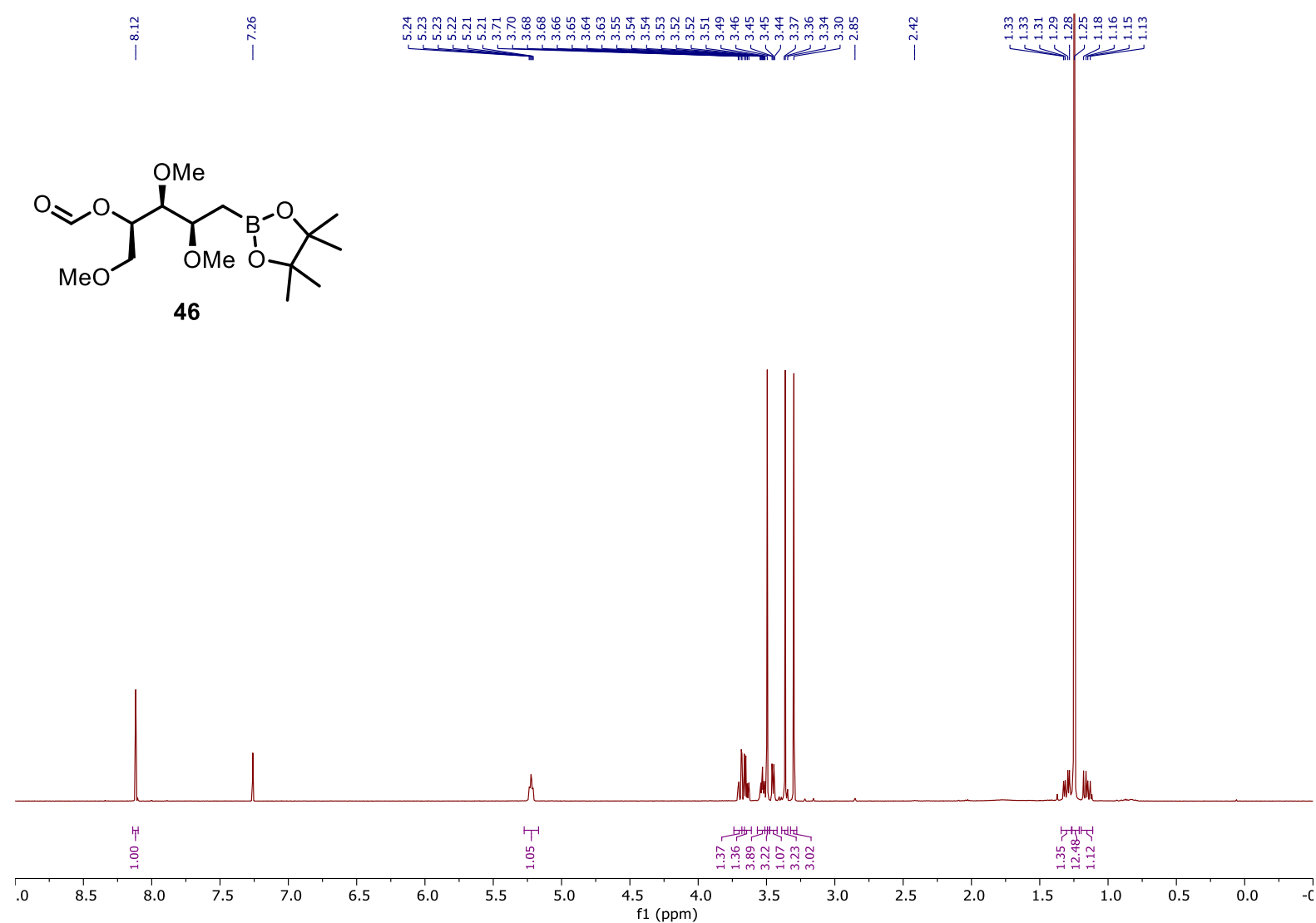

${ }^{13} \mathrm{C}$ NMR (101 MHz, $\mathrm{CDCl}_{3}$ ) of compound 46 (see procedure):

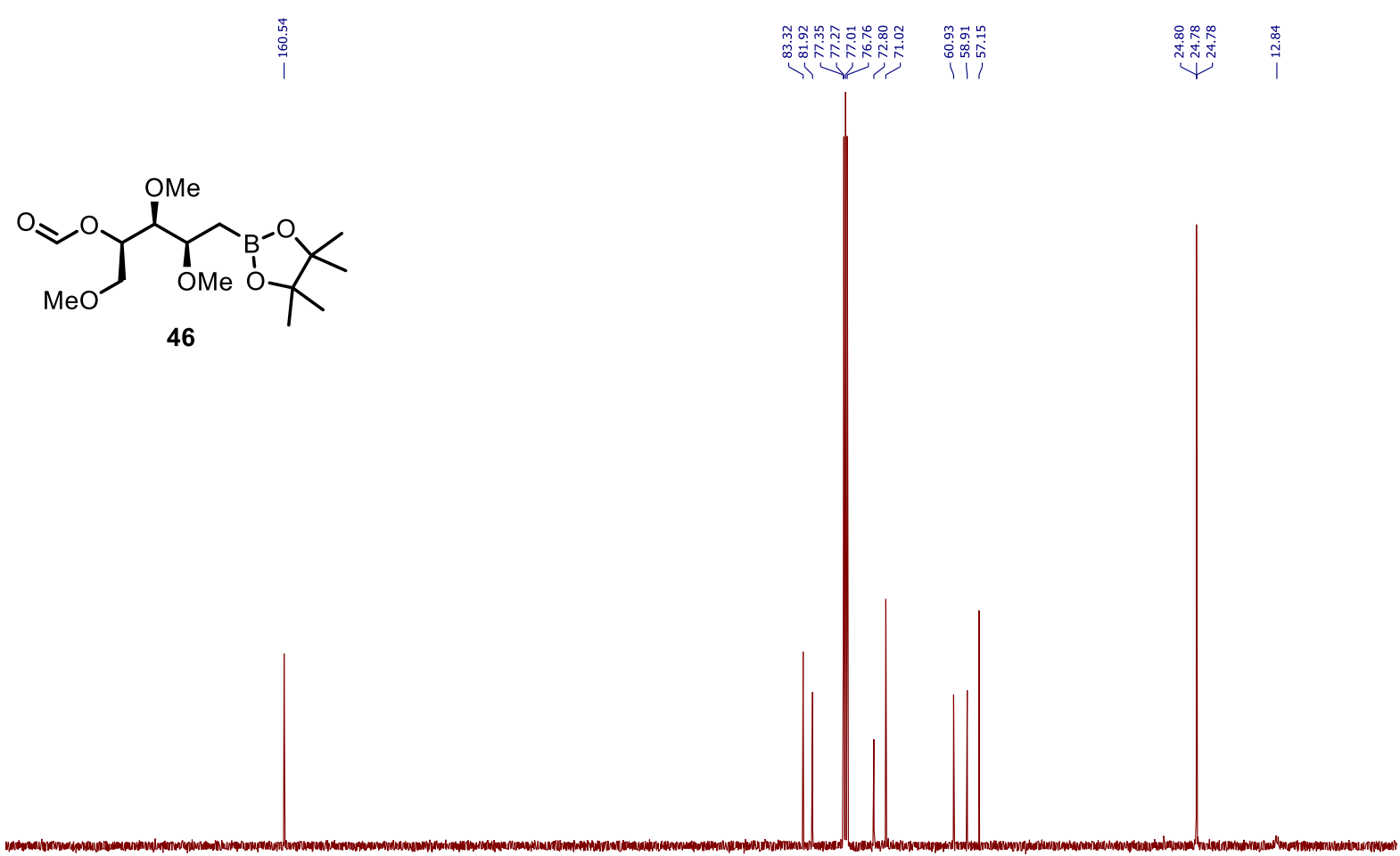

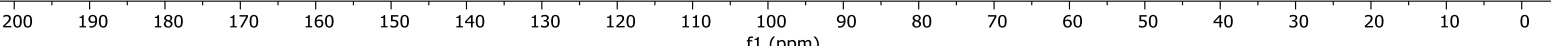


${ }^{1} \mathrm{H}$ NMR (400 MHz, $\mathrm{CDCl}_{3}$ ) of compound $\mathbf{6 b}$ (see procedure):

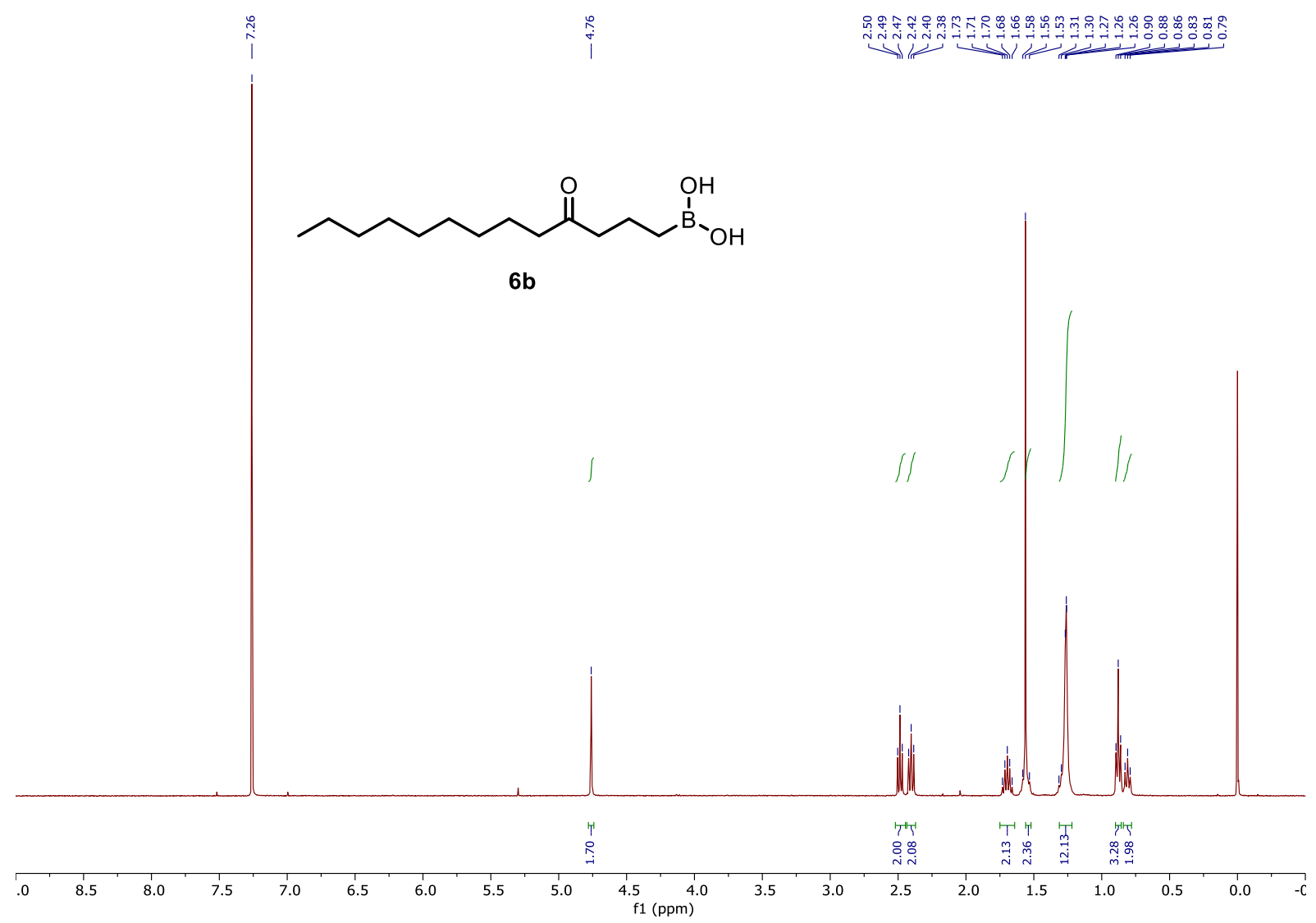

${ }^{13} \mathrm{C}$ NMR (101 MHz, $\left.\mathrm{CDCl}_{3}\right)$ of compound $\mathbf{6 b}$ (see procedure)

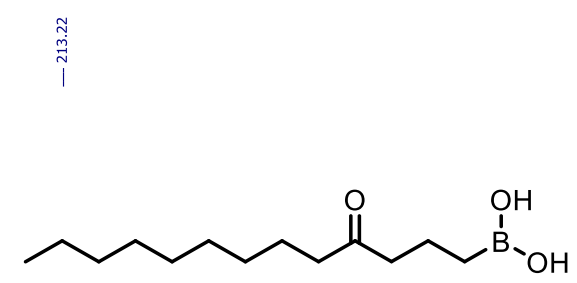

6b
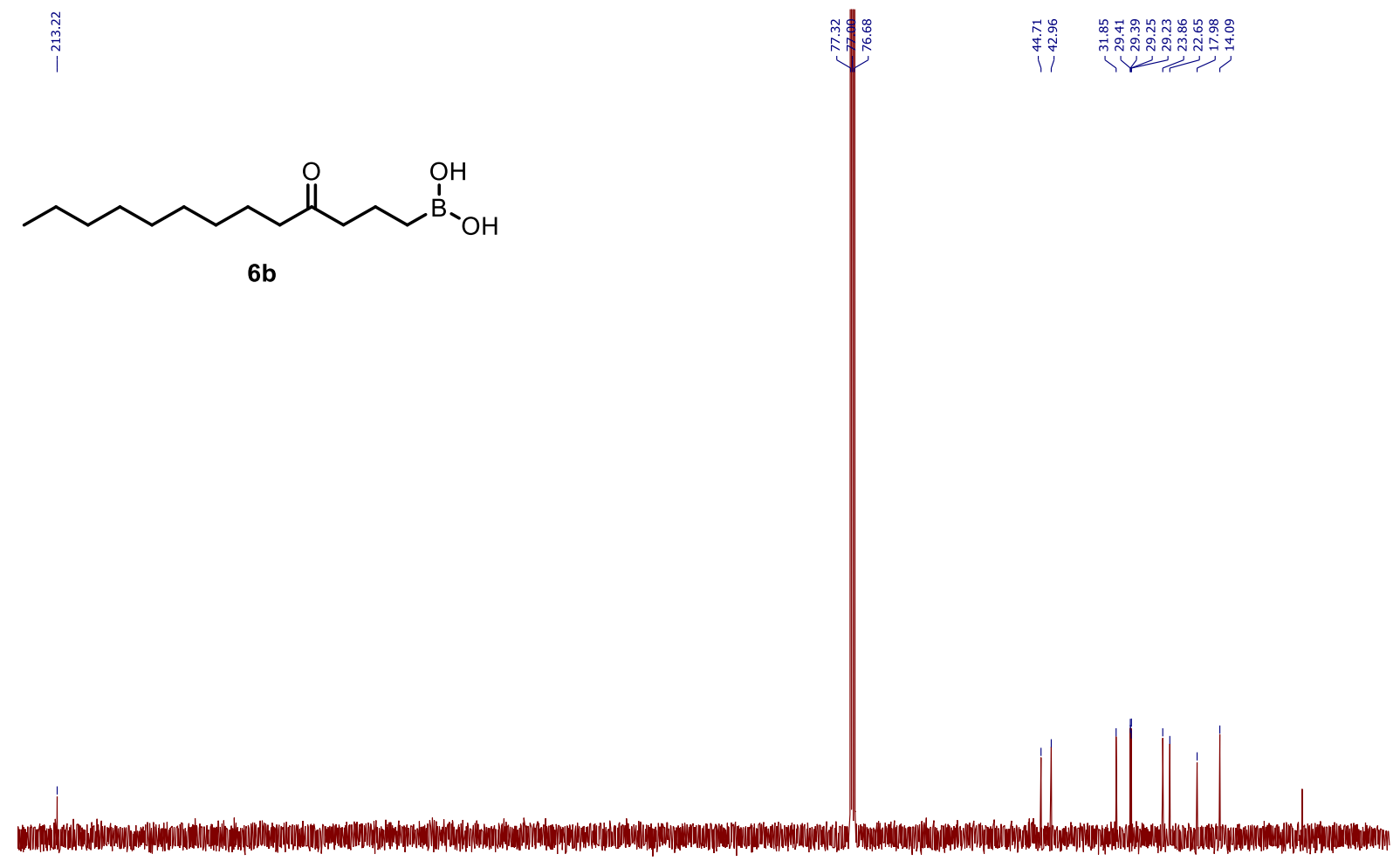

\begin{tabular}{rllllllllllllllllllllllll}
\hline 20 & 210 & 200 & 190 & 180 & 170 & 160 & 150 & 140 & 130 & 120 & 110 & 100 & 10 & 80 & 70 & 60 & 50 & 40 & 30 & 20 & 10 & 0 & -10
\end{tabular} 
${ }^{11} \mathrm{~B}$ NMR (128 MHz, $\mathrm{CDCl}_{3}$ ) of compound $\mathbf{6 b}$ (see procedure):

$\stackrel{\substack{\infty \\ \text { \} }}{\mid}$

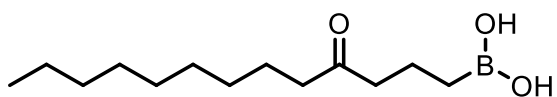

6b

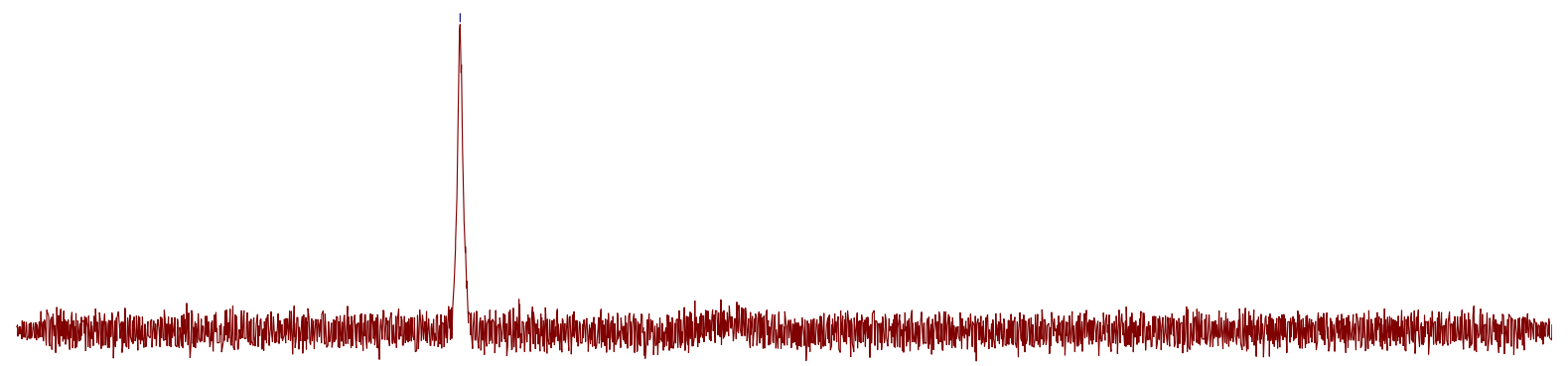

\begin{tabular}{lllllllllllllllllllll}
\hline 90 & 80 & 70 & 60 & 50 & 40 & 30 & 20 & 10 & 0 & $\begin{array}{c}1 \\
\mathrm{f} 1(0 \mathrm{pm})\end{array}$ & -20 & -30 & -40 & -50 & -60 & -70 & -80 & -90 & -100 & -11
\end{tabular} 
${ }^{1} \mathrm{H}$ NMR (400 MHz, $\mathrm{CDCl}_{3}$ ) of compound $\mathbf{6 c}$ (see procedure):

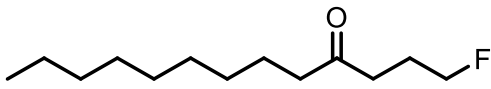

$6 c$
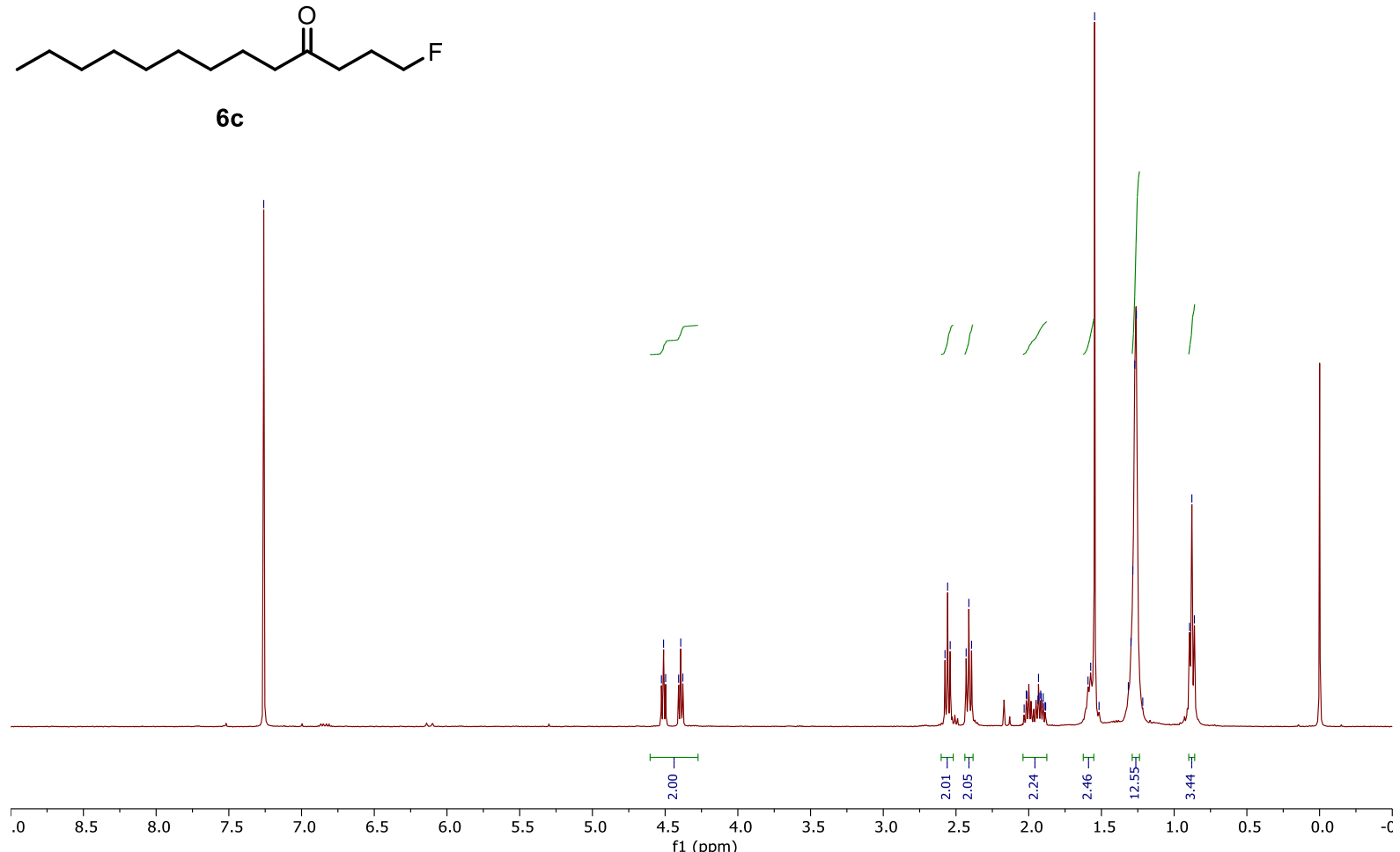

${ }^{13} \mathrm{C}$ NMR (101 MHz, $\mathrm{CDCl}_{3}$ ) of compound $\mathbf{6 c}$ (see procedure):

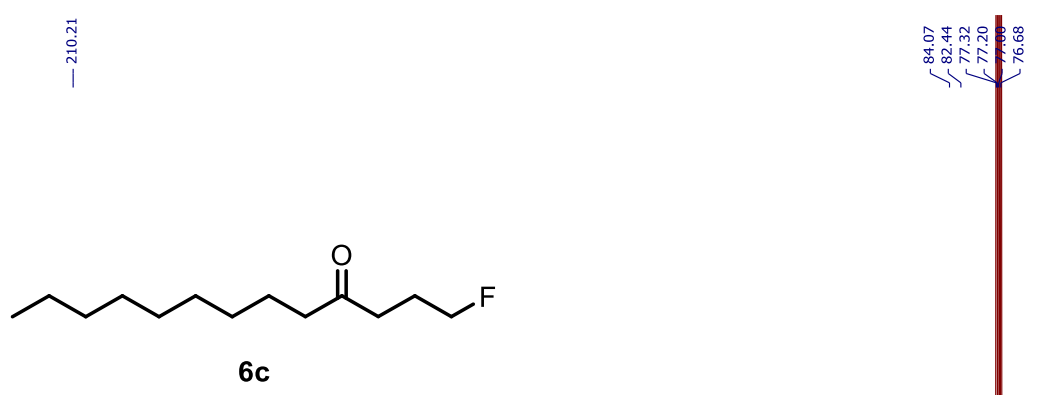

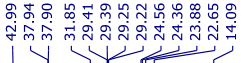

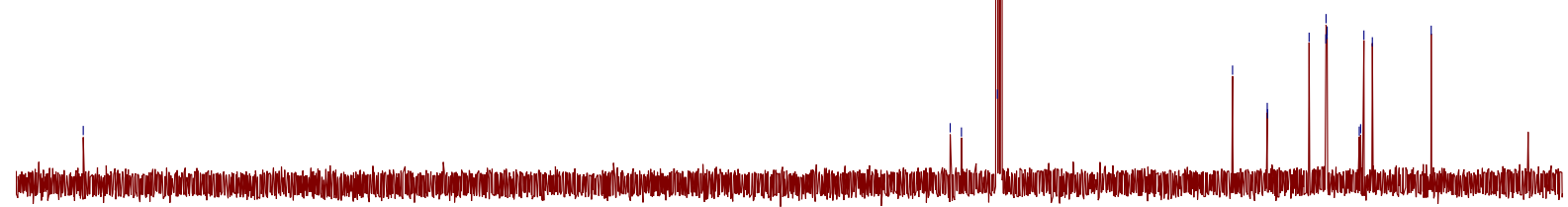

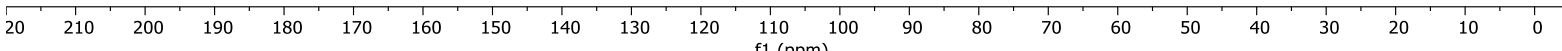


${ }^{19} \mathrm{~F}$ NMR (377 MHz, $\mathrm{CDCl}_{3}$ ) of compound $\mathbf{6 c}$ (see procedure)

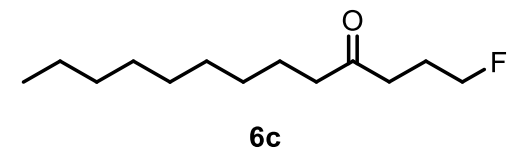

6c 
${ }^{1} \mathrm{H}$ NMR (400 MHz, $\mathrm{CDCl}_{3}$ ) of compound $\mathbf{6 d}$ (see procedure):

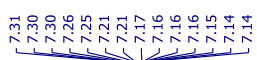

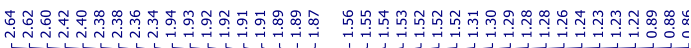

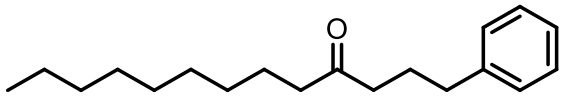

$6 d$

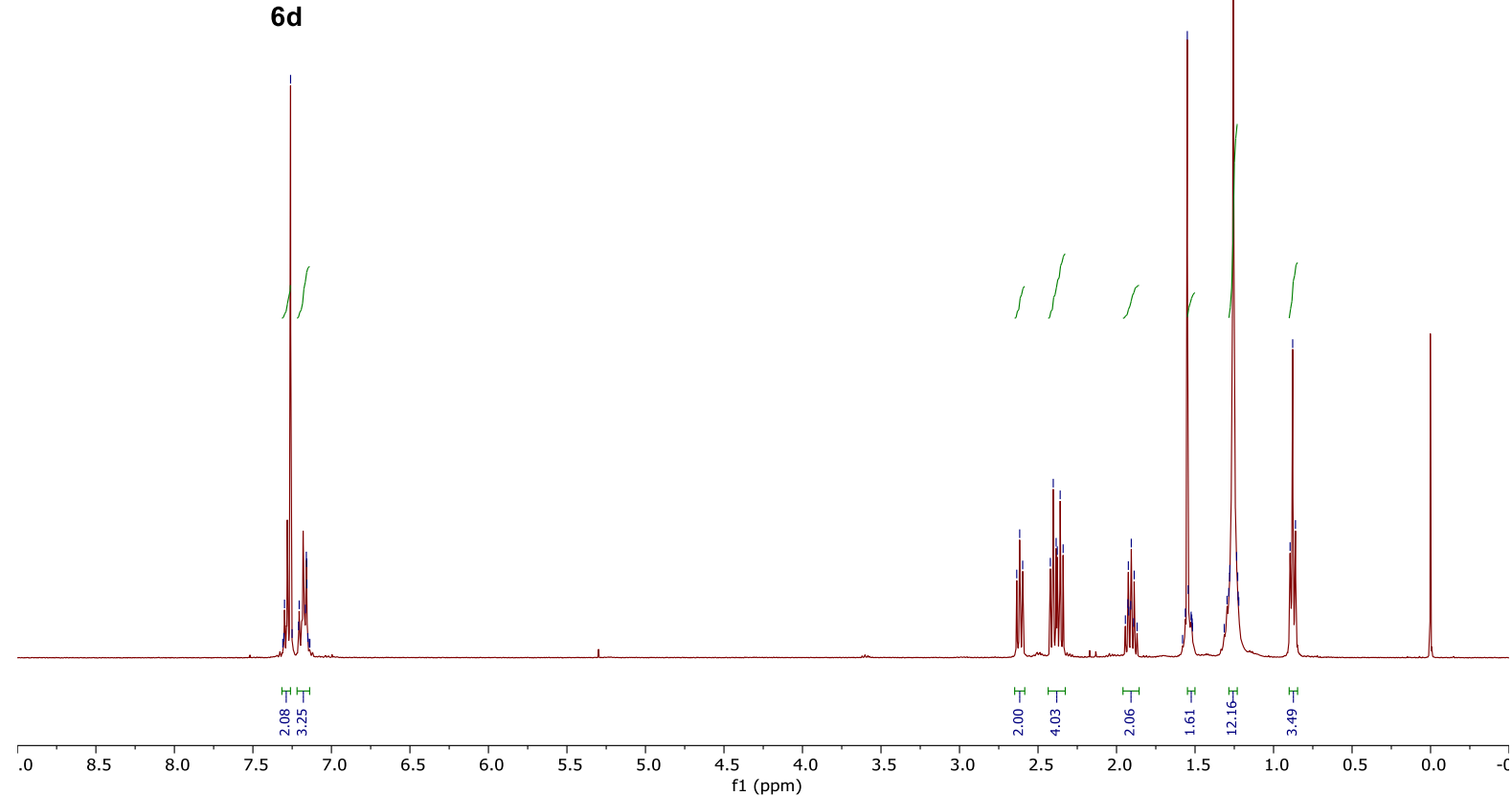

${ }^{13} \mathrm{C}$ NMR (101 MHz, $\mathrm{CDCl}_{3}$ ) of compound $\mathbf{6 d}$ (see procedure):
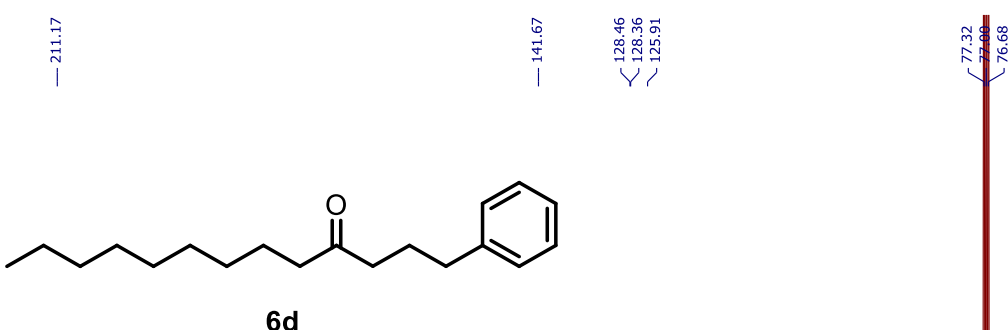

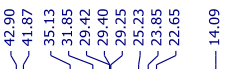

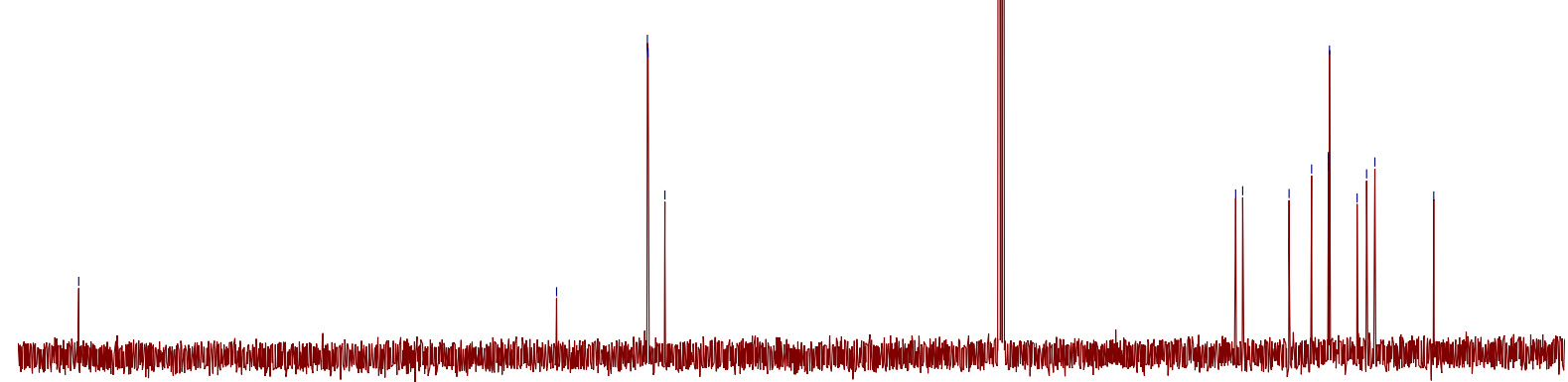

\begin{tabular}{lllllllllllllllllllllll}
\hline & 20 & 200 & 190 & 180 & 170 & 160 & 150 & 140 & 130 & 120 & 110 & 100 & 90 & 80 & 70 & 60 & 50 & 40 & 30 & 20 & 10 & 0
\end{tabular} 